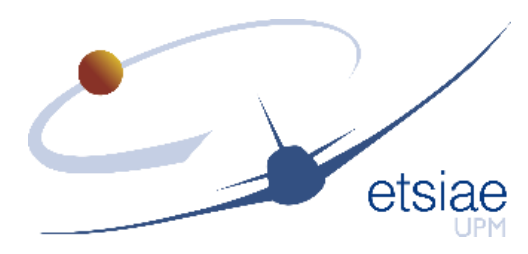

Universidad Politécnica de Madrid

Escuela Técnica Superior de Ingeniería Aeronáutica y del Espacio

\title{
ESTUDIO DE CONFIGURACIONES NO CONVENCIONALES DE LOS ESTABILIZADORES PARA AVIONES \\ DE TRANSPORTE AÉREO COMERCIAL
}

\author{
Tesis Doctoral
}

\author{
Alejandro Sánchez Carmona \\ Ingeniero Aeronáutico
}



Departamento de Aeronaves y Vehículos Espaciales

Escuela Técnica Superior de Ingeniería Aeronáutica y del Espacio

\section{ESTUDIO DE CONFIGURACIONES NO CONVENCIONALES DE LOS ESTABILIZADORES PARA AVIONES DE TRANSPORTE AÉREO COMERCIAL}

Alejandro Sánchez Carmona Ingeniero Aeronáutico

Cristina Cuerno Rejado Doctora en Ingeniería Aeronáutica 



\section{ÍNDICE}

RESUMEN.

$\mathrm{XV}$

ABSTRACT

$\mathrm{XVI}$

NOMENCLATURA

XVII

ACRÓNIMOS

XXVII

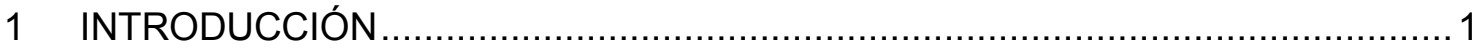

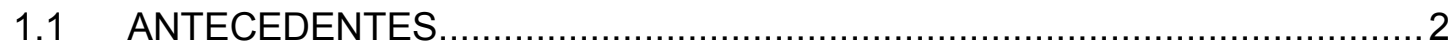

1.2 ESTADO DEL ARTE DE CONFIGURACIONES DE COLA NO

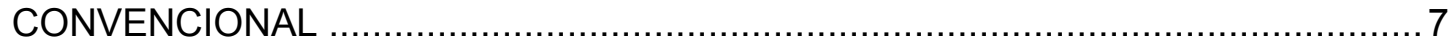

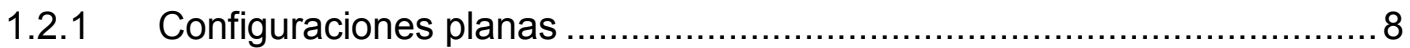

1.2.2 Configuraciones no planas ........................................................... 10

1.3 HERRAMIENTAS CLÁSICAS DE DISEÑO CONCEPTUAL Y PRELIMINAR17

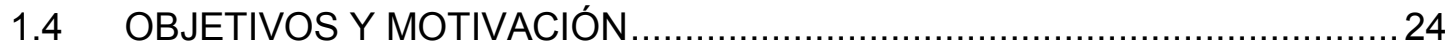

2 DISEÑO CONCEPTUAL DE LAS CONFIGURACIONES DE COLA

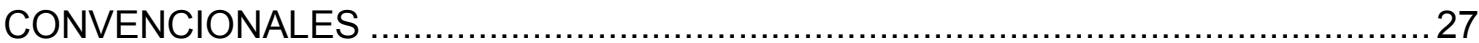

2.1 CARACTERIZACIÓN DE LAS CONFIGURACIONES DE COLA

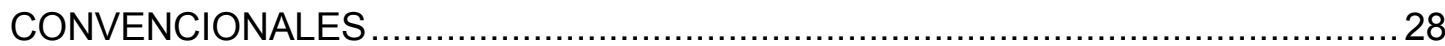

2.2 CORRELACIONES Y ANÁLISIS DE DEPENDENCIA .................................37

2.2.1 Parámetros generales ............................................................... 38

2.2.2 Fuselaje posterior ........................................................................ 43

2.2.3 Estabilizador horizontal ............................................................... 45

2.2.4 Estabilizador vertical ................................................................... 51

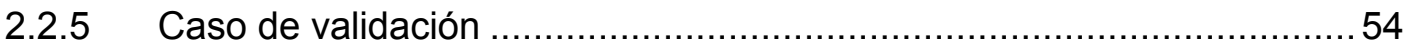

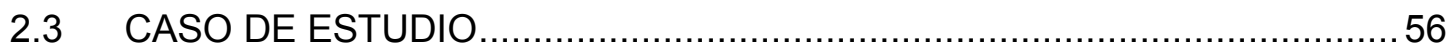

2.3.1 Base de datos CeRAS: CSR-01 .................................................... 56

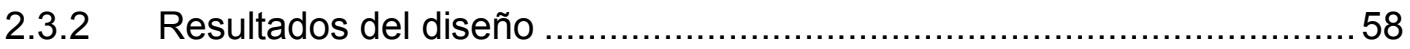

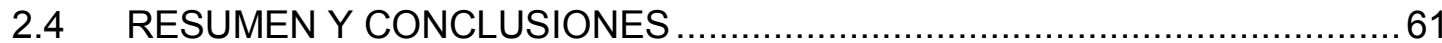

3 DISEÑO CONCEPTUAL DE CONFIGURACIONES DE ESTABILIZADORES DE COLA NO CONVENCIONALES

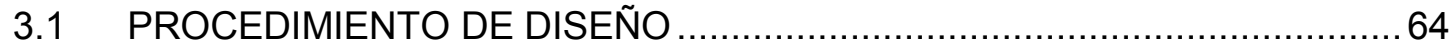

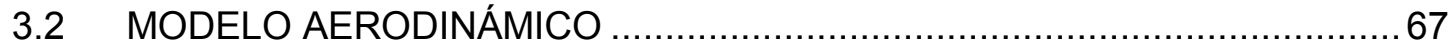

3.2.1 Derivadas de estabilidad en configuración convencional ......................67

3.2.2 Coeficientes de fuerzas en configuraciones no convencionales: el efecto del diedro.

3.2.3 Ecuaciones de momentos........................................................... 72

3.2.4 Comparación con otras metodologías................................................ 75 
3.2.5 Derivadas de estabilidad estática en crucero para configuraciones de cola

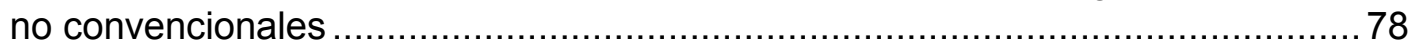

3.2.6 Cargas en la cola en maniobras simétricas .....................................78

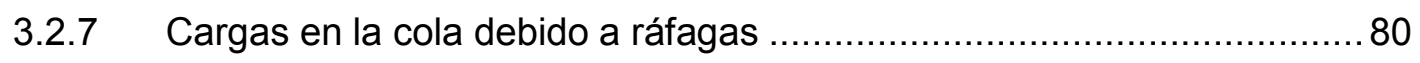

3.2.8 Cargas en la cola en maniobras asimétricas ................................... 81

3.3 MODELO ESTRUCTURAL Y DE PESOS .............................................. 83

3.3.1 Método basado en regresiones estadísticas .................................... 83

3.3.2 Método casi analítico en material metálico ......................................... 84

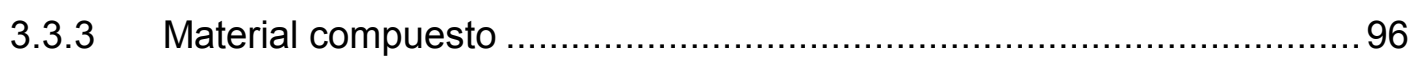

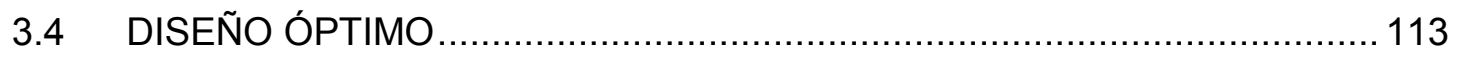

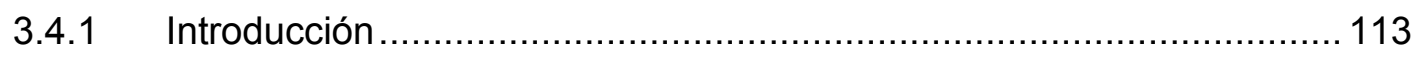

3.4.2 Planteamiento del problema matemático .......................................... 114

3.4.3 Métodos de ajuste: redes neuronales ............................................ 116

3.4.4 Algoritmos de optimización ........................................................ 122

3.4.5 Criterios de finalización de búsqueda ............................................ 125

3.4.6 Monotonía y actividad ......................................................... 125

3.5 RESUMEN Y CONCLUSIONES ..................................................... 127

4 RESULTADOS DEL DISEÑO DE CONFIGURACIONES NO CONVENCIONALES

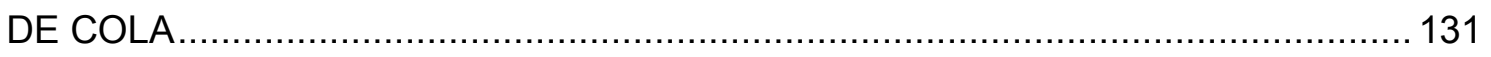

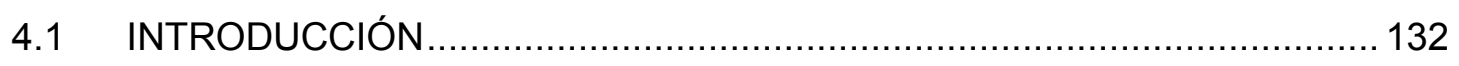

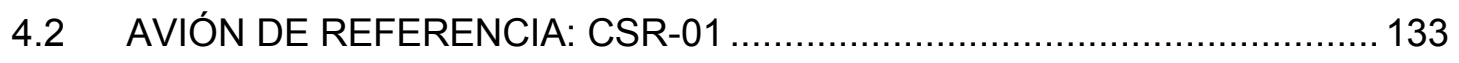

4.2.1 Ruta de diseño .................................................................. 138

4.2.2 Derivadas de estabilidad en crucero........................................... 139

4.2.3 Fallo del motor crítico en ascenso .................................................. 141

4.2.4 Aterrizaje con viento cruzado..................................................... 142

4.2.5 Estimación de peso basado en regresiones estadísiticas de los estabilizadores.................................................................................. 143

4.2.6 Estimación de peso basado en métodos casi analíticos de los

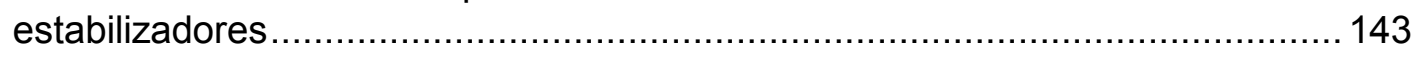

4.3 DIMENSIONADO DE UNA CONFIGURACIÓN DE COLA EN V.................. 154

4.3.1 Parámetros de diseño.......................................................... 154

4.3.2 Restricciones del espacio de diseño ............................................. 156

4.3.3 Función objetivo: el peso .......................................................... 172

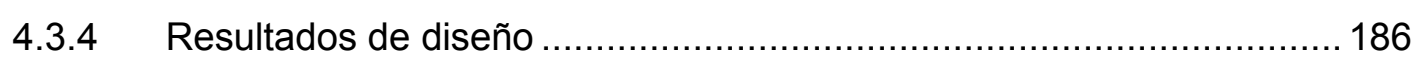

4.4 APLICACIÓN A OTRAS CONFIGURACIONES NO CONVENCIONALES 194

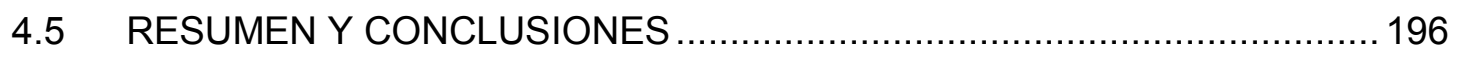

5 CONCLUSIONES Y TRABAJOS FUTUROS ......................................... 199 
5.1 CARACTERIZACIÓN DE CONFIGURACIONES DE COLA

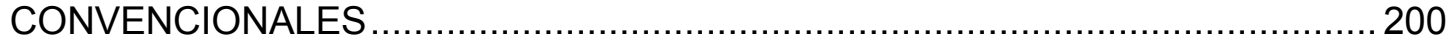

5.2 METODOLOGÍA DE DISEÑO DE CONFIGURACIONES DE COLA NO CONVENCIONALES

5.3 RESULTADOS PARA LA COLA EN V. 203

5.4 FUTUROS TRABAJOS 205

6 REFERENCIAS

.... i 


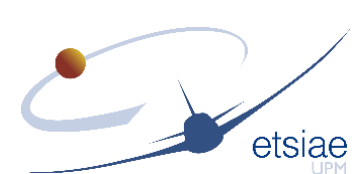

\section{ÍNDICE DE FIGURAS}

Figura 1-1 Imagen del X-48B, avión de configuración blended wing body desarrollado por la NASA para investigación. Fuente: (Thomas 2009) ......................................... 3

Figura 1-2 Definición de clasificación de las configuraciones más habituales. Fuente: (Torenbeek 1982).

Figura 1-3 Recopilación de diferentes configuraciones de cola. Fuente: (Raymer 2012).

Figura 1-4 Ejemplos de configuraciones no planas: (a) superficies verticales múltiples, Fuente: (Gall 2010a), (b) superficies cerradas, Fuente: (Cazals 2009a) y (c) diedro variable, Fuente: (Cazals 2009b). 7

Figura 1-5 Comparativa de un perfil NACA 64010 sin timón con morphing (arriba) con el timón con morphing (abajo). Fuente: (Castillo-Acero, Cuerno-Rejado y Gómez-Tierno 2014).

Figura 1-6 Diseño conceptual de un actuador sweeping jet. Fuente: (Seele et al. 2012).

Figura 1-7 Spoilers sobre un estabilizador horizontal para eliminar el timón de

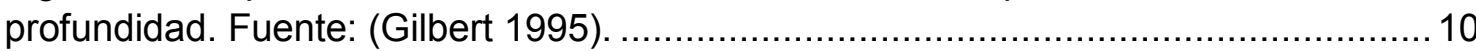

Figura 1-8 Configuraciones de cola no planas abiertas en patentes. Fuente: (a) (Cazals y Sagne 2010) y (b) (Gall 2010b).

Figura 1-9 Configuración box wing propuesta por la NASA y Lockheed Martin's. Fuente: (Gipson 2015).

Figura 1-10 Configuración box wing propuesta por la NASA y Lockheed Martin's. Fuente: (NASA 2011)

Figura 1-11 Configuración de cola en anillo de un avión de fabricación casera. Fuente: («Ring Tail debuts» 2011).

Figura 1-12 Ejemplos de configuraciones de cola cerradas. Fuente: (a) (Cazals y Druot 2012), (b) (Cazals 2009c) y (c) (Cazals 2009d).

Figura 1-13 Ejemplo de configuración de cola con dispositivos de punta de ala. Fuente: (Cazals 2009b).

Figura 2-1 Definición del ángulo de guarda (TSA) y del ángulo de upsweep (UA), parámetros característicos del fuselaje posterior. Fuente: elaboración propia. ............31

Figura 2-2 Función de densidad de probabilidad F de Fisher-Snedecor para grados de libertad 3 y 50 , donde el área a la derecha de la azul es la probabilidad de que la regresión no sea extrapolable a la población. Fuente: elaboración propia. 36

Figura 2-3 Clasificación de las aeronaves de acuerdo con el segmento de vuelo. Fuente: elaboración propia.

Figura 2-4 Correlaciones lineales que relacionan el peso máximo de despegue con la superficie alar del avión de acuerdo con la configuración de cola. Fuente: elaboración propia.

Figura 2-5 Correlaciones que relacionan el peso máximo de despegue con la superficie alar del avión de acuerdo con el segmento de vuelo. Fuente: elaboración propia.......40 


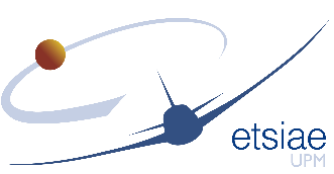

Figura 2-6 Correlaciones que relacionan el peso máximo de despegue con la superficie alar del avión de acuerdo con el número de motores del avión. Fuente: elaboración propia. 40

Figura 2-7 Correlaciones que relacionan el parámetro de volumen global del avión con el peso máximo de despegue de acuerdo con la configuración de cola. Fuente: elaboración propia.

Figura 2-8 Correlaciones que relacionan el parámetro de volumen global del avión con el peso máximo de despegue de acuerdo con el número de motores del avión. Fuente: elaboración propia.

Figura 2-9 Correlaciones que relacionan el parámetro de volumen global del avión con el peso máximo de despegue de acuerdo con si llevan (Sí) o no (NO) motores en el cono de cola. Fuente: elaboración propia.

Figura 2-10 Correlaciones que relacionan la superficie del estabilizador horizontal y la superficie alar de acuerdo con el número de motores. Fuente: elaboración propia. ....46

Figura 2-11 Correlaciones que relacionan la superficie del estabilizador horizontal y la superficie alar de acuerdo con si llevan (Sí) o no (NO) motores en el cono de cola. Fuente: elaboración propia. 46

Figura 2-12 Correlaciones que relacionan el brazo de momentos del estabilizador horizontal y la longitud del fuselaje de acuerdo con si llevan (Sí) o no (NO) motores en el cono de cola. Fuente: elaboración propia. 47

Figura 2-13 Correlaciones que relacionan el parámetro de resistencia inducida y el peso máximo de despegue en función del fabricante. Fuente: elaboración propia. 48

Figura 2-14 Correlaciones que relacionan el parámetro de resistencia inducida y el peso máximo de despegue en función del segmento de vuelo. Fuente: elaboración propia.48

Figura 2-15 Correlaciones que relacionan las superficies del estabilizador vertical y la alar en función del número de motores instalados. Fuente: elaboración propia. 51

Figura 2-16 Correlaciones que relacionan el producto de la superficie del estabilizador vertical por su brazo de momentos y el producto del empuje de un motor en despegue por la distancia al plano de simetría del avión del motor crítico en función del número de motores instalados. Fuente: elaboración propia.

Figura 2-17 Tres vistas del CSR-01. Fuente: (ILR y RWTH-Aechen-University 2014).57

Figura 3-1 Valores de K para determinar la pendiente de la curva de coeficiente de fuerza lateral en función del ángulo de resbalamiento. Fuente: (Purser y Campbell 1945) ....69

Figura 3-2 Comparación de los coeficientes de fuerza longitudinal $C L \alpha$ y lateral $C Y \beta$ entre Tornado y un método semi-empírico. Fuente: elaboración propia.

Figura 3-3 Comparativa entre los métodos semi-empíricos de Torenbeek y Roskam con el software Tornado y la combinación propuesta de estudio entre Tornado y Roskam para el cálculo del coeficiente de sustentación para un rango de deflexiones del control de borde de salida. Fuente: elaboración propia.

Figura 3-4 Esquema de fuerzas actuando en el avión de cola no convencional cuando vuela con ángulo de balance, tomando el ejemplo de configuración de cola en V. Fuente: elaboración propia. 


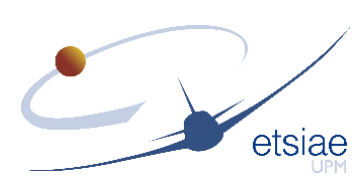

Figura 3-5 Comparación de métodos para el cálculo del coeficiente de sustentación y de momento de cabeceo entre Tornado, Torenbeek y el método propuesto en vuelo de crucero a Mach 0,6, (a), y en segundo segmento, (b). Fuente: elaboración propia. ....77

Figura 3-6 Composición de los elementos de la estructura de un ala de un avión. Fuente: (Torenbeek 2013). 84

Figura 3-7 Dimensiones del cajón de torsión. Fuente: elaboración propia. .87

Figura 3-8 Sección de un elemento del panel de extradós y definición de los parámetros geométricos. Fuente: elaboración propia.

Figura 3-9 Sección de un elemento del panel de extradós. Fuente: (Farrar 1949)......89

Figura 3-10 Curvas de diseño para secciones de larguerillos en $Z$ para distintos valores del factor de Farrar. Fuente: (Farrar 1949)

Figura 3-11 Curvas para determinar los factores $k s s, R h$ y $R d$ para estimar el esfuerzo crítico de pandeo elástico. Fuente: (Kuhn 1956).

Figura 3-12 Corrección por plasticidad del esfuerzo crítico de pandeo a cortadura de una chapa para las aleaciones de aluminio indicadas en la gráfica. Fuente: (Kuhn 1956). 91

Figura 3-13 Esquema de la geometría de una sección que incluye el cordón superior de un larguero, extendiéndose según la dirección de las flechas. Fuente: elaboración propia.

Figura 3-14 Estado de cortadura al que está sometido el alma del larguero dividido entre un estado de cortadura pura y un estado de tensión diagonal pura, con un factor de tensión diagonal $k$. Fuente: elaboración propia.

Figura 3-15 Numeración de láminas dentro del laminado. Fuente: elaboración propia.

Figura 3-16 Secciones típicas de larguerillos. De izquierda a derecha: sección en J, en $\mathrm{L}$, en $\mathrm{C}$ y en $\mathrm{Z}$. Fuente: elaboración propia. 100

Figura 3-17 Esquema de un larguerillo en Z, donde y es la posición del eje neutro. Fuente: elaboración propia. 100

Figura 3-18 Chapa rectangular sometida a carga biaxial. Fuente: elaboración propia. 102

Figura 3-19 Chapa simplemente apoyada sometida a compresión y cortadura. Fuente: elaboración propia. 103

Figura 3-20 Esquema de un panel de extradós incluyendo definiciones de algunos parámetros geométricos. Fuente: elaboración propia. 107

Figura 3-21 Variación del espesor del revestimiento en función de los parámetros $\lambda$ y $A R$ para el Caso 1 (izquierda) y Caso 2 (derecha). Fuente: elaboración propia. 109

Figura 3-22 Variación del ala del larguerillo en función de los parámetros $\lambda$ y $A R$ para el Caso 1 (izquierda) y Caso 2 (derecha). Fuente: elaboración propia. 110

Figura 3-23 Variación del área del panel en función de los parámetros $\lambda$ y $A R$ para el Caso 1 (izquierda) y Caso 2 (derecha). Fuente: elaboración propia.

Figura 3-24 Esquema de red neuronal del tipo feedforward. Fuente: (Gengiskanhg 2004) 


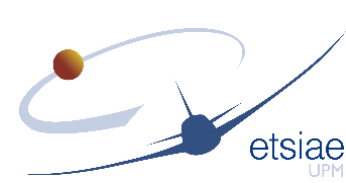

Figura 3-25 Funciones de activación tipo sigmoide y tangente hiperbólica. Fuente: elaboración propia. 119

Figura 3-26 Esquema de una red neuronal con una capa oculta, indicando la nomenclatura de los pesos para la primera neurona de la capa oculta y para la neurona de salida. Fuente: elaboración propia. 120

Figura 3-27 Evolución del valor de la función objetivo a cada paso de iteración hasta alcanzar el óptimo. Fuente: elaboración propia. 121

Figura 3-28 Evolución del valor de la función objetivo en un caso en el que se produce overfitting. La línea naranja representa la evolución del error en el conjunto de entrenamiento, y la amarilla en el conjunto de validación. Fuente: elaboración propia.

Figura 3-29 Evolución del valor de la función objetivo en un caso en el que se elige el punto de capacidad óptima. La línea naranja representa la evolución del error en el conjunto de entrenamiento, la amarilla en el conjunto de validación, y la naranja el de prueba. Fuente: elaboración propia.

Figura 4-1 Forma en planta del ala del avión CSR-01. Fuente: elaboración propia... 134 Figura 4-2 Vistas del avión CSR-01. Fuente: (ILR y RWTH-Aechen-University 2014). 134

Figura 4-3 Perfiles del ala del CSR-01. Fuente: (ILR y RWTH-Aechen-University 2014). 135

Figura 4-4 Polar del avión CSR-01 en configuración limpia en función del número de Mach de vuelo. Fuente: elaboración propia. 136

Figura 4-5 Polar del avión CSR-01 en configuración de despegue, ascenso, aproximación y aproximación con el tren de aterrizaje extendido. Fuente: elaboración propia. 136

Figura 4-6 Estimación de la deflexión máxima del timón de dirección del CSR-01. Fuente: elaboración propia. 137

Figura 4-7 Envolvente de maniobra para $22000 \mathrm{ft}$ a peso máximo de despegue. Fuente: elaboración propia. 140

Figura 4-8 Resultado de Tornado tras alcanzar la condición de equilibrio para el fallo del motor crítico tras el despegue. Fuente: elaboración propia.

Figura 4-9 Resultado de Tornado tras alcanzar la condición de equilibrio aterrizaje con viento cruzado. Fuente: elaboración propia. 143

Figura 4-10 Distribución de momento flector a lo largo de la envergadura del estabilizador horizontal del CSR-01 para las maniobras simétricas. Fuente: elaboración propia. 145

Figura 4-11 Distribución de fuerza cortante a lo largo de la envergadura del estabilizador horizontal del CSR-01 para las maniobras simétricas. Fuente: elaboración propia. .. 145

Figura 4-12 Distribución de momento torsor a lo largo de la envergadura del estabilizador horizontal del CSR-01 para las maniobras simétricas. Fuente: elaboración propia. .. 146

Figura 4-13 Distribución de momento flector a lo largo de la envergadura del estabilizador vertical del CSR-01 para las maniobras asimétricas. Fuente: elaboración propia. 146 


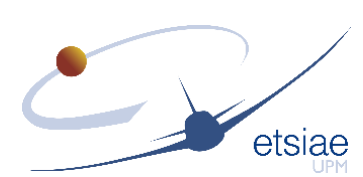

Figura 4-14 Distribución de fuerza cortante lo largo de la envergadura del estabilizador vertical del CSR-01 para las maniobras asimétricas. Fuente: elaboración propia. .... 147

Figura 4-15 Distribución de momento torsor lo largo de la envergadura del estabilizador vertical del CSR-01 para las maniobras asimétricas. Fuente: elaboración propia. .... 147

Figura 4-16 Perfil NACA 0012 del estabilizador horizontal del CSR-01 adimensionalizado con la cuerda y remarcado el cajón de torsión interior. Fuente: elaboración propia. 149

Figura 4-17 Carga por unidad de longitud y flujo cortante a lo largo de la semienvergadura del estabilizador horizontal del CSR-01 para las maniobras simétricas. Fuente: elaboración propia. 151

Figura 4-18 Perfil NACA 0009 del estabilizador vertical del CSR-01 adimensionalizado con la cuerda y remarcado el cajón de torsión interior. Fuente: elaboración propia. . 152

Figura 4-19 Carga por unidad de longitud y flujo cortante a lo largo de la envergadura del estabilizador vertical del CSR-01 para las maniobras asimétricas. Fuente: elaboración propia.

Figura 4-20 Definición de la envergadura, $b$, cuerda en la raíz, $C r$ y ángulo de diedro, $\Gamma$, de una cola en $\mathrm{V}$ y dónde se sitúan los controles. Fuente: elaboración propia. 155

Figura 4-21 Análisis del efecto de la envergadura, cuerda en la raíz y el parámetro de estrechamiento al variar el ángulo de diedro de la cola en $V$ en la deflexión del timón de profundidad, situado en el control interior, para equilibrar en condiciones de aterrizaje. Fuente: elaboración propia. 157

Figura 4-22 Análisis del efecto de la envergadura, cuerda en la raíz y el parámetro de estrechamiento al variar el ángulo de diedro de la cola en $V$ en la deflexión del timón de profundidad, situado en el control interior, para equilibrar en condiciones de segundo segmento. Fuente: elaboración propia. 158

Figura 4-23 Análisis del efecto de la envergadura, cuerda en la raíz y el parámetro de estrechamiento al variar el ángulo de diedro de la cola en $V$ en la deflexión del timón de profundidad, situado en el control interior, para equilibrar en condiciones de crucero. Fuente: elaboración propia. 158

Figura 4-24 Esquema explicativo del efecto de variar la envergadura en la deflexión de equilibrado del control longitudinal en las tres condiciones de vuelo: crucero, segundo segmento y aterrizaje. Fuente: elaboración propia.

Figura 4-25 Ángulo de calado de la cola para equilibrar el CSR-01 en el punto medio de la condición de crucero. Fuente: elaboración propia. 160

Figura 4-26 Ángulo de deflexión del timón de profundidad para equilibrar el avión en el segundo segmento, considerando el ángulo de calado de la cola determinado para la condición de crucero. Fuente: elaboración propia. 161

Figura 4-27 Ángulo de deflexión del timón de profundidad para equilibrar el avión en aterrizaje, considerando el ángulo de calado de la cola determinado para la condición de crucero. Fuente: elaboración propia. 161

Figura 4-28 Análisis del efecto de la envergadura, la cuerda en la raíz, el parámetro de estrechamiento en función de la variación del diedro de la derivada estática longitudinal en la condición de crucero para una cola en V. Fuente: elaboración propia. 162 


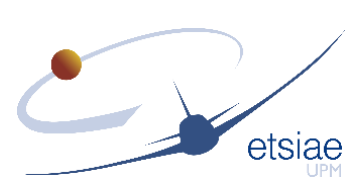

Figura 4-29 Análisis del efecto de la envergadura, la cuerda en la raíz, el parámetro de estrechamiento en función de la variación del diedro de la derivada estática lateral en la condición de crucero para una cola en V. Fuente: elaboración propia. 163

Figura 4-30 Resultados del ajuste de las derivadas de estabilidad estática longitudinal en crucero por medio de una red neuronal de una capa oculta con cinco neuronas. Fuente: elaboración propia. 164

Figura 4-31 Resultados del ajuste de las derivadas de estabilidad estática lateral en crucero por medio de una red neuronal de una capa oculta con tres neuronas. Fuente: elaboración propia. 164

Figura 4-32 Análisis del efecto de la envergadura, cuerda en la raíz y parámetro de estrechamiento con la variación del diedro de la cola en $V$ en la condición del control de la aeronave tras el fallo del motor crítico en el segundo segmento. Fuente: elaboración propia.

Figura 4-33 Resultados del ajuste de las deflexiones del timón de dirección para controlar el avión tras el fallo del motor crítico en el segundo segmento por medio de una red neuronal de una capa oculta con seis neuronas. Fuente: elaboración propia. 166

Figura 4-34 Análisis del efecto de la envergadura, cuerda en la raíz y parámetro de estrechamiento con la variación del diedro de la cola en $V$ en la condición del control de la aeronave en aterrizaje con viento cruzado. Fuente: elaboración propia. 167

Figura 4-35 Resultados del ajuste de las deflexiones del timón de dirección para controlar el avión en aterrizaje con viento cruzado por medio de una red neuronal de una capa oculta con cinco neuronas. Fuente: elaboración propia. 168

Figura 4-36 Espacio de diseño posible definido por las restricciones impuestas por las derivadas de estabilidad estáticas en crucero, tanto longitudinal $c m \alpha$ y lateral $c n \beta$, y por el control del avión tras el fallo del motor crítico en el segundo segmento (CEF). Fuente: elaboración propia. 169

Figura 4-37 Espacio de diseño posible definido por las restricciones impuestas por las derivadas de estabilidad estáticas en crucero, tanto longitudinal $c m \alpha$ y lateral $c n \beta$, y por el control del avión en el aterrizaje con viento cruzado, definiendo un mínimo para el diedro (CwL MIN). Fuente: elaboración propia.

Figura 4-38 Definición de la envergadura del timón de dirección propuesto para ser rediseñado. Fuente: elaboración propia.

Figura 4-39 Tamaño mínimo del timón de dirección para que la restricción impuesta por el control del avión tras el fallo del motor crítico en el segundo segmento coincida con la restricción impuesta por la estabilidad estática lateral en crucero. Fuente: elaboración propia. 171

Figura 4-40 Espacio de diseño posible definido por las restricciones impuestas por las derivadas de estabilidad estáticas en crucero, tanto longitudinal $C m \alpha$ y lateral $C n \beta$, y por el control del avión en el aterrizaje con viento cruzado con el nuevo tamaño del timón de dirección, definiendo un máximo para el diedro (CwL MAX). Fuente: elaboración propia.

Figura 4-41 Dependencia del peso determinado combinando las fórmulas para colas convencionales en función de la cuerda en la raíz. Fuente: elaboración propia. 173 


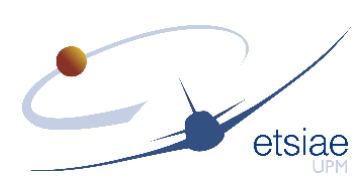

Figura 4-42 Dependencia del peso determinado combinando las fórmulas para colas convencionales en función del diedro. Fuente: elaboración propia. 174

Figura 4-43 Dependencia del peso determinado combinando las fórmulas para colas convencionales en función de la envergadura. Fuente: elaboración propia. 174

Figura 4-44 Dependencia del peso determinado combinando las fórmulas para colas convencionales en función del parámetro de estrechamiento. Fuente: elaboración propia.

Figura 4-45 Dependencia del peso determinado combinando las fórmulas para colas convencionales en función de la cuerda en la raíz en puntos situados sobre la restricción impuesta por la derivada de estabilidad longitudinal en crucero. Fuente: elaboración propia.

Figura 4-46 Dependencia del peso determinado combinando las fórmulas para colas convencionales en función de la envergadura en puntos situados sobre la restricción impuesta por la derivada de estabilidad longitudinal en crucero. Fuente: elaboración propia.

Figura 4-47 Dependencia del peso determinado combinando las fórmulas para colas convencionales en función del parámetro de estrechamiento en puntos situados sobre la restricción impuesta por la derivada de estabilidad longitudinal en crucero. Fuente: elaboración propia. 176

Figura 4-48 Dependencia del peso determinado combinando las fórmulas para colas convencionales en función de la cuerda en la raíz en puntos situados sobre la restricción impuesta por la derivada de estabilidad lateral en crucero. Fuente: elaboración propia.

Figura 4-49 Dependencia del peso determinado combinando las fórmulas para colas convencionales en función de la envergadura en puntos situados sobre la restricción impuesta por la derivada de estabilidad lateral en crucero. Fuente: elaboración propia.

Figura 4-50 Dependencia del peso determinado combinando las fórmulas para colas convencionales en función del parámetro de estrechamiento en puntos situados sobre la restricción impuesta por la derivada de estabilidad lateral en crucero. Fuente: elaboración propia.

Figura 4-51 Cargas introducidas en la cola en las maniobras consideradas, analizando el efecto de variar la cuerda en la raíz, (a) para $\Gamma=30^{\circ}, \mathrm{Cr}=3,7 \mathrm{~m}, b=12 \mathrm{~m}$ y $\lambda=0,32$ y (b) para $\Gamma=30^{\circ}, \mathrm{Cr}=4,5 \mathrm{~m}, b=12 \mathrm{~m}$ y $\lambda=0,32$. Fuente: elaboración propia..... 181

Figura 4-52 Cargas introducidas en la cola en las maniobras consideradas, analizando el efecto de variar el parámetro de estrechamiento, (a) para $\Gamma=30^{\circ}, \mathrm{Cr}=4,5 \mathrm{~m}, \mathrm{~b}=$ $12 m$ y $\lambda=0,32$ y (b) para $\Gamma=30^{\circ}, C r=4,5 m, b=12 m$ y $\lambda=0,36$. Fuente: elaboración propia.

Figura 4-53 Cargas introducidas en la cola en las maniobras consideradas, analizando el efecto de variar la envergadura, (a) para $\Gamma=30^{\circ}, C r=4,1 m, b=22 \mathrm{~m}$ y $\lambda=0,32 \mathrm{y}$ (b) para $\Gamma=30^{\circ}, C r=4,1 m, b=26 m$ y $\lambda=0,32$. Fuente: elaboración propia.

Figura 4-54 Cargas introducidas en la cola en las maniobras consideradas, analizando el efecto de variar el diedro, (a) para $\Gamma=30^{\circ}, C r=4,1 m, b=12 m$ y $\lambda=0,32$ y (b) para $\Gamma=50^{\circ}, C r=4,1 m, b=12 m$ y $\lambda=0,32$. Fuente: elaboración propia. 184 


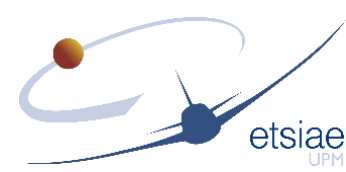

Figura 4-55 Peso del estabilizador en función del diedro para las maniobras seleccionadas. Fuente: elaboración propia. 185

Figura 4-56 Peso del estabilizador en función de la cuerda en la raíz para las maniobras seleccionadas. Fuente: elaboración propia. 185

Figura 4-57 Peso del estabilizador en función de la envergadura para las maniobras seleccionadas. Fuente: elaboración propia. 186

Figura 4-58 Peso del estabilizador en función del parámetro de estrechamiento para las maniobras seleccionadas. Fuente: elaboración propia 186

Figura 4-59 Relación que presentan las variables de diseño en los puntos de intersección entre la restricción impuesta por la derivada estática en crucero lateral y longitudinal. Fuente: elaboración propia. 187

Figura 4-60 Peso estimado por el método basado en las regresiones para colas convencionales que presentan las variables de diseño en los puntos de intersección entre la restricción impuesta por la derivada estática en crucero lateral y longitudinal. Fuente: elaboración propia. 188

Figura 4-61 Superficie de la cola en $V$ correspondiente a las variables de diseño en los puntos de intersección entre la restricción impuesta por la derivada estática en crucero lateral y longitudinal. Fuente: elaboración propia. 189

Figura 4-62 Coeficiente de resistencia sin sustentación de la cola en $V$ correspondiente a las variables de diseño en los puntos de intersección entre la restricción impuesta por la derivada estática en crucero lateral y longitudinal. Fuente: elaboración propia. .... 190

Figura 4-63 Incremento del combustible consumido en la ruta de diseño de la cola en $\mathrm{V}$ correspondiente a las variables de diseño en los puntos de intersección entre la restricción impuesta por la derivada estática en crucero lateral y longitudinal calculando el peso por medio de las regresiones estadísticas. Fuente: elaboración propia. 191

Figura 4-64 Peso estimado por el método basado los métodos casi analíticos que presentan las variables de diseño en los puntos de intersección entre la restricción impuesta por la derivada estática en crucero lateral y longitudinal. Fuente: elaboración propia. 192

Figura 4-65 Incremento del combustible consumido en la ruta de diseño de la cola en $\mathrm{V}$ correspondiente a las variables de diseño en los puntos de intersección entre la restricción impuesta por la derivada estática en crucero lateral y longitudinal calculando el peso por medio del método casi analítico. Fuente: elaboración propia.

Figura 4-66 Definición de la envergadura, $b 1$ y $b 2$, cuerda en la raíz, Croot y ángulo de diedro, $\Gamma$, de una cola en $U$ y dónde se sitúan los controles. Fuente: elaboración propia. 


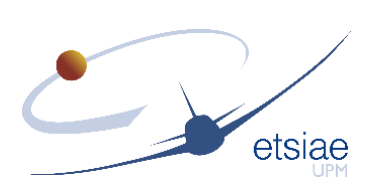

\section{ÍNDICE DE TABLAS}

Tabla 1-1 Clasificación de las configuraciones de cola más habituales. Fuente: elaboración propia.

Tabla 2-1 Modelos de avión incluidos en la base de datos de estudio de configuraciones de cola convencional. Fuente: elaboración propia.

Tabla 2-2 Parámetros considerados en la base de datos clasificados según su naturaleza. Fuente: elaboración propia.

Tabla 2-3 Fronteras establecidas según el segmento de vuelo. Fuente: elaboración propia.

Tabla 2-4 Números de Mach de crucero de diseño para los aviones clasificados en función del tipo de motor (turbofán o turbohélice) en columnas y según el segmento de vuelo por filas. Se incluye la cantidad de aviones que pertenecen a cada categoría, el promedio de los números de Mach y la desviación típica. Fuente: elaboración propia.

Tabla 2-5 Esbeltez del cono de cola y fracción de longitud de fuselaje que ocupa en función de diferentes criterios: configuración de cola, segmento de vuelo, número de motores, posición de los motores y tipo de planta propulsora. Se incluye la cantidad de aviones que pertenecen a cada categoría, el promedio de los parámetros y su desviación típica. Fuente: elaboración propia.

Tabla 2-6 Ángulo de guarda y ángulo de upsweep en función de diferentes criterios: configuración de cola, segmento de vuelo, número de motores, posición de los motores y tipo de planta propulsora. Se incluye la cantidad de aviones que pertenecen a cada categoría, el promedio de los parámetros y su desviación típica. Fuente: elaboración propia.

Tabla 2-7 Flecha de los puntos $1 / 4$ y parámetro de estrechamiento del estabilizador horizontal en función de diferentes criterios: configuración de cola, segmento de vuelo, número de motores, posición de los motores y tipo de planta propulsora. Se incluye la cantidad de aviones que pertenecen a cada categoría, el promedio de los parámetros y su desviación típica. Fuente: elaboración propia.

Tabla 2-8 Alargamiento del estabilizador horizontal y relación entre las superficies del timón de profundidad y del estabilizador en función de diferentes criterios: configuración de cola, segmento de vuelo, número de motores, posición de los motores y tipo de planta propulsora. Se incluye la cantidad de aviones que pertenecen a cada categoría, el promedio de los parámetros y su desviación típica. Fuente: elaboración propia. ........50

Tabla 2-9 Flecha de los puntos $1 / 4$ y parámetro de estrechamiento del estabilizador vertical en función de diferentes criterios: configuración de cola, segmento de vuelo, número de motores, posición de los motores y tipo de planta propulsora. Se incluye la cantidad de aviones que pertenecen a cada categoría, el promedio de los parámetros y su desviación típica. Fuente: elaboración propia.

Tabla 2-10 Alargamiento del estabilizador vertical y relación entre las superficies del timón de dirección y del estabilizador en función de diferentes criterios: configuración de cola, segmento de vuelo, número de motores, posición de los motores y tipo de planta propulsora. Se incluye la cantidad de aviones que pertenecen a cada categoría, el promedio de los parámetros y su desviación típica. Fuente: elaboración propia. 54 


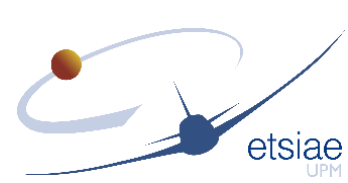

Tabla 2-11 Principales datos característicos del CSR-01. Fuente: elaboración propia. .56

Tabla 2-12 Datos de los estabilizadores del CSR-01. Fuente: elaboración propia. .....57

Tabla 2-13 Resultados del proceso de diseño del estabilizador horizontal del CSR-01. En las celdas donde aparecen dos valores, los que están antes de la barra (/) se han obtenido basados en los valores del ala estimados que se encuentran en la tabla y los que están después de la barra (/) están basados en los datos reales del ala, también incluidos en la tabla. Fuente: elaboración propia.

Tabla 3-1 Propiedades de las láminas y orientación utilizados en los laminados del revestimiento y los larguerillos. Fuente: elaboración propia. 109

Tabla 3-2 Resultados para los casos de carga considerados según el método de Farrar para factor de Farrar óptimo y el propuesto para materiales compuestos. Fuente: elaboración propia. 112

Tabla 4-1 Parámetros del avión CSR-01 incluidos en la base de datos CeRAS. Fuente: elaboración propia.

Tabla 4-2 Parámetros geométricos del avión CSR-01 sobre los dispositivos hipersustentadores. Fuente: elaboración propia. 135

Tabla 4-3 Parámetros del diedro, torsión, espesor relativo y perfil por secciones del ala del CSR-01 indicadas por la distancia al plano de simetría $(y)$. Fuente: elaboración propia. 135

Tabla 4-4 Valores del coeficiente de resistencia sin sustentación de los estabilizadores referidos a la superficie alar del CSR-01. Fuente: elaboración propia. 138

Tabla 4-5 Valores de los parámetros de ajuste del modelo de estimación de pesos de costillas, sujeciones y uniones. Fuente: elaboración propia. 150

Tabla 4-6 Valores de los parámetros de ajuste del modelo de estimación de pesos de elementos secundarios por unidad de superficie. Fuente: elaboración propia. 150

Tabla 4-7 Valores de los parámetros de ajuste del modelo de estimación de pesos de elementos secundarios por unidad de superficie. Fuente: elaboración propia......

Tabla 4-8 Pesos resultantes del estabilizador horizontal para cada una de las maniobras simétricas. Fuente: elaboración propia.

Tabla 4-9 Pesos resultantes del estabilizador vertical para cada una de las maniobras asimétricas. Fuente: elaboración propia.

Tabla 4-10 Intervalo de búsqueda de los parámetros de diseño. Fuente: elaboración propia. 


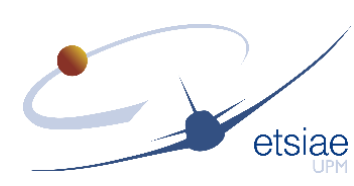

\section{RESUMEN}

En esta tesis se presenta una metodología de diseño de configuraciones de cola no convencionales que permita llevar a cabo estudios de viabilidad al nivel de diseño conceptual. Para poder acometerlos se parte de una caracterización de las configuraciones de cola que más se utilizan en los aviones de transporte comercial (configuración convencional). Esta caracterización se lleva a cabo por medio de procedimientos clásicos en el diseño conceptual. Estos incluyen la construcción de regresiones que relacionen los valores de parámetros característicos de los aviones o incluso se pueden describir las configuraciones por medio de valores promedios y desviaciones típicas de ciertas variables. Los resultados de esta caracterización permiten establecer un procedimiento de dimensionado de los estabilizadores de cola en configuración convencional, que se componen de una superficie horizontal y otra vertical.

Una vez definida y caracterizada la configuración convencional, se ha realizado un análisis del estado del arte de configuraciones de cola no convencionales, tanto desde el punto de vista de geometrías no utilizadas en los empenajes de aviones de transporte comercial, como desde el punto de vista de sistemas o técnicas que no se hayan implementado comercialmente hasta ahora. El foco se pone sobre las geometrías no convencionales que se identifiquen como novedosas y potencialmente beneficiosas, siendo el objetivo establecer un procedimiento de dimensionado de estas configuraciones.

Las superficies estabilizadoras cumplen una serie de funciones, que deben ser consideradas en el dimensionado de las nuevas configuraciones. Estas funciones se cuantifican a nivel de diseño en la normativa de certificación aplicable. La base de certificación recoge los requisitos mínimos con los que hay que demostrar el cumplimiento para que el proyecto de avión obtenga su certificado de tipo, y las aeronaves de serie, su certificado de aeronavegabilidad, que les autoriza para realizar operaciones. Es por ello que es de vital importancia que el procedimiento de diseño contemple estos requisitos. Entre otros, caben destacar las condiciones de control del avión tras el fallo del motor crítico en el segundo segmento y en el aterrizaje con viento cruzado.

Además de considerarse los aspectos reflejados en la normativa, el estudio debe analizar la viabilidad del uso de configuraciones no convencionales. El punto sobre el que se pueden extraer conclusiones a nivel de diseño conceptual es el consumo de combustible para la ruta de diseño. Este consumo depende, en aspectos que se refieran a las superficies de cola, del peso y de la resistencia aerodinámica de los estabilizadores. Por tanto, la metodología de dimensionado debe estimar ambas magnitudes para las configuraciones novedosas, permitiendo extraer conclusiones sobre las ventajas en términos de consumo que puedan tener.

Como caso de validación de la metodología propuesta, se ha seleccionado la configuración de cola en $\mathrm{V}$, que es sobre la que se han realizado más estudios en el pasado y permite comparar los resultados obtenidos. El consumo de combustible resultante del procedimiento de diseño coincide con los estimados en otros proyectos de investigación para este tipo de configuraciones en su implementación en aviones de transporte comercial, validando de esta manera la utilización de esta metodología para el dimensionado de otras configuraciones de cola no convencionales. 


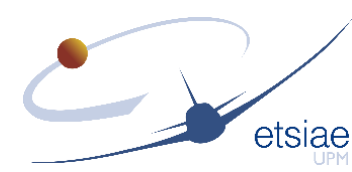

\section{ABSTRACT}

In this thesis, a methodology for the design of unconventional tail configurations is presented, which allows carrying out feasibility studies at conceptual design stages. In order to undertake them, the first step is a characterization of the tail configurations that are most used in commercial transport aircraft (conventional configuration). This characterization is carried out by means of classical procedures in the conceptual design. These include the construction of regressions that relate the values of typical parameters of the aircraft or even the configurations can be described by means of average values and standard deviations of certain variables. The results of this characterization allow to establish a procedure for sizing the tail stabilizers of conventional configuration, which are composed of a horizontal and a vertical surface.

Once the conventional configuration has been defined and characterized, an analysis of the state of the art of unconventional tail configurations has been carried out, both from the point of view of geometries not used in the empennages of commercial transport aircraft, and from the point of view of systems or techniques that have not been commercially implemented so far. The focus is placed on the unconventional geometries that are identified as novel and potentially advantageous, thus the objective of this thesis is to establish a procedure for sizing these configurations.

The stabilizing surfaces fulfill a series of functions, which must be considered in the dimensioning of the new configurations. These functions are quantified at the design level in the applicable certification regulations. The certification base includes the minimum requirements with which compliance has to be demonstrated in order for the aircraft project to obtain its type certificate, and the aircraft in series to obtain their airworthiness certificates, that authorizes them to carry out operations. That is why it is vitally important that the design procedure takes into account these requirements. Among others, it is worth noting the aircraft control requirements after the failure of the critical engine in climbing and in the crosswind landing.

In addition to considering the aspects reflected in the regulations, the study should analyze the feasibility of using non-conventional configurations. The point on which conclusions can be drawn at the conceptual design level is fuel consumption for the design route. This consumption depends on, in aspects that refer to the tail surfaces, the weight and the aerodynamic drag of the stabilizers. Therefore, the sizing methodology must estimate both magnitudes for the novel configurations, allowing conclusions to be drawn about the advantages in terms of consumption they may have.

As a validation case of the proposed methodology, the Vee-tail configuration has been selected, that is the one on which more studies have been carried out in the past and allows the results obtained to be compared. The fuel consumption resulting from the design procedure coincides with those estimated in other research projects for this type of configuration in its implementation in commercial transport aircraft, validating the use of this methodology for the sizing of other non-conventional tail configurations. 


\section{NOMENCLATURA}

\section{Símbolo}

$A_{i j}$

$\overline{A_{l J}}$

$A_{L}$

$A_{M}$

$A_{s}$

$A R$

$\overline{A R}$

Alargamiento de un elemento del panel

$A R_{\text {eff }}$

Alargamiento efectivo del estabilizador vertical

$A R_{H}$

$A R_{V}$

$A R^{\prime}{ }_{V}$

Doble del alargamiento del estabilizador vertical
Área del larguero

Área del montante

Área del larguerillo

Alargamiento del pane

\begin{tabular}{cc}
$a_{H}$ & $\begin{array}{c}\text { Pendiente de la curva de sustentación de la cola referida a su propia } \\
\text { superficie }\end{array}$ \\
\hline$b$ & Envergadura de la cola en $\mathrm{V}$ \\
\hline$b_{c}$ & Ancho de la sección del conjunto chapa y larguerillo \\
\hline$b_{H}$ & Ancho del elemento $i$ de un larguerillo \\
\hline$b_{i}$ & Ancho del ala del larguerillo \\
\hline$b_{S}$ & Ancho del cajón de torsión
\end{tabular}




\begin{tabular}{|c|c|}
\hline$b_{V}$ & Envergadura del estabilizador vertical \\
\hline$b_{w}$ & Envergadura alar \\
\hline$c_{D}$ & Coeficiente de resistencia aerodinámica \\
\hline$c_{D 0 H}$ & Coeficiente de resistencia parásita del estabilizador horizontal \\
\hline$c_{e}$ & Consumo específico del motor \\
\hline$\frac{c_{\text {elev }}}{c}$ & $\begin{array}{l}\text { Cociente de cuerda del timón de profundidad respecto a la cuerda del } \\
\text { estabilizador }\end{array}$ \\
\hline$c_{F}$ & Coeficiente de fricción \\
\hline$c_{L}$ & Coeficiente de sustentación \\
\hline$c_{L_{A-T}}$ & Coeficiente de sustentación del avión sin contar la cola \\
\hline$c_{L H}$ & Coeficiente de sustentación del estabilizador horizontal \\
\hline$c_{L T}$ & Coeficiente de sustentación de la cola \\
\hline$c_{L \alpha}$ & Pendiente de la curva de sustentación \\
\hline$c_{L \alpha_{t}}$ & $\begin{array}{l}\text { Derivada del coeficiente de sustentación de la cola respecto del ángulo } \\
\text { de ataque }\end{array}$ \\
\hline
\end{tabular}

\begin{tabular}{cc}
\hline$c_{l \alpha}$ & $\begin{array}{c}\text { Derivada del coeficiente de sustentación del perfil respecto del ángulo de } \\
\text { ataque }\end{array}$ \\
\hline$c_{m_{A-T}}$ & Coeficiente de momento de cabeceo eliminando la contribución de la \\
cola
\end{tabular}




\begin{tabular}{|c|c|}
\hline$c_{m \alpha_{H}}$ & $\begin{array}{c}\text { Derivada del coeficiente de momento de cabeceo de la cola respecto del } \\
\text { ángulo de ataque }\end{array}$ \\
\hline$c_{n_{A-T}}$ & Coeficiente de momento de guiñada del avión sin contar con la cola \\
\hline$c_{n \beta}$ & $\begin{array}{l}\text { Derivada del coeficiente de momento de guiñada respecto del ángulo de } \\
\text { resbalamiento }\end{array}$ \\
\hline$c_{n \beta_{A-V}}$ & $\begin{array}{l}\text { Derivada del coeficiente de momento de guiñada sin contar el } \\
\text { estabilizador vertical respecto del ángulo de resbalamiento }\end{array}$ \\
\hline$c_{n \beta_{V}}$ & $\begin{array}{c}\text { Derivada del coeficiente de momento de guiñada del estabilizador } \\
\text { vertical respecto del ángulo de resbalamiento }\end{array}$ \\
\hline$c_{r H}$ & Cuerda en la raíz del estabilizador horizontal \\
\hline$C_{r}$ & Cuerda en la raíz de la cola en $\mathrm{V}$ \\
\hline$\frac{c_{r u d d}}{c}$ & $\begin{array}{l}\text { Cociente entre la cuerda del timón de dirección respecto de la cuerda del } \\
\text { estabilizador vertical }\end{array}$ \\
\hline$c_{r w}$ & Cuerda en la raíz del ala \\
\hline$c_{t}$ & Cuerda en la punta \\
\hline$c_{Y T}$ & Coeficiente de fuerza lateral de la cola \\
\hline$c_{Y_{\beta V}}$ & $\begin{array}{l}\text { Derivada del coeficiente de fuerza lateral del estabilizador vertical } \\
\text { respecto del ángulo de resbalamiento }\end{array}$ \\
\hline$C M A_{H}$ & Cuerda media aerodinámica del estabilizador horizontal \\
\hline$C M A_{w}$ & Cuerda media aerodinámica del ala \\
\hline$C M G$ & Cuerda media geométrica \\
\hline$D_{H}$ & Resistencia aerodinámica del estabilizador horizontal \\
\hline$D_{H i}$ & Parámetro de Resistencia inducida del estabilizador horizontal \\
\hline$D_{i j}$ & Rigidez a flexión del laminado \\
\hline$\overline{D_{l j}}$ & Rigidez pseudo-adimensional a flexión del laminado \\
\hline$D_{\text {indH }}$ & Resistencia inducida del estabilizador horizontal \\
\hline
\end{tabular}




\begin{tabular}{|c|c|}
\hline$d_{s}$ & Longitud del ala del larguerillo \\
\hline$E$ & Módulo de Young \\
\hline$E_{L}$ & Módulo de Young en dirección longitudinal a las fibras \\
\hline$E_{T}$ & Módulo de Young en dirección transversal a las fibras \\
\hline$\overline{E_{l j}}$ & Constantes $E$ pseudo-adimensionales para la carga crítica a cortadura \\
\hline$E A_{e q}$ & Rigidez equivalente en el plano \\
\hline$\overline{E A_{e q}}$ & Rigidez equivalente pseudo-dimensional en el plano \\
\hline$E_{b} I_{e q}$ & Rigidez equivalente a flexión \\
\hline$\overline{E_{b} I_{e q}}$ & Rigidez equivalente pseudo-dimensional a flexión \\
\hline$F$ & Factor de Farrar \\
\hline$F_{\text {crip }}$ & Esfuerzo admisible por crippling \\
\hline$F_{e}$ & Factor estadístico de la función $F$ de Fisher-Snedecor \\
\hline$F_{c}$ & Fuerza cortante \\
\hline$F_{S U}$ & Esfuerzo último de cortadura \\
\hline$G_{L T}$ & Módulo de cortadura \\
\hline$g$ & Gravedad \\
\hline$h$ & Espesor de la lámina \\
\hline$H_{\text {fus }}$ & Altura del fuselaje \\
\hline$h_{L}$ & Espesor de las láminas del revestimiento \\
\hline$h_{L L}$ & Espesor de las láminas de los larguerillos \\
\hline$h_{s}$ & Altura del larguerillo \\
\hline$h_{t}$ & Altura del cajón de torsión \\
\hline$I$ & Momento de inercia de la sección \\
\hline
\end{tabular}


Parámetro corrector de la derivada de la fuerza lateral de la cola en $\mathrm{V}$

$K$ respecto al resbalamiento o Parámetro de alcance de la ecuación de Breguet

\begin{tabular}{|c|c|}
\hline$K_{g}$ & Factor de atenuación de ráfagas \\
\hline$K_{\rho H}$ & Factor de densidad del estabilizador horizontal \\
\hline$K_{\rho V}$ & Factor de densidad del estabilizador vertical \\
\hline$k$ & Factor de tensión diagonal \\
\hline$k_{r}$ & Factor de respuesta \\
\hline$L$ & Sustentación \\
\hline$L_{c}$ & Paso entre costillas \\
\hline$L_{f u s}$ & Longitud del fuselaje \\
\hline$L_{T H}$ & Brazo de momentos del estabilizador horizontal \\
\hline$L_{T}$ & Brazo de momentos de la cola \\
\hline$L_{t}$ & Sustentación de la cola \\
\hline$L_{T V}$ & Brazo de momentos del estabilizador vertical \\
\hline$M$ & Número de Mach \\
\hline$M_{f}$ & Momento flector \\
\hline$M_{\text {Tor }}$ & Momento torsor \\
\hline MTOW & Peso máximo de despegue \\
\hline$n$ & Factor de carga \\
\hline$n_{e}$ & Número de datos tomado para construir una regresión \\
\hline$n_{L i}$ & Número de láminas el elemento $i$ del larguerillo \\
\hline$N$ & Carga de compresión por unidad de longitud \\
\hline
\end{tabular}




\begin{tabular}{|c|c|}
\hline$N_{x}$ & Carga de compresión \\
\hline$N_{\text {xcrit }}$ & Carga de pandeo a compresión pura \\
\hline$N_{x s k i n}$ & Carga de compresión absorbida por el revestimiento \\
\hline$N_{x y}$ & Carga de cortadura \\
\hline$N_{\text {xycrit }}$ & Carga de pandeo a cortadura pura \\
\hline$P$ & Probabilidad de que una regresión no sea extrapolable a la población \\
\hline$p$ & Grados de libertad de la función $F$ de Fisher-Snedecor \\
\hline$Q$ & Matriz de rigidez de un laminado \\
\hline$q$ & Flujo cortante \\
\hline$q_{H} / q$ & Cociente de presión dinámica entre el estabilizador horizontal y el ala \\
\hline$q_{T}$ & Flujo cortante debido al momento torsor \\
\hline$q_{T} / q$ & Cociente de presión dinámica entre la cola y el ala \\
\hline$q_{V} / q$ & Cociente de presión dinámica entre el estabilizador vertical y el ala \\
\hline$R$ & Alcance \\
\hline$R_{g}$ & Radio de giro \\
\hline$R^{2}$ & Coeficiente de determinación \\
\hline$R F_{\text {length }}$ & Longitud del fuselaje posterior \\
\hline$S$ & Resistencia a cortadura de una lámina \\
\hline$S_{H}$ & Superficie del estabilizador horizontal \\
\hline$S_{t}$ & Superficie de la cola \\
\hline$S_{V}$ & Superficie del estabilizador vertical \\
\hline$S_{w}$ & Superficie alar \\
\hline
\end{tabular}


Suma de los cuadrados de la mínima distancia a la curva de regresión de

los puntos conocidos

\begin{tabular}{|c|c|}
\hline$S S_{R}$ & Suma de los cuadrados de la resta entre los valores \\
\hline$\left(\frac{t}{c}\right)_{\max }$ & Espesor relativo máximo \\
\hline$T$ & Empuje \\
\hline$T_{T O e}$ & Empuje de un motor al despegue \\
\hline$T S A$ & Ángulo de guarda \\
\hline$T S S$ & $\begin{array}{l}\text { Suma de los cuadrados de la resta de los puntos de la curva de } \\
\text { regresión y la media de los valores de los puntos conocidos }\end{array}$ \\
\hline$t$ & Espesor del revestimiento \\
\hline$t_{i}$ & Espesor del elemento $i$ de un larguerillo \\
\hline$t_{s}$ & Espesor de los larguerillos \\
\hline$U_{\max }$ & Intensidad de ráfaga \\
\hline$U A$ & Ángulo de upsweep del fuselaje posterior \\
\hline$V$ & Velocidad de vuelo \\
\hline$V_{A}$ & Velocidad de maniobra de proyecto \\
\hline$V_{D}$ & Velocidad de picado de proyecto \\
\hline$V_{e H}$ & Coeficiente de volumen del estabilizador horizontal \\
\hline$V_{e V}$ & Coeficiente de volumen del estabilizador vertical \\
\hline$V_{M C}$ & Velocidad mínima de control del avión \\
\hline Vol & Parámetro de volumen global del avión \\
\hline$W$ & Peso del avión \\
\hline$W_{\text {attach }}$ & Peso de las sujeciones \\
\hline$W_{\text {joints }}$ & Peso de las uniones \\
\hline
\end{tabular}




\begin{tabular}{|c|c|}
\hline$W_{\text {rib }}$ & Peso de costillas \\
\hline $\bar{X}$ & Media aritmética e la variable aleatoria $X$ \\
\hline$X^{c}$ & Resistencia a compresión en la dirección $x$ de una lámina \\
\hline$X^{t}$ & Resistencia a tracción en la dirección $x$ de una lámina \\
\hline$x_{c a}$ & Posición del centro aerodinámico del avión \\
\hline$x_{\text {baCMA }}$ & $\begin{array}{l}\text { Posición longitudinal del borde de ataque de la cuerda media } \\
\text { aerodinámica }\end{array}$ \\
\hline$x_{c g}$ & Posición del centro de gravedad del avión \\
\hline$x_{c g L}$ & Posición del centro de gravedad del avión en aterrizaje \\
\hline$x_{c g T o}$ & Posición del centro de gravedad del avión en despegue \\
\hline $\bar{Y}$ & Media aritmética de la variable aleatoria $Y$ \\
\hline$Y^{c}$ & Resistencia a compresión en la dirección y de una lámina \\
\hline$Y^{t}$ & Resistencia a tracción en la dirección y de una lámina \\
\hline $\bar{y}$ & Posición del eje neutro \\
\hline$Y_{A-T}$ & Fuerza lateral del avión sin contar con la cola \\
\hline$y_{C M A}$ & Posición lateral de la cuerda media aerodinámica \\
\hline$y_{e}$ & Posición lateral del motor crítico \\
\hline$Y_{T}$ & Fuerza lateral de la cola del avión \\
\hline$z_{e}$ & $\begin{array}{l}\text { Distancia vertical entre el punto de aplicación del empuje y el centro de } \\
\text { gravedad }\end{array}$ \\
\hline$\alpha$ & Ángulo de ataque \\
\hline$\alpha_{d}$ & Ángulo de tensión diagonal \\
\hline$\alpha_{f}$ & Ángulo de ataque del fuselaje \\
\hline$\alpha_{\text {tail }}$ & Ángulo de ataque de la cola \\
\hline
\end{tabular}




\begin{tabular}{|c|c|}
\hline$\beta$ & Ángulo de resbalamiento \\
\hline$\Gamma$ & Ángulo de diedro \\
\hline$\gamma_{x y}$ & Deformación angular fuera del plano $x y$ \\
\hline$\epsilon$ & Ángulo de deflexión de estela \\
\hline$\epsilon_{x}, \epsilon_{y}$ & Deformaciones en el plano $x y$ \\
\hline$\Delta c_{L_{H L D}}$ & $\begin{array}{c}\text { Contribución al coeficiente de sustentación de los dispositivos } \\
\text { hipersustentadores }\end{array}$ \\
\hline$\Delta c_{m_{H L D}}$ & $\begin{array}{l}\text { Contribución al coeficiente de momento de cabeceo de los dispositivos } \\
\text { hipersustentadores }\end{array}$ \\
\hline$\Delta C_{m_{f u s}}$ & Contribución al coeficiente de momento de cabeceo del fuselaje \\
\hline$\Delta \epsilon_{f}$ & $\begin{array}{l}\text { Incremento del ángulo de deflexión de estela debido a la deflexión de los } \\
\text { flaps }\end{array}$ \\
\hline$\delta_{\text {asym }}$ & Deflexión antisimétrica del timón de dirección \\
\hline$\delta_{c}$ & Deflexión de un control \\
\hline$\delta_{e}$ & Deflexión del timón de profundidad del estabilizador horizontal \\
\hline$\delta_{f}$ & Deflexión de los dispositivos hipersustentadores \\
\hline$\delta_{r}$ & Deflexión del timón de dirección del estabilizador vertical \\
\hline$\delta_{\text {sym }}$ & Deflexión simétrica del timón de profundidad \\
\hline$\kappa$ & Curvatura \\
\hline$\Lambda_{0,25 H}$ & Flecha de los puntos $1 / 4$ del estabilizador horizontal \\
\hline$\Lambda_{0,25 V}$ & Flecha de los puntos $1 / 4$ del estabilizador vertical \\
\hline$\Lambda_{b a}$ & Flecha del borde de ataque \\
\hline$\lambda$ & $\begin{array}{l}\text { Parámetro de estrechamiento de la cola en } \mathrm{V} \text { o relación entre la carga a } \\
\text { compresión que absorbe el revestimiento con la total de la sección. }\end{array}$ \\
\hline$\lambda_{H}$ & Parámetro de estrechamiento del estabilizador horizontal \\
\hline
\end{tabular}




\begin{tabular}{|c|c|}
\hline$\lambda_{w}$ & Parámetro de estrechamiento de un ala \\
\hline$\mu$ & Parámetro másico del avión para la ráfaga vertical \\
\hline$\mu_{v}$ & Parámetro másico del estabilizador vertical para la ráfaga lateral \\
\hline$v$ & Coeficiente de Poisson \\
\hline$\rho$ & Densidad del aire \\
\hline$\rho_{\text {mat }}$ & Densidad del material de fabricación del estabilizador horizontal y vertical \\
\hline$\rho_{x y}$ & Coeficiente de correlación de Pearson \\
\hline$\sigma_{V}$ & Deflexión de estela lateral sobre el estabilizador vertical \\
\hline$\sigma_{x}$ & Desviación típica de la variable aleatoria $X$ \\
\hline$\sigma_{x y}$ & Covarianza de las variables aleatorias $X$ y $Y$ \\
\hline$\sigma_{y}$ & Desviación típica de la variable aleatoria $Y$ \\
\hline$\tau_{\text {admisible }}$ & Esfuerzo admisible a cortadura \\
\hline$\tau_{c r}$ & Esfuerzo crítico de pandeo a cortadura \\
\hline$\tau_{r}$ & Efectividad del timón de dirección del estabilizador vertical \\
\hline$\phi$ & Ángulo de balance \\
\hline
\end{tabular}

$\Phi \quad$ Factor de eficiencia aerodinámica de la polar

$\Omega_{f l e} \quad$ Peso específico por unidad de superficie de la estructura de borde de ataque fijo

$\Omega_{f t e} \quad$ Peso específico por unidad de superficie de la estructura de borde de salida fijo

$\Omega_{t e f} \quad$ Peso específico por unidad de superficie de los controles de borde de salida 


\section{ACRÓNIMOS}

\begin{tabular}{|c|c|c|}
\hline Siglas & Definición en inglés & Definición en castellano \\
\hline ALC & Airplane Life Cycle & Ciclo de vida del avión \\
\hline CeRAS & $\begin{array}{c}\text { Central Reference Aircraft Data } \\
\text { System }\end{array}$ & - \\
\hline CEF & Critical Engine Failure & Fallo del motor crítico \\
\hline CFD & Computational Fluids Dynamics & $\begin{array}{l}\text { Dinámica de Fluidos } \\
\text { Computacional }\end{array}$ \\
\hline EASA & European Aviation Safety Agency & $\begin{array}{c}\text { Agencia Europea de Seguridad } \\
\text { Aérea }\end{array}$ \\
\hline FAR & Federal Aviation Regulation & - \\
\hline FEM & Finite Element Method & Método de Elementos Finitos \\
\hline GA & Genetic Algorithm & Algoritmo genético \\
\hline $1+D$ & - & Investigación y Desarrollo \\
\hline LCC & Life Cycle Cost & Coste del ciclo de vida del avión \\
\hline NASA & $\begin{array}{c}\text { National Aeronautics and Space } \\
\text { Administration }\end{array}$ & - \\
\hline $\begin{array}{l}\mathrm{OACI} / \\
\mathrm{ICAO}\end{array}$ & $\begin{array}{c}\text { International Civil Aviation } \\
\text { Organisation }\end{array}$ & $\begin{array}{l}\text { Organización de Aviación Civil } \\
\text { Internacional }\end{array}$ \\
\hline RPAS & Remoted Piloted Aircraft System & Sistema Aéreo no Tripulado \\
\hline VLM & Vortex-Lattice Method & Método de Vortex-Lattice \\
\hline
\end{tabular}





\subsection{ANTECEDENTES}

En la actualidad, existe una concienciación creciente por parte de la mayoría de los gobiernos de todo el mundo acerca de la emisión de gases contaminantes a la atmósfera (ACARE (The Advisory Council for Aeronautics Research in Europe) 2010; Busquin et al. 2001; Darecki et al. 2011; European Parliament 2009). El impacto medioambiental de las aeronaves recae en dos aspectos principales: emisión de ruido y emisión de partículas y gases contaminantes que contribuyen al cambio climático (Lee et al. 2010; Macintosh y Wallace 2009). Por otra parte, el crecimiento del tráfico aéreo es un continuo a lo largo de los años. De hecho, en 2017 se produjo un $4 \%$ más de crecimiento que en 2016, lo que corresponde con un ritmo de crecimiento mayor que el experimentado en los últimos años (Eurocontrol 2018). En Europa, esto se debe principalmente al auge de las compañías de bajo coste, la recuperación del tráfico ruso y de los viajes al norte de África. Sin embargo, el tráfico en el oeste de Europa se resiente un poco debido a las incertidumbres del mercado, tales como las negociaciones del Brexit, haciendo que las previsiones de crecimiento entre el año 2020 y 2024 se sitúen por debajo del $2 \%$. Desde el punto de vista internacional, en los países de economías en pleno desarrollo, como puede ser China o India, el crecimiento anual previsto hasta el año 2034 se sitúa en hasta un $6 \%$, siendo el segmento que más crece el que corresponde a aviones de pasillo único con un alcance inferior a $3000 \mathrm{NM}$ (Schulz 2018; Boeing 2017).

La convivencia de este crecimiento de tráfico aéreo con la intención de reducir las emisiones de $\mathrm{CO}_{2}$, principalmente, lleva a la Organización de Aviación Civil Internacional (OACl, o ICAO por sus siglas en inglés) a acordar en octubre de 2016 una resolución que marca una ruta de reducción de las emisiones de gases contaminantes por parte de la aviación internacional a partir del año 2021 (ICAO 2016). Esta resolución establece un esquema de reducción de emisiones por encima del $80 \%$ del nivel que haya en 2020 durante el periodo de 2021 a 2035 (European Commission 2017). Además, esta resolución está en línea con los objetivos marcados en los Acuerdos de París sobre el cambio climático (European Commission 2016).

Para permitir este desarrollo del mercado y la reducción de emisiones, las actuaciones de los aviones de transporte se han ido mejorando de manera muy importante durante las últimas décadas debido, especialmente, a desarrollos en áreas tecnológicas clave tales como aerodinámica, propulsión, emisiones de ruido y su disminución, estructuras, aviónica, materiales, etc. (Anderson 2002; Martinez-Val y Perez 2009). Un ejemplo de esta evolución de los aviones de transporte comercial es el análisis comparativo del parámetro de alcance, ya que es un buen descriptor de las actuaciones de un avión. Este parámetro casi se ha doblado en su valor con respecto a los inicios de los aviones con motor a reacción (Martinez-Val, Palacin y Perez 2008).

A pesar de esta clara evolución que han tenido los aviones de reacción a lo largo de su historia, la configuración general del avión apenas ha cambiado. Esta disposición general de los elementos se puede caracterizar por un fuselaje esbelto, un ala de alto alargamiento, dos superficies estabilizadoras unidas al cono de cola y los motores situados o bajo el ala o en el fuselaje posterior (Anderson 2002). Por tanto, se abre un campo de estudio en cuanto a la configuración de la aeronave se refiere, pudiendo analizar configuraciones no convencionales que potencialmente tengan beneficios desde el punto de vista de actuaciones o emisiones de gases contaminantes.

Existen numerosos estudios que contemplan el cambio de configuración del avión y las consecuencias que tendría en las actuaciones de este. Estas configuraciones 
novedosas suponen un cambio drástico en el aspecto del avión, como pueden ser las configuraciones joined-wing, ala volante, blended wing body o box-wing (Kroo 2005; Sharma 2015; Frota et al. 2010; Torenbeek 2013; Garcia-Benitez, Cuerno-Rejado y Gomez-Blanco 2016). En la Figura 1-1 se incluye un ejemplo de un blended wing body, en concreto el X-48B desarrollado por la NASA. Sin embargo, a pesar de los potenciales beneficios que presentan estas configuraciones, las compañías aéreas no se lanzan a comercializar estos aviones debido al cambio tan drástico que suponen. Otra posible aproximación es intentar buscar configuraciones no convencionales que supongan un cambio menor en el aspecto de la aeronave, de tal forma que sirva como un paso intermedio hacia estas configuraciones completamente diferentes a las empleadas hasta ahora. Por ejemplo, se pueden buscar configuraciones de cola no convencionales. Solamente el fuselaje del avión contribuye alrededor de un $28 \%$ a la resistencia parásita del avión y el empenaje en un 14\% (Anderson 1999). Estas cifras son orientativas, pero hacen ver la posible repercusión que podría tener una nueva configuración de cola que reduzca la resistencia del fuselaje posterior y estabilizadores de cola. Una reducción de la resistencia de la aeronave supone un ahorro en consumo de combustible y, en consecuencia, una reducción de emisiones de gases contaminantes, que llevaría a diseñar un avión con un menor impacto negativo en el medio ambiente.

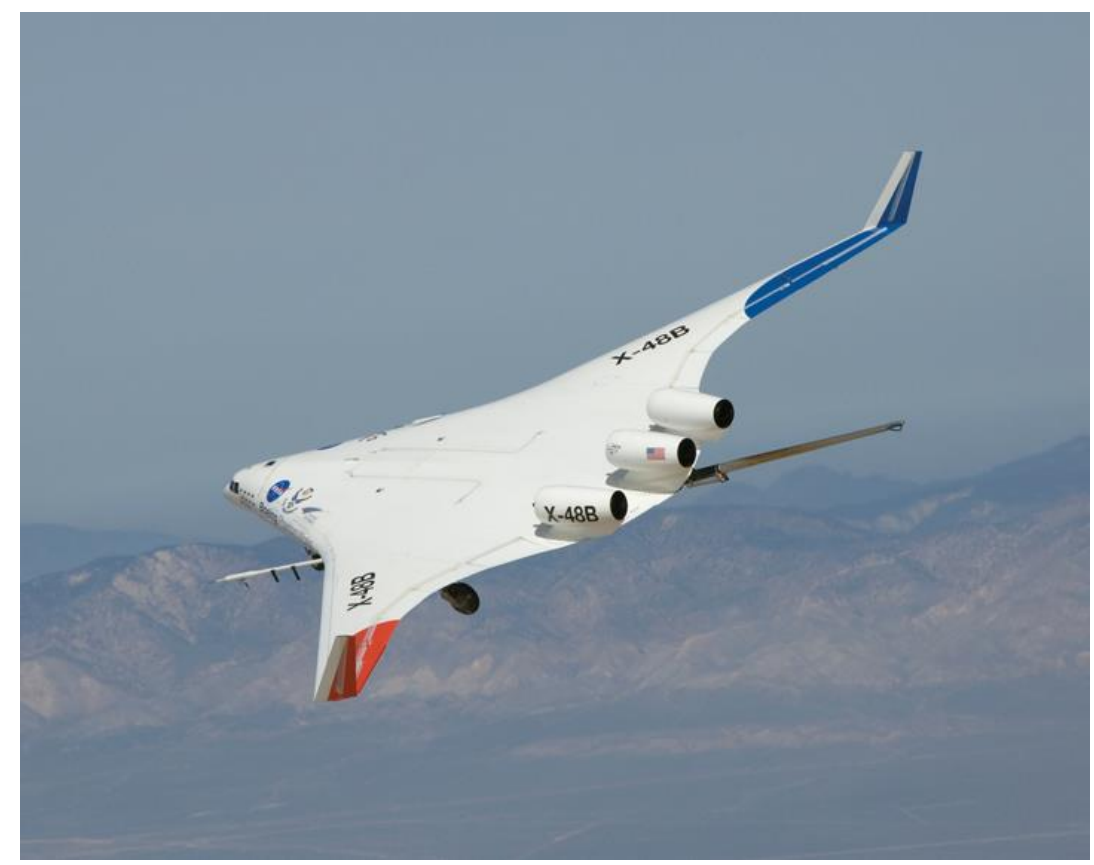

Figura 1-1 Imagen del X-48B, avión de configuración blended wing body desarrollado por la NASA para investigación. Fuente: (Thomas 2009).

La configuración de cola convencional utilizada en los aviones de transporte comercial consta de un estabilizador horizontal y uno vertical, ortogonales entre sí. Las principales funciones que tiene la cola horizontal es equilibrar longitudinalmente el avión, garantizar la estabilidad estática longitudinal, proporcionar un comportamiento dinámico longitudinal aceptable y producir fuerzas que permitan maniobrar en el plano vertical. Por otro lado, el estabilizador vertical debe asegurar el control del avión tras el fallo del motor crítico en el segundo segmento, garantizar la estabilidad estática lateral, proporcionar un comportamiento dinámico lateral aceptable, permitir el aterrizaje con ciertas condiciones de viento cruzado y producir las fuerzas necesarias para maniobrar fuera del plano vertical (Torenbeek 1982). Aparte de esta configuración convencional, existen muchas otras cuyas funciones son la conjunción de las de ambas superficies anteriormente descritas. Las configuraciones de cola más habituales se pueden 
clasificar en varios grupos: A, B y C, según diversos autores (Raymer 2012; Torenbeek 1982). Además, dentro de estos grupos se pueden encontrar varios subgrupos recogidos en la Tabla 1-1. Por supuesto, existen muchas otras configuraciones de cola que se pueden diseñar que no están recogidas en la clasificación anterior. Un ejemplo de varias de ellas se recoge en la Figura 1-3.

\begin{tabular}{cc}
\hline A-1 & Convencional \\
\hline A-2 & Cruciforme \\
A-3 & Cola en T \\
B-1 & Cola en H \\
\hline B-2 & Boom-mounted \\
\hline C & Cola en V \\
\hline
\end{tabular}

Tabla 1-1 Clasificación de las configuraciones de cola más habituales. Fuente: elaboración propia.

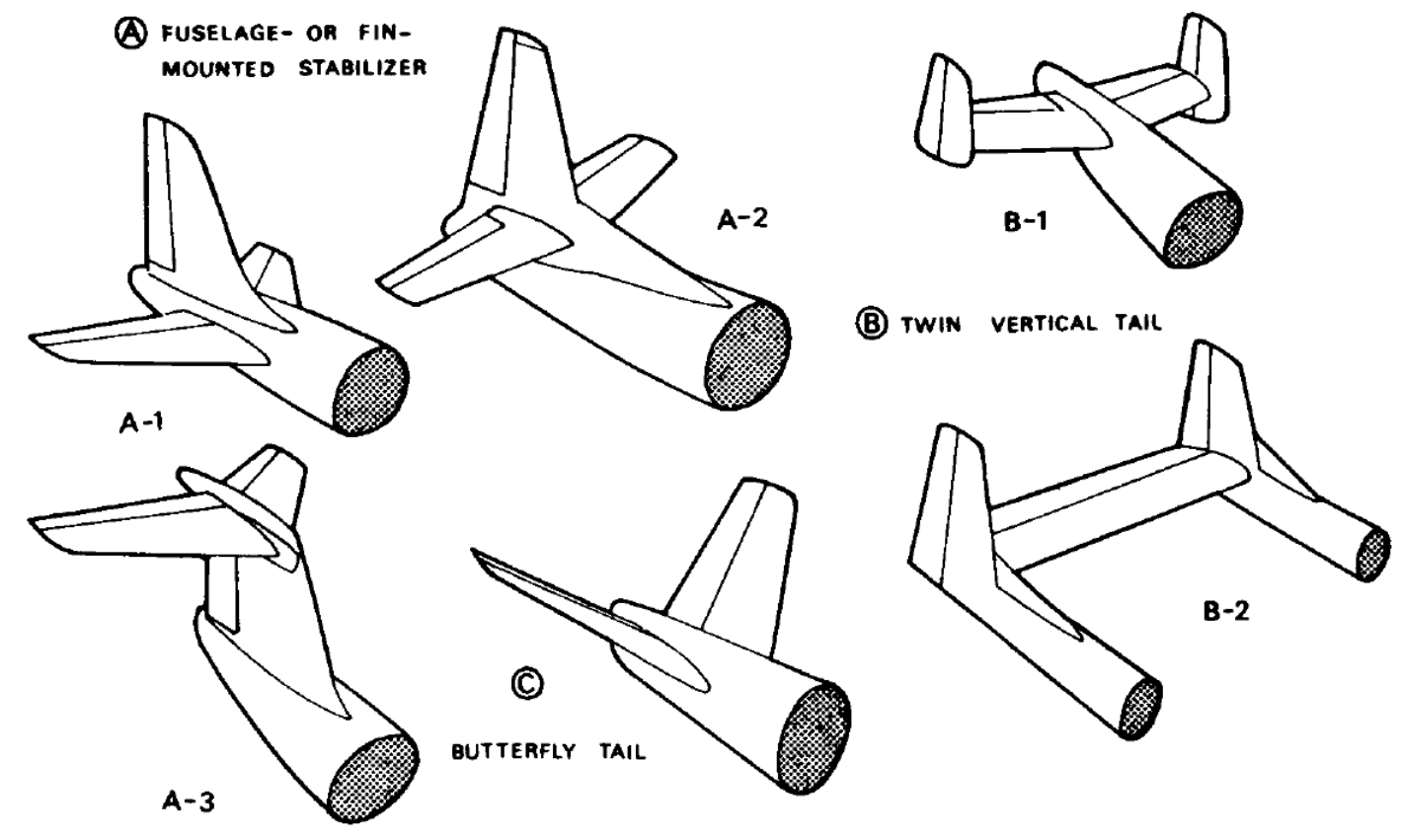

Figura 1-2 Definición de clasificación de las configuraciones más habituales. Fuente:

(Torenbeek 1982). 


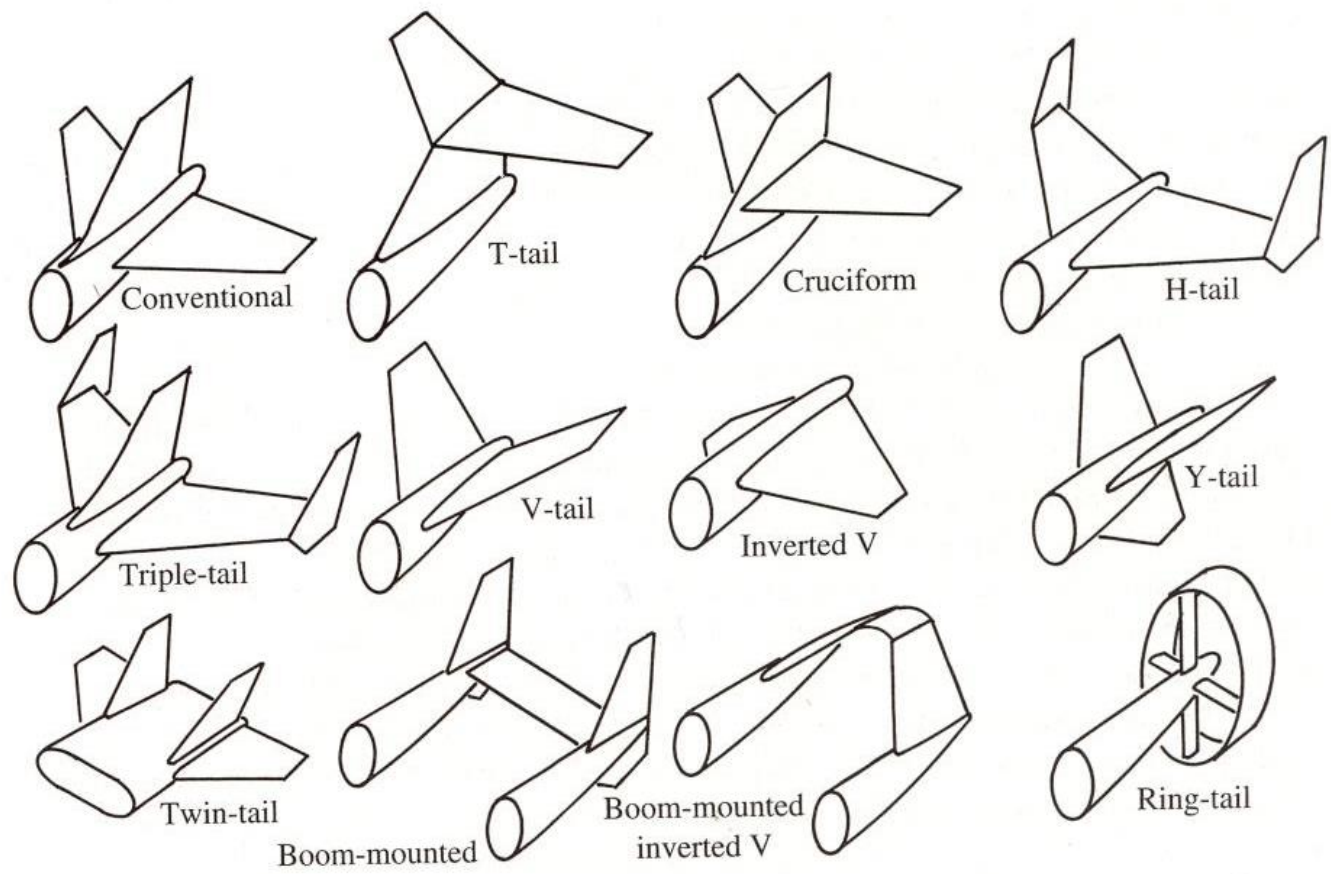

Figura 1-3 Recopilación de diferentes configuraciones de cola. Fuente: (Raymer 2012).

En el pasado se han realizado numerosos estudios para mejorar el rendimiento de las superficies de cola convencional (Viswanath 1996; Abbas, Dias y Cabello 2004; Lucas, Velazquez y Vega 2011). Los estudios se pueden dividir en dos principales líneas de investigación: investigaciones detalladas del campo fluido alrededor del cono de cola y estabilizadores $y$, por otro lado, estudios encaminados a encontrar nuevas configuraciones. A pesar de ser dos líneas distintas, pueden estar fuertemente ligadas, ya que un mejor análisis de lo que ocurre en el fluido alrededor del fuselaje posterior puede desembocar en la selección de una nueva configuración de cola no convencional que reduzca la resistencia aerodinámica.

Tal y como se ha comentado previamente, una reducción de la resistencia parásita de las superficies de cola supone un menor peso de combustible necesario para realizar la misión del avión. El consumo de combustible es una de las principales contribuciones al coste directo de operación de una aeronave de transporte comercial (Torenbeek 1982). Este combustible puede verse reducido por diversos factores. Entre ellos se incluye la mejora del consumo específico del motor o una correcta elección de la trayectoria de vuelo, como puede ser volar a la altitud de mínimo consumo entre otras. Estas vías cada vez tienen menos margen de mejora, ya que los motores han evolucionado mucho a lo largo de la historia y las rutas de vuelo cada vez están más saturadas y no se puede conseguir que todos los aviones vuelen en sus condiciones óptimas de consumo. Otras dos estrategias para reducir el consumo de combustible son la reducción de la resistencia aerodinámica del avión, como ya se ha expuesto anteriormente, o la reducción de peso del mismo. Una reducción de la resistencia aerodinámica supone que los motores del avión tendrán que desarrollar menos empuje para volar en una cierta condición, a igualdad del resto de parámetros relevantes del avión, y, en consecuencia, gastarán menos combustible. En cuanto a la reducción de peso, centrado en las posibilidades del cono de cola y estabilizadores, las principales evoluciones se atribuyen al uso de materiales compuestos, que presentan una densidad menor que los materiales metálicos, pero pueden desarrollar las mismas funciones que estos últimos. El impacto que tiene la reducción de peso en el coste de operación de una aeronave es un tema 
estudiado con anterioridad. Un estudio realizado por la aerolínea SAS Scandinavian Airlines, estima que el coste de combustible necesario para operar un A330 durante su vida útil, aproximadamente 25 años, por kilogramo de masa volada es de entre 1,500 y $2,000 € / \mathrm{kg}$. Teniendo en cuenta que el peso de la estructura es del orden del $20-30 \%$ del peso de la aeronave, se puede concluir que una reducción de peso supone un ahorro económico considerable (Kaufmann 2008). 


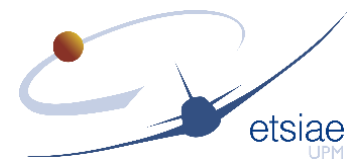

\subsection{ESTADO DEL ARTE DE CONFIGURACIONES DE COLA NO CONVENCIONAL}

Por todo lo expuesto hasta ahora, en este apartado se va a realizar un análisis del estado del arte de diferentes configuraciones y dispositivos de cola no convencionales. El número de documentos es bastante elevado, por lo que se ha decidido clasificarlos en cuatro grupos principales: configuraciones planas, configuraciones no planas, tailless y canard. Esta clasificación se ha inspirado en la que hacen otros autores para clasificar la configuración innovadoras pero que afectan a la disposición completa del avión, no centrado en la cola (Torenbeek 2007; Kroo 2005).

Las configuraciones planas son las que se utilizan habitualmente en los aviones de transporte comercial. Engloban las colas de configuración convencional, cola en T y cruciforme. Los avances que se recopilan en este grupo están relacionados con ciertos sistemas y dispositivos utilizados sobre estas configuraciones, que permanecen inalteradas. Las mejoras que introducen estos dispositivos suponen reducción de peso o mejoras en la eficiencia de los controles, entre otros aspectos.

Los conceptos de configuraciones no planas agrupan una gran variedad de colas, como pueden ser biplanos, superficies cerradas, winglets o colas en anillo. A su vez, estas configuraciones se pueden clasificar atendiendo al aspecto general de las mismas. Se ha decidido establecer las siguientes tres categorías: superficies abiertas, superficies cerradas y dispositivos de punta. En el primer grupo se incluyen las configuraciones que contemplen más de una superficie vertical, pero que no llegan a formar una superficie cerrada. Las superficies cerradas son configuraciones que presentan varias superficies horizontales y/o verticales que están conectadas de alguna manera, formando una superficie cerrada. En la categoría de dispositivos de punta se incluyen dispositivos de punta de superficie, tales como los winglets o similares. Es cierto que la frontera entre este grupo y el primero puede no estar claramente definido, ya que un winglet de gran tamaño podría pasar a considerarse como una configuración de superficie abierta. La Figura 1-4 recoge un ejemplo de cada una de estas tres categorías.

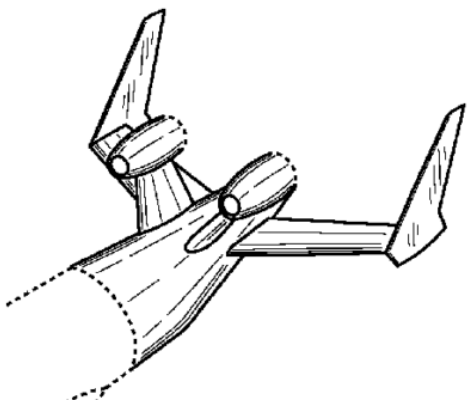

(a)

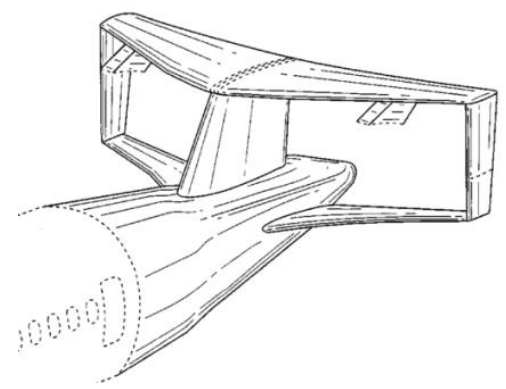

(b)

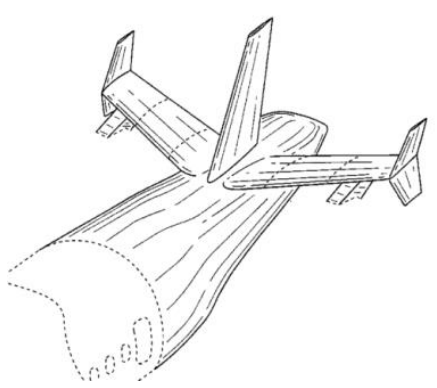

(c)

Figura 1-4 Ejemplos de configuraciones no planas: (a) superficies verticales múltiples, Fuente: (Gall 2010a), (b) superficies cerradas, Fuente: (Cazals 2009a) y (c) diedro variable, Fuente: (Cazals 2009b).

Por último, en la categoría de aviones tailless se incluyen los diseños que no tienen ningún tipo de superficie en la cola, y en la categoría canard los aviones que tienen los estabilizadores situados por delante del ala. Estas categorías no se van a analizar en este estado del arte de configuraciones de cola no convencionales ya que se escapan de los objetivos de esta tesis y no se pueden considerar estrictamente no 
convencionales, como la configuración canard, o son inviables para su adaptación a un avión convencional, como la tailless.

\subsubsection{Configuraciones planas}

Los dispositivos y tecnologías recogidos en esta sección son muy diversos, pero todos ellos tratan de proveer a las superficies de cola de algún beneficio desde varios puntos de vista. Como se ha indicado anteriormente, estos dispositivos se aplican sobre aviones de transporte comercial con las configuraciones de cola más habituales, como son las convencionales, cola en $\mathrm{T}$ e incluso cruciforme.

Una de las principales líneas de investigación son las estructuras inteligentes. Este tipo de estructuras tratan de aprovecharse del hecho de que las fuerzas aerodinámicas generadas sobre las superficies de cola dependen de la geometría de esta, y viceversa. La disciplina que considera esta interacción entre geometría y estructura es la aeroelasticidad. Por tanto, la idea es controlar y adecuar la geometría para conseguir unas superficies de cola tan eficientes como sea posible. El modo de controlar esta geometría puede ser activo o pasivo. El primero de ellos se produce por medio de una serie de actuadores, mientras que el segundo se lleva a cabo exclusivamente por la acción del viento.

El concepto de adaptar la geometría para mejorar la eficiencia de una superficie sustentadora recibe el nombre de morphing. Existen estudios que tratan de aplicar este concepto a un estabilizador vertical completo (Trapani y Guo 2008), o únicamente al timón de dirección (Castillo-Acero, Cuerno-Rejado y Gómez-Tierno 2014). En el primero de los casos, se intenta diseñar un estabilizador vertical que no necesite del timón de dirección, ya que modificando la geometría del mismo mediante actuadores se pueden acometer las funciones que recalaban sobre el timón. Esto podría reducir la complejidad estructural del estabilizador y en consecuencia su peso, de acuerdo con las investigaciones realizadas. A pesar de ello, surgen varias desventajas como podrían ser si realmente el sistema de actuadores será más eficiente que el timón de dirección clásico o que son necesarios una serie de refuerzos estructurales, principalmente en el borde de ataque, para mantener las deformaciones de la geometría dentro de los límites deseables. Por tanto, se concluye que nuevos materiales más avanzados serían necesarios para poder implementar esta solución en los aviones de transporte comercial. En el caso de que únicamente se aplique este concepto al timón de dirección, lo que supone una solución menos radical que la anterior. Con esta idea la eficiencia del estabilizador se ve mejorada, que se estima en un beneficio potencial del $16 \%$ en términos de fuerza lateral con respecto a una configuración convencional Figura 1-5. 

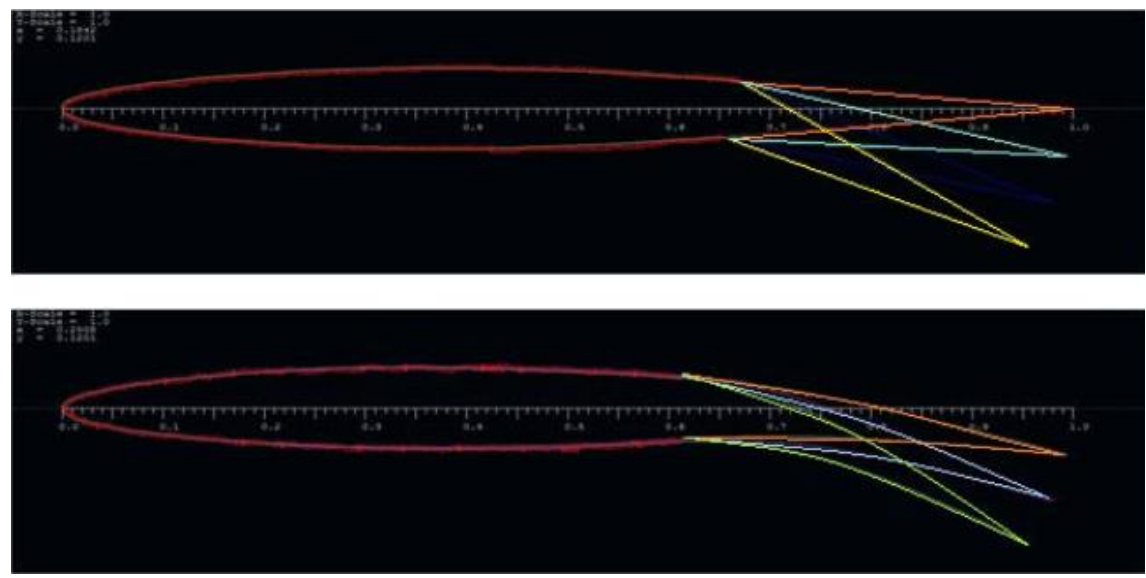

Figura 1-5 Comparativa de un perfil NACA 64010 sin timón con morphing (arriba) con el timón con morphing (abajo). Fuente: (Castillo-Acero, Cuerno-Rejado y Gómez-Tierno 2014).

Otra línea de investigación trata sobre el control del flujo de aire alrededor de las superficies de cola. Gobernar este flujo es útil para mejorar la eficiencia de las superficies de control, evitando fenómenos perjudiciales como la separación de la capa límite o el bataneo. Uno de estos dispositivos se centra en controlar activamente la capa límite a lo largo del timón de profundidad (Meister, Pfennig y Held 1999). El sistema consiste en una serie de perforaciones en el borde de ataque del control por donde el aire se succiona, de tal forma que se fuerza a que le flujo sea laminar. Además, este mismo sistema puede servir de sistema de deshielo, cambiando el sentido de paso del aire a través de las perforaciones y expulsar aire caliente. Este sistema debería mejorar la efectividad del control y reduce la resistencia de la superficie vertical, aunque no está cuantificado el grado de mejora del sistema. Un sistema similar investigado desde hace tiempo son los conocidos como actuadores sweeping jets (Seele et al. 2012). Este sistema consiste en colocar una tobera que esté emitiendo un flujo de aire justo en el eje de charnela del timón de dirección para conseguir que se adhiera la capa límite a la superficie del control, mejorando así su efectivad en torno a un $30 \%$. El esquema del camino que sigue el flujo de aire se incluye en la Figura 1-6. La principal desventaja que presentan estos sistemas es que los actuadores tienen que trabajar en una determinada condición del fluido, siendo las velocidades al menos 3 veces mayor que la del flujo libre pero mantenerse subsónico, preferiblemente.

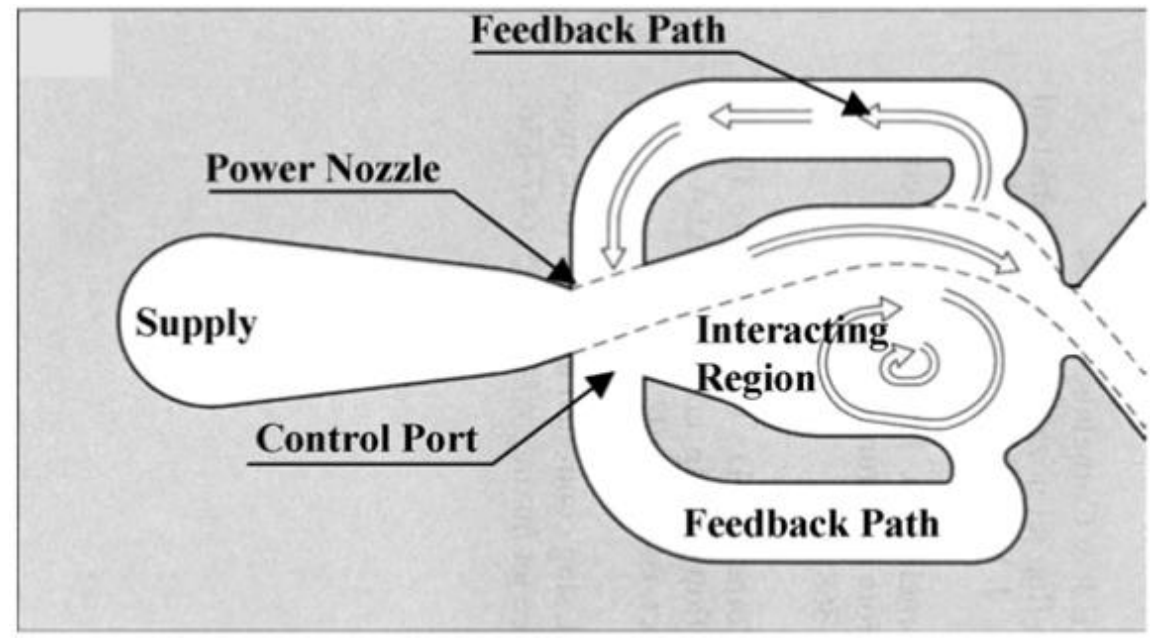

Figura 1-6 Diseño conceptual de un actuador sweeping jet. Fuente: (Seele et al. 2012). 
También existen sistemas que tratan de controlar el flujo de aire alrededor de un estabilizador horizontal con el fin de prescindir del timón de profundidad (Gilbert 1995). Este sistema en concreto instala una serie de spoilers a lo largo del extradós e intradós del borde de ataque de la superficie, tal y como se muestra en la Figura 1-7. La deflexión variable de los spoilers permite controlar la sustentación sobre la superficie de cola. El principal inconveniente que puede presentar este sistema es el posible peso y complejidad mayores que podría presentar el conjunto de actuadores necesarios. Además, la alteración de la geometría del perfil justo en el pico de succión del mismo debería estudiarse con detenimiento antes de instalar este dispositivo en un estabilizador de un avión de transporte comercial.

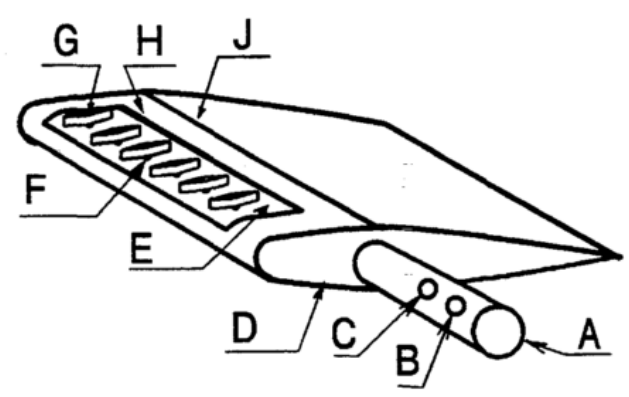

Figura 1-7 Spoilers sobre un estabilizador horizontal para eliminar el timón de profundidad. Fuente: (Gilbert 1995).

La superficie estabilizadora horizontal se dimensiona para una cierta condición crítica, por lo que para la mayor parte de la duración del vuelo el tamaño está sobredimensionado. Por tanto, se plantean estudios que permitan al estabilizador horizontal cambiar su superficie para optimizar el tamaño en diferentes fases de vuelo al mismo tiempo (Couffignal y Vinches 2012). Esta patente consiste en que haya una parte central fija del estabilizador y, al menos, una superficie móvil que deslice sobre la anterior desde el interior para aumentar o disminuir la superficie en planta del estabilizador, incluyendo una serie de flaps para evitar la discontinuidad entre las superficies fija y las móviles. De nuevo, a pesar de que el área se está intentando optimizar, el peso adicional necesario para llevar esa superficie móvil y el sistema que la mueve podría no compensar los beneficios en términos de resistencia aerodinámica.

Desde el punto de vista de la estructura, también se han producido mejoras en investigación. Hoy en día, el uso de materiales compuestos está muy extendido en elementos estructurales cada vez de mayor importancia. En concreto en las superficies de cola, existen varios trabajos que muestran la investigación que se ha realizado en este campo, desde el diseño de nuevos conceptos de costillas para un estabilizador horizontal (Zuniga Sagredo 2008), evoluciones en el cajón de torsión también para estabilizadores horizontales (Law 2006) e incluso en la integración completa de la estructura de un estabilizador (Ramírez Blanco et al. 2012). El objetivo principal de estas nuevas técnicas y procedimientos de fabricación es el de aumentar el porcentaje de material compuesto que se utiliza en la estructura y establecer mejoras desde el punto de vista de las inspecciones y otras tareas de mantenimiento.

\subsubsection{Configuraciones no planas}

\subsubsection{Superficies abiertas}

Este grupo engloba todas las soluciones que son aplicables a las configuraciones de cola que sean no planas, pero no formen una superficie cerrada. Estos diseños se 
encuentran en fases conceptuales para aviones de transporte comercial, pero se pueden indicar algunos potenciales beneficios que presentan algunas de estas opciones a priori.

La configuración de cola en U es un ejemplo característico de esta clasificación. Esta opción ha sido estudiada en varios trabajos, donde se ha llegado a la conclusión que la cola en U no presenta una potencial reducción de resistencia (Carrier y Gebhardt 2005). Sin embargo, las actuaciones que se desarrollan con esta configuración son similares a las de una cola convencional, incluso habiendo considerado una cola completamente flexible con conceptos de morphing (Cesnik, Su y Fellow 2005). Por tanto, las colas en $U$ pueden ser empleadas por otro motivo que no sea mejorar la resistencia o actuaciones de la cola, por ejemplo, para reducir el ruido de motores montados en la cola. A pesar de estos potenciales beneficios, los estudios deben desarrollarse un paso más para poder extraer conclusiones más relevantes y que finalmente se puedan aplicar a los aviones de transporte comercial. Aun así, varias investigaciones que se han desarrollado en el marco europeo tratan sobre esta configuración en U. Es el caso del proyecto NACRE (Frota et al. 2010). Este proyecto trata de integrar y validar tecnologías que habiliten nuevos conceptos de avión que puedan ser potencialmente desarrollados. Por tanto, no se concentra en una configuración en concreto, sino que presenta soluciones genéricas a nivel de componentes del avión, como por ejemplo el ala, la cabina o el fuselaje, que puedan ser aplicadas para generar una nueva configuración de avión. Como resultado, se desarrollaron un conjunto de configuraciones no convencionales. En varias de ellas, la denominada Pro Green y Simple Flying Bus, se contempló la posibilidad de instalar empenajes innovadores. Concretamente, en la Pro Green, que se enfocó con el objetivo de reducir el impacto medioambiental del avión, se decidió desarrollar el concepto de una cola con múltiples estabilizadores verticales. Por otro lado, en la Simple Flying Bus se trabajó con la idea de diseñar un avión con los menores costes de fabricación posibles, y se contempló la posibilidad de utilizar la cola en $\mathrm{V}$, ya que otros proyectos previos habían trabajado ya con esta configuración en concreto, como es el proyecto NEFA FP5 (Mirat 2006).

La configuración de cola en $\mathrm{V}$ es la más sencilla que se puede incluir en las configuraciones no planas abiertas. Es cierto que está configuración está mucho más extendida en el campo de las aeronaves pilotadas por control remoto, como por ejemplo el Northrop Grumman Global Hawk o el General Atomics MQ-9 Reaper. Sin embargo, existe un avión tripulado con esta configuración que fue fabricado en serie, como es el caso del Beechcraft Bonanza Model 35. Este avión es pertenece al segmento de aviación general, tiene seis asientos y se introdujo en el mercado en el año 1947. En principio, la cola en $\mathrm{V}$ presenta potenciales beneficios en términos de resistencia aerodinámica y de peso, ya que son necesarias únicamente dos superficies de cola en lugar de las tres que presenta la configuración convencional. Puesto que las aplicaciones son principalmente para aviones pilotados por control remoto, los estudios se centran en investigar en este contexto. Tratan de comparar las derivadas de estabilidad direccionales entre una configuración convencional y una cola en $\mathrm{V}$, llevando a cabo ensayos en túnel aerodinámico a bajos números de Reynolds (Musa et al. 2015, 2014). Precisamente por el número de Reynolds empleado, los resultados son útiles para los RPAS (Remoted Piloted Aircraft System) que lleven esta configuración. También se han llevado a cabo estudios semejantes que realizan las mismas comparaciones pero por medio de técnicas de mecánica de fluidos computacional (CFD), de nuevo para números de Reynolds bajos (Zhang et al. 2008, 2013). Incluso esta configuración se ha estudiado desde el punto de vista del desarrollo de modelos dinámicos para RPAS que tienen en cuenta distintos modelos de fallo, con el objetivo 
de construir autopilotos tolerantes al fallo para este tipo de aeronaves (GarcíaHernández, Cuerno-Rejado y Pérez-Cortés 2017).

Tal y como se ha comentado anteriormente, la cola en $V$ se ha estudiado también para ser instalada en aviones de transporte comercial. Es el caso del proyecto NEFA FP5 (Mirat 2006), que concluía que se podía reducir el área total de la cola en un $20 \%$ a través de esta configuración, pero que la complejidad añadida a los sistemas resultaba en no tener beneficios desde el punto de vista de costes o peso comparado con la configuración de cola convencional. También hay estudios que tratan sobre configuraciones de blended wing-body que presentan este tipo de cola, analizando el efecto de variar el ángulo de diedro del estabilizador modelizando las fuerzas aerodinámicas por medio de programas basados en métodos vortex-lattice (VLM), por lo que solamente se trabajaron con condiciones de velocidad inferiores a $50 \mathrm{~m} / \mathrm{s}$ (Song et al. 2014).

Por último, se encuentran configuraciones de nuevo en el mundo de los RPAS que se pueden recoger en esta categoría, como es el caso de las colas con tail boom. Esta configuración consiste en alejar del fuselaje las superficies estabilizadoras por medio de unas barras que reciben el nombre de booms, tal y como se muestra en la Figura 1-2 en la clasificación B-2. No se va a hacer mucho hincapié en esta configuración, ya que se recurre a ella cuando el fuselaje es demasiado corto y se quiere mejorar la estabilidad de la aeronave alejando las superficies de control del centro de gravedad. Este caso no aplica a la aviación comercial, por tanto, no se contemplará en este estudio. Sí que existen algunas patentes que recogen diseños de cola innovadores que estarían bajo el paraguas de esta clasificación de configuraciones no planas abiertas. Algunas de ellas se incluyen en la Figura 1-8. Estos diseños no están desarrollados, únicamente están patentados ante una posible inclusión en la aviación comercial del futuro.

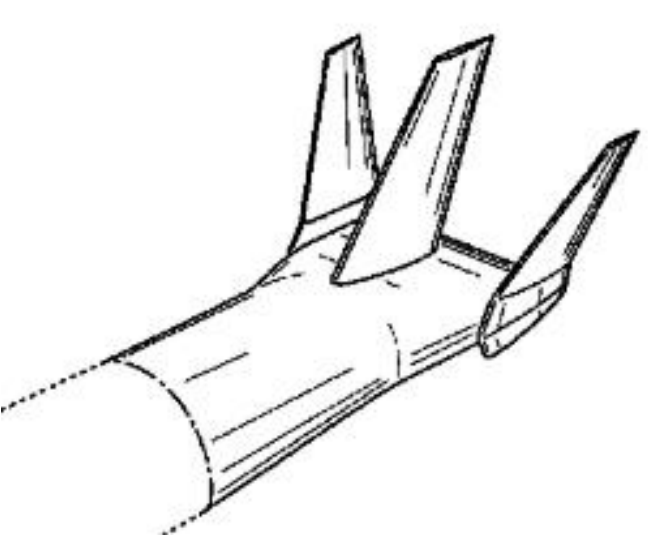

(a)

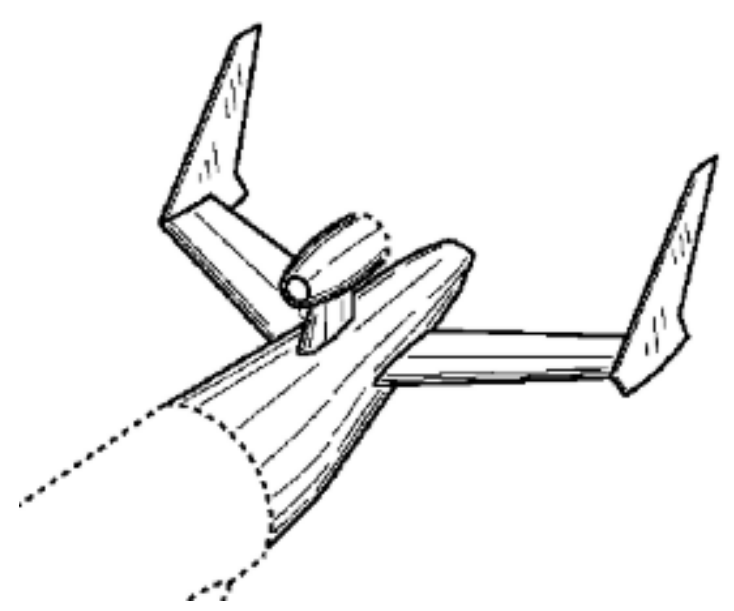

(b)

Figura 1-8 Configuraciones de cola no planas abiertas en patentes. Fuente: (a) (Cazals y Sagne 2010) y (b) (Gall 2010b).

\subsubsection{Superficies cerradas}

Las propiedades aerodinámicas de las configuraciones no planas cerradas son bastante interesantes. En este grupo se incluyen las configuraciones box-wing, joined-wing, y colas en anillo. Estas configuraciones aparecen con el objetivo de reducir los vórtices de punta de ala. Se muestra un diseño conceptual de un avión con configuración box wing elaborado por la empresa Lockheed Martin en la Figura 1-9. Tanto esta 


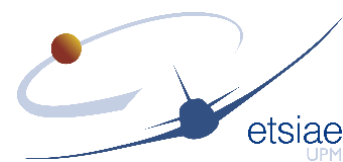

configuración como la joined wing consisten en contactar el ala principal con un ala situada en el fuselaje posterior con flecha inversa, con la diferencia de que la configuración box wing necesita de una superficie vertical para realizar esa conexión, pero la joined wing no, como se puede ver en la Figura 1-10. Por ejemplo, las superficies en anillo reducen la resistencia de vórtices en alrededor de un $50 \%$ con respecto a la configuración convencional de la misma envergadura (Kroo 2005). Además, la configuración box wing alcanza el mínimo de resistencia inducida en cuanto a configuraciones no planas se refiere, para una envergadura dada. Estas configuraciones no solo afectan a la disposición de las superficies de cola, sino a la del avión completo. Como se ha expuesto previamente, se quiere focalizar el estudio en las configuraciones no convencionales de cola.

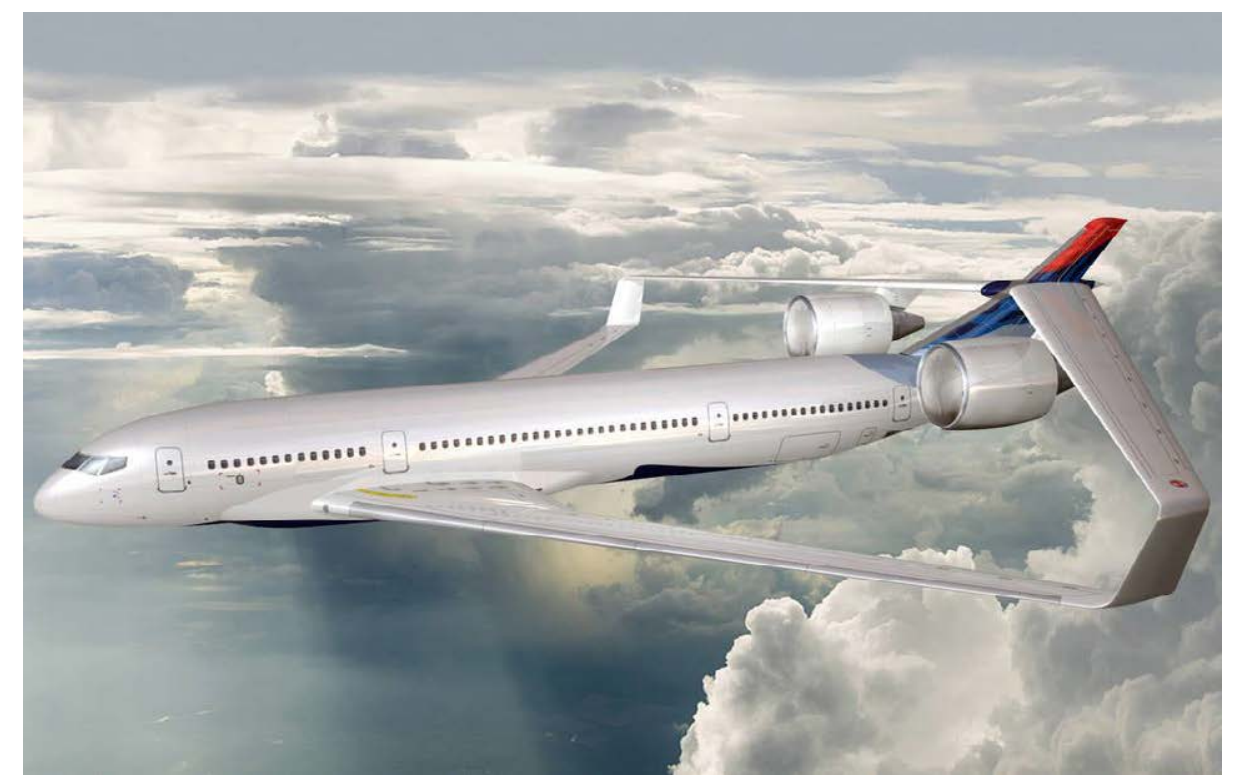

Figura 1-9 Configuración box wing propuesta por la NASA y Lockheed Martin's. Fuente: (Gipson 2015).

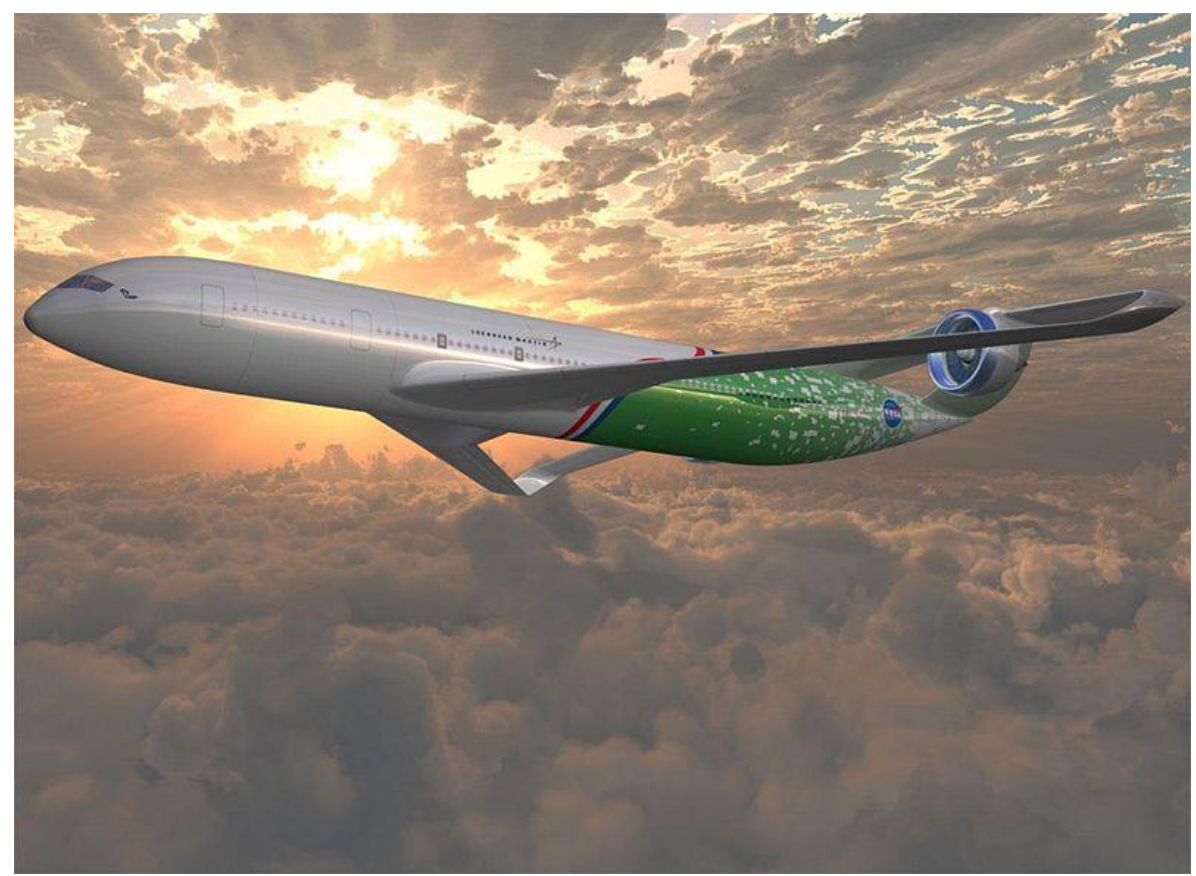

Figura 1-10 Configuración box wing propuesta por la NASA y Lockheed Martin's. Fuente: (NASA 2011) 
Analizando las posibilidades que tendrían estos conceptos de superficies cerradas para ser aplicadas exclusivamente a las configuraciones de cola, sin modificar la configuración alar, la principal opción es la cola en anillo. Desde el punto de vista estructural, la rigidez de la cola se ve incrementada con respecto a una configuración convencional, por el mero hecho de que la superficie esté cerrada. Por tanto, podría presentarse ventajas en cuanto a reducción de peso se refiere. Sin embargo, se plantean varios inconvenientes. Los timones de dirección y profundidad son más difíciles de implementar, además de que se produciría un acoplamiento entre el control lateraldireccional y el longitudinal. Por otra parte, habría que realizar un estudio sobre cómo afecta la estela del ala sobre la efectividad de la cola en anillo. De hecho, esta configuración se ha probado únicamente en un avión ultraligero de fabricación casera (Figura 1-11). El autor del prototipo indica una serie de problemas que ha tenido para la fabricación de la cola para poder darle la forma a los perfiles y que esta cola presenta un buen comportamiento frente a la pérdida, a pesar de que la resistencia aerodinámica es superior a la de una cola convencional equivalente. Por tanto, se puede decir que esta configuración de cola no está lo suficientemente madura como para ser implementada en un avión de transporte comercial.

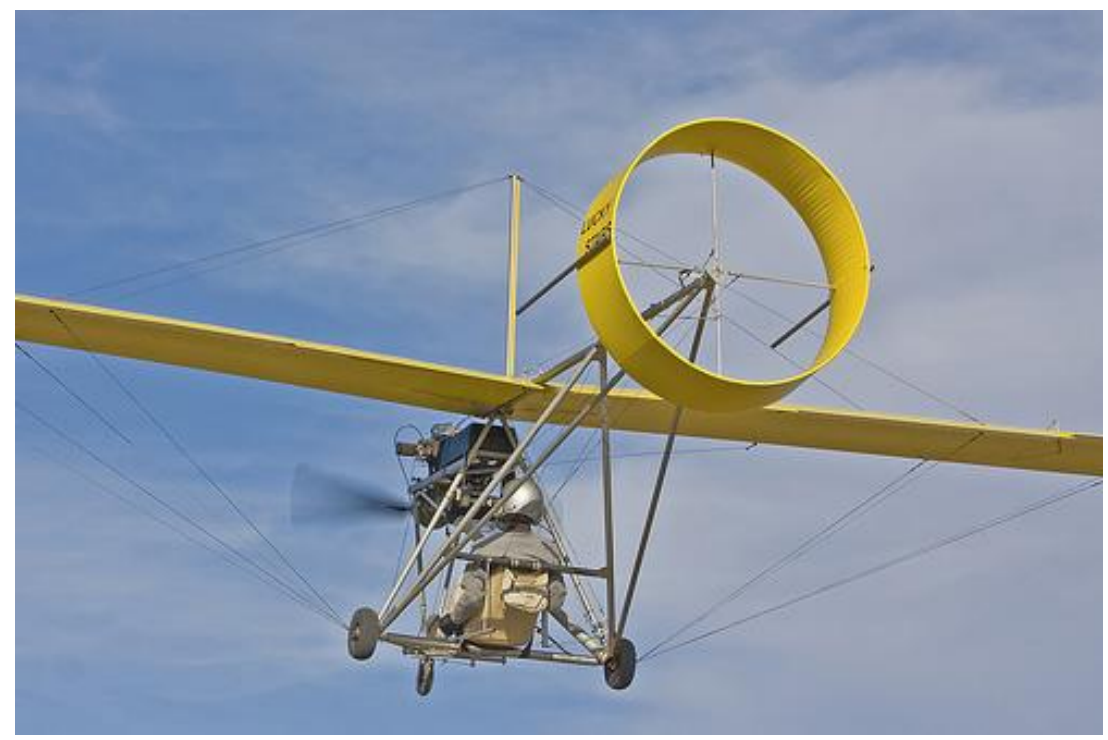

Figura 1-11 Configuración de cola en anillo de un avión de fabricación casera. Fuente: («Ring Tail debuts» 2011)

Aparte de la cola en anillo, existen patentes que contemplan configuraciones de cola cerradas que se basan en las configuraciones alares como la box wing, pero que solamente afectan al cono de cola. Estas geometrías no están estudiadas en profundidad, por lo que sería necesario plantear un análisis de ventajas e inconvenientes que tendrían cada una de estas ideas. En la Figura 1-12 se incluyen algunas de las opciones que se han encontrado en la literatura abierta. 


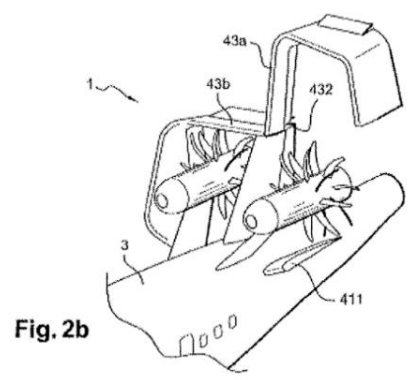

(a)

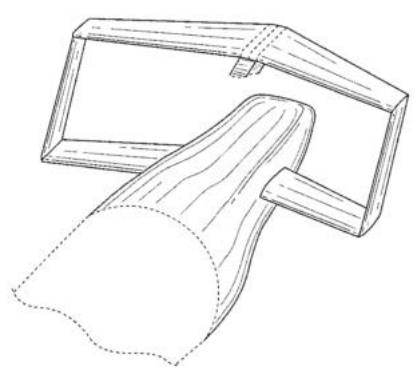

(b)

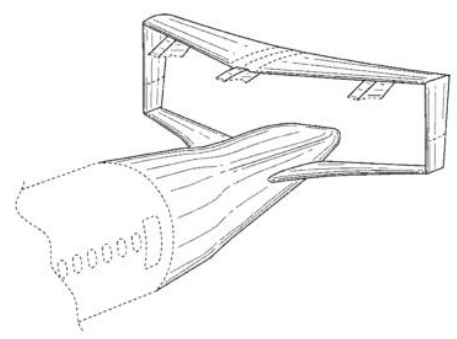

(c)

Figura 1-12 Ejemplos de configuraciones de cola cerradas. Fuente: (a) (Cazals y Druot 2012), (b) (Cazals 2009c) y (c) (Cazals 2009d)

\subsubsection{Dispositivos de punta de ala}

Los dispositivos de punta de ala, como pueden ser los winglets entre otros, tienen el objetivo de reducir la resistencia inducida del ala (Mclean 2005). A pesar de ello se presentan una serie de desventajas que también se deben contemplar a la hora de utilizar estos dispositivos. Estas giran en torno a dos conceptos: el incremento de resistencia parásita y el incremento de peso. El primero de ellos se produce porque el área mojada aumenta. El segundo se produce por el incremento de peso que introduce el mismo dispositivo y los refuerzos necesarios para soportarlos. Por tanto, como en la mayoría de las circunstancias, es necesario realizar un análisis de beneficios y perjuicios que puede tener instalar estos dispositivos.

Hoy en día se utilizan estos dispositivos principalmente en el ala principal, ya que la resistencia inducida generada por ella es mucho mayor que la que se genera en el estabilizador horizontal, aunque los beneficios serían exactamente igual en estas superficies, eso sí, a mucha menor escala. Es por ello que existen estudios que han analizado los beneficios que pueden tener diferentes geometrías de dispositivos de punta de ala en la reducción de resistencia inducida (Cone 1962; Luciano, Antonio y Giovanni 2014). Una variable importante es el ángulo que forma el dispositivo de punta de ala con el plano sustentador. De hecho, en estos estudios se analiza el efecto de colocarlos en una cola en $\mathrm{V}$. Una evolución lógica de estos dispositivos es hacia configuraciones abiertas como la cola en $U$ e incluso, si se extienden los bordes marginales de los dispositivos de forma paralela al plano sustentador, a una configuración en $\mathrm{C}$ o cerrada. Debido a esto, se comentaba previamente que la frontera entre esta categoría y las anteriores es un poco difusa. Lo que ocurre es que se le ha querido dar un trato por separado, ya que se pueden encontrar diseños que incluyen este tipo de dispositivos en estabilizadores horizontales de aviones de transporte comercial. De nuevo, estos diseños únicamente están sobre el papel y patentados, como el de la Figura 1-13. 


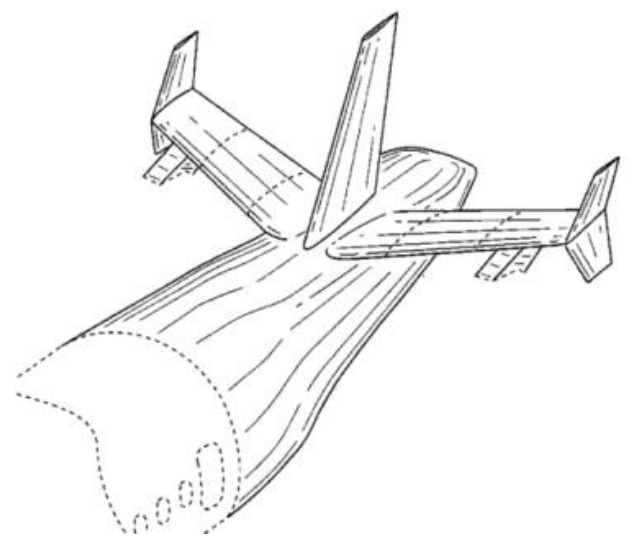

Figura 1-13 Ejemplo de configuración de cola con dispositivos de punta de ala. Fuente: (Cazals 2009b). 


\subsection{HERRAMIENTAS CLÁSICAS DE DISEÑO CONCEPTUAL Y PRELIMINAR}

El diseño y fabricación de un nuevo avión de transporte por parte de una empresa comienza por establecer las características esenciales a alto nivel del producto, el concepto general del mismo y, posteriormente, sigue con una selección de geometría y sistemas detallados, así como, por último, de la fabricación y distribución del mismo. La propuesta de diseño del nuevo avión normalmente lo lleva un departamento de la compañía en concreto, que incluyen aspectos técnicos, tecnológicos o de negocio. Este departamento tiene un trabajo esencial en la definición de nuevos productos y su éxito tiene una influencia clara en el futuro y eficacia de la empresa. Este departamento se encarga, principalmente, de tareas entre las que se incluyen las siguientes: proyectos futuros, desarrollo de procedimientos y herramientas, habilitación de tecnologías y evaluación de la competencia. La primera de estas tareas engloba las actividades relacionadas con estudios de viabilidad y propuestas novedosas de diseños distintos a los que se llevaban a cabo en la empresa. Debido al carácter general de estos estudios, las actividades tienen una fuerte componente multidisciplinar, por lo que el departamento debe tener miembros ingenieros expertos en diferentes campos tales como física del vuelo, estructuras o sistemas. En la siguiente categoría se incluye el desarrollo de procedimientos y herramientas que sirvan para el dimensionado geométrico, análisis de actuaciones, estimación de pesos y de costes. En general, estas herramientas permanecen bajo la protección de la compañía y no son accesibles, ya que muestran las capacidades de la empresa en cuestión. En cuanto a la habilitación de tecnologías, las empresas tienen departamentos de investigación y desarrollo que desarrollan nuevas tecnologías principalmente relacionadas con la investigación operacional, análisis de mercados o capacidades de fabricación. Es por ello que el departamento de diseños futuros se encarga de identificar estas tecnologías que ayuden a alcanzar los objetivos futuros de la compañía y colabora en el desarrollo de las mismas con el departamento de I+D. Por último, también se desarrollan tareas relacionadas con el análisis del mercado para situar a la empresa en comparación con sus competidoras. Por eso, se realizan estudios exploratorios anuales e incluso se trata de modelizar aviones de la competencia y construir bases de datos, periódicamente actualizadas, que recogen los aviones del estado del arte actual.

Todas las tareas relacionadas con el diseño, fabricación, puesta en servicio y operación de un avión recibe el nombre de proyecto. Existen dos motivos principales por los que una compañía se embarca en un nuevo proyecto de avión: una petición expresa de un cliente para cubrir una necesidad de mercado, tras el visto bueno del fabricante claro está, o por el resultado de alguno de los estudios realizados por la compañía por parte del departamento de diseño futuros, tal y como se ha expuesto anteriormente. Un proyecto típico consta de las siguientes etapas (Torenbeek 1982):

1. Planificación y diseño conceptual: en esta fase se determinan las especificaciones iniciales.

2. Diseño preliminar: de entre las opciones planteadas en el diseño conceptual, se trata de determinar la opción que se adecuará más al mercado.

3. Diseño detallado: una vez seleccionada la configuración final, se finaliza el diseño y la integración de sistemas de cara a ensayos y certificación.

4. Fabricación y adquisición y/o venta: en esta fase se fabrica el avión y se entrega a los clientes.

5. Operación y soporte: una vez ya se ha entregado el avión a los clientes, se realizan las actividades de operación y además se da soporte a dicho cliente. Es habitual que esta fase y la anterior se superpongan. 
6. Baja de servicio: marca el fin de la vida operativa del avión. Las actividades de esta fase consisten en la destrucción y correcta eliminación de los residuos.

A partir de las seis fases del proyecto de avión indicadas, se puede plantear la definición de ciclo de vida del avión (ALC): tiempo transcurrido durante las seis fases del proyecto. A su vez, es posible determinar el coste del ciclo de vida del avión (LCC), que es el coste total del programa durante el ciclo de vida del avión. A su vez, el coste total del ciclo de vida se suele dividir en cuatro términos: coste de investigación, desarrollo, ensayos y evaluación; coste de adquisición; coste operativo y coste de eliminación.

Las tareas de diseño se encuadran en alguna de las tres primeras etapas del proyecto de un avión, dependiendo del nivel de detalle y especificidad de las mismas. Analizando las contribuciones a los costes del ciclo de vida, únicamente un pequeño porcentaje pertenece a las fases de diseño. A pesar de que son las fases que menor coste suponen para la compañía, precisamente son las responsables de comprometer el coste del ciclo de vida de un avión, ya que las decisiones que se tomen afectarán al resto de fases del programa. Por tanto, las principales posibilidades de un diseñador para reducir el coste del ciclo de vida de un avión es actuar en estas fases iniciales de diseño. Independientemente del motivo que ha causado el inicio del programa, todo proyecto parte de unas especificaciones iniciales. Estas pueden venir requeridas por el cliente o incluso por las líneas de trabajo de la compañía. Algunos de los parámetros clásicos que se suelen fijar como especificaciones iniciales son las siguientes:

- Carga de pago, tanto el tipo como el peso.

- Alcance y/o autonomía.

- Velocidad y altitud de vuelo.

- Requisitos de ascensos y de maniobras.

- Distancia de despegue y aterrizaje.

Durante la etapa de diseño conceptual, un grupo reducido de personas se encarga de proponer múltiples configuraciones que puedan cumplir, a priori, con las especificaciones iniciales basándose en la experiencia previa de la compañía y del propio grupo de trabajo. En esta fase se produce una modificación continua de los parámetros básicos del avión mediante un proceso iterativo, haciendo uso de estimaciones rápidas para determinar las características a priori de las propuestas. Un aspecto a considerar que puede ser pasado por alto habitualmente es que esta etapa está condicionada por las especificaciones iniciales impuestas por el departamento de diseños futuros. Es por ello que las decisiones que se tomaron para fijar estas especificaciones suponen un pequeño porcentaje del coste del proyecto del avión, pero compromete el coste total del programa. Si se desarrolla un nuevo avión para el que no existe un mercado, el proyecto estará condenado al fracaso. El equipo de diseño del proyecto no puede ser totalmente responsable de este fracaso si se ha dado el visto bueno a decisiones equivocadas, por lo que dedicar más recursos humanos para el diseño avanzado no es necesariamente la solución para evitar errores de juicio sobre las características del mercado. Aunque el desarrollo del concepto no es, a veces, considerado como parte del proyecto del avión, es por lo menos tan importante para el éxito de un programa como la fase de diseño de detalle (Torenbeek 2013).

La duración característica de la etapa de diseño conceptual suele estar entre los nueve y los doce meses para un avión de transporte comercial. Esta fase inicial del proyecto es la más creativa, ya que se van a definir la geometría de los elementos principales, su posición y las relaciones que habrá entre ellos, de tal forma que satisfagan las especificaciones iniciales del diseño. Además, como se ha comentado anteriormente, se suelen llevar paralelamente varios diseños, contemplando posibilidades más convencionales y otros diseños más arriesgados e innovadores. Hay que resaltar en 


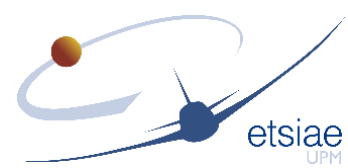

cuanto a los diseños más innovadores que los costes no recurrentes de un proyecto de avión son bastante elevados, de tal forma que una dificultad técnica menor, en principio, puede poner en peligro la viabilidad comercial del producto. Debido a ello, la mayoría de los diseños de aviones comerciales civiles pecan de cierto conservadurismo, basándose en los procedimientos que ya han funcionado en el pasado para otros diseños y que han acabado en buen puerto. Sin embargo, este enfoque puede llevar a la empresa a perder oportunidades de mercado por no utilizar tecnologías que estaban suficientemente maduras, pero suponían un cierto grado de incertidumbre comparado con los procedimientos y técnicas usados anteriormente.

Retomando las actividades recogidas en la fase de diseño conceptual, el objetivo es explorar las diversas configuraciones consideradas con el fin de determinar cuál es la disposición general que es técnicamente más adecuada y económicamente viable. Para alcanzar esta configuración será necesario realizar estimaciones iniciales de pesos y actuaciones, así como disponer de dibujos de carácter general de los principales elementos de la aeronave, o incluso de ciertas secciones de estos. Por otra parte, el centrado del avión es un aspecto relevante que considerar en estas etapas también. Como regla general, las herramientas de diseño son de carácter semi-empírico, usando procedimientos de precisión baja o media junto con técnicas de optimización con capacidades básicas, ya que la definición de la geometría no está a un nivel de detalle muy elevado. Estas técnicas de diseño conceptual se validan con bases de datos estadísticas, manuales de diseño conceptual y tendencias históricas de los diseños, estimando las mejoras que se han producido en ciertas tecnologías. Los errores característicos que se asumen en estas etapas son entre un 5\% y un 10\% (Torenbeek 1982, 2013).

Las técnicas de diseño basadas en bases de datos estadísticas y tendencias históricas han sido de gran ayuda para los diseñadores de configuraciones convencionales, ya que permiten obtener un dimensionado inicial de una serie de datos del avión con una gran facilidad. Una de las primeras publicaciones donde aparece una metodología de dimensionado a base de regresiones construidas a partir de una base de datos surge en los años 80 (Roskam 1986). A partir de ahí, muchos otros autores construyen correlaciones para establecer tendencias en los diseños de aviones, centrándose en algún segmento o algún tipo de avión en concreto, ya sean biturbofanes (Kallinderis, Vouvakos y Menounou 2009), biturbohélices (Vouvakos, Kallinderis y Menounou 2010; Ibrahim 2004) o aviones ligeros (Ibrahim y Mohnot 2006). La primera tarea para poder desarrollar esta metodología es recopilar toda la información posible acerca de los aviones que se quieren incluir en la base de datos y sobre los parámetros de estos que se quieran analizar. El principal inconveniente que tiene esta técnica es que, si se quiere proponer un diseño innovador, no habrá información suficiente en el estado del arte como para construir algún tipo de regresión que sirva de apoyo al diseñador. Por otra parte, se encuentran varios manuales de diseño conceptual que sirven como guía y como validación de las propuestas realizadas. Estos manuales han ido actualizándose a lo largo del tiempo y se recogen fundamentalmente en libros (Torenbeek 1982; Roskam 1989; Nicolai y Carichner 2010; Jenkinson, Simpkin y Rhodes 1999; Sadraey 2013; Torenbeek 2013; Raymer 1989; Nicolai 1975).

Como se ha expuesto anteriormente, una de las principales características de esta etapa es el carácter iterativo de las actividades. Se propone un dimensionado inicial para generar un diseño básico para los principales elementos del avión, tales como ala, fuselaje, góndolas o superficies de cola, entre otros. Una vez analizado este dimensionado inicial, se propondrán modificaciones en aquellos elementos que supongan una cierta mejora en algún aspecto determinado. De nuevo, se analizará la nueva configuración alcanzada y se volverán a implementar una serie de cambios, Así hasta que se alcance una configuración definitiva. Es cierto que, en el diseño de configuraciones convencionales, las soluciones finales alcanzadas son muy semejantes 
a las propuestas iniciales. Sin embargo, en el diseño de configuraciones novedosas, los cambios en las propuestas son mucho más acusados, debido a la naturaleza de las técnicas que se emplean en estas etapas, que están poco contrastadas al no haber aviones semejantes en el mercado. Por esto, no suele ser demasiado útil realizar una optimización completa y muy detallada de una configuración de avión en estas etapas iniciales del proyecto (Bishop y Page 1973).

Una vez se ha decidido en concepto básico del avión y se han reducido las propuestas, idealmente a una única configuración, el proyecto entra en la etapa de diseño preliminar. A diferencia con la fase de diseño conceptual, en este caso el avión se analiza como un completo, definiendo todos los subsistemas y demás elementos a un nivel de detalle mayor, buscando soluciones de compromiso y optimizadas. En esta etapa ya comienzan a participar en el diseño especialistas en campos más específicos, que tratan de refinar el diseño, siguiendo las directrices de los ingenieros que definieron el concepto inicial del avión. Para alcanzar este nivel de detalle, serán necesario herramientas de cálculo más sofisticadas y específicas, tanto para las estimaciones de pesos y de costes como de aerodinámica y actuaciones.

En esta fase de diseño preliminar se pueden definir dos grandes objetivos: definir por completo el diseño y validar el mismo. Las primeras actividades se dirigen a diseñar por completo la aeronave, partiendo del diseño básico que salió de la etapa de diseño conceptual. En el caso que aún se conservasen más de una configuración, en estas fases iniciales de esta etapa el equipo debería decantarse por una sola opción. Posteriormente se realizan estudios de sensibilidad paramétricos, sobre los que basarse para obtener una solución lo más optimizada posible. Entre los aspectos que se deben determinar se encuentran los requisitos volumétricos, dimensiones principales, pesos de los diferentes elementos, características aerodinámicas, las cargas sobre la estructura y actuaciones del avión. Una vez se han determinado todas estas características, se pasa a la fase de validación del diseño. Para ello se echa mano de herramientas de alta precisión, simulaciones e incluso ensayos. Algunas de estas herramientas de alta precisión y simulaciones incluyen programas de mecánica de fluidos computacional (CFD) y de cálculo estructural por medio de elementos finitos (FEM). En general, este tipo de programas proporcionan estimaciones más precisas que las herramientas que se utilizan en la fase de diseño conceptual. Aun así, el grado de incertidumbre suele ser demasiado elevado, por lo que siempre se deberán validar los resultados mediante ensayos en túnel aerodinámico o en vuelo, para el caso de los programas CFD, o algún tipo de ensayo estructural, para los de FEM.

Por último, en la fase de diseño detallado el personal involucrado pasa a ser mucho más amplio, del orden de miles de personas, donde se forman equipos para trabajar en diversos aspectos de la configuración finalmente seleccionada. Los procesos y métodos de cálculo son mucho más refinados en esta fase y por lo tanto mucho más costosos que en las fases previas. Una vez estos estudios están lo suficientemente avanzados, se puede proceder a la fabricación de prototipos para los primeros ensayos de certificación. Una vez se han pasado estos ensayos, se adquiere el certificado de tipo (en el caso de aviación civil) donde se demuestra la aeronavegabilidad del modelo. Esta última etapa se solapa con la fase de fabricación y suele durar típicamente entre cinco y ocho años para un avión de transporte comercial, hasta producir el primer avión en serie.

Por todo lo que se ha expuesto hasta ahora, los fabricantes utilizan una serie de herramientas para acometer el diseño conceptual y preliminar de una aeronave que no están disponibles para ser adquiridas debido a que incluyen información sobre los procedimientos que sigue la compañía, su experiencia y conocimientos, que de estar disponibles para la competencia les daría una ventaja importante. A pesar de ello, existen otras herramientas que se han desarrollado por otras empresas, centros de 
investigación e incluso por universidades. Dependiendo del enfoque que se le haya dado a cada una de estas herramientas, dispondrán de diferentes funcionalidades, tales como módulos de estimación de pesos, de optimización o de actuaciones. Además, estos métodos dispondrán de diversas precisiones en función de la etapa de diseño para la cual se hayan pensado. Para las primeras etapas de diseño conceptual, la prioridad es que sean métodos rápidos, de tiempos de implementación y de cálculo reducidos, de tal forma que sean flexibles a múltiples cambios. Por tanto, este requisito de rapidez va en contra del de precisión, es decir, que estas herramientas tendrán una precisión relativamente baja; es el caso de los métodos basados en correlaciones estadísticas y los que se incluyen en la mayoría de los manuales de diseño, que se basan en correcciones semi-empíricas a modelos físicos, que se construyen a partir de la experiencia de los diseñadores o como resultado de ciertos ensayos específicos. A medida que el conocimiento de la aeronave va creciendo, la complejidad y precisión de las herramientas de diseño utilizadas también deben ir creciendo. De ahí, que se deba realizar una transición entre estos métodos semi-empíricos a herramientas computacionales complejas que incluso tengan en cuenta los resultados de ensayos más sofisticados. Debido al carácter rápido y genérico de las metodologías empleadas en las primeras etapas de diseño, se ignorarán ciertos detalles de la geometría de la aeronave, tales como uniones o remaches, y se utilizarán modelos que esquematicen la estructura interna, tanto primaria como secundaria. Las herramientas típicamente deben tener carácter modular para dotarlas de flexibilidad necesaria en estas etapas iniciales de diseño conceptual.

A partir de la década de los 80 se inició una tendencia en la que los centros de investigación y universidades empiezan a trabajar en el campo del diseño conceptual y preliminar de aviones, en aspectos complementarios a los que se desarrollan por los propios fabricantes de aviones. Por tanto, se quiere recoger a continuación el conjunto de herramientas más relevantes, tanto desarrolladas en empresas como en centros de investigación o universidades, que estén disponibles para el diseño de aviones. El fin es analizar las capacidades del estado del arte de estas herramientas y poder concluir con los objetivos que se presentan en esta tesis:

1. Configuration Development System, CDS: definido por Daniel P. Raymer (Raymer 1989) y desarrollado por Rockwell International. Permitía desarrollar la configuración como una colección de componentes, cada uno de los cuales se definía tridimensionalmente a través de secciones transversales. Disponía de un módulo de análisis aerodinámico.

2. AirCraft SYNThesis, ACSYNT: este código fue un desarrollo conjunto de NASA y Virginia Polythecnic Institute y aún sigue en uso, siendo comercializado por la empresa Phoenyx Integration desde 1997. Admite síntesis de la configuración, cálculo de la misión, optimización y cálculo de costes (Jayaram, Myklebust y Gelhausen 1992).

3. Aircraft Design and Analysis System, ADAS: este sistema fue desarrollado en la Universidad de Delft. La geometría estaba basada en AutoCAD. En la actualidad no está en funcionamiento (Bil 1988).

4. FLight OPtimization System, FLOPS: es un código que aún está en funcionamiento y tiene un gran parecido con ACSYNT, con gran cantidad de módulos para cálculo de ruido, actuaciones en despegue y otras características (Mccullers 1984).

5. RDS: Este código es obra de Daniel P. Raymer. Contiene un método básico de dimensionado que se basa en la metodología del propio autor publicada en su libro "Aircraft Design: A Conceptual Approach" (Raymer 2012). Permite analizar ciertas actuaciones, cálculo de pesos, costes y algunas funciones de optimización. Dispone de un módulo de CAD en tres dimensiones que ayuda a la visualización de los diseños (Raymer 2016).

6. Advanced Aircraft Analysis, AAA: la versión inicial de esta herramienta fue el resultado de informatizar los 8 volúmenes de la obra de Jan Roskam, por parte de 
la Universidad de Kansas. Los módulos principales están centrados en cálculo de pesos, aerodinámica, geometría, costes, estabilidad y control, dinámica, propulsión, estructuras y cargas (DAR corporation [sin fecha]).

7. Preliminary Aircraft Design and Optimization, PrADO: a finales de los años noventa el Institut für Aerodynamik und Strömungstechnik del centro de investigación alemán DLR (Deutsches Zentrum für Luft- und Raumfahrt), desarrolló una herramienta de diseño preliminar y optimización de aviones llamada PrADO en colaboración con la Universidad de Braunschweig. Esta herramienta está fundamentalmente centrada en el análisis de las características aerodinámicas y estructurales, partiendo de métodos de vortex lattice, métodos de paneles, métodos de Euler y llegando a métodos Navier-Stokes que combinan con herramientas de elementos finitos (como NASTRAN) para el cálculo estructural. Han aplicado esta herramienta en algunas configuraciones no convencionales, así como para un avión supersónico de transporte (Seubert 1997).

8. Pacelab APD: se trata de una herramienta comercial relativamente reciente creada por la empresa de software PACE para satisfacer las necesidades de analistas de aviones, ingenieros de diseño conceptual y preliminar, integradores de aeronaves y expertos en subsistemas. Pacelab APD se integra en una plataforma más amplia llamada Pacelab Suite que está diseñada para dar soporte al lanzamiento al mercado de productos. Pacelab APD permite generar un diseño conceptual parametrizado partiendo de las estimaciones de métodos como los de Torenbeek o Raymer, o bien de métodos propios del usuario, para pasar al cálculo de actuaciones, análisis y optimización del diseño («Pacelab SysArc - TXT» [sin fecha]). Además, existen numerosas publicaciones en revistas y congresos que llevan a cabo estudios utilizando esta herramienta (Cinar, Emeneth y Mavris 2016; Ganesh 2018; Cinar et al. 2017).

9. Common parametric Aircraft Configuration Schema, CPACS: se trata de un estándar de definición de datos para sistemas aeronáuticos desarrollado por el centro de investigación alemán DLR (Deutsches Zentrum für Luft- und Raumfahrt) en el año 2005, de uso interno y que han aplicado en trabajos de investigación que desarrollan dentro del campo del diseño de aviones, helicópteros y motores, así como para estudios de impacto climático. Permite llevar a cabo análisis de optimización multidisciplinar, simulaciones distribuidas y trabajo colaborativo («DLR - Air Tansportation Systems - CPACS» [sin fecha]).

10. Multidisciplinary Integrated Conceptual Aircraft Design and Optimization (MICADO): es un entorno altamente automatizado desarrollado desde 2010 por el Institut für Luft -und-Raumfahrt (ILR) de la RWTH Aachen University que permite llevar a cabo el diseño conceptual de un avión de transporte comercial a través de un proceso iterativo, al cual se le incorporan estrategias de optimización, con una mínima intervención del usuario (Risse et al. 2012).

11. Central Reference Aircraft data System, CeRAS: es una base de datos centralizada que recoge datos de diseño de aviones comerciales de transporte con la finalidad de que se puedan utilizar en proyectos de investigación sobre el diseño conceptual y preliminar de aviones, así como en la evaluación e integración de tecnologías. El desarrollo se está llevando cabo en la RWTH Aachen University (Risse et al. 2012; ILR y RWTH-Aechen-University 2014)

12. Aircraft Preliminary Sizing Tool, PreSTo: el objetivo de la herramienta PreSTo es la realización de los cálculos necesarios para el diseño conceptual partiendo de requisitos iniciales y llegando a una definición geométrica completa del avión, una estimación de las masas, la polar en crucero y el coste directo de operación. La herramienta, de libre disposición, se basa en la metodología de diseño conceptual utilizada con propósitos docentes en la Universidad de Ciencias Aplicadas de Hamburgo y se aplica a través de una hoja de cálculo. Se están preparando interfaces para otros códigos y un sistema de visualización 3D para la geometría (Seeckt y Scholz 2010). 
13. Computerised Environment for Aircraft Synthesis and Integrated Optimisation Methods, CEASIOM: es una herramienta-marco de diseño conceptual que integra otras herramientas como: CAD \& generación de mallas, CFD, análisis de estabilidad y control, análisis aerodinámico, análisis aeroelástico, etc. Todos los módulos individuales comparten un diseño de avión común, pero que es una entrada al entorno y no un resultado, y que es optimizado por CEASIOM (Afsar y Salam 2013; Richardson et al. 2011; Cavagna, Ricci y Travaglini 2011; «CEASIOM - Conceptual Aircraft Design Tool» [sin fecha]).

14. openMDAO: se trata de un entorno de diseño, análisis y optimización multidisciplinar. El entorno está diseñado para ayudar a vincular diferentes elementos de código con el fin de poder llevar a cabo análisis combinados en los que se haga uso de diversas disciplinas, con diferentes niveles de precisión, y gestionar las interacciones entre ellos. OpenMDAO está específicamente diseñado para gestionar el flujo de datos que se va generando, incluyendo ciertos algoritmos de optimización (Moore, Naylor y Gray 2008).

15. Aircraft Design and Analysis Software, ADAS: se desarrolló en el Departamento de Ingeniería Aeroespacial de la Universidad de Nápoles por Fabrizio Nicolosi y Giuseppe Paduano durante cinco años. El origen de la herramienta era con fines académicos, pero a medida que la fueron evolucionando se mostró que podían llevarse a cabo estudios de investigación y que podía servir como apoyo a la industria (Nicolosi y Paduano 2011).

Todas estas herramientas permiten al usuario, de una manera u otra, trabajar en el diseño de una configuración de avión, por regla general convencional, aunque alguna de ellas se puede adaptar o está pensada a configuraciones no convencionales. Algunas son de difícil acceso y otras funcionan como "caja negra", por lo que no se pueden conocer con exactitud los procedimientos internos que siguen, y mucho menos adaptarlos a otras necesidades que tenga el usuario. Debido a eso existe esta gran variedad de herramientas, ya que cada centro de investigación o universidad se ha visto en la obligación de desarrollar su propia metodología, dándole un enfoque concreto y que se adecúe a las líneas de investigación que se desarrollan en cada centro. Lo que sí se ha percibido es que ninguna de ellas permite generar una configuración convencional $\mathrm{y}$, posteriormente, dimensionar una configuración no convencional, a determinar por el usuario, que sea semejante en misión a la anterior. El objetivo de esta herramienta sería analizar la viabilidad de las configuraciones no convencionales, al ser comparadas con las convencionales competidoras. 


\subsection{OBJETIVOS Y MOTIVACIÓN}

Debido al marco legislativo actual, en el que se quiere tender hacia tecnologías que tengan cada vez un menor impacto en el medioambiente, está justificado embarcarse en un estudio para analizar si los aviones del futuro pueden contribuir a este objetivo. Parece ser que esta configuración convencional ha alcanzado un límite asintótico en productividad y capacidad, en un tamaño similar al del A380 (Martínez-Val, CuernoRejado, et al. 1994). Por tanto, es necesario plantear nuevas estrategias para poder cumplir con las restricciones de emisiones de gases contaminantes a la atmósfera. Una de esas estrategias es la adopción de configuraciones no convencionales. Como se ha mostrado, la mayoría de los estudios sobre configuraciones no convencionales están dirigidos al ala o al avión completo, siendo las configuraciones no planas el máximo exponente en innovación. Así que una opción que supone un paso en la dirección de poder adoptar aviones con configuraciones totalmente no convencionales es actuar únicamente en el fuselaje posterior, proponiendo configuraciones de cola que sustituyan a las que se emplean hoy en día.

Con esta tesis se pretende determinar la viabilidad, así como la conveniencia del uso de configuraciones de cola innovadoras en la aviación comercial como un medio alternativo a las configuraciones convencionales, formadas por un estabilizador vertical y un horizontal en una posición baja, principalmente. Este estudio debe enfocarse desde las etapas de diseño conceptual y, a lo sumo, preliminar, ya que para poder concluir sobre las principales ventajas o inconvenientes que presenten estas configuraciones no es necesario llegar al nivel de detalle que se alcanza en las últimas etapas de diseño detallado.

Previo al estudio de configuraciones de cola no convencionales, será necesario caracterizar claramente las configuraciones convencionales clásicas. Esta caracterización se llevará a cabo mediante la recopilación de información sobre un gran número de aeronaves, con la idea de extraer tendencias de diseño en las superficies de cola dependiendo de diferentes aspectos, tales como el número de motores, posición de los mismos, segmento de vuelo, etc. A partir de esta base de datos también se podrá estudiar la dependencia de los parámetros de diseño entre sí.

Para determinar los beneficios potenciales que proporcionarán las configuraciones innovadoras, es necesario seleccionar una aeronave de referencia de la que se conozcan todas sus actuaciones. La aeronave que se va a seleccionar va a ser el avión piloto del proyecto CeRAS, presentado entre las herramientas de diseño de aviones mencionadas en el apartado 1.3. Sobre este avión ficticio se conoce toda la geometría, comportamiento aerodinámico, sistemas, actuaciones, contaminación y costes directos de operación. Esta aeronave será para la cual se realizará el estudio de viabilidad de las configuraciones no convencionales de cola.

Como objetivo transversal, y añadido al ya citado, se plantean las bases de una nueva metodología que permita determinar el dimensionado de configuraciones no convencionales en el fuselaje posterior a partir de unos datos de entrada sobre la misión, además de generar el avión de fuselaje posterior convencional equivalente para permitir la comparación entre ambos. Esto complementaría a las herramientas clásicas de diseño conceptual de aviones en dos aspectos principales: la rapidez del diseño a partir de unos pocos datos de la misión y la comparación con configuraciones convencionales. Ahondando más en los estudios que se han realizado hasta ahora sobre configuraciones de cola no convencionales, hay un aspecto crucial que no ha sido tenido en cuenta hasta 
ahora, como es la regulación vigente. El procedimiento de diseño debe estar sustentado en las funciones y requisitos de certificación que apliquen a las superficies de cola.

En este contexto, la originalidad de la tesis se fundamenta en la profundización en el diseño de configuraciones de cola no convencionales y en el análisis de ventajas e inconvenientes concluyendo así la viabilidad o no de dichas configuraciones, basando el diseño en la normativa de certificación vigente. Adicionalmente, el objetivo transversal mencionado anteriormente, la nueva metodología de diseño conceptual comparativa entre configuraciones convencionales y no convencionales aportaría un grado más de transcendencia, ya que permitiría comparar de una forma sencilla y rápida la adecuación del uso de una configuración u otra dependiendo de la misión que se desee llevar a cabo con la aeronave. 
2 DISEÑO CONCEPTUAL DE LAS CONFIGURACIONES DE COLA CONVENCIONALES 


\subsection{CARACTERIZACIÓN DE LAS CONFIGURACIONES DE COLA CONVENCIONALES}

En este capítulo se va a acometer la caracterización de las configuraciones de cola convencional. Esta fase inicial en el estudio de configuraciones no convencionales es esencial para extraer tendencias en los diseños de las colas que actualmente se usan en la aviación comercial, pudiendo así definir lo que se considera convencional.

Para ello, se ha construido una base de datos que contiene parámetros de aviones de transporte aéreo comercial (Sanchez-Carmona 2014). Esta base de datos tiene que ser lo más amplia posible, tanto en número de aviones como en el de parámetros, de esta forma las conclusiones extraídas sobre la caracterización de colas convencionales estarán mejor fundamentadas. Los dos principales fabricantes de aeronaves del mundo son Airbus y Boeing, por lo que todos sus aviones se han incluido en la base de datos. Atendiendo al segmento de aviación regional, tanto Bombardier como Embraer tienen un papel destacado, es por ello que sus aviones también hayan sido incluidos en el estudio. Por otra parte, se han incluido fabricantes de aviones que tienen un rol más secundario pero que no dejan de ser importantes en aquellos países a los que pertenecen, Rusia y China. Finalmente, también se han considerado para el estudio aviones de fabricantes más antiguos, con la idea de contemplar la posible evolución de los diseños a lo largo de la historia. La Tabla 2-1 recoge los modelos de avión que se han incluido en la base de datos organizados por la nacionalidad del fabricante y por fabricantes, a su vez.

En cuanto a los parámetros que se consideren para incluir en la base de datos, deben ser tales que permitan alcanzar el objetivo marcado, es decir, caracterizar las configuraciones de cola convencionales. Es por lo que estos parámetros incluyen geometría, pesos e incluso información acerca de la misión que realiza el avión. Debido a la diversa naturaleza de los mismos, se pueden englobar en cuatros grandes grupos: generales, fuselaje posterior, estabilizador horizontal y estabilizador vertical. En la Tabla 2-2 se recogen todos los parámetros que han sido incluidos en la base de datos, clasificados de acuerdo con las categorías que se acaban de definir. Hay que indicar que no siempre ha sido posible determinar la totalidad de los parámetros para todos y cada uno de los aviones debido a la ausencia de información o baja calidad de ciertas fuentes. Además, no todos los parámetros aplican a todos los aviones, ya que algunos de ellos vienen marcados por alguna configuración en concreto.

Los parámetros generales incluyen aquellos que no son específicos del cono de cola o de los estabilizadores, pero tienen cierta relevancia a la hora de extraer tendencias en los diseños. La fecha del primer vuelo es uno de los parámetros que se incluyen en este grupo. Se ha considerado entre estos parámetros ya que permite clasificar los aviones por épocas y analizar si ha habido evolución de ciertos parámetros a lo largo de la historia. Dentro de este grupo también encontramos variables que describen la geometría externa del avión, tales como las dimensiones del fuselaje, la envergadura y superficie alares. Además, se ha calculado la cuerda media aerodinámica del ala ya que es de especial relevancia para el dimensionado de los estabilizadores de cola. Por último, se han incluido el número de pasajeros, el alcance, el tipo de planta propulsora y el peso máximo al despegue. 


\begin{tabular}{|c|c|c|}
\hline Nacionalidad del fabricante & Fabricante & Modelos \\
\hline \multirow{8}{*}{ Europa } & Airbus & $\begin{array}{c}\text { A300, A310, A318, A319, } \\
\text { A320, A321, A330, A340, } \\
\text { A350, A380 }\end{array}$ \\
\hline & ATR & ATR 72 \\
\hline & BAE & Jetstream 41, 146 \\
\hline & BAC & 111 \\
\hline & Britten Norman & Trislander \\
\hline & Sud Aviation & Caravelle \\
\hline & $\begin{array}{l}\text { Vickers } \\
\text { Armstrong }\end{array}$ & VC10 \\
\hline & Saab & 2000,340 \\
\hline \multirow[t]{2}{*}{ Estados Unidos } & Boeing & $\begin{array}{c}\text { B707, B720, B717, B727, } \\
\text { B737, B747, B757, B767, } \\
\text { B777, B787, MD11, DC9, } \\
\text { DC10, MD87 }\end{array}$ \\
\hline & Lockheed & L1011 Tristar \\
\hline \multirow{4}{*}{ Rusia } & Ilyushin & IL 96 \\
\hline & Sukhoi & Superjet 100 \\
\hline & Irkut & MS21 \\
\hline & Antonov & An 26, An 28 \\
\hline \multirow{2}{*}{ Canadá } & Bombardier & CRJ 900, CS300, Q400 \\
\hline & $\mathrm{DHC}$ & Twin Otter Series 400 \\
\hline Brasil & Embraer & $\begin{array}{c}\text { ERJ 135, ERJ 145, } \\
\text { Emb } 190\end{array}$ \\
\hline \multirow{2}{*}{ China } & Xian & MA 700, MA 60 \\
\hline & Comac & C919 \\
\hline
\end{tabular}

Tabla 2-1 Modelos de avión incluidos en la base de datos de estudio de configuraciones de cola convencional. Fuente: elaboración propia. 


\begin{tabular}{|c|c|}
\hline & Parámetros \\
\hline \multirow{14}{*}{ Generales } & Fecha del primer vuelo \\
\hline & Segmento de vuelo \\
\hline & Planta propulsora \\
\hline & Número de motores \\
\hline & Alcance \\
\hline & Número de pasajeros \\
\hline & Peso máximo al despegue \\
\hline & Superficie alar \\
\hline & Envergadura alar \\
\hline & Cuerda media aerodinámica del ala \\
\hline & Longitud de fuselaje \\
\hline & Altura del fuselaje \\
\hline & Anchura del fuselaje \\
\hline & Número de Mach de crucero \\
\hline \multirow{3}{*}{ Fuselaje posterior } & $\begin{array}{l}\text { Longitud del fuselaje posterior } \\
\text { Ánqulo de quarda }\end{array}$ \\
\hline & Esbeltez del fuselaje posterior \\
\hline & Ángulo de upsweep \\
\hline \multirow{12}{*}{ Estabilizador horizontal } & Posición longitudinal \\
\hline & Superficie de referencia \\
\hline & Envergadura \\
\hline & Cuerda en la raíz \\
\hline & Cuerda en la punta \\
\hline & Cuerda media aerodinámica \\
\hline & Flecha de la línea de puntos $1 / 4$ \\
\hline & Diedro \\
\hline & Parámetro de estrechamiento \\
\hline & Alargamiento \\
\hline & Brazo de momentos \\
\hline & Coeficiente de volumen \\
\hline \multirow{11}{*}{ Estabilizador vertical } & Posición longitudinal \\
\hline & Superficie de referencia \\
\hline & Envergadura \\
\hline & Cuerda en la raíz \\
\hline & Cuerda en la punta \\
\hline & Cuerda media aerodinámica \\
\hline & Flecha de la línea de puntos $1 / 4$ \\
\hline & Parámetro de estrechamiento \\
\hline & Alargamiento \\
\hline & Brazo de momentos \\
\hline & Coeficiente de volumen \\
\hline
\end{tabular}

Tabla 2-2 Parámetros considerados en la base de datos clasificados según su naturaleza. Fuente: elaboración propia.

En la categoría de fuselaje posterior, o cono de cola, se han considerado los parámetros que describen la geometría del fuselaje desde que termina la zona cilíndrica hasta el final del mismo. En este estudio se han tenido en cuenta cuatro: longitud de esta sección de fuselaje, su esbeltez, el ángulo de guarda y el ángulo de upsweep. Hay que hacer una mención especial a los dos últimos. El ángulo de guarda también recibe el nombre de tail strike angle. Este ángulo viene definido por el máximo ángulo de cabeceo que puede alcanzar el avión en despegue hasta que un punto del cono de cola toca con la pista. El ángulo de upsweep del fuselaje posterior es el ángulo que forman la recta que une los centros de las secciones de inicio y fin del cono de cola en una vista lateral del 


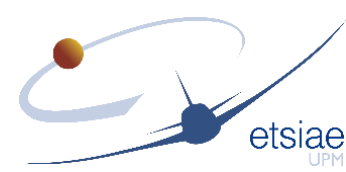

avión, con respecto a una recta horizontal paralela a la línea media del fuselaje (Figura 2-1).

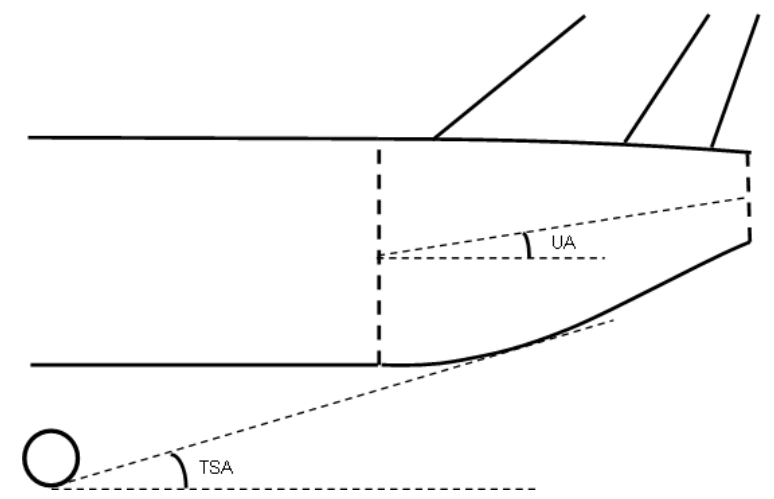

Figura 2-1 Definición del ángulo de guarda (TSA) y del ángulo de upsweep (UA), parámetros característicos del fuselaje posterior. Fuente: elaboración propia.

En cuanto a las superficies estabilizadoras de cola, los parámetros son equivalentes en ambos casos salvo el diedro, que para el estabilizador vertical siempre es $90^{\circ}$, por lo que no se ha considerado relevante incluirlo en la base de datos. La superficie de referencia se ha considerado como la superficie en planta, en el caso del estabilizador horizontal, y como la superficie proyectada sobre el plano de simetría, en el del vertical. El resto de los parámetros se han obtenido siguiendo las definiciones habituales del diseño conceptual de aviones. Se quiere destacar la cuerda media aerodinámica, que se define como la cuerda que debe tener un ala rectangular sin estrechamiento equivalente que desarrolle el mismo coeficiente de sustentación y el mismo coeficiente de momento de cabeceo que el ala de la que se quiere determinar su cuerda media aerodinámica (Yates 1952). Siguiendo esta definición para alas de simple estrechamiento, con parámetro de estrechamiento $\lambda_{w}$ y cuerda en la raíz $c_{r w}$, que son una buena aproximación de la geometría que presentan tanto los estabilizadores verticales como horizontales, la expresión por la cual se obtiene esta cuerda es la siguiente:

$$
C M A_{w}=\frac{2}{3} c_{r w} \frac{1+\lambda_{w}+\lambda_{w}^{2}}{1+\lambda_{w}}
$$

Además, también se pueden obtener para este tipo de alas de simple estrechamiento las coordenadas $x$ e $y$ del borde de ataque de esta cuerda, ya que se puede asumir que el centro aerodinámico se encuentra en el punto $1 / 4$ de esta cuerda. Las expresiones que correspondientes de estas coordenadas se indican a continuación, donde la flecha del borde de ataque de la superficie se define como $\Lambda_{b a}$ :

$$
\begin{gathered}
x_{\text {baCMA }}=y_{C M A} \tan \Lambda_{b a} \\
y_{C M A}=\frac{b_{w}}{6} \frac{1+2 \lambda_{w}}{1+\lambda_{w}}
\end{gathered}
$$

La información necesaria para rellenar la base de datos se ha obtenido de diversas fuentes. Las fuentes más fiables son las que se pueden consultar en las propias páginas web de los fabricantes. Allí se pueden encontrar documentos tales como los Airplane Characteristics for Airport Planning o informes comerciales, que suelen incluir una 
imagen a tres vistas de la aeronave. Por otra parte, también se puede obtener información en la literatura abierta. La principal fuente es el libro Jane's: All the World's Aircraft, que recoge datos de diversa naturaleza sobre una gran variedad de aviones. Por supuesto, estas fuentes no recogen toda la información necesaria de todas las aeronaves consideradas para el estudio. La información ha tenido que ser complementada con otras fuentes de menor fiabilidad a priori, como webs no oficiales, revistas, otros estudios similares, etc. Por último, existen datos que se pueden obtener a partir de otros mediante expresiones matemáticas. Hay situaciones en las que este dato además se ha encontrado en alguna fuente, por lo que sirve para analizar la veracidad de la fuente. Se ha llevado una trazabilidad del origen de los datos, ya que podría ser la causa de una anomalía en una tendencia de diseño.

Como se ha comentado en el párrafo anterior, algunos datos se pueden determinar mediante relaciones a partir de otros. De hecho, se han establecido algunos parámetros adicionales a los comentados anteriormente que, a pesar de no poderse consultar en ninguna fuente de forma directa, se ha observado que tienen una especial relevancia en el diseño de las configuraciones de cola convencionales en la aviación comercial.

El primero de ellos se ha bautizado con el nombre de parámetro de volumen global. Es el volumen que encierra el paralelepípedo definido por el conjunto ala-fuselaje:

$$
V o l=H_{f u s} L_{f u s} b_{w}
$$

Se ha determinado por semejanza a otros estudios previos cuyo objetivo era el diseño conceptual de aviones comerciales basado en correlaciones entre diferentes parámetros (Kallinderis, Vouvakos y Menounou 2009).

Otro de los parámetros derivados más relevantes propuestos en el análisis se obtiene a partir de la expresión de la resistencia inducida por torbellinos de la cola horizontal. Para determinarlo, se combina la ecuación de momentos del avión completo [ 2-5], despreciando el momento que produce el empuje por no estar alineado con el centro de gravedad, con la expresión de la resistencia inducida por torbellinos del estabilizador horizontal [ 2-6 ]. Despejando el coeficiente de sustentación de la cola horizontal de la ecuación de momentos y sustituyendo en la expresión de la resistencia inducida se obtiene la expresión [ 2-7 ].

$$
\begin{gathered}
c_{L} \frac{x_{c g}-x_{c a}}{C M A_{w}}+c_{m a c}-V_{e H} \frac{q_{H}}{q} c_{L H}=0 \\
D_{H}=\frac{1}{2} \rho V^{2} S_{H} \frac{q_{H}}{q}\left(c_{D O H}+\frac{1}{\pi A R_{H} \Phi} c_{L H}^{2}\right) \\
D_{\text {indH }}=\frac{1}{2} \rho V^{2} S_{H} \frac{1}{\pi A R_{H} \Phi} \frac{1}{V_{e H}^{2}} \frac{q}{q_{H}}\left(c_{L} \frac{x_{c g}-x_{c a}}{C M A_{w}}+c_{m a c}\right)^{2}
\end{gathered}
$$

Sustituyendo la superficie del estabilizador por $b_{H}^{2} / A R_{H}$ y usando el equilibrio de fuerzas verticales del avión completo para reemplazar el coeficiente de sustentación, se alcanza la expresión [ 2-8 ].

$$
D_{i n d H}=\frac{1}{2} \rho V^{2} \frac{q}{\pi \Phi q_{H}} \frac{b_{H}^{2}}{V_{e H}^{2} A R_{H}^{2}}\left(\frac{2 W}{\rho V^{2} S_{w}} \frac{x_{c g}-x_{c a}}{C M A_{w}}+c_{m a c}\right)^{2}
$$

Como primera aproximación, el coeficiente de momento aerodinámico del avión no se ha tenido en cuenta, debido a que el otro término del paréntesis se ha considerado como 


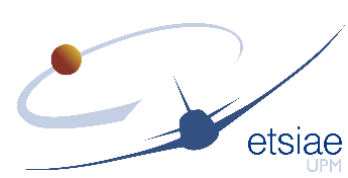

dominante $\mathrm{y}$, además, es un parámetro difícil de obtener en la literatura abierta para todos los aviones. Por otro lado, el peso representativo del avión que se ha tomado es el $M T O W$. Por lo que sacando del paréntesis el $M T O W$ y la $M A C_{W}$ se obtiene la expresión [2-9].

$$
D_{\text {ind }} \cong \frac{1}{2} \rho V^{2} \frac{q}{\pi \Phi q_{\mathrm{H}}} \frac{b_{H}^{2} M T O W^{2}}{V_{e H}^{2} A R_{H}^{2} C M A_{w}^{2}}\left(\frac{2}{\rho V^{2} S_{w}}\left(x_{c g}-x_{c a}\right)\right)^{2}
$$

El parámetro al que se quería llegar se ha llamado $D_{H i}$ y su expresión es la [ 2-10 ]. Más adelante se verá la utilidad que tiene este parámetro y las tendencias de diseño que muestra en etapas de diseño conceptual de un estabilizador horizontal de configuración convencional.

$$
D_{H i}=\frac{b_{H} M T O W}{A R_{H} V_{e H} C M A_{w}}
$$

Por otra parte, desde el punto de vista del estabilizador vertical de la aeronave, uno de los criterios de diseño impuesto por las normas de certificación que lo dimensionan es el control del avión tras el fallo del motor crítico en segundo segmento tras el despegue. Planteando el equilibrio de momentos en guiñada una vez ya ha fallado el motor crítico, se llega a la expresión [ 2-11].

$$
\frac{c_{Y_{\beta V}} \frac{q_{V}}{q} S_{V}}{S_{w} b_{w}}=\left(\frac{T_{T O e} y_{e} c_{L}}{W}+b_{w} c_{n_{\beta_{A-V}}} \beta\right) \cdot \frac{1}{b_{w} L_{T V}\left(-\left(\beta-\sigma_{v}\right)+\delta_{r} \tau_{r}\right)}
$$

En esta ecuación aparecen varios términos que pueden ser susceptibles de establecerse relaciones entre ellos. Pasando el brazo de momentos $L_{T V}$ al lado izquierdo de la igualdad, aparece el producto de la superficie del estabilizador vertical por este brazo, $S_{V} L_{T V}$, dividido por la superficie alar y su envergadura, definiendo así el coeficiente de volumen. En el otro lado del igual, se puede tomar el momento que produce el motor crítico como parámetro representativo: $T_{e} y_{e}$, donde $T_{e}$ es el empuje máximo en despegue de un motor y $y_{e}$ es la distancia lateral entre el motor crítico y el plano de simetría del avión. Se presentarán regresiones entre el numerador del coeficiente de volumen y el momento que produce la asimetría de empuje, ya que son los únicos con los que se han obtenido buenos resultados. El resto de las combinaciones posibles inspiradas en esta ecuación no han derivado en correlaciones con un nivel de significación suficiente.

Tanto los parámetros que se pueden consultar directamente en alguna de las fuentes como los parámetros derivados de estos, sirven para caracterizar y dimensionar las superficies estabilizadoras de los aviones de transporte aéreo comercial. Una forma habitual de establecer relaciones entre los parámetros para obtener un diseño conceptual de la superficie es mediante correlaciones o regresiones. Estas técnicas estadísticas tienen un uso extendido en el diseño conceptual de aviones desde hace tiempo, como se ha expuesto en la introducción (Roskam 1986; Kallinderis, Vouvakos y Menounou 2009; Vouvakos, Kallinderis y Menounou 2010). Las regresiones permiten determinar una relación entre dos parámetros de diseño, asumiendo la hipótesis de que son variables aleatorias de distribución normal. Asociada a la misma, se puede obtener el coeficiente de correlación de Pearson, el error estadístico o típico y el nivel de significación. El coeficiente de correlación de Pearson es una medida de la relación lineal entre dos variables aleatorias cuantitativas. En el caso de que se esté estudiando 
dos variables aleatorias $X$ e $Y$ sobre una muestra de una población estadística, el coeficiente de correlación de Pearson viene dado por la expresión [2-12 ] y con la letra $\rho_{x y}$.

$$
\rho_{x y}=\frac{\sigma_{x y}}{\sigma_{x} \sigma_{y}}
$$

donde $\sigma_{x y}$ es la covarianza de $(X, Y), \sigma_{x}$ y $\sigma_{y}$ son las desviaciones típicas de las variables $X$ e $Y$ respectivamente. La covarianza de dos variables aleatorias se determina mediante la expresión [ 2-13 ] y la desviación típica por la [ 2-14], siendo $\bar{X}$ e $\bar{Y}$ las medias aritméticas de las variables aleatorias $X$ e $Y$ y $n$ el tamaño de la muestra.

$$
\begin{gathered}
\sigma_{x y}=\frac{1}{n} \sum_{i=1}^{n}\left(X_{i}-\bar{X}\right)\left(Y_{i}-\bar{Y}\right) \\
\sigma_{x}=\sqrt{\frac{1}{n} \sum_{i=1}^{n}\left(X_{i}-\bar{X}\right)^{2}}
\end{gathered}
$$

Sustituyendo las expresiones de la covarianza y las desviaciones típicas en la ecuación del coeficiente de Pearson, y tomando cuadrados a ambos lados, se llega a la expresión [ 2-15], donde $R^{2}$ es el coeficiente de determinación, que se va a emplear para establecer la calidad de las correlaciones calculadas.

$$
R^{2}=\frac{\left(\sum_{i=1}^{n}\left(X_{i}-\bar{X}\right)\left(Y_{i}-\bar{Y}\right)\right)^{2}}{\sum_{i=1}^{n}\left(X_{i}-\bar{X}\right)^{2} \sum_{i=1}^{n}\left(Y_{i}-\bar{Y}\right)^{2}}
$$

El coeficiente $R^{2}$ está comprendido en el rango [0,1]. Cuanto más cerca se encuentre el valor de 1, más relación lineal habrá entre las variables aleatorias. Está claro que es posible que la relación entre los parámetros no sea lineal, y sea necesario implementar otro tipo de regresión. Para obtener las ecuaciones de las regresiones se utiliza el ajuste por mínimos cuadrados. En el caso de las regresiones no lineales, el coeficiente de correlación se obtiene de forma diferente; se hace uso del coeficiente de determinación. El coeficiente de determinación se obtiene mediante la ecuación [ 2-16 ], donde $S S_{E}$ es la suma de los cuadrados de la mínima distancia a la curva en los puntos conocidos y $S S_{R}$ es la suma de los cuadrados de la resta de los puntos de la curva estimada y la media de los valores de los puntos conocidos.

$$
R^{2}=1-\frac{S S_{E}}{S S_{R}}
$$

En algunos casos no es suficiente con analizar el coeficiente de determinación para concluir que existe una relación significante entre las dos variables. Una forma de mostrar la adecuación de un modelo de ajuste es mediante el error típico, que se obtiene como sigue:

$$
e^{2}=\frac{S S_{E}}{n_{e}-p-1}
$$

donde $n_{e}$ es el número de datos tomado y $p$ es el número de grados de libertad del modelo de ajuste, es decir, el número de coeficientes a ajustar (por ejemplo, en una regresión lineal se ajustan dos parámetros). Esta variable mide la desviación típica de los residuos y por tanto mide el grado de dispersión de los resultados. En este estudio se va a trabajar con la hipótesis de que el error típico es constante a lo largo de la curva 
de regresión, definiendo así por tanto una distribución normal alrededor de cada punto de la curva de desviación típica e. De ahí, se puede garantizar que, tomando un intervalo de dos veces el error típico a cada lado de la curva de regresión, el 95\% de los puntos deberían ser interiores al mismo. De esta forma se establece un intervalo de confianza del $95 \%$ alrededor de la regresión. Por tanto, cuanto menor sea el error típico comparado con el valor que tome la regresión en cada punto, más estrecho es el intervalo de confianza, menor es la dispersión de los puntos y mayor fiabilidad tienen los resultados estimados mediante la regresión en relación con la muestra.

Por otra parte, en la mayoría de los casos no se dispone del $100 \%$ de los datos que conforman la población. Lo normal es trabajar con una muestra. Por tanto, es necesario medir hasta qué punto los resultados obtenidos para la muestra tomada son extrapolables a la población. Para ello se define el estadístico $F_{e}$ de la siguiente forma:

$$
F_{e}=\frac{M S_{R}}{M S_{E}}=\frac{\frac{S S_{R}}{p}}{\frac{S S_{E}}{n-p-1}}
$$

donde $S S_{R}$ es la suma de los cuadrados de la resta de los valores estimados mediante la regresión y la media de los datos. Con esta variable se puede medir la probabilidad de error de que la regresión sea un buen estimador de los datos. Para ello, basta con medir el área que queda a la derecha de la función de distribución $F$ de Fisher-Snedecor, representada en la Figura 2-2, entrando en $x$ con el valor de $F_{e}$, con un primer grado de libertad igual $p-1$ y un segundo grado de libertad igual a $n-p-1$. Estos dos coeficientes llamados grados de libertad hacen cambiar la forma a la función. Se considera que la regresión tiene un nivel de significancia muy alto si la probabilidad resultante, que es el área tomada mediante las operaciones anteriormente mencionadas (que llamaremos $P$ de aquí en adelante), se encuentra por debajo del 0,001. Si esto se produce, se podrá asegurar que la correlación establecida es representativa de la población, y no sólo de la muestra. Toda la información indicada acerca de las variables estadísticas necesarias para analizar la inferencia de las regresiones se puede consultar y ampliar en la referencia (Mason, Gunst y Hess 2003).

Por tanto, para dar una información estadística completa de cada una de las regresiones que se construyan, se van a proporcionar la ecuación de la regresión, el coeficiente de determinación, el error típico y la probabilidad de que la regresión no sea extrapolable a la población, es decir, el nivel de significación. El coeficiente de determinación vendrá indicado por medio del valor del $R^{2}$, mientras que el nivel de significación por medio del valor de $P$. Sin embargo, el error típico vendrá indicado al final de la ecuación de regresión tras un símbolo de \pm y multiplicado por 2 , ya que tal y como se ha comentado, de esta forma se asegura que el $95 \%$ de los datos se encontrará en el intervalo proporcionado. 


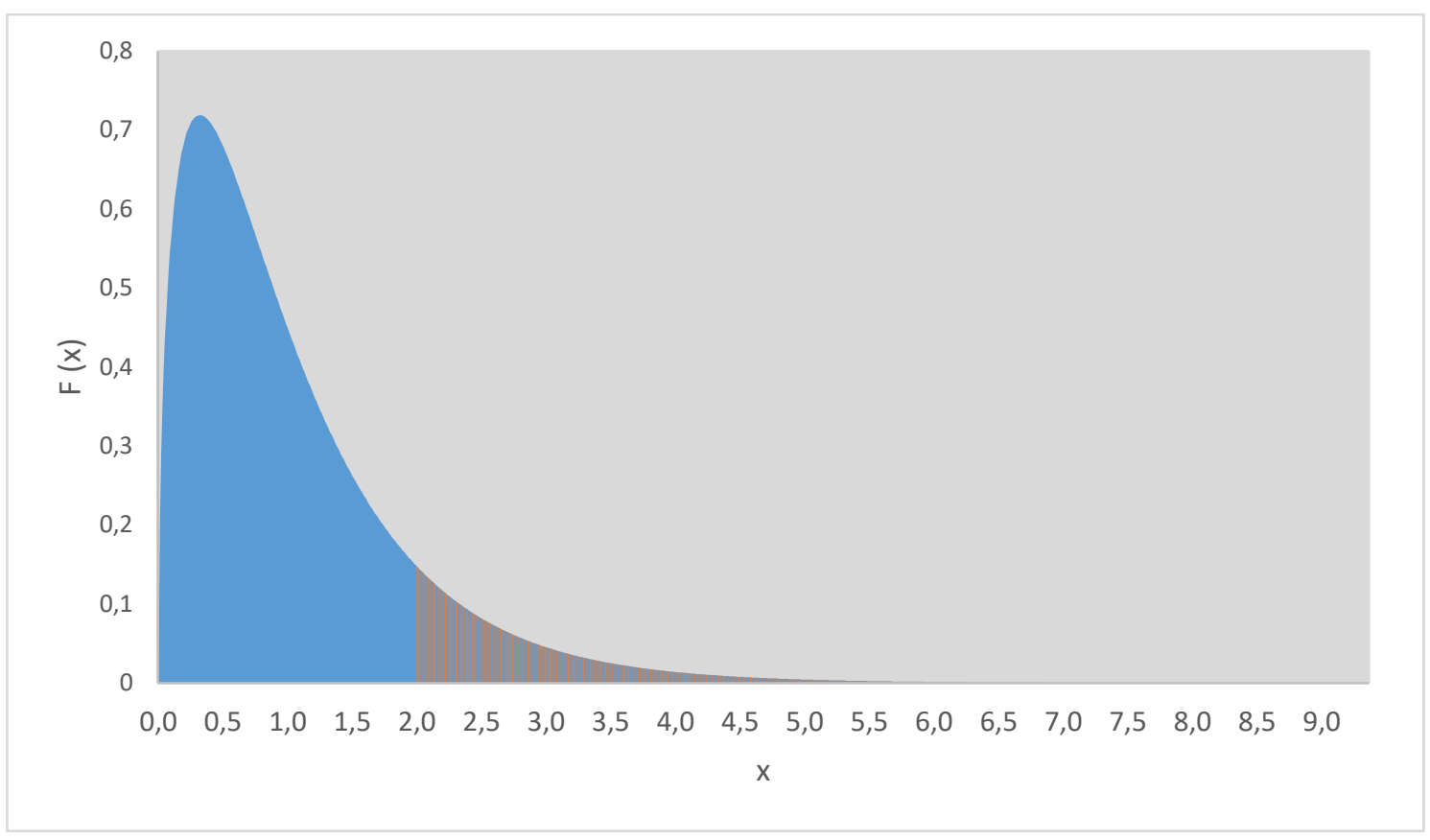

Figura 2-2 Función de densidad de probabilidad F de Fisher-Snedecor para grados de libertad 3 y 50, donde el área a la derecha de la azul es la probabilidad de que la regresión no sea extrapolable a la población. Fuente: elaboración propia.

Por último, en el caso de que queramos ver valores representativos de un parámetro en concreto, no la relación entre dos variables, se puede estudiar el promedio. Este en sí mismo puede no representar un valor típico, ya que podría haber una gran dispersión de los datos, así que es necesario analizar también la desviación típica. Si la desviación típica es pequeña comparada con el valor medio, entonces se podrá decir que este es un valor representativo del parámetro que se está estudiando. Por supuesto, el número de aviones que se incluyen en la muestra también es una variable a tener en cuenta, y así saber si el promedio involucra a muchas aeronaves o tan sólo a unas pocas. 


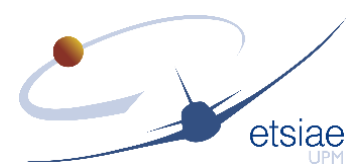

\subsection{CORRELACIONES Y ANÁLISIS DE DEPENDENCIA}

Una vez se han presentado los parámetros que van a estar contenidos en la base de datos, el procedimiento por el cual se van a establecer relaciones entre ellos y la magnitud que se va a utilizar para evaluar la validez de las correlaciones, se puede pasar a construir las regresiones entre los diferentes parámetros. En este caso, se han considerado como objeto de estudio aquellas correlaciones que han alcanzado un valor del coeficiente $\mathrm{R}^{2}$ superior a 0,8 . A pesar de ello, alguna de ellas que no ha alcanzado este valor, se ha contemplado porque ha permitido extraer alguna conclusión interesante, precisamente porque se esperaba que funcionase adecuadamente, y finalmente no ha sido así. Debido a la gran diversidad de aeronaves que están contenidas en la base de datos, se ha decidido clasificarlas con el objetivo de extraer conclusiones asociadas a ciertas propiedades características del avión, como pueden ser el segmento de vuelo, la configuración de cola, el número de motores o la posición de estos.

El segmento de vuelo agrupa las aeronaves según su alcance. Se han establecido cuatro categorías: aviones regionales, de pasillo único, de medio alcance y de largo alcance. A pesar de que las fronteras entre cada una de las categorías son un poco difusas, se ha decidido establecer los límites en alcance indicados en la Tabla 2-3. De la misma forma, en la Figura 2-3 se muestra qué aviones corresponden a qué categoría en función del número de pasajeros típico que pueden llevar cada uno. Se puede ver que hay varias aeronaves que se encuentran justo en la frontera, de ahí que las categorías sean un poco arbitrarias, ya que dependiendo del criterio es posible que una aeronave pertenezca a una u otra.

\begin{tabular}{|cc|}
\hline Segmento de vuelo & Alcance \\
\hline Regional & $\mathrm{R}<2,000 \mathrm{NM}$ \\
\hline Pasillo único & $2,000 \mathrm{NM}<\mathrm{R}<3,500 \mathrm{NM}$ \\
\hline Medio alcance & $3,500 \mathrm{NM}<\mathrm{R}<6,000 \mathrm{NM}$ \\
\hline Largo alcance & $\mathrm{R}>6,000 \mathrm{NM}$ \\
\hline
\end{tabular}

Tabla 2-3 Fronteras establecidas según el segmento de vuelo. Fuente: elaboración propia.

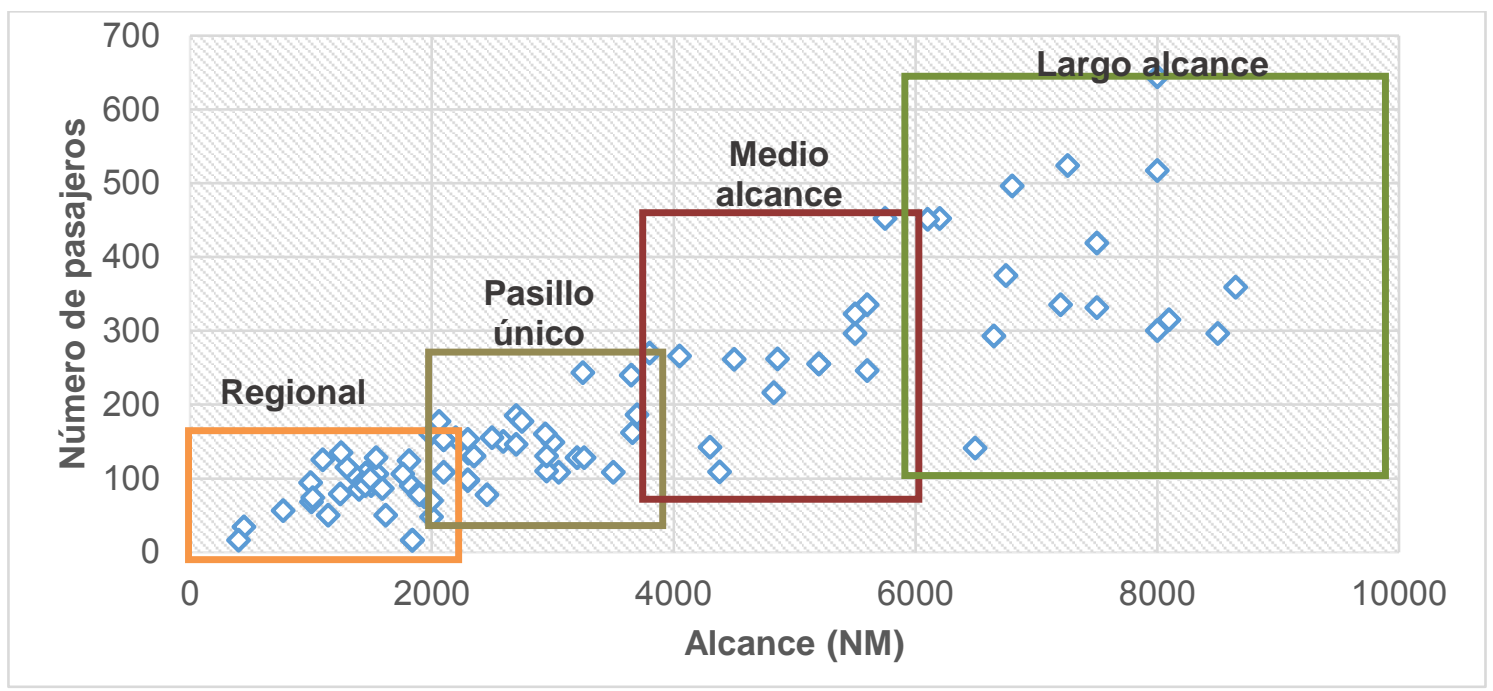

Figura 2-3 Clasificación de las aeronaves de acuerdo con el segmento de vuelo. Fuente: elaboración propia. 
En cuanto a la clasificación en función de la configuración de cola, tan sólo se han considerado las opciones que se encuentran más extendidas en la aviación de transporte comercial, como son la configuración convencional y cola en T. La principal diferencia entre ellas es la posición vertical del estabilizador horizontal, ya que en la cola convencional se encuentra sujeto al cono de cola y en la configuración de cola en T se encuentra sujeto a la parte superior del estabilizador vertical. Es cierto que existen aviones comerciales con configuración cruciforme, es decir, que el estabilizador horizontal se posiciona en un punto intermedio del estabilizador vertical. A pesar de ello, no se ha contemplado en el estudio ya que apenas hay aviones de transporte comercial que dispongan sus estabilizadores en esta posición, por lo que no se pueden extraer conclusiones estadísticas sobre los mismos.

Por último, el número de motores y su posición son características del avión que afecta al diseño de este, de ahí que se hayan considerado interesantes para este estudio. De hecho, el número de motores puede estar estrechamente relacionado con la configuración de cola, ya que lo habitual es utilizar la configuración en T si se llevan instalados tres motores, de tal forma que uno de ellos está situado en el plano de simetría embebido en el estabilizador vertical o en el cono de cola. También la posición de los motores afecta a la configuración de cola, ya que, en el caso de aviones con motores bajo las alas, el diseño del estabilizador vertical queda dimensionado por el control de la aeronave tras el fallo de motor crítico en el segundo segmento. Por otra parte, si se instalan dos motores, uno a cada lado del cono de cola, lo habitual también es decantarse por una configuración de cola en T. Por todo ello, el número de motores y su posición son características complementarias a la configuración de cola que se debían contemplar en este estudio sobre aviones de transporte comercial.

\subsubsection{Parámetros generales}

Uno de los parámetros más relevantes en la fase de diseño conceptual de un avión es la carga alar. La carga alar afecta a la mayoría de las actuaciones del avión, tales como el crucero, el despegue o el aterrizaje, pero también es un parámetro que influye en las cualidades del vuelo, como por ejemplo la velocidad de entrada en pérdida o el comportamiento frente a ráfagas verticales. Se define como el cociente entre el peso de la aeronave y su superficie alar. Es por ello, que se ha propuesto para este estudio establecer una correlación entre el peso máximo de despegue y la superficie alar. La Figura 2-4 muestra los resultados obtenidos si se clasifican los aviones según la configuración de cola.

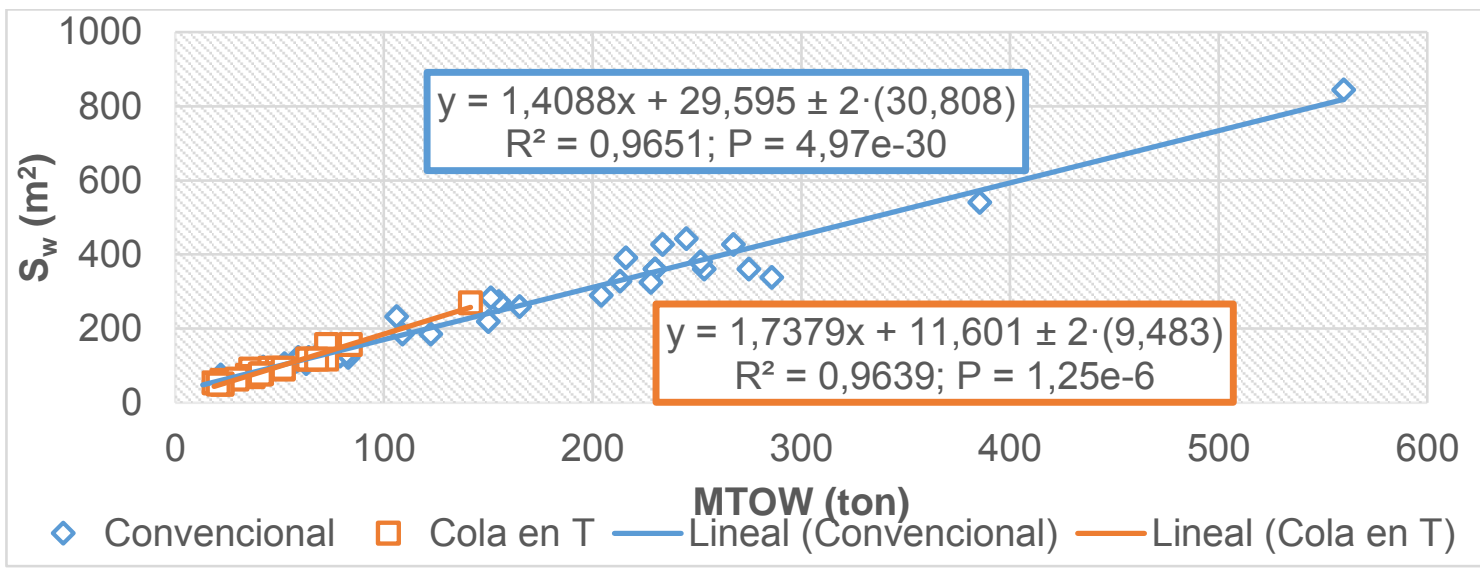

Figura 2-4 Correlaciones lineales que relacionan el peso máximo de despegue con la superficie alar del avión de acuerdo con la configuración de cola. Fuente: elaboración propia. 
Como se podía esperar, esta correlación presenta buenos resultados en ambas categorías, ya que es una relación clásica en diseño conceptual utilizada por otros autores anteriormente, pero estableciendo una relación no lineal conocida como square cube law, que en esencia es una relación potencial entre las variables (Torenbeek 1982). En este caso, se han establecido correlaciones lineales porque son intrínsecamente más simples y con un $\mathrm{R}^{2}$ superior al establecido como válido y también superior a la correlación potencial. Además, el error asociado a las regresiones se indica en la propia gráfica multiplicado por dos, para garantizar que el $95 \%$ de las muestras estén dentro del intervalo asumiendo la hipótesis de que las variables son aleatorias y de distribución normal, tal y como se comentó en la sección anterior. Este error es alrededor de un $10 \%$ del valor medio en ambos casos, siendo ligeramente mayor que este en la categoría de aviones de configuración convencional. Además, se observa como en ambas regresiones el nivel de significación es muy alto, ya que la probabilidad de que estas no sean extrapolables a la población completa es inferior al 0,001 establecido como límite.

Analizando los resultados, se observa como la recta asociada a las aeronaves de cola en T se sitúa ligeramente por encima de la correspondiente a cola convencional. Con esto se puede concluir que, de media, los aviones de configuración de cola en T presentan una menor carga alar, lo que supone una peor respuesta frente a ráfagas verticales, ya que para un mismo peso máximo de despegue estos aviones tienen mayor superficie alar.

Si clasificamos los aviones según el segmento de vuelo, los resultados se muestran en la Figura 2-5. En este caso se observa como sí que funciona mejor la correlación del tipo square cube law para los segmentos pasillo único y medio alcance. Algunos autores indican que el exponente de la regresión potencial depende del tipo de motor, diferenciando entre turbofán y turbohélice (Torenbeek 1982), correspondiendo 0,8 y 0,6 estos valores respectivamente. Aquí se muestra que no sólo depende del tipo de planta propulsora, sino que también del segmento de vuelo, precisamente creciendo los valores del exponente desde 0,5 hasta 0,88 a medida que aumenta el peso máximo de despegue. Precisamente los exponentes varían entre los mismos valores que para el tipo de planta propulsora, de acuerdo con la referencia anteriormente citada. Sin embargo, para el caso del segmento regional y de largo alcance, se ha presentado una relación lineal porque supone una mejor aproximación para ambos segmentos. De nuevo, en la figura se indican los valores del error típico asociados a cada una de las regresiones y de las probabilidades de que la regresión no sea significativa. 


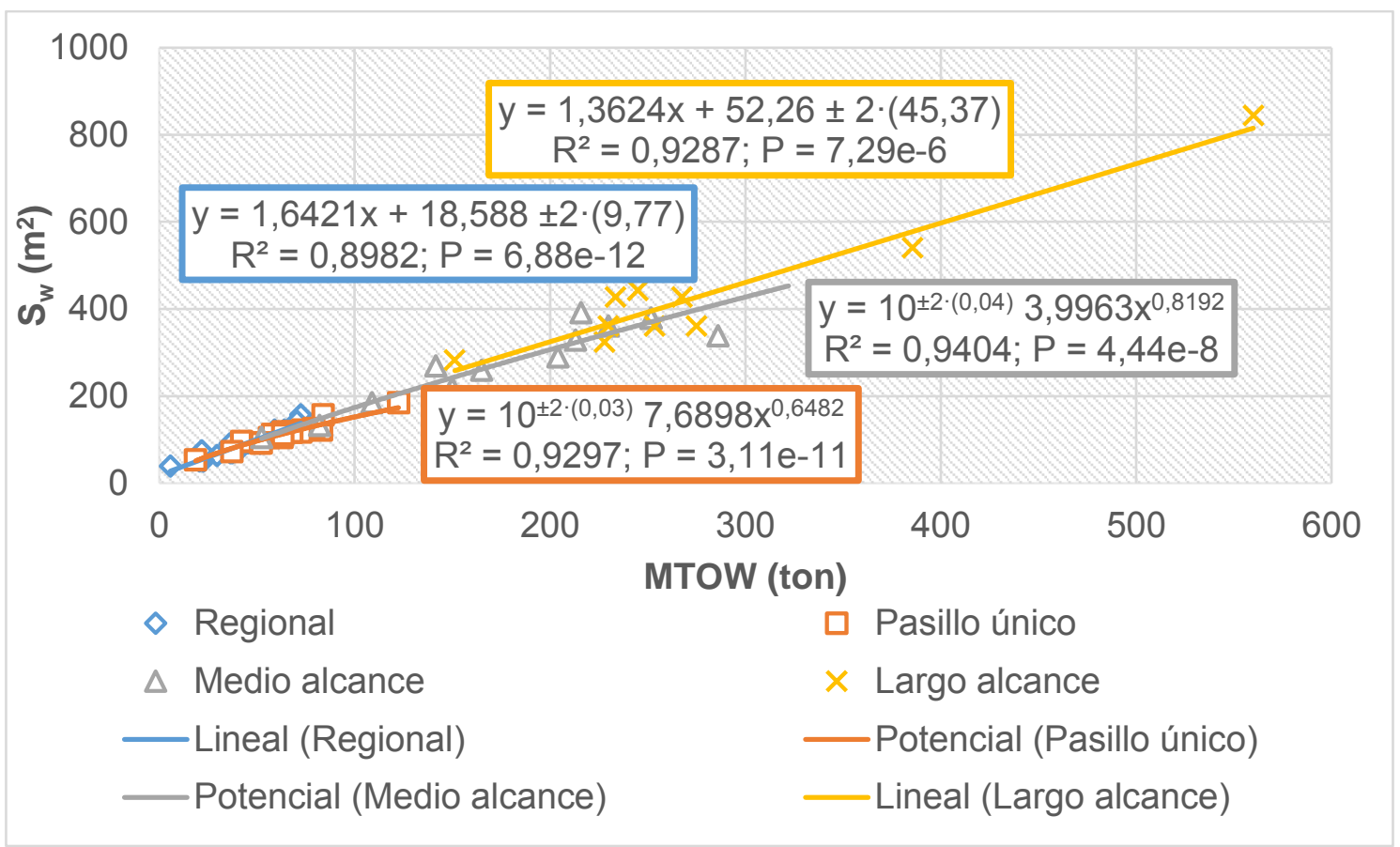

Figura 2-5 Correlaciones que relacionan el peso máximo de despegue con la superficie alar del avión de acuerdo con el segmento de vuelo. Fuente: elaboración propia.

Por último, se presenta la dependencia entre el peso máximo de despegue y la superficie alar clasificando los aviones dependiendo del número de motores en la Figura 2-6. De nuevo se observa como la regresión potencial presenta mejor coeficiente de correlación que la regresión lineal para el caso de tres y cuatro motores, sin embargo, para el caso de dos motores se utiliza la lineal. También se puede concluir que, para un mismo valor de superficie alar, superior a $200 \mathrm{~m}^{2}$, el peso del avión es mayor si se instalan tres motores que si lo hacen un número par de ellos. Nuevamente, se incluyen al final de las ecuaciones de regresión los errores típicos asociados a cada aproximación y los niveles de significación.

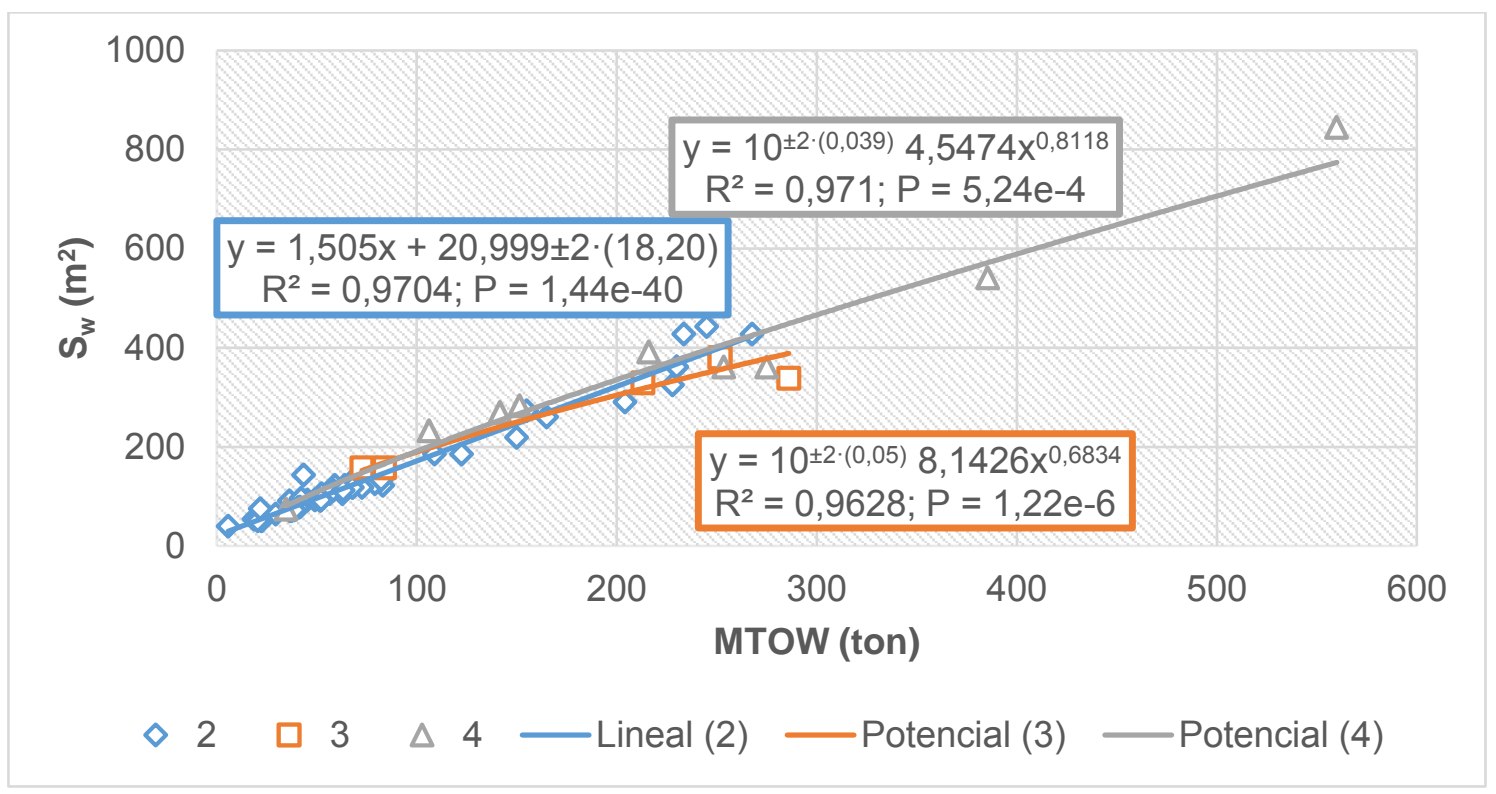

Figura 2-6 Correlaciones que relacionan el peso máximo de despegue con la superficie alar del avión de acuerdo con el número de motores del avión. Fuente: elaboración propia. 
Por otra parte, se puede establecer una relación entre el tamaño total de la aeronave y el peso máximo de despegue. El tamaño se va a representar como el parámetro de volumen global presentado en la sección anterior, es decir, el producto de la envergadura alar por la altura y por la longitud del fuselaje. De nuevo, se trata de buscar qué clasificación de la muestra da mejores resultados a la hora de establecer esta relación. Se ha visto que las mejores de entre las que se han tenido en cuenta han sido el número de motores y la posición de estos. La Figura 2-7 muestra los resultados según la configuración de cola, la Figura 2-8 muestra los resultados obtenidos en función del número de motores, y la Figura 2-9 los correspondientes a la clasificación según la posición del motor. En esta última se contemplan dos posibilidades: que tengan motores en el fuselaje posterior o que no los tenga, correspondiendo en la leyenda sí o NO respectivamente.

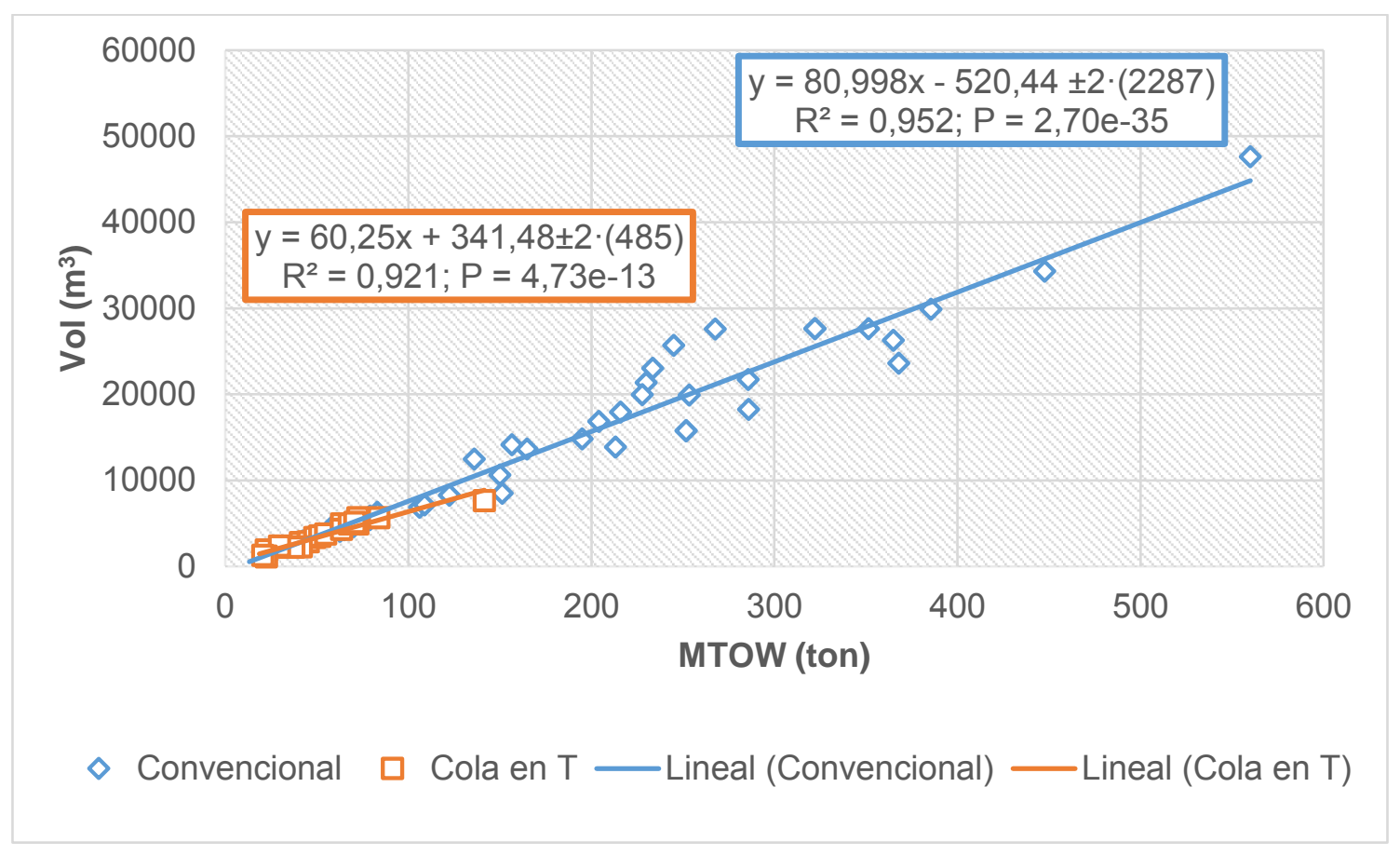

Figura 2-7 Correlaciones que relacionan el parámetro de volumen global del avión con el peso máximo de despegue de acuerdo con la configuración de cola. Fuente: elaboración propia. 


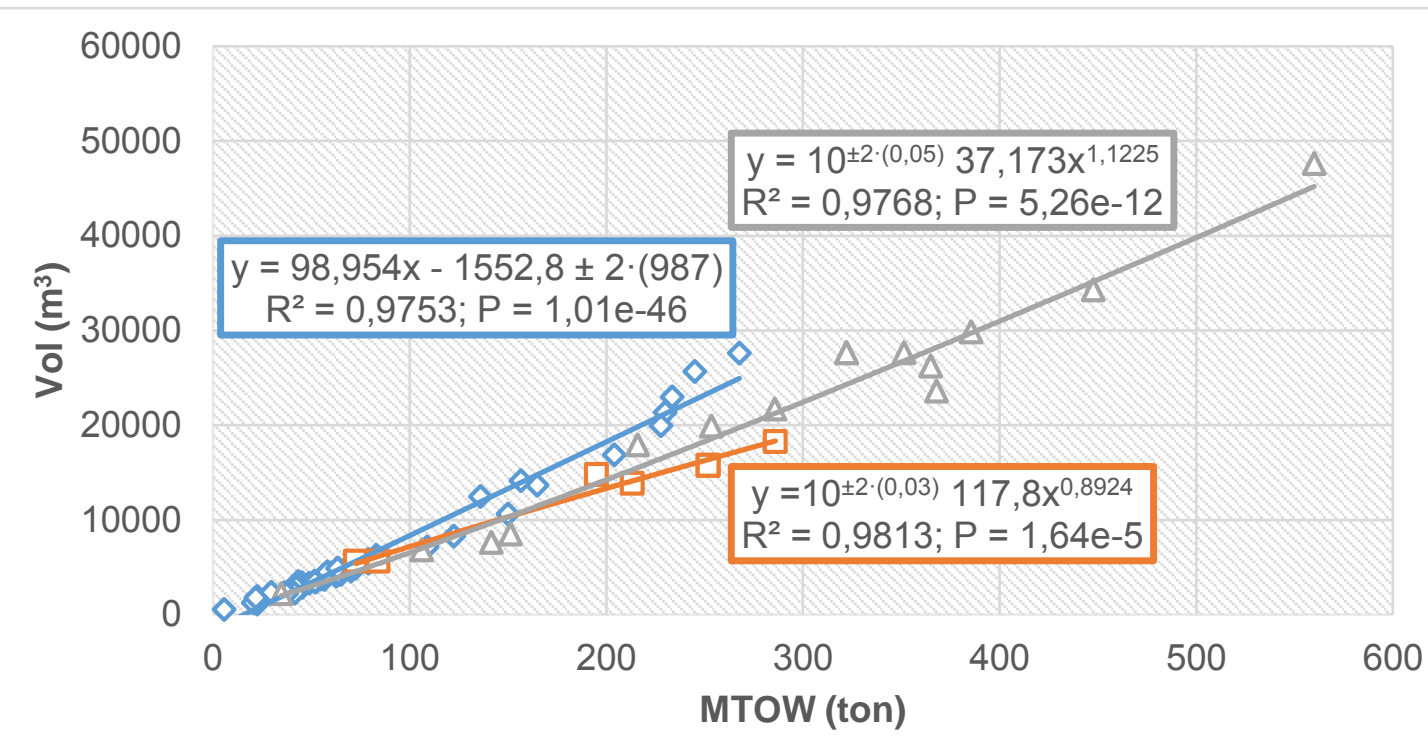

$\diamond 2 \square 3 \Delta 4-$ Lineal (2) - Potencial (3) — Potencial (4)

Figura 2-8 Correlaciones que relacionan el parámetro de volumen global del avión con el peso máximo de despegue de acuerdo con el número de motores del avión. Fuente: elaboración propia.

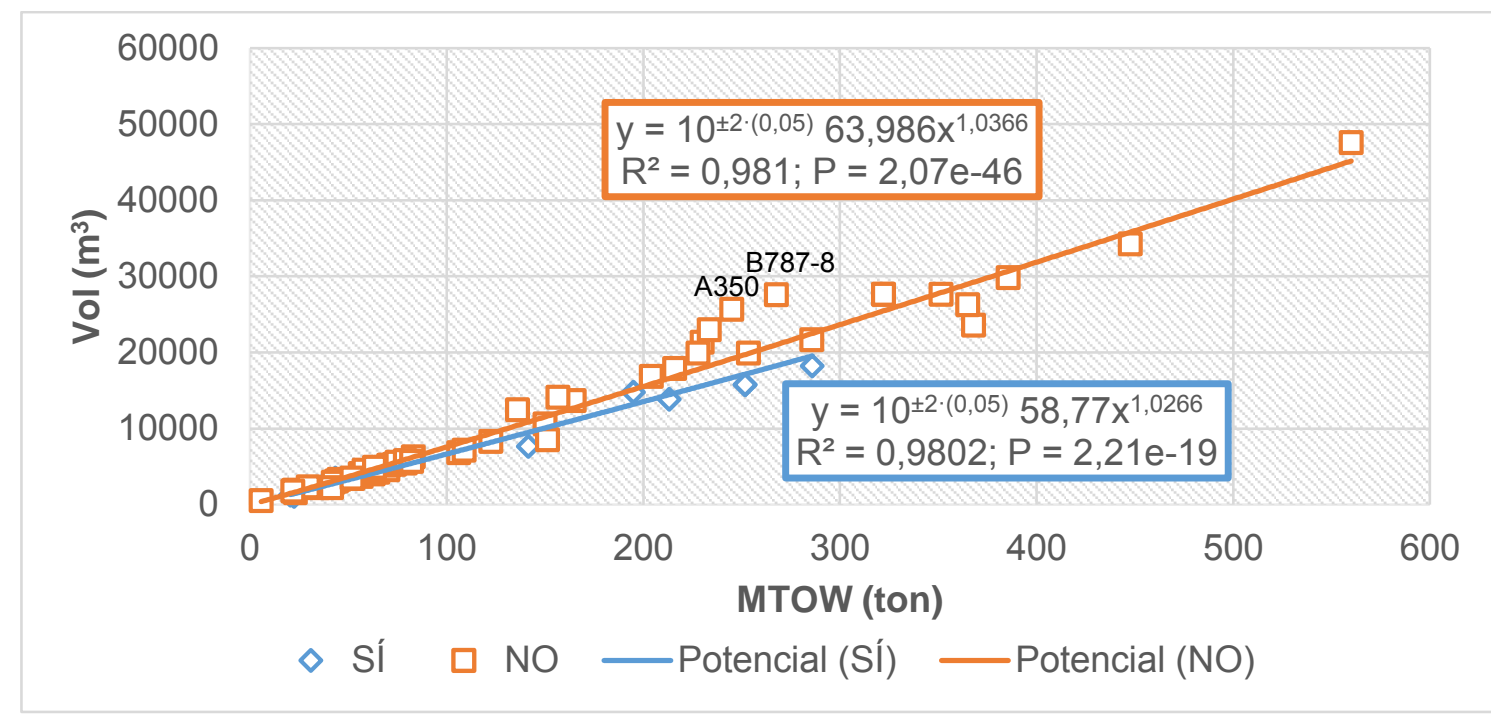

Figura 2-9 Correlaciones que relacionan el parámetro de volumen global del avión con el peso máximo de despegue de acuerdo con si llevan (Sí) o no (NO) motores en el cono de cola.

Fuente: elaboración propia.

A la vista de las regresiones mostradas en las figuras, se puede concluir que el número de motores afecta a la relación que existe entre el tamaño del avión y el peso máximo de despegue, siendo la opción más pesada la trimotor y la más ligera la bimotor. De la misma forma, si la configuración del avión es tal que se lleva al menos un motor instalado en el fuselaje posterior del avión, se obtiene una aeronave más pesada que si se decide instalarlos bajo el ala. También se observa como en la regresión correspondiente a los aviones que no llevan motores en el fuselaje posterior, encontramos una serie de puntos que se encuentran sustancialmente por encima de la curva. Se ha visto que estos aviones son más modernos que el resto (tales como el A350 y el B787-8), de tal forma que los avances que se obtienen en el uso de materiales compuestos en elementos 


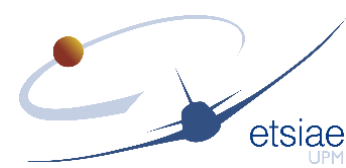

estructurales llevan a un avión que presenta mayor tamaño pero menor peso máximo de despegue.

Por último, se va a presentar un análisis sobre las velocidades de crucero para las que se diseñan los aviones en función del principal criterio de clasificación que se ha visto que afecta a esta variable, como es el segmento de vuelo. En la Tabla 2-4 se muestran los promedios y las desviaciones típicas obtenidas para cada uno de los casos. Como es lógico, se han separado los resultados por un lado las aeronaves que llevan turbofán y por otro las que llevan turbohélice. Se observa cómo, aunque todos se encuentran en torno a 0,8 , el promedio del número de Mach de crucero para los aviones equipados con motores turbofán crece a medida que el alcance aumenta. Sin embargo, los aviones turbohélice presentan una mayor variedad de números de Mach, como muestra la desviación típica, eso sí, alrededor de un valor próximo a 0,4.

\begin{tabular}{|c|ccc|ccc|}
\hline $\begin{array}{c}\text { Segmento de } \\
\text { vuelo }\end{array}$ & Cantidad & Promedio & $\begin{array}{c}\text { Desviación } \\
\text { típica }\end{array}$ & Cantidad & Promedio & $\begin{array}{c}\text { Desviación } \\
\text { típica }\end{array}$ \\
\hline $\begin{array}{c}\text { Regional } \\
\text { Pasillo único }\end{array}$ & 19 & 0,79 & 0,03 & 6 & 0,38 & 0,17 \\
\hline $\begin{array}{c}\text { Medio } \\
\text { alcance }\end{array}$ & 19 & 0,80 & 0,03 & 1 & 0,40 & - \\
$\begin{array}{c}\text { Largo } \\
\text { alcance }\end{array}$ & 12 & 0,82 & 0,03 & - & - & - \\
\hline
\end{tabular}

Tabla 2-4 Números de Mach de crucero de diseño para los aviones clasificados en función del tipo de motor (turbofán o turbohélice) en columnas y según el segmento de vuelo por filas. Se incluye la cantidad de aviones que pertenecen a cada categoría, el promedio de los números de Mach y la desviación típica. Fuente: elaboración propia.

\subsubsection{Fuselaje posterior}

Para el estudio recogido en este documento, el fuselaje posterior, o cono de cola, se considerará desde que termina la zona cilíndrica del fuselaje hasta el final de este, tal y como se ha descrito anteriormente. Desde el punto de vista de este tramo de fuselaje, se van a extraer conclusiones acerca de su geometría. Se van a analizar parámetros tales como la esbeltez del cono de cola, el porcentaje de longitud que ocupa este con respecto a la longitud del fuselaje o el ángulo de guarda. De igual modo que se ha hecho con el número de Mach de crucero, los resultados se presentarán en forma de tabla, comparando los valores medios y las desviaciones típicas obtenidas para diferentes criterios de clasificación con el objetivo de esclarecer cómo afectan al diseño del fuselaje posterior y al cono de cola. La Tabla 2-5 recoge los resultados obtenidos para la esbeltez del fuselaje posterior, medida como el cociente entre la longitud de este tramo y la altura del fuselaje; y la fracción que ocupa el cono de cola en relación al fuselaje completo. Se puede observar como apenas hay diferencias en los valores medios entre las diferentes categorías en ambos parámetros, concluyendo que aproximadamente la esbeltez del cono de cola es 3,4 y la fracción de longitud que ocupa está en torno al $37 \%$. Si bien, se pueden notar pequeñas diferencias para alguna de las categorías. Por ejemplo, es reseñable que la esbeltez del cono de cola es ligeramente mayor en el caso de los aviones tetramotores que para el resto, alrededor de un 7\%; así como que la fracción de longitud del cono de cola es sensiblemente menor, sobre un $6 \%$, en el segmento de vuelo de largo alcance. Conclusiones semejantes se pueden extraer en la categoría de tipo de planta propulsora, ya que se puede decir que el cono de cola de los turbohélices es ligeramente más esbelto, casi un $10 \%$ mayor, y ocupa una fracción de fuselaje un $6 \%$ mayor. Llama la atención que las diferencias más acusadas aparezcan en 
categorías diferentes a la de configuración de cola o posición de los motores, ya que a priori cabía pensar que la disposición de los estabilizadores y si los motores se instalan en el fuselaje posterior o no eran aspectos que deberían afectar en mayor medida a estos dos parámetros.

\begin{tabular}{|c|c|c|c|ccc|}
\hline & \multicolumn{2}{|c|}{ Esbeltez del cono de cola } & \multicolumn{3}{c|}{$\begin{array}{c}\text { Fracción de longitud de } \\
\text { fuselaje del cono de cola }\end{array}$} \\
\hline Criterio & Cantidad & Promedio & $\begin{array}{c}\text { Desviación } \\
\text { típica }\end{array}$ & Cantidad & Promedio & $\begin{array}{c}\text { Desviación } \\
\text { típica }\end{array}$ \\
\hline Convencional & 34 & 3,45 & 0,401 & 34 & 0,371 & 0,049 \\
\hline Cola en T & 13 & 3,36 & 0,379 & 13 & 0,351 & 0,074 \\
\hline Regional & 18 & 3,42 & 0,381 & 18 & 0,371 & 0,081 \\
\hline $\begin{array}{c}\text { Pasillo único } \\
\text { Medio }\end{array}$ & 11 & 3,50 & 0,187 & 11 & 0,371 & 0,041 \\
\hline $\begin{array}{c}\text { alcance } \\
\text { Largo }\end{array}$ & 13 & 3,43 & 0,405 & 13 & 0,369 & 0,053 \\
\hline alcance & 7 & 3,50 & 0,503 & 7 & 0,347 & 0,049 \\
\hline 2 motores & 36 & 3,39 & 0,360 & 36 & 0,360 & 0,067 \\
\hline 3 motores & 6 & 3,40 & 0,465 & 6 & 0,381 & 0,051 \\
\hline 4 motores & 8 & 3,64 & 0,412 & 8 & 0,394 & 0,031 \\
\hline $\begin{array}{c}\text { Motores en } \\
\text { cola: Sín }\end{array}$ & 13 & 3,34 & 0,451 & 13 & 0,361 & 0,075 \\
\hline $\begin{array}{c}\text { Motores en } \\
\text { cola: NO }\end{array}$ & 35 & 3,48 & 0,361 & 35 & 0,374 & 0,057 \\
\hline Turbofán & 44 & 3,40 & 0,386 & 44 & 0,366 & 0,057 \\
\hline Turbohélice & 6 & 3,71 & 0,227 & 6 & 0,387 & 0,090 \\
\hline
\end{tabular}

Tabla 2-5 Esbeltez del cono de cola y fracción de longitud de fuselaje que ocupa en función de diferentes criterios: configuración de cola, segmento de vuelo, número de motores, posición de los motores y tipo de planta propulsora. Se incluye la cantidad de aviones que pertenecen a cada categoría, el promedio de los parámetros y su desviación típica. Fuente: elaboración propia.

Por otra parte, se van a comentar los resultados obtenidos tras el análisis de los ángulos de guarda y upsweep. De nuevo, los valores medios y las desviaciones típicas obtenidas en función de los diferentes criterios de clasificación se recogen en la Tabla 2-6. Se puede ver como no se extraen tendencias claras en ninguna de las categorías. De hecho, los valores tienen una gran dispersión, ya que la desviación típica es prácticamente del mismo orden de magnitud que el promedio. A pesar de esto, se puede decir que el ángulo de guarda en los aviones que llevan instalados motores en la cola de media es mayor que los que no lo llevan; aproximadamente un $22 \%$ mayor. Diferencias similares se encuentran al comparar los aviones equipados con motores turbofán y los que no, siendo el ángulo de guarda mayor en los primeros. En cuanto al ángulo de upsweep, las desviaciones típicas son aún mayores comparadas con los promedios que en el caso del ángulo de guarda, por lo que lo máximo que se puede asegurar es que los valores oscilan entre 3 y 4 grados. 


\begin{tabular}{|c|c|c|c|c|c|c|}
\hline \multirow[b]{2}{*}{ Criterio } & \multicolumn{3}{|c|}{ Ángulo de guarda (ํ) } & \multicolumn{3}{|c|}{ Ángulo de upsweep () } \\
\hline & Cantidad & Promedio & $\begin{array}{l}\text { Desviación } \\
\text { típica }\end{array}$ & Cantidad & Promedio & $\begin{array}{c}\text { Desviación } \\
\text { típica }\end{array}$ \\
\hline Convencional & 33 & 11,52 & 2,45 & 34 & 4,26 & 2,26 \\
\hline Cola en $\mathrm{T}$ & 13 & 11,52 & 3,10 & 13 & 2,99 & 1,28 \\
\hline Regional & 18 & 11,36 & 2,98 & 18 & 4,21 & 2,91 \\
\hline Pasillo único & 10 & 10,68 & 1,98 & 11 & 3,73 & 1,10 \\
\hline $\begin{array}{l}\text { Medio } \\
\text { alcance }\end{array}$ & 13 & 12,36 & 2,59 & 13 & 3,77 & 1,81 \\
\hline $\begin{array}{l}\text { Largo } \\
\text { alcance }\end{array}$ & 7 & 11,31 & 1,60 & 7 & 3,64 & 1,16 \\
\hline 2 motores & 35 & 11,23 & 2,58 & 36 & 4,13 & 2,21 \\
\hline 3 motores & 6 & 12,54 & 1,83 & 6 & 2,41 & 1,21 \\
\hline 4 motores & 8 & 12,20 & 2,72 & 8 & 3,76 & 1,53 \\
\hline $\begin{array}{l}\text { Motores en } \\
\text { cola: Sí }\end{array}$ & 13 & 13,37 & 1,68 & 13 & 3,08 & 1,12 \\
\hline $\begin{array}{l}\text { Motores en } \\
\text { cola: NO }\end{array}$ & 34 & 10,91 & 2,55 & 35 & 4,32 & 2,20 \\
\hline Turbofán & 43 & 11,83 & 2,49 & 44 & 3,66 & 1,34 \\
\hline Turbohélice & 6 & 9,52 & 2,01 & 6 & 5,37 & 4,85 \\
\hline
\end{tabular}

Tabla 2-6 Ángulo de guarda y ángulo de upsweep en función de diferentes criterios: configuración de cola, segmento de vuelo, número de motores, posición de los motores y tipo de planta propulsora. Se incluye la cantidad de aviones que pertenecen a cada categoría, el promedio de los parámetros y su desviación típica. Fuente: elaboración propia.

\subsubsection{Estabilizador horizontal}

En cuanto al estabilizador horizontal, se pueden plantear relaciones clásicas como son su área frente a la superficie alar. Sin embargo, esta relación depende fuertemente del criterio de clasificación. En concreto, si se clasifican los aviones según el número de motores o su posición, el coeficiente de determinación obtenido es sustancialmente mejor que en cualquiera de las otras categorías. Los resultados se muestran en la Figura 2-10 y la Figura 2-11. Se observa cómo los aviones que llevan instalados tres motores se comportan de forma diferente al resto, de ahí que se haya optado por presentar las regresiones en función de la posición de los motores, donde se estratifican las dos tendencias. Además, los aviones que llevan algún motor en el fuselaje posterior necesitan mayor superficie de estabilizador horizontal que los que los llevan bajo las alas, para una misma superficie alar. Esto es debido a que el centro de gravedad de los aviones que llevan motores en cola está más retrasado que en el caso de que no lo hacen, siendo el brazo de momentos de las superficies de cola menor y, por lo tanto, la superficie del estabilizador debe ser mayor para alcanzar un mismo valor del coeficiente de volumen. En ambas figuras se incluyen los resultados correspondientes al error típico y a la probabilidad de que la regresión no sea significativa en cada una de las correlaciones construidas. 


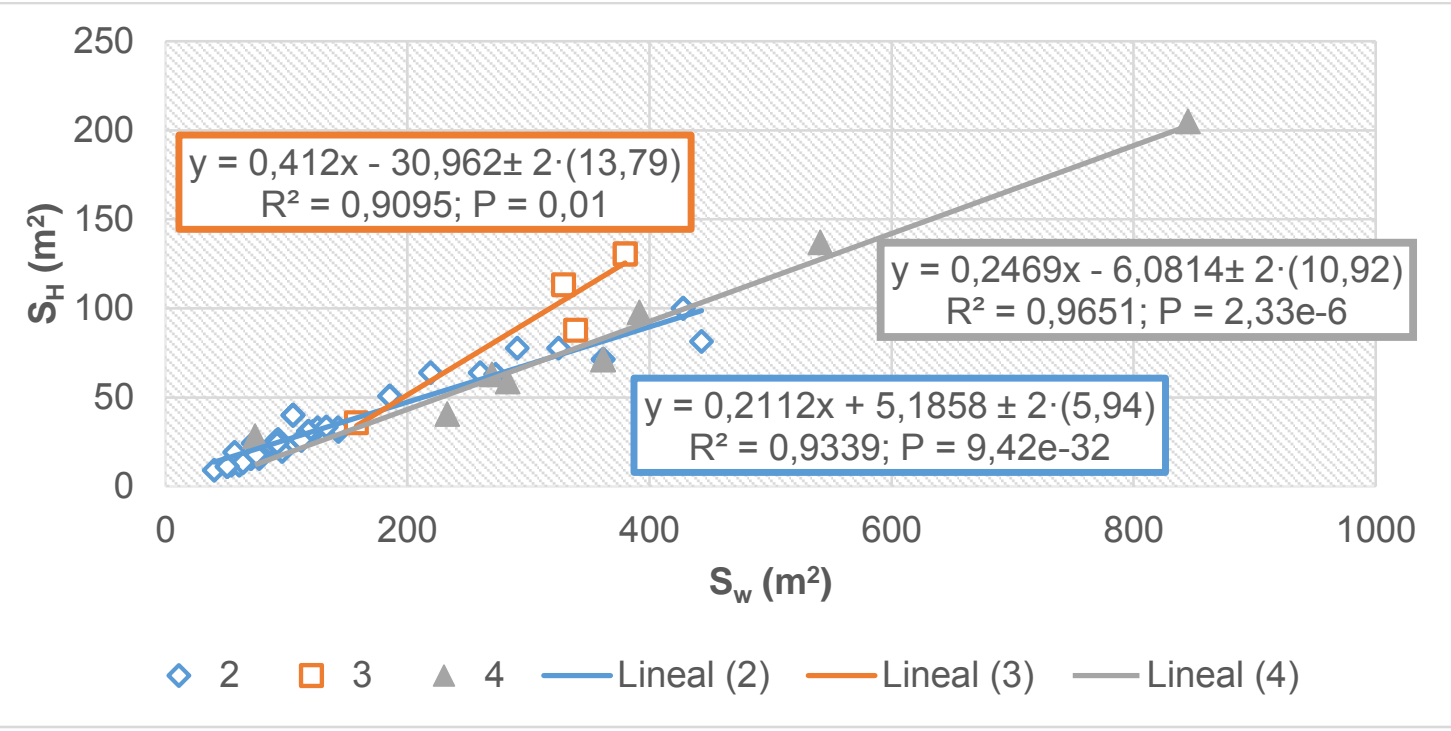

Figura 2-10 Correlaciones que relacionan la superficie del estabilizador horizontal y la superficie alar de acuerdo con el número de motores. Fuente: elaboración propia.

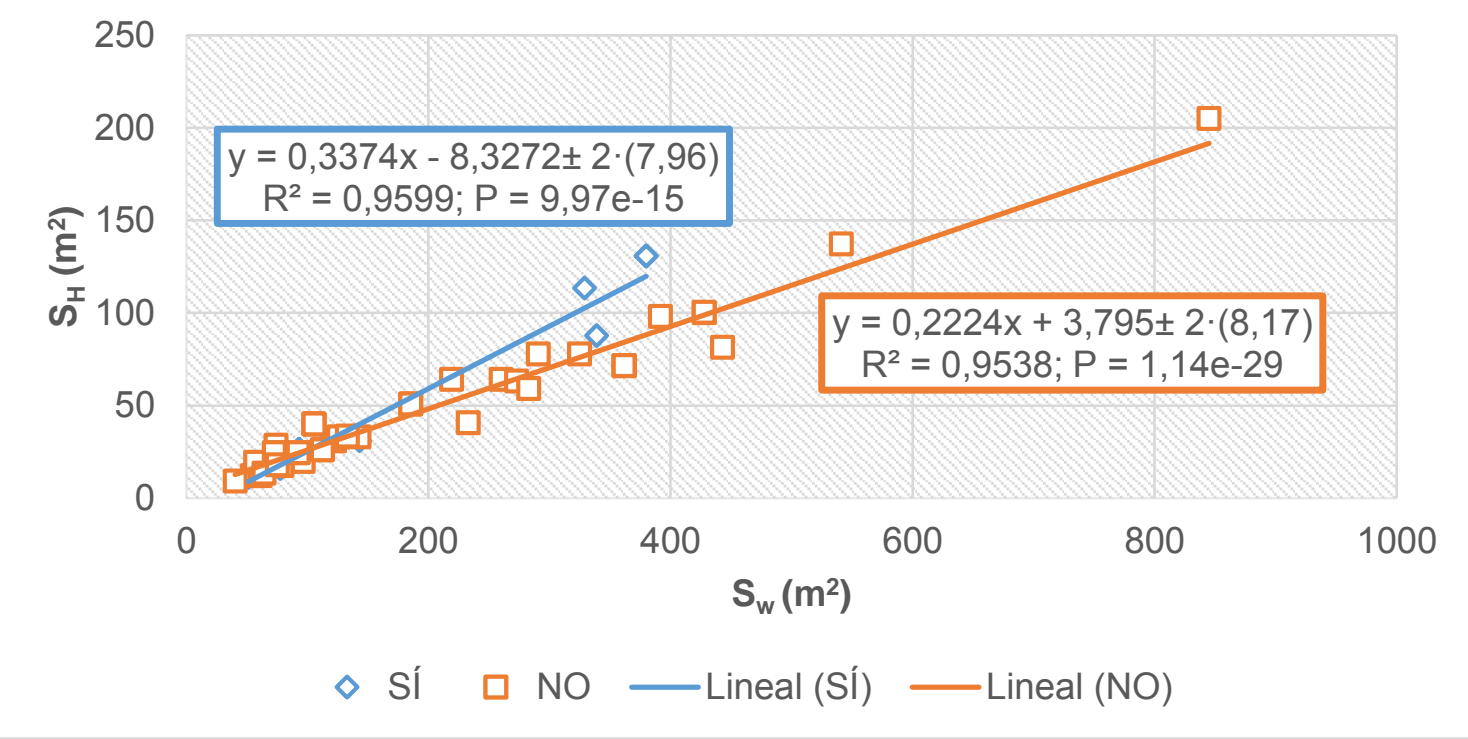

Figura 2-11 Correlaciones que relacionan la superficie del estabilizador horizontal y la superficie alar de acuerdo con si llevan (Sí) o no (NO) motores en el cono de cola. Fuente: elaboración propia.

El brazo de momentos del estabilizador horizontal es un parámetro de relevancia a la hora del diseño de las superficies de cola. Por ello, se ha buscado una regresión que permita determinar su valor en función de otros parámetros de diseño, en concreto, la longitud del fuselaje. Se ha visto que esta correlación funciona bien siguiendo varios criterios de clasificación, tales como la configuración de cola o el número de motores, en el caso de que este sea un número par. Sin embargo, la que mejor resultados presenta es la que se incluye en la Figura 2-12, de acuerdo a la posición de los motores. Se puede observar como la pendiente correspondiente a los aviones que no llevan ningún motor en el fuselaje posterior es mayor que la correspondiente a los aviones que sí los llevan. Este hecho junto con que la recta de los aviones que no llevan motores en cola está por encima permite concluir que el brazo de momentos en los aviones que tienen motores instalados en cola es menor que el resto, para una misma longitud de fuselaje. Esto se debe a que estos aviones tienen el centro de gravedad más retrasado 


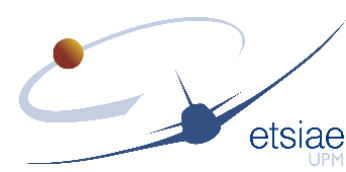

y, por lo tanto, el ala también lo está. Así, la distancia entre el punto $1 / 4$ de la cuerda media aerodinámica del ala y el punto $1 / 4$ de la cuerda media geométrica del estabilizador horizontal es menor. Esto concuerda con los resultados obtenidos anteriormente para el área del estabilizador horizontal, ya que se ha visto cómo los aviones con motores en cola presentan una mayor superficie del estabilizador que los demás, para una misma superficie alar. De esta forma, desde el punto de vista de la estabilidad estática longitudinal, ambos efectos se compensan. En la figura se incluyen los resultados correspondientes al error típico y a la probabilidad de que la regresión no sea significativa en cada una de las correlaciones construidas.

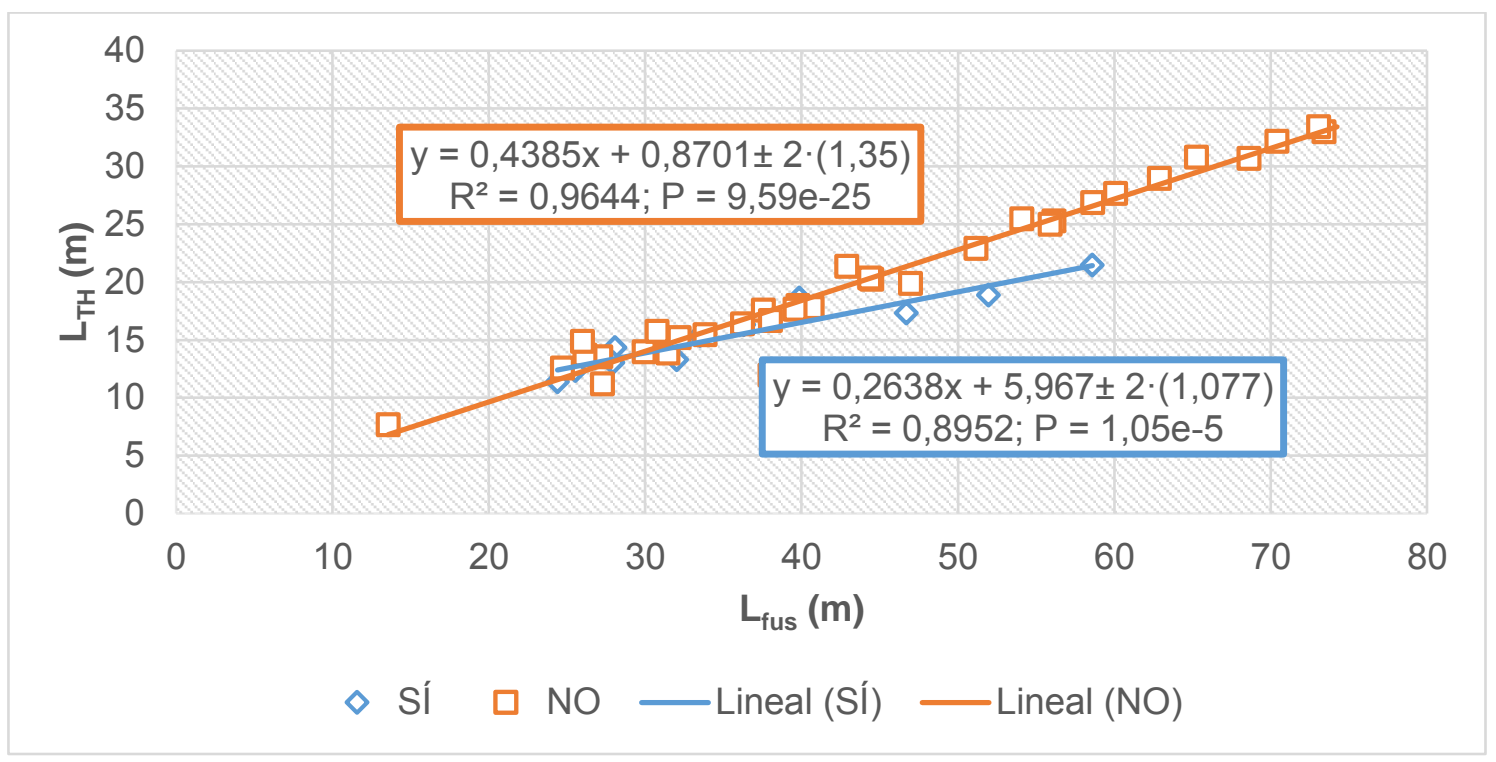

Figura 2-12 Correlaciones que relacionan el brazo de momentos del estabilizador horizontal y la longitud del fuselaje de acuerdo con si llevan (SÍ) o no (NO) motores en el cono de cola.

Fuente: elaboración propia.

También es posible establecer relaciones entre el parámetro de resistencia inducida presentado en apartados anteriores y el peso máximo de despegue. Esta relación funciona especialmente bien cuando los aviones se clasifican según el fabricante. En la Figura 2-13 se muestran los resultados obtenidos para los cuatro fabricantes más relevantes de los que se encontraban en la base de datos, como son Boeing, Airbus, Embraer y Bombardier. Las regresiones obtenidas para los dos últimos son menos vinculantes debido al número más reducido de aviones que se han considerado en este estudio. Sin embargo, sí que se pueden extraer conclusiones a la vista de las regresiones que presentan los aviones de Boeing y Airbus. Se observa como los aviones del fabricante estadounidense presentan valores del parámetro de resistencia inducida mayores que para los del fabricante europeo, salvo alguna excepción. En la figura se incluyen los resultados correspondientes al error típico y a la probabilidad de que la regresión no sea significativa en cada una de las correlaciones construidas, salvo para los fabricantes Embraer y Bombardier debido a que las regresiones tan solo se componen de tres puntos en cada caso. 

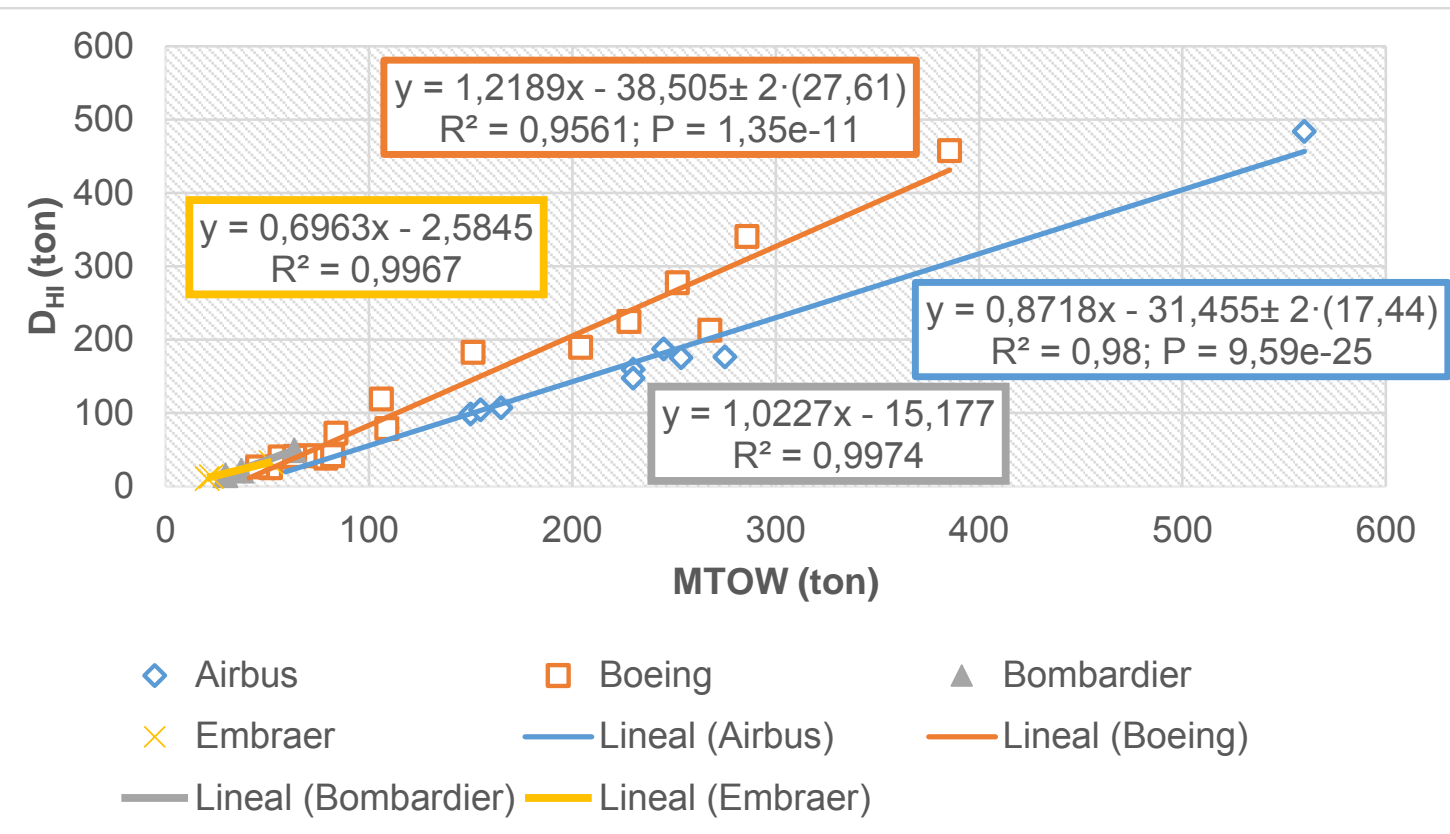

Figura 2-13 Correlaciones que relacionan el parámetro de resistencia inducida y el peso máximo de despegue en función del fabricante. Fuente: elaboración propia.

Asimismo, se puede comparar los resultados obtenidos en función del segmento de vuelo (Figura 2-14). Se ha decidido mostrar también estas regresiones debido a que se puede ver, en las que se han presentado hasta ahora, que tienen una dispersión considerable a bajos valores de peso máximo de despegue. Esto nos permitirá estimar mejor este parámetro a bajos valores de peso, como son los segmentos de pasillo único y regional. Sin embargo, en la figura se observa como las correlaciones no son buenas para los dos segmentos de mayor peso máximo de despegue, de ahí que para estos sea preferible usar alguna de las que depende del fabricante. En la figura se incluyen los resultados correspondientes al error típico y a la probabilidad de que la regresión no sea significativa en cada una de las correlaciones construidas.

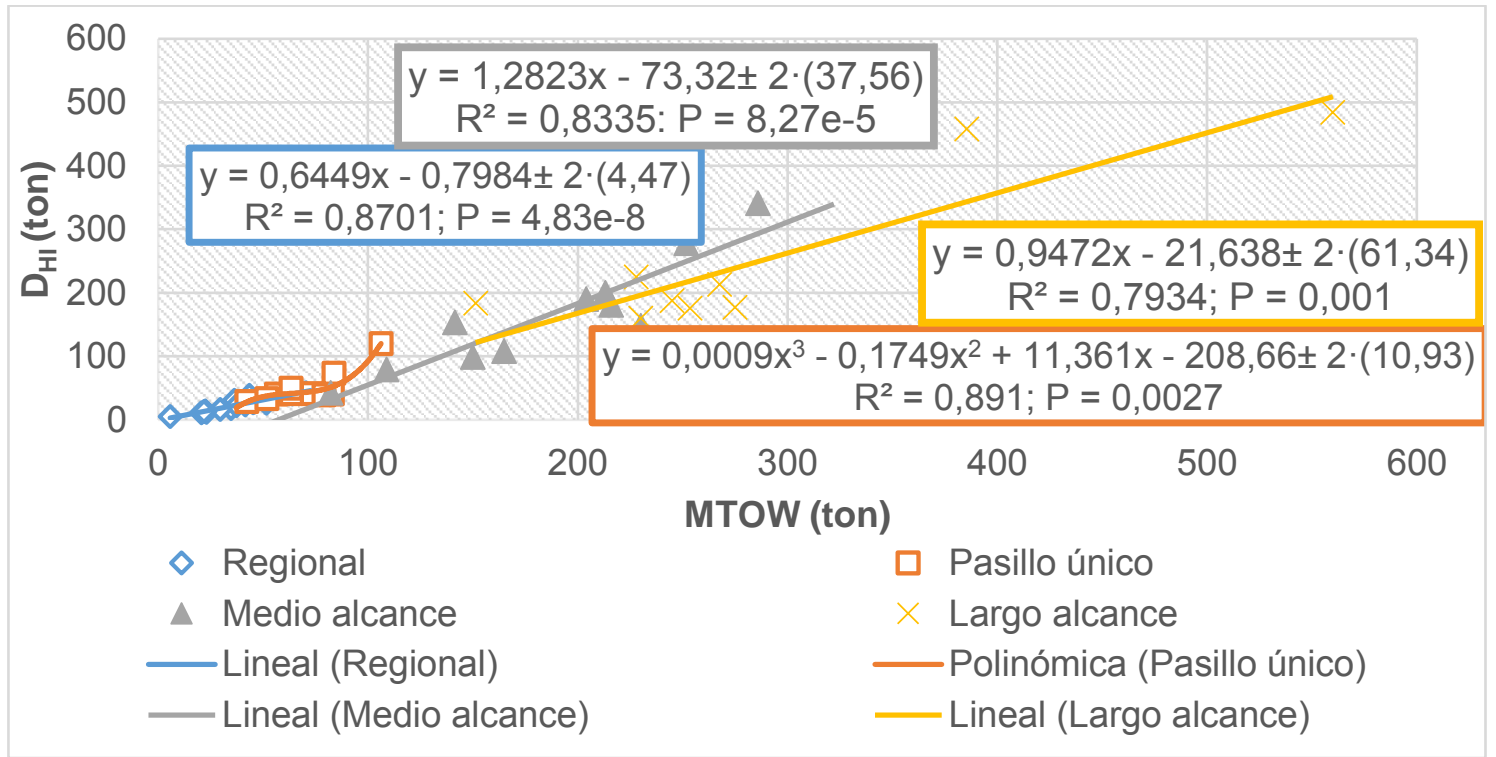

Figura 2-14 Correlaciones que relacionan el parámetro de resistencia inducida y el peso máximo de despegue en función del segmento de vuelo. Fuente: elaboración propia. 
Para terminar con la caracterización de los estabilizadores horizontales se contemplan una serie de parámetros, de los cuales se van a estudiar sus promedios y desviaciones típicas, tales como el alargamiento, parámetro de estrechamiento, flecha o superficie adimensional del timón de profundidad. Respecto a la flecha de la línea de puntos $1 / 4 \mathrm{y}$ el parámetro de estrechamiento del estabilizador horizontal, se recogen los resultados en la Tabla 2-7. Se observa cómo la flecha es mayor en los segmentos de vuelo cuya velocidad de crucero es mayor, como es el caso de medio y largo alcance, y es menor para los segmentos regional y pasillo único. Es cierto que en la mayoría de los grupos de clasificación se obtienen desviaciones típicas de un orden de magnitud próximo al del promedio, lo cual deja una gran variabilidad a los resultados. Sin embargo, se hace notar la poca variabilidad que se presenta en los aviones trimotores, fijando la flecha de los puntos $1 / 4$ alrededor de $35^{\circ}$.

\begin{tabular}{|c|c|c|c|c|c|c|}
\hline & \multicolumn{3}{|c|}{ Flecha puntos $1 / 4\left({ }^{\circ}\right)$} & \multicolumn{3}{|c|}{ Parámetro de estrechamiento } \\
\hline Criterio & Cantidad & Promedio & $\begin{array}{l}\text { Desviación } \\
\text { típica }\end{array}$ & Cantidad & Promedio & $\begin{array}{l}\text { Desviación } \\
\text { típica }\end{array}$ \\
\hline Convencional & 45 & 30,32 & 6,63 & 45 & 0,376 & 0,093 \\
\hline Cola en $\mathbf{T}$ & 24 & 26,10 & 9,59 & 24 & 0,462 & 0,129 \\
\hline Regional & 26 & 22,67 & 10,53 & 26 & 0,467 & 0,165 \\
\hline Pasillo único & 21 & 29,70 & 5,85 & 21 & 0,390 & 0,097 \\
\hline $\begin{array}{l}\text { Medio } \\
\text { alcance }\end{array}$ & 14 & 33,65 & 2,70 & 14 & 0,383 & 0,127 \\
\hline $\begin{array}{l}\text { Largo } \\
\text { alcance }\end{array}$ & 11 & 33,17 & 2,70 & 11 & 0,356 & 0,055 \\
\hline 2 motores & 57 & 27,19 & 8,84 & 57 & 0,417 & 0,139 \\
\hline 3 motores & 6 & 35,81 & 0,91 & 6 & 0,355 & 0,022 \\
\hline 4 motores & 10 & 31,74 & 5,44 & 10 & 0,416 & 0,126 \\
\hline $\begin{array}{l}\text { Motores en } \\
\text { cola: Sí }\end{array}$ & 24 & 30,66 & 5,55 & 24 & 0,411 & 0,110 \\
\hline $\begin{array}{l}\text { Motores en } \\
\text { cola: NO }\end{array}$ & 47 & 27,22 & 9,57 & 47 & 0,414 & 0,145 \\
\hline Turbofán & 66 & 30,90 & 4,19 & 66 & 0,388 & 0,097 \\
\hline Turbohélice & 7 & 6,06 & 4,50 & 7 & 0,637 & 0,203 \\
\hline
\end{tabular}

Tabla 2-7 Flecha de los puntos $1 / 4$ y parámetro de estrechamiento del estabilizador horizontal en función de diferentes criterios: configuración de cola, segmento de vuelo, número de motores, posición de los motores y tipo de planta propulsora. Se incluye la cantidad de aviones que pertenecen a cada categoría, el promedio de los parámetros y su desviación típica. Fuente: elaboración propia.

Analizando el parámetro de estrechamiento, las aeronaves con cola en T presentan un valor más alto, de media, que las de cola convencional. Esto se da por la imposición geométrica que tiene el hecho de que esté instalado en la punta del estabilizador vertical. Desde el punto de vista del segmento de vuelo, el parámetro de estrechamiento decrece a medida que el alcance crece. Además, se encuentran notables diferencias en la clasificación acorde al número de motores, ya que los aviones trimotores presentan un menor parámetro de estrechamiento. Esto se debe a que la instalación de un motor en el fuselaje posterior lleva a un mayor peso del cono de cola. En consecuencia, las soluciones de diseño del estabilizador horizontal son tales que tengan un peso más 
reducido para intentar compensar el aumento que provoca el motor de cola. Es por ello por lo que tanto el parámetro de estrechamiento como el alargamiento, como se puede ver en la Tabla $2-8$, son menores, alrededor de un $13 \%$ en ambos casos, que en el resto de los aviones. Analizando el alargamiento del estabilizador horizontal en el resto de las categorías, en ninguna se encuentran diferencias importantes salvo en los aviones de medio alcance, que presentan un alargamiento un $10 \%$ menor que el del resto. De nuevo, las desviaciones típicas obtenidas no son lo suficientemente pequeñas como para estratificar los resultados en categorías claras, solapándose las horquillas correspondientes a cada grupo. De ahí que la única conclusión que se puede extraer es que el alargamiento del estabilizador horizontal se sitúa en torno a 4,5. Por último, se ha estudiado el valor del cociente entre la superficie del timón de profundidad o elevador y la superficie del estabilizador horizontal, también incluido en la Tabla 2-8. Aquí sí que se encuentran diferencias en función del criterio de clasificación. Se observa cómo los aviones de configuración de cola en $\mathrm{T}$ presentan un mayor valor de este cociente. Además, el cociente se reduce a medida que el alcance del avión crece. Los aviones que instalan motores en cola también presentan un ratio mayor.

\begin{tabular}{|c|c|c|c|c|c|c|}
\hline \multirow[b]{2}{*}{ Criterio } & \multicolumn{3}{|c|}{ Alargamiento } & \multicolumn{3}{|c|}{$\mathbf{S}_{\text {elev }} / \boldsymbol{S}_{\mathrm{H}}$} \\
\hline & Cantidad & Promedio & $\begin{array}{l}\text { Desviación } \\
\text { típica }\end{array}$ & Cantidad & Promedio & $\begin{array}{l}\text { Desviación } \\
\text { típica }\end{array}$ \\
\hline Convencional & 45 & 4,61 & 0,770 & 36 & 0,247 & 0,037 \\
\hline Cola en $T$ & 24 & 4,55 & 0,604 & 24 & 0,341 & 0,045 \\
\hline Regional & 26 & 4,77 & 0,566 & 25 & 0,331 & 0,052 \\
\hline Pasillo único & 21 & 4,62 & 0,700 & 17 & 0,285 & 0,052 \\
\hline $\begin{array}{l}\text { Medio } \\
\text { alcance }\end{array}$ & 14 & 4,19 & 0,835 & 12 & 0,239 & 0,051 \\
\hline $\begin{array}{l}\text { Largo } \\
\text { alcance }\end{array}$ & 11 & 4,64 & 0,687 & 9 & 0,237 & 0,013 \\
\hline 2 motores & 57 & 4,75 & 0,589 & 49 & 0,303 & 0,056 \\
\hline 3 motores & 6 & 3,67 & 0,293 & 6 & 0,221 & 0,035 \\
\hline 4 motores & 10 & 4,19 & 0,874 & 9 & 0,246 & 0,061 \\
\hline $\begin{array}{l}\text { Motores en } \\
\text { cola: Sí }\end{array}$ & 24 & 4,45 & 0,640 & 24 & 0,315 & 0,069 \\
\hline $\begin{array}{l}\text { Motores en } \\
\text { cola: NO }\end{array}$ & 47 & 4,68 & 0,706 & 38 & 0,268 & 0,050 \\
\hline Turbofán & 66 & 4,57 & 0,709 & 57 & 0,282 & 0,062 \\
\hline Turbohélice & 7 & 4,71 & 0,597 & 7 & 0,329 & 0,038 \\
\hline
\end{tabular}

Tabla 2-8 Alargamiento del estabilizador horizontal y relación entre las superficies del timón de profundidad y del estabilizador en función de diferentes criterios: configuración de cola, segmento de vuelo, número de motores, posición de los motores y tipo de planta propulsora. Se incluye la cantidad de aviones que pertenecen a cada categoría, el promedio de los parámetros y su desviación típica. Fuente: elaboración propia. 


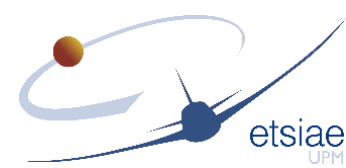

\subsubsection{Estabilizador vertical}

En este apartado se va a tratar de caracterizar el estabilizador vertical. Al igual que en el caso del horizontal, se llevará a cabo tanto por medio de relaciones entre parámetros como de valores típicos para algunos otros. Primeramente, se va a establecer la relación análoga a la que se presentó para el estabilizador horizontal, como es la que se produce entre el área del estabilizador y la superficie alar. Esta relación depende fuertemente del número de motores, como se puede ver en la Figura 2-15. Se puede ver cómo los aviones que tienen 3 motores tienen estabilizadores verticales de mayor superficie que el resto, para una misma superficie alar. Esto se debe a que al estar uno de los motores embebidos en la cola vertical, se necesita un mayor tamaño de la deriva. En la figura se incluyen los resultados correspondientes al error típico y a la probabilidad de que la regresión no sea significativa en cada una de las correlaciones construidas.

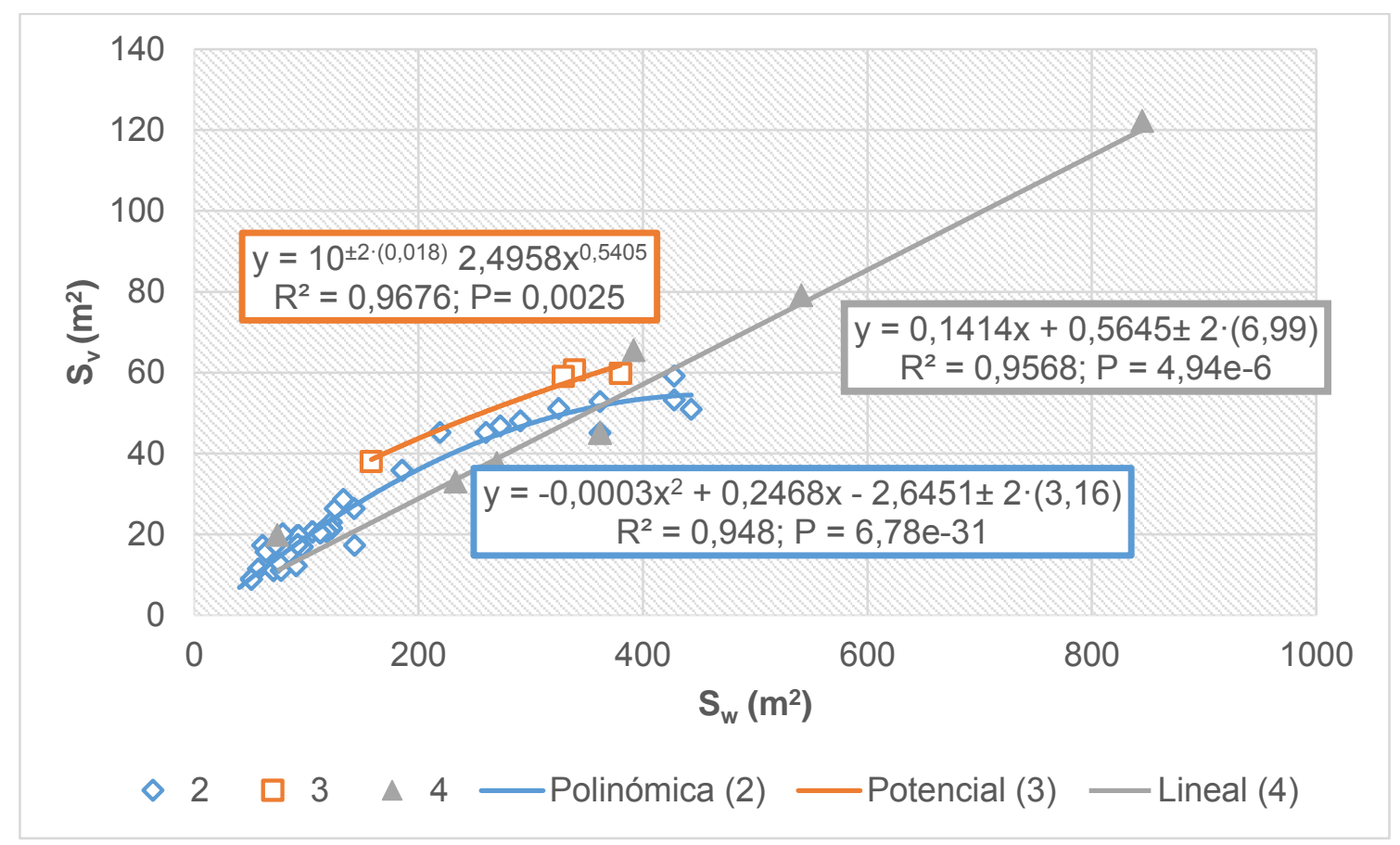

Figura 2-15 Correlaciones que relacionan las superficies del estabilizador vertical y la alar en función del número de motores instalados. Fuente: elaboración propia.

Por otra parte, tal y como se ha indicado en el apartado 2.1, dedicado a la descripción de los parámetros, se incluyó la ecuación que rige el dimensionado del estabilizador vertical regido por el control lateral tras el fallo de motor crítico en despegue. De ahí se puede extraer una relación entre el producto de la superficie del estabilizador por el brazo de momentos y el momento generado por la asimetría de empuje, que es el producto de la distancia al plano de simetría del avión del motor crítico por la fuerza resultante de la asimetría de empuje, que se puede aproximar por el empuje al despegue de un motor (Torenbeek 1982). Los resultados se muestran en la Figura 2-16. De nuevo, se ha visto cómo la categoría donde se obtenían mejores resultados es la que depende del número de motores, mostrando una separación clara entre los aviones trimotores y el resto. Al igual que ocurría en la regresión anterior, el hecho de que los aviones trimotores tengan un motor en el interior de la deriva vertical condiciona el diseño de la misma, diferenciándose de forma evidente de los aviones con un número de motores par. En la figura se incluyen los resultados correspondientes al error típico y a la probabilidad de que la regresión no sea significativa en cada una de las correlaciones construidas. 


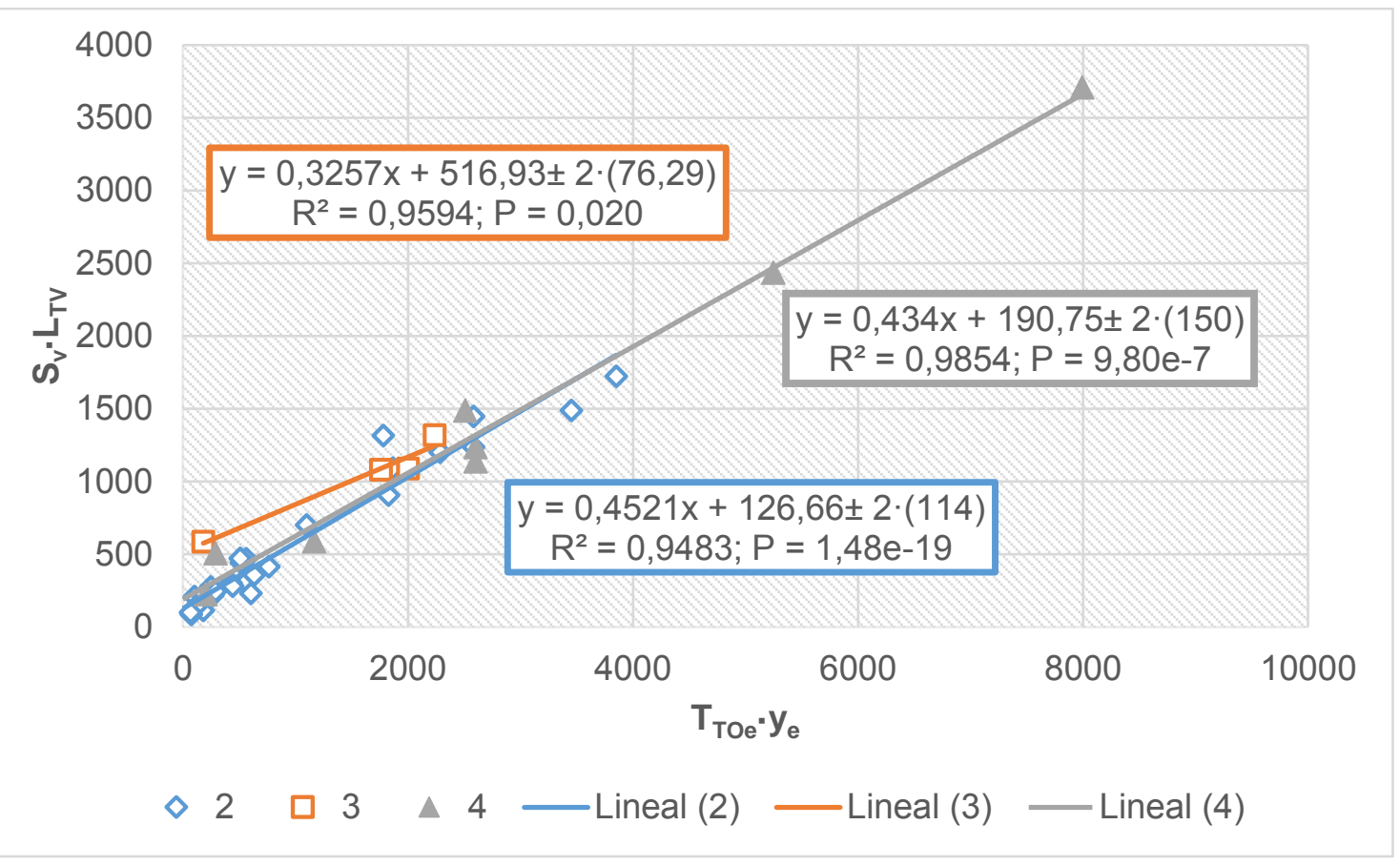

Figura 2-16 Correlaciones que relacionan el producto de la superficie del estabilizador vertical por su brazo de momentos y el producto del empuje de un motor en despegue por la distancia al plano de simetría del avión del motor crítico en función del número de motores instalados.

Fuente: elaboración propia.

A continuación, en la Tabla 2-9 se incluyen los resultados obtenidos para la flecha de línea de puntos $1 / 4$ de la deriva vertical y del parámetro de estrechamiento de acuerdo con los diferentes criterios contemplados en este estudio. Como se puede ver, los valores de la flecha son bastante mayores que para el caso del estabilizador horizontal. Además, las desviaciones típicas son un orden de magnitud inferior a los promedios, por lo que se puede decir que apenas presentan dispersión los resultados. Analizando las categorías, se encuentran importantes diferencias en la clasificación en función de la configuración de cola, pudiendo concluir que las aeronaves con cola en T presentan derivas verticales de mayor flecha que las de los aviones de configuración convencional. Algo similar ocurre en la categoría dependiente del número de motores, siendo los trimotores los que mayores valores de flecha presentan. Nuevamente se aprecia cómo condiciona el motor de cola el diseño del estabilizador vertical. En cuanto al parámetro de estrechamiento, la primera conclusión que se puede extraer es la gran dispersión que presentan la mayoría de las categorías, ya que los valores de desviación típica son del orden de los valores promedios. A pesar de ello, aquí también se pueden encontrar diferencias comparando las diferentes configuraciones de cola. En este caso los aviones de configuración en $\mathrm{T}$ tienen un parámetro de estrechamiento mayor, aproximadamente el doble, lo cual está justificado por el hecho de que la cuerda en la punta debe ser lo suficientemente grande como para sostener el estabilizador horizontal. La misma explicación aplica al caso de los aviones con motores en cola, ya que la mayoría de ellos son de configuración en $\mathrm{T}$, de ahí que también se aprecie esta diferencia con los aviones que los llevan bajo las alas, a pesar de que una pequeña parte de los aviones con motores bajo las alas usan la configuración de cola en T. Por último, es destacable la deferencia que se encuentra entre los aviones trimotores y el resto. En este caso ocurre justo lo contrario que en los aviones de cola en $\mathrm{T}$, ya que la restricción geométrica aparece en la cuerda en la raíz al tener que albergar el motor que se sitúa en el plano de simetría. De ahí que la cuerda en la raíz se mayor que en el resto de los aviones, y, en consecuencia, el parámetro de estrechamiento es menor. 


\begin{tabular}{|c|c|c|c|c|c|c|}
\hline \multirow[b]{2}{*}{ Criterio } & \multicolumn{3}{|c|}{ Flecha puntos $1 / 4\left(^{(}\right)$} & \multicolumn{3}{|c|}{ Parámetro de estrechamiento } \\
\hline & Cantidad & Promedio & $\begin{array}{l}\text { Desviación } \\
\text { típica }\end{array}$ & Cantidad & Promedio & $\begin{array}{c}\text { Desviación } \\
\text { típica }\end{array}$ \\
\hline Convencional & 44 & 36,94 & 4,277 & 44 & 0,337 & 0,070 \\
\hline Cola en $T$ & 22 & 41,35 & 4,899 & 22 & 0,698 & 0,099 \\
\hline Regional & 24 & 37,79 & 6,168 & 24 & 0,570 & 0,193 \\
\hline Pasillo único & 20 & 38,07 & 5,197 & 20 & 0,438 & 0,206 \\
\hline $\begin{array}{l}\text { Medio } \\
\text { alcance }\end{array}$ & 14 & 39,08 & 2,711 & 14 & 0,371 & 0,140 \\
\hline $\begin{array}{l}\text { Largo } \\
\text { alcance }\end{array}$ & 10 & 39,69 & 3,106 & 10 & 0,347 & 0,053 \\
\hline 2 motores & 54 & 38,16 & 4,695 & 54 & 0,464 & 0,199 \\
\hline 3 motores & 6 & 41,27 & 5,791 & 6 & 0,393 & 0,140 \\
\hline 4 motores & 9 & 38,29 & 5,231 & 9 & 0,453 & 0,178 \\
\hline $\begin{array}{l}\text { Motores en } \\
\text { cola: Sí }\end{array}$ & 23 & 40,99 & 3,452 & 23 & 0,613 & 0,186 \\
\hline $\begin{array}{l}\text { Motores en } \\
\text { cola: NO }\end{array}$ & 44 & 36,72 & 4,466 & 44 & 0,361 & 0,118 \\
\hline Turbofán & 64 & 39,08 & 4,085 & 64 & 0,453 & 0,193 \\
\hline Turbohélice & 5 & 30,30 & 7,040 & 5 & 0,499 & 0,186 \\
\hline
\end{tabular}

Tabla 2-9 Flecha de los puntos $1 / 4$ y parámetro de estrechamiento del estabilizador vertical en función de diferentes criterios: configuración de cola, segmento de vuelo, número de motores, posición de los motores y tipo de planta propulsora. Se incluye la cantidad de aviones que pertenecen a cada categoría, el promedio de los parámetros y su desviación típica. Fuente: elaboración propia.

Por último, se va a analizar el alargamiento y el cociente entre el área del timón de dirección y la superficie de la deriva vertical. Los resultados se incluyen en la Tabla 2-10. Como se puede apreciar, el alargamiento tiene valores que se pueden considerar como representativos ya que la desviación típica es, por poco, un orden de magnitud inferior a los promedios. En este caso también se encuentran las principales diferencias en las categorías de configuración de cola y en la que depende de la posición de los motores. Los aviones de configuración de cola en T presentan un menor alargamiento que los aviones de configuración convencional, alrededor de un $36 \%$ menor, con el fin de reducir las cargas generadas en el encastre de la deriva vertical, ya que tiene que soportar sus propias cargas y transmitir al fuselaje las cargas del estabilizador horizontal. De igual forma encontramos esta diferencia en los aviones que llevan motores en cola con respecto a los que los llevan bajo las alas, ya que los primeros utilizan mayoritariamente la configuración de cola en $\mathrm{T}$. En cuanto a la relación entre las superficies de timón de dirección y la deriva vertical, la principal diferencia se puede encontrar en la clasificación según el número de motores. Los aviones trimotores presentan un cociente menor, ya que buena parte del estabilizador vertical está ocupado por el motor que está embebido en la deriva. 


\begin{tabular}{|c|c|c|c|c|c|c|}
\hline \multirow[b]{2}{*}{ Criterio } & \multicolumn{3}{|c|}{ Alargamiento } & \multicolumn{3}{|c|}{$S_{\text {rud }} / S_{H}$} \\
\hline & Cantidad & Promedio & $\begin{array}{c}\text { Desviación } \\
\text { típica }\end{array}$ & Cantidad & Promedio & $\begin{array}{l}\text { Desviación } \\
\text { típica }\end{array}$ \\
\hline Convencional & 44 & 1,718 & 0,150 & 31 & 0,277 & 0,084 \\
\hline Cola en $T$ & 22 & 1,097 & 0,123 & 22 & 0,255 & 0,052 \\
\hline Regional & 24 & 1,307 & 0,309 & 23 & 0,282 & 0,058 \\
\hline Pasillo único & 20 & 1,544 & 0,369 & 14 & 0,295 & 0,051 \\
\hline $\begin{array}{l}\text { Medio } \\
\text { alcance }\end{array}$ & 14 & 1,670 & 0,202 & 9 & 0,185 & 0,088 \\
\hline $\begin{array}{l}\text { Largo } \\
\text { alcance }\end{array}$ & 10 & 1,669 & 0,187 & 9 & 0,286 & 0,060 \\
\hline 2 motores & 54 & 1,494 & 0,335 & 41 & 0,294 & 0,045 \\
\hline 3 motores & 6 & 1,599 & 0,380 & 6 & 0,135 & 0,048 \\
\hline 4 motores & 9 & 1,501 & 0,258 & 9 & 0,247 & 0,077 \\
\hline $\begin{array}{l}\text { Motores en } \\
\text { cola: Sí }\end{array}$ & 23 & 1,245 & 0,290 & 23 & 0,235 & 0,071 \\
\hline $\begin{array}{l}\text { Motores en } \\
\text { cola: NO }\end{array}$ & 44 & 1,663 & 0,225 & 31 & 0,300 & 0,056 \\
\hline Turbofán & 64 & 1,503 & 0,334 & 51 & 0,263 & 0,069 \\
\hline Turbohélice & 5 & 1,512 & 0,226 & 5 & 0,335 & 0,059 \\
\hline
\end{tabular}

Tabla 2-10 Alargamiento del estabilizador vertical y relación entre las superficies del timón de dirección y del estabilizador en función de diferentes criterios: configuración de cola, segmento de vuelo, número de motores, posición de los motores y tipo de planta propulsora. Se incluye la cantidad de aviones que pertenecen a cada categoría, el promedio de los parámetros y su desviación típica. Fuente: elaboración propia.

\subsubsection{Caso de validación}

En este apartado se va a acometer un caso de validación de las correlaciones construidas. Lo primero que se va a hacer para llevar a cabo esta tarea es que se va a extraer de la base de datos un avión, en concreto el Boeing B737-8MAX, actualizándose así las ecuaciones de todas las regresiones en las que entraba este avión. Posteriormente se dimensionará el estabilizador horizontal de este avión partiendo de unos parámetros generales iniciales, como son el peso máximo de despegue, el número de pasajeros, el alcance de diseño y las dimensiones del fuselaje. A partir de estos datos se propone dar los pasos siguientes para diseñar el estabilizador horizontal:

1. Entrando en la Figura 2-7 en la categoría de cola convencional con el peso máximo de despegue, se obtiene $\mathrm{Vol}=6,044 \mathrm{~m}^{3}$, que presenta un error relativo con respecto al valor del avión real del $6 \%$.

2. Aplicando la definición del parámetro de volumen global, $\mathrm{Vol}=L_{f u s} H_{f u s} b_{w}$, se puede obtener la envergadura alar $b_{w}=38 \mathrm{~m}$, que presenta un error relativo del $6 \%$.

3. La superficie alar de puede obtener de la Figura 2-4, en la serie correspondiente a la configuración convencional de cola, partiendo del peso máximo de despegue. El valor correspondiente es $S_{w}=145 \mathrm{~m}^{2}$, al que se le asocia un error relativo respecto del B737-8MAX del 8,9\%.

4. Es necesario estimar la cuerda en la raíz del ala a partir del peso máximo de despegue, por medio de una regresión incluida en (Kallinderis, Vouvakos y 


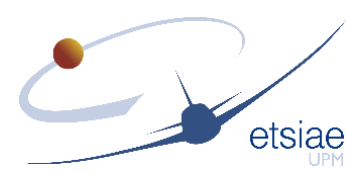

Menounou 2009), alcanzándose un resultado de $6,47 \mathrm{~m}$, que presenta un porcentaje de error relativo del $9,8 \%$.

5. Asumiendo un ala equivalente de forma en planta trapecial, es posible determinar la cuerda media aerodinámica del ala por medio de las relaciones matemáticas correspondientes (Yates 1952), siendo su valor de 4,43 m y el error relativo resultante del $1,6 \%$.

6. Por medio de la regresión de la Figura 2-10, para los aviones bimotores, la forma en planta del estabilizador horizontal se puede determinar $S_{H}=36,3 \mathrm{~m}^{2}$ con un error relativo del $8,3 \%$.

7. El siguiente paso es determinar el brazo de momentos del estabilizador. Se consigue usando la regresión de la Figura 2-12, resultando en $L_{T H}=18,2 \mathrm{~m}$ y un error relativo del $2,9 \%$.

8. Ahora se puede calcular el coeficiente de volumen del estabilizador horizontal aplicando su definición: $V_{e H}=1,027$, que corresponde con un error relativo respecto del avión original del 0,8\%.

9. Por último, para determinar el alargamiento y la envergadura del estabilizador es necesario hallar el parámetro de resistencia inducida $D_{H i}=43,55$ ton por medio de la correlación incluida en la Figura 2-14 para los aviones de medio alcance. Aplicando la definición de este parámetro, se obtiene una envergadura de $15 \mathrm{~m}$ y un alargamiento de 6,23 , con unos errores relativos del $3,4 \%$ y del $1,3 \%$, respectivamente.

A la vista de los resultados obtenidos y del error relativo cometido respecto del avión que se ha tomado para realizar este proceso de validación, se puede decir que los métodos utilizados son adecuados para el diseño conceptual debido a la precisión, superior al $90 \%$, y del tiempo de cálculo, que es muy bajo. De hecho, el error medio de los parámetros estimados del estabilizador horizontal está por debajo del 4,6\%, incluyendo una excelente aproximación del coeficiente de volumen. Las ecuaciones de las correlaciones que se han utilizado para llevar a cabo este caso de validación, al suprimir el avión B737-8MAX, se incluyen en la referencia (Cuerno-Rejado y SanchezCarmona 2016). 


\subsection{CASO DE ESTUDIO}

En este capítulo se va a tratar de poner de manifiesto la utilidad de las regresiones y valores característicos de los parámetros por medio de un caso de ejemplo. Se trata de dimensionar el estabilizador horizontal de un avión de referencia. Este avión será un avión englobado en la categoría de pasillo único, de configuración convencional, con dos motores bajo las alas y equipado con turbofanes. Para ello se va a hacer uso de la base de datos CeRAS, a la cual se dedica una sección específica para explicar en qué consiste y el fin con la que se creó.

\subsubsection{Base de datos CeRAS: CSR-01}

CeRAS viene de las siglas en inglés Central Reference Aircraft data System y es una base de datos diseñada para albergar datos de diseño de aviones de transporte comercial. La idea es que estos datos se utilicen para proyectos de investigación relacionados con el diseño conceptual y/o preliminar de aviones. Esta base de datos surge debido a la gran variabilidad que presentan los datos que se pueden encontrar en diferentes fuentes acerca de parámetros de diseño de aviones de transporte comercial. De esta manera, se podría utilizar una referencia común para todos los proyectos de diseño, pudiendo extraer conclusiones más relevantes y que no dependan de la fuente de información de la que beban los datos de los aviones. La construcción de esta base de datos, a la cual se accede vía web, ha sido impulsada por el $I L R$, dentro de la universidad RWTH de Aachen, con la colaboración de la oficina de proyectos futuros de Airbus.

A día de hoy, la base de datos está compuesta por dos aviones, uno de corto alcance y otro de largo alcance, aunque solamente se disponen de los datos del de corto alcance, el CSR-01. La Tabla 2-11 recoge las características generales más importantes de esta aeronave (Risse 2014), que se pueden consultar en la base de datos.

\begin{tabular}{|ccc|}
\hline Parámetro & Unidades & Valor \\
\hline Alcance de diseño & $\mathrm{NM}$ & 2750 \\
\hline Pasajeros de diseño & - & 150 \\
\hline Mach de crucero & - & 0,78 \\
\hline Carga alar & $\mathrm{kg} / \mathrm{m}^{2}$ & 629,1 \\
\hline Ratio empuje-peso & - & 0,312 \\
\hline Peso máximo de despegue & $\mathrm{kg}$ & 77000 \\
\hline Peso máximo de aterrizaje & $\mathrm{kg}$ & 64500 \\
\hline Peso vacío operativo & $\mathrm{kg}$ & 42100 \\
\hline Peso máximo sin combustible & $\mathrm{kg}$ & 62100 \\
\hline Peso máximo de combustible & $\mathrm{kg}$ & 18700 \\
\hline Superficie alar & $\mathrm{m}^{2}$ & 122,41 \\
\hline Envergadura alar & $\mathrm{m}$ & 34,07 \\
\hline Cuerda media aerodinámica & $\mathrm{m}$ & 4,18 \\
\hline Empuje estático a nivel del mar & $\mathrm{kN}$ & 117,88 \\
\hline
\end{tabular}

Tabla 2-11 Principales datos característicos del CSR-01. Fuente: elaboración propia. 
Como se puede comprobar, este avión pertenece al segmento de pasillo único, siguiendo el criterio establecido para clasificar las aeronaves de la base de datos en función del alcance de la ruta de diseño. Además, se puede acceder a una imagen tres vistas que se adjunta en la Figura 2-17. Como se observa, es un avión de configuración de cola convencional, con dos motores turbofán bajo las alas. Su longitud del fuselaje es de 37,49 metros, siendo el alto y el ancho de este 4,14 y 4 metros, respectivamente.
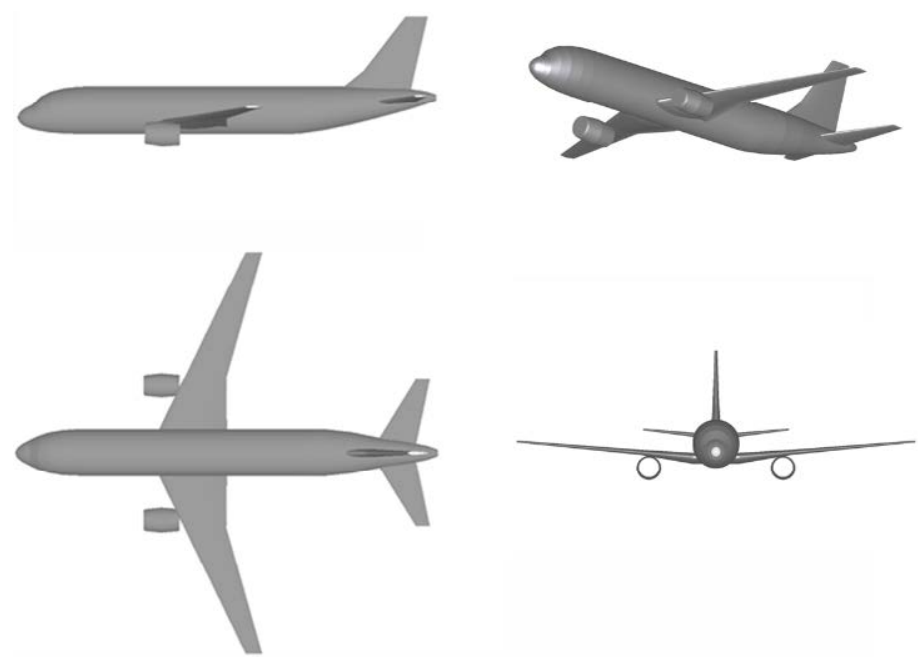

Figura 2-17 Tres vistas del CSR-01. Fuente: (ILR y RWTH-Aechen-University 2014).

Para el estudio que se quiere llevar a cabo es necesario conocer los datos de los estabilizadores, que son los que se van a utilizar para comprobar las tendencias extraídas de la base de datos construida en esta tesis. Algunos de ellos se pueden consultar directamente en la base de datos, como son la superficie, la envergadura, el alargamiento, el parámetro de estrechamiento o la flecha de la línea de puntos $1 / 4$. Sin embargo, existen otros parámetros característicos que van a ser objeto del estudio que se deben determinar a partir de la imagen a tres vistas, de la misma forma que se hizo con todos y cada una de las aeronaves que se han contemplado, como son los brazos de momentos de los estabilizadores. A partir de ahí se puede calcular el coeficiente de volumen de estos. Todos estos parámetros se incluyen en la Tabla 2-12.

\begin{tabular}{|cccc|}
\hline Parámetro & Unidades & $\begin{array}{c}\text { Estabilizador } \\
\text { horizontal }\end{array}$ & $\begin{array}{c}\text { Estabilizador } \\
\text { vertical }\end{array}$ \\
\hline Superficie & $\mathrm{m}^{2}$ & 32,23 & 28,21 \\
\hline Envergadura & $\mathrm{m}$ & 12,46 & 6,86 \\
\hline Alargamiento & - & 4,82 & 1,67 \\
\hline Estrechamiento & - & 0,32 & 0,32 \\
\hline Flecha puntos $1 / 4$ & 0 & 28,47 & 35,39 \\
\hline Brazo de momentos & $\mathrm{m}$ & 17,27 & 16,69 \\
\hline Coeficiente de volumen & - & 1,088 & 0,113 \\
\hline
\end{tabular}

Tabla 2-12 Datos de los estabilizadores del CSR-01. Fuente: elaboración propia. 


\subsubsection{Resultados del diseño}

En este apartado se van a mostrar los resultados que se obtienen al hacer uso de las correlaciones y promedios presentados hasta ahora para dimensionar el estabilizador horizontal del CSR-01. Para ello se partirá del valor del peso máximo de despegue, de las dimensiones del fuselaje y del ala. De todas formas, además del dimensionado del estabilizador horizontal, primeramente, se van a mostrar los resultados que se obtendrían para determinar la superficie y la envergadura alares para este avión, con el objetivo de validar las correlaciones construidas para la estimación de ciertos parámetros que se han incluido en la categoría de generales.

Para estimar la superficie alar se debe utilizar la regresión que la relaciona con el peso máximo de despegue. En el fondo lo que se está intentando estimar es la carga alar del avión. Es por ello que se debe utilizar los aviones que sean semejantes en misión, es decir, que pertenezcan al mismo segmento de vuelo. De hecho, la regresión construida para este segmento presenta un coeficiente de correlación superior a 90\%. Entrando con el peso máximo de despegue de 77 toneladas se obtiene una superficie alar de $128,45 \mathrm{~m}^{2}$, lo cual supone un 4,94\% de error con respecto al valor real del CSR-01. Por tanto, podemos concluir que la estimación es buena para la superficie alar. Continuando con los parámetros generales, nos quedaría determinar la envergadura alar. Para ello se hará uso de la correlación construida para relacionar el parámetro de volumen global con el peso máximo de despegue. Puesto que las dimensiones del fuselaje se consideran conocidas a priori, ya que vendrá determinado por el número de pasajeros que se quieran transportar, del parámetro de volumen global se puede despejar la envergadura alar. En este caso, el criterio de clasificación adecuado para determinar el valor de este parámetro es el que depende del número de motores, porque es el que mejor separa las tendencias de los aviones, tal y como se ha visto anteriormente. Entrando con el peso máximo de despegue en esta regresión, se obtiene un valor del parámetro de volumen global de $6066 \mathrm{~m}^{3}$, y de ahí se determina una envergadura alar de $36,77 \mathrm{~m}$, lo cual supone un error del $7,95 \%$ con respecto al valor real de la envergadura del CSR-01. De nuevo se puede decir que esta regresión es una buena forma de aproximar la envergadura alar.

A continuación, se va a proceder a dimensionar el estabilizador horizontal del CSR-01. Para ello, primeramente, se hace uso de la regresión que relaciona la superficie del estabilizador con la superficie alar. En este punto se contemplan dos opciones: utilizar la superficie alar real o la estimada previamente. Este último camino serviría para mostrar un proceso de dimensionado completo basado en correlaciones. Para estas primeras etapas del dimensionado del estabilizador se van a llevar en paralelo ambos caminos, pero en el momento de determinar el coeficiente de volumen, sería necesario conocer el valor de la cuerda media aerodinámica del ala. Esta correlación no se ha incluido en el estudio ya que este se centra en las superficies de cola. Sin embargo, otros autores han construido correlaciones que estiman las cuerdas del ala en función de la superficie alar, para aviones biturbofán (Kallinderis, Vouvakos y Menounou 2009). De ahí se puede obtener tanto la cuerda en la raíz como la cuerda en la punta del ala si se conoce la superficie alar. Posteriormente, se puede construir un ala equivalente de simple estrechamiento que tenga las mismas cuerdas en la punta y en la raíz. Este ala equivalente nos permitirá determinar la cuerda media aerodinámica, definida en (Yates 1952), sin más que haciendo uso de la expresión correspondiente a alas de simple estrechamiento, que se recuerda a continuación: 


$$
C M A_{w}=\frac{2}{3} c_{r w} \frac{1+\lambda_{w}+\lambda_{w}^{2}}{1+\lambda_{w}}
$$

Así, se determina que la cuerda media aerodinámica puede ser 4,305 m o 4,219 m, en función si la superficie alar con la que se entra en las regresiones es la estimada previamente o la real del CSR-01, respectivamente. Como se puede ver, para ambos procedimientos el error es asumible; un 3\% en el primero y un $0,9 \%$ en el segundo. Además, en este caso el ala equivalente aproxima bien la cuerda media aerodinámica, aunque no hay garantías que para otros aviones se puedan obtener unos resultados tan ajustados.

Ahora ya se está en disposición para dimensionar el estabilizador horizontal. Como se ha dicho anteriormente, se llevarán en paralelo los cálculos partiendo de la superficie alar real, por un lado, y de la superficie alar estimada, por otro. La regresión que se utiliza para determinar la superficie del estabilizador horizontal es la que depende de la posición de los motores, ya que se ha visto con los resultados mostrados en los apartados previos que el hecho de instalar los motores en el fuselaje posterior o en las alas condiciona el dimensionado de los estabilizadores. De esta forma la superficie del estabilizador resulta en $32,36 \mathrm{~m}^{2}$ o en $31,02 \mathrm{~m}^{2}$, en función de si se basa en el área estimada o en la real, respectivamente. Como se ve, ambas aproximaciones se ajustan bastante a la realidad, ya que presentan un error relativo del $0,41 \%$ y del $-3,76 \%$, respectivamente. Seguidamente, se puede determinar el brazo de momentos del estabilizador haciendo uso de la regresión que lo relaciona con la longitud del fuselaje. Puesto que esta última se ha considerado como conocida ya que se puede estimar a partir del número de pasajeros que se quieren transportar en la misión de diseño, se obtiene que el brazo de momentos es $17,31 \mathrm{~m}$. Al igual que antes, la regresión que se ha escogido es la correspondiente a los aviones que llevan los motores bajo las alas. Comparando con el valor real del avión se tiene un error relativo del $0,23 \%$. En este momento se está en disposición de hallar el coeficiente de volumen del estabilizador horizontal. De nuevo, se puede estimar un valor basado en los resultados de las correlaciones para el ala y otro en los valores reales de la misma. En el primero de ellos, se llega a un coeficiente de volumen de 1,033, que supone un error relativo del $-6,89 \%$. Por el otro lado, se obtiene un coeficiente de volumen de 1,049, que corresponde a un error relativo del $-3,55 \%$. De nuevo en ambos casos los resultados se encuentran por debajo de un $10 \%$ de error relativo, que es el margen habitual asumido en etapas de diseño conceptual. Por último, para estimar el alargamiento y la envergadura del estabilizador, se utiliza el parámetro de resistencia inducida del estabilizador horizontal de cola, para el cual se estableció una regresión con el peso máximo de despegue. De nuevo y al igual que en el caso de la carga alar, se utiliza la regresión que se construyó para los aviones de pasillo único, ya que se encuentran comportamientos diferentes en función del rango de pesos y de la misión de diseño. El valor del parámetro de resistencia inducida se estima en 40,034 para el CSR-01, lo que significa un error relativo del $-8,53 \%$ con respecto al real. Aplicando la definición de este parámetro y la del alargamiento, es posible despejar la envergadura del estabilizador horizontal. En este caso, los resultados son independientes de los datos del ala que se tomen como referencia, tanto los estimados como los reales. Se obtiene una envergadura del estabilizador de 13,6, lo que supone un error relativo del $9,15 \%$; y en consecuencia un alargamiento de 5,71 , con un error relativo del $18 \%$.

Puesto que este último paso resulta en un error relativo en el alargamiento mayor de lo esperado, se puede presentar un procedimiento alternativo basado en los promedios 
determinados para ciertos valores y comprobar qué parámetro de resistencia inducida se obtendría por este camino. Escogiendo los valores promedio determinados en los apartados previos en la categoría de motores bajo las alas, para la flecha de puntos $1 / 4$, el parámetro de estrechamiento y el alargamiento del estabilizador horizontal, este quedaría dimensionado con los correspondientes valores: $27,22^{\circ}, 0,414$ y 4,68 , respectivamente. Como se puede ver, el mayor error relativo se encuentra en el parámetro de estrechamiento, debido a que ya se comentó que había mucha dispersión en los resultados. Sin embargo, en la flecha se aprecia un erro relativo del $-4,39 \%$ y en el alargamiento de $-2,9 \%$. Ahora es posible hallar la envergadura del estabilizador sin más que aplicando la definición del alargamiento y utilizar la estimación de la superficie de cola. De nuevo, si los datos del ala están basados en las estimaciones realizadas se obtiene una envergadura de $12,31 \mathrm{~m}$, que supone una mejor aproximación que por el método anterior, ya que se reduce el error hasta el -1,23\%. En este caso, el parámetro de resistencia inducida está sobrestimado en un 6,08\%. Por el otro lado, si se utilizan los datos reales del ala del CSR-01, la envergadura estimada pasa a ser 12,05 m, correspondiente a un error relativo del $-3,31 \%$, y el parámetro de resistencia inducida estaría sobrestimado en un $3,25 \%$.

\begin{tabular}{|ccccc|}
\hline Parámetro & Unidades & Real & Estimado & Error relativo \\
\hline Superficie alar & $\mathrm{m}^{2}$ & 122,41 & 128,45 & $4,94 \%$ \\
\hline Envergadura alar & $\mathrm{m}$ & 34,07 & 35,01 & $2,77 \%$ \\
\hline CMA del ala & $\mathrm{m}$ & 4,18 & 4,31 & $2,99 \%$ \\
\hline Área del estabilizador & $\mathrm{m}^{2}$ & 32,23 & $32,36 / 31,02$ & $0,41 \% /-3,76 \%$ \\
\hline Brazo de momentos & $\mathrm{m}$ & 17,27 & 17,31 & $0,23 \%$ \\
\hline Coeficiente de volumen & - & 1,088 & $1,013 / 1,049$ & $-6,89 \% /-3,55 \%$ \\
\hline Alargamiento & - & 4,88 & 4,68 & $-2,90 \%$ \\
\hline Envergadura & $\mathrm{m}$ & 12,46 & $12,31 / 12,05$ & $-1,23 \% /-3,31 \%$ \\
\hline $\begin{array}{c}\text { Parámetro de } \\
\text { resistencia inducida }\end{array}$ & ton & 43,77 & $46,43 / 45,19$ & $6,08 \% / 3,25 \%$ \\
\hline
\end{tabular}

Tabla 2-13 Resultados del proceso de diseño del estabilizador horizontal del CSR-01. En las celdas donde aparecen dos valores, los que están antes de la barra (/) se han obtenido basados en los valores del ala estimados que se encuentran en la tabla y los que están después de la barra (/) están basados en los datos reales del ala, también incluidos en la tabla. Fuente: elaboración propia. 


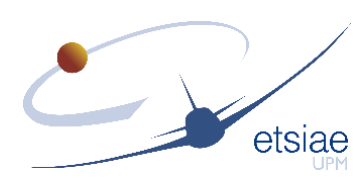

\subsection{RESUMEN Y CONCLUSIONES}

En este capítulo se han mostrado las tendencias de diseño que presentan las configuraciones de cola más extendidas en los aviones de transporte comercial, como son la configuración convencional y la cola en $\mathrm{T}$. Se han construido regresiones que relacionan parámetros de diseño de diferente índole, tanto generales del avión como específicos del fuselaje posterior y los estabilizadores. Estas regresiones tienen la especial aportación de que se han construido siguiendo diferentes criterios de clasificación, lo cual le da un valor añadido ya que permite extraer conclusiones de diseño de esos criterios. Además, se ha comprobado que el nivel de significación obtenido en todas ellas es muy alto, ya que la probabilidad de que no sean aplicables a otros aviones está muy por debajo del límite establecido. Se ha visto como la mayoría de las correlaciones que utilizan como variable dependiente el peso máximo de despegue deben usarse con la clasificación según el segmento de vuelo. Esto se debe a que el segmento de vuelo agrupa las aeronaves con misiones semejantes y es de esperar que las variables dependientes del peso estén relacionadas con la misión del avión. Sin embargo, en el diseño de los estabilizadores tiene especial relevancia la clasificación en función del número de motores y la posición de estos. En cuanto a la clasificación por número de motores, la principal diferencia se encuentra en los trimotores, ya que deben llevar un motor en el plano de simetría que normalmente está en el cono de cola. Como es obvio, este motor condiciona el diseño de los estabilizadores de cola. De la misma forma, el hecho de llevar, o no, los motores instalados en el fuselaje posterior afecta al diseño de las superficies de cola, de ahí que las regresiones que clasifican las aeronaves según este criterio mayoritariamente tienen mejor resultado. De la misma forma, las tendencias de diseño establecidas mediante promedios y desviaciones típicas enriquecen los aspectos relacionados con variables adimensionales o ángulos, tales como los alargamientos o las flechas, por ejemplo. Es cierto que una de las conclusiones que se extrae al analizar estas tablas es que hay bastante dispersión de valores, por lo que el valor promedio hallado podría no ser representativo. Pero sí que ha servido para comparar diferentes categorías y establecer tendencias en los diseños. También es destacable el estudio de los parámetros relacionados con el fuselaje posterior, que hasta ahora no se había analizado en la literatura abierta. Por último, se ha llevado a cabo un caso de estudio de un avión cuyos datos son abiertos a la comunidad científica y que se encuentra en una base de datos dedicada a la validación de procesos de diseño conceptual, como el que se presenta en este capítulo. Los resultados se agrupan en la Tabla $2-13$, donde se puede ver que los errores relativos se encuentran por debajo del 10\%, que es el margen asumido habitualmente en los procedimientos de diseño conceptual. Cabe destacar que el procedimiento aquí presentado, junto con el caso de validación, están publicados en la referencia (Cuerno-Rejado y Sanchez-Carmona 2016). 
RESUMEN Y CONCLUSIONES 
3 DISEÑO CONCEPTUAL DE CONFIGURACIONES DE ESTABILIZADORES DE COLA NO CONVENCIONALES 


\subsection{PROCEDIMIENTO DE DISEÑO}

En este capítulo se va a describir el proceso de diseño conceptual que se va a seguir para dimensionar una superficie de cola no convencional. El punto de partida se fijará en que esta superficie de cola debe cumplir las mismas funciones que las que tenía la configuración convencional. Repasando las funciones del estabilizador horizontal tenemos las siguientes:

- Garantizar la estabilidad estática longitudinal.

- Garantizar el equilibrado longitudinal del avión en todos los puntos de la envolvente.

- Proporcionar capacidad de maniobra en el plano vertical.

- Proporcionar una estabilidad dinámica longitudinal dentro de límites.

Por otra parte, si se recopilan las principales funciones del estabilizador vertical se encuentran las que siguen:

- Asegurar el control de la aeronave tras el fallo de motor crítico en el segundo segmento.

- Garantizar la estabilidad estática lateral.

- Garantizar modos de comportamiento dinámico lateral-direccional dentro de ciertos límites.

- Proporcionar capacidad de maniobra fuera del plano vertical.

- Garantizar el aterrizaje con viento cruzado.

Todas estas funciones vienen recogidas en la normativa de aeronavegabilidad aplicable a los aviones de transporte comercial de más de $5700 \mathrm{~kg}$ de peso máximo de despegue. A nivel europeo, el código es el CS-25 (EASA 2018) y en Estados Unidos es el FAR-25 («Code of Federal Regulations: Title 14 - Chapter I - Subchapter C - Part 25» 2017). Ambas regulaciones son análogas, en la subparte B Vuelo, los artículos del 25.171 al 25.181 tratan el tema de la estabilidad de la aeronave. Las condiciones de equilibrado se encuentran en el artículo 25.161. El aterrizaje con viento cruzado se contempla en los artículos 25.233 y 25.237. Por último, los artículos que tratan con las actuaciones y controlabilidad de la aeronave tras el fallo de motor crítico son el 25.121 y el 25.147. Inspirándose en estos artículos y funciones, es posible establecer unos criterios que restrinjan el espacio de diseño admisible para cualquier superficie de cola no convencional. Como primera aproximación al problema, se van a omitir los requisitos de estabilidad dinámica. Esto se comprobará, en su caso, una vez obtenida la primera propuesta de diseño a nivel conceptual, ya que necesita de un conocimiento más detallado del avión, como pueden ser los momentos de inercia, para poder ser evaluado adecuadamente. Entonces, los requisitos seleccionados son los siguientes cuatro:

- Garantizar estabilidad estática lateral y longitudinal en condiciones de crucero.

- Controlar la aeronave tras el fallo de motor crítico en segundo segmento.

- Controlar la aeronave en aterrizaje con viento cruzado.

- Garantizar capacidad de equilibrado longitudinal en cualquiera de las condiciones de vuelo anteriormente mencionadas.

El primero de los criterios se va a implementar por medio de comparación con un avión de referencia. Tal y como se ha comentado en el capítulo dedicado al planteamiento de los objetivos, el dimensionado de la configuración no convencional se hará sobre un avión de referencia, sustituyendo sus superficies estabilizadoras por la nueva propuesta. La idea es caracterizar plenamente este avión de tal forma que se determinen las 


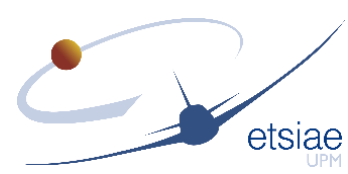

derivadas de estabilidad estáticas tanto longitudinales como lateral-direccionales en las condiciones de crucero y utilizar estos valores como los mínimos exigibles al avión con la superficie de cola no convencional. De esta forma se cumplirían los requisitos mínimos de estabilidad estática indicados en la norma, ya que se asume que el avión de referencia los cumplía, al ser un diseño certificado.

El segundo y el tercer criterio de dimensionado indican que la aeronave debe ser controlable en las situaciones mencionadas. El código de certificación dice que esto se produce si una vez equilibrada la aeronave en todos los ejes, el ángulo de balance resultante no excede los $5^{\circ}$. Además, en el caso del aterrizaje con viento cruzado, se indica que el avión debe ser controlable con un viento a $90^{\circ}$ de $20 \mathrm{kt}$ o igual a un $20 \%$ de la velocidad de entrada en pérdida, el que sea mayor, pero en ningún caso exceder los $25 \mathrm{kt}$. Es por ello que para establecer el requisito de diseño se va a considerar que la velocidad de viento cruzado va a ser $25 \mathrm{kt}$, que es el límite superior y, en consecuencia, los resultados serán conservativos. En este estudio, ambas maniobras se van a considerar como planas, es decir, que no se va a tener en cuenta la deflexión de los alerones, ya que esto genera unas cargas de nuevo conservativas (Lomax 1996). Desde el punto de vista del fallo de motor crítico, el ángulo de resbalamiento es un grado de libertad. Si el motor que falla se sitúa a la derecha del piloto, el avión puede permanecer en vuelo con valores negativos de ángulo de resbalamiento si tiene una superficie de cola menor que la necesaria para volar con ángulo de resbalamiento nulo en esa condición (EASA 2018). En realidad, el ángulo de resbalamiento no es totalmente libre ya que está limitado por el ángulo de balance resultante del equilibrado, el cual está limitado por la normativa de certificación que se ha mencionado anteriormente. Por tanto, con la premisa de ser conservativos, se ha decidido equilibrar la aeronave a ángulo de resbalamiento nulo en lugar de buscar el ángulo de resbalamiento necesario para alcanzar el ángulo de balance límite, a pesar de que podría existir una superficie de cola menor que cumpla el requisito con un ángulo de balance no nulo. Si al imponer la condición de ángulo de resbalamiento nulo no se cumpliese el requisito en ángulo de balance, entonces sí se buscaría el límite en ángulo de resbalamiento.

Por último, el cuarto requisito se traduce en que la superficie de cola no convencional tenga potencia de mando suficiente como para equilibrar la aeronave longitudinalmente tanto en las condiciones de crucero, como en las de segundo segmento y de aterrizaje con viento cruzado. Por supuesto, en el caso de estas dos últimas condiciones, hay que tener en cuenta que los dispositivos hipersustentadores estén desplegados en un cierto ángulo y afectan al equilibrado longitudinal de la aeronave.

Estos criterios aquí mencionados únicamente restringen el espacio de diseño posible de las superficies de cola no convencionales. Sin embargo, no permiten determinar el dimensionado óptimo. Para ello se debe establecer una función objetivo que minimizar. En este caso se va a considerar que el diseño debe ser tal que se minimice el peso de la estructura de cola. La estructura de la superficie de cola debe ser tal que soporte las cargas aerodinámicas generadas en sí misma. A falta de información acerca de las configuraciones de cola no convencionales, a continuación se enumeran los casos de carga críticos clásicos considerados para la configuración de cola convencional, divididos en los casos de carga para el estabilizador vertical y los correspondientes a los del estabilizador horizontal (Lomax 1996; Raymer 1989):

- Para el estabilizador vertical: fallo de motor crítico, balanceo holandés, ráfaga lateral, maniobras de guiñada y velocidad mínima de control en tierra. 
- Para el estabilizador horizontal: ráfaga longitudinal, deflexión máxima del control longitudinal y maniobras de cabeceo.

A la vista de lo que se ha comentado en este apartado, será necesario construir un modelo aerodinámico que permita determinar las restricciones impuestas por los criterios establecidos basados en las normas de certificación para aviones de transporte comercial. Además, este modelo aerodinámico debe permitir estimar las cargas generadas en las superficies de cola, necesarias para hallar el peso necesario en estructura de la cola. Por tanto, también hace falta desarrollar un modelo estructural que, entrando con los casos de carga permita estimar el peso de la estructura. Ambos modelos deben retener el efecto que tienen sobre las restricciones y sobre el peso la variación de los parámetros que se consideren como de diseño para la configuración de cola no convencional que se quiera dimensionar. Por último, una vez establecidos todos los modelos y los parámetros de diseño, será necesario establecer un procedimiento de optimización que tenga en cuenta las restricciones impuestas y minimice la función objetivo, en este caso el peso, en el espacio de diseño definido por los parámetros de dimensionado. Por tanto, en los apartados siguientes de este capítulo se trata de presentar los modelos aerodinámicos y estructurales contemplados en este estudio que servirán para construir las fronteras del espacio de diseño y la función objetivo a minimizar. También se presentarán métodos que serán útiles para encontrar el mínimo de la función objetivo, dependiendo de las características de esta. 


\subsection{MODELO AERODINÁMICO}

\subsubsection{Derivadas de estabilidad en configuración convencional}

En este capítulo se van a recoger las expresiones de las derivadas de estabilidad estática longitudinal y lateral que se van a considerar en este estudio, para recordar de qué términos dependen y que parámetros afectan a los valores que alcanzan. De esta forma, se podrá determinar cuáles son los parámetros sobre los que merece la pena realizar la optimización.

Se va a partir de la pendiente de curva de sustentación de una superficie de cola horizontal referida a una superficie de referencia que, en general, es distinta a la superficie del estabilizador:

$$
c_{L \alpha_{H}}=a_{H} \frac{S_{H}}{S_{w}}
$$

donde $a_{t}$ es la pendiente de la curva del estabilizador horizontal, $S_{H}$ es su área en planta y $S_{w}$ es la superficie de referencia, que corresponde a la superficie alar. A su vez, la pendiente de la curva de sustentación se puede determinar por medio de una expresión semi-empírica siguiendo el método DATCOM (Torenbeek 1982):

$$
a_{H}=\frac{2 \pi /\left(1-M^{2}\right)^{0,5}}{\frac{2}{A R_{H}\left(1-M^{2}\right)^{0,5}}+\sqrt{\frac{1}{\left(\frac{c_{l \alpha}}{2 \pi}\right)^{2}\left(1-M^{2}\right) \cos ^{2} \Lambda_{\beta}}+\left(\frac{2}{\left(1-M^{2}\right)^{0,5} A R_{H}}\right)^{2}}}
$$

donde $\Lambda_{\beta}$ se determina a partir de $\tan \Lambda_{\beta}=\tan \Lambda_{0,5} /\left(1-M^{2}\right)^{0,5}$. La contribución del estabilizador horizontal a la derivada del coeficiente de momento de cabeceo con respecto del ángulo de ataque, también llamada derivada de estabilidad estática longitudinal, se puede determinar como sigue:

$$
c_{m \alpha_{H}}=-a_{H} V_{e H}
$$

Por otra parte, la derivada de la fuerza lateral con respecto del ángulo de resbalamiento es:

$$
c_{Y \beta_{V}}=-a_{V} \frac{S_{V}}{S_{w}}
$$

donde $a_{V}$ es la pendiente de la curva de sustentación del estabilizador vertical, $S_{V}$ es su área y $S_{w}$ la superficie alar, al igual que antes. Es posible determinar el parámetro $a_{V}$ mediante la ecuación [ 3-5 ], para velocidades bajas.

$$
a_{V}=\frac{2 \pi A R_{V}^{\prime}}{2+\sqrt{\frac{A R_{e f f}^{2}}{\left(\frac{c_{l \alpha}}{2 \pi}\right)^{2}}\left(1+\tan ^{2} \Lambda_{0,5 V}\right)+4}}
$$

$A R_{\text {eff }}$ es el alargamiento efectivo del estabilizador vertical. Este alargamiento es mayor que el geométrico porque el fuselaje y el estabilizador horizontal hacen un efecto de winglet, tomando el valor de $1,55 A R_{V}$ (Perkins y Hage 1949). Además, en el numerador 
aparece un alargamiento que corresponde con el doble del geométrico, que es $b_{V}^{2} / S_{V}$. Por último, la derivada del coeficiente de guiñada con respecto del ángulo de resbalamiento, también llamada derivada de estabilidad estática lateral, partiendo de la expresión anterior, es:

$$
c_{n \beta_{V}}=a_{V} V_{e V}
$$

\subsubsection{Coeficientes de fuerzas en configuraciones no convencionales: el efecto del diedro}

Como se ha visto en el apartado de la introducción dedicado al estado del arte de configuraciones no convencionales, una de las formas más habituales de construir estas configuraciones es dándole diedro a un estabilizador horizontal, llegando así a una configuración de cola en $\mathrm{V}$ y pudiendo eliminar así el estabilizador vertical. El comportamiento aerodinámico de este tipo de superficies de cola no se puede estimar directamente con las expresiones para configuraciones convencionales presentadas en el apartado anterior. Es por ello que en esta sección se va a tratar de analizar varias metodologías por medio de las cuales se pueden estimar tanto las derivadas de estabilidad de colas en $\mathrm{V}$ como la distribución de fuerza a lo largo de la envergadura, lo cual será necesario para el dimensionado de la estructura y la determinación del peso de la misma.

\subsubsection{Métodos semi-empíricos}

Las asunciones básicas que se realizan para alcanzar expresiones sencillas para las colas en $V$ son considerarlas como un ala con diedro (Phillips, Hansen y Nelson 2006). La distribución de sustentación a lo largo de la envergadura se puede determinar mediante una teoría de línea sustentadora para alas sin flecha y sin diedro, asumiendo que esto no tiene efectos sobre los resultados. Además, el ángulo de resbalamiento induce un ángulo de ataque diferente en cada una de las dos superficies de la cola. Se realizará la hipótesis de que estos incrementos son iguales en ambas superficies, pero de signo contrario. La magnitud de estos incrementos será igual a la que se produce para esos mismos ángulos de ataque en el mismo ala, pero con diedro nulo. Estas hipótesis son cada vez más inexactas a medida que el ángulo de diedro es mayor. Por último, para simplificar aún más el análisis, las características longitudinales y direccionales se consideran independientes y se supone aerodinámica lineal.

Con estas hipótesis, se llega a las siguientes expresiones para las derivadas de la sustentación y de la fuerza lateral con respecto al ángulo de ataque y de resbalamiento respectivamente (Purser y Campbell 1945):

$$
\begin{gathered}
\left(c_{L \alpha}\right)_{V-\text { tail }} \cong\left(c_{L \alpha}\right)_{\Gamma=0} \cos ^{2} \Gamma \\
\left(c_{Y \beta}\right)_{V-\text { tail }} \cong-K\left(c_{L \alpha}\right)_{\Gamma=0} \sin ^{2} \Gamma
\end{gathered}
$$

El parámetro $K$ es la relación entre el coeficiente de sustentación de la cola medido en un plano normal al plano definido por las cuerdas en cada sección de la cola $\left(c_{L}\right.$ en la figura) y la suma de los cambios en el coeficiente de sustentación normal a cada sección de la cola cuando esta tiene guiñada ( $c_{L}^{\prime}$ en la figura). Los valores que toma este parámetro en función del alargamiento y del parámetro de estrechamiento se muestran en la Figura 3-1. 


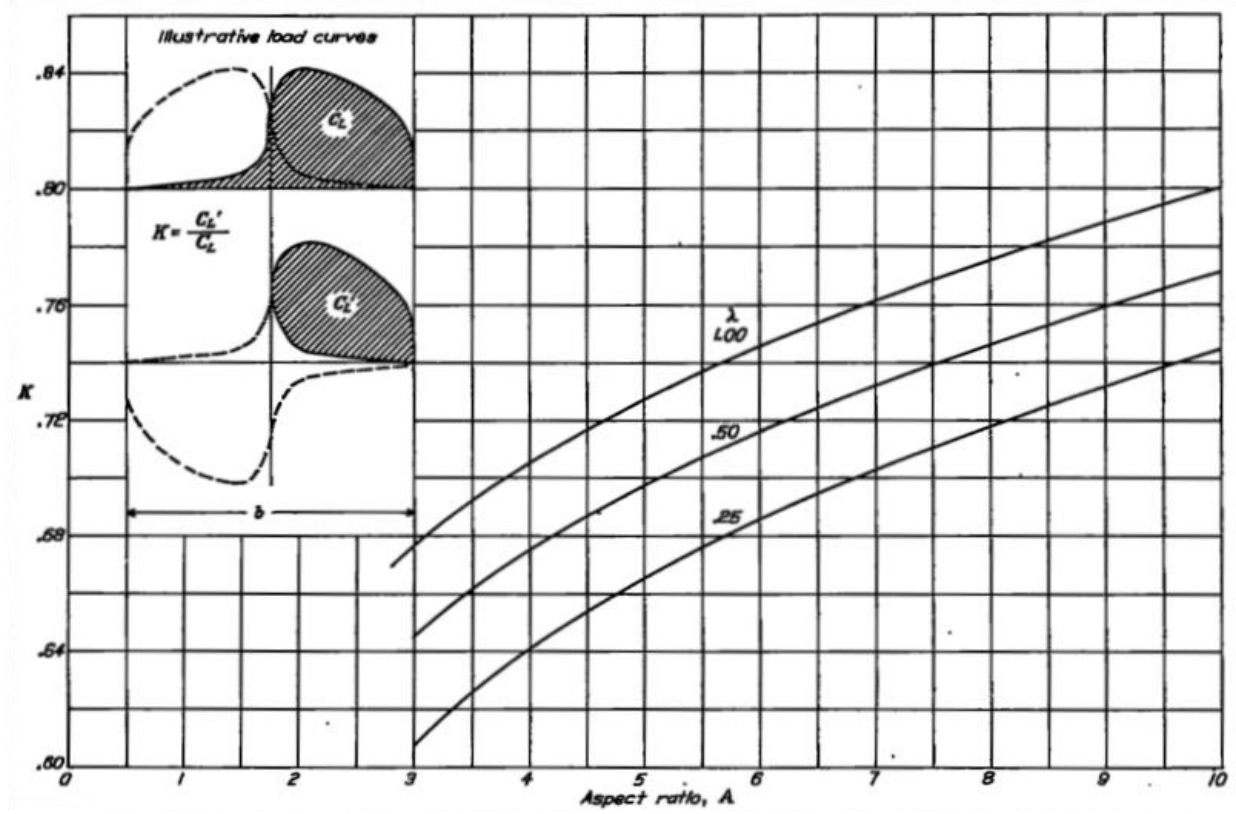

Figura 3-1 Valores de K para determinar la pendiente de la curva de coeficiente de fuerza lateral en función del ángulo de resbalamiento. Fuente: (Purser y Campbell 1945)

Esta metodología permite estimar los coeficientes $c_{L \alpha}$ y $c_{Y \beta}$ de una cola con un ángulo de diedro elevado. Con estos dos coeficientes y aplicando la definición de las derivadas estáticas de estabilidad definidas en las ecuaciones [3-3 ] y [3-6 ], es posible determinar semi-empíricamente los valores de estos coeficientes. El principal inconveniente de estos métodos es que no permiten estimar la distribución de fuerza a lo largo de la semienvergadura de la superficie de cola. Por ello, estos métodos se usarán para validar por comparación aquellos que finalmente admitan tanto determinar los coeficientes de fuerzas y momentos como la distribución de fuerza a lo largo de la superficie.

\subsubsection{Combinación de métodos semi-empíricos con métodos VLM}

La opción que se ha contemplado para resolver el problema anteriormente mencionado es el uso de un programa basado en métodos VLM, como es el Tornado (Melin 2000). Este software es un método Vortex Lattice para aerodinámica lineal que se pensó para ser utilizado en aplicaciones de diseño conceptual. Modelando cualquier superficie sustentadora como placas planas, Tornado puede resolver la mayoría de las derivadas de estabilidad para un amplio rango de geometrías. A su vez, permite determinar la distribución de fuerzas a lo largo de la envergadura y resolver varias alas a la vez. Tornado está pensado para aerodinámica a bajas velocidades, es decir, en régimen incompresible. Sin embargo, tiene la posibilidad de incorporar la corrección de PrandtlGlauert para mejorar los resultados en el régimen de subsónico alto (Polhamus 1971). 
A pesar de que las especificaciones del software indican la amplia versatilidad y la validez para varias geometrías, se ha realizado una comparación entre los coeficientes estimados mediante Tornado y mediante los métodos semi-empíricos anteriormente expuestos variando el diedro de un ala de forma en planta determinada. Los resultados se muestran en la Figura 3-2. Ambos métodos son prácticamente iguales a bajos valores del ángulo de diedro. A medida que este crece, las curvas se van separando a pesar de que siguen una tendencia similar. El coeficiente de fuerza longitudinal presenta menor diferencia respecto a la refernecia que el lateral, situándose ambos en torno al 15\% para un ángulo de diedro alrededor de $40^{\circ}$. Hay que tener en cuenta que el propio método semi-empírico restringe su validez a ángulos de diedro inferiores a $45^{\circ}$, ya que próximos a este valor el método se hace demasiado impreciso. Por tanto, se puede decir que el Tornado presenta un comportamiento aceptable al estimar el efecto del ángulo de diedro.
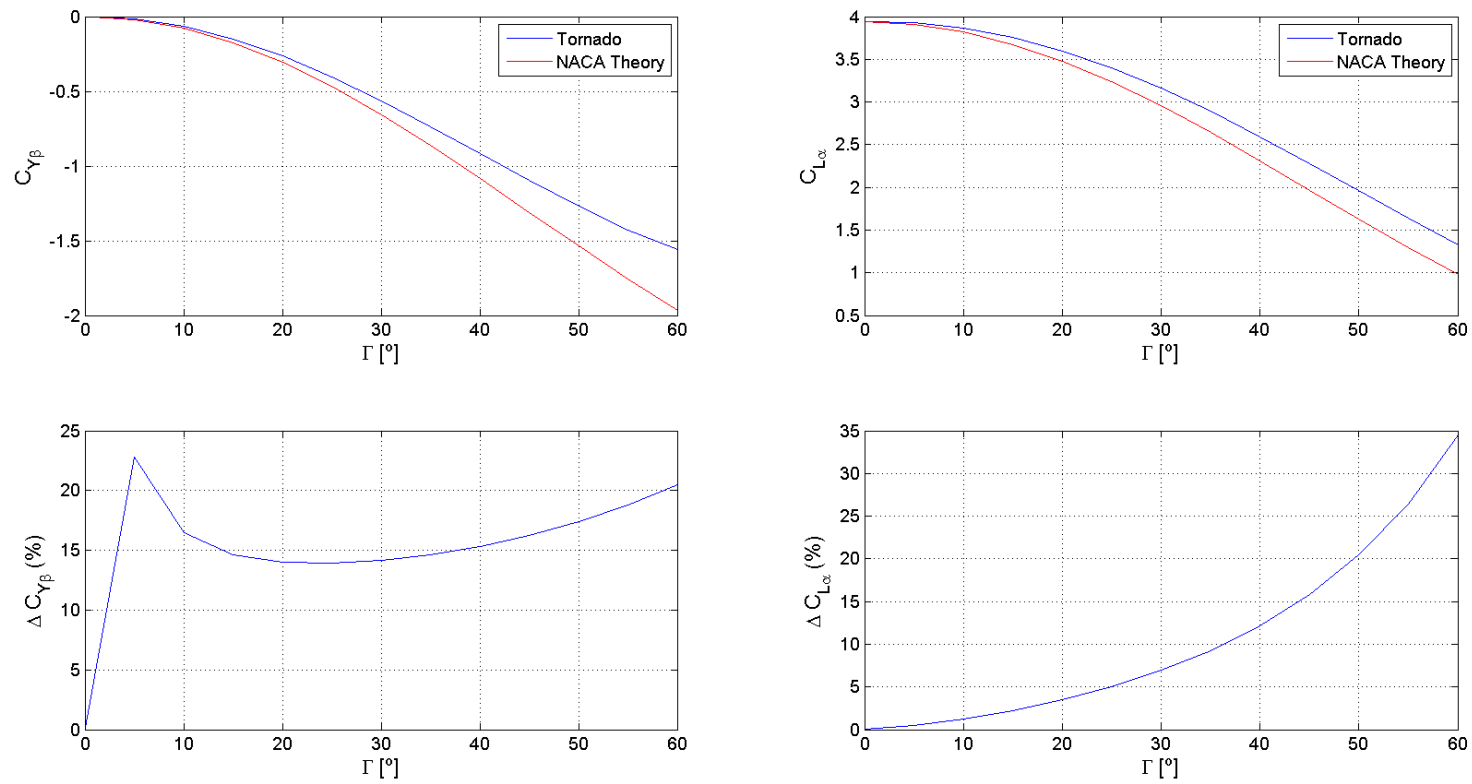

Figura 3-2 Comparación de los coeficientes de fuerza longitudinal $C_{L \alpha}$ y lateral $C_{Y \beta}$ entre Tornado y un método semi-empírico. Fuente: elaboración propia.

El software Tornado se basa en aerodinámica lineal para determinar los coeficientes de fuerzas y momentos. En concreto, los coeficientes de fuerzas que dependen de la deflexión del control no retienen efectos no lineales, que comienzan a presentarse a partir de deflexiones de $15^{\circ}$ aproximadamente. Para corregir estas imprecisiones, se ha propuesto una modificación en el código del software, de tal forma que retenga estos efectos a ángulos de deflexión altos. Se trata de incluir los factores de efectividad del timón de los métodos semi-empíricos por medio de una deflexión efectiva, que es igual al producto de la deflexión por el factor de efectividad (Roskam 1987). Se han comprobado los resultados del modelo mediante la comparación de cuatro métodos: Tornado, dos métodos semi-empíricos basados en las referencias (Torenbeek 1982; Roskam 1987) y la combinación entre Tornado y con la deflexión efectiva. Los resultados se muestran en la Figura 3-3, obtenidos para un ala de simple estrechamiento a ángulo de ataque nulo variando el ángulo de deflexión de un dispositivo de borde de salida. Se observa como Tornado no retiene efectos no lineales y la pendiente es constante para todos los valores de la deflexión. Sin embargo, los métodos semiempíricos no presentan una pendiente constante en función de la deflexión, sino que se reduce para valores de deflexión superiores a $15^{\circ}$ debido a los efectos no lineales. Con la corrección propuesta al Tornado, ahora el software sí que retiene los efectos no lineales y la estimación de la derivada de la fuerza con respecto al ángulo de deflexión 


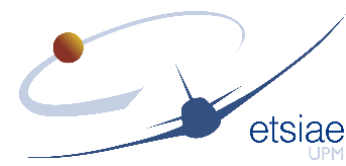

se sitúa con un error inferior al $10 \%$ con respecto a los métodos semi-empíricos considerados históricamente como válidos en el diseño conceptual de aviones. Por tanto se considera como válida la corrección propuesta del Tornado.

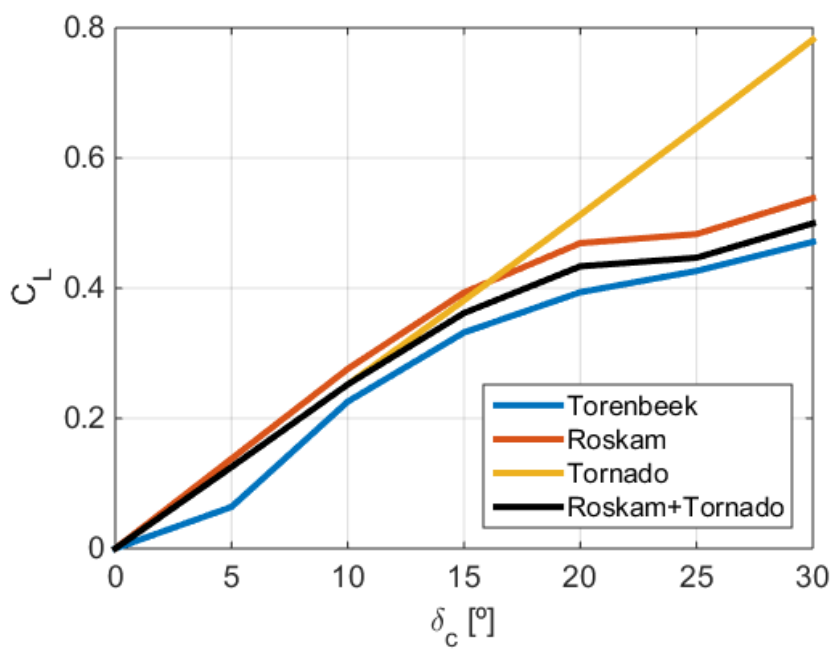

Figura 3-3 Comparativa entre los métodos semi-empíricos de Torenbeek y Roskam con el software Tornado y la combinación propuesta de estudio entre Tornado y Roskam para el cálculo del coeficiente de sustentación para un rango de deflexiones del control de borde de salida. Fuente: elaboración propia.

Por todo lo presentado en esta sección, se concluye que Tornado se puede utilizar para determinar las derivadas de las fuerzas y los momentos de un ala aislada. Es cierto que en las descripciones incluidas por los diseñadores del programa indican que es posible resolver varias alas al mismo tiempo, pero se ha comprobado que la interacción entre ambas alas no la simula adecuadamente, comparado con métodos semi-empíricos clásicos. El problema es que no tiene en cuenta de la misma forma la deflexión de estela. Además, tampoco contempla efectos de interacción entre ala y fuselaje. De hecho, no permite modelar el fuselaje.

Por tanto, se va a realizar una combinación de métodos semi-empíricos clásicos (Torenbeek 1982; Roskam 1987) para estimar las contribuciones de los elementos que se puedan modelizar por este procedimiento y, los términos que estén relacionados con la superficie de cola aislada, se harán por medio de Tornado, con la corrección de las deflexiones propuesta. Esto implica una serie de variaciones que hay que considerar a la hora de tratar tanto los datos de entrada al software como los de salida. La primera de ellas es con respecto al ángulo de ataque de entrada a la superficie, ya que la cola se encuentra inmersa en la estela del ala principal. De ahí, que por medio de los métodos semi-empíricos incluidos en las referencias indicadas se puede estimar el ángulo de ataque que ve la cola, medido respecto al plano $x y$ del avión es:

$$
\alpha_{\text {tail }}=\alpha_{f}\left(1-\frac{d \epsilon}{d \alpha}\right)+\Delta \epsilon_{f}
$$

donde la derivada de la deflexión de estela respecto del ángulo de ataque del ala $d \epsilon / d \alpha$ depende de la geometría del ala y de la distancia de esta a la superficie de cola y $\Delta \epsilon_{f}$ depende de la deflexión de los flaps del ala, siendo nula esta contribución si no están deflectados.

Por otra parte, hay que estimar de alguna manera el efecto que tiene el fuselaje en los coeficientes de fuerzas y momentos de la cola. Tanto para el caso del estabilizador 
horizontal (Torenbeek 1982) como para el del vertical (Jones et al. 1993; Ciliberti, Nicolosi y Della Vecchia 2014) se establecen una serie de factores correctores para estimar el efecto de interferencia con el fuselaje. Estas correcciones habrá que realizarlas de la misma forma a los resultados extraídos de Tornado.

En conclusión, se va a utilizar el software Tornado para resolver las derivadas de coeficientes de fuerzas y de momentos y las distribuciones a lo largo de la envergadura de fuerzas y de momentos de alas aisladas, en concreto, de las superficies de cola no convencional. Resultados que serán corregidos de la forma que aquí se ha descrito para poder ser integrados en los coeficientes de fuerzas y de momentos del avión completo.

\subsubsection{Ecuaciones de momentos}

En este apartado se van a presentar las ecuaciones de equilibrio momentos que se van a necesitar para llevar a cabo este estudio y cómo estimar cada uno de los términos que aparecen en las ecuaciones. Estas son las de equilibrio tanto longitudinal como lateral. Se comenzará por presentar la longitudinal:

$$
\frac{1}{2} \rho V^{2} S_{w} C M A_{w} c_{m_{A-T}}-\frac{q_{T}}{q} \frac{1}{2} \rho V^{2} S_{w} L_{T} c_{L T}+T z_{e}=0
$$

donde $\rho$ es la densidad del aire, $V$ es la velocidad de vuelo de la aeronave, $S_{w}$ es el área en planta del ala, $M A C_{w}$ es la cuerda media aerodinámica del ala, $q_{T} / q$ es el cociente de presiones dinámicas entre la cola y el ala, $L_{T}$ es el brazo de momentos de la cola, $c_{L T}$ es el coeficiente de sustentación de la superficie de cola, $T$ es el empuje necesario para volar en esta condición de vuelo de equilibrio y $z_{e}$ es la distancia vertical entre la línea donde se aplica la resultante de empuje y el centro de gravedad. En [ 3-10 ], el coeficiente $c_{m_{A-T}}$ representa el coeficiente de momento de cabeceo del avión eliminando la contribución de la cola. Este coeficiente se va a determinar mediante métodos semiempíricos habitualmente utilizados para aviones de transporte comercial (Torenbeek 1982) como sigue:

$$
c_{m_{A-T}}=c_{m_{\text {Tor }}}+c_{m_{\text {airfoil }}}+\Delta c_{m_{H L D}}+\Delta c_{m_{\text {fus }}}+\frac{c_{L}\left(x_{c g}-x_{c a}\right)}{C M A_{w}}
$$

En la ecuación anterior, $c_{m_{T o r}}$ y $c_{m_{\text {airfoil }}}$ son las contribuciones al coeficiente de momento de cabeceo de la distribución del ángulo de torsión del ala y los coeficientes de momentos de los perfiles utilizados a lo largo de la envergadura, respectivamente. Por otra parte, los términos $\Delta c_{m_{H L D}}$ y $\Delta c_{m_{f u s}}$ son los incrementos de momento de cabeceo que producen los dispositivos hipersustentadores, en caso de estar deflectados, y el propio fuselaje. La contribución de los dispositivos hipersustentadores se tendrá en cuenta en las condiciones de segundo segmento y aterrizaje. Además, $x_{c g}$ y $x_{c a}$ son las posiciones longitudinales del centro de gravedad y del centro aerodinámico. Por último, el $c_{L}$ es el coeficiente de sustentación del avión completo. Este se determina mediante el equilibrio de fuerzas verticales:

$$
\frac{1}{2} \rho V^{2} S_{w} c_{L} \cong W
$$

De esta forma, el peso del avión, $W$, queda equilibrado con la fuerza de sustentación del avión, teniendo en cuenta que el ángulo de ascenso $\gamma$ sea pequeño y su coseno se pueda aproximar a uno. Para poder alcanzar el coeficiente de sustentación necesario para cumplir esta ecuación, es necesario determinar el ángulo de ataque del avión 


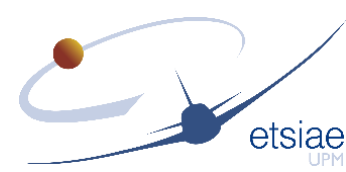

correspondiente, $\alpha$, y la deflexión del control longitudinal, $\delta_{\text {sym }}$, instalado en la cola para, al tiempo, cumplir la ecuación de momentos longitudinal [ 3-10 ]. La dependencia del coeficiente de sustentación con estos dos parámetros se presenta a continuación:

$$
c_{L}=c_{L_{A-T}}(\alpha)+\Delta c_{L_{H L D}}\left(\delta_{f}\right)+\frac{q_{T}}{q} c_{L T}\left(\alpha, \delta_{\text {sym }}\right)
$$

En esta ecuación se indican las dependencias de cada término entre paréntesis, donde el coeficiente $c_{L_{A-T}}$ depende exclusivamente del ángulo de ataque, ya que es el coeficiente de sustentación del conjunto ala fuselaje. La contribución de los dispositivos hipersustentadores de nuevo se tendrá en cuenta en las condiciones de vuelo donde se lleven desplegados. En primera aproximación, el coeficiente de sustentación de estos dispositivos dependerá del ángulo de deflexión del estos, para el cual se considerará un valor característico para condición de vuelo en la que se utilicen. Para determinar el incremento se hará uso de métodos semi-empíricos de la literatura abierta (Torenbeek 1982). En cuanto al coeficiente de sustentación de la cola, depende tanto del ángulo de ataque como de la deflexión del control longitudinal. El ángulo de ataque que ve la cola no es el mismo que el del ala debido a la deflexión de la estela que se produce por estar la cola aguas abajo de la corriente que le llega al ala. Sin embargo, el ángulo de ataque de la cola depende del que vea el ala y se ha modelizado, de nuevo, siguiendo metodologías clásicas de diseño conceptual de aviones (Torenbeek 1982). A pesar de que el ángulo de ataque de la cola se va a determinar mediante esta metodología, puesto que será necesario la distribución de fuerzas y de momentos a lo largo de la envergadura, se va a utilizar Tornado para hallar el coeficiente de sustentación que genera la superficie de cola en las condiciones en las que se encuentre la misma. Las condiciones de ángulo de ataque vendrán dadas por la deflexión de estela y el ángulo de ataque correspondiente del ala. Por supuesto, la dependencia con el ángulo de deflexión del control se tendrá en cuenta mediante la corrección de ángulo de control efectivo expuesta en el apartado anterior, de tal forma que se retengan los comportamientos no lineales en ángulos de deflexión elevados.

En resumen, el sistema que se debe resolver para determinar la deflexión del control longitudinal de la cola es el siguiente:

$$
\left\{\begin{array}{l}
\frac{1}{2} \rho V^{2} S_{w}\left[c_{L_{A-T}}(\alpha)+\Delta c_{L_{H L D}}\left(\delta_{f}\right)+\frac{q_{T}}{q} c_{L T}\left(\alpha, \delta_{s y m}\right)\right]=W \cos \gamma \\
\frac{1}{2} \rho V^{2} S_{W} C M A_{w} c_{m_{A-T}}(\alpha)-\frac{q_{T}}{q} \frac{1}{2} \rho V^{2} S_{w} L_{T} c_{L T}\left(\alpha, \delta_{s y m}\right)+T z_{e}=0
\end{array}\right.
$$

Las incógnitas en este sistema son el ángulo de ataque y la deflexión del control longitudinal. Estas ecuaciones no se pueden resolver analíticamente ya que, tal y como se ha comentado anteriormente, el coeficiente de sustentación de la cola se resuelve mediante Tornado, con las correcciones semi-empíricas correspondientes. Hay que tener en cuenta que las deflexiones de los controles deben darse de tal forma que una deflexión positiva del control simétrico sea tal que produzca un movimiento de cabeceo hacia abajo del morro del avión. El sistema se puede resolver haciendo uso de un método de Newton. Para ello se debe calcular la matriz Jacobiana del sistema, que de nuevo debe determinarse numéricamente, como puede ser por el método de diferencias finitas centradas.

Seguidamente, se puede determinar la ecuación de momentos de guiñada. La expresión final es la siguiente: 


$$
\frac{1}{2} \rho V^{2} S_{w} b_{w} c_{n_{A-T}}-\frac{1}{2} \rho V^{2} S_{w} L_{T} c_{Y T}+T_{e} y_{e}=0
$$

En la ecuación anterior, la envergadura alar viene representada por el símbolo $b_{w}, y_{e}$ es la distancia lateral entre el punto donde se aplica la resultante del motor crítico que no está inoperativo durante el segundo segmento y el plano de simetría del avión, y la resultante de fuerzas correspondiente a la situación de fallo de motor crítico es el término $T_{e}$, que se considerará igual al empuje en despegue de un motor. Por tanto, el último sumando representa el momento de guiñada generado por la asimetría de empuja tras el fallo de motor crítico. Por supuesto, este término aplicará en las condiciones de vuelo donde tenga sentido, es decir, en la condición de fallo de motor crítico en segundo segmento. El coeficiente de momento de guiñada del avión sin tener en cuenta la contribución de la cola, $c_{n_{A-T}}$, se puede determinar mediante métodos semi-empíricos clásicos de la literatura abierta. La principal contribución al momento de guiñada del avión sin la cola es debida al fuselaje y su valor depende del ángulo de resbalamiento. Por otra parte, la contribución de la fuerza lateral de cola, $c_{Y T}$, se va a estimar mediante el uso de Tornado. Esta fuerza debe ser tal que cumpla la ecuación [ 3-15 ]. Este coeficiente depende de la deflexión de la superficie de la cola dedicada al control lateral del avión, $\delta_{a s y m}$. El signo de esta deflexión se toma positivo si genera momento de guiñada positivo. Recordando la normativa aplicable, el equilibrio de momentos laterales del avión debe ser tal que el ángulo de balance resultante sea menor o igual a $5^{\circ}$, tanto en la condición de fallo de motor crítico en segundo segmento como en el aterrizaje con viento cruzado. Para determinar este ángulo de balance, se hace uso del esquema de fuerzas representado en la Figura 3-4, donde el ángulo de balance viene representado por $\phi$.

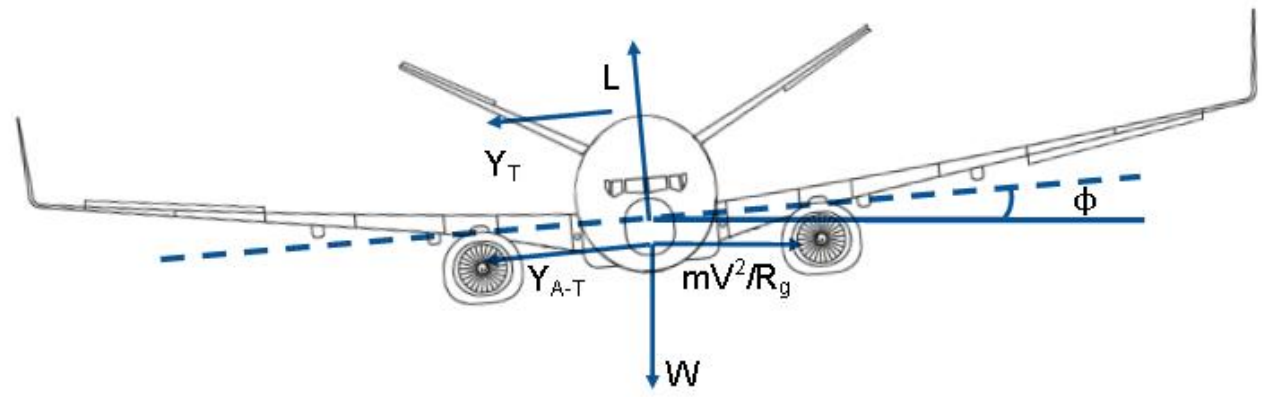

Figura 3-4 Esquema de fuerzas actuando en el avión de cola no convencional cuando vuela con ángulo de balance, tomando el ejemplo de configuración de cola en V. Fuente: elaboración propia.

De ahí, se establece el siguiente equilibrio de fuerzas laterales:

$$
Y_{A-T}+W \sin \phi=Y_{T}+\frac{W}{g} \frac{V^{2}}{R_{g}} \cos \phi
$$

y de fuerzas verticales, como sigue:

$$
L=W \cos \phi+\frac{W}{g} \frac{V^{2}}{R_{g}} \sin \phi
$$

De nuevo, la fuerza lateral generada por el avión sin considerar la cola, $Y_{A-T}$, se calcula mediante métodos semi-empíricos. Este término tiene dos contribuciones principales: la del fuselaje y la del ángulo de diedro del ala, que a su vez dependen del ángulo de 


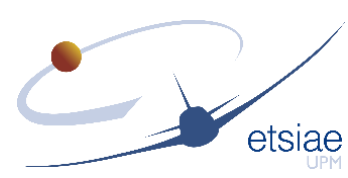

resbalamiento. El término de fuerza lateral de la cola, $Y_{T}$, se determina mediante Tornado. $R$ representa el radio de giro de la maniobra. Asumiendo que el ángulo de balance es pequeño, es posible simplificar la ecuación llegando a la expresión final siguiente para el ángulo de balance:

$$
\phi=\frac{Y_{T}-Y_{A-T}}{W} \pm \sqrt{\left(Y_{T}-Y_{A-T}\right)^{2}-4\left(1-\frac{L}{W}\right)}
$$

\subsubsection{Comparación con otras metodologías}

Se ha realizado un proceso de comparación para analizar la fiabilidad de la metodología propuesta que combina aproximaciones semi-empíricas para estimar los coeficientes aerodinámicos de cualquier elemento del avión salvo las superficies de cola con métodos VLM para el cálculo de los coeficientes aerodinámicos de la cola. La comparación se ha realizado entre el método combinado propuesto con el software Tornado, simulando tanto el ala como las superficies de cola, y con un método puramente semi-empírico como es el método de Torenbeek. Para ello, se va a escoger un avión de referencia para el cual se va a estimar el coeficiente de sustentación y de momento de cabeceo por medio de los tres métodos propuestos para dos condiciones de vuelo: crucero y segundo segmento. El método de referencia será el de Torenbeek, ya que es el que más extendido tiene su uso en etapas de diseño conceptual de aviones de transporte comercial, por lo que se asume que los resultados obtenidos mediante este método son adecuados. Esta metodología estima las contribuciones de las superficies de cola de la misma forma que el ala principal, es decir, basándose en ecuaciones semi-empíricas. Esta es la principal diferencia con la metodología propuesta en este estudio. Es cierto, que para poder resolver el problema completo es necesario conocer las curvas de coeficientes aerodinámicos de los perfiles instalados en el ala y en la cola. Empezando por estos últimos, no son un problema ya que son perfiles simétricos NACA, por lo que sus curvas son ampliamente conocidas y fácilmente consultables. Sin embargo, los perfiles del ala son más diferentes de un avión a otro. En el caso en concreto del avión de referencia escogido para llevar a cabo este estudio comparativo, que es el mismo que se utilizó como caso de estudio en el capítulo anterior, el CSR-01, la información de la que se dispone es de la geometría del perfil en tres secciones diferentes de la envergadura del ala. Por tanto, es necesario estimar los coeficientes aerodinámicos asociados a cada uno de esos perfiles. Para ello se ha hecho uso del software X-Foil («XFOIL 6.99. Subsonic Airfoil Development System» 2013). Los resultados de esta estimación sirven tanto para la metodología de Torenbeek como para la combinación propuesta en el estudio, ya que en ambos casos es necesario considerar los perfiles instalados en el ala. Por último, con Tornado se pueden simular varias alas en el mismo problema, por lo que se ha generado un modelo de ala y superficies de cola de forma que el software resuelva todo de una misma vez. El principal inconveniente es que no se puede tener en cuenta el fuselaje porque el software está desarrollado exclusivamente para superficies sustentadoras, con lo cual de partida ya se puede ver que los resultados pueden diferir de los que se obtengan con el método de Torenbeek. Además, Tornado tampoco considera los coeficientes de momento de cabeceo de los perfiles, pero sí aproxima los de sustentación de los mismos. Por tanto, para que la comparación tenga más sentido, se ha decidido añadir estos términos correspondientes a los momentos de cabeceo de los perfiles. Debido a la limitación en número de Mach de Tornado, y haciendo uso de la corrección de Prandtl-Glauert, se ha fijado el número de Mach de crucero en 0,6. En cuanto a la condición de segundo segmento, se ha escogido la velocidad que resulta de incrementar en un $20 \%$ la 
velocidad de entrada en pérdida en configuración de despegue del CSR-01, porque es la que se establece en las normas que se debe alcanzar tras finalizar el despegue y comenzar el segundo segmento (CS 25.107 (EASA 2018) y FAR 25.107 («Code of Federal Regulations: Title 14 - Chapter I - Subchapter C - Part 25» 2017)), datos que se pueden consultar en la base de datos de CeRAS, que corresponde a un Mach de 0,278. En esta condición de vuelo se llevan los flaps desplegados, tomando como ángulo de deflexión un valor típico de $10^{\circ}$. La Figura 3-5 muestra los resultados obtenidos de los coeficientes de sustentación y de momento de cabeceo para los tres métodos contemplados para las dos condiciones de vuelo. A la vista de los gráficos, se ve como Tornado no se aproxima lo suficiente a las curvas obtenidas por Torenbeek que, como se comentó anteriormente, es la curva de referencia. Sin embargo, analizando los resultados obtenidos por medio del método combinado propuesto en este estudio, las curvas están prácticamente superpuestas en todo el rango de ángulos de ataque contemplados. Aun así, la curva de coeficiente de sustentación presenta menos diferencia con la referencia que la curva de momentos. Si se toma la condición de crucero al Mach considerado y a un peso característico, el coeficiente de sustentación es de 0,5 y el ángulo de ataque necesario para alcanzar ese coeficiente por medio del método propuesto es un $0,14 \%$ inferior que el que se obtendría por medio del método de Torenbeek. La misma comparación se puede realizar con el coeficiente de sustentación necesario para el vuelo en segundo segmento, donde se encuentra una desviación del método propuesto respecto del de Torenbeek del -0,25\%. En el caso de las curvas de momentos, las diferencias son algo mayores, como se puede apreciar a simple vista. También se observa como a velocidades inferiores las curvas se parecen más entre sí que para el Mach de crucero. De hecho, para el ángulo de ataque necesario para alcanzar el coeficiente de sustentación necesario para el vuelo en segundo segmento, el coeficiente de momento obtenido con el método combinado es un 1,8\% mayor que el obtenido por Torenbeek. Por el otro lado, esta misma comparación se puede realizar en el vuelo de crucero, donde aquí se encuentra una diferencia del $12 \%$. Por tanto, se ha comprobado que la metodología propuesta de combinación entre métodos semi-empíricos y VLM para las superficies de cola presenta resultados muy próximos a los que se obtienen por medio de métodos clásicos usados en etapas de diseño conceptual de aviones de transporte comercial. 


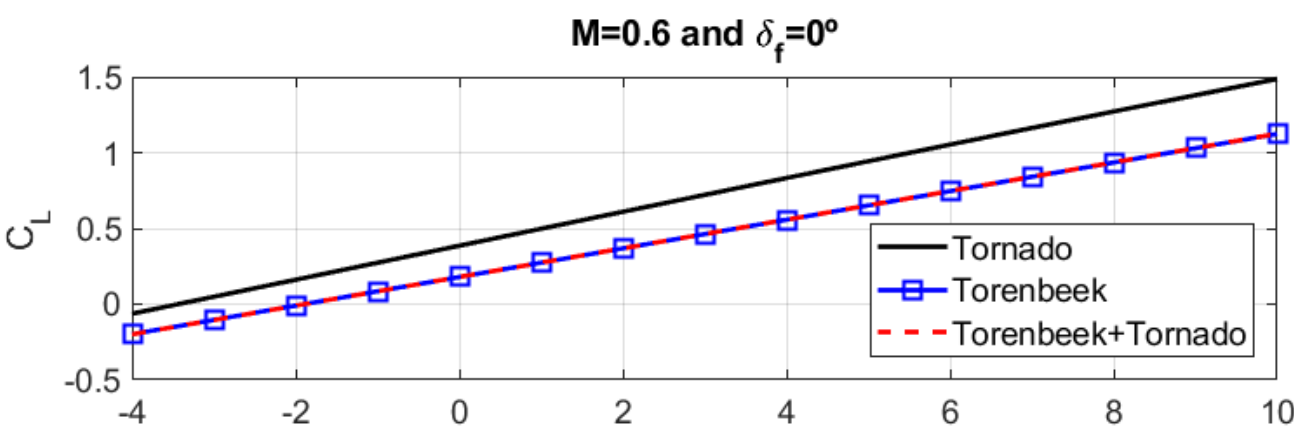

$\alpha\left[^{\circ}\right]$

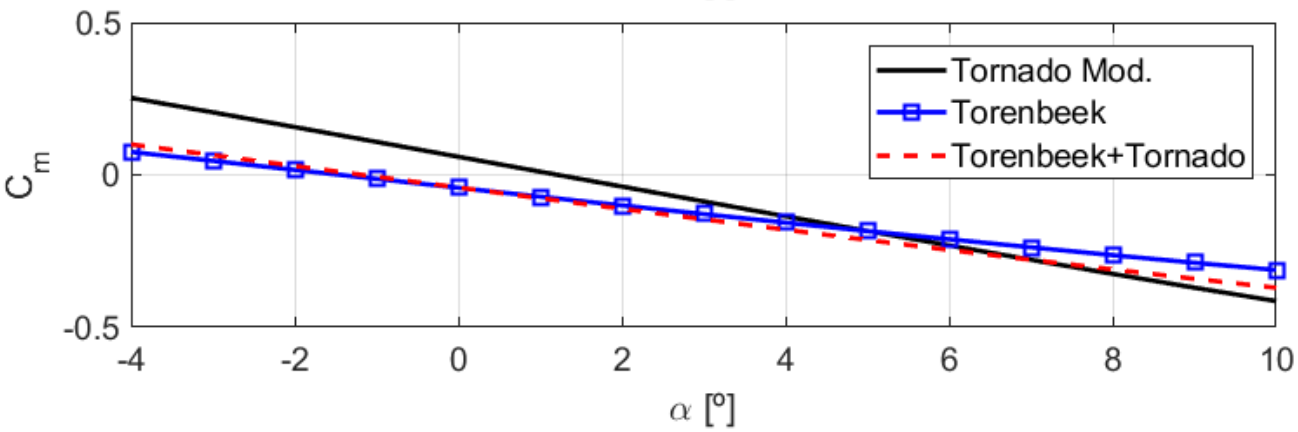

(a)

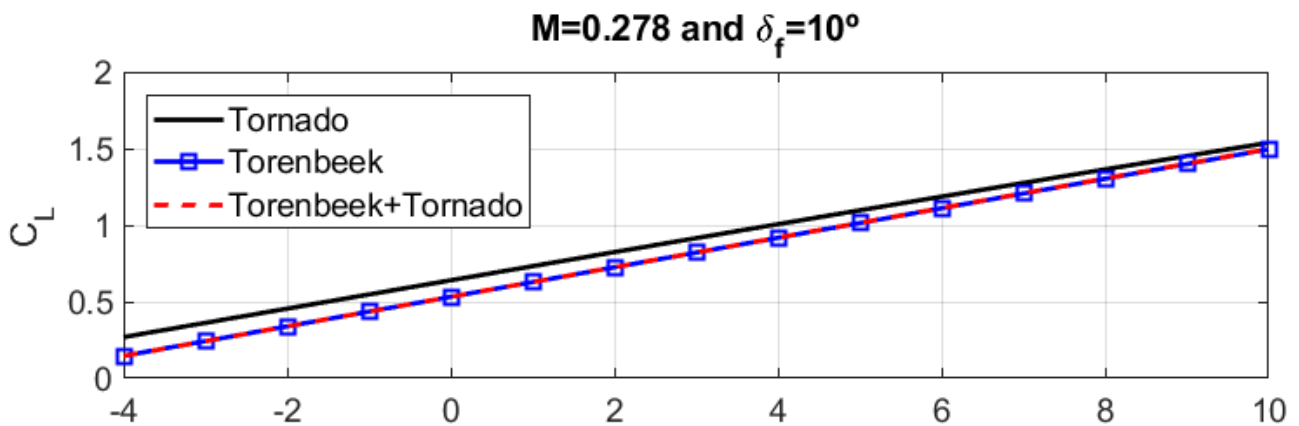

$\left.\alpha{ }^{\circ}\right]$

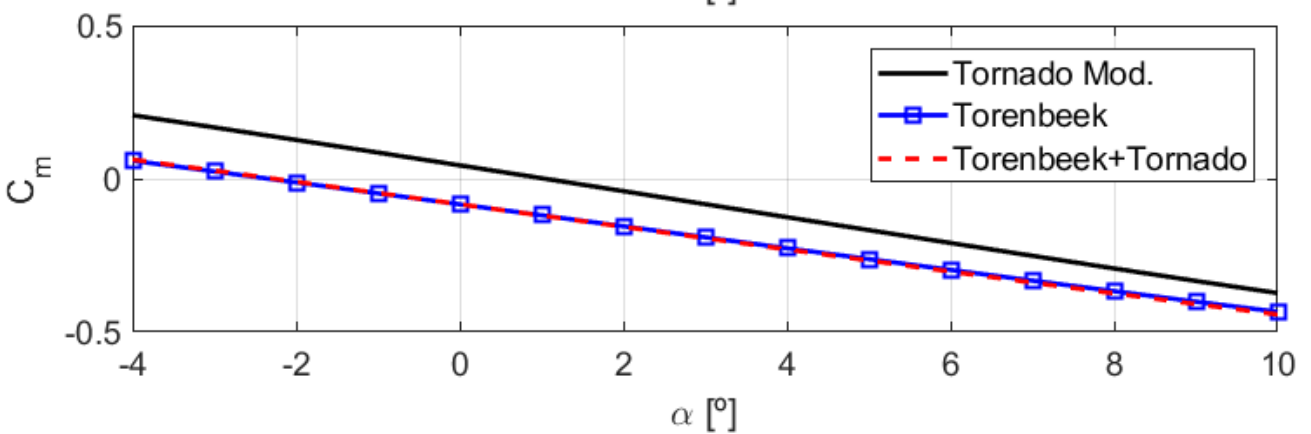

(b)

Figura 3-5 Comparación de métodos para el cálculo del coeficiente de sustentación y de momento de cabeceo entre Tornado, Torenbeek y el método propuesto en vuelo de crucero a Mach 0,6, (a), y en segundo segmento, (b). Fuente: elaboración propia. 


\subsubsection{Derivadas de estabilidad estática en crucero para configuraciones de cola no convencionales}

Las derivadas de estabilidad que se van a utilizar para caracterizar las propiedades de estabilidad estática en crucero son $c_{m \alpha}$, para la estabilidad estática longitudinal, y $c_{n \beta}$ para la lateral. Para determinar estos coeficientes es necesario plantear las ecuaciones de momentos longitudinal y lateral y derivarlas respecto del ángulo de ataque y del ángulo de resbalamiento, respectivamente. La derivada de estabilidad estática longitudinal se obtiene derivando respecto del ángulo de ataque, la segunda ecuación del sistema [ 3-14 ], una vez se hayan determinado los ángulos de equilibrio de la condición de crucero. De la misma forma, derivando la ecuación [ 3-15 ] respecto del ángulo de resbalamiento en torno a la deflexión de control de equilibrio, se estima la derivada de estabilidad estática lateral. En ambos casos, puesto que aparecen términos que se estiman mediante Tornado, es necesario hacer uso de algún esquema de derivación numérico, como puede ser el de diferencias finitas centradas.

\subsubsection{Cargas en la cola en maniobras simétricas}

Siguiendo la definición habitual de maniobras simétricas, se consideran como tal las que tan solo entran en juego la sustentación y el momento de cabeceo del avión. Por tanto, se asume que las fuerzas laterales son despreciables o nulas. Además, se toma la hipótesis de que el avión no modifica su altitud ni su número de Mach de vuelo. Las condiciones de vuelo que se van a contemplar para analizar las cargas que se producen en la cola durante maniobras simétricas se pueden dividir en dos: sin aceleración de cabeceo o estacionarias y maniobras de cabeceo abruptas o con aceleración de cabeceo (Lomax 1996).

Las condiciones de vuelo estacionarias son aquellas en las que no se produce aceleración de cabeceo del avión. Por ejemplo, un viraje estacionario se puede considerar como una maniobra simétrica, a pesar de que la aeronave sufre una aceleración lateral debido al giro. También se incluye en esta categoría las maniobras de cambio de nivel de vuelo sin aceleración de cabeceo. Este tipo de condiciones se resuelven encontrando la solución al sistema de ecuaciones formado por la ecuación de fuerzas verticales y la de momentos de cabeceo del avión, ambas igualadas a cero, ya que no se producen aceleraciones. En el apartado 3.2.3 se ha presentado el caso particular de un vuelo de ascenso, con ángulo de ascenso pequeño. En otro tipo de maniobras donde el factor de carga no esté próximo a uno, se deberán modificar estas ecuaciones de tal forma que el peso del avión quede multiplicado por el factor de carga correspondiente a dicha maniobra. La solución del sistema determinará la deflexión necesaria del timón de profundidad y el ángulo de ataque al cual se debe situar el avión. En esta condición de equilibrio, la cola está desarrollando una fuerza que debe ser soportada por la estructura de la misma y su unión al fuselaje, y estructura adyacente.

El otro tipo de maniobras que se consideran simétricas son aquellas que se producen al deflectar de forma rápida el timón de profundidad. Dentro de estas maniobras se deben considerar dos tipos: deflexión unchecked del timón a la velocidad $V_{\mathrm{A}} \mathrm{y}$ deflexión checked del timón a velocidades entre $\mathrm{V}_{\mathrm{A}} \mathrm{y} \mathrm{V}_{\mathrm{D}}$. Las maniobras unchecked suponen una deflexión súbita del timón que produzca una aceleración de cabeceo máxima positiva (morro hacia arriba) del avión cuando el avión se encuentra volando en vuelo rectilíneo y estacionario a la velocidad de maniobra de proyecto $V_{A}$, de acuerdo con FAR 25.331 («Code of Federal Regulations: Title 14 - Chapter I - Subchapter C - Part 25» 2017) o con CS 25.331 (EASA 2018). La respuesta dinámica del avión debe ser tenida en cuenta para el cálculo de la carga en la cola resultante. A pesar de ello, si sobre el centro de 


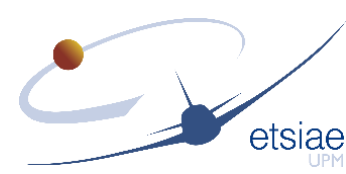

gravedad se produce una aceleración vertical que excede el factor de carga límite positivo de maniobra, no se deben considerar las cargas que se produzcan tras alcanzar dicho factor de carga. Para alcanzar esta aceleración de cabeceo máxima se debe deflectar el timón desde la posición de equilibrio para vuelo rectilíneo y uniforme hasta la deflexión máxima, manteniéndose en esa posición tras alcanzar el tope. La deflexión súbita del timón se puede modelizar habitualmente de dos formas, a su vez, como son una ley de deflexión lineal o exponencial hasta el tope. La deflexión checked del timón consiste en alcanzar el tope del timón, pero posteriormente se vuelve a la posición de equilibrio. Esta ley de deflexión se modela por medio de una sinusoide que alcanza la deflexión máxima del timón. Las características de que debe tener dicha sinusoide se recogen en la normativa aplicable, concretamente en el artículo CS 25.331 (EASA 2018). En el caso de la normativa de Estados Unidos, el código indica que esta maniobra debe realizarse de tal forma que no se exceda el factor de carga límite, bien positivo o negativo. La aceleración que aparece en el avión no debe ser inferior a un valor especificado en las normas, tanto para las maniobras de cabeceo con aceleración positiva o negativa. Los detalles se incluyen en el artículo FAR 25.331 («Code of Federal Regulations: Title 14 - Chapter I - Subchapter C - Part 25» 2017). Existen tres modelizaciones típicas, como son la función rampa, la exponencial o la sinusoidal. La primera función se compone de dos rampas, de tal forma que pasado un cierto tiempo establecido en las normas se reduzca la deflexión. Las otras dos representaciones son tales que permiten ajustar el ritmo de la subida, en el caso de la exponencial, o la frecuencia, en el caso de la sinusoidal.

Para modelizar las cargas generadas en la cola tras una maniobra con aceleración de cabeceo producida por una deflexión unchecked, existe una simplificación que no requiere resolver el comportamiento dinámico de la aeronave. El incremento de carga en la cola y en el momento de cabeceo debido a la deflexión abrupta del control se puede dividir en dos efectos: el incremento de ángulo de ataque que se produce y el incremento de deflexión en sí mismo. Tras una serie de operaciones recogidas en (Lomax 1996) se alcanza la siguiente expresión:

$$
L_{t}=L_{t, n=1}+k_{r} L_{t \delta_{e}} \Delta \delta_{\text {emax }}
$$

El primer sumando de la expresión anterior corresponde a la fuerza generada en la cola para el equilibrado del avión en crucero y el segundo sumando al incremento de fuerza generado por el incremento de deflexión entre la de equilibrio y la máxima. Esta contribución debe reducirse por medio de un factor de respuesta $k_{r}$, que se puede tomar igual a 0,9 para ser conservativos. Este parámetro debe reducirse en su valor si se considera la deflexión abrupta modelizada por medio de una ley exponencial. Además, se recomienda el uso de este factor únicamente si se ha alcanzado la combinación de velocidad y altitud crítica, en términos de carga.

En este estudio, tal y como se ha expuesto anteriormente, se van a estimar las cargas aerodinámicas por medio del software Tornado. Este software no admite una entrada en forma de función del tiempo para los controles del avión. Por tanto, se va a recurrir al método simplificado para determinar las fuerzas generadas tras una deflexión súbita del timón. Además, tampoco se está seguro de que la condición que se vaya a analizar vaya a ser la crítica en términos de velocidad y altitud; de ahí que se haya tomado la decisión de ser aún más conservativos que el modelo presentado al tomar el factor de respuesta como igual a la unidad. En consecuencia, es suficiente con introducir la 
deflexión máxima del timón, tras equilibrar el avión en una condición de crucero a $\mathrm{V}_{\mathrm{A}}$, para estimar la carga que se genera en la cola.

Al igual que ocurre con las maniobras con aceleración de cabeceo, las maniobras checked también se pueden modelizar de forma simplificada para estimar la carga generada en la cola. La expresión que resulta del modelo simplificado recogido en (Lomax 1996) es:

$$
L_{t}=L_{t, b a l}+L_{t \delta_{e}} \Delta \delta_{e c m}
$$

La fuerza de la cola necesaria para equilibrar el avión a un cierto factor de carga, en general distinto de uno, corresponde al primer sumando de la expresión anterior. El segundo sumando representa el incremento de fuerza que se genera en la cola por incrementar el ángulo de deflexión del timón con respecto a la posición de equilibrio en un valor igual al siguiente:

$$
\Delta \delta_{e c m}=-(1+C B F)\left(\delta_{e b a l}-\delta_{\text {etrim }}\right)
$$

donde $C B F$ es un factor que se toma igual a cero cuando el control vuelve a la posición de equilibrio y 0,5 cuando se deflecta el timón, $\delta_{\text {ebal }}$ corresponde al ángulo de deflexión del timón para equilibrar la aeronave en la condición de vuelo al factor de carga considerado y $\delta_{\text {etrim }}$ al ángulo que equilibra el avión para factor de carga uno, es decir, al inicio de la maniobra.

Las maniobras checked se pueden considerar para calcular las cargas que generan en la cola por medio del software Tornado de la misma forma que en el caso de maniobras con aceleración de cabeceo, es decir, haciendo uso del modelo simplificado. Sin embargo, el principal inconveniente que presentan es que deben evaluarse para todas las velocidades situadas entre $V_{A}$ y $V_{D}$. Esto choca de lleno con las limitaciones del software, ya que no es capaz de estimar cargas con precisión en condiciones de vuelo que supongan velocidades transónicas. Es por esto que tan solo se pueden evaluar este tipo de maniobras a las velocidades que no alcancen dicho régimen.

\subsubsection{Cargas en la cola debido a ráfagas}

Las condiciones atmosféricas inducen cargas en los estabilizadores que deben ser consideradas. Concretamente, el avión vuela inmerso en un fluido que presenta turbulencias en forma de ráfagas. Por tanto, se deben modelizar las ráfagas y el comportamiento del avión frente a ellas para poder estimar las cargas inducidas en la cola. El modelo que se va a utilizar para definir la ráfaga parte del Criterio de Pratt (Pratt y Walker 1953; Pratt 1953), que supone una ráfaga consinuidal de semilongitud de onda 12,5 CMG, y la aparición del factor de atenuación de ráfagas, $K_{g}$, relacionándose con una ráfaga instantánea equivalente. El factor de atenuación y el factor de carga que aparece con este modelo son los siguientes:

$$
K_{g}=\frac{0,88 \mu}{5,3+\mu}
$$




$$
\Delta n=K_{g} \frac{\rho_{0} U_{\max } V c_{L \alpha}}{2 W / S_{w}}
$$

donde $U_{\max }$ es la velocidad de intensidad de ráfaga y $\mu=2 W /\left(\rho c_{L \alpha} C M G g S_{w}\right)$ es el parámetro másico del avión. El valor de la intensidad de ráfaga depende de la velocidad de vuelo según se establece en las normas de certificación. A partir del año 2000 , fecha en la cual los códigos europeo y norteamericano incluyen unos cambios notables, el modelo de cálculo de los factores de carga experimentados por el avión ante ráfagas verticales, positivas y negativas se ha modificado, siendo menos intuitivo. Se ha decidido utilizar el modelo antiguo que permite ser integrado de mejor forma con la metodología que se ha presentado para el cálculo de cargas aerodinámicas manteniendo resultados conservativos y en el margen de error del resto de la herramienta. Por otra parte, el ángulo de ataque que se induce en el avión por la aparición de la ráfaga y la carga que soporta la cola son (Lomax 1996):

$$
\begin{gathered}
\Delta \alpha_{w}=\frac{180 K_{g} U_{\max }}{1,69 V \pi} \\
L_{t}=L_{t, n=1}+\left(1-\frac{d \epsilon}{d \alpha}\right) \Delta \alpha_{w} L_{t \alpha}
\end{gathered}
$$

Para exportar este modelo al software Tornado, no hay más que equilibrar la aeronave en una cierta condición de crucero y, tras ello, introducir un incremento de ángulo de ataque igual al que se define en la ecuación [ 3-24 ]. De esta forma se puede determinar la carga que se genera en la cola en estas condiciones.

Este mismo modelo se puede aplicar para estimar las cargas que se generan en la cola si aparece una ráfaga lateral, sin más que sustituyendo el parámetro másico por el siguiente (Lomax 1996):

$$
\mu_{v}=2 I_{Z} /\left(\rho c_{Y \beta_{V}} C M G_{V} g S_{V} L_{T V}^{2}\right)
$$

En consecuencia, la carga que aparece en la cola vertical es:

$$
L_{V}=\frac{K_{g} \rho U_{\max } V c_{Y \beta_{V}} S_{V}}{2}
$$

\subsubsection{Cargas en la cola en maniobras asimétricas}

El avión debe diseñarse para soportar las cargas que resultan de las maniobras de guiñada que se indican a continuación en velocidades entre $V_{M C}$ y $V_{D}$, incluyendo estas, según se recoge en el artículo CS 25.351 (EASA 2018) o en el FAR 25.351 («Code of Federal Regulations: Title 14 - Chapter I - Subchapter C - Part 25» 2017). Los momentos aerodinámicos que se generan alrededor del centro de gravedad deben equilibrarse de forma conservativa. Además, se debe considerar que la velocidad de guiñada es nula, ya que esta hipótesis es conservativa con las cargas que se generan, y que no se realizan con las alas a nivel. Las maniobras asimétricas que afectan a la cola que se generan por la deflexión del timón de dirección se pueden reducir a tres condiciones, 
que se deben aplicar entre las velocidades de mínimo control del avión y la velocidad de picado de proyecto, y son:

- Maniobra 1: con el avión en un vuelo sin aceleración y sin guiñada, se supone que el timón de dirección se deflecta rápidamente hasta el máximo de su deflexión o hasta la deflexión que suponga una fuerza en el pedal igual a $1335 \mathrm{~N}$.

- Maniobra 2: con el timón de dirección deflectado al máximo o en el ángulo correspondiente a que el piloto tenga que hacer una fuerza en el pedal igual a $1335 \mathrm{~N}$, se asume que el avión gira alrededor del eje z hasta un ángulo de resbalamiento resultante.

- Maniobra 3: Con al avión equilibrado en guiñada para el ángulo del timón de dirección anterior, se supone que el control vuelve a cero.

La maniobra 1 se puede modelizar fácilmente con el software Tornado, ya que no es más que indicar la deflexión máxima del timón de dirección. Además, puesto que se han corregido los resultados del software para que contemple los efectos no lineales que se produce a altas deflexiones, el resultado debería ser preciso para velocidades que no entren en el régimen transónico.

La maniobra 2 se simplifica siguiendo las indicaciones incluidas en (Lomax 1996), donde se dice que se puede estimar las cargas generadas por esta maniobra como la suma de las que se producen por el hecho de tener el timón deflectado al máximo y las que se producen por volar al ángulo de resbalamiento estacionario que se alcanza tras esa deflexión.

En el caso de la maniobra 3, se puede determinar las cargas sin más que restar las condiciones de ángulo de resbalamiento estacionario con deflexión máxima y la de ángulo de resbalamiento estacionario con deflexión nula. Es decir, que las cargas de esta maniobra se pueden aproximar por las que generan exclusivamente el hecho de volar al ángulo de resbalamiento estacionario.

Otra condición de vuelo que introduce cargas en la cola es el control del avión tras el fallo del motor crítico, recogido en el artículo FAR 25.367 («Code of Federal Regulations: Title 14 - Chapter I - Subchapter C - Part 25» 2017) o CS 25.367 (EASA 2018). Las ecuaciones necesarias para resolver esta condición ya se han presentado anteriormente, pero la norma contempla dos situaciones de carga que deben estudiarse: la deflexión del timón de dirección necesaria para anular el momento de guiñada generado por la asimetría de empuje volando con ángulo de resbalamiento nulo, y el ángulo de resbalamiento necesario para que el momento de guiñada se anule sin que sea necesario deflexión del timón alguna.

Tanto en las maniobras 2 y 3 como en la de control del avión tras el fallo del motor crítico sin deflexión del timón, el ángulo de resbalamiento resultante puede situarse por encima de los valores en los que tiene validez (aerodinámica lineal) el software Tornado, que se va a utilizar para calcular las distribuciones de fuerzas y momentos a lo largo de la envergadura de la cola. Es por ello, que se ha decidido sustituir estas condiciones por un ángulo de resbalamiento constante e igual a $10^{\circ}$, en lugar de hallar el correspondiente estacionario en cada caso. Es una simplificación que viene obligada por las herramientas de cálculo seleccionadas, que podrían ser actualizadas en el futuro. 


\subsection{MODELO ESTRUCTURAL Y DE PESOS}

La estimación del peso de la estructura es un aspecto fundamental en el diseño conceptual de un avión de transporte comercial. En estas etapas del diseño, se desea una herramienta lo más precisa posible y que permita realizar un estudio de sensibilidad ante las variaciones de ciertos parámetros de diseño. En cuanto a la precisión, siempre se pueden utilizar modelos complicados basados en métodos de elementos finitos que estimen el peso de la estructura con un error en torno al 1-2\%. Sin embargo, para poder calcularlo es necesario conocer en detalle la geometría y el diseño de la estructura, lo cual no suelen estar definidos en estas etapas iniciales de diseño conceptual. Estas herramientas son más apropiadas para diseño preliminar o detallado, donde el conocimiento de la configuración está mucho más avanzado y definido. Por tanto, es necesario desarrollar una metodología de estimación de pesos que sea lo suficientemente precisa pero que el tiempo que tarde en implementarse, en calcular y en hacer variaciones de los valores de los parámetros de diseño sea reducido. Con estas premisas se pueden encontrar dos tipos de metodologías: métodos estadísticos y métodos casi-analíticos. En este capítulo se abordarán ambos tipos de métodos para la estimación del peso de configuraciones de cola no convencionales.

\subsubsection{Método basado en regresiones estadísticas}

El primer método que se va a presentar para determinar el peso de la estructura de una configuración de cola no convencional es el que se basa en una serie de regresiones que establecen una dependencia entre el peso de la estructura y el valor de ciertos parámetros de diseño (Sadraey 2013). Estas regresiones se construyeron para estimar el peso de los estabilizadores de cola de una configuración convencional, es decir, el estabilizador vertical y horizontal. Sin embargo, estas ecuaciones se pueden extrapolar para las configuraciones no convencionales sin más que utilizar la proyección horizontal de la superficie de cola como si fuera un estabilizador horizontal y la proyección vertical como si fuera un estabilizador vertical, pudiendo así entrar en las expresiones diseñadas para las configuraciones convencionales. Esta técnica se ha aplicado principalmente a la configuración de cola en V (Roskam 1999). Las ecuaciones se han construido basándose en cuatro principios fundamentales: la relación directa que existe entre el peso de un componente y su densidad media, datos reales publicados sobre pesos de componentes, factores empíricos y ecuaciones empíricas publicadas en la literatura abierta. Los dos primeros principios hacen que la técnica sea precisa y fiable. Pero, los dos últimos indican que es necesario ajustar una serie de parámetros para calibrar los resultados.

En cuanto a la regresión utilizada para la estimación del peso de un estabilizador horizontal, se ha considerado que el peso es una función que depende del área de la forma en planta del mismo, del espesor relativo máximo, de la configuración de cola, del material de fabricación, de la cuerda del control longitudinal, y de otros parámetros geométricos de la cola tales como el alargamiento, parámetro de estrechamiento y flecha (Sadraey 2013). La expresión que estima el peso en kilogramos es como sigue:

$$
W_{H}=S_{H} C M A_{H}\left(\frac{t}{c}\right)_{\max } \rho_{\text {mat }} K_{\rho H}\left(\frac{A R_{H}}{\cos \left(\Lambda_{0,25 H}\right)}\right)^{0,6} \lambda_{H}^{0,04} V_{e H}^{0,3}\left(\frac{c_{e l e v}}{c}\right)^{0,4}
$$

donde $\rho_{\text {mat }}$ es la densidad del material de fabricación y $K_{\rho H}$ es el factor de densidad del estabilizador horizontal. Ambos parámetros son constantes que se deben ajustar para obtener resultados precisos para estabilizadores horizontales reales. 
El peso del estabilizador vertical se considera función de nuevo del área de la forma en planta del mismo, del espesor relativo máximo, del material, del coeficiente de volumen, del alargamiento, del parámetro de estrechamiento y de la flecha. La expresión que corresponde a la estimación del peso del estabilizador vertical en kilogramos es la siguiente:

$$
W_{V}=S_{V} C M A_{V}\left(\frac{t}{c}\right)_{\text {max }} \rho_{\text {mat }} K_{\rho V}\left(\frac{A R_{V}}{\cos \left(\Lambda_{0,25 V}\right)}\right)^{0,6} \lambda_{V}^{0,04} V_{e V}^{0,2}\left(\frac{c_{r u d d}}{c}\right)^{0,4}
$$

En esta expresión, nuevamente las constantes $\rho_{\text {mat }}$ y $K_{\rho V}$ son la densidad del material y el factor de densidad del estabilizador vertical que deben ser ajustados para que la ecuación tenga resultados fiables.

A pesar de que la densidad de los materiales utilizados y los factores de densidad de los estabilizadores sean parámetros a ajustar, hay que hacer notar que no pueden tomar cualquier valor, sino que en la referencia de donde se obtuvieron las expresiones vienen indicados unos rangos aproximados entre los que se deberían encontrar los parámetros una vez ajustados (Sadraey 2013).

\subsubsection{Método casi analítico en material metálico}

El método casi analítico que se ha desarrollado en este apartado está basado en el método presentado en la referencia (Torenbeek 2013) que está pensado para estimar el peso de alas de aviones civiles. Este se basa en dimensionar el cajón de torsión de un ala de tal forma que resista las fuerzas aerodinámicas para factor de carga último. En la Figura 3-6 se muestran los principales componentes de la estructura de un ala. Si se puede estimar el peso de cada uno de estos componentes, la suma de todos ellos dará como resultado la estimación del peso del ala.

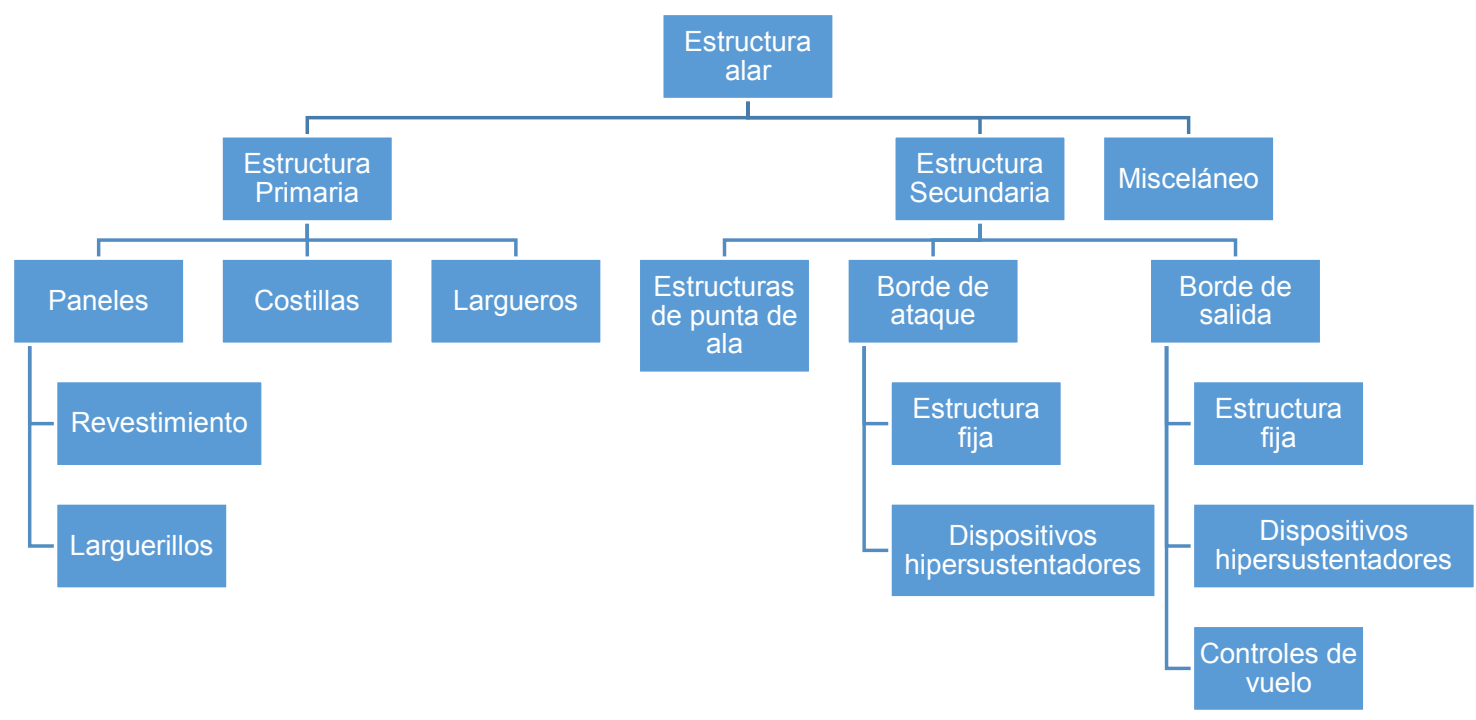

Figura 3-6 Composición de los elementos de la estructura de un ala de un avión. Fuente:

(Torenbeek 2013).

La estructura primaria de un ala se compone de una serie de costillas distribuidas a lo largo de la envergadura, formando un cajón de torsión cerrado por los largueros y por paneles. Los paneles cierran el cajón por la parte superior e inferior, y están formados por revestimiento y rigidizadores longitudinales llamados larguerillos. Los largueros rigidizan y limitan el cajón de torsión por la parte frontal y trasera, siendo las costillas las que lo cierran en la dirección de la envergadura. El cajón de torsión absorbe las cargas 


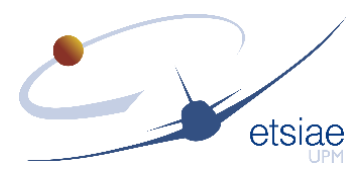

aerodinámicas, las de inercia y las de tierra que actúan sobre el ala y, a su vez, recibe cargas de las estructuras secundarias y de los elementos instalados por fuera del ala como pueden ser los motores. La combinación de todas estas cargas transmite unos momentos flector y torsor y fuerza cortante al cajón de torsión. Por tanto, es posible determinar la cantidad de material que es necesario para soportar las cargas críticas, pudiendo estimar el peso de la estructura primaria de forma ideal. Se puede incrementar este peso para tener en cuenta principios de tolerancia al daño o de fail-safe e incluso consideraciones operacionales o de mantenimiento. La estructura secundaria se compone de elementos situados por delante del larguero anterior y por detrás del posterior. Estos elementos se pueden clasificar en estructuras fijas y móviles, como pueden ser los dispositivos hipersustentadores o los controles de vuelo. En el caso de la estructura secundaria, no se puede determinar su peso de una forma analítica, en consecuencia, es necesario recurrir a la estadística. Por último, la categoría miscelánea engloba elementos tales como los carenados o la pintura. Algunos de ellos se pueden incluir en alguna de las categorías anteriores y otros incluso los fabricantes no los consideran como parte del peso del ala. Sin embargo, el método los incluye para completar todos los elementos.

Para el cálculo del peso de la estructura del ala, el método se basa en las siguientes hipótesis:

- Es aplicable a alas de gran alargamiento con estructura primaria de cajón de torsión principalmente compuesta por aleaciones de aluminio. A pesar de ello se incluyen algunas directrices para corregir por el uso de materiales compuestos.

- El ala se considera una estructura rígida estática. Se incluye una tentativa de corrección para soportar las deformaciones aeroelásticas.

- Los casos de carga que se consideran son la maniobra estacionaria y las ráfagas, entre los cuales se coge el caso de carga crítico.

- El momento flector es absorbido por los paneles de extradós sometidos a compresión y los de intradós sometidos a tracción. Los paneles que se componen de revestimiento y larguerillos son sustituidos por un revestimiento sin rigidizadores de espesor equivalente.

- Las cargas de cortadura son transmitidas por los largueros rigidizados usando un espesor de revestimiento equivalente.

- Los momentos torsores no se tienen en cuenta explícitamente. En cambio, el peso del cajón se corrige para alcanzar una rigidez a torsión suficiente.

- El peso de las costillas se estima mediante regresiones estadísticas en primera aproximación.

- Se centra en predecir con precisión las cargas de los paneles rigidizados situados en el encastre del ala, donde se produce el momento flector máximo.

- El peso se determina por integración del peso por unidad de longitud del cajón de torsión a lo largo de la envergadura.

- El resto de los elementos no ideales se consideran como correcciones al peso anterior.

- La estructura secundaria se determina mediante regresiones estadísticas.

Siguiendo todas las directrices del método especificadas en la referencia, se ha comprobado que es posible estimar el peso de un ala con una precisión bastante elevada. Se ha tomado como ejemplo el ala del CSR-01, avión del cual se conocen todos los datos necesarios para implementar el método. Es cierto que ha sido necesario corregir los resultados con los factores indicados en la propia referencia para considerar 
los elementos estructurales fabricados en material compuesto. El peso del ala del CSR01 que se puede encontrar en la base de datos CeRAS es de $8097 \mathrm{~kg}$, alcanzando un peso de $8411 \mathrm{~kg}$ por el método para alas aquí indicado. El error relativo es del 3,88\%, con lo que se concluye que este método es muy adecuado para ser implementado en etapas de diseño conceptual de aviones.

Sin embargo, este método no es directamente aplicable para estimar el peso de las superficies de cola, tanto convencionales como no convencionales. Lo primero es identificar los elementos estructurales que aplican a una superficie de cola. La estructura primaria se va a considerar igual que la de un ala, es decir, compuesta por paneles, largueros y costillas. En cuanto a la estructura secundaria, no habría que considerar los elementos móviles de borde de ataque, ya que solamente se contemplan los controles de vuelo de borde de salida. Además, no se llevan dispositivos hipersustentadores en la cola, por lo que esta contribución también debe ser eliminada. Por último, no todos los elementos que se engloban en la categoría miscelánea del ala son equivalentes en las superficies de cola. Por ejemplo, los refuerzos por colgar los motores bajo las alas no se consideran en la cola. Sin embargo, extensiones de punta de ala podrían ser aplicables en algunas configuraciones de cola. En resumen, habría que ver elemento por elemento cuales se considerarían en esta categoría en función de la configuración de cola a estudiar.

Por otra parte, también se van a modificar las hipótesis de partida con las que se van a trabajar para estimar el peso de la estructura, quedando como siguen:

- Es aplicable a alas con estructura primaria de cajón de torsión principalmente compuesta por aleaciones de aluminio. A pesar de ello se incluyen algunas directrices para corregir por el uso de materiales compuestos.

- El estabilizador se considera una estructura rígida estática.

- Los casos de carga considerados son los descritos en los apartados 3.2.6, 3.2.7 y 3.2.8.

- El cajón de torsión soporta momentos flectores, momentos torsores y fuerzas cortantes.

- El cajón se considera aproximadamente rectangular, de altura $h_{t}$ y longitud $b_{t}$, siendo la altura el espesor máximo del perfil en cada sección y la longitud la distancia entre los largueros. Además, se compondrá de dos cordones situados en las posiciones del larguero anterior y posterior respectivamente; y el panel se formará por una chapa rigidizada por larguerillos de sección en $Z$ de esbeltez 0,3(Farrar 1949) (ver Figura 3-7).

- Los largueros se formarán por dos angulares aprisionando una chapa, que son precisamente los cordones del cajón de torsión. A su vez, la chapa está rigidizada en la dirección de la envergadura mediante una serie de angulares llamados montantes, situados cada 0,5 metros, que corresponde aproximadamente con un montante por cada tramo de larguero entre costillas (hipótesis basada en el estado del arte).

- El área de los cordones se considerará pequeña en comparación con el área del panel de cada sección. Además, se diseñará todo el panel para que soporte el flujo cortante máximo de entre los que se alcancen justo pasado el cordón anterior y justo antes del posterior, para ser conservativos.

- El panel de extradós se diseñará para soportar las cargas $N$ y $q$ a factor de carga último y se seleccionará el caso crítico de entre los casos de carga contemplados. 
- Se considerará que el área del panel de intradós es igual a la del extradós, por ser conservativos.

- El peso de las costillas se estima mediante regresiones estadísticas en primera aproximación.

- El resto de los elementos no ideales se consideran como correcciones al peso anterior.

- La estructura secundaria se determina mediante regresiones estadísticas.

Con este modelo de estructura, se recuerda que un momento flector $M_{f}$ genera una carga de compresión por unidad de longitud $N$ igual a $M_{f} / h_{t} b_{t}$, que un momento torsor $M_{\text {Tor }}$ genera un flujo cortante $q_{T}$ igual a $M_{\text {Tor }} / 2 h_{t} b_{t}$, que junto con el que se alcanza por la fuerza cortante tras el larguero es el flujo cortante de diseño $q$ y será igual a $\left|q_{T}\right|+$ $\left|F_{c} / 2 h_{t}\right|$.

De esta forma, los cambios que se producen con respecto a la metodología presentada en (Torenbeek 2013) para alas afectan principalmente a la forma de dimensionar el cajón de torsión. Además, ahora sí se tienen en cuenta los momentos torsores y se diseñará el panel de tal forma que soporten un flujo cortante $q_{\text {caso }}$ y una carga de compresión por unidad de longitud $N_{\text {caso }}$.

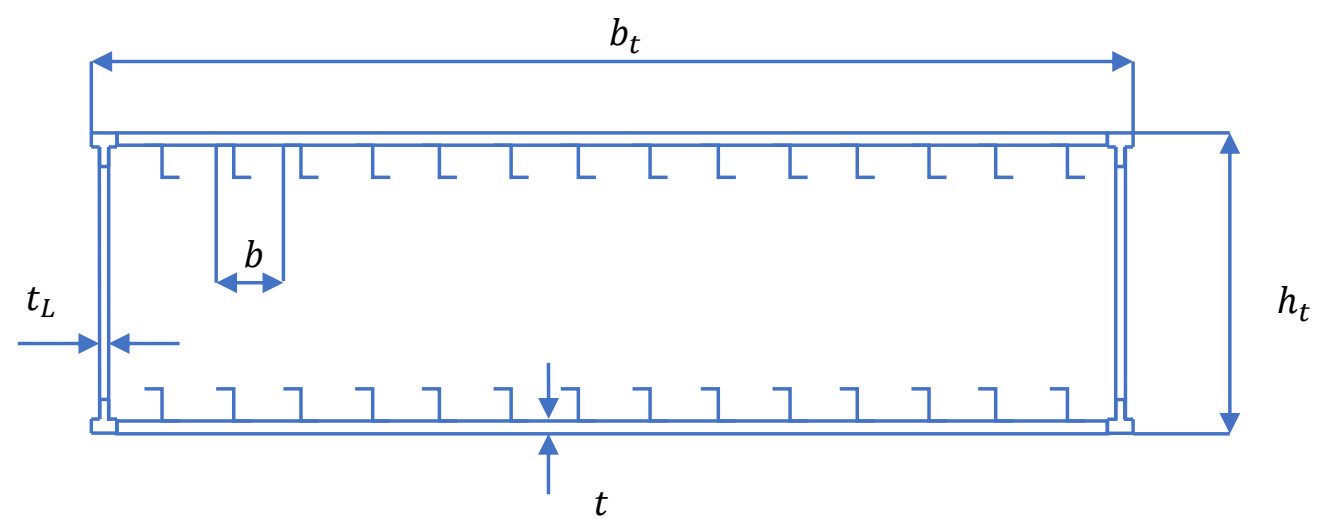

Figura 3-7 Dimensiones del cajón de torsión. Fuente: elaboración propia.

\subsubsection{Dimensionado de un panel del extradós}

El dimensionado de un panel de longitud $L$, con rigidizadores en $Z$, metálico de aleación de aluminio y sometido a una carga de compresión por unidad de longitud $N_{\max }$, es un problema ya resuelto, a nivel de diseño conceptual, en la referencia (Farrar 1949). El método propuesto por Farrar consiste en diseñar un panel rigidizado minimizando su peso. Se asumirá que el panel tiene un número de larguerillos suficientemente alto como para que el esfuerzo de inestabilidad sea igual al que tuviera un panel infinitamente ancho, lo cual se puede garantizar habiendo unos tres o cuatro larguerillos. Se supone también que las costillas proporcionan apoyos simples. Un panel rigidizado de este tipo podrá fallar de muchas formas. Un caso podría ser que empezase a pandear la chapa que se encuentra entre los larguerillos. También podría ocurrir que el alma o las alas de los larguerillos se deformasen y se saliesen de su plano o incluso que el larguerillo rotase entero como sólido rígido, provocando el fallo del panel. Por otro lado, el panel podría fallar globalmente, es decir, que pandease la estructura completa de chapa y larguerillos. Este último fallo se considera el pandeo global del panel como columna. El resto de los anteriores se consideran pandeos locales de la estructura, ya que afectan a una parte del panel. Además de estos fallos, en panel podría fallar porque pandease la chapa que se encuentra entre remaches o que la posición de la fila de remaches 
induzca otros modos de fallos. Se supondrá que estos fallos que dependen de los remaches no se producirán ya que son evitables si los remaches se colocan de forma adecuada. La metodología propuesta por Farrar consiste en dimensionar el panel para que el inicio del pandeo local se produzca al mismo tiempo que el pandeo global de la estructura, porque es la que resulta en un mínimo peso (Farrar 1949). El pandeo como columna se determina a partir del esfuerzo crítico de Euler para una viga de sección como la de la Figura 3-8, formada por una chapa, que compone el revestimiento, y un larguerillo de sección en Z. A su vez, el esfuerzo de Euler, que es el crítico en vigas esbeltas, viene dado por la expresión siguiente (Niu 1988), siendo $L$ la longitud del panel en la dirección de la envergadura e $I$ el momento de inercia de la sección:

$$
\sigma_{\text {Euler }}=\frac{\pi^{2} E I}{L_{c}^{2}}
$$

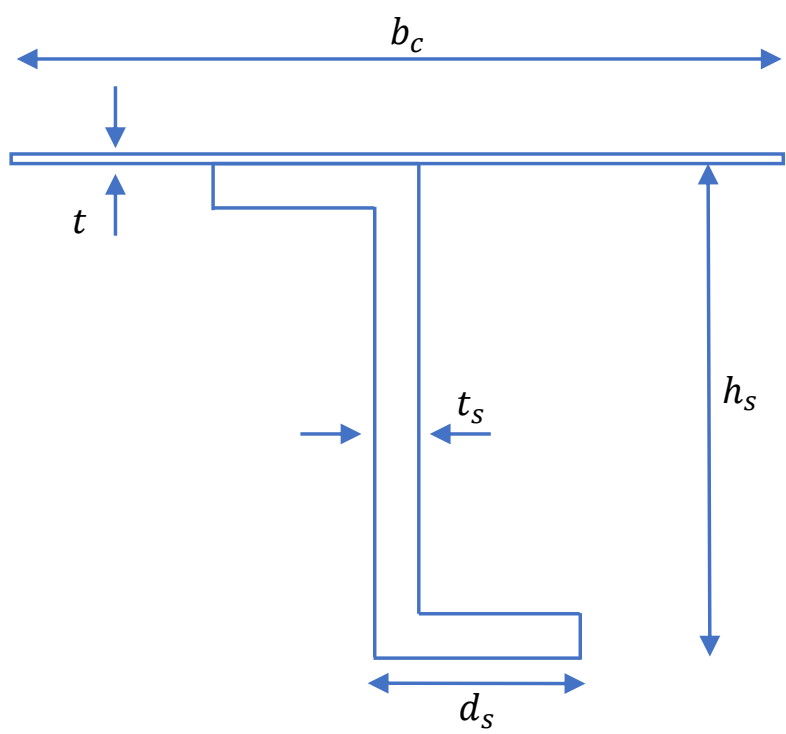

Figura 3-8 Sección de un elemento del panel de extradós y definición de los parámetros geométricos. Fuente: elaboración propia.

Por otra parte, el pandeo local es una mezcla de los que se han expuesto anteriormente, ya que en general no se pueden dar de forma simultánea dependiendo de la geometría del laerguerillo. Sin embargo, se determinó teóricamente el inicio de pandeo local para una amplia combinación de chapa y larguerillos (Argyris 1947). Los resultados para larguerillos en $Z$ de esbeltez 0,3 se muestran en la Figura 3-9. Aquí se representa el esfuerzo de pandeo local, $f_{b}$, adimensionalizado con un esfuerzo de referencia, $f_{0}$, que toma el valor $3,62 E\left(t / b_{c}\right)^{2}$, siguiendo la nomenclatura de la Figura 3-8, frente al área del larguerillo, $A_{s}$, dividida por el área de la chapa, $b t$, para diversos valores del cociente entre el espesor del larguerillo y de la chapa. Las partes superiores de las curvas corresponden al fallo de la chapa que se encuentra entre los larguerillos y las partes inferiores corresponden al fallo local del larguerillo. El quiebro de las curvas es justo donde se inician a la vez ambos tipos de pandeo local. 


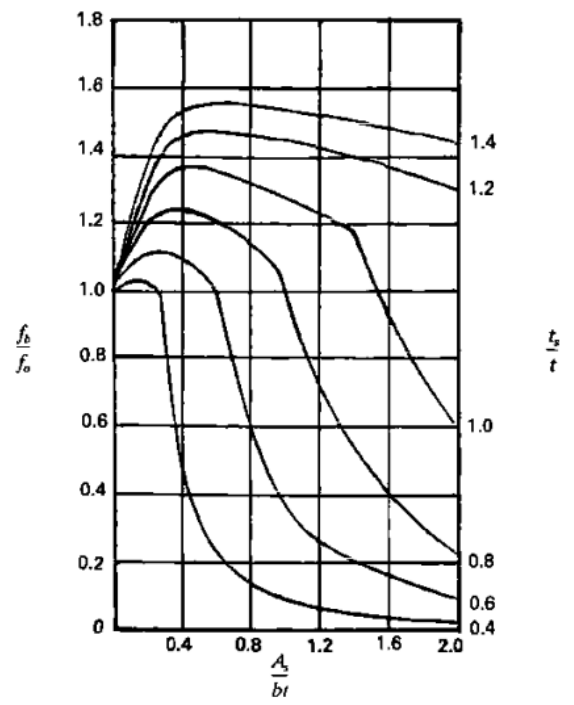

Figura 3-9 Sección de un elemento del panel de extradós. Fuente: (Farrar 1949).

Imponiendo que el pandeo global y el local se producen al mismo tiempo, es posible extraer unas curvas de nivel a factor de Farrar constante, definiéndose este como el cociente entre el esfuerzo medio soportado por el panel $N_{\max } b_{c} /\left(A_{s}+b t\right)$ y un esfuerzo de referencia igual a $\left(N_{\max } E / L_{c}\right)^{0,5}$. Maximizar el factor de Farrar implica maximizar el esfuerzo medio soportado, lo cual minimiza el área de la sección y en consecuencia el peso. Si se construyen unas curvas siguiendo la línea de gradiente mínimo de esas curvas de nivel se alcanzan las curvas representadas en la Figura 3-10. A la vista de las curvas, el factor de Farrar máximo toma el valor 0,95. Este máximo usualmente lleva a un panel con un número de larguerillos demasiado elevado comparado con los aviones semejantes, por lo que en la práctica se alcanzan valores del factor de Farrar en torno a 0,88 , como una solución de compromiso entre el peso ideal y cuestiones de fabricabilidad. Una vez seleccionado el factor de Farrar, el resto del panel queda dimensionado según indiquen las curvas de la Figura 3-10.

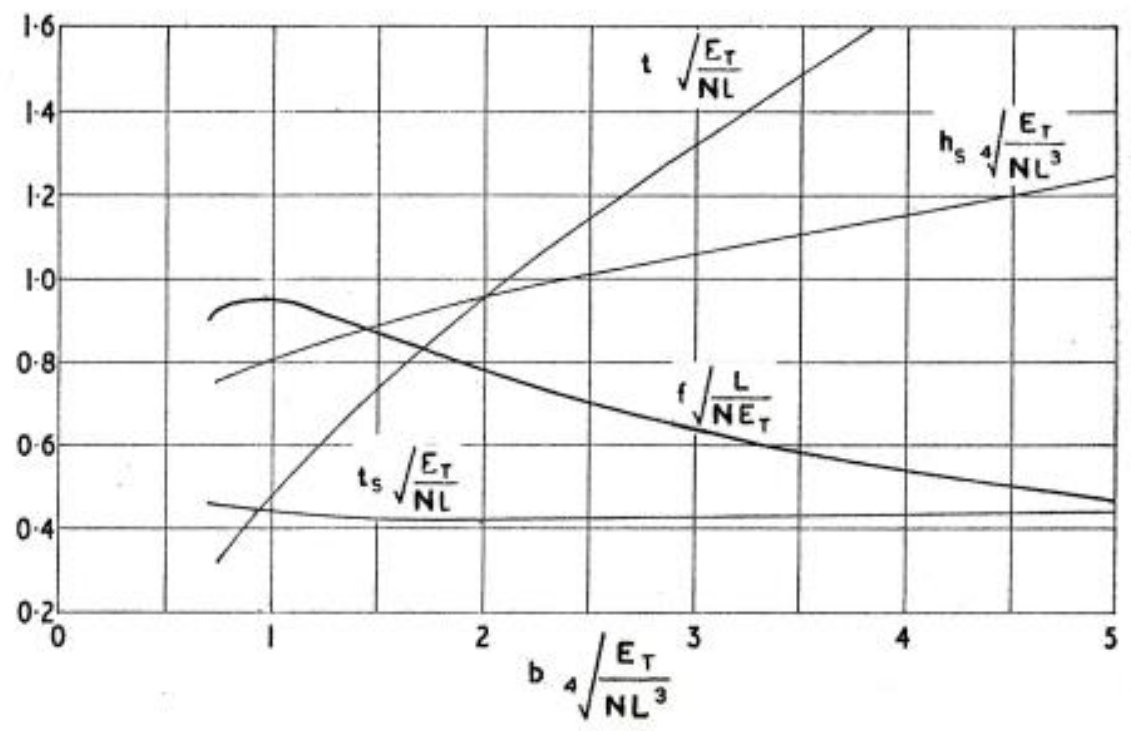

Figura 3-10 Curvas de diseño para secciones de larguerillos en Z para distintos valores del factor de Farrar. Fuente: (Farrar 1949).

Hasta ahora solamente se ha tenido en cuenta la carga a compresión $N_{\max }$ que debe soportar el panel. Para esfuerzos combinados entre compresión y cortadura para 
paneles simplemente apoyados se determinará la capacidad de soportar esos esfuerzos mediante la elipse de cargas, ya que se demostró experimentalmente que los casos de carga forman una elipse como se indica a continuación (Niu 1988):

$$
\left(\frac{q}{q_{\max }}\right)^{2}+\left(\frac{N}{N_{\max }}\right)^{2}=1
$$

En la ecuación anterior, el flujo cortante máximo que puede soportar el panel en ausencia de compresión es $q_{\max }$, y $N_{\max }$ es la carga de compresión por unidad de longitud máxima que puede soportar el panel en ausencia de cortadura, que es para la cual se ha diseñado el panel de Farrar anteriormente. Por tanto, dado un caso de carga $N_{\text {caso }}$ y $q_{\text {caso }}$, el panel podrá soportarlo si el punto no se encuentra fuera de la elipse, y sí lo hará si se encuentra en el interior o sobre ella. Para determinar el flujo cortante máximo que puede soportar el panel en ausencia de compresión, se debe tener en cuenta que en estos paneles no se está permitiendo el pandeo antes del fallo del mismo, por lo que este flujo cortante será el mínimo entre el admisible y el crítico de pandeo, es decir:

$$
q_{\max }=t \min \left(F_{S U}, \tau_{c r}\right)
$$

El esfuerzo crítico de pandeo de una chapa que mide $h x d$ se puede determinar mediante la siguiente expresión (Kuhn 1956):

$$
\tau_{\text {cr,elastico }}=k_{s S} E\left(\frac{t}{d_{c}}\right)^{2}\left[R_{h}+\frac{1}{2}\left(R_{d}-R_{h}\right)\left(\frac{d_{c}}{h_{c}}\right)^{3}\right]
$$

donde $k_{s s}$ es un factor que se extrae de la Figura 3-11, donde también vienen definidos $d_{c}$ y $h_{c}$, y los coeficientes $R_{h}$ y $R_{d}$ se obtienen de la Figura 3-11, en función del tipo de apoyo que tenga la chapa en el lado que mide $h$ y el que mide $d$, respectivamente.
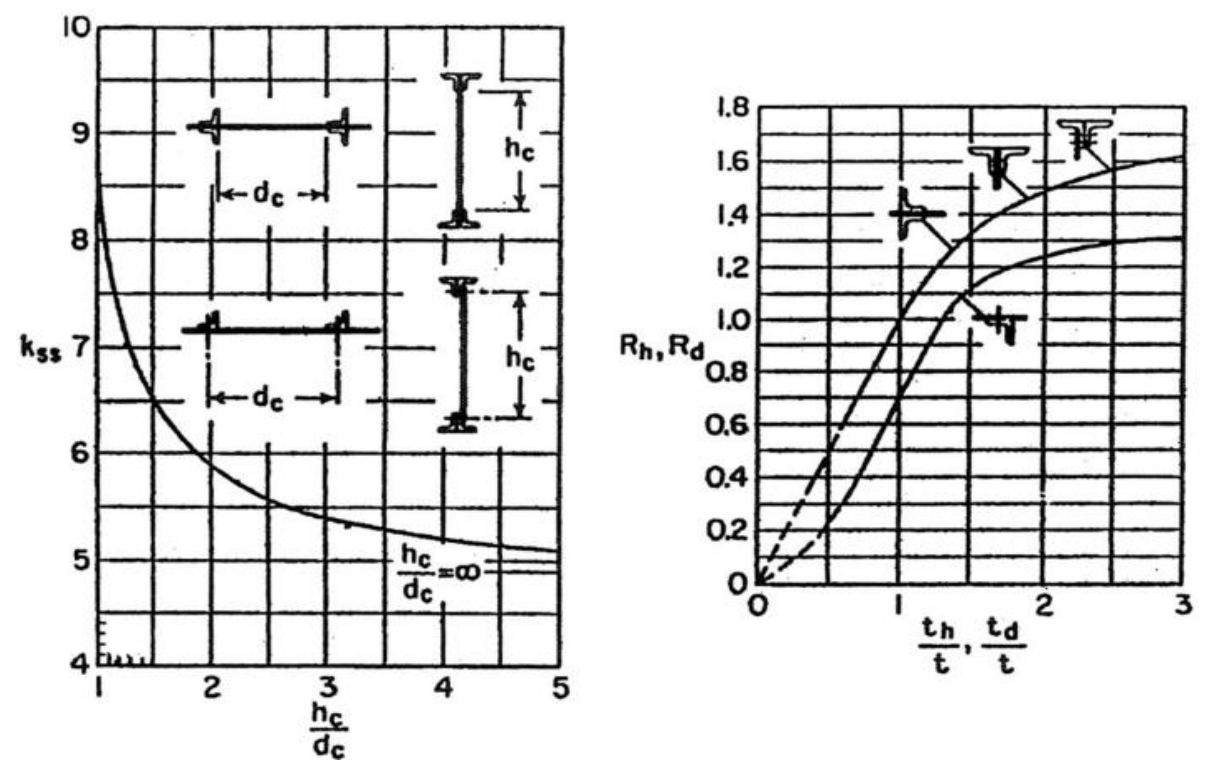

Figura 3-11 Curvas para determinar los factores $k_{s s}, R_{h}$ y $R_{d}$ para estimar el esfuerzo crítico de pandeo elástico. Fuente: (Kuhn 1956).

El esfuerzo crítico de pandeo de una chapa sometida a cortadura presentado anteriormente parte de la hipótesis de que el material tiene un comportamiento elástico. 
Para tener en cuenta efectos plásticos se puede corregir el resultado anterior según la Figura 3-12.

En el caso del panel del extradós, el lado de la chapa que corresponde a $h$ es el que mide $L$, que se apoya en los larguerillos, y el que concierne a $d$ es el que mide $b$, que se apoya en las costillas. Ambos apoyos se consideran apoyos simples que no aprisionan la chapa por los dos lados, por lo que la curva que se debe utilizar para determinar los coeficientes $R_{h}$ y $R_{d}$ es la inferior de las dos que aparecen en el gráfico. Por otra parte, se considerará que si el cociente entre $L$ y $b$ es mayor de cinco, el valor de $k_{S S}$ es de 4,9 .

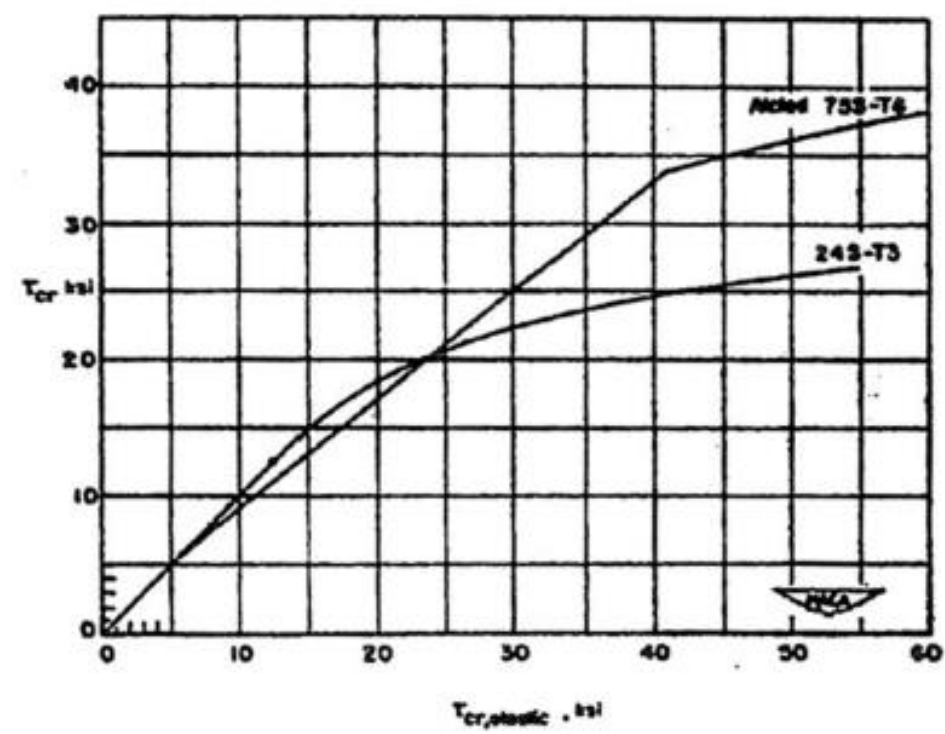

Figura 3-12 Corrección por plasticidad del esfuerzo crítico de pandeo a cortadura de una chapa para las aleaciones de aluminio indicadas en la gráfica. Fuente: (Kuhn 1956).

En la práctica, se considerarán diversos casos de carga que darán lugar a diferentes combinaciones de flujo cortante y carga de compresión por unidad de longitud. Estos casos se pueden representar en un espacio de coordenadas $(N, q)$. Los puntos que aquí se incluyen deben estar incrementados en el margen de seguridad establecido. El diseño del panel trata de determinar la elipse que pase por el punto que haga que todos los demás casos queden dentro o sobre la elipse. Este punto recibe el nombre de caso de carga crítico. Para ello, primeramente se determina el cociente entre el flujo cortante máximo en ausencia de compresión y la carga de compresión máxima por unidad de longitud en ausencia de cortante, haciendo uso de la expresión [ 3-33 ] y la definición del factor de Farrar, imponiendo un determinado valor del factor de Farrar. Seguidamente, se puede hallar el valor de $q_{\max }$ y $N_{\max }$ entrando en la ecuación de la elipse con este cociente y el valor del caso de carga crítico. Una vez determinada la carga de compresión máxima por unidad de longitud en ausencia de cortadura, se procede a dimensionar el panel siguiendo las curvas de la Figura 3-10.

\subsubsection{Dimensionado del alma del larguero}

Como se ha indicado en las hipótesis de partida, los largueros están formados por cuatro angulares que cada dos aprisionan dos extremos de una chapa, que a su vez está rigidizada en la dirección de la envergadura cada cierta distancia por otros dos angulares que aprisionan la chapa llamados montantes (ver Figura 3-13). El espesor del alma del larguero $t_{L}$, es decir, el de la chapa aprisionada, se puede determinar asumiendo que se encuentra sometida a un estado de tensión que se puede dividir entre un estado de 
tensión diagonal pura y un estado de cortadura pura (Kuhn, Peterson y Levin 1952). Se define un factor $k$ que determina la proporción de tensión diagonal que hay en relación al estado de cargas completo y, en consecuencia, el estado de cortadura pura ocurre en una proporción de $1-k$ (Figura 3-14).

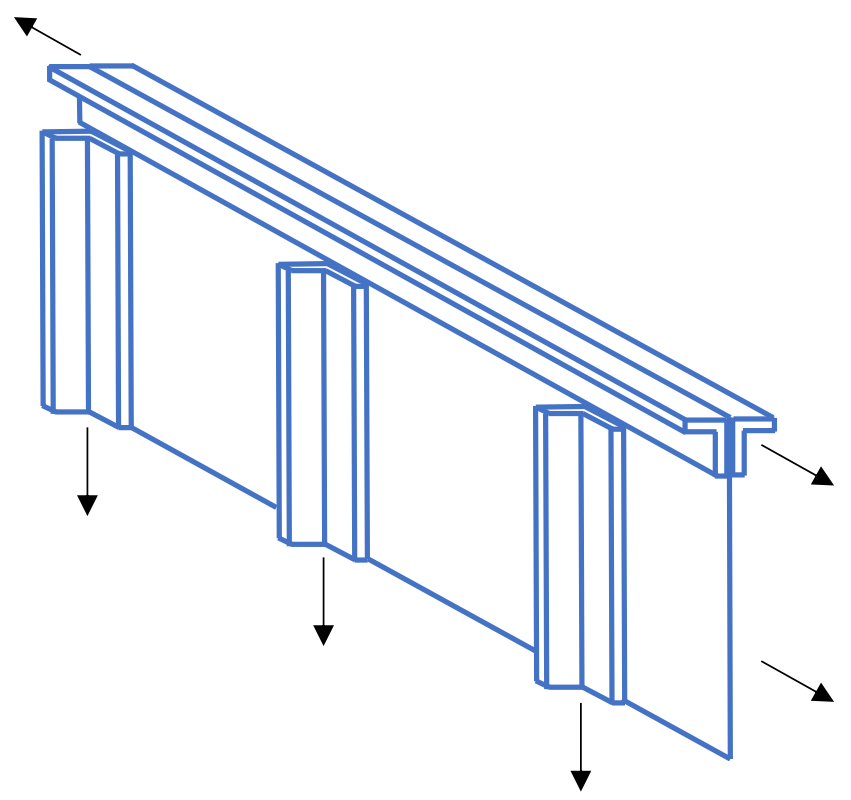

Figura 3-13 Esquema de la geometría de una sección que incluye el cordón superior de un larguero, extendiéndose según la dirección de las flechas. Fuente: elaboración propia.
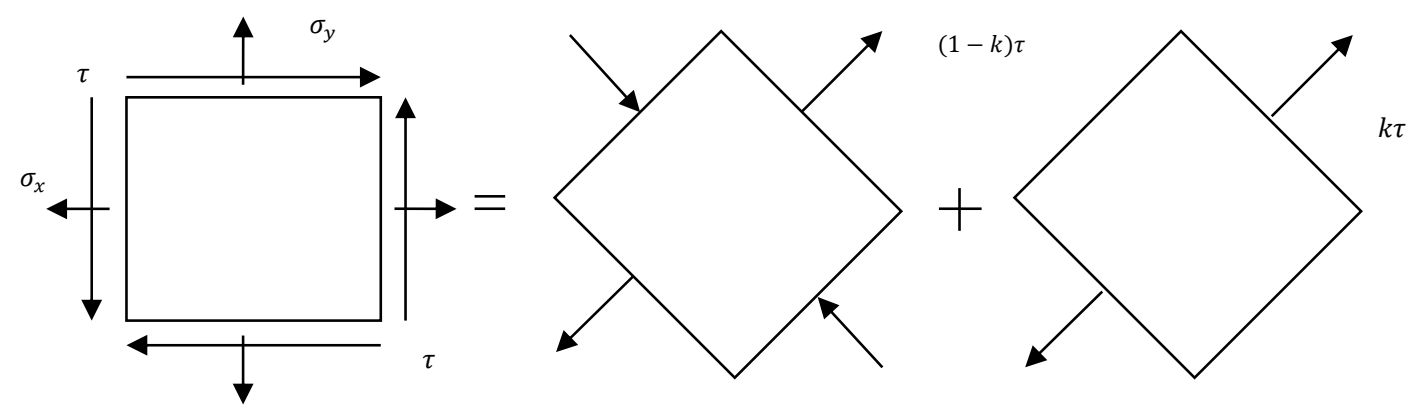

Figura 3-14 Estado de cortadura al que está sometido el alma del larguero dividido entre un estado de cortadura pura y un estado de tensión diagonal pura, con un factor de tensión diagonal $k$. Fuente: elaboración propia.

El flujo de cortadura que dimensiona el alma del larguero es el que corresponde al caso de carga crítico de la elipse de cargas. A partir de este flujo, se puede determinar el esfuerzo de cortadura $\tau$ que será el flujo cortante dividido por el espesor del alma del larguero. Hay que tener en cuenta que las cargas para las que hay que dimensionar son las cargas últimas, normalmente incrementadas en un cierto margen de seguridad, por ejemplo, el $5 \%$. Por otro lado, este esfuerzo debe ser igual al admisible, que se puede determinar para el estado de tensión descrito anteriormente mediante el criterio de Von Misses, alcanzándose la siguiente expresión: 


$$
\tau_{\text {admisible }}=\frac{F_{S U}}{\sqrt{1+k^{2}\left(\frac{4}{3 \sin ^{2} 2 \alpha_{d}}-1\right)}}
$$

Los parámetros $k$ y $\alpha$, siendo este último el ángulo de tensión diagonal, dependen de los espesores del alma, cordones y montantes de una cierta forma determinada por la teoría de Kuhn. El factor de tensión diagonal se determina de la siguiente forma:

$$
k=\tanh \left(0,5 \log \left(\frac{\tau_{\text {admisible }}}{\tau_{c r}}\right)\right)
$$

El esfuerzo crítico del denominador corresponde con el de una chapa sometido a cortadura, ecuación [ 3-33 ]. Para poder estimarlo es necesario suponer a priori una relación entre los espesores de los angulares y de la chapa, ya que la geometría de los angulares no está determinada. Este cociente se tomará como 1,5 en primera aproximación. El ángulo de tensión diagonal, siguiendo la misma teoría, se llega a que se puede estimar como sigue:

$$
\tan ^{4} \alpha_{d}=\frac{1+\frac{h t}{2 A_{L}}}{1+\frac{d t}{A_{M}}}
$$

En la expresión anterior, $A_{L}$ es el área de un cordón y $A_{M}$ el área de la sección de un montante. Puesto que las áreas de los cordones no son conocidas, en primera aproximación se puede considerar que el ángulo de tensión diagonal es de $45^{\circ}$. De esta forma, queda un sistema de ecuaciones para determinar el factor de tensión diagonal y el espesor del alma del larguero, que hay que resolver numéricamente.

\subsubsection{Peso del cajón de torsión}

El procedimiento anteriormente descrito dimensiona el alma de los largueros y el panel de extradós para una sección determinada de la envergadura del ala. Sin más que considerando la distribución de momentos y fuerzas a lo largo de la envergadura, se podrá realizar este procedimiento en varias secciones del ala. Asumiendo que el panel del intradós tiene la misma área que la del extradós e integrando las áreas totales a lo largo de la envergadura, se determinará el volumen total del cajón de torsión del ala; que multiplicado por la densidad del material utilizado se obtendrá su peso.

\subsubsection{Peso de las costillas}

Las costillas dan forma al perfil del estabilizador y sirven de apoyo a los paneles, además de transmitir las cargas sobre el extradós y el intradós a los largueros. Incluso algunas costillas reciben cargas adicionales causadas por las superficies de control de vuelo 0 transmiten las cargas al fuselaje. Como es lógico, el peso de las costillas depende de estas funciones que se han descrito, entre otras. Por tanto, solamente unas cuantas de ellas se pueden considerar como típicas, pudiendo estimar su peso. Incluso si se pudiera estimar con precisión el peso de una costilla, sigue existiendo el problema de determinar cuántas de ellas componen la totalidad de la superficie para poder estimar el peso final de las mismas. Se podría intentar optimizar la posición de estas para minimizar el peso del cajón de torsión, pero en la práctica se encuentran muchas otras restricciones que obligan a colocar costillas en ciertas posiciones, como por ejemplo la instalación de las superficies de control. Así que es más realista tomar un valor medio de paso entre 
costillas, que suele variar entre 0,5 y 1 metro aproximadamente (Torenbeek 2013). En esta misma referencia se puede encontrar una regresión que permite estimar el peso de las costillas, inicialmente obtenida para alas que se puede adaptar ajustando una serie de coeficientes para las superficies de cola:

$$
W_{\text {rib }}=k_{\text {rib }} \rho g S\left(t_{\text {ref }}+\frac{t_{r}+t_{t}}{2}\right)
$$

Las hipótesis básicas que se han seguido para alcanzar esta expresión son las siguientes:

- El peso por unidad de longitud del área de las costillas es constante a lo largo de la envergadura.

- Para un paso entre costillas constante, el número de costillas es proporcional a la envergadura. El peso de una costilla es proporcional entonces al producto de la cuerda media de la superficie, al espesor de una sección media y a la envergadura, es decir, es proporcional al volumen de la superficie estabilizadora.

- Si el paso entre costillas es proporcional a espesor medio de la superficie, el número de costillas por unidad de longitud es inversamente proporcional al mismo. El peso de la costilla es entonces proporcional a la cuerda media multiplicado por la envergadura, es decir, al área de la superficie.

Asumiendo que habrá costillas tanto como las descritas en el segundo punto como las del tercero y que es necesario ajustar los parámetros $k_{\text {rib }}$ y $t_{r e f}$, se sugiere la expresión [ 3-37 ] anteriormente mostrada. Se recomiendan unos valores típicos, para un ala, de los parámetros $k_{\text {rib }}=0,5 \times 10^{-3}$ y $t_{r e f}=1 \mathrm{~m}$. Para el caso de superficies de cola se ha decidido tomar los valores $k_{\text {rib }}=0,3 \times 10^{-3}$ y $t_{\text {ref }}=0,45 \mathrm{~m}$, que son ligeramente inferiores debido a que las cargas que deben soportar los estabilizadores son menores y el tamaño de los mismos también es menor. Estos valores se ajustaron para el caso de referencia del CSR-01.

\subsubsection{Peso de las estructuras secundarias y misceláneas}

A pesar de que las estructuras secundarias pueden contribuir en un porcentaje considerable al peso de la superficie, la mayoría de los métodos publicados tratan estas contribuciones de forma superficial debido a la dificultad de estimarlos. En la referencia (Torenbeek 2013) se incluyen regresiones estadísticas para estimar el peso de estos elementos, pero obtenidas para alas. De nuevo, se han adaptado estas regresiones para que se puedan aplicar a los estabilizadores de cola.

Comenzando por el peso de las estructuras de borde de ataque fijo, corresponden a la estructura que se instala por delante del cajón de torsión para dar forma al borde de ataque cuando no hay dispositivos ahí instalados. Estos paneles no suelen estar diseñados para soportar ninguna de las cargas generadas por los momentos flector y torsor ni la fuerza cortante. La carga principal de diseño es la presión aerodinámica sobre la superficie. La expresión propuesta para el peso específico por unidad de superficie es la siguiente:

$$
\Omega_{f l e}=3,15 k_{f l e} \Omega_{r e f}\left(\frac{q_{D}}{q_{\text {ref }}}\right)^{0,25}\left(\frac{b M T O W}{\cos \Lambda_{0,25} W_{\text {ref }} b_{\text {ref }}}\right)^{0,145}
$$




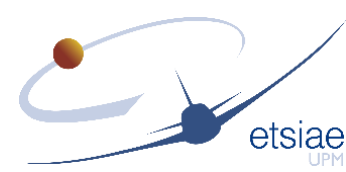

En esta ecuación hay una serie de coeficientes constantes que toman los siguientes valores: $k_{\text {fle }}=1, \Omega_{\text {ref }}=56 \mathrm{~N} / \mathrm{m}^{2}, q_{\text {ref }}=30 \mathrm{kN} / \mathrm{m}^{2}, W_{\text {ref }}=10^{6} \mathrm{~N}$ y $b_{\text {ref }}=50 \mathrm{~m}$. La presión dinámica viene representada por $q_{D}$.

De la misma manera es posible determinar el peso específico por unidad de superficie de la estructura de borde de salida fija, que corresponde a la que se encuentra tras el cajón de torsión cuando no se consideran los controles de vuelo de borde de salida. Se encargan de sujetar los dispositivos de borde de salida, así como marcar los ejes de charnela. La expresión que permite estimar el peso es:

$$
\Omega_{f t e}=2,6 \Omega_{\text {ref }}\left(\frac{\text { bMTOW }}{\cos \Lambda_{0,25} W_{\text {ref }} b_{\text {ref }}}\right)^{0,0544}
$$

Los valores de los parámetros de referencia son los mismos que para la estructura de borde de ataque fijo.

En cuanto a los dispositivos de control de vuelo, su peso es más difícil de determinar, ya que la estructura depende de las cargas que soporta el control, del tamaño del mismo, de la configuración y del actuador utilizados. En etapas de diseño conceptual, buena parte de esta información no es conocida. Por tanto, el método propuesto puede arrastrar errores de hasta el $20 \%$. Se puede mejorar la precisión si se dispone de información de controles que soporten cargas similares y tengan dimensiones análogas a las que se están diseñando. La regresión que se propone en la referencia es la siguiente:

$$
\Omega_{\text {tef }}=1,7 k_{\text {sup }} k_{\text {slot }} \Omega_{\text {ref }}\left(1+\left(\frac{\text { MTOW }}{W_{\text {ref }}}\right)^{0,35}\right)
$$

El parámetro $k_{\text {sup }}$ depende de la sujeción del eje de movimiento. Para dispositivos de un solo eje con soportes simples se toma el valor 1 . Por otra parte, el parámetro $k_{\text {slot }}$ cambia su valor dependiendo del número de ranuras que tenga el dispositivo. Para el dispositivo simplemente ranurado toma el valor 1 . El resto de los parámetros constantes de la ecuación son iguales a los descritos anteriormente.

Para tener en cuenta ciertos elementos que se han considerado dentro de la categoría miscelánea se propone incrementar el peso de la estructura secundaria en un $10 \%$. Es un factor corrector indicado en la referencia de donde se han obtenido todas las regresiones, que, si se dispusiera de más información, puede ser calibrado para mejorar el ajuste de los resultados.

Por último, se puede corregir el peso de la estructura por el debido a ciertas uniones entre los elementos, refuerzos de costillas y otros elementos similares; y también por el debido a los remaches y a otras restricciones de fabricación. En cuanto al peso de las sujeciones, se puede determinar como sigue:

$$
W_{\text {attach }}=3 \times 10^{-4} n_{u l t} \text { MTOW } \frac{S_{t}}{S_{w}}
$$

Puesto que este incremento de peso estaba determinado para un ala, se ha decidido que el peso de estos elementos para las superficies de cola será proporcional a la superficie, de ahí que aparezca el cociente entre superficies al final de la ecuación. Por 
otra parte, el peso debido a remaches y a restricciones de fabricación se puede estimar mediante la expresión siguiente, donde el factor $\delta_{i d}$ típicamente toma el valor $10^{-3} \mathrm{~m}$ :

$$
W_{\text {joints }}=1,2 \rho g S_{\text {tail }} \delta_{\text {id }}
$$

\subsubsection{Peso de la superficie de cola}

El peso total de la superficie de cola se obtendrá sumando todas las contribuciones determinadas hasta ahora. Hay que tener en cuenta que los estabilizadores están fabricados en un porcentaje mayor que en las alas de material compuesto. Por eso, en los elementos que parten de regresiones estadísticas, es decir, las costillas y las estructuras secundarias, se aplican factores reductores. El principal dilema surge en el peso del cajón de torsión, ya que la metodología desarrollada está basada en que la estructura está fabricada en aleaciones de aluminio. En primera aproximación, el peso del cajón de torsión se va a corregir por un factor que corresponde al cambio de densidad entre el aluminio y el material compuesto. En el apartado siguiente se presentará una de las contribuciones novedosas de esta tesis que es una metodología de diseño de paneles específica para materiales compuestos y comprobar hasta qué punto esta hipótesis es fiable o no. Por tanto, con estas hipótesis, el peso total de la superficie de cola se determina de la siguiente manera:

$$
\begin{gathered}
W_{\text {tail }}=W_{\text {box }}+W_{\text {rib }}+1,1\left(\Omega_{f l e} S_{\text {fle }} C_{f l e}+\Omega_{f t e} S_{f t e} C_{f t e}+\Omega_{t e f} S_{\text {tef }} C_{\text {tef }}\right) \\
+W_{\text {attach }}+W_{\text {joints }}
\end{gathered}
$$

Los coeficientes de corrección utilizados para contemplar la fabricación en material compuesto son los siguientes: $C_{f l e}=0,80, C_{f t e}=0,80$ y $C_{t e f}=0,85$. Para determinar la superficie que abarcan cada uno de los elementos de la estructura secundaria, se ha relacionado con la superficie total de la cola, aproximando que el borde de ataque fijo supone un $4 \%$ del total, el borde de salida fijo un $3 \%$ y los controles de vuelo un $25 \%$.

Como se puede intuir, la obtención de este peso es un procedimiento iterativo, ya que las cargas trasmitidas al cajón de torsión dependen del propio peso de la estructura. Es decir, que los momentos flector y torsor y la fuerza cortante que llega a cada sección depende de las cargas aerodinámicas y de las de inercia. Por tanto, es necesario dar una condición inicial al peso de la estructura e iterar hasta que el proceso converja. Además, para determinar las cargas en cada una de las secciones de la envergadura, hay que suponer cómo se distribuye la carga generada por el peso de la estructura a lo largo del ala. Se seguirá la hipótesis de que esta distribución es proporcional a la cuerda elevado a 1,2, hipótesis habitual en diseño conceptual. Habrá que determinar el factor de proporcionalidad sin más que ajustando que la integral del peso a lo largo de la envergadura es el peso total de la cola. Por tanto, cabe destacar que este factor va variando en cada paso de iteración del proceso de convergencia.

\subsubsection{Material compuesto}

En el apartado anterior se ha expuesto un procedimiento de diseño de un cajón de torsión de una superficie de cola en material metálico. Sin embargo, en la actualidad, las superficies de cola de los aviones de transporte aéreo comercial están construidas principalmente de material compuesto. Es por ello que es necesario dar un paso más en el modelo estructural y desarrollar un procedimiento de diseño del cajón de torsión con material compuesto. 


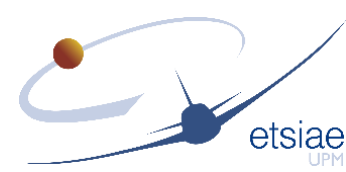

En esta sección se expone el modelo de material compuesto que se ha seguido para el diseño de colas no convencionales. En este caso únicamente se va a considerar un panel del extradós, con el objetivo de comprobar si los ahorros contemplados en el apartado anterior debido al uso de materiales compuestos en lugar de aleaciones de aluminio se corresponden con los que se obtendrían si se diseña ese panel directamente en material compuesto. Las cargas que soporta este panel de extradós se pueden reducir a una combinación de compresión y cortadura. Para poder modelar el comportamiento del panel es necesario suponer una composición determinada de cada uno de ellos, es decir, la selección de una fibra, una matriz y un apilado de dichas láminas. Una vez conocido todo esto, el comportamiento mecánico se va a determinar mediante la Teoría de Laminado Clásica y los diferentes criterios clásicos de fallo y de resistencia máxima (Kassapoglou 2013). Con ello, el dimensionado del panel consistirá en determinar el tamaño de las alas y del alma de los larguerillos, el número de ellos y el espesor de las láminas de material compuesto que se deben utilizar de tal forma que soporte las cargas consideradas con el mínimo área posible.

\subsubsection{Teoría de Laminado Clásica}

Las propiedades de un laminado compuesto por láminas de espesor delgado comparado con las otras dimensiones de la estructura se pueden asumir como un estado de tensión plana. Esto conlleva que los esfuerzos fuera de su propio plano son aproximadamente nulos. Además, suponiendo que el material es ortotrópico, como es el caso de una lámina aislada, la matriz de rigidez es la siguiente:

$$
\left(\begin{array}{c}
\sigma_{x} \\
\sigma_{y} \\
\tau_{x y}
\end{array}\right)=\left[\begin{array}{ccc}
Q_{x x} & Q_{x y} & 0 \\
Q_{x y} & Q_{y y} & 0 \\
0 & 0 & Q_{s s}
\end{array}\right]\left(\begin{array}{c}
\epsilon_{x} \\
\epsilon_{y} \\
\gamma_{x y}
\end{array}\right)
$$

Es posible determinar los valores de la matriz de rigidez en función de las propiedades del laminado, en concreto del módulo de Young a lo largo de las fibras, es decir, en dirección longitudinal $\left(E_{L}\right)$, del módulo de Young en dirección transversal a las fibras $\left(E_{T}\right)$, el módulo de cortadura $\left(G_{L T}\right)$ y los coeficientes de Poisson $\left(v_{L T}\right.$ y $\left.v_{T L}\right)$.

$$
\begin{gathered}
Q_{x x}=\frac{E_{L}}{1-v_{L T} v_{T L}} \\
Q_{y y}=\frac{E_{T}}{1-v_{L T} v_{T L}} \\
Q_{x y}=\frac{v_{L T} E_{T}}{1-v_{L T} v_{T L}}=\frac{v_{T L} E_{L}}{1-v_{L T} v_{T L}} \\
Q_{S S}=G_{L T}
\end{gathered}
$$

Ahora se puede determinar la matriz de rigidez para un laminado compuesto por láminas de cualquier orientación:

$$
\begin{gathered}
Q_{11}(\theta)=m^{4} Q_{x x}+n^{4} Q_{y y}+2 m^{2} n^{2} Q_{x y}+4 m^{2} n^{2} Q_{s s} \\
Q_{22}(\theta)=n^{4} Q_{x x}+m^{4} Q_{y y}+2 m^{2} n^{2} Q_{x y}+4 m^{2} n^{2} Q_{s s} \\
Q_{12}(\theta)=m^{2} n^{2} Q_{x x}+m^{2} n^{2} Q_{y y}+\left(m^{4}+n^{4}\right) Q_{x y}-4 m^{2} n^{2} Q_{s s} \\
Q_{66}(\theta)=m^{2} n^{2} Q_{x x}+m^{2} n^{2} Q_{y y}-2 m^{2} n^{2} Q_{x y}+\left(m^{2}-n^{2}\right)^{2} Q_{s s}
\end{gathered}
$$




$$
\begin{aligned}
& Q_{16}(\theta)=m^{3} n Q_{x x}-m n^{3} Q_{y y}+\left(m n^{3}-m^{3} n\right) Q_{x y}+2\left(m n^{3}-m^{3} n\right) Q_{s s} \\
& Q_{26}(\theta)=m n^{3} Q_{x x}-m^{3} n Q_{y y}+\left(m^{3} n-m n^{3}\right) Q_{x y}+2\left(m^{3} n-m n^{3}\right) Q_{s s}
\end{aligned}
$$

Donde $m=\cos \theta$ y $n=\sin \theta$. Para obtener las fuerzas resultantes sólo hay que integrar la ecuación [ 3-44 ] a lo largo del espesor del laminado. Ya que la rigidez es constante en cada lámina, aunque no tiene porqué ser igual en cada lámina, la integración puede sustituirse por un sumatorio respecto de $z$ :

$$
\begin{aligned}
\left(\begin{array}{c}
N_{x} \\
N_{y} \\
N_{x y}
\end{array}\right) & =\left[\begin{array}{lll}
A_{11} & A_{12} & A_{16} \\
A_{12} & A_{22} & A_{26} \\
A_{16} & A_{26} & A_{66}
\end{array}\right]\left(\begin{array}{c}
\epsilon_{x} \\
\epsilon_{y} \\
\gamma_{x y}
\end{array}\right) \\
A_{i j} & =\Sigma_{k=1}^{n} Q_{i j}\left(z_{k}-z_{k-1}\right)
\end{aligned}
$$

Donde $i, j=1,2,6$ y el sumatorio se hace sobre todas las láminas $(n)$ siguiendo el esquema de la Figura 3-15.

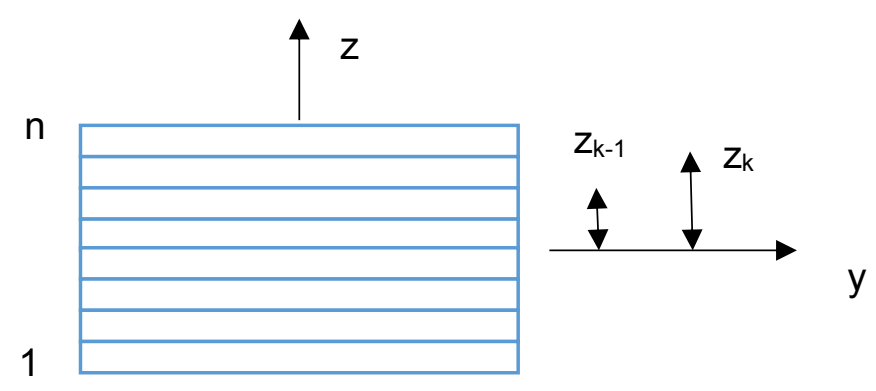

Figura 3-15 Numeración de láminas dentro del laminado. Fuente: elaboración propia.

Para un laminado sometido a momento flector, se contemplan las hipótesis de Kirchoff en la teoría de placas: secciones planas permanecen planas y perpendiculares al eje neutro. Considerando el desplazamiento fuera del propio plano del laminado como $w$, las curvaturas se definen:

$$
\begin{gathered}
\kappa_{x}=-\frac{\partial^{2} w}{\partial x^{2}} \\
\kappa_{y}=-\frac{\partial^{2} w}{\partial y^{2}} \\
\kappa_{x y}=-2 \frac{\partial^{2} w}{\partial x \partial y}
\end{gathered}
$$

suponiendo que, en una situación de momento flector puro con pequeñas deformaciones, éstas son proporcionales a las curvaturas y varían linealmente a lo largo del espesor del laminado. Con estas hipótesis se llega a las ecuaciones constitutivas para este estado de carga:

$$
\begin{aligned}
\left(\begin{array}{c}
M_{x} \\
M_{y} \\
M_{x y}
\end{array}\right) & =\left[\begin{array}{lll}
D_{11} & D_{12} & D_{16} \\
D_{12} & D_{22} & D_{26} \\
D_{16} & D_{26} & D_{66}
\end{array}\right]\left(\begin{array}{c}
\kappa_{x} \\
\kappa_{y} \\
\kappa_{x y}
\end{array}\right) \\
D_{i j} & =\sum_{k=1}^{n} \frac{Q_{i j}}{3}\left(z_{k}^{3}-z_{k-1}^{3}\right)
\end{aligned}
$$


Donde $i, j=1,2,6$ y el sumatorio se hace sobre todas las láminas $(n)$ siguiendo el esquema de la Figura 3-15.

Las ecuaciones [ 3-55 ] y [ 3-61 ] representan las cargas en situaciones de deformaciones como membrana pura en el primer caso y deformaciones del laminado por momento flector puro en el segundo caso. En una situación desacoplada, las deformaciones en el plano sólo se pueden causar por cargas en el mismo plano y las curvaturas sólo por momentos flectores. En el caso de un caso de cargas acoplado, las deformaciones se expresan según las siguientes ecuaciones:

$$
\begin{gathered}
\epsilon_{x}=\epsilon_{x 0}+z \kappa_{x} \\
\epsilon_{y}=\epsilon_{y 0}+z \kappa_{y} \\
\gamma_{x y}=\gamma_{x y 0}+z \kappa_{x y}
\end{gathered}
$$

Por lo que las ecuaciones constitutivas generalizadas son:

$$
\begin{gathered}
\left(\begin{array}{c}
N_{x} \\
N_{y} \\
N_{x y} \\
M_{x} \\
M_{y} \\
M_{x y}
\end{array}\right)=\left[\begin{array}{llllll}
A_{11} & A_{12} & A_{16} & B_{11} & B_{12} & B_{16} \\
A_{12} & A_{22} & A_{26} & B_{12} & B_{22} & B_{26} \\
A_{16} & A_{26} & A_{66} & B_{16} & B_{26} & B_{66} \\
B_{11} & B_{12} & B_{16} & D_{11} & D_{12} & D_{16} \\
B_{12} & B_{22} & B_{26} & D_{12} & D_{22} & D_{26} \\
B_{16} & B_{26} & B_{66} & D_{16} & D_{26} & D_{66}
\end{array}\right]\left(\begin{array}{c}
\epsilon_{x} \\
\epsilon_{y} \\
\gamma_{x y} \\
\kappa_{x} \\
\kappa_{y} \\
\kappa_{x y}
\end{array}\right) \\
B_{i j}=\sum_{k=1}^{n} \frac{Q_{i j}}{2}\left(z_{k}^{2}-z_{k-1}^{2}\right)
\end{gathered}
$$

Donde $i, j=1,2,6$ y el sumatorio se hace sobre todas las láminas $(n)$ siguiendo el esquema de la Figura 3-15. Estos coeficientes se anulan si el laminado es simétrico.

Para el caso que se va a tratar en este estudio, se va a asumir que los laminados están compuestos por láminas delgadas que suponen un estado de tensión plana. Además, solamente se va a trabajar con laminados simétricos compuestos por láminas de un mismo espesor $h$. Por tanto, las ecuaciones constitutivas quedan de la siguiente forma:

$$
\left(\begin{array}{c}
N_{x} \\
N_{y} \\
N_{x y} \\
M_{x} \\
M_{y} \\
M_{x y}
\end{array}\right)=\left[\begin{array}{rrrrrl}
A_{11} & A_{12} & A_{16} & 0 & 0 & 0 \\
A_{12} & A_{22} & A_{26} & 0 & 0 & 0 \\
A_{16} & A_{26} & A_{66} & 0 & 0 & 0 \\
0 & 0 & 0 & D_{11} & D_{12} & D_{16} \\
0 & 0 & 0 & D_{12} & D_{22} & D_{26} \\
0 & 0 & 0 & D_{16} & D_{26} & D_{66}
\end{array}\right]\left(\begin{array}{c}
\epsilon_{x} \\
\epsilon_{y} \\
\gamma_{x y} \\
\kappa_{x} \\
\kappa_{y} \\
\kappa_{x y}
\end{array}\right)
$$

Así, se pueden simplificar las ecuaciones [ 3-56 ] y [ 3-61 ] como se muestra a continuación:

$$
\begin{gathered}
A_{i j}=h \Sigma_{k-1}^{n} Q_{i j} \\
D_{i j}=h^{3} \sum_{k-1}^{n} \frac{Q_{i j}}{3}\left(\left(k-\frac{n}{2}\right)^{3}-\left(k-1-\frac{n}{2}\right)^{3}\right)
\end{gathered}
$$

Por lo que se pueden definir las siguientes matrices de pseudo-rigidez:

$$
\overline{A_{\imath \jmath}}=\Sigma_{k-1}^{n} Q_{i j}
$$




$$
\overline{D_{l j}}=\sum_{k-1}^{n} \frac{Q_{i j}}{3}\left(\left(k-\frac{n}{2}\right)^{3}-\left(k-1-\frac{n}{2}\right)^{3}\right)
$$

Las hipótesis con las que se han alcanzado estas ecuaciones son aplicables a todos los laminados con los que se van a construir tanto el revestimiento como los larguerillos, pero cada uno con una orientación diferente decidida a priori. A pesar de que el laminado presenta todas sus láminas del mismo espesor, pueden ser diferentes entre los larguerillos y el revestimiento. Para diferenciarlos, se llamará $h_{L}$ al espesor de las láminas que forman el revestimiento y $h_{L L}$ al de las láminas del larguerillo.

\subsubsection{Comportamiento de un elemento rigidizador}

Los elementos rigidizadores usados en los revestimientos de extradós e intradós del cajón de torsión reciben el nombre de larguerillos, tal y como se ha comentado previamente. Estos pueden tener una gran variedad de geometrías de sección transversal (Figura 3-16).
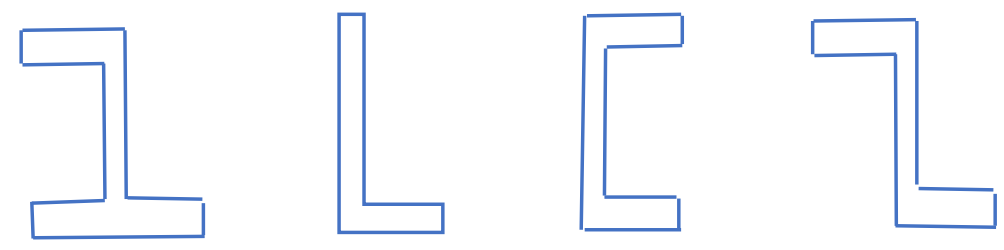

Figura 3-16 Secciones típicas de larguerillos. De izquierda a derecha: sección en J, en L, en C y en Z. Fuente: elaboración propia.

Para determinar el comportamiento que tienen estos elementos frente a cargas, es necesario hacer uso de algunas definiciones. Puesto que el larguerillo que se va a modelar es el que tiene sección en Z, la Figura 3-17 muestra la nomenclatura que se va a necesitar para los cálculos posteriores. El motivo por el que se va a modelar este larguerillo es para permitir la comparación con los procedimientos habituales para material metálico, en este caso el método de Farrar, los cuales están aplicados a larguerillos con esta sección en concreto.

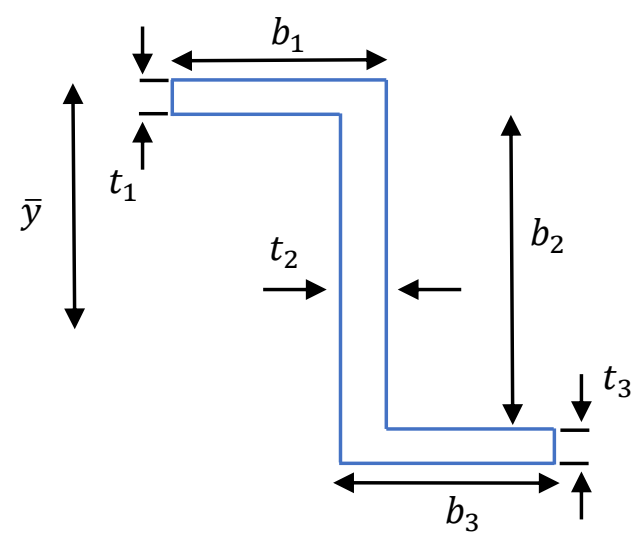

Figura 3-17 Esquema de un larguerillo en Z, donde $\bar{y}$ es la posición del eje neutro. Fuente: elaboración propia.

Las rigideces a carga axial y a pandeo de un elemento rigidizador son muy utilizadas en diseño conceptual y en el análisis de estructuras. Existen algunas diferencias 


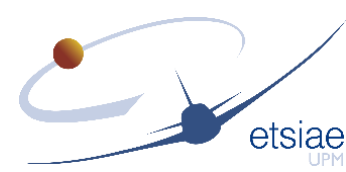

importantes con respecto a los larguerillos fabricados en material metálico, ya que cada uno de los elementos que lo componen, alma y alas, pueden tener diferentes laminados y en consecuencia diferentes rigideces. Estas diferencias se evidencian aún más al determinar la posición del eje neutro:

$$
\bar{y}=\frac{\Sigma(E A y)_{i}}{\Sigma(E A)_{i}}
$$

Hay que reseñar que $E$ puede ser tanto el módulo a cargas axiales como a momentos flectores, y en general serán diferentes. Por tanto, hay que separar el problema axial del de momentos flectores, ya que en estos casos se utilizará el parámetro $E_{b} I$ en lugar del $E A$. Para determinar la rigidez a cargas axiales se asumirá que los laminados son simétricos y balanceados (Kassapoglou 2013). De esta forma se llega a que el módulo de cada uno de los elementos que componen el rigidizador es:

$$
E_{i}=\frac{1}{t_{i}}\left(A_{11}-\frac{A_{12}^{2}}{A_{22}}\right)
$$

$\mathrm{Y}$, por tanto, la rigidez de cada uno de los elementos es la siguiente:

$$
(E A)_{i}=E_{i} b_{i} t_{i}
$$

Con esto se puede determinar la rigidez axial que presenta el larguerillo completo, asumiendo que, si se somete a una carga axial pura, la carga por unidad de longitud se reparte de acuerdo con la rigidez axial de cada uno de los elementos. Así se puede definir una rigidez axial equivalente del rigidizador completo como la suma de las rigideces de cada uno de los elementos:

$$
(E A)_{e q}=\Sigma_{i}(E A)_{i}
$$

Se puede dar un paso más si se aplican las hipótesis de que los laminados tienen todos los mismos espesores de lámina, que los larguerillos tienen la misma esbeltez que los de Farrar $\left(h_{s}=d_{s} / 0,3\right.$ en la Figura 3-8) y que los espesores son pequeños comparados con las longitudes transversales. Con esto se puede definir la rigidez equivalente como sigue:

$$
(E A)_{e q}=\Sigma_{i=1}^{3}(E A)_{i}=d_{s} h_{L L}\left(E_{1} n_{L 1}+\frac{E_{2} n_{L 2}}{0,3}+E_{3} n_{L 3}\right)=d_{s} h_{L L} \overline{E A_{e q}}
$$

En la ecuación anterior, $n_{L i}$ es el número de láminas que componen el elemento $i$. Como se puede ver, el parámetro $\overline{E A_{e q}}$, llamado de pseudo-rigidez, únicamente depende del número de láminas que componen los laminados del rigidizador y de la orientación de las mismas, es decir, es independiente de la geometría del larguerillo en Z. Por tanto, se puede determinar su valor sin haber diseñado el larguerillo todavía.

Este mismo procedimiento se puede seguir para determinar la rigidez equivalente para momentos flectores. Cada uno de los elementos del larguerillo contribuyen a esta rigidez de la siguiente forma:

$$
\left(E_{b} I\right)_{i}=E_{b i}\left(\frac{\text { ancho }_{i} \times \text { alto }_{i}^{3}}{12}+A_{i} d_{i}^{2}\right)
$$


donde $d_{i}$ es la distancia del centro de gravedad de cada uno de los elementos al eje neutro, $A_{i}$ es el área y $E_{b i}$ se puede hallar como sigue:

$$
E_{b i}=\frac{12}{t_{i}^{3}\left(d_{11}\right)_{i}}
$$

El coeficiente $\left(d_{11}\right)_{i}$ es el elemento 11 de la inversa de la matriz $D$ para el elemento $i$. De la misma forma que antes, se puede definir una rigidez equivalente sin más que sumando las rigideces de cada uno de los elementos (Kassapoglou 2013):

$$
E_{b} I_{e q}=\Sigma_{i}\left(E_{b} A\right)_{i}
$$

De nuevo, aplicando las mismas hipótesis anteriores se llega a un parámetro de pseudorigidez que es independiente de la geometría del larguerillo:

$$
\begin{gathered}
\left(E_{b} I\right)_{e q}=\Sigma_{i=1}^{3}\left(E_{b} I\right)_{i} \cong d_{s}^{3} h_{L L}\left(\frac{E_{b 3} n_{L 3}}{0,3^{2}}+E_{b 2} n_{L 2}\left(\frac{1}{12 \times 0,3^{3}}+\frac{1}{0,3 \times 0,6^{2}}\right)\right) \\
=d_{s} h_{L L} \overline{E_{b} I_{e q}}
\end{gathered}
$$

\subsubsection{Pandeo global}

Primeramente, se va a analizar la carga crítica que inicia el pandeo del revestimiento sin elementos rigidizadores, es decir, sin larguerillos. La ecuación que gobierna el comportamiento de una placa rectangular sometida a una carga biaxial (Figura 3-18) es la siguiente:

$$
D_{11} \frac{\partial^{4} w}{\partial x^{4}}+2\left(D_{12}+2 D_{66}\right) \frac{\partial^{4} w}{\partial x^{2} \partial y^{2}}+D_{22} \frac{\partial^{4} w}{\partial y^{4}}=N_{x} \frac{\partial^{2} w}{\partial x^{2}}+N_{y} \frac{\partial^{2} w}{\partial y^{2}}
$$

Donde $w$ es el desplazamiento fuera del plano de la placa. Esta expresión supone que los términos de acoplamiento de momento flector y torsión $D_{16}$ y $D_{26}$ son despreciables comparados con los términos restantes $D_{11}, D_{12}, D_{22}$ y $D_{66}$. La placa se supone simplemente apoyada en todos los lados. Las cargas $N_{x}$ y $N_{y}$ se indican en la Figura 3-18.

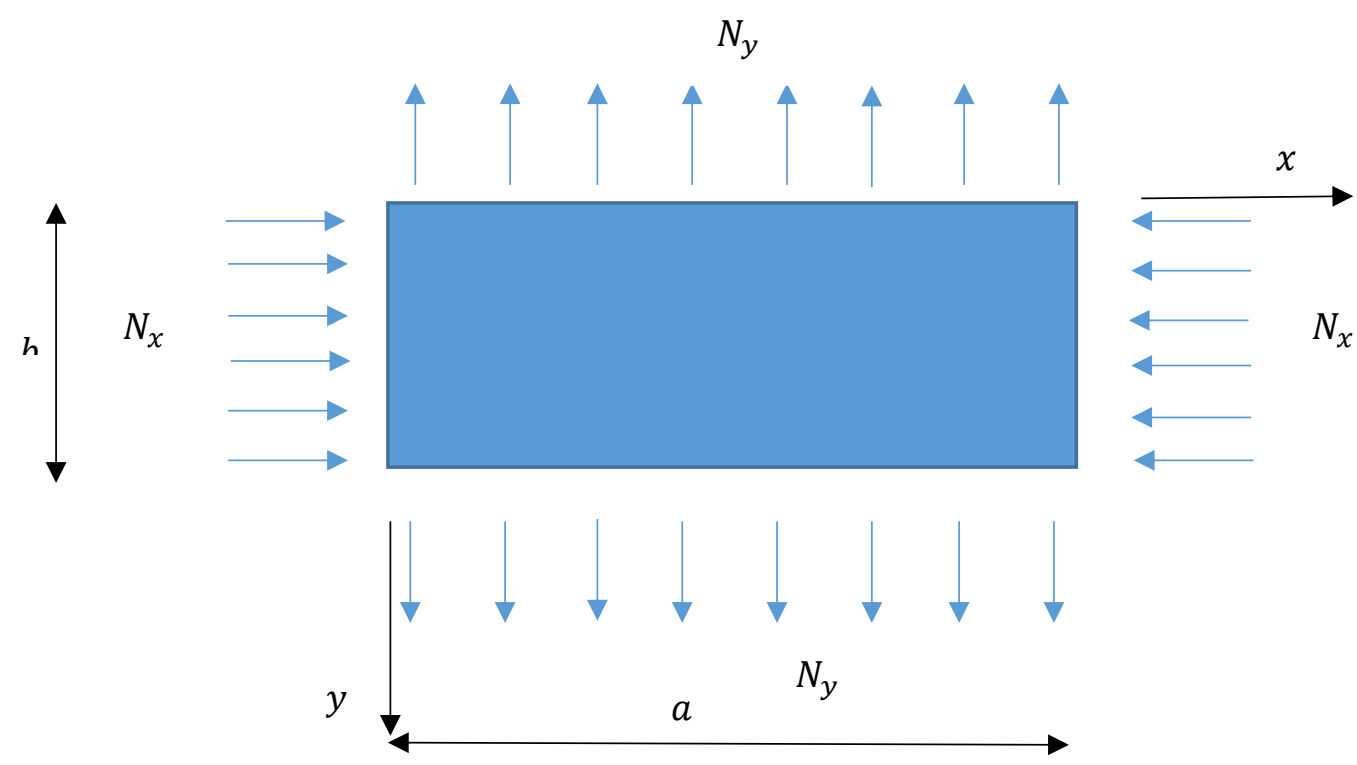

Figura 3-18 Chapa rectangular sometida a carga biaxial. Fuente: elaboración propia. 
De ahí, las condiciones de contorno son:

$$
\begin{aligned}
& w=M_{x}=-D_{11} \frac{\partial^{2} w}{\partial x^{2}}-D_{12} \frac{\partial^{2} w}{\partial y^{2}}=0 ; \text { en } x=0 \text { y } x=a \\
& w=M_{y}=-D_{12} \frac{\partial^{2} w}{\partial x^{2}}-D_{22} \frac{\partial^{2} w}{\partial y^{2}}=0 ; \text { en } y=0 \text { y } y=b
\end{aligned}
$$

La solución de la ecuación para la carga crítica de pandeo es la siguiente, sabiendo que $k=N_{y} / N_{x}$ :

$$
\begin{gathered}
N_{0}=\pi^{2}\left[D_{11} m^{4}+2\left(D_{12}+2 D_{66}\right) m^{2} n^{2}(A R)^{2}+D_{22} n^{4}(A R)^{4}\right] \\
/\left(a^{2}\left(m^{2}+k n^{2}(A R)^{2}\right)\right.
\end{gathered}
$$

La carga $N_{0}$ es función del número de semi-ondas $m$ en la dirección $x$ y $n$ en la dirección $y$. Cambios en los valores de $m$ y $n$ suponen diferentes modos de fallo de la placa. En el caso de que sólo haya carga de compresión $N_{x}$, la ecuación [ 3-84 ] cambia haciendo cero el valor de $k$. Además, minimizando en función del valor de $m$ y $n$ se obtendría la carga crítica que produce el pandeo. Para $n$ el valor que minimiza $N_{0}$ es 1 . Para el caso de $m$, el valor que minimiza $N_{0}$ es $m^{*}=\left(\frac{D_{22}}{D_{11}}\right)^{0,25} A R$. Sustituyendo ambos valores en la ecuación se determina la expresión de la carga crítica de pandeo a compresión.

Para dar un paso más en el procedimiento, se va a determinar la carga crítica de pandeo de una placa sometida a una carga de compresión combinada con cortadura. Este caso de cargas es el típico de una sección del extradós del cajón de torsión de un ala. La Figura 3-19 representa el esquema de cargas descrito. Se van a suponer que los lados de la placa están simplemente apoyados. La ecuación que rige el comportamiento de la placa es la siguiente:

$$
D_{11} \frac{\partial^{4} w}{\partial x^{4}}+2\left(D_{12}+2 D_{66}\right) \frac{\partial^{4} w}{\partial x^{2} \partial y^{2}}+D_{22} \frac{\partial^{4} w}{\partial y^{4}}=N_{x} \frac{\partial^{2} w}{\partial x^{2}}+2 N_{x y} \frac{\partial^{2} w}{\partial x \partial y}
$$

Las condiciones de contorno coinciden con el caso anterior, indicadas en las ecuaciones [ 3-82 ] y [ 3-83 ].

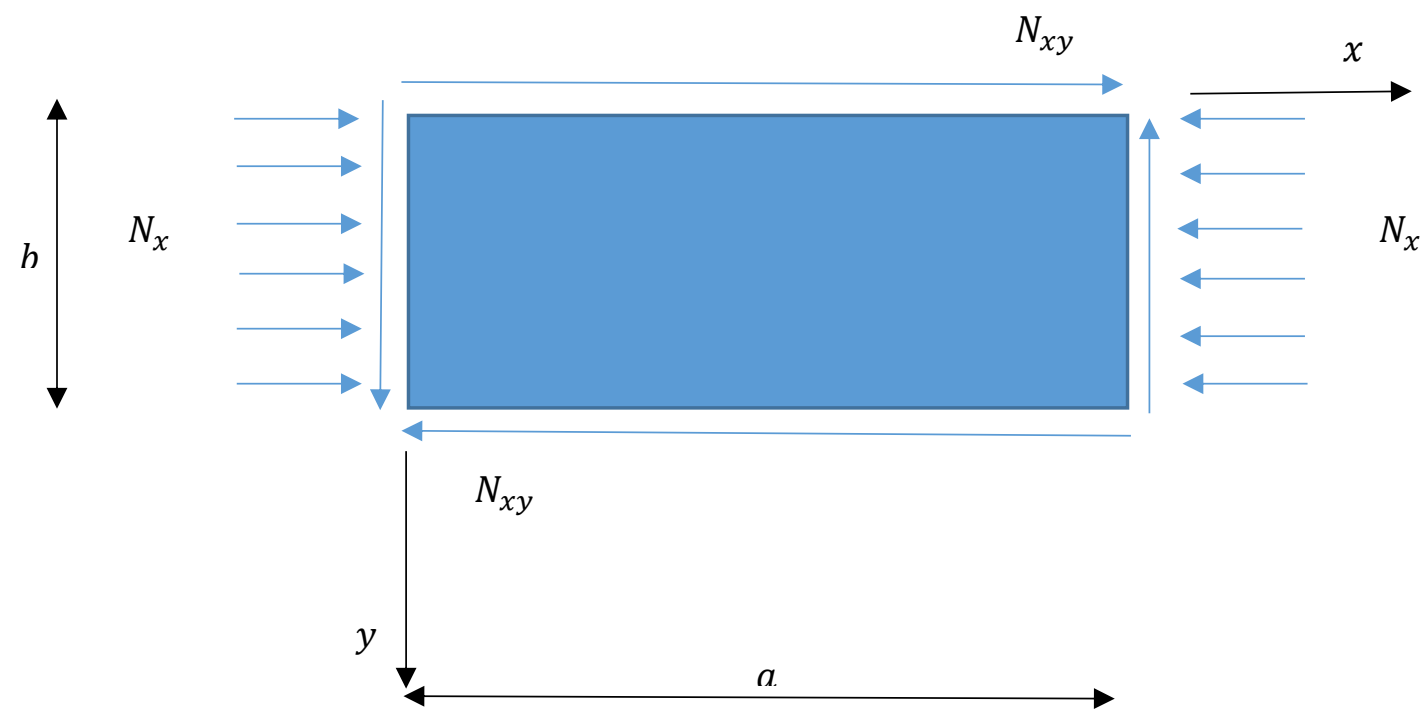

Figura 3-19 Chapa simplemente apoyada sometida a compresión y cortadura. Fuente: elaboración propia. 
Para resolver el problema se va a suponer un desplazamiento fuera del plano de la chapa de la forma:

$$
w=w_{1} \sin \left(\frac{\pi x}{a}\right) \sin \left(\frac{\pi y}{b}\right)+w_{2} \sin \left(\frac{2 \pi x}{a}\right) \sin \left(\frac{2 \pi y}{b}\right)
$$

Se hallará la solución del problema mediante el procedimiento de mínima energía (Kassapoglou 2013). Así se obtiene la carga crítica de pandeo, suponiendo que $k=$ $N_{x y} / N_{x}$, que tiene la siguiente expresión:

$$
N_{0}=\frac{\frac{\pi^{2}}{a^{2}}\left[D_{11}+2\left(D_{12}+2 D_{66}\right)(A R)^{2}+D_{22}(A R)^{4}\right]}{\left(2-\frac{8192}{81 \pi^{4}} k^{2}(A R)^{2}\right)}\left[5 \pm \sqrt{9+\frac{\frac{65536}{81} k^{2}}{\pi^{4}}(A R)^{2}}\right]
$$

El valor de la carga crítica de pandeo será el mínimo de los valores de la expresión anterior. Se puede analizar esta expresión dependiendo del valor de $k$. Si $k=0$ se tiene el caso de compresión pura $\left(N_{x c r i t}\right)$. En este caso, la expresión resultante es igual a la obtenida en [ 3-84 ] para compresión pura $(k=0)$ con $m=1$, es decir, para alargamientos grandes y/o para una relación entre $D_{22}$ y $D_{11}$ grande. Analizando un caso en concreto, las dos expresiones tienen la misma validez hasta un alargamiento de 1,5 aproximadamente.

Por otra parte, si consideramos valores de $k \rightarrow \infty$, es decir, compresión despreciable frente a cortadura, el valor de la carga crítica quedaría de la siguiente forma:

$$
N_{\text {xycrit }}= \pm \frac{9 \pi^{4} b}{32 a^{3}}\left(D_{11}+2\left(D_{12}+2 D_{66}\right)(A R)^{2}+D_{22}(A R)^{4}\right)
$$

Esta expresión es una aproximación a la carga crítica de pandeo de una placa rectangular sometida a cortadura. Típicamente, esta expresión es un 30\% mayor que la solución exacta. Para mejorar la precisión basta con suponer más términos en el desplazamiento en la ecuación [ 3-86 ]. Reteniendo términos hasta orden 3 tanto en el seno como en el coseno, se puede alcanzar la siguiente expresión (Kassapoglou 1997):

$$
N_{x y c r i t}=\min \left\{\begin{array}{c}
\frac{45 \pi^{4}}{32 A R a^{2}} \frac{D_{11}+2\left(D_{12}+2 D_{66}\right) A R^{2}+D_{22} A R^{4}}{\sqrt{\frac{706}{25}-C_{53} \frac{C_{45}+C_{34}}{C_{34 C_{45}}}}} \\
\frac{\pi^{4}}{128 A R a^{2}} \frac{\sqrt{E_{11} E_{22} E_{33} E_{44}}}{\sqrt{\frac{81}{625} E_{11} E_{22}+\frac{E_{11} E_{33}}{25}+\frac{E_{33} E_{44}}{81}+\frac{E_{22} E_{44}}{25}}}
\end{array}\right\}
$$

Los valores de los coeficientes que aparecen en la ecuación se incluyen en la misma referencia.

Para determinar la combinación de cargas críticas que producen el pandeo de la chapa se hace uso de la siguiente expresión:

$$
\frac{N_{x}}{N_{x c r i t}}+\left(\frac{N_{x y}}{N_{x y c r i t}}\right)^{2}=1
$$

Esta curva representa el límite de estabilidad de la placa. Si nuestro caso de carga se sitúa fuera de la parábola de cargas límite, se puede decir que la chapa está pandeada. 


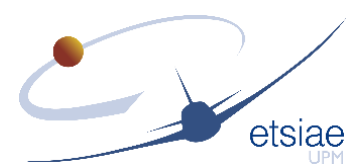

En caso contrario, si el caso de carga de encuentra en el interior de la curva, la placa permanece sin pandear.

En el caso de que el revestimiento esté rigidizado por larguerillos, hay que contemplar que los rigidizadores contribuyen a soportar las cargas. Para ello, no hay más que asumir que el larguerillo incrementa el valor del elemento 11 de la matriz $D$ en una cantidad igual a su rigidez equivalente frente a momentos flectores, es decir:

$$
D_{11}=D_{11 s k i n}+\frac{E_{b} I_{e q}}{b}
$$

De nuevo, considerando la hipótesis de que todas las láminas tienen el mismo espesor, que las del revestimiento tienen espesor $h_{L}$ y las del larguerillo $h_{L L}$, se pueden extraer los parámetros geométricos:

$$
D_{11}=h_{L}^{3}\left(\overline{D_{11}}+\frac{d_{s}^{3} h_{L L} \overline{E_{b} I_{e q}}}{h_{L}^{3} b}\right)
$$

\subsubsection{Pandeo local}

El pandeo local es una mezcla de diferentes modos de fallo, al igual que ocurría en el caso de estructuras metálicas. El comportamiento real debe determinarse experimentalmente, pero aquí se presenta un modelo teórico que corresponde a diferentes modos que se van a aplicar separadamente.

Antes de presentar el modelo, es necesario explicar cómo se reparte la carga entre los elementos de una sección. Las cargas de compresión son absorbidas tanto por los larguerillos como por el revestimiento, sin embargo, se va a trabajar con la hipótesis de que las cargas de cortadura son soportadas únicamente por el revestimiento. Esto significa que el conjunto revestimiento y larguerillos contribuyen a alcanzar la carga de pandeo a compresión, pero el revestimiento a su vez está sometido a una combinación de compresión y cortadura. Se puede definir un parámetro $\lambda$ que establezca la relación entre la compresión por unidad de longitud que absorbe el revestimiento en relación con la total de la sección (Kassapoglou 2013):

$$
\lambda=\frac{N_{x}}{N_{\text {xskin }}}=1+\frac{E A_{e q}}{A_{11} b}
$$

donde b es el ancho de un elemento de la sección según la

Figura 3-20.

El primer modo de pandeo local contemplado es el que produce el pandeo del revestimiento situado entre dos rigidizadores consecutivos, por lo que se puede modelizar como el pandeo global, pero tomando las dimensiones de esta placa. Por otra parte, el pandeo local de los rigidizadores se puede tomar como el pandeo como columna de una viga de sección en $Z$, sin tener en cuenta el revestimiento; es decir, se puede suponer que falla al esfuerzo crítico de Euler, considerando apoyos simples en los extremos (Kassapoglou 2013):

$$
P_{c r}=\frac{\pi^{2} E_{b} I}{L^{2}}
$$




\subsubsection{Fallo por crippling}

El esfuerzo de fallo por crippling para el alma y las alas de un rigidizador se obtiene como sigue (Kassapoglou 1997):

1. Para las alas:

$$
F_{\text {crip }}=\left\{\begin{array}{c}
F_{\text {cu }} ; \frac{b}{t}<2 \\
F_{c u} \frac{1,6753}{\left(\frac{b}{t}\right)^{0,7245}} ; \frac{b}{t} \geq 2
\end{array}\right.
$$

2. Para el alma:

$$
F_{\text {crip }}=\left\{\begin{array}{c}
F_{\text {cu }} ; \frac{b}{t}<8 \\
F_{c u} \frac{9,8307}{\left(\frac{b}{t}\right)^{1,099}} ; \frac{b}{t} \geq 8
\end{array}\right.
$$

En ambos casos se considera que $F_{c u}$ es el esfuerzo máximo a compresión para cada uno de los elementos, que se obtiene por el criterio de fallo de la primera lámina, siguiendo la teoría de fallo Tsai-Wu (Tsai y Wu 1971). Este criterio es el resultado de un intento matemático de generalizar el criterio de fallo de Tsai-Hill creando un ajuste de la curva basado en una teoría de tensores y teniendo en cuenta que los materiales compuestos tienen distintas resistencias a compresión y a tracción. Esto significa que este criterio no está completamente basado en fenómenos físicos, pero incluye ese ajuste. De hecho, uno de los coeficientes desconocidos en el criterio se obtiene imponiendo que si el material fuera isótropo se debe recuperar el criterio de Von Mises.

$$
\frac{\sigma_{x}^{2}}{X^{t} X^{c}}+\frac{\sigma_{y}^{2}}{Y^{t} Y^{c}}-\sqrt{\frac{1}{X^{t} X^{c}} \frac{1}{Y^{t} Y^{c}}} \sigma_{y} \sigma_{x}+\left(\frac{1}{X^{t}}-\frac{1}{X^{c}}\right) \sigma_{x}+\left(\frac{1}{Y^{t}}-\frac{1}{Y^{c}}\right) \sigma_{y}+\frac{\tau_{x y}^{2}}{S^{2}}=1
$$

A pesar de que no es un modelo basado plenamente en fenómenos físicos, se ha comprobado mediante ensayos que proporciona un ajuste bastante bueno, excepto para estado de cargas biaxiales a compresión. Las resistencias a tracción y compresión en ambas direcciones, y la resistencia a cortadura son datos de entrada a la ecuación y propiedades asociadas al laminado.

\subsubsection{Aplicación al diseño de un panel de extradós}

Se va a recordar la definición de los parámetros geométricos del panel del extradós para llevar a cabo de forma coherente los modos de fallo descritos anteriormente para un panel de extradós sometido a compresión y cortadura. En la

Figura 3-20 se muestras las definiciones más importantes. 


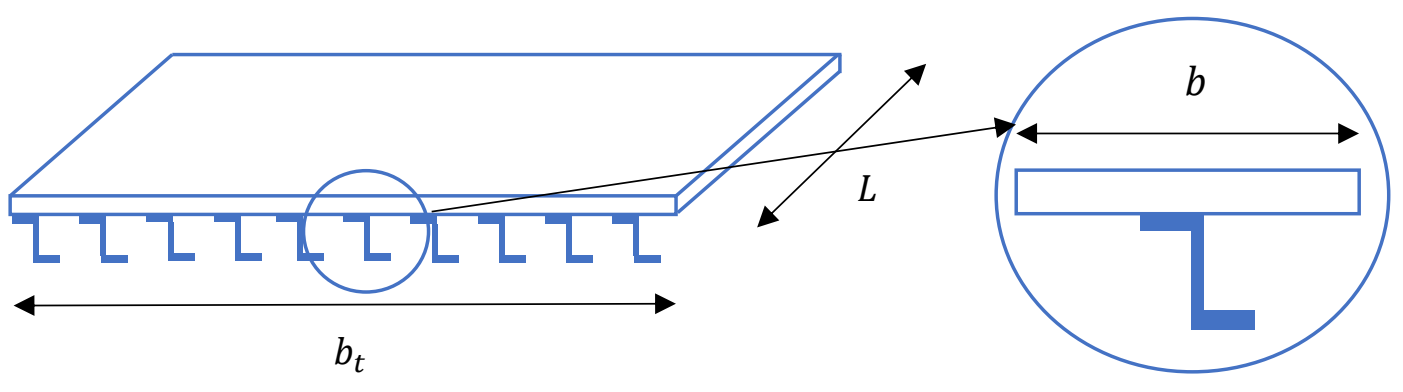

Figura 3-20 Esquema de un panel de extradós incluyendo definiciones de algunos parámetros geométricos. Fuente: elaboración propia.

El pandeo global del panel se va a considerar siguiendo la ecuación [ 3-90 ], asumiendo que si el caso de cargas se encuentra sobre la parábola es que la estructura falla. Las cargas por unidad de longitud críticas se modelarán siguiendo las ecuaciones [ 3-84 ] y [ 3-89 ] para los casos de compresión pura y cortadura pura, respectivamente. Para el caso de compresión pura, $k$ toma el valor $0, n$ el valor 1 y $m$ el valor óptimo $m^{*}$. Puesto que el objetivo último es compararse con la metodología de Farrar para materiales metálicos, se van a plantear las mismas hipótesis aplicadas al panel. Se va a suponer que el pandeo local y global del panel ocurren al mismo tiempo. Debido a que se han descrito dos modos de pandeo local, se utilizará el pandeo entre rigidizadores para imponer este criterio de diseño y se comprobará que el otro modo ocurre más tarde. Con este criterio quedan dos grados de libertad, que serán $\lambda$ y $\overline{A R}=L / b$, es decir, el porcentaje de carga a compresión que soportan los larguerillos y el número de ellos. Por tanto, el procedimiento de diseño sería el siguiente, dado un caso de carga en concreto y para un panel de ciertas dimensiones:

1. Primero se seleccionan las propiedades mecánicas de todas las láminas (tipo de fibra, porcentaje de la misma, etc.) y se deciden en qué orientación, número de láminas y orden se van a seguir para cada uno de los laminados.

2. Posteriormente se fijan los valores de los dos grados de libertad. Se debe indicar que $\lambda$ puede tomar valores a partir de 1 y que $\overline{A R}$ tiene que tomar valores múltiplos de $A R$ para que se obtenga un número de larguerillos natural.

3. Después de eso, de la condición de pandeo local del revestimiento entre larguerillos se puede determinar el espesor de las láminas del revestimiento $h_{L}$.

4. Una vez conocido el espesor de las láminas del revestimiento, de la condición de pandeo global se puede determinar el valor de $E_{b} I_{e q}$ necesario para soportar el caso de carga.

5. De la definición de $\lambda$ y $\overline{A R}$ se puede obtener la rigidez axial equivalente, $E A_{e q}$, necesaria.

6. Por otro lado, con los datos de los laminados de los larguerillos, se puede calcular los valores de las pseudo-rigideces $\overline{E A_{e q}}$ y $\overline{E_{b} I_{e q}}$.

7. Después, aplicando la relación entre las rigideces con dimensiones y las pseudoadimensionales, se puede determinar la medida de las alas de los larguerillos en Z:

$$
d_{s}=\sqrt{E_{b} I_{e q} / E A_{e q} \times \overline{E A_{e q}} / \overline{E_{b} I_{e q}}}
$$


8. Tras esto, se pueden obtener el resto de las variables, entre las que están el espesor de las láminas de los larguerillos y el área total de la sección.

9. Se vuelve al paso 2 para otros valores de los grados de libertad, con el objetivo de buscar qué combinación de parámetros resulta en un área mínima.

Para obtener resultados más reales, se han impuesto una serie de restricciones a la búsqueda:

- El tamaño de los larguerillos no debe ser tal que se exceda el paso entre ellos.

- El espesor de ningún laminado puede ser inferior a 0,381 mm, por temas de costes en la fabricación (Kassapoglou 1997).

- Los larguerillos no pueden fallar por crippling.

- Los larguerillos no pueden absorber más carga que la de fallo como columna para cada uno de ellos.

A continuación, se va a realizar un ejemplo de aplicación numérica del procedimiento de diseño anteriormente descrito. El diseño consiste en dimensionar un panel rigidizado con larguerillos sometido a una combinación de compresión y cortadura. Precisamente, los paneles del extradós de una superficie de cola están sometidos a este caso de cargas (Baker y Scott 2016). De nuevo, se seleccionará el caso de estudio partiendo de los datos de los que se disponen en la base de datos CeRAS para al avión CSR-01. Si embargo, hay un inconveniente puesto que la base de datos no recoge casos de carga de los estabilizadores de cola. Por tanto, se ha decidido adaptar los datos que se conocen sobre el ala principal para estimar un caso de carga para el estabilizador horizontal. Se ha seleccionado un panel situado al $40 \%$ de la semienvergadura del ala, que por sus dimensiones podría ser del estabilizador, y se han seleccionado las cargas que le corresponderían a esa sección, pero divididas en un factor de diez, ya que es un valor de relación típica entre las fuerzas del ala y del estabilizador. Por tanto, el ancho del panel mide 1,72 metros y la altura del cajón de torsión es de 0,42 metros, ya que se conocen las posiciones de los largueros. La única variable que se ha tenido que estimar es el paso entre costillas, que de la literatura abierta se ha fijado e 0,75 metros (Torenbeek 2013). Por último, el caso de carga que resulta de los datos incluidos en CeRAS, incrementados en un margen de seguridad del $10 \%$, es de $104 \mathrm{kN} / \mathrm{m}$ de compresión por unidad de longitud y $46,7 \mathrm{kN} / \mathrm{m}$ de flujo cortante. Para analizar la sensibilidad que tiene el diseño a cambios en los valores de las cargas, se analizará también un caso de carga obtenido arbitrariamente dividiendo por dos el caso anterior. Así, se tienen el Caso 1 y Caso 2, respectivamente.

El objetivo principal de este caso de estudio es analizar si la hipótesis realizada en el diseño de materiales metálicos para adaptar los resultados a materiales compuestos de aplicar un factor reductor igual al cociente entre las densidades del material compuesto y aleación de aluminio utilizados es realista o no lo es. De esta forma se determinará si el ahorro en peso de material es proporcional a la densidad o es necesario considerar otros factores en etapas de diseño conceptual.

Siguiendo el esquema de la metodología presentada, lo primero es determinar las propiedades del material a utilizar, la orientación de las láminas, orden y el número de ellas que se van a considerar. La orientación se ha seleccionado de tal forma que se obtengan laminados simétricos y se han basado en otros laminados utilizados para presentar y validar procedimientos de diseño de materiales compuestos (Loughlan 1994). Además, se ha seleccionado un material compuesto utilizado en estructuras aeronáuticas como es el Hexcel 8552 AS4, cuyas propiedades se han incluido en la Tabla 3-1(Marlett et al. 2011). 


\begin{tabular}{|c|c|c|c|}
\hline & $\begin{array}{c}\text { Revestimiento y ala } \\
\text { inferior del } \\
\text { larguerillo }\end{array}$ & $\begin{array}{l}\text { Ala superior } \\
\text { del larguerillo }\end{array}$ & Alma del larguerillo \\
\hline$E_{L}[\mathrm{GPa}]$ & 124 & 124 & 124 \\
\hline$E_{t}[\mathrm{GPa}]$ & 11 & 11 & 11 \\
\hline$v_{21}$ & 0,33 & 0,33 & 0,33 \\
\hline$G$ [GPa] & 5 & 5 & 5 \\
\hline Orientación [] & {$[45,-45,-45,45,0,0]_{s}$} & {$\left[45,-45,0_{3}\right]_{\mathrm{s}}$} & {$[45,-45,45,-45,-45]_{\mathrm{s}}$} \\
\hline
\end{tabular}

Tabla 3-1 Propiedades de las láminas y orientación utilizados en los laminados del revestimiento y los larguerillos. Fuente: elaboración propia.

El siguiente paso del procedimiento es establecer el intervalo de variación de los grados de libertad. El parámetro $\lambda$ tomará valores entre 1 y 10 y el parámetro $\overline{A R}$ entre $A R$ y $50 A R$, con un paso de $A R$, de tal forma que se garantice que el número de larguerillos sea natural, que es precisamente el cociente entre $\overline{A R}$ y $A R$. En la Figura 3-21 se muestran los resultados obtenidos para el espesor del revestimiento para distintos valores de los grados de libertad y para ambos casos de carga contemplados. Valores más altos del parámetro $\lambda$ indican que el revestimiento soporta menos carga, por lo que el espesor decrece. De la misma forma, si el número de larguerillos crece, el paso entre ellos es menor $y$, en consecuencia, el espesor que hace pandear el revestimiento entre larguerillos para una misma carga también es menor. Es importante hacer notar que la solución para $\lambda=1$ supone que toda la carga se la lleva el revestimiento, por tanto, significa que no hay larguerillos en la sección. Por ello, a pesar de que matemáticamente podría tener solución, físicamente no tendría sentido resolver el problema para este caso con un parámetro $\overline{A R}$ mayor que $A R$. De ahí que en las gráficas se haya resuelto el caso para $\lambda=1$ y $\overline{A R}=A R$, y a partir de este punto se ha trazado una línea horizontal, asumiendo que el inicio de esa recta es el único punto obtenido realmente, y que representan el espesor necesario para soportar los casos de carga sin la necesidad de rigidizadores. Como es lógico, si se instalan rigidizadores, el espesor del revestimiento decrece es menor que cuando no los hay.
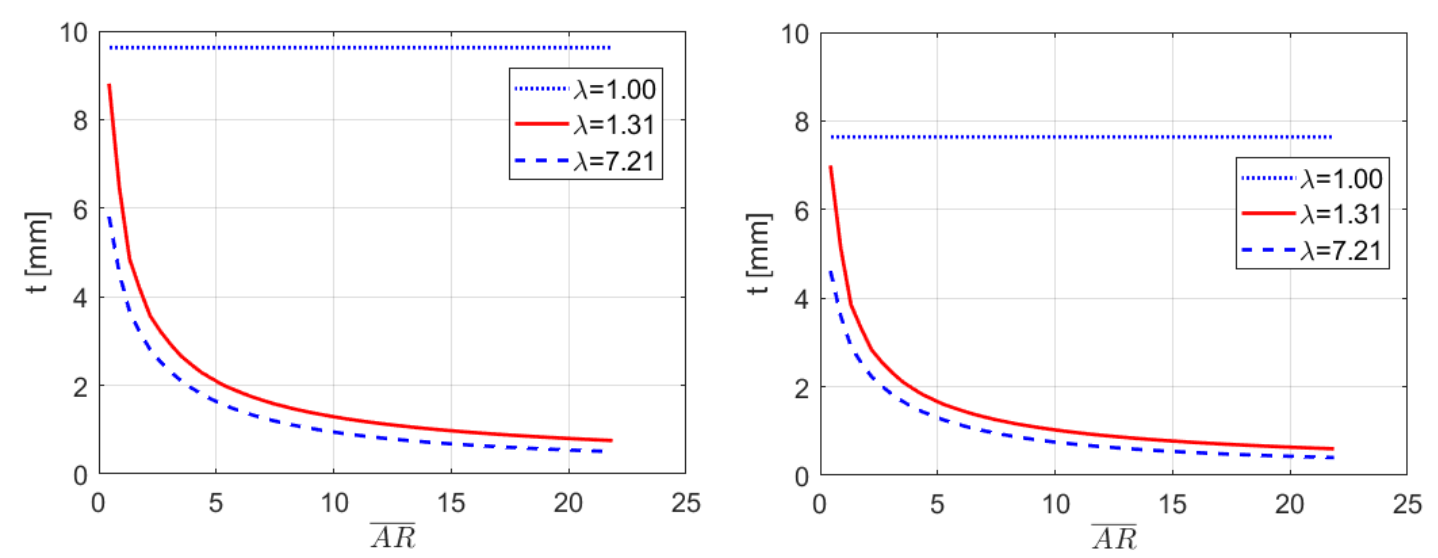

Figura 3-21 Variación del espesor del revestimiento en función de los parámetros $\lambda$ y $\overline{A R}$ para el Caso 1 (izquierda) y Caso 2 (derecha). Fuente: elaboración propia.

Después de esto, haciendo uso de la condición de pandeo global, es posible determinar la rigidez $E_{b} I$ de cada uno de los larguerillos. Además, a través de la definición del parámetro $\lambda$ se puede hallar la rigidez axial de los mismos. El siguiente paso es calcular las pseudo-rigideces equivalentes de los larguerillos de acuerdo con las propiedades del material incluidas en la Tabla $3-1$. Estos resultados son $2,38 \mathrm{MPa}$ para $\overline{E A_{e q}}$ y 
3,96 MPa para $\overline{E_{b} I_{e q}}$. Una vez se han calculado estas variables, se puede hallar el tamaño de las alas del larguerillo por medio de las definiciones de los parámetros de pseudo-rigidez siguiendo la ecuación [ 3-98 ]. Los resultados se muestran en la Figura 3-22. Al igual que antes, los resultados para $\lambda=1$ corresponden al tamaño asociado a no tener larguerillos, es decir, es una recta horizontal que está sobre el eje de abscisas. Para el resto de las combinaciones de los grados de libertad, el tamaño de las alas crece a medida que aumenta el número de larguerillos. Esto se debe a que la rigidez axial decrece más rápidamente con el número de larguerillos de lo que lo hace la rigidez $E_{b} I$. Por eso, la longitud de las alas y consecuentemente la altura del alma necesitan ser más grandes para aumentar el momento de inercia, a pesar de que el área se reduzca porque el espesor así lo hace. El mismo razonamiento es aplicable para explicar el comportamiento con las variaciones del parámetro $\lambda$. La rigidez axial crece más rápido $\operatorname{con} \lambda$ que la rigidez a pandeo. Por tanto, la longitud de las alas tiene que ser menor para permitir un incremento mayor en área, debido al aumento de espesor, que del momento de inercia.
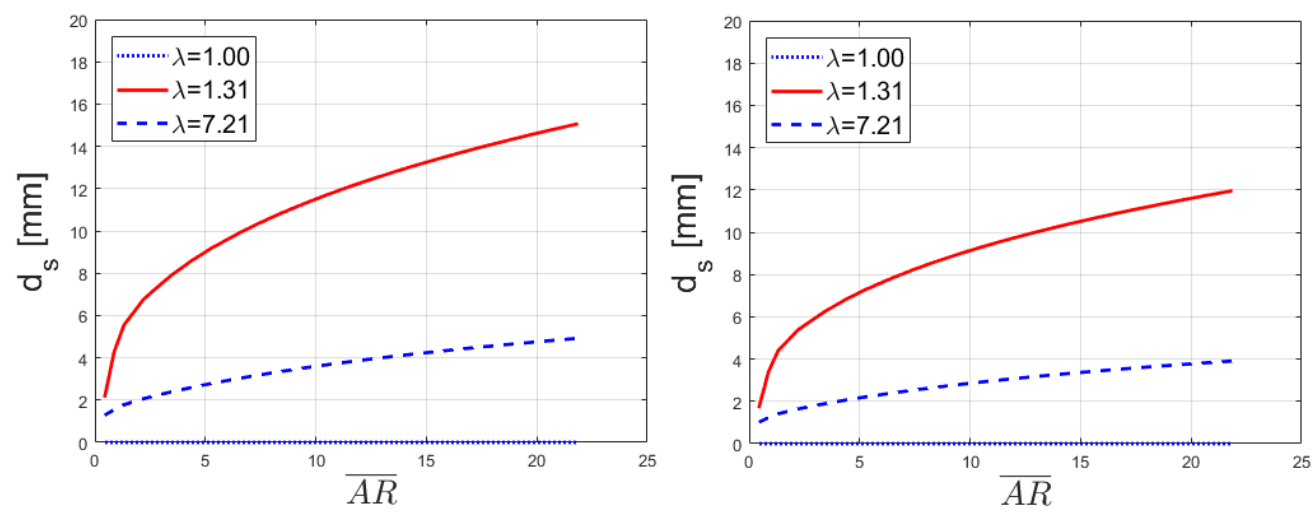

Figura 3-22 Variación del ala del larguerillo en función de los parámetros $\lambda$ y $\overline{A R}$ para el Caso 1 (izquierda) y Caso 2 (derecha). Fuente: elaboración propia.

Finalmente, se puede calcular el área del panel necesaria para soportar el estado de cargas con las hipótesis de diseño contempladas. Los resultados se representan en la Figura 3-23. El área requerida aumenta con el parámetro $\lambda$ para un número de larguerillos constante y distinto de cero, que sería el caso de $\lambda=1$. Como era de esperar, el área requerida para el Caso 2 es menor que para el Caso 1. Cabe destacar que las soluciones que se encuentran por debajo de la recta horizontal, que define la situación donde no hay larguerillos y toda la carga la soporta el revestimiento, son una muestra de que un panel rigidizado puede soportar la misma carga con un área menor que la que corresponde a un panel no rigidizado. Por tanto, es necesario encontrar la combinación de los parámetros $\lambda$ y $\overline{A R}$ que minimicen el área del panel. Se puede observar como cada una de las curvas representadas en la Figura 3-23 se extienden hasta puntos diferentes dependiendo del valor de $\lambda$. Esto se debe a que alguna de las restricciones impuestas se alcanza. Si el número de larguerillos es suficientemente alto, los espesores del alma y las alas son tan pequeños que se pueden alcanzar las limitaciones de fallo por crippling, por fabricación o por el otro modo de pandeo local. 

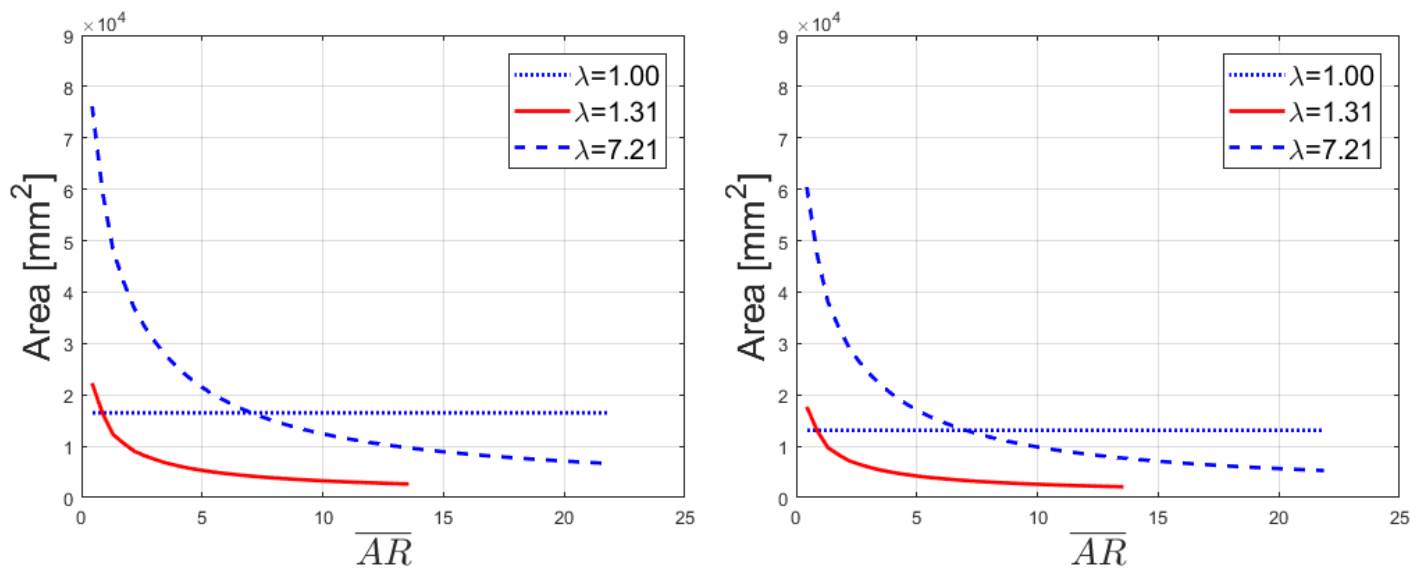

Figura 3-23 Variación del área del panel en función de los parámetros $\lambda$ y $\overline{A R}$ para el Caso 1 (izquierda) y Caso 2 (derecha). Fuente: elaboración propia.

El proceso de búsqueda del mínimo es simple desde el punto de vista matemático, ya que la función de área es monótona con los parámetros de los que depende. Por tanto, el mínimo siempre se alcanza en el contorno de búsqueda. Como es lógico, las curvas anteriormente mostradas son una versión reducida de la malla real que se ha utilizado para encontrar el mínimo. Sobre esta malla, el mínimo se alcanza en los valores de los parámetros $\lambda=1,62$ y $\overline{A R}=21,86$ en ambos casos de carga.

\subsubsection{Comparativa con el procedimiento para material metálico}

Como se ha comentado anteriormente, el objetivo es determinar el ahorro de peso que se produce por el uso de material compuesto en lugar de las aleaciones de aluminio típicas utilizadas en las estructuras aeronáuticas. En concreto, se van a comparar los resultados con los obtenidos para los mismos casos de carga si se diseña el panel siguiendo el método de Farrar expuesto en la sección dedicada al diseño de estructuras metálicas. Puesto que el mínimo peso se obtiene para el factor de Farrar óptimo, y este valor para los larguerillos de sección en $Z$ es de 0,95. Los resultados tanto del procedimiento presentado para material compuesto como los obtenidos por Farrar para ambos casos de carga considerados se incluyen en la Tabla 3-2. En ella se incluyen las principales variables geométricas, el número de larguerillos y el peso del panel. La última variable calculada para el caso de Farrar óptimo (Peso del panel compuesto) corresponde al peso del panel basado en la geometría obtenida con la metodología de Farrar para material metálico pero utilizando la densidad del material compuesto utilizado en este estudio, $1600 \mathrm{~kg} / \mathrm{m}^{3}$, en lugar de la típica de aluminio utilizado en aeronáutica, como puede ser $2800 \mathrm{~kg} / \mathrm{m}^{3}$. 


\begin{tabular}{|c|c|c|c|c|c|}
\hline & Unidades & \multicolumn{2}{c}{ Farrar óptimo } & \multicolumn{2}{c|}{ Método propuesto } \\
\hline & $\mathrm{mm}$ & 0,55 & 0,39 & 0,71 & 0,56 \\
\hline $\boldsymbol{t}$ & $\mathrm{mm}$ & 0,59 & 0,41 & 0,39 & 0,39 \\
\hline $\boldsymbol{t}_{s}$ & $\mathrm{~mm}$ & 24,70 & 20,77 & 36,74 & 29,16 \\
\hline $\boldsymbol{h}_{s}$ & $\mathrm{~mm}$ & 28,45 & 23,92 & 34,30 & 34,30 \\
\hline $\boldsymbol{b}$ & & 62 & 73 & 51 & 51 \\
\hline $\begin{array}{c}\text { № } \\
\text { larguerillos }\end{array}$ & $\mathrm{kg}$ & 5,04 & 3,53 & - & - \\
\hline $\begin{array}{c}\text { Peso del } \\
\text { panel } \\
\text { metálico }\end{array}$ & $\mathrm{kg}$ & 2,88 & 2,02 & 2,83 & 2,25 \\
\hline $\begin{array}{c}\text { Peso del } \\
\text { panel } \\
\text { compuesto }\end{array}$ & $\mathrm{kg}$ & & & & \\
\hline
\end{tabular}

Tabla 3-2 Resultados para los casos de carga considerados según el método de Farrar para factor de Farrar óptimo y el propuesto para materiales compuestos. Fuente: elaboración propia.

Se puede comprobar como el método predice ahorros en cuestión de peso de entre un $30 \%$ y un $40 \%$ respecto al material de aleación de aluminio, dependiendo del caso de carga. Esta reducción de peso coincide con los factores característicos de ahorro contemplados en diseño conceptual cuando se diseña en material compuesto respecto estructuras metálicas de aluminio sometidas a una combinación de compresión y cortadura, como es el caso de los paneles de extradós de las superficies de cola (Baker y Scott 2016; Niu 1992). Además, cuando se compara la última fila de la Tabla 3-2, se comprueba que la reducción de pesos, a pesar de estar comprendida entre unos factores característicos, no es exactamente proporcional a la reducción de densidad, depende claramente del caso de carga.

Se han llevado a cabo más ensayos para diferentes casos de carga. En un rango de unas 15 veces mayor y 5 veces menor que las cargas correspondientes al Caso 1, el material y el laminado propuestos en este estudio resulta en soluciones que presentan un comportamiento similar al que se ha mostrado aquí. Sin embargo, si el caso de carga es suficientemente diferente solo en $N_{x}$ o en $N_{x y}$ o en la relación $N_{x} / N_{x y}$, el método dimensiona paneles con una sección demasiado pesada en comparación con la análoga de material metálico como para considerar los resultados como válidos. El problema se encuentra en la selección del material y/o en la orientación de las láminas, que alguno o ambos dos no son adecuados. Sería necesario cambiar el material o cambiar el número de láminas o su orientación para alcanzar resultados aceptables. Mediante un procedimiento de prueba-error, es posible encontrar estas orientaciones para que los resultados que se presentan para los casos de carga aquí contemplados sean semejantes a los obtenidos si se cambian las cargas en alguno de los sentidos que se ha comentado. Por tanto, sería necesario establecer un procedimiento más automatizado de selección de número de láminas y de su orientación, en función del caso de carga, para que el procedimiento sea completo. Este problema es más amplio que el que se ha presentado en esta sección, y precisamente es el que ya se ha acometido por otros autores (Kassapoglou 1997; Liu, Butler y Kim 2008; Degenhardt et al. 2008). El principal problema que tienen estas metodologías es que no están pensadas para etapas de diseño conceptual, ya que conllevan demasiado tiempo de cálculo para estas etapas. 


\subsection{DISEÑO ÓPTIMO}

\subsubsection{Introducción}

Los diseñadores de aviones, al igual que en muchas otras disciplinas de la ingeniería, siempre han tratado de que cada uno de sus nuevos diseños sean lo mejor posible, utilizando todas las herramientas de las que disponen para alcanzar ese óptimo. Desde los hermanos Wright hasta que aparecieron los primeros ordenadores con capacidad gran capacidad de cálculo, se utilizaban técnicas de optimización muy rudimentarias, que únicamente podían plantearse para unas cuantas variables. Tras la aparición de los ordenadores, gracias a la capacidad de llevar a cabo cálculos repetitivos, las técnicas evolucionaron más rápidamente (Wright y Wright 2001; Holt 1982; SobieszczanskiSobieski y Haftka 1997). Estas nuevas técnicas se pueden agrupar bajo el paraguas del concepto: Multidisciplinary Design Optimization o MDO. Esta disciplina es una metodología de diseño de sistemas de ingeniería complejos que están gobernados por fenómenos físicos y que interaccionan con otros sistemas (Sobieszczanski-Sobieski 1995).

MDO permite al diseñador trabajar sobre un gran número de variables que están afectadas por restricciones y funciones de diferentes disciplinas. En el mundo de la aeronáutica, se han aplicado estas técnicas a varios estudios sobre diseño de aviones de muy diferente naturaleza (Hepperle 2008; Martins y Lambe 2013; Lyu y Martins 2014; Ceruti, Voloshin y Marzocca 2014; Diedrich y Willcox 2005; Raymer 2002; Park et al. 2015; Martins, Kenway y Brooks 2016; Papageorgiou et al. 2018). Además, no solo se aplica MDO al diseño de aviones, sino que existen muchas otras disciplinas en las que se utilizan, tales como en barcos (Peri y Campana 2003; Kalavalapally, Penmetsa y Grandhi 2006), naves espaciales (Cai et al. 2010; Braun, Moore y Kroo 1997) o automóviles (Kokkolaras et al. 2004; McAllister y Simpson 2003).

La principal motivación para utilizar técnicas de MDO es que el mejor diseño de un sistema multidisciplinar únicamente se puede determinar si se tienen en cuenta todas las interacciones entre las diferentes disciplinas que afectan al problema. Esta interacción no se puede considerar de forma arbitraria, sino que es necesario darle un tratamiento matemático adecuado. Una de las consideraciones más importantes a la hora de implementar técnicas MDO es cómo organizar el análisis de los modelos y el programa de optimización. De esta forma, la combinación de la formulación del problema y la estrategia de organización reciben el nombre de la arquitectura de MDO. La arquitectura utilizada puede ser monolítica, si se va a resolver únicamente un problema de optimización. Si el problema se partiera en varios subproblemas, donde cada uno de ellos tiene una serie de variables y restricciones, se estaría ante una arquitectura distribuida (Martins y Lambe 2013).

En el caso de esta tesis, la estructura será monolítica, ya que solo se va a considerar un problema de optimización. Además, debido a que el problema se refiere a etapas de diseño conceptual, el objetivo principal es no utilizar técnicas o metodologías muy complejas, que requieran un gran coste computacional de cálculo. Por tanto, se buscarán estrategias como puede ser el analizar la topología del espacio de diseño y de la función objetivo, para simplificar al máximo el problema. A pesar de ello, en este apartado se expondrán tanto técnicas de simplificación como algoritmos de optimización; ya que, en el fondo, se está resolviendo un problema de programación no lineal (Bazaraa y Setty 1979). 


\subsubsection{Planteamiento del problema matemático}

Los modelos de un diseño se describen en forma de variables de diseño, parámetros y constantes. Con todas ellas se puede construir una función objetivo sobre la cual se trata de buscar el óptimo. Además, esta función objetivo está sometida a restricciones impuestas al enfrentar el modelo con la realidad. Por tanto, se puede decir que para optimizar un diseño se deben seguir los siguientes pasos (Papalambros y Wilde 2017):

1. Seleccionar el conjunto de variables que se van a utilizar para describir el modelo.

2. Elegir la función objetivo, expresada en términos de las variables de diseño, sobre la que se va a buscar el máximo o el mínimo.

3. Determinar el conjunto de restricciones, de nuevo expresadas en función de las variables de diseño, que deben satisfacerse para que el diseño sea adecuado.

4. Encontrar la combinación de valores de las variables de diseño que minimizan o maximizan la función objetivo cumpliendo con las restricciones.

Desde un punto de vista matemático, formalmente se agruparán las variables de diseño $x_{1}, x_{2}, x_{3}, \ldots, x_{n}$ en un vector $\boldsymbol{x}=\left(x_{1}, x_{2}, \ldots, x_{n}\right)^{T}$, que pertenece a un subconjunto $\mathcal{X}$ del espacio real $n$-dimensional $\mathbb{R}^{n}$. Teniendo en cuenta que la función objetivo tiene que ser cuantificable y expresada en términos de las variables de diseño, podemos definir la función objetivo como $f(\boldsymbol{x})$. Las restricciones se pueden expresar como relaciones funcionales de las variables de diseño, separando las inecuaciones, $g(\boldsymbol{x}) \leq 0$, de las igualdades, $h(\boldsymbol{x})=0$, utilizando la forma negativa-nula. Se asume que las funciones de las restricciones tienen una salida eEscalar, pero sería perfectamente generalizable si la salida fuese un vector. El conjunto de variables de diseño que satisfacen estas restricciones forma lo que se llama como el dominio factible del problema. Con todo esto, el problema de diseño quedaría definido de la siguiente forma (Martins y Lambe 2013):

$$
\begin{gathered}
\min f(\boldsymbol{x}) \\
\text { sujeto a } \boldsymbol{h}(\boldsymbol{x})=\mathbf{0} \\
\boldsymbol{g}(\boldsymbol{x}) \leq \mathbf{0} \\
\boldsymbol{x} \in X \subseteq \mathbb{R}^{n}
\end{gathered}
$$

En la ecuación anterior, las funciones $\boldsymbol{h}$ y $\boldsymbol{g}$ reciben el nombre de sistema de restricciones y $\mathcal{X}$ el de conjunto restringido. Tanto la función $f$ como las $\boldsymbol{h}$ y $\boldsymbol{g}$ pueden ser expresadas en formas muy diferentes. Una forma es que vengan dadas por expresiones algebraicas que dependen de las variables de diseño. Estas relaciones normalmente se derivan directamente de ecuaciones básicas o leyes físicas. El inconveniente es que normalmente estos modelos básicos no describen el problema fielmente y tienen que ser corregidos con datos experimentales o empíricos. Entonces, se pueden extraer las relaciones sin más que haciendo un ajuste de una curva a los datos medidos. Otra posibilidad es que el sistema no tenga ecuaciones como tal, pero formalmente sean el resultado de un proceso complejo que involucre varios pasos de iteración y de cálculo que, habitualmente, solamente se pueden hacer con un programa de ordenador. En estos casos se suele usar el término modelo de simulación. En la práctica, los modelos matemáticos son una mezcla de todos los casos, es decir, habrá tanto ecuaciones algebraicas, como puramente experimentales y modelos de simulación (Papalambros y Wilde 2017). 


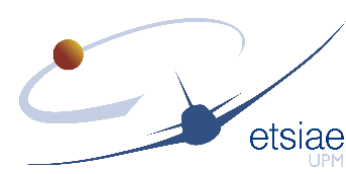

A veces, por la naturaleza del modelo de diseño, surgen dos funciones objetivo, que pueden ser una competitiva con respecto a la otra. De tal forma que sea necesario encontrar una solución de compromiso. Si mantenemos más de una función objetivo, el proceso trata de buscar un óptimo en una función vectorial en lugar de una eEscalar. Las herramientas necesarias para plantear y resolver el problema matemático son bastante extensas y representan una teoría de optimización específica (Miettinen 1999).

Otro aspecto importante sobre el problema planteado en [ 3-99 ] es determinar cuándo el problema tiene solución. La existencia es una cuestión importante en la teoría de optimización y difícil de calcular. Salvo casos especiales, si asumimos que el problema está bien planteado y basado en modelos matemáticos tratados con rigor y con significado ingenieril detrás, se podrá suponer que el problema tiene una solución real y creíble. En estos casos se dice que el problema está bien planteado, ya que se asume que tiene solución. A pesar de ello, existen propiedades matemáticas que está asociadas al modelo y a su solución, que pueden ser utilizadas para comprobar si el problema está adecuadamente planteado. Puede ocurrir que el problema no esté planteado correctamente porque el modelo no se ha formulado adecuadamente. Entonces, este análisis matemático puede ayudar a clarificar el problema de ingeniería y realizar las modificaciones oportunas.

Por tanto, suponiendo que estamos trabajando sobre un problema bien planteado, las restricciones y el conjunto restringido definen una región dentro de un espacio de $n$ dimensiones, como ya se ha comentado anteriormente. El espacio factible de las variables de diseño está acotado por una serie de límites o contorno. Dentro de ese espacio de diseño es donde hay que encontrar el óptimo. Aquí se puede definir el concepto de actividad (Papalambros y Wilde 2017). Una restricción se considera activa si al eliminarla el óptimo cambia de posición. Esto lleva a que el óptimo se alcanza sobre esta restricción. En el caso de una restricción definida por una inecuación, significa que en el óptimo la restricción se cumple como si fuera una igualdad. El concepto de actividad es muy relevante en procedimiento de diseño óptimo, ya que una restricción activa representa un requisito de diseño que tiene una influencia directa en el óptimo. Esta información es muy interesante para el diseñador. No hay que confundir las restricciones inactivas con las redundantes. Las inactivas son aquellas donde no se alcanza el óptimo, pero sí restringen el espacio de diseño. En cambio, las redundantes no aportan información adicional, es decir, se encuentran fuera del espacio factible. Por supuesto, una variación de ciertos parámetros de diseño puede convertir restricciones de un tipo a otro, pasando una redundante a activa, o viceversa.

El espacio de diseño, a su vez, puede tener diferentes topografías. La función objetivo puede alcanzar el óptimo sobre el contorno o en un punto interior. En el primero de los casos alguna de las restricciones está activa, en cambio, en el segundo la solución no depende de las restricciones seleccionadas para el problema. Además, la función objetivo puede tener más de un óptimo. Si el óptimo es únicamente relativo a los valores de la función en los puntos localmente próximos a este, se dice que es un óptimo local. De entre todos los óptimos locales, el que tenga el valor mínimo, o máximo, es el mínimo, o máximo, global. Determinar si el óptimo alcanzado por un algoritmo de optimización es local o global no es sencillo, a pesar de ser un aspecto crucial. Normalmente, se asume que el conocimiento sobre el problema es suficientemente extenso como para poder realizar un buen juicio crítico de la solución obtenida (Wilde 1978). 
Una vez completado el modelo, se pueden utilizar métodos matemáticos para encontrar la solución del problema. Por este motivo tiene especial relevancia construir de forma adecuada el modelo, porque el método matemático pierde de vista el sentido físico del problema y puede alcanzar una solución inadecuada. De hecho, un estudio físico apropiado del problema puede simplificar de una forma drástica el método matemático a utilizar para resolverlo. Y, por otra parte, propiedades matemáticas básicas pueden ayudar analizar si el problema está incompleto. Por ejemplo, los principios de monotonía se utilizan para llevar a cabo una reducción de los grados de libertad del modelo antes de implementar ningún algoritmo de optimización (Papalambros 1979). Es por eso por lo que el método de resolución y el problema a resolver tienen que ir de la mano.

Para construir modelos a menudo se necesitan incorporar datos experimentales o determinados numéricamente, que normalmente están dado en forma de tabla 0 matrices. En el objetivo de esta tesis, tal y como se ha podido ver en los modelos aerodinámicos y de pesos, el caso es el de generar un conjunto de datos por medio de procedimientos matemáticos complejos realizados mediante ordenador. Esos puntos representan una función, a pesar de que no tengan una ecuación algebraica explícitamente. Desde el punto de vista matemático, tiene considerables ventajas convertir estos puntos a una forma algebraica. Esto es lo que generalmente se conoce como ajustar una curva. Puede ocurrir que incluir el modelo numérico completo en los pasos de iteración del método de optimización sea computacionalmente más caro que generar una malla de puntos y ajustar una curva al mismo con una precisión adecuada. Estos nuevos modelos generados reciben el nombre de modelos de orden reducido, modelos subrogados o metamodelos, en función del método que se haya seguido para obtenerlos. De esta forma se pueden sustituir el conjunto de puntos o el modelo numérico completo por estos modelos subrogados. Esto puede reducir de forma drástica el tiempo de computación si el problema de diseño es muy amplio (Sasena, Papalambros y Goovaerts 2001; Liem, Mader y Martins 2015; Kumar y Ghosh 2014).

\subsubsection{Métodos de ajuste: redes neuronales}

Los métodos para ajustar una curva a una serie de puntos dependen del número de puntos y de los grados de libertad que tiene la curva. Por ejemplo, si se quieren ajustar $n+1$ puntos con un polinomio de grado $n$, el procedimiento es sencillo, ya que el polinomio tiene el mismo número de coeficientes que puntos tiene el modelo, por lo que se tiene un sistema de $n+1$ ecuaciones con el mismo número de incógnitas. Sin embargo, si el número de puntos es mayor que los grados de libertad que tiene la curva de ajuste, lo deseable sería utilizar la información extra para conseguir un mejor ajuste de la curva (Draper 1981). Una forma de obtener esta curva es determinando los valores absolutos de las desviaciones de los puntos con respecto a la curva y encontrar la que minimiza la suma de estas desviaciones. Otra opción, que normalmente es más utilizada, es encontrar la curva que minimiza la suma de los cuadrados de las desviaciones, lo que se conoce por ajuste por mínimos cuadrados (Lawson y Hanson 1995). Por supuesto, esta técnica se puede utilizar para cualquier tipo de función que represente la curva, no necesariamente un polinomio. Esta técnica es la misma que se ha utilizado para determinar las regresiones del Capítulo 2. Por tanto, también son aplicables las técnicas y procedimientos para determinar si el ajuste es suficientemente bueno. Factores como el $R^{2}$ o el error típico permiten cuantificar la bondad del ajuste. Como es de esperar, dependiendo de la ecuación de la curva de ajuste el problema se puede complicar, en función de si el problema que resulta es lineal, intrínsecamente lineal (si se puede linealizar el problema con alguna transformación) o no lineal. Lógicamente, los problemas de ajuste por mínimos cuadrados no lineales son más 


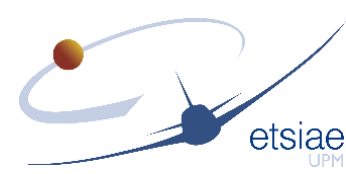

difíciles de implementar. A pesar de que la mayoría de los comportamientos de las funciones con las que se trabaja en ingeniería, en general, y en esta tesis, en particular, son no lineales, para construir un modelo subrogado puede no ser necesario una precisión muy alta siempre y cuando el ajuste siga la misma tendencia que los puntos y el estudio de actividad de las restricciones y monotonía de la función objetivo lleve a una determinación del óptimo preliminar. Por supuesto, más adelante se puede refinar este ajuste para mejorar la precisión del óptimo en su caso.

El ajuste por mínimos cuadrados tiene la principal ventaja de su sencillez para construir el modelo de optimización para determinar los coeficientes del ajuste. Sin embargo, tiene el principal inconveniente que es necesario una inspección previa de los datos para establecer a priori el tipo de ecuación que se quiere ajustar y que se prevé que va a seguir la tendencia de los puntos. Esta tarea puede no ser obvia. Siempre se puede utilizar un polinomio de un grado suficientemente alto para conseguir un buen ajuste a los puntos. El inconveniente que puede surgir es lo que se conoce como overfitting. Esto lleva a que la curva se aproxime mucho a los puntos, pero la interpolación entre los mismos se separe demasiado de la tendencia que siguen. Este comportamiento es signo de que se debe cambiar la curva porque tenemos un sobreajuste. Por tanto, si no se tiene claro el tipo de función que sigue la tendencia de los puntos, lo que puede ocurrir más fácilmente en un problema de varias dimensiones, es recomendable utilizar otro tipo de técnicas que permiten automatizar este proceso de aproximación al problema de ajuste.

Una técnica para evitar tener que seleccionar previamente la ecuación a ajustar a los puntos es el uso de redes neuronales (Hagan, Demuth y Beale 1996). El objetivo final que hay detrás de las redes neuronales es el mismo que en el ajuste no lineal por mínimos cuadrados: encontrar una serie de coeficientes que minimizan el error de la función al ser comparado con los puntos a ajustar. Lo que cambia es el álgebra que hay detrás de ellas. Esta técnica junto con otras semejantes de machine learning se han empleado en el mundo de la aviación para generar modelos de ajuste para ensayos en túnel aerodinámico (Norgaard, Jorgensen y Ross 1997; Dupuis, Jouhaud y Sagaut 2018) o para el diseño de aviones y vehículos espaciales (Jules y Lin 2002; Secco y Mattos 2015).

Una red neuronal consiste en un conjunto de nodos o neuronas que están conectados entre sí de una cierta forma. Esa conectividad viene dada por su arquitectura, que establece el nivel de interconexión entre las diferentes neuronas. Si los nodos únicamente tienen conexiones de salida, se consideran que son los nodos de entrada al modelo. En cambio, si solamente tienen conexiones de entrada, son los que se sitúan al final de la red y son los nodos de salida. El resto de los nodos se encuentran en lo que se consideran como capas ocultas de la red. Las capas las forman las neuronas que se encuentran a una misma distancia de los nodos de entrada o de salida. 


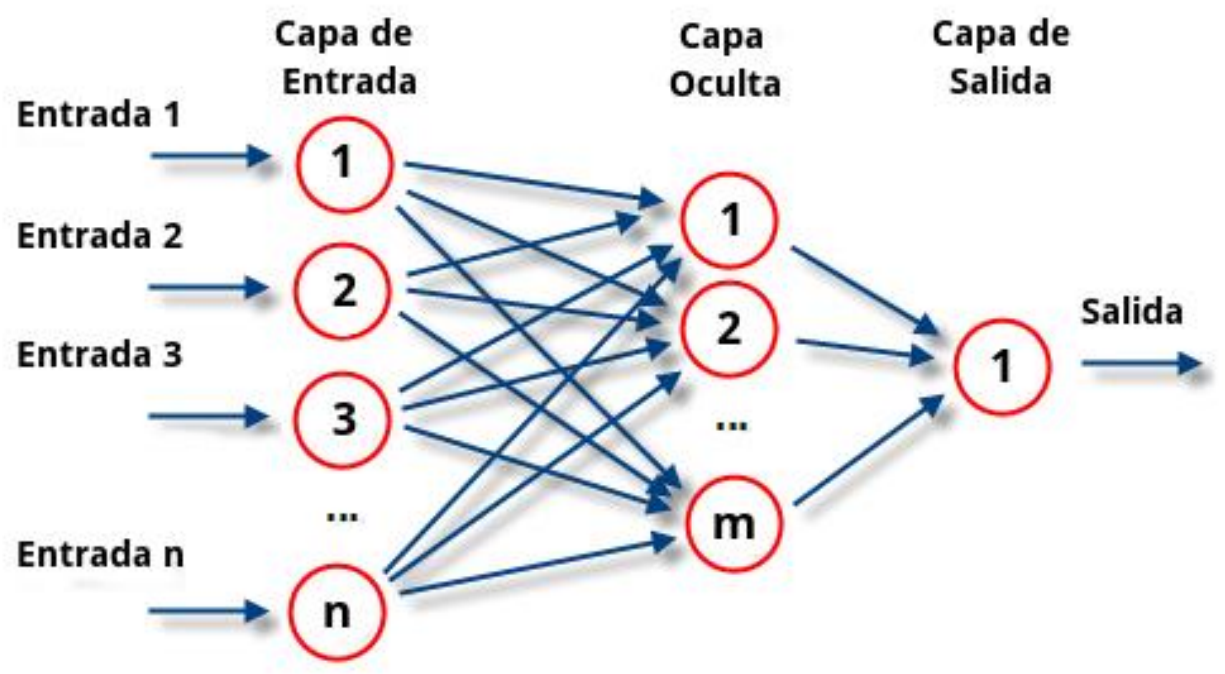

Figura 3-24 Esquema de red neuronal del tipo feedforward. Fuente: (Gengiskanhg 2004)

Las redes neuronales se pueden clasificar principalmente, de acuerdo con el tipo de conectividad, en dos tipos: las redes cuyas neuronas únicamente se conectan con las neuronas que se encuentran en capas posteriores, llamadas feedforward, o las que se conectan con cualquiera de las otras neuronas, también llamadas redes recurrentes. Desde el punto de vista de la aplicación de las redes neuronales a un ajuste por regresión, el número de entradas a la red neuronal coincide con el número de variables de las que depende la función a ajustar, y el número de salidas corresponde con las dimensiones de la función a ajustar. En el caso que ocupa a esta tesis, el número de salidas siempre será uno y el tipo de red será feedforward con una única capa oculta, ya que habitualmente es suficiente para problemas de regresión (Martínez-Estudillo 2005).

Cada neurona se caracteriza por un valor numérico denominado estado de activación y representado por $y_{i j}$, donde el índice $i$ indica el número de la capa y el índice $j$ el número de neurona dentro de la capa. Además, existe una función de salida o de activación que cambia el estado por medio de una función $f_{i j}\left(y_{i j}\right)$. Por regla general, las funciones de activación son diferentes dependiendo de si corresponden a neuronas de la capa oculta o de la capa de salida. Normalmente, para un problema de regresión, la función de salida es lineal o la identidad. Sin embargo, existen muchas otras funciones de activación como pueden ser la sigmoide o la tangente hiperbólica (ver Figura 3-25), que se reservan habitualmente para las capas intermedias (Matlab 1997; Beale y Demuth 1994). 


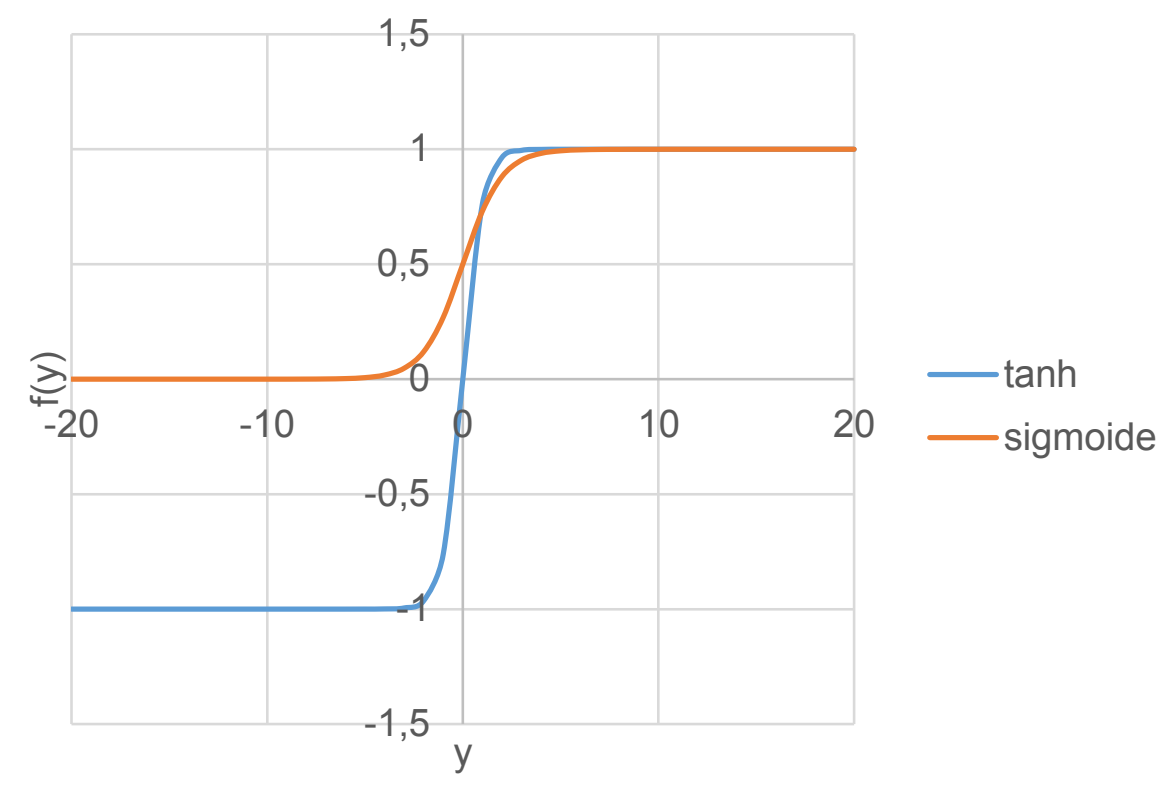

Figura 3-25 Funciones de activación tipo sigmoide y tangente hiperbólica. Fuente: elaboración propia.

Contemplando la nomenclatura indicada en el párrafo anterior, que únicamente se va a trabajar con una capa oculta y que las relaciones entre neuronas se producen hacia adelante en la red, la relación más sencilla que se puede suponer entre las neuronas es del tipo lineal. Tomando como ejemplo la Figura 3-26, el estado de activación de una neurona es el siguiente:

$$
y_{i j}=f_{i j}\left(\sum_{k=1}^{n_{i-1}}\left(w_{i j, k} \times y_{i-1, k}\right)+b_{i j}\right)
$$

En la ecuación anterior los coeficientes $w_{i j, k}$ reciben el nombre de pesos y los $b_{i j}$ son los bias. Los pesos y los bias forman la relación lineal entre los estados de activación de la capa anterior con la actual. De esta forma, cada neurona $i j$ tiene $n_{i-1}$ relaciones lineales, es decir, se relaciona con el número de neuronas de la capa anterior $n_{i-1}$. En consecuencia, cada neurona $i j$ tiene asociado un número de pesos igual al número de neuronas de la capa anterior. Precisamente el índice $k$ de la ecuación representa este número. Los bias que corresponderían a cada una de las relaciones con las neuronas de la capa anterior se pueden agrupar en uno solo para cada neurona $j$ de la capa $i$, que es el $b_{i j}$. En el caso que se muestra en la figura, las neuronas de la primera capa representan los datos de entrada, que en este caso serían tres. Al ser la capa de salida de una sola neurona, quiere decir que la función que estamos intentando ajustar es eEscalar. Como se ha comentado anteriormente, en cada neurona hay una función de activación $f_{i j}$ que modifica el estado de activación. De ahí, que en la ecuación [ 3-100 ] aparezca dicha función; a pesar de que esa función pueda ser la identidad, como suele ser el caso en las capas de entrada y de salida. 


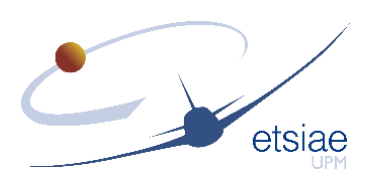

DISEÑO ÓPTIMO

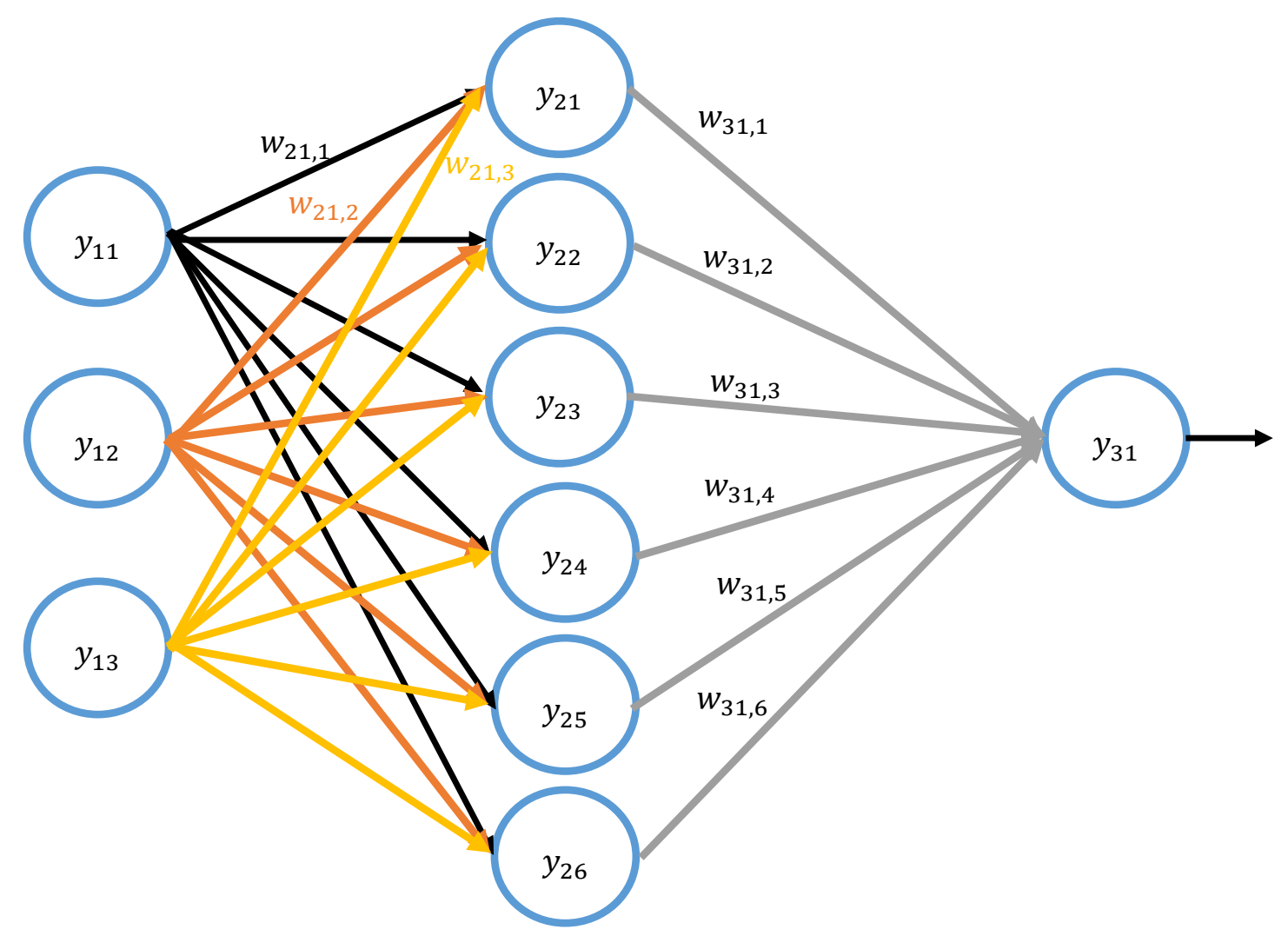

Figura 3-26 Esquema de una red neuronal con una capa oculta, indicando la nomenclatura de los pesos para la primera neurona de la capa oculta y para la neurona de salida. Fuente: elaboración propia.

Los coeficientes de pesos y bias son los que hay que determinar para que la función de salida se ajuste lo más posible a la serie de puntos de entrada. Para ello, se puede seguir un ajuste por mínimos cuadrados, donde la función objetivo es la suma de los cuadrados de las distancias entre los valores estimados mediante la red neuronal y los puntos reales, y las incógnitas son los pesos y los bias. Como se puede deducir del esquema presentado, aumentar el número de neuronas hace aumentar drásticamente el número de incógnitas del problema de optimización que hay que resolver. En el apartado 3.4.4 se van a presentar una serie de esquemas de búsqueda del óptimo que serían aplicables a este problema. Puesto que la resolución de este problema de optimización no presenta ninguna restricción en el espacio de las variables de diseño, simplemente habría que aplicar uno de estos esquemas para alcanzar el óptimo. Es por ello que no se va a hacer mayor hincapié en la resolución de este problema de mínimos cuadrados. Este procedimiento aquí descrito de tratar de ajustar el modelo a la salida es lo que recibe el nombre de aprendizaje supervisado o entrenamiento de una red neuronal (Nielsen 2015; Goodfellow, Bengio y Courville 2016). Si el algoritmo converge 


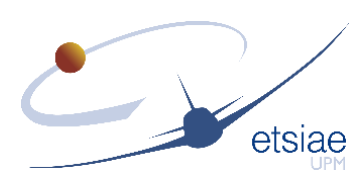

como debe, en cada paso de iteración la función objetivo decrece, tal y como muestra cualitativamente la Figura 3-27.

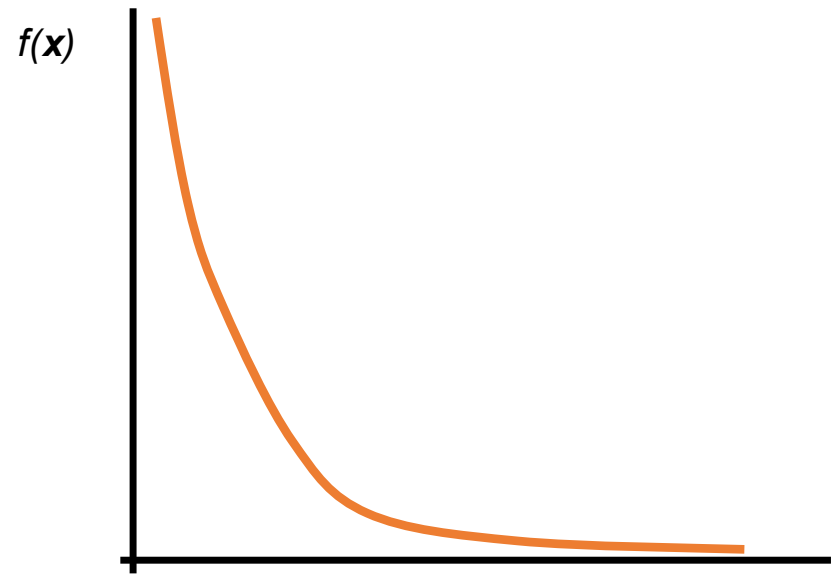

iteraciones

Figura 3-27 Evolución del valor de la función objetivo a cada paso de iteración hasta alcanzar el óptimo. Fuente: elaboración propia.

Al igual que ocurre en cualquier problema de ajuste por mínimos cuadrados, puede producirse el efecto de sobreajuste o overfitting, anteriormente descrito. Una forma de saber que se ha producido este fenómeno es ir evaluando la suma del error cuadrático en un conjunto de puntos distinto del utilizado para el entrenamiento, de tal forma que lo que se observa es que la función objetivo sigue disminuyendo su valor, pero la suma del error cuadrático en estos otros puntos comienza a crecer (Figura 3-28).

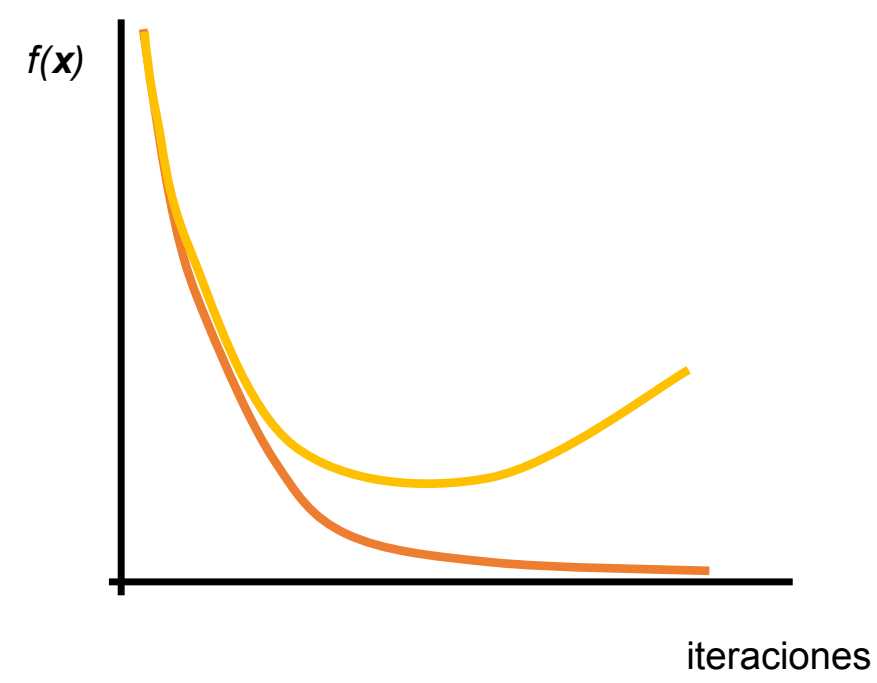

Figura 3-28 Evolución del valor de la función objetivo en un caso en el que se produce overfitting. La línea naranja representa la evolución del error en el conjunto de entrenamiento, y la amarilla en el conjunto de validación. Fuente: elaboración propia.

Para evitar este efecto, lo que se hace es dividir la muestra en tres conjuntos de puntos: el conjunto de entrenamiento, el de validación y el de prueba. El primero de ellos se utiliza de la forma como se ha explicado hasta ahora, es decir, es sobre el cual se trata de minimizar la función objetivo. Paralelamente, en cada paso de iteración se evalúa el error cuadrático sobre el conjunto de validación. El propósito es ir comprobando que el error también se va reduciendo sobre esta muestra. Si esto ocurre, el procedimiento sigue hasta que la función alcance su valor mínimo. Sin embargo, puede ocurrir el efecto de sobreajuste llegado un cierto paso de iteración. 
Como se ha visto antes, esto se percibe al comenzar a aumentar el error sobre el conjunto de validación. Entonces, la solución que se recomienda escoger es la que corresponde al mínimo en el conjunto de validación (Goodfellow, Bengio y Courville 2016), donde la distancia entre el error en el conjunto de entrenamiento y validación es mínima. Este punto también se conoce como el de capacidad óptima del modelo, ya que si se escogen soluciones que se corresponden a pasos de iteración por debajo de este se encuentra un modelo que tiene demasiado error en cualquiera de los conjuntos; y si se escogen puntos por encima del de capacidad óptima, aparece el efecto de overfitting. Por último, el conjunto de prueba sirve para confirmar que la solución escogida tiene el mismo comportamiento en una serie de puntos que no se han utilizado para el entrenamiento. Si el entrenamiento ha alcanzado una solución satisfactoria, el comportamiento a lo largo de las iteraciones debería ser como se muestra en la Figura 3-29, aproximadamente. En la práctica, el conjunto total de datos se divide de forma aleatoria en los tres subconjuntos, de tal forma que aproximadamente se cumpla la proporción 80,10 y 10 por ciento, para el de entrenamiento, validación y prueba, respectivamente.

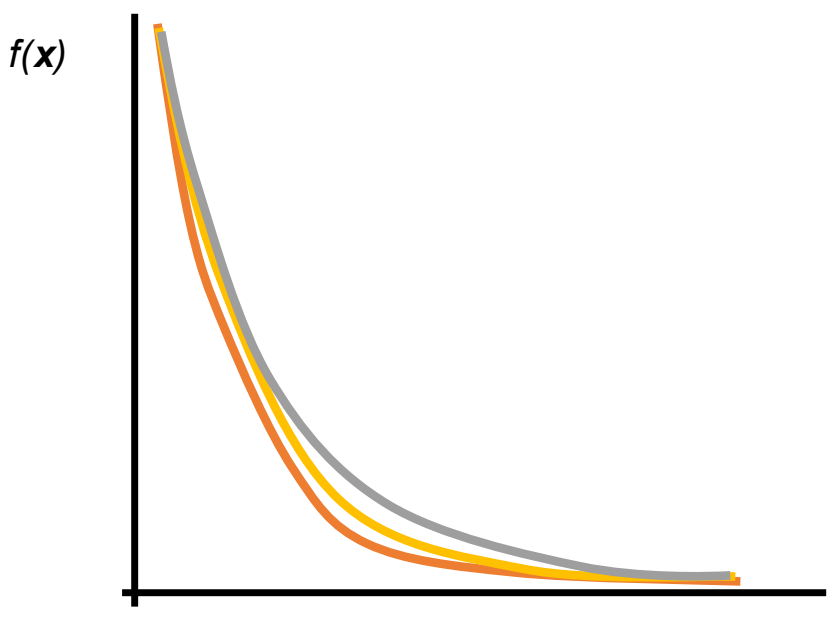

iteraciones

Figura 3-29 Evolución del valor de la función objetivo en un caso en el que se elige el punto de capacidad óptima. La línea naranja representa la evolución del error en el conjunto de entrenamiento, la amarilla en el conjunto de validación, y la naranja el de prueba. Fuente: elaboración propia.

\subsubsection{Algoritmos de optimización}

En este apartado se describen sucintamente una serie de algoritmos de optimización, que sirven para determinar el óptimo de una función objetivo (Gill y Murray 1978). Estos algoritmos son aplicables, en el caso de esta tesis, tanto para el cálculo del modelo de ajuste como para encontrar el mínimo de la función de peso. En el caso del modelo de ajuste, la función objetivo es la que se obtiene por la suma de los cuadrados de los residuos, tal y como se ha comentado previamente. En el caso del óptimo de la función de pesos para las configuraciones de cola no convencionales, dependerá del modelo de pesos y estructural seleccionado de entre los que se presentaron en la sección 3.3. Para generalizar la formulación matemática en todos los esquemas que se presenten, se considerará que las incógnitas se agrupan en una variable que recibirá el nombre de $\boldsymbol{x}$, y la función objetivo el nombre de $f$. En el caso de que se esté entrenando una red neuronal, las incógnitas corresponden con el conjunto de pesos y bias de la red que se haya construido. Sin embargo, en el caso de la función de peso, las $x$ corresponden con las variables de diseño sobre las que se esté buscando el óptimo. 


\subsubsection{Método del gradiente}

Como muchos otros métodos, es un proceso iterativo que parte desde una condición inicial, evalúa la función y da un paso hacia una dirección donde el valor de la función sea menor. Para encontrar la dirección en la que dar el paso, es necesario explorar cómo es la función alrededor del punto. La forma obvia de mostrar una dirección en la que la función decrece es mediante la diferencial de primer orden de la función:

$$
\partial f=\nabla f\left(x_{0}\right) \partial x>0
$$

Entonces, la dirección de perturbación por la que la función desciende es tal que:

$$
\partial x=-\nabla f^{T}\left(x_{0}\right)
$$

Independientemente del signo del vector gradiente, moverse a lo largo de la dirección negativa del gradiente será una dirección descendente, por tanto:

$$
\partial f=-\left|\nabla f\left(x_{0}\right)\right|^{2}<0
$$

Para construir el procedimiento iterativo, sin más que dar un paso de una longitud pequeña en la dirección del gradiente negativo, se obtiene el siguiente punto de iteración:

$$
\boldsymbol{x}_{k+1}=\boldsymbol{x}_{k}-\alpha_{k} \nabla f^{T}\left(\boldsymbol{x}_{k}\right)
$$

donde $k$ es el paso de iteración actual, $\alpha_{k}$ la longitud del paso $k$ y $k+1$ es el paso de iteración siguiente. La longitud de cada paso puede considerarse constante durante todo el procedimiento o irse ajustando a cada paso. En el caso de que se decida dar una longitud del paso constante, al procedimiento se le llama método del gradiente descendente. A medida que el algoritmo se aproxima al mínimo, el gradiente tiende a cero y la convergencia se hace más lenta (Papalambros y Wilde 2017). Por eso, se puede mejorar ese problema haciendo variable la longitud del paso en la dirección del gradiente, recibiendo el nombre de método del gradiente. De hecho, se puede determinar la longitud óptima que debe tomarse para que la función alcance el menor valor posible en la dirección del gradiente. Resolviendo este problema, se obtiene:

$$
\boldsymbol{x}_{k+1}=\boldsymbol{x}_{k}-\left(\frac{\nabla f\left(\boldsymbol{x}_{k}\right) \nabla f^{T}\left(\boldsymbol{x}_{k}\right)}{\nabla f\left(\boldsymbol{x}_{k}\right) \boldsymbol{H}_{k} \nabla f^{T}\left(\boldsymbol{x}_{k}\right)}\right) \nabla f^{T}\left(\boldsymbol{x}_{k}\right)
$$

Donde $\boldsymbol{H}_{k}$ es la matriz Hessiana de la función $f$ en el punto $\boldsymbol{x}_{k}$.

\subsubsection{Método de Newton}

Como se ha comentado anteriormente, en el procedimiento del método del gradiente descendente la convergencia se hace lenta cuando se está próximo a un punto estacionario. Una forma de mejorar esa convergencia es utilizar una aproximación de mayor orden:

$$
f\left(\boldsymbol{x}_{k+1}\right)=f\left(\boldsymbol{x}_{k}\right)+\nabla f_{k} \partial \boldsymbol{x}_{k}+\frac{1}{2} \partial \boldsymbol{x}_{k}^{T} \boldsymbol{H}_{k} \partial \boldsymbol{x}_{k}
$$

El punto que minimiza esta función se extrae de la condición de punto estacionario: 


$$
\nabla f^{T}\left(\boldsymbol{x}_{k}\right)+\boldsymbol{H}_{k} \partial \boldsymbol{x}_{k}=\mathbf{0}
$$

Si la matriz Hessiana es invertible, el paso de iteración queda:

$$
\boldsymbol{x}_{k+1}=\boldsymbol{x}_{k}-\boldsymbol{H}_{k}^{-1} \nabla f^{T}\left(\boldsymbol{x}_{k}\right)
$$

Si la función es localmente convexa, la matriz Hessiana es definida-positiva y el paso de iteración resulta en un punto donde la función adquiere un valor menor. Sin embargo, si la matriz es definida-negativa el método hace moverse hacia puntos donde los valores de la función son mayores. Por tanto, esto significa que este procedimiento es más preciso en las zonas próximas a un mínimo (Papalambros y Wilde 2017).

Una variante de este método es el llamado Gauss-Newton (Lai, Kek y Tay 2017), que consiste en aproximar la matriz Hessiana por: $\boldsymbol{J}_{k}^{T} \boldsymbol{J}_{k}$, evitando así calcular segundas derivadas que, en general, es computacionalmente más costoso.

\subsubsection{Método de Levenberg-Marquardt}

Este método surgió como consecuencia de los estudios que realizaron los matemáticos Levenberg y Marquardt para resolver problemas de mínimos cuadrados (Levenberg 1944; Marquardt 1963). En el algoritmo de Gauss-Newton la aproximación de la matriz Hessiana puede ser singular, pero se puede invertir de la siguiente forma:

$$
\boldsymbol{G}=\boldsymbol{H}+\mu \boldsymbol{I}
$$

Así, los autovalores de esta matriz son los que había antes incrementados en el parámetro $\mu$. El esquema finalmente quedaría:

$$
\boldsymbol{x}_{k+1}=\boldsymbol{x}_{k}-\left[\boldsymbol{J}_{k}^{T} \boldsymbol{J}_{k}+\mu_{k} \boldsymbol{I}\right]^{-1} \nabla f^{T}\left(\boldsymbol{x}_{k}\right)
$$

El parámetro $\mu$ determina cuánto se parece el procedimiento al método de GaussNewton. Para valores tendiendo a cero, el método se convierte en el de Gauss-Newton. Sin embargo, para valores del parámetro tendiendo a infinito, idealmente, el término dominante en el corchete es el segundo sumando, cuya inversa es directamente el inverso del parámetro $\mu$. Es decir, el siguiente paso de iteración sería un factor pequeño multiplicado por el gradiente. En conclusión, estaría aproximándose al método del gradiente descendente. Por lo tanto, ajustando el valor del parámetro se puede disfrutar de las ventajas del método del gradiente descendente cuando se está lejos de la solución y del método de Gauss-Newton cuando se está cerca del óptimo.

\subsubsection{Otros métodos}

Existen muchos otros métodos que se pueden utilizar para alcanzar el óptimo de una función que se basan en diferentes estrategias de búsquedas. Uno de los más conocidos es el método del gradiente conjugado, que es una variante del método del gradiente, y el método del gradiente conjugado escalado, que es una modificación del anterior, a su vez (Møller 1993). Por otra parte, otros métodos tratan de buscar una buena aproximación de la matriz Hessiana, para tratar de evitar su inversa, al igual que en el caso del método de Gauss-Newton. Estos métodos reciben el nombre de métodos Quasi-Newton (Gill y Murray 1972; Shanno 1970b). Entre ellos se encuentran aproximaciones clásicas como las de los métodos DFP, dado ese nombre por las iniciales de los autores que la desarrollaron (Davidon 1959; Fletcher y Powel 1963), y BFGS, también bautizado así por las iniciales de los autores (Broyden 1970; Fletcher 
1970; Goldfarb 1970; Shanno 1970a). Por último, se pueden encontrar métodos que no necesitan información acerca del gradiente para alcanzar el óptimo, como pueden ser, entre otros, los métodos heurísticos (Hare, Nutini y Tesfamariam 2013).

\subsubsection{Criterios de finalización de búsqueda}

Los criterios para determinar cuándo se debe parar el algoritmo de búsqueda del mínimo de una función deben contemplar diversas situaciones:

- Se alcanza una buena estimación de la solución.

- Se ha encontrado un punto de no progreso o de progreso lento.

- Se ha excedido un número aceptable de iteraciones.

- No existe una solución aceptable.

- El algoritmo alcanza una iteración cíclica.

Con eso se trata de establecer cuánto de lejos se encuentra del óptimo y si la secuencia es convergente. Idealmente, habría que medir la distancia entre el punto que se encuentra el algoritmo y el óptimo. Como es lógico, el óptimo no se conoce, por lo que se pueden utilizar las siguientes condiciones, donde $k$ representa el paso de iteración:

$$
\begin{gathered}
\left|f_{k}-f_{k+1}\right|<\epsilon \\
|| x_{k}-x_{k+1}||<\epsilon
\end{gathered}
$$

También se puede imponer la condición de que el gradiente de la función tiende a cero, asumiendo que en ese punto se tiene un mínimo, ya que, salvo raras excepciones, no se debería alcanzar un punto estacionario que no lo sea. Por tanto, otra condición sería:

$$
|| \nabla f_{k} \|<\epsilon
$$

En los casos en los que los algoritmos estén resolviendo problemas sometidos a ciertas restricciones, es necesario comprobar que no se incumplen las mismas, donde la función $\boldsymbol{g}_{k}$ engloba todas las restricciones del problema:

$$
|| \boldsymbol{g}_{k}||<\epsilon
$$

Determinar un criterio de finalización de la búsqueda del óptimo es una cuestión de experiencia y de adaptación a cada problema, por eso que no se amplían más conceptos sobre este tema aparte de los criterios clásicos expuestos aquí. Más información y referencias se pueden encontrar en (Papalambros y Wilde 2017; Boyd y Vandenberghe 2009).

\subsubsection{Monotonía y actividad}

Aunque ya se ha presentado someramente el concepto de actividad, en este apartado se va a retomar para resaltar la importancia y la utilidad que tiene (Chan 2006). Como se ha expuesto previamente, se considera que una restricción es activa si al eliminarla el óptimo cambia de posición. En caso contrario se dice que la restricción es inactiva, siempre y cuando no sea redundante. La utilidad que tiene este fenómeno es que cualquier restricción que se demuestre ser activa permite reducir los grados de libertad del problema en al menos uno y, en consecuencia, eliminar una variable del modelo. Por otro lado, cualquier restricción que se demuestre ser inactiva se puede dejar de 
tener en cuenta para la resolución del problema. Ambas simplificaciones reducen el tiempo de computación de obtención del óptimo.

Por otra parte, la monotonía de la función objetivo y de las restricciones pueden ser explotadas para simplificar el problema o mejorar la compresión de este (Hansen, Jaumard y Lu 1989; Papalambros 1979; Chan 2006). Lo primero es enunciar el Teorema de la Monotonía: dada una función $f(x)$ y las consistentes funciones de restricción $g_{i}(x)$, si todas ellas son crecientes o todas decrecientes con respecto de $x$, el dominio del problema de minimización no está bien restringido. Esta definición no es muy útil en sí misma, aunque la negación de las hipótesis lleva a construir los principios de monotonía. El primer principio de monotonía (MP1) nos indica: en un problema de minimización bien restringido, cualquier variable creciente está restringida por debajo por al menos una restricción activa no creciente. El principal valor del MP1 es que nos permite probar que una restricción es activa sin tener que determinar el óptimo primero. Las restricciones que hacen que el problema esté correctamente restringido reciben el nombre de críticas. Es decir, que la criticidad es un caso particular del concepto de actividad de una restricción.

Existen varios conceptos derivados del análisis de la monotonía aplicado a restricciones definidas mediante una inecuación, que son las que aparecen en esta tesis. Estos conceptos son: criticidad condicional, criticidad múltiple, dominancia (Wilde 1975), relajación y no criticidad. Para entender el primer concepto se supone una variable es monótona para la función objetivo y, a su vez, tiene una monotonía opuesta para una serie de restricciones. Entonces, si estas restricciones no son críticas para ninguna otra variable, por el MP1, al menos una de ellas es crítica, aunque no se sepa cuál de ellas. Este conjunto de restricciones recibe el nombre de condicionalmente críticas para la variable en cuestión. Si el número de restricciones condicionalmente críticas es igual a uno, se recupera el caso de una restricción crítica. Si alguna de estas restricciones es crítica para varias variables, se dice que es múltiplemente crítica. Este tipo de restricciones complican la comprensión del problema y la determinación de si está correctamente restringido. Por tanto, es recomendable tratar de eliminar este tipo de restricciones. Por otra parte, es posible probar que una restricción es inactiva. Si dos restricciones son tales que $g_{2}(\boldsymbol{x})<g_{1}(\boldsymbol{x}) \leq 0$ para todos los valores factibles de $\boldsymbol{x}$, entonces la restricción $g_{2}$ no puede ser activa sin violar la $g_{1}$. Si esto ocurre, se dice que $g_{2}$ está dominada por $g_{1}$ y recibe el nombre de restricción redundante. Esto se demuestra mediante el Teorema de Dominancia (Papalambros y Wilde 2017). Otra forma de determinar la actividad de una restricción es resolver el problema sin tener en cuenta la restricción y comprobar si el óptimo infringe la restricción. El hecho de obviar una restricción recibe el nombre de relajación de la restricción. Por medio del Teorema de Relajación se puede demostrar que si el óptimo de un problema con una restricción relajada incumple esta, entonces la restricción es activa (Papalambros y Wilde 2017). En consecuencia, si el óptimo del problema relajado no incumple la restricción relajada, se puede decir que, si el mínimo es único, la restricción es inactiva. Esta estrategia puede ser interesante si el problema relajado es mucho más simple de resolver que el restringido. Por último, el concepto de no criticidad aplica a restricciones que crecen con respecto a una cierta variable si la función objetivo también lo hace, por supuesto asumiendo un problema de minimización y donde las restricciones se definen siguiendo la forma nula negativa $\left(g_{i}(\boldsymbol{x}) \leq 0\right)$. Si una restricción no es crítica respecto una variable y no depende de ninguna otra, se concluye que es inactiva o inconsistente (Teorema de no Criticidad) (Papalambros y Wilde 2017). 


\subsection{RESUMEN Y CONCLUSIONES}

En este capítulo se han presentado todas las herramientas necesarias para poder construir y resolver el problema de dimensionado óptimo de una configuración de cola no convencional. El principal valor añadido es que los criterios de diseño se basan en la normativa de certificación aplicable, lo que es totalmente necesario para que los resultados que se obtengan tengan un punto de vista realista. Para poder cumplir los requisitos basados en la normativa de certificación, es necesario desarrollar un modelo aerodinámico para traducir a ecuaciones esos requisitos. Este modelo debe poder estimar tanto derivadas de coeficientes aerodinámicos como distribuciones de fuerza a lo largo de la superficie de cola. Esto lleva a seleccionar un método de Vortex Lattice, en concreto se utilizará el software Tornado que tiene implementado este método. Este tipo de software presenta una serie de limitaciones, siendo la principal de ellas que se basa en aerodinámica lineal, por tanto, no es posible estimar con precisión regímenes de vuelo transónicos. Como máximo, se puede alcanzar un régimen subsónico alto con la corrección de Prandtl-Glauert. A pesar de ello, esto únicamente afecta a los requisitos que se impongan sobre el vuelo de crucero, ya que varias de las restricciones aplican en maniobras de despegue y aterrizaje, donde el régimen se puede considerar incompresible. Para intentar subsanar las limitaciones de Tornado, se ha combinado con metodologías semi-empíricas clásicas utilizadas en diseño conceptual de aviones de transporte comercial. De esta forma, se ha construido un modelo aerodinámico donde exclusivamente se ha utilizado Tornado para resolver la aerodinámica de la superficie de cola y la metodología semi-empírica para el resto de los elementos del avión, tales como el ala, la interacción con el fuselaje e incluso para corregir las deflexiones de los controles y deflexión de estela que entran a Tornado para considerar comportamiento no lineal. Este modelo se ha enfocado de tal forma que es perfectamente utilizable para casi cualquier geometría de cola. Es cierto que es posible que algunos de los coeficientes correctores semi-empíricos, como puede ser la interacción entre la cola y el fuselaje, dependerán de la geometría considerada. Incluso es posible que para alguna configuración en concreto no sea posible establecer algunos términos como el anterior mediante factores semi-empíricos, pero siempre será factible utilizar un programa de CFD o realizar una serie de ensayos en túnel aerodinámico para determinar estos términos que no sean directamente estimables mediante correcciones semi-empíricas. De la misma forma, se deben utilizar estas herramientas para poder extender la metodología a números de Mach de vuelo que correspondan a régimen compresible, tanto para estimar las derivadas de los coeficientes aerodinámicos como las fuerzas y momentos correspondientes. Sin embargo, se ha comprobado que la metodología propuesta se comporta de la misma forma que los métodos semi-empíricos de diseño conceptual utilizados habitualmente, en el rango de números de Mach considerados en este estudio. Esta nueva metodología, aplicada a una configuración de cola en V, ha sido recientemente publicada (Sanchez-Carmona y Cuerno-Rejado 2018b).

La función objetivo que se quiere optimizar será el peso, por tanto, es necesario construir un modelo de estimación de pesos también. Este modelo se puede basar en ecuaciones estadísticas o en las cargas que debe soportar, dimensionando así más en detalle la estructura. Las ecuaciones estadísticas son más fáciles de implementar, pero tienen el inconveniente de que están construidas para las configuraciones de cola convencionales, por lo que su extensión a configuraciones no convencionales debe hacerse con mucho cuidado. De ahí que, dependiendo de la configuración a estudio, sea más recomendable dimensionar la estructura en función de las cargas que debe soportar. Esto es generalizable a cualquier geometría, siempre y cuando las cargas 
estén adecuadamente modelizadas. Puesto que las cargas se basan en el modelo aerodinámico, los resultados están condicionados por las limitaciones que tienen este módulo. La metodología de diseño conceptual desarrollada parte de las que se utilizan para dimensionar la estructura del ala, elemento que está mucho más estudiado que las superficies de cola. Se ha adaptado esta metodología para las configuraciones de cola no convencionales, analizando la aplicabilidad de cada uno de los términos que se contemplan en la metodología. Además, se ha dejado libre la posibilidad de seleccionar el factor de Farrar que se desee para diseñar la estructura, en contraposición de algunos modelos que se encuentran en la literatura abierta que establecen uno fijo. El principal inconveniente que tiene este modelo es que está pensado para materiales metálicos, que son los que se usan en la fabricación del ala. La primera aproximación que se ha hecho es corregir el peso final por un factor determinado por la diferencia de densidades entre los materiales metálicos utilizados en el ala y los materiales compuestos utilizados en las superficies de cola. Se ha querido comprobar la validez de esta hipótesis desarrollando un método de dimensionado de un panel rigidizado extrapolando los mismos conceptos que se emplean en el método para las aleaciones de aluminio. Se ha concluido que aplicar un factor corrector constante e igual al cociente de densidades no es preciso, ya que el peso de la estructura depende del caso de cargas en concreto, como era de esperar. Este método de dimensionado del panel en material compuesto ha sido aceptado para ser publicado en una revista indexada en JCR (SanchezCarmona y Cuerno-Rejado 2018a). El problema es que este modelo que contempla el dimensionado de la estructura directamente en material compuesto no está completo, ya que únicamente se ha estudiado el diseño de un panel rigidizado sometido a compresión y cortadura. Sería necesario estudiar el comportamiento de un panel en un estado de tensión diagonal, para dimensionar los largueros, y encontrar un procedimiento para estimar el peso de las costillas de los estabilizadores. Además, la metodología para aleaciones de aluminio incluye algunos términos basados en estudios estadísticos, que no serían directamente aplicables a material compuesto, y deberían ser revisados. Por ejemplo, el peso de remaches se establece mediante una correlación que depende del peso máximo de despegue del avión. Puesto que los requisitos de remachado en materiales compuestos son distintos, es de esperar que esa regresión no sea válida para las configuraciones de cola. Debido a esto, los métodos que se contemplarán para el caso de estudio serán el estadístico y el basado en cargas para material metálico, con la corrección de densidad, asumiendo las posibles imprecisiones de los mismos.

Los requisitos basados en la certificación vigente establecen una serie de restricciones al diseño, y se trata de minimizar la función objetivo considerando esas limitaciones. De ahí que se hayan presentado una serie de herramientas para resolver el problema de optimización. El primer paso sería establecer las variables de diseño, que dependerán de la configuración no convencional de estudio. Posteriormente, habría que analizar las restricciones de acuerdo con las variables seleccionadas. Para ello, se pueden utilizar modelos de ajuste para trabajar más cómodamente con las limitaciones, ya que, por el modelo aerodinámico propuesto, no es posible determinarlas analíticamente. Una posibilidad, que tiene la principal ventaja que no es necesario suponer ninguna forma de la función a priori, es utilizar redes neuronales para construir la regresión. Esta opción también facilita el trabajo si las funciones dependen de varias variables. Por último, habría que buscar el óptimo sobre la función objetivo sometida a las restricciones. Se han presentado una serie de algoritmos de optimización, aunque en la medida de lo posible se hará un análisis del problema desde el punto de vista de la topología de las funciones involucradas, para intentar simplificarlo lo más posible y extraer conclusiones 
DISEÑO CONCEPTUAL DE CONFIGURACIONES DE ESTABILIZADORES DE COLA NO CONVENCIONALES

más relevantes. Para ello se han descrito un conjunto de estrategias y definiciones que pueden ayudar a este propósito, como es el estudio de monotonía y criticidad. 
RESUMEN Y CONCLUSIONES 


\section{RESULTADOS DEL DISEÑO DE CONFIGURACIONES NO CONVENCIONALES DE COLA}




\subsection{INTRODUCCIÓN}

En este capítulo se va a mostrar un caso de aplicación de dimensionado de una configuración de cola no convencional. El objetivo es diseñar la cola de tal forma que desarrolle las mismas funciones que las de una cola convencional de un avión de referencia, de tal forma que se pueda sustituir una por la otra. Finalmente, se compararán el resultado y la cola convencional con el fin de extraer una serie de conclusiones. El primer paso para proceder al diseño es la selección de los parámetros de diseño. Estos parámetros deben caracterizar adecuadamente la configuración. Posteriormente, se definirá el espacio de diseño factible que cumple con las restricciones del problema. Se recuerda que las condiciones que se van a contemplar para garantizar que la configuración no convencional cumple las mismas funciones que la convencional son las siguientes:

- Asegurar que las derivadas de estabilidad estática en crucero son iguales que las del avión de referencia, o tales que el avión tenga un comportamiento estático más estable.

- Permitir el control de la aeronave tras el fallo del motor crítico en el segundo segmento. Para ello la deflexión del timón de dirección debe poder anular el momento de guiñada generado por el desequilibrio de empuje con un ángulo de balance resultante inferior a $5^{\circ}$ y con un ángulo de deflexión del control inferior al límite.

- Permitir el control del avión en condiciones de aterrizaje con viento cruzado. Para ello la deflexión del timón de dirección debe poder anular el momento de guiñada generado por el desequilibrio de empuje con un ángulo de balance resultante inferior a $5^{\circ}$ y con un ángulo de deflexión del control inferior al límite.

- El ángulo del timón de profundidad para el trimado del avión no debe exceder los límites del control.

Hay que añadir que se pueden establecer más condiciones que complementan a las anteriores y restringirían más el espacio de diseño. Sin embargo, se ha decidido empezar por estas como una primera aproximación para la etapa de diseño conceptual, que es el marco en el que se ha querido desarrollar el estudio.

Dentro del espacio de diseño factible, se procederá a determinar el punto donde se optimiza la función objetivo. Esta función será el peso de la cola, que se puede estimar por cualquiera de los métodos presentados en el capítulo 3.3. Se recuerda que los procedimientos contemplados serán dos: el basado en correlaciones estadísticas y el basado en la metodología de Farrar, corrigiendo con un factor porque los materiales empleados no son metálicos, sino materiales compuestos.

Una vez establecido el diseño final, la comparativa permitirá al diseñador extraer una serie de conclusiones desde diversos puntos de vista. Se puede determinar si la configuración seleccionada resulta tener un peso menor que la convencional o saber si genera una menor resistencia parásita. Estos dos aspectos son los principales, ya que se ha visto que son las dos variables que describen si se va a producir un ahorro de combustible o no. 


\subsection{AVIÓN DE REFERENCIA: CSR-01}

En este apartado se van a recopilar los datos y cálculos necesarios relacionados con el avión de referencia. Al igual que ocurrió en el capítulo dedicado a la caracterización de las configuraciones convencionales mediante regresiones, el avión de referencia se va a tomar de la base de datos CeRAS. En concreto, se ha elegido el único avión con información completa disponible, el CSR-01. Como se ha comentado en capítulos anteriores, en la base de datos es posible encontrar información acerca de su geometría, pesos y actuaciones. En la Tabla 4-1 se incluyen los principales datos de carácter general que van a ser necesarios para el desarrollo del estudio. Además, en la Figura 4-1 y Figura 4-2 se muestran la forma en planta del ala y las vistas del avión, incluyendo los dispositivos hipersustentadores y las posiciones de los largueros anterior y posterior.

\begin{tabular}{|c|c|c|}
\hline Parámetro & Unidades & Valor \\
\hline Superficie alar & {$\left[\mathrm{m}^{2}\right]$} & 122,40 \\
\hline Envergadura alar & {$\left[\mathrm{m}^{2}\right]$} & 12,46 \\
\hline Cuerda media aerodinámica del ala & {$\left[\mathrm{m}^{2}\right]$} & 3,46 \\
\hline $\begin{array}{c}\text { Separación en el eje z cuerpo entre la línea de actuación } \\
\text { de los motores y el centro de gravedad }\end{array}$ & {$[\mathrm{m}]$} & 1,32 \\
\hline $\begin{array}{l}\text { Distancia lateral entre el motor crítico y el plano de } \\
\text { simetría }\end{array}$ & {$[\mathrm{m}]$} & 5 \\
\hline Brazo de momentos del estabilizador horizontal & {$[\mathrm{m}]$} & 17,57 \\
\hline Brazo de momentos del estabilizador vertical & {$[\mathrm{m}]$} & 16,88 \\
\hline Empuje del motor crítico en despegue & {$[\mathrm{kN}]$} & 117 \\
\hline Posición del centro aerodinámico del avión & {$[\% \mathrm{CMA}]$} & 28,6 \\
\hline Posición longitudinal del centro de gravedad al despegue & {$[\% \mathrm{CMA}]$} & 31 \\
\hline Posición longitudinal del centro de gravedad al aterrizaje & {$[\% \mathrm{CMA}]$} & 31,75 \\
\hline Peso máximo de despegue & $\mathrm{kg}$ & 77000 \\
\hline Peso máximo de aterrizaje & $\mathrm{kg}$ & 64500 \\
\hline Peso vacío operativo & $\mathrm{kg}$ & 42100 \\
\hline Peso máximo sin combustible & $\mathrm{kg}$ & 62100 \\
\hline Peso máximo de combustible & $\mathrm{kg}$ & 18700 \\
\hline Carga de pago de diseño & $\mathrm{kg}$ & 13608 \\
\hline Peso del estabilizador horizontal & $\mathrm{kg}$ & 682 \\
\hline Peso del estabilizador vertical & $\mathrm{kg}$ & 522 \\
\hline
\end{tabular}

Tabla 4-1 Parámetros del avión CSR-01 incluidos en la base de datos CeRAS. Fuente: elaboración propia. 


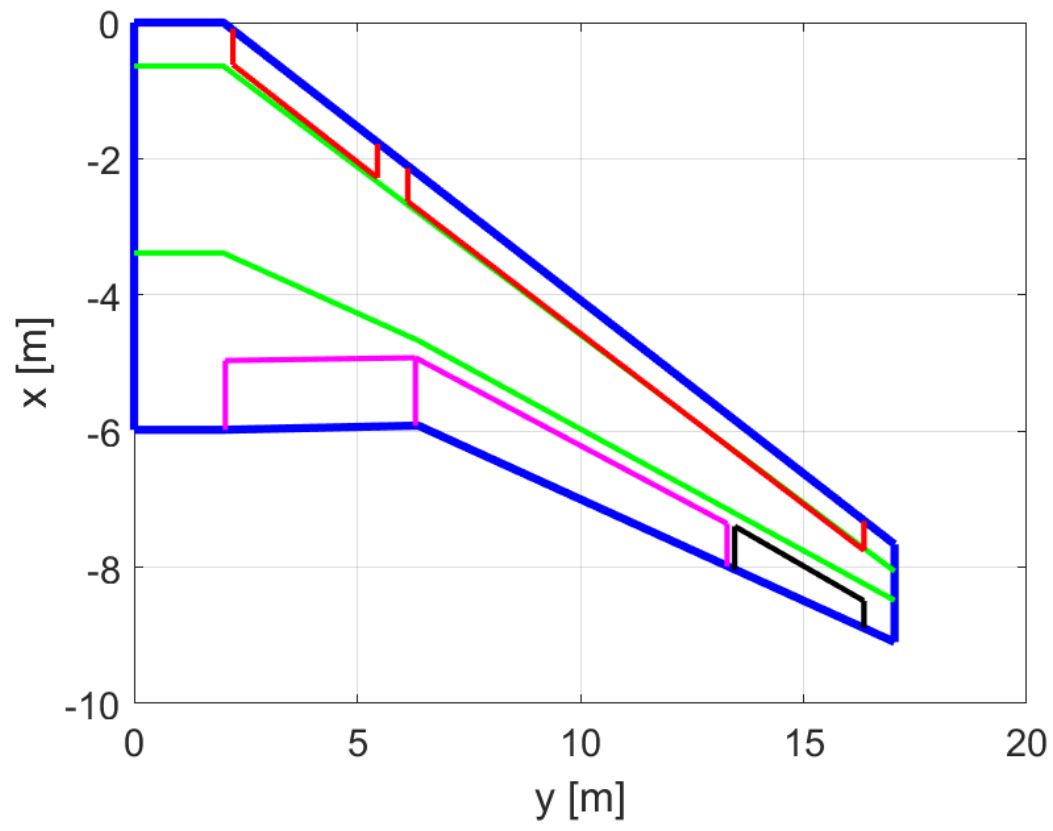

Figura 4-1 Forma en planta del ala del avión CSR-01. Fuente: elaboración propia.
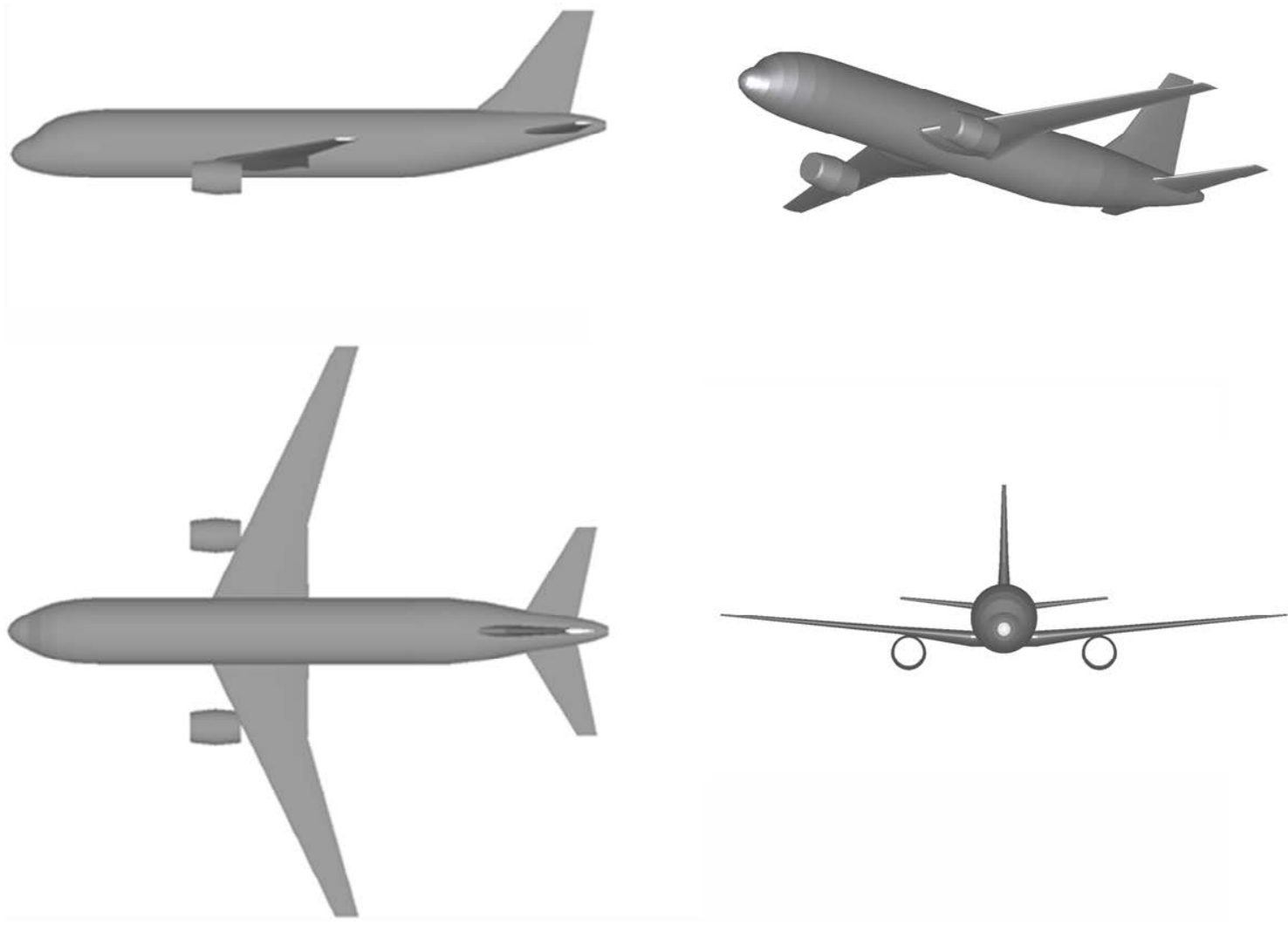

Figura 4-2 Vistas del avión CSR-01. Fuente: (ILR y RWTH-Aechen-University 2014).

A la vista de la forma en planta del ala, de la información que se recoge en la base de datos y de las directrices de diseño que se pueden encontrar en los libros de diseño conceptual (Torenbeek 1982; Sadraey 2013; Nicolai y Carichner 2010), se pueden determinar las propiedades geométricas de los dispositivos hipersustentadores del ala. Esta información se recoge en la Tabla 4-2. Por terminar con la información sobre el ala, la base de datos también incluye las geometrías de los perfiles utilizados por secciones, la ley de torsión que siguen estos perfiles y el ángulo de diedro (Tabla 4-3). Las 
geometrías y nomenclatura de los perfiles se reflejan en la Figura 4-3. Se quiere añadir que en la base de datos no aparece más que la geometría de los perfiles, por lo que se han estimado sus propiedades aerodinámicas por medio del software X-FOIL.

\begin{tabular}{|lccc|}
\hline & $\begin{array}{c}\text { Posición en porcentaje de } \\
\text { semienvergadura }\end{array}$ & $\begin{array}{c}\text { Porcentaje de } \\
\text { cuerda }\end{array}$ & $\begin{array}{c}\text { Extensión de } \\
\text { cuerda }\end{array}$ \\
\hline Slat interior & $13 \%-32 \%$ & $11 \%$ & $10 \%$ \\
\hline Slat exterior & $36 \%-96 \%$ & $25 \%$ & $10 \%$ \\
\hline Flap interior & $12 \%-37 \%$ & $23 \%$ & $10 \%$ \\
\hline Flap exterior & $37 \%-78 \%$ & $27 \%$ & $22 \%$ \\
\hline
\end{tabular}

Tabla 4-2 Parámetros geométricos del avión CSR-01 sobre los dispositivos hipersustentadores.

Fuente: elaboración propia.

\begin{tabular}{|cccc|}
\hline & $y=2 m$ & $y=6,37 m$ & $y=17,04 m$ \\
\hline Ángulo de diedro & $0^{\circ}$ & $5^{\circ}$ & $5^{\circ}$ \\
\hline Ángulo de incidencia & $3,5^{\circ}$ & $0,5^{\circ}$ & $0,3^{\circ}$ \\
\hline Espesor relativo & $15 \%$ & $12 \%$ & $11 \%$ \\
\hline Perfil aerodinámico & $\mathrm{F} 15-15$ & $\mathrm{~F} 15-12$ & $\mathrm{~F} 15-11$ \\
\hline
\end{tabular}

Tabla 4-3 Parámetros del diedro, torsión, espesor relativo y perfil por secciones del ala del CSR-01 indicadas por la distancia al plano de simetría (y). Fuente: elaboración propia.

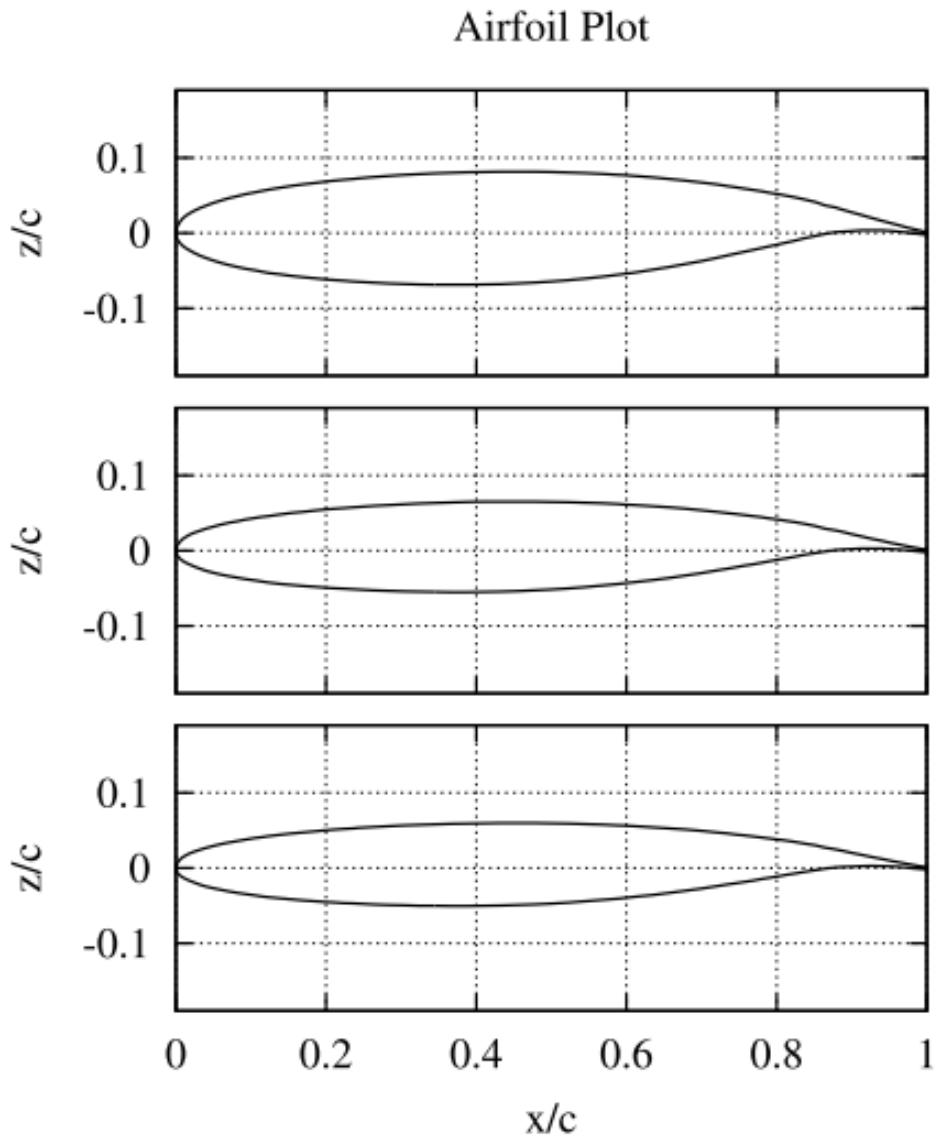

F15-15

F15-12

F15-11

Figura 4-3 Perfiles del ala del CSR-01. Fuente: (ILR y RWTH-Aechen-University 2014). 
Adicionalmente, la base de datos incluye información acerca de la polar del avión dependiendo del número de Mach de vuelo y de la configuración de vuelo. En la Figura 4-4 se agrupan las polares en configuración limpia y dependiendo del número de Mach, y en la Figura 4-5 se muestran las polares en configuraciones de despegue, ascenso, aproximación y aproximación con el tren extendido, respectivamente.

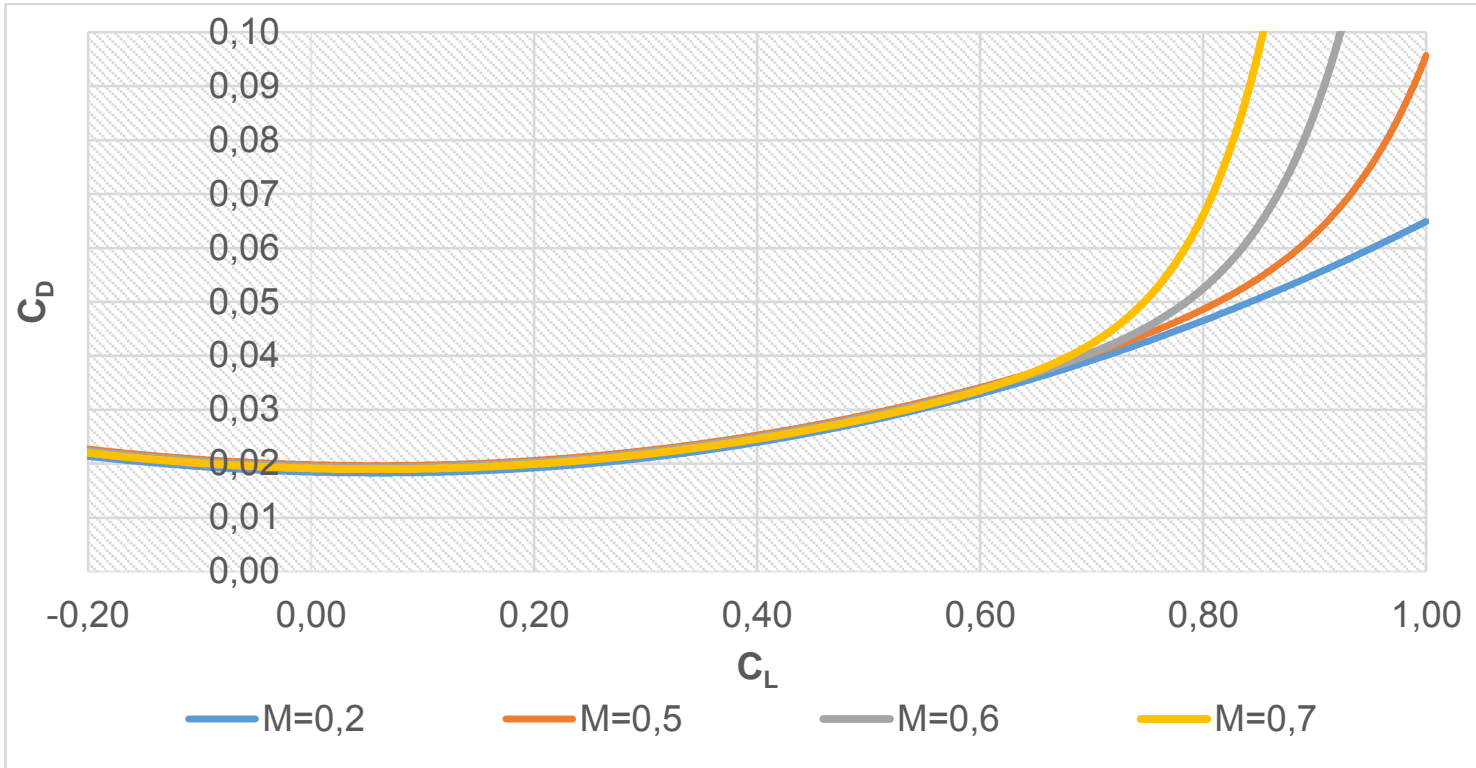

Figura 4-4 Polar del avión CSR-01 en configuración limpia en función del número de Mach de vuelo. Fuente: elaboración propia.

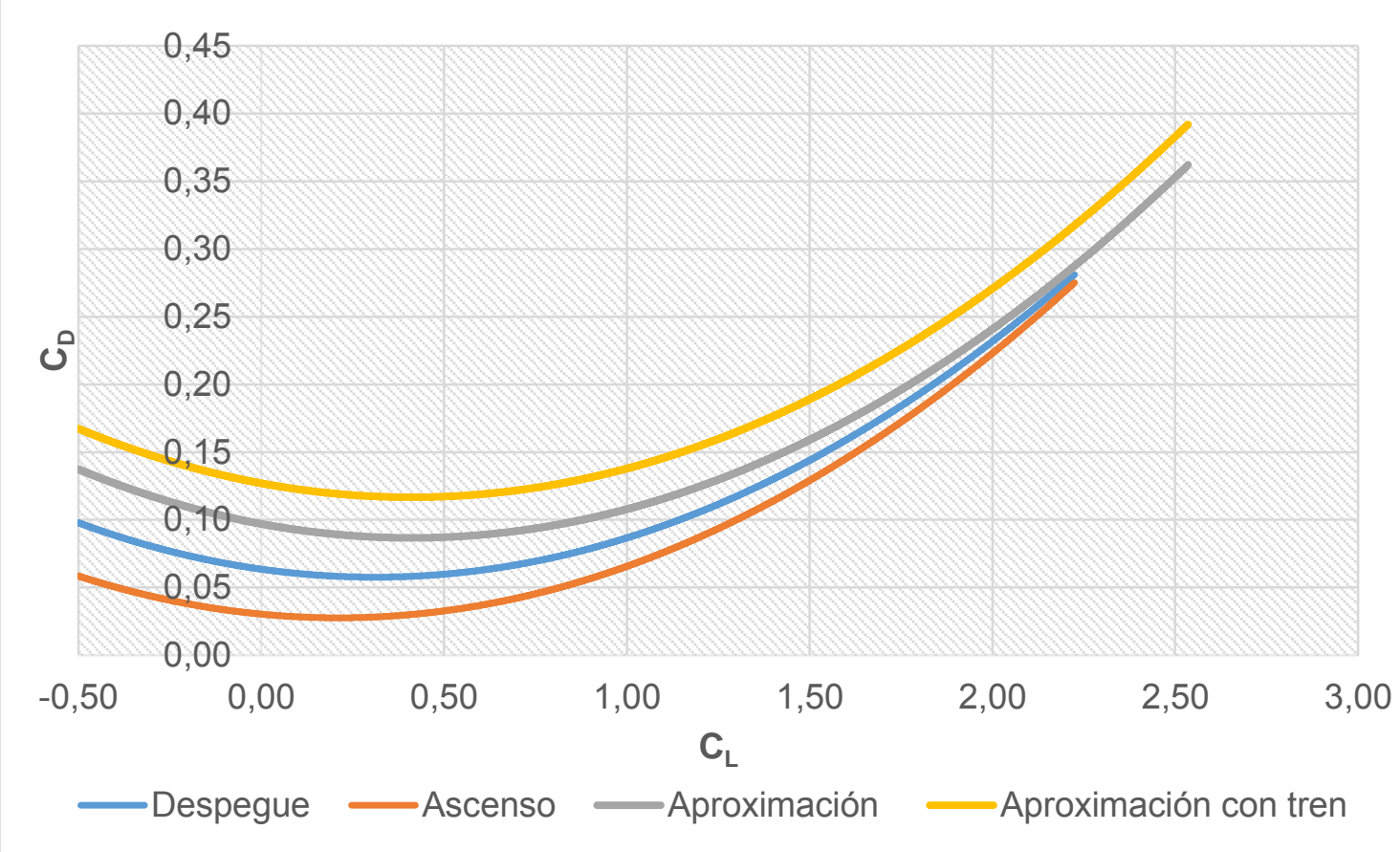

Figura 4-5 Polar del avión CSR-01 en configuración de despegue, ascenso, aproximación y aproximación con el tren de aterrizaje extendido. Fuente: elaboración propia.

En cuanto a los estabilizadores de cola, las geometrías externas están perfectamente definidas en la base de datos, sin embargo, existen una serie de datos que no están incluidos en la base de datos pero que son necesarios para el estudio, como por ejemplo las deflexiones máximas de los controles. Para ello, se ha recopilado información de 


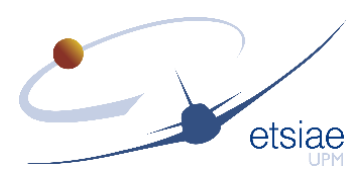

aviones semejantes, como es el caso del Airbus A320. Para las deflexiones de los controles, se ha encontrado un documento que describe los controles de vuelo de donde se ha podido extraer esta información (Airbus 2005). Ahí se indica que el timón de profundidad tiene un rango de deflexiones comprendido en el intervalo $\left[-17^{\circ}, 30^{\circ}\right]$. Por otra parte, la deflexión máxima del timón de dirección depende de la velocidad de vuelo, ya que la regulación establece que la fuerza aplicada por el piloto no debe exceder 667 $\mathrm{N}$ (EASA 2018). La deflexión máxima extraída del documento se incluye en la Figura 4-6. No están definidos los porcentajes de cuerda que ocupan los timones de dirección y de profundidad. Por tanto, se ha decidido que ocupen todo el borde de salida en un porcentaje de cuerda del 25\%, a la vista del estado del arte. En cuanto a los perfiles aerodinámicos que componen los estabilizadores, se ha considerado que el horizontal lleva un NACA 0012 y el vertical un NACA 0009.

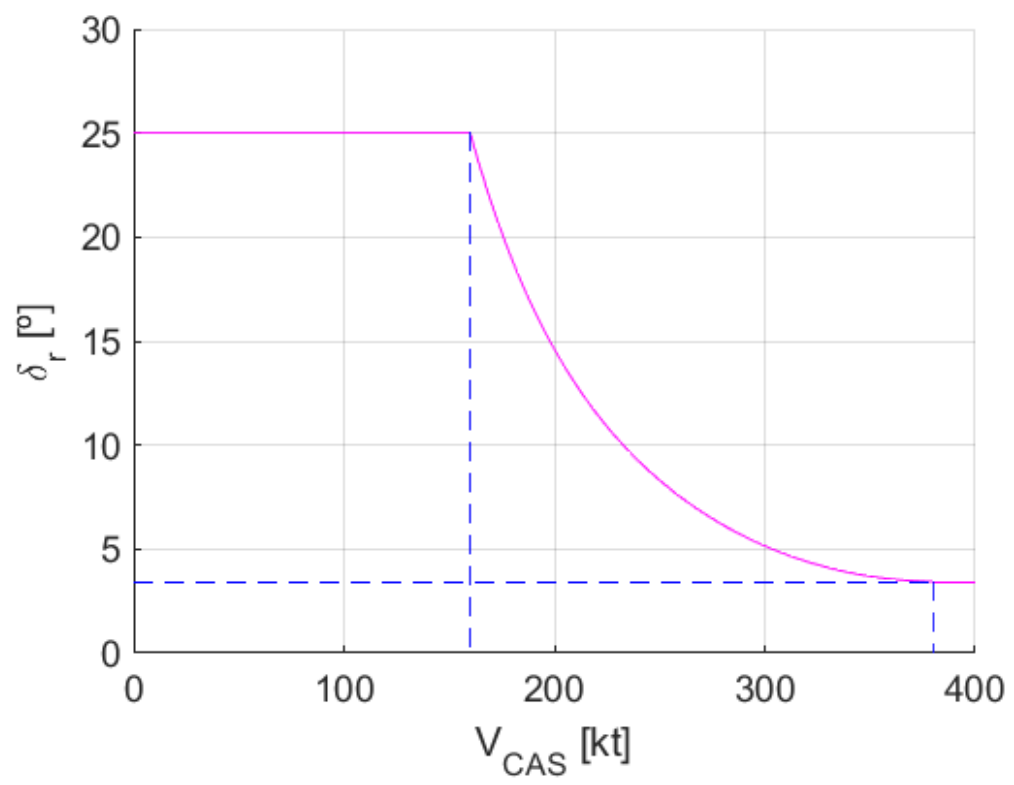

Figura 4-6 Estimación de la deflexión máxima del timón de dirección del CSR-01. Fuente: elaboración propia.

Gracias a que están completamente definidas las geometrías de los estabilizadores de cola, se puede estimar la contribución que presentan estas superficies a la resistencia sin sustentación en crucero del CSR-01. Para ello, se va a utilizar la aproximación que se propone en la referencia (Torenbeek 1982), tanto para el estabilizador horizontal como vertical:

$$
c_{D} S_{w}=2 c_{F}\left(1+2,75 t / c \cos ^{2} \Lambda_{0,5}\right) S
$$

En la expresión anterior, el parámetro $S$ representa la superficie en planta de cada uno de los estabilizadores, incluyendo el tramo de superficie que se encuentra en el interior del fuselaje o del otro estabilizador. De esta forma se tiene en cuenta el incremento de resistencia asociado a la interacción con el fuselaje y con la otra superficie (Torenbeek 1982). El coeficiente de fricción, $C_{F}$, depende del número de Reynolds basado en la cuerda media geométrica de cada uno de los estabilizadores. Para el estabilizador vertical, el coeficiente de fricción es aproximadamente 0,0037 , para un número de Reynolds de vuelo de $2,57 \times 10^{7}$. En el caso del estabilizador horizontal el número de Reynolds es de $1,58 \times 10^{7}$ y el coeficiente de fricción correspondiente es de 0,0042 . El 
espesor relativo $t / c$ de las superficies es 0,12 y 0,09 para el estabilizador horizontal y vertical, respectivamente. Por último, las flechas de puntos $1 / 2$ de las superficies son $28^{\circ}$ para el estabilizador horizontal y $35^{\circ}$ para el vertical. Con todo esto, se puede determinar la contribución al coeficiente de resistencia sin sustentación del CSR-01, $C_{D 0}$, sin más que pasar dividiendo la superficie alar al otro lado del igual. Los resultados que se obtienen se incluyen en la Tabla 4-4. La suma de estas dos contribuciones supone aproximadamente un $17 \%$ de la resistencia sin sustentación que presenta el avión en el vuelo de crucero, siendo esta última obtenida de la información que se encuentra en la base de datos CeRAS.

\begin{tabular}{|ccc|} 
& Estabilizador vertical & Estabilizador Horizontal \\
\hline $\boldsymbol{c}_{\boldsymbol{D} 0}$ & 0,0014 & 0,0019 \\
\hline
\end{tabular}

Tabla 4-4 Valores del coeficiente de resistencia sin sustentación de los estabilizadores referidos a la superficie alar del CSR-01. Fuente: elaboración propia.

Desde el punto de vista del modelo aerodinámico expuesto en el capítulo anterior, existe una limitación en Mach de crucero para poder estimar con fiabilidad las propiedades aerodinámicas y las cargas generadas en la cola. Por ello, se ha analizado la envolvente de maniobra del CSR-01 para diferentes pesos y altitudes. Asumiendo que ese Mach límite es 0,6 , correspondiente a un régimen subsónico alto, se ha buscado la altitud para la cual la velocidad máxima de maniobra $V_{A}$ coincide con dicho Mach límite, que corresponde a $22000 \mathrm{ft}$ aproximadamente. Precisamente se ha buscado que la velocidad de maniobra quede en ese Mach de crucero porque uno de los casos de carga mencionados que se incluyen en la normativa es la deflexión máxima del timón de profundidad a la velocidad máxima de maniobra. Como es lógico, sería necesario comprobar muchos más casos de carga que se producen en régimen transónico, por lo que sería necesario hacer uso de otras herramientas de cálculo aerodinámico como pueden ser los programas de CFD. Sin embargo, esto no cambia conceptualmente la metodología de estimación del peso de la estructura, únicamente que habría una mayor variedad de casos de carga.

\subsubsection{Ruta de diseño}

En este apartado se va a realizar un análisis de la ruta de diseño que se indica en la base de datos CeRAS. La misión de diseño consiste en transportar $13608 \mathrm{~kg}$ de carga de pago a $2750 \mathrm{MN}$. El número Mach de crucero es 0,78 y la altitud de $35000 \mathrm{ft}$. En la base de datos se dice que esta misión se realiza con un peso al despegue de $74102 \mathrm{~kg}$, ligeramente inferior al peso máximo de despegue. Además, el peso del combustible de reserva es de $3258 \mathrm{~kg}$ y el que se consume en el viaje es de $14992 \mathrm{~kg}$, excluyendo lo que se consume en el taxi antes del despegue y tras el aterrizaje.

Se quiere presentar un método de estimación del consumo de combustible que permita ser utilizado para estimar el consumo de combustible en esta misma misión, pero llevada a cabo por el avión con la configuración de cola no convencional. En una primera aproximación, se va a utilizar la ecuación de Breguet para estimar el consumo de combustible en la ruta de diseño, aproximando todo el viaje como si fuese crucero (Torenbeek 1982; Martínez-Val, Pérez, et al. 1994). De esta forma, la ecuación de Breguet para el alcance de la ruta de diseño queda como sigue:

$$
R=K \ln \frac{T O W}{L W}
$$




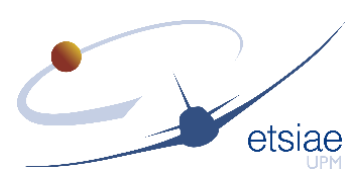

La $K$ de la ecuación de Breguet se estima, para un avión turbofán, de la siguiente forma:

$$
K=\frac{V}{c_{e}} \frac{c_{L}}{c_{D}}
$$

En la definición aparecen la velocidad de vuelo, $V$, el consumo específico, $c_{e}$, y la eficiencia aerodinámica, $c_{L} / c_{D}$. La velocidad de vuelo viene fijada por la misión de diseño. El consumo específico de los motores se encuentra en la base de datos, siendo de $16,03 \mathrm{mg} / \mathrm{Ns}$ para la condición de crucero de la ruta de diseño. La eficiencia aerodinámica corresponde a la media de la ruta del avión y se puede estimar en el punto en el que el CSR-01 ha consumido la mitad del combustible del viaje. En ese punto, el avión tiene un peso equivalente a la semisuma entre el peso de despegue y el peso de aterrizaje. El peso de aterrizaje incluye el peso vacío operativo, la carga de pago y las reservas de combustible. Precisamente el peso de reservas corresponde a un 5,53\% del peso de aterrizaje, a la vista de los datos del CSR-01 que se pueden encontrar en la base de datos CeRAS para la misión de diseño. Realizando el equilibrio de fuerzas verticales en este punto medio del crucero, es posible determinar el coeficiente de sustentación necesario y, en consecuencia, el coeficiente de resistencia del punto medio del crucero. Hay que hacer notar que la resolución del problema es iterativa, ya que la estimación de la $K$ de Breguet depende del peso de despegue y este depende de la $K$ a través de la ecuación [ 4-2 ]. De esta forma, se puede estimar la $K$ de Breguet, que toma el valor de $22291 \mathrm{~km}$ en nuestro caso.

Con el valor de la $K$, ya se puede entrar en la ecuación [ 4-2 ] para despejar el peso de despegue y el peso de combustible. El primero de ellos es $74101 \mathrm{~kg}$ y el segundo $15135 \mathrm{~kg}$. Estos resultados suponen un error relativo con respecto a los que se proporcionan en la base de datos del $-0,001 \%$ para el peso de despegue y del $0,95 \%$ para el peso de combustible del viaje. De esta forma, se puede concluir que el método propuesto para la estimación del peso de combustible es una buena aproximación al real en la ruta de diseño del CSR-01.

\subsubsection{Derivadas de estabilidad en crucero}

La condición de crucero seleccionada de nuevo está condicionada por las capacidades de Tornado. Estas condiciones de crucero servirán para determinar las derivadas de estabilidad estática e imponer, posteriormente, que el avión con las nuevas superficies de cola tenga, al menos, estas mismas propiedades. En este caso, se puede asumir que, si para la condición de crucero seleccionada el avión de cola no convencional presenta unas propiedades de estabilidad semejantes, es de esperar que también lo sean en otras condiciones de vuelo en crucero. Por tanto, para el cálculo de las derivadas de estabilidad estática en crucero, es menos crítica la limitación que presenta el modelo aerodinámico. Así que se ha seleccionado un Mach de crucero de 0,6 a la altitud de $22000 \mathrm{ft}$. 


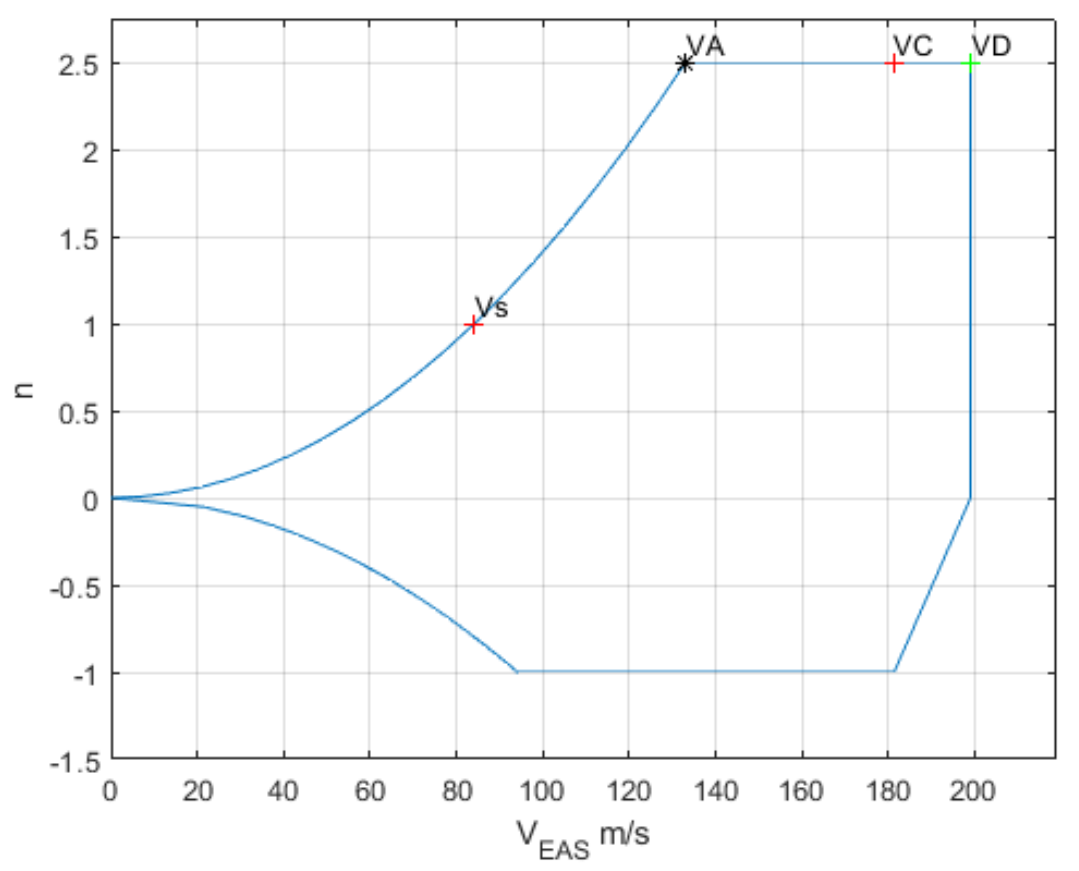

Figura 4-7 Envolvente de maniobra para 22000 ft a peso máximo de despegue. Fuente: elaboración propia.

Para las condiciones de crucero descritas anteriormente, se deben estimar las derivadas de estabilidad estática, que serán los valores que deberán igualar, al menos, la nueva configuración de cola que se proponga. Para ello, lo primero es determinar las condiciones de equilibrio, tanto de fuerzas como de momentos, es decir, resolver el sistema [ 3-14 ]. Primeramente, del equilibrio de fuerzas verticales es necesario extraer el valor del coeficiente de sustentación que debe alcanzar la aeronave para igualar el peso. El peso que se va a tomar es el correspondiente a consumir la mitad del combustible necesario para el viaje. En consecuencia, el coeficiente de sustentación medio del crucero resultante es de 0,49. Este coeficiente nos permite hallar la resistencia que está desarrollando el CSR-01 con los datos de polar anteriormente expuestos. Del equilibrio de fuerzas longitudinales se extrae el valor del empuje, que será igual al de la resistencia para las condiciones de crucero descritas. Con todo ello, ya se dispone de todos los datos necesarios para resolver el sistema salvo las expresiones que relacionan el coeficiente de sustentación y el coeficiente de momentos, sin considerar la contribución de la cola, con el ángulo de ataque. Estas ecuaciones se pueden estimar siguiendo los métodos clásicos de diseño conceptual de aviones (Roskam 1987; Torenbeek 1982). La curva de sustentación que se obtiene es: $c_{L_{A-T}}=4,983 \alpha+0,183$, y la de momentos es: $c_{m_{A-T}}=0.0680 \alpha+0.12092$, para una posición del centro de gravedad igual al $30,6 \%$ de la cuerda media aerodinámica, sacado de los datos disponibles en la base de datos para las condiciones de crucero seleccionadas.

La solución del sistema [ 3-14 ] se obtiene mediante un método de Newton, determinando el jacobiano de las ecuaciones por medio de un esquema de diferencias finitas centradas. En apenas dos o tres pasos de iteración, dependiendo de la condición inicial que se seleccione, se alcanza la solución: $\alpha=4,39^{\circ}$ y $\delta_{\text {sym }}=-4,04^{\circ}$. Hay que indicar que esta solución se ha alcanzado con un ángulo de incidencia del estabilizador horizontal igual a $-0,1^{\circ}$, que es para el cual se disponen cálculos de la polar. En consecuencia, se pueden determinar las derivadas de estabilidad estáticas alrededor de este punto de equilibrio. Para la derivada estática longitudinal es $c_{m \alpha}=-2,45$. Para determinar la derivada de estabilidad estática lateral se debe derivar con respecto del 


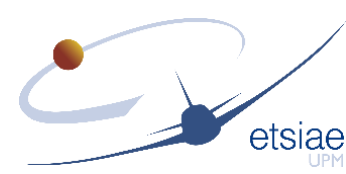

ángulo de resbalamiento la ecuación [ 3-15 ], que se satisface inmediatamente ya que no hay asimetría de empuje ni ángulo de resbalamiento. El coeficiente de momento de guiñada del avión, sin considerar la contribución de la cola, es igual a $c_{n_{A-T}}=-0,1396 \beta$ $y$, en consecuencia, la derivada de estabilidad estática lateral toma el valor $c_{n \beta}=0,155$.

\subsubsection{Fallo del motor crítico en ascenso}

En el vuelo de ascenso tras el despegue, se considera que el tren de aterrizaje ya está recogido pero los dispositivos hipersustentadores están extendidos. El CSR-01 lleva instalados slats, en el borde de ataque, y flaps, en el borde de salida, tal y como se puede observar en la Figura 4-1. Estos dispositivos afectan tanto a la curva de sustentación del avión como a la curva de momentos. Ambas contribuciones se pueden tener en cuenta mediante los mismos métodos clásicos de diseño conceptual por los que se obtuvieron estas curvas para el vuelo de crucero (Roskam 1987; Torenbeek 1982). Para ello, es necesario la información que se recoge en la Tabla 4-2. La contribución a las curvas de coeficientes de sustentación y de momentos no solamente dependen de estas variables geométricas, sino también de la deflexión de los dispositivos. Se ha considerado que en estas condiciones los flaps se deflectan $10^{\circ} \mathrm{y}$ los slats $15^{\circ}$.

De nuevo, el primer paso es equilibrar longitudinalmente la aeronave, de la misma forma que se ha realizado para el vuelo de crucero, pero con los datos de esta condición de vuelo. Cabe destacar que el centro de gravedad se considera que se encuentra al $31 \%$ de la cuerda media aerodinámica y que la velocidad es de $94 \mathrm{~m} / \mathrm{s}$, que corresponde con la velocidad de mínimo control del avión. Además, el peso de esta condición es el máximo de despegue decrementado en un $2 \%$, por el consumo durante la carrera de despegue, y el ángulo de ascenso se considera que es el mínimo considerado en las normas con fallo de motor, es decir, un ascenso del 2,4\% para los aviones bimotor. Los resultados obtenidos para el equilibrado longitudinal son un ángulo de ataque de $3,22^{\circ}$ y una deflexión del timón de profundidad de $0,40^{\circ}$.

Tras resolver el equilibrado longitudinal, se pasa a determinar el equilibrado lateral. Para ello, hay que encontrar la solución a la ecuación [ 3-15 ], es decir, la deflexión del control antisimétrico para anular el momento de guiñada resultante sobre el avión. En este caso, el control antisimétrico se encuentra en el estabilizador vertical y tiene que estar deflectado $7,74^{\circ}$ para anular el momento de guiñada del avión completo, resultando en un ángulo de balance de $1,57^{\circ}$. Como era de esperar, el ángulo de balance se encuentra por debajo de lo $5^{\circ}$ que marca la normativa como el límite para estas situaciones. En la Figura 4-8 se muestra el modelo devuelto por el software Tornado en la situación de equilibrio, tanto longitudinal como en guiñada. Se pueden ver ambas superficies estabilizadoras en colores distintos y dos puntos resaltados en rojo y negro. El primero de ellos es el que toma Tornado como centro de gravedad y el segundo el que toma como referencia para calcular momentos, es decir, el centro aerodinámico, datos que se recogen en la Tabla 4-1. 


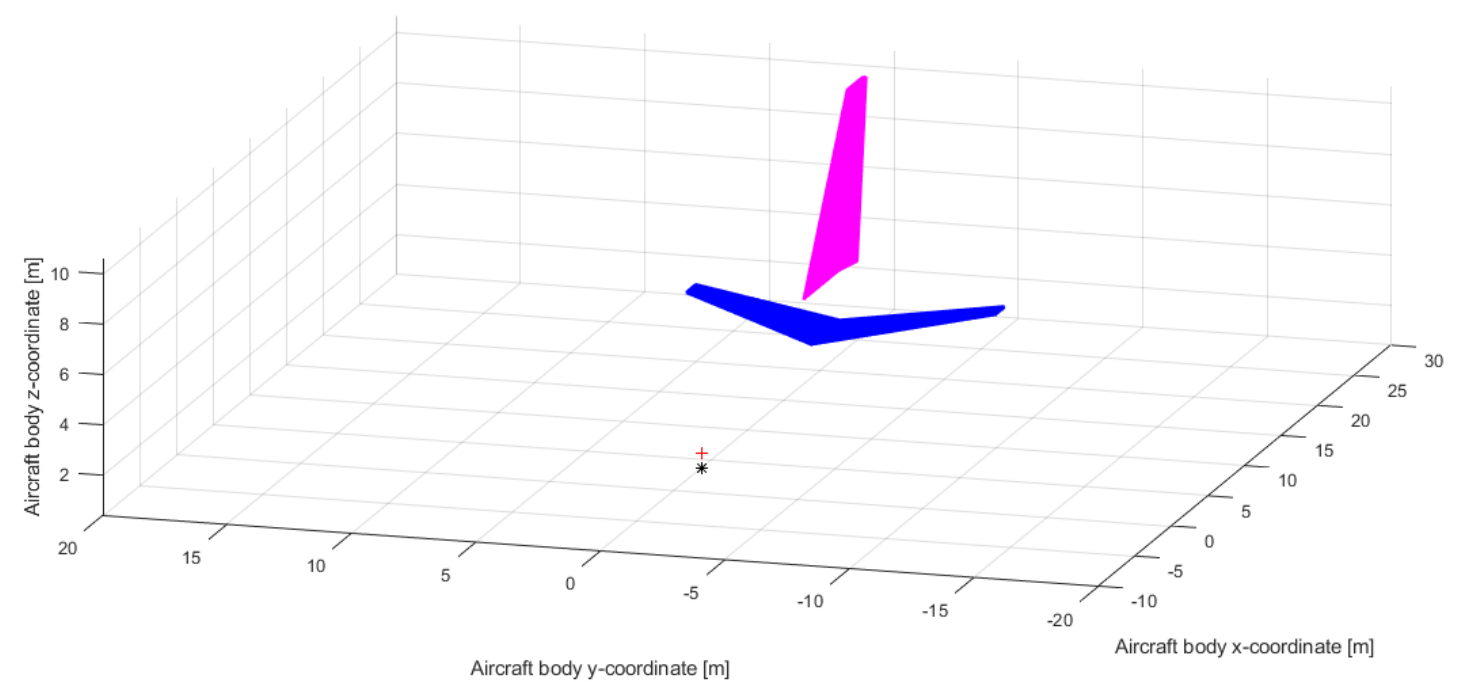

Figura 4-8 Resultado de Tornado tras alcanzar la condición de equilibrio para el fallo del motor crítico tras el despegue. Fuente: elaboración propia.

\subsubsection{Aterrizaje con viento cruzado}

La condición de aterrizaje con viento cruzado se resuelve de una forma similar a la del fallo de motor crítico, pero considerando las condiciones particulares de este caso. Puesto que la normativa establece que el viento cruzado para el cual se dimensiona el estabilizador vertical no debe superar los $25 \mathrm{kt}$, como se indica en el artículo 25.237 de las normas de certificación (EASA 2018), se ha tomado precisamente este valor límite para el estudio. La velocidad para la cual se ha aplicado esta condición corresponde con la que resulta de incrementar en un $30 \%$ la velocidad de entrada en pérdida en configuración de aterrizaje. Para ello, es necesario establecer el coeficiente máximo de sustentación en condiciones de aterrizaje y el peso de aterrizaje, asumiendo que la altitud es la correspondiente a nivel del mar. El coeficiente de sustentación máximo en condiciones de aterrizaje se ha podido extraer de la base de datos CeRAS. El peso del avión en estas condiciones se ha estimado en el peso máximo de aterrizaje, ya que era el mayor peso para el cual se disponía de un centro de gravedad más retrasado, de acuerdo con la base de datos. Así, la velocidad a la cual vuela el avión es de 71,16 m/s. A esta velocidad, el coeficiente de sustentación necesario para equilibrar el peso es de 1,67. Planteando el equilibrio de fuerzas se puede determinar el ángulo de descenso del avión, que será máximo si el motor no produce empuje. Este ángulo resulta ser de unos $6^{\circ}$ aproximadamente, por lo que la aproximación de que la sustentación debe equilibrar todo el peso sigue siendo aplicable. Al igual que en el caso del fallo del motor crítico en el segundo segmento, en esta condición se llevan deflectados las superficies hipersustentadoras, flaps y slats. Ahora, las deflexiones corresponden a $35^{\circ}$ de flap y $25^{\circ}$ de slats.

La resolución del equilibrado longitudinal lleva a un ángulo de ataque de $0,31^{\circ} \mathrm{y}$ un ángulo de deflexión del timón de profundidad de $0,47^{\circ}$. Por otra parte, el ángulo de deflexión del timón de dirección para equilibrar el avión en las condiciones de viento cruzado que se consideran es de $11,1^{\circ}$. De nuevo, se incluye la geometría generada por Tornado en la condición de equilibrio en la Figura 4-9. 


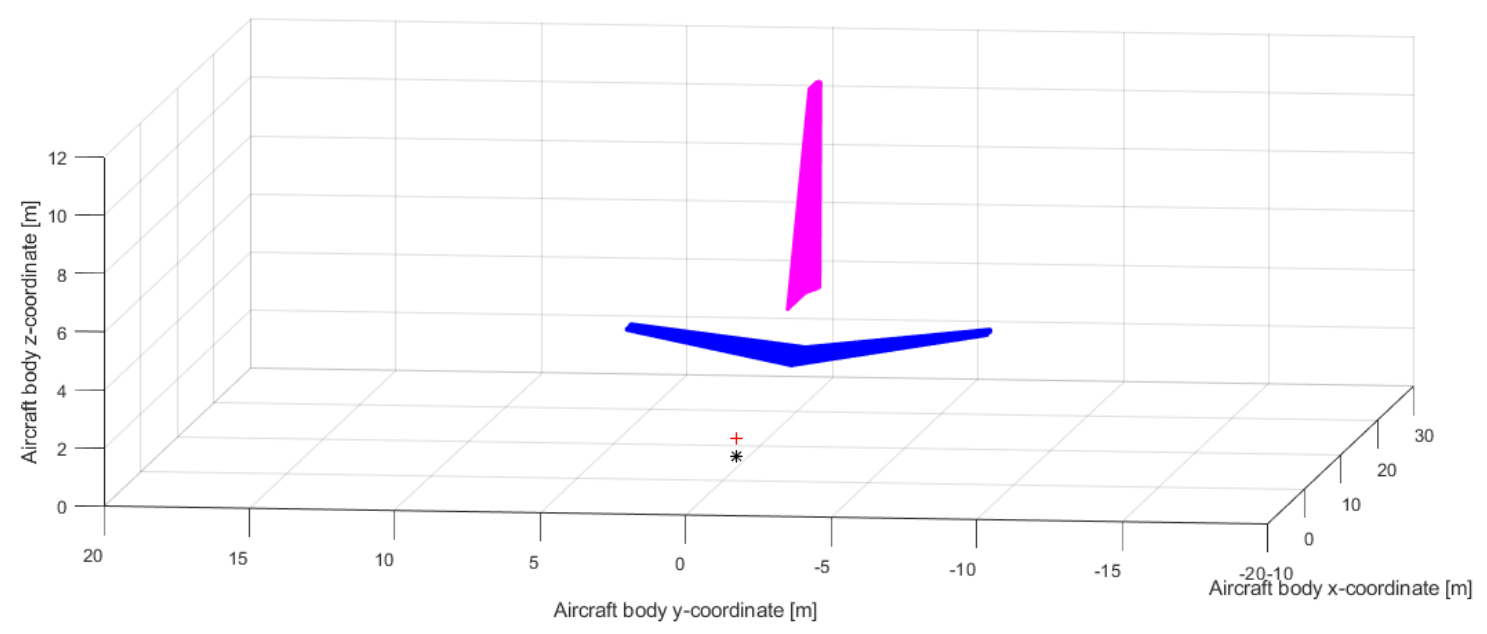

Figura 4-9 Resultado de Tornado tras alcanzar la condición de equilibrio aterrizaje con viento cruzado. Fuente: elaboración propia.

\subsubsection{Estimación de peso basado en regresiones estadísiticas de los estabilizadores}

La estimación de pesos basado en las ecuaciones [3-28 ] y [3-29 ] pasa por determinar los valores de la densidad de los materiales utilizados en la fabricación de los estabilizadores y los parámetros de densidad del CSR-01, dando por conocidas el resto de variables geométricas. Asumiendo que el material que más se utiliza en las superficies de cola es material compuesto en fibra de carbono, se puede estimar una densidad del material en $1900 \mathrm{~kg} / \mathrm{m}^{3}$ para ambas superficies de cola (Baker y Scott 2016). Para calcular los parámetros de densidad $K_{\rho H}$ y $K_{\rho V}$ de los estabilizadores horizontal y vertical, respectivamente, se ha impuesto que el peso resultante de las ecuaciones sea el que se puede encontrar en la base de datos CeRAS para las superficies de cola del CSR-01 (Tabla 4-1). En consecuencia, el factor de densidad para el estabilizador horizontal toma el valor de 0,0218 y el del vertical 0,034 . Tal y como se ha comentado en el capítulo 3.3.1, tanto la densidad de los materiales como los parámetros de densidad deben situarse en unos valores característicos para poder ser considerados como adecuados (Sadraey 2013). Se ha comprobado, que los resultados aquí obtenidos se encuentran en esos intervalos, validándose así la aplicación de este método.

\subsubsection{Estimación de peso basado en métodos casi analíticos de los estabilizadores}

El peso calculado por medio del método casi analítico necesita de las cargas aerodinámicas generadas en las superficies de cola. A la vista de las limitaciones que presenta el software Tornado se han decidido contemplar las siguientes maniobras, a la altitud seleccionada de crucero, de $22000 \mathrm{ft}$ :

1. Equilibrado longitudinal del avión en un viraje estacionario a $V_{A}$ y a factor de carga límite, como maniobra simétrica estacionaria.

2. Deflexión súbita del timón de profundidad en una condición de equilibrio de vuelo de crucero a $V_{A}$, como maniobra simétrica con aceleración de cabeceo unchecked. 
3. Equilibrado lateral del avión tras el fallo del motor crítico en segundo segmento con ángulo de resbalamiento nulo. Esta maniobra se ha modelizado a nivel del mar, no a la altitud de crucero.

4. Vuelo a $\bigvee_{A}$ con ángulo de resbalamiento igual a $10^{\circ}$, sin deflexión del timón de dirección, que corresponde a la maniobra 2 de las expuestas en las maniobras asimétricas del apartado 3.2.8.

5. Vuelo a $V_{A}$ con ángulo de resbalamiento igual a $10^{\circ}$ y deflexión del timón de dirección máxima en el sentido que hiciera al avión moverse hacia ángulos de resbalamiento nulo, correspondiente a la maniobra 3 de las que se indican en el apartado 3.2.8.

6. Ráfaga lateral a $\mathrm{V}_{\mathrm{A}}$.

7. Ráfaga vertical positiva a $V_{A}$.

8. Vuelo a $V_{A}$ con ángulo de resbalamiento nulo, pero deflexión máxima del timón de dirección, como maniobra 1 dentro de las asimétricas de las que se recogen en el apartado 3.2.8.

Los números de la lista anterior se van a utilizar para identificar las condiciones de vuelo a partir de ahora. De esta forma, la condición de vuelo 1 es la que corresponde al equilibrado longitudinal del avión en un viraje estacionario a $V_{A}$ y a factor de carga límite, y así sucesivamente.

Las cargas que se generan en los estabilizadores generarán compresión en el extradós de la superficie o en el intradós dependiendo del sentido de la fuerza resultante. En principio, dependiendo de la maniobra en concreto, tanto el extradós como el intradós podría estar sometido a compresión. Por tanto, puesto que en las hipótesis del cálculo de pesos se considera que tanto el extradós como el intradós tienen la misma geometría, se escogerá el caso de carga crítico independientemente de donde genere la compresión, es decir, no se tendrá en cuenta el sentido de la resultante, sino el valor absoluto de la carga.

Los resultados de las maniobras que se han enumerado anteriormente se van a separar entre los que inducen cargas en el estabilizador horizontal y los que las inducen en el vertical. Estas cargas se van a presentar en forma de distribución del momento flector, fuerza cortante y momento torsor, que son las que se van a necesitar para determinar el flujo cortante y carga de compresión por unidad de longitud que debe soportar cada sección de la superficie estabilizadora. Los resultados de momento flector se recogen en la Figura 4-10, los de fuerza cortante en la Figura 4-11 y los de momento torsor en la Figura 4-12. En todas las figuras se incluye una leyenda que indica bajo qué maniobra se han generado esas cargas, refiriéndose a la lista anterior. Se puede ver como la condición 1 es la más crítica en términos de momento torsor. Sin embargo, la condición 2 es la crítica atendiendo al momento flector y fuerza cortante, por lo que hay fuertes indicios de que esta maniobra va a ser la que dimensione el estabilizador horizontal.

En cuanto a las maniobras asimétricas, son las que inducen cargas en el estabilizador vertical. Los resultados se reflejan en la Figura 4-13, Figura 4-14 y Figura 4-15 para el momento flector, fuerza cortante y momento torsor, respectivamente. En este caso, la condición 4 es la que genera más fuerza cortante y momento flector de las que se han considerado, además de ser casi igual que la que más momento torsor introduce, que es la condición 5. Por tanto, parece que la condición 4 es la más crítica de todas.

Independientemente de las intuiciones que se han reflejado de cuáles serían las cargas críticas tanto para el dimensionado del estabilizador vertical como el horizontal, se van 
a comprobar todas ellas, ya que cada maniobra lleva asociada un factor de carga diferente y puede afectar al resultado final en términos de peso.

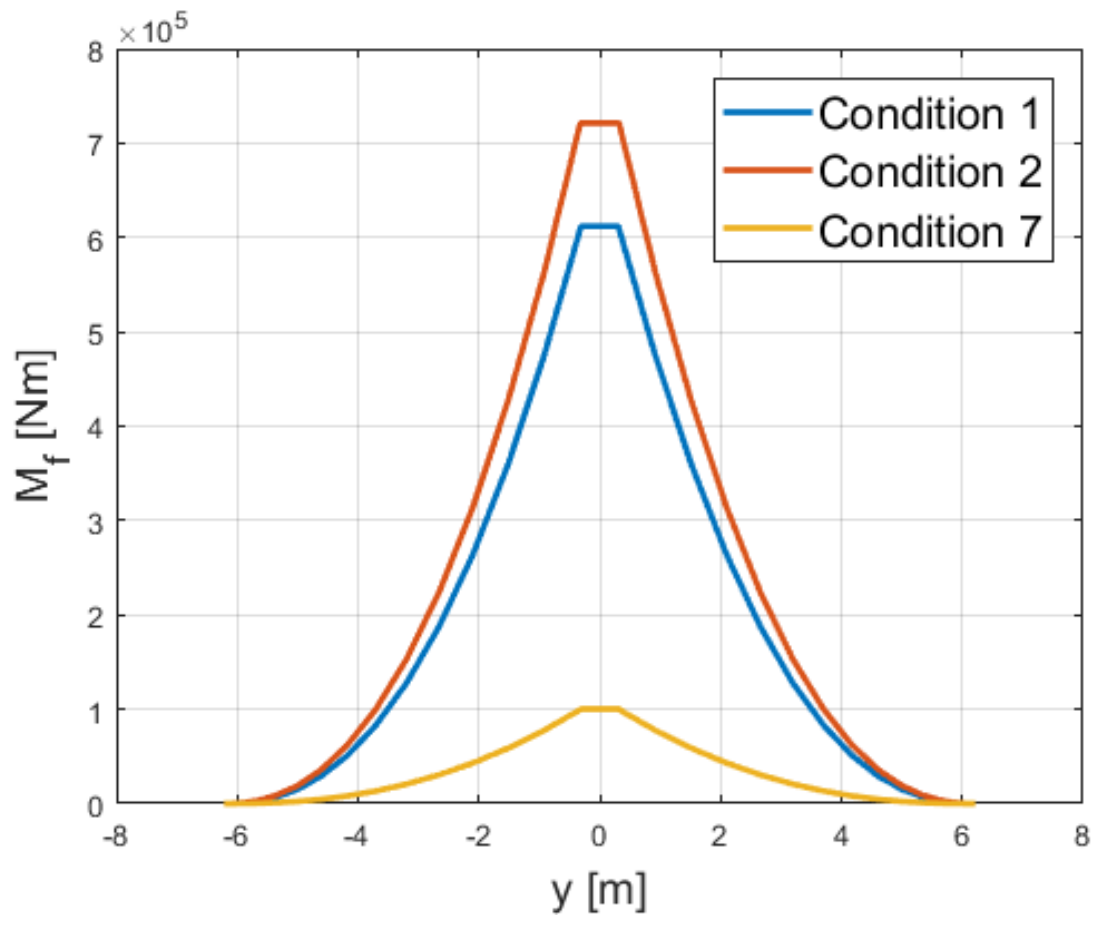

Figura 4-10 Distribución de momento flector a lo largo de la envergadura del estabilizador horizontal del CSR-01 para las maniobras simétricas. Fuente: elaboración propia.

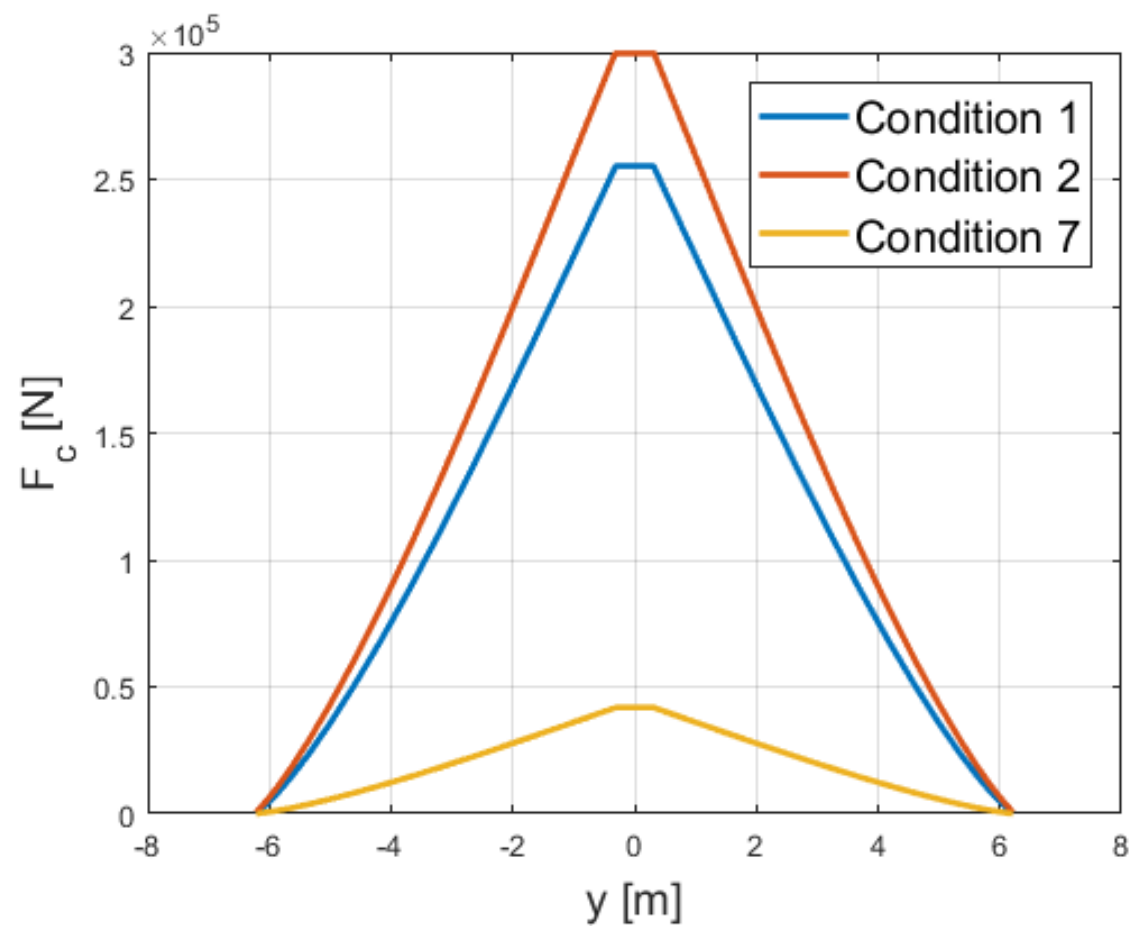

Figura 4-11 Distribución de fuerza cortante a lo largo de la envergadura del estabilizador horizontal del CSR-01 para las maniobras simétricas. Fuente: elaboración propia. 


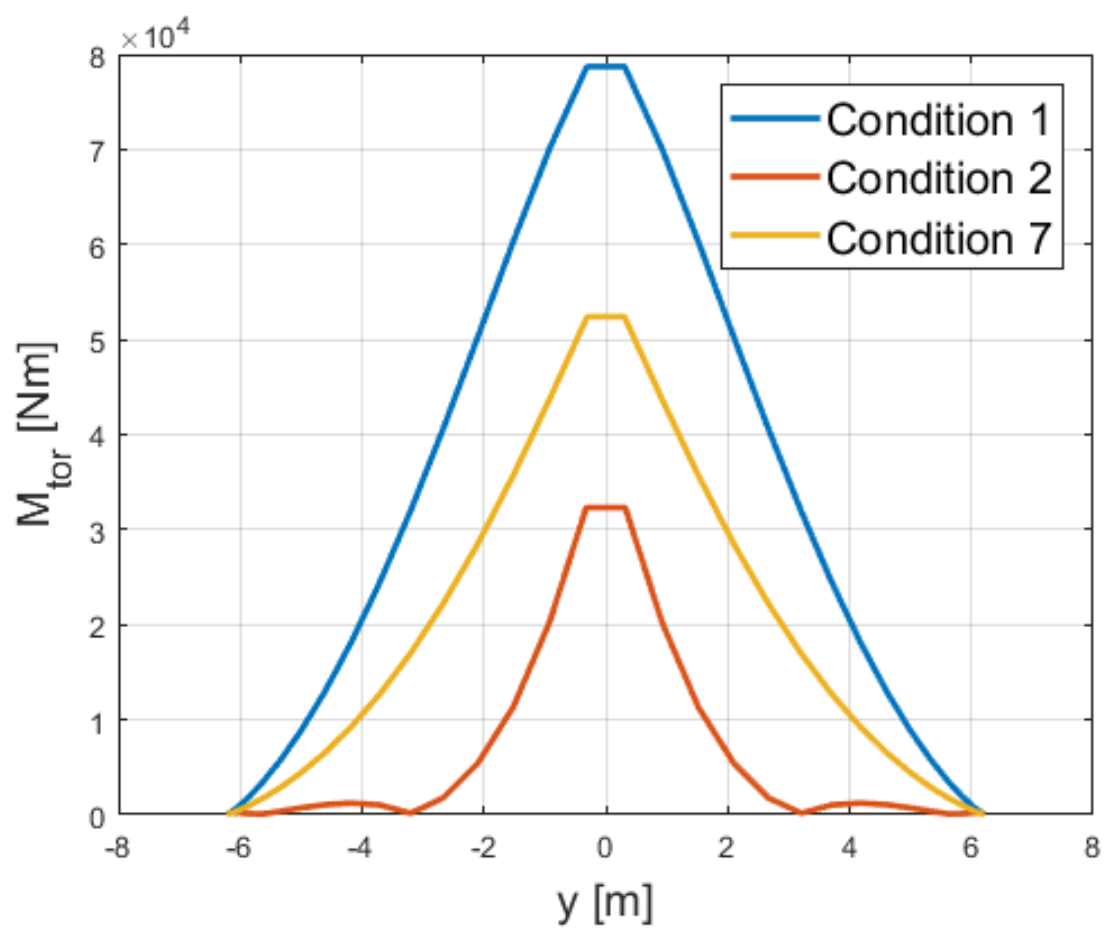

Figura 4-12 Distribución de momento torsor a lo largo de la envergadura del estabilizador horizontal del CSR-01 para las maniobras simétricas. Fuente: elaboración propia.

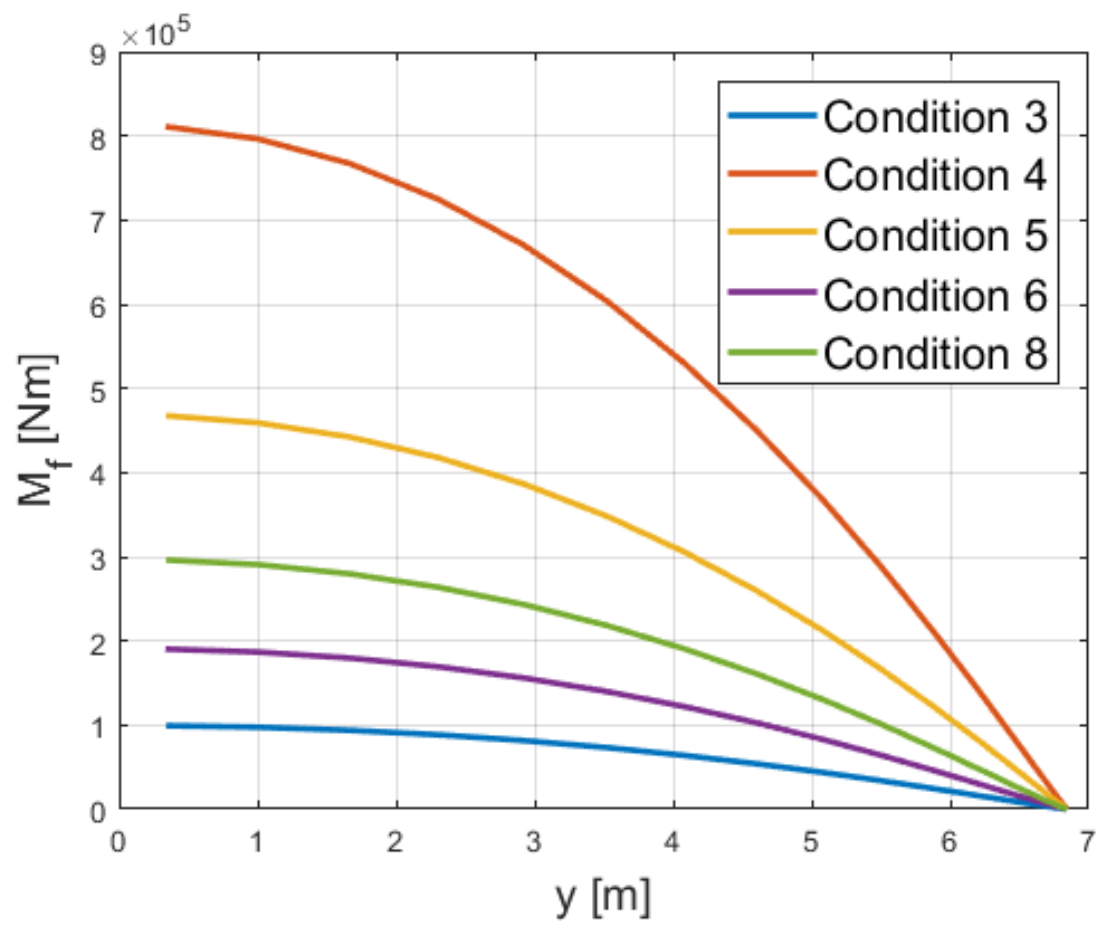

Figura 4-13 Distribución de momento flector a lo largo de la envergadura del estabilizador vertical del CSR-01 para las maniobras asimétricas. Fuente: elaboración propia. 


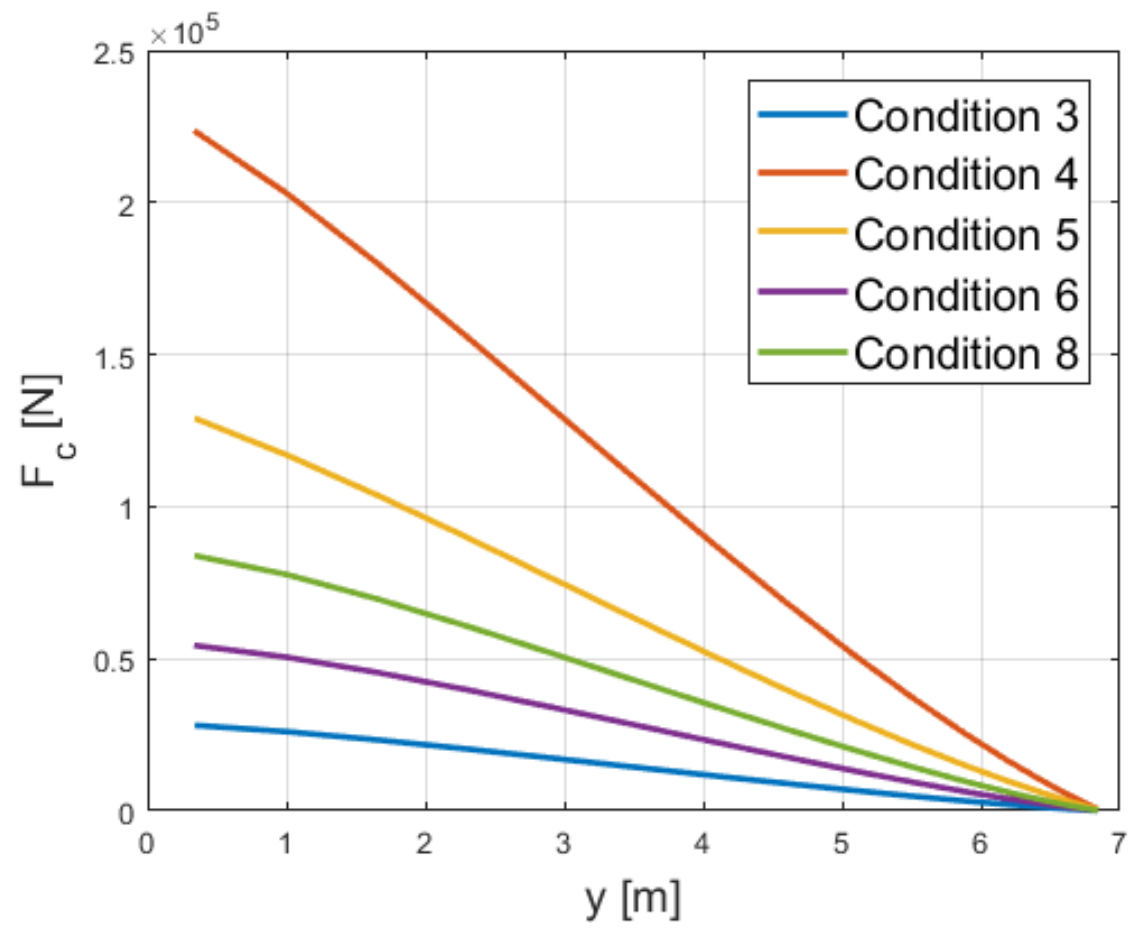

Figura 4-14 Distribución de fuerza cortante lo largo de la envergadura del estabilizador vertical del CSR-01 para las maniobras asimétricas. Fuente: elaboración propia.

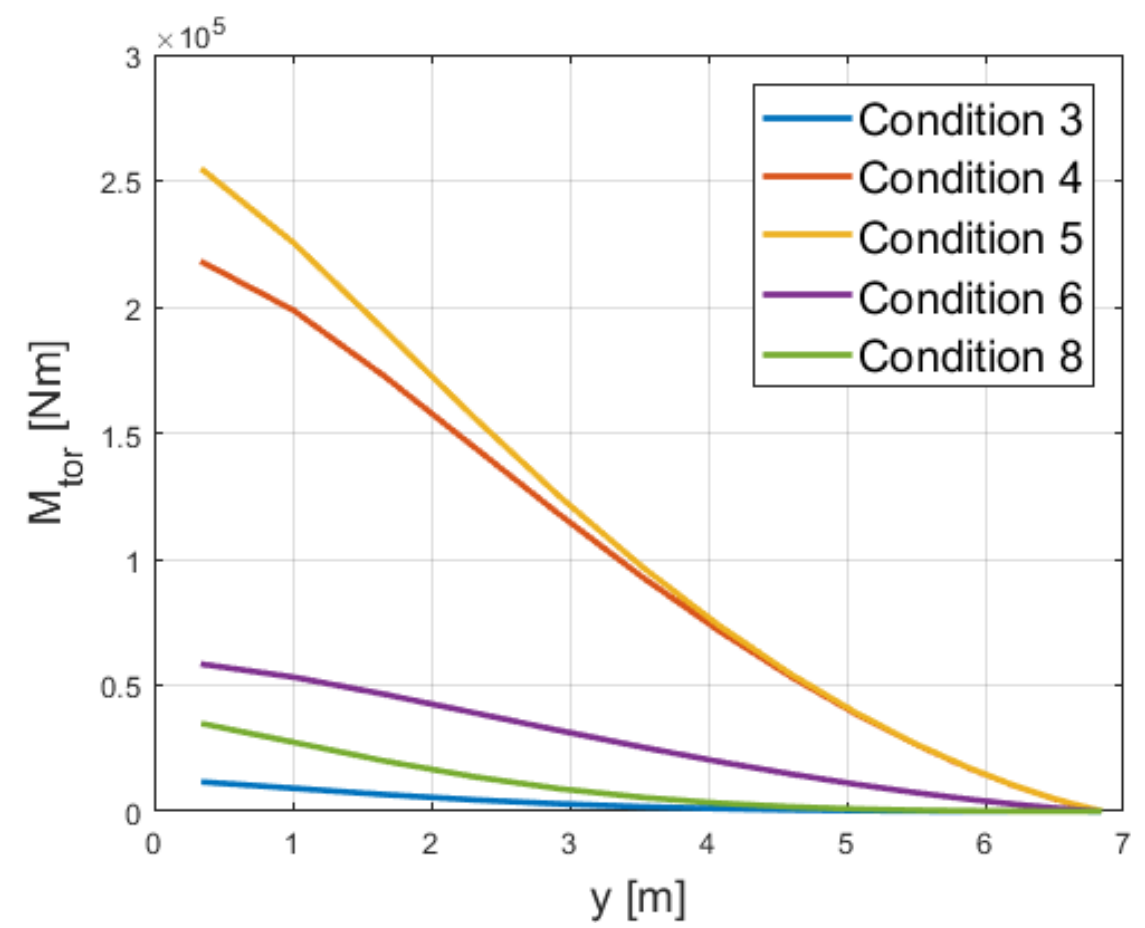

Figura 4-15 Distribución de momento torsor lo largo de la envergadura del estabilizador vertical del CSR-01 para las maniobras asimétricas. Fuente: elaboración propia. 
En primer lugar se va a estimar el peso del estabilizador horizontal para validar el proceso de dimensionado de la estructura presentado. A partir de las fuerzas y momentos de cada uno de los casos de carga, se determinan la fuerza por unidad de longitud y flujo cortante que generan sobre cada sección de la estructura. Para ello se hace uso de las expresiones que se presentaron, basadas en las correspondientes hipótesis, que a continuación se recuerdan:

$$
\begin{gathered}
N=\frac{M_{f}}{h_{t} b_{t}} \\
q=\left|\frac{M_{t o r}}{2 h_{t} b_{t}}\right|+\left|\frac{F_{c}}{2 h_{t}}\right|
\end{gathered}
$$

Se quiere hacer notar que las cargas determinadas a partir de las maniobras son cargas límites, es decir, que se deben incrementar en un determinado factor establecido en las normas para pasar a ser cargas últimas, que es para las que se debe dimensionar la estructura. Este factor es normalmente de 1,5. Aun así, se ha decidido dejar un margen de seguridad para ser conservativo, por lo que se ha incrementado en un $10 \%$ los valores de las cargas últimas obtenidos.

No se debe olvidar que el propio peso de la estructura sometido al factor de carga correspondiente a la maniobra también introduce un momento flector, torsor y fuerza cortante. El problema es que para este punto aún no se conoce el valor del peso, porque depende del dimensionado de la estructura. Por tanto, se debe realizar un proceso iterativo de convergencia hasta que el peso estimado al inicio para determinar las cargas a las que está sometido el estabilizador coincida con lo que pesa la estructura una vez dimensionada.

Las dimensiones del cajón de torsión se basan en el perfil aerodinámico del estabilizador, en el tamaño de la cuerda y en la posición de los largueros. Empezando por esto último, se ha supuesto que el larguero anterior se encuentra al $15 \%$ de la cuerda y el posterior al $55 \%$, de tal forma que haya espacio suficiente para instalar los controles. Como se ha comentado en las hipótesis de partida del modelo estructural, se va a considerar que el cajón de torsión es rectangular. Entonces, para establecer sus dimensiones se debe intersecar el perfil por un plano vertical que pase por el $15 \%$ de la cuerda y por otro que pase por el larguero posterior. De esta forma el panel de extradós se define por la unión de los puntos de corte de estos dos planos con el extradós del perfil, y el intradós del panel por la unión de los puntos de corte con el intradós del perfil. La representación gráfica de lo que se acaba de explicar se muestra en la Figura 4-16. Se puede observar como el cajón es prácticamente rectangular siguiendo el procedimiento descrito para definir el mismo. Así, se pueden estimar las dimensiones del cajón, en cada una de las secciones de envergadura correspondientes, necesarias para estimar las cargas que se generan en cada sección de la superficie. 

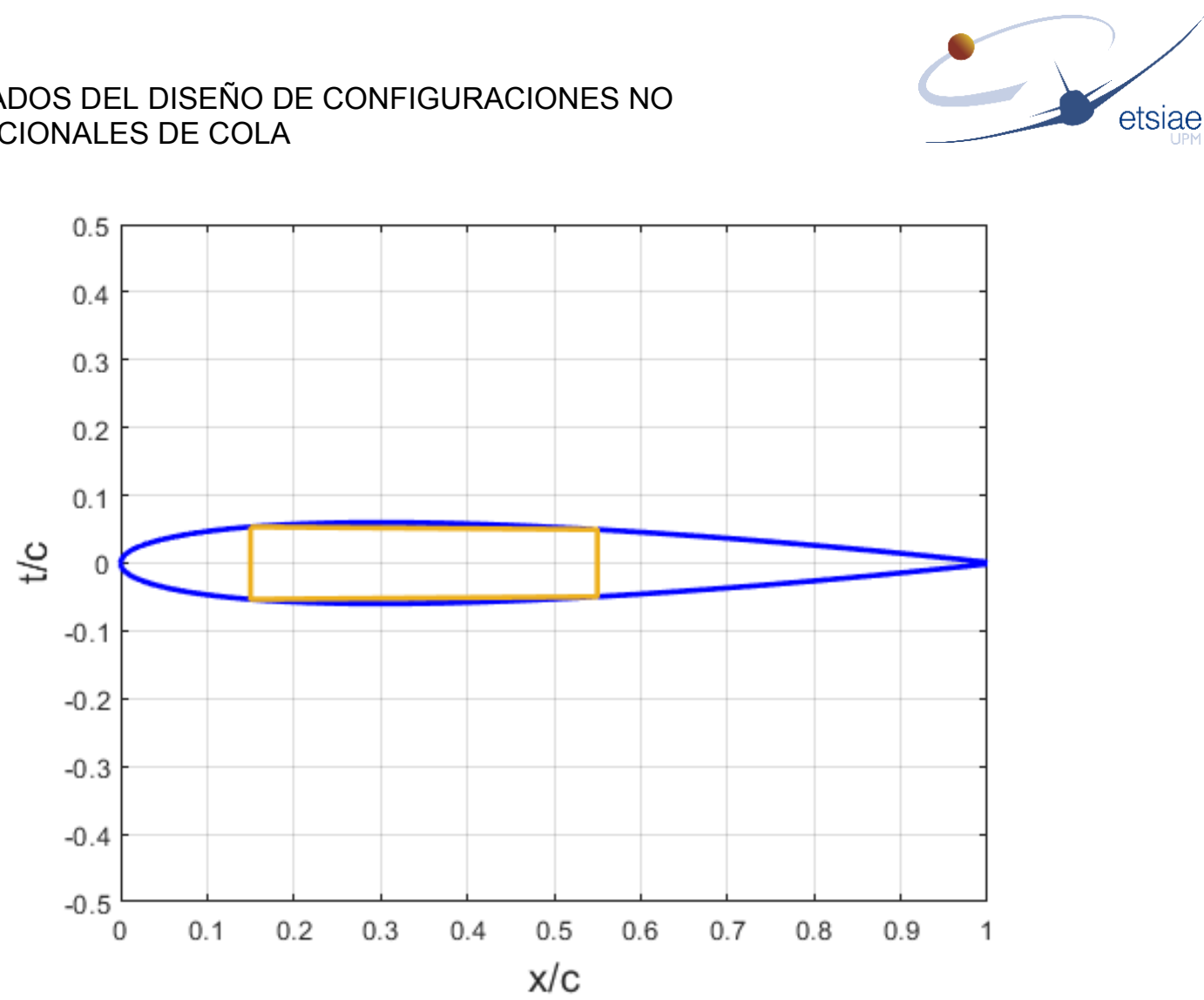

Figura 4-16 Perfil NACA 0012 del estabilizador horizontal del CSR-01 adimensionalizado con la cuerda y remarcado el cajón de torsión interior. Fuente: elaboración propia.

El panel de extradós, o intradós, se va a dimensionar a factor de Farrar óptimo en un principio, es decir, 0,95, a pesar de que se sabe que el número de larguerillos resultantes será elevado. Analizando el resultado de pesos, se comprobará si es necesario reducir el factor. Se asumirá que las costillas distan entre sí un metro (según estructuras de aviones semejantes), por lo que el panel tiene las medidas del ancho del cajón por un metro de largo. El alma de los largueros se va a dimensionar asumiendo que se encuentran en un estado de tensión diagonal, siguiendo el procedimiento descrito en 3.3.2.2, y que ambos son iguales. Se va a suponer que los montantes que rigidizan el alma de los largueros se encuentran separados entre sí una distancia igual a 0,5 m, a la vista del estado del arte.

Por último, para estimar el peso de la estructura es necesario añadir el peso de las costillas y el peso de la estructura secundaria y miscelánea. Ambas contribuciones se determinan por medio de regresiones estadísticas que se han adaptado al estabilizador horizontal. Comenzando por el peso de las costillas, en la Tabla 4-5 se recogen los valores que se han escogido para los parámetros de los que depende el peso de las costillas. La selección de estos parámetros se ha llevado a cabo siguiendo las recomendaciones incluidas en la referencia donde se describe el método aplicado a las alas de un avión (Torenbeek 2013). En la misma Tabla 4-5 también se incluyen una serie de parámetros que son necesarios para estimar el peso de las sujeciones y de las uniones, siguiendo las ecuaciones que se han presentado en el apartado 3.3.2.5. La densidad del material que se ha utilizado para todos estos elementos es la misma que se ha utilizado en el modelo de pesos basado íntegramente en regresiones estadísticas, es decir, de $1900 \mathrm{~kg} / \mathrm{m}^{3}$. El peso de las sujeciones depende del peso máximo de despegue y del factor de carga último, que corresponde a incrementar el límite en un $50 \%$, es decir, que alcanza el valor de 3,75 . 


\begin{tabular}{|cc|}
\hline Parámetro & Valor \\
\hline $\boldsymbol{k}_{\text {rib }}$ & 0,003 \\
\hline $\boldsymbol{t}_{\text {ref }}$ & $0,45 \mathrm{~m}$ \\
\hline $\boldsymbol{t}_{\text {root }}$ & $0,12 C_{r} \mathrm{~m}$ \\
\hline $\boldsymbol{t}_{\boldsymbol{t i p}}$ & $0,12 c_{t} \mathrm{~m}$ \\
\hline $\boldsymbol{\delta}_{\boldsymbol{i d}}$ & $10^{-3}$ \\
\hline
\end{tabular}

Tabla 4-5 Valores de los parámetros de ajuste del modelo de estimación de pesos de costillas, sujeciones y uniones. Fuente: elaboración propia.

En cuanto al peso de la estructura secundaria, se incluyen en la Tabla 4-6 los valores de los parámetros que se deben seleccionar para poder aplicar las regresiones indicadas en el apartado 3.3.2.5, que corresponden al peso específico por unidad de superficie de cada uno de estos elementos. De nuevo, para la selección de los valores se han seguido las indicaciones de la referencia (Torenbeek 2013).

\begin{tabular}{|cc|}
\hline Parámetro & Valor \\
\hline $\boldsymbol{k}_{\text {fle }}$ & 1 \\
\hline $\boldsymbol{\Omega}_{\text {ref }}$ & $56 \mathrm{~N} / \mathrm{m}^{2}$ \\
\hline $\boldsymbol{q}_{\boldsymbol{D}}$ & $13218 \mathrm{~N} / \mathrm{m}^{2}$ \\
\hline $\boldsymbol{q}_{\text {ref }}$ & $30000 \mathrm{~N} / \mathrm{m}^{2}$ \\
\hline $\boldsymbol{b}_{\text {st }}$ & $b / \cos \Lambda_{0,25} \mathrm{~m}$ \\
\hline $\boldsymbol{W}_{\text {ref }}$ & $10^{6} \mathrm{~N}$ \\
\hline $\boldsymbol{b}_{\text {ref }}$ & $50 \mathrm{~m}$ \\
\hline $\boldsymbol{k}_{\text {sup }}$ & 1 \\
\hline $\boldsymbol{k}_{\text {slot }}$ & 1 \\
\hline
\end{tabular}

Tabla 4-6 Valores de los parámetros de ajuste del modelo de estimación de pesos de elementos secundarios por unidad de superficie. Fuente: elaboración propia.

Por último, será necesario indicar cómo se van a calcular las superficies de los elementos secundarios, ya que para determinar su peso se debe multiplicar el peso por unidad de superficie por la superficie de cada uno de ellos. Los elementos secundarios que se van a considerar en el estabilizador horizontal son un borde de ataque fijo, borde de salida fijo y dispositivo de control de borde de salida. En la Tabla 4-7 se recogen cómo se van a determinar sus superficies, aproximándolo a partir de la información e imágenes que se encuentran en la base de datos CeRAS. Además, se van a aplicar una serie de factores correctores que se deben a que estos elementos están fabricados en material compuesto, en lugar de con aleaciones de aluminio. Estas constantes también se basan en las recomendaciones que se incluyen en la referencia (Torenbeek 2013), ya que a pesar de que el modelo está desarrollado para alas principales de aviones de transporte comercial, es habitual que estos elementos estén fabricados en material compuesto en las propias alas. 


\begin{tabular}{|cc|}
\hline Parámetro & Valor \\
\hline $\boldsymbol{S}_{\boldsymbol{f l e}}$ & $0,04 S_{t} \mathrm{~m}^{2}$ \\
\hline $\boldsymbol{S}_{\boldsymbol{f t e}}$ & $0,03 S_{t} \mathrm{~m}^{2}$ \\
\hline $\boldsymbol{S}_{\boldsymbol{t e f}}$ & $0,25 S_{t} \mathrm{~m}^{2}$ \\
\hline $\boldsymbol{C}_{\boldsymbol{f l e}}$ & 0,80 \\
\hline $\boldsymbol{C}_{\boldsymbol{f t e}}$ & 0,80 \\
\hline $\boldsymbol{C}_{\boldsymbol{t} \text { ef }}$ & 0,85 \\
\hline
\end{tabular}

Tabla 4-7 Valores de los parámetros de ajuste del modelo de estimación de pesos de elementos secundarios por unidad de superficie. Fuente: elaboración propia.

Una vez el proceso iterativo ha convergido a un cierto peso, para las tres condiciones de maniobras simétricas que se han contemplado, las distribuciones de fuerza de compresión por unidad de longitud y flujo cortante resultantes para cada una de las condiciones se muestran en la Figura 4-17, y los pesos finales en la Tabla 4-8. Se puede comprobar que la condición 2 es la que resulta en el mayor peso del estabilizador, por lo que se corrobora que es la condición crítica. Comparando con el peso del estabilizador que se recoge en la base de datos, que es de $682 \mathrm{~kg}$, el resultado subestima el valor de referencia en un $5,6 \%$ aproximadamente. Además, se observa cómo afecta el caso de carga al peso del cajón de torsión, siendo sensiblemente menor para la condición 7, que es la que menor carga introduce a la superficie. El resto de los elementos que componen el peso se mantienen aproximadamente constantes, ya que se determinan por medio de correlaciones estadísticas. Es cierto que se aprecia una pequeña variabilidad, pero se debe al proceso de convergencia, que hace que existan esas diferencias.

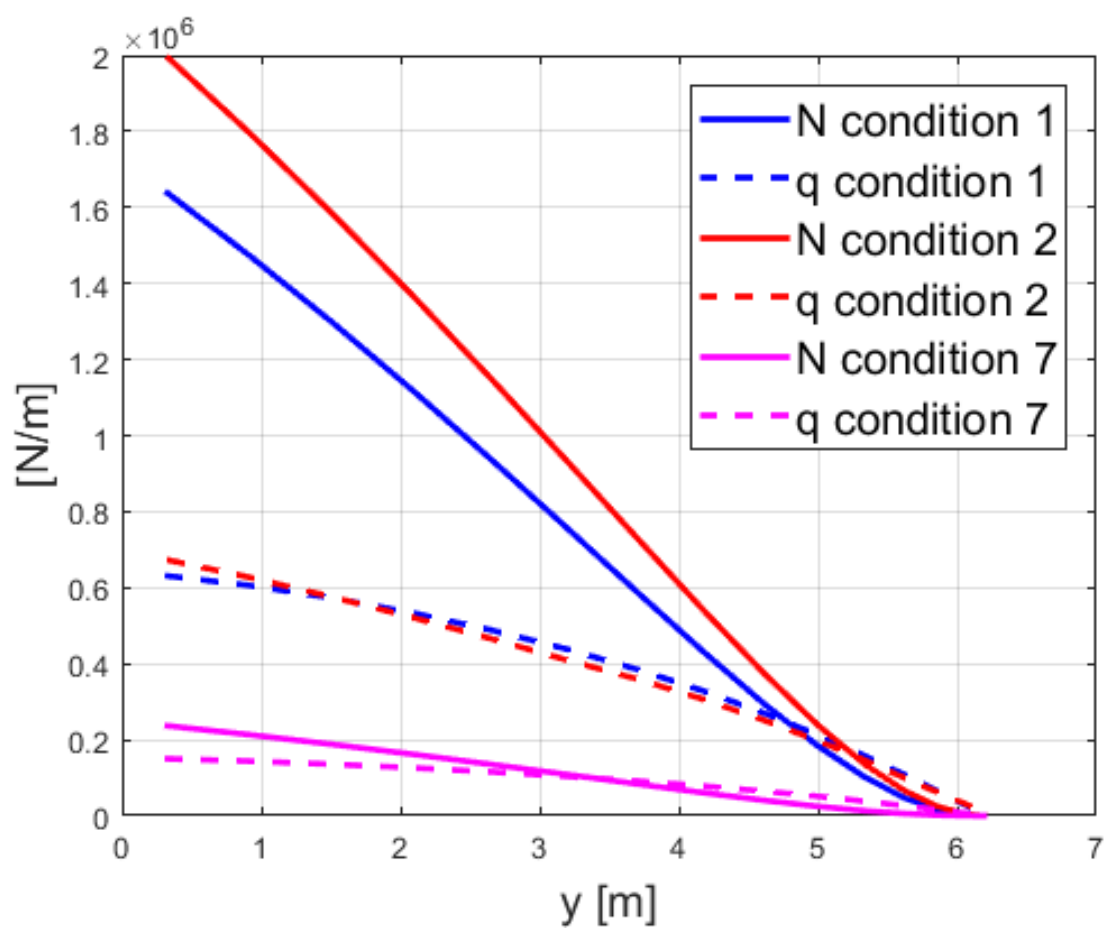

Figura 4-17 Carga por unidad de longitud y flujo cortante a lo largo de la semienvergadura del estabilizador horizontal del CSR-01 para las maniobras simétricas. Fuente: elaboración propia. 


\begin{tabular}{|lcc|}
\hline & Peso del cajón & Peso del estabilizador \\
\hline Condición 1 & $225 \mathrm{~kg}$ & $637 \mathrm{~kg}$ \\
\hline Condición 2 & $246 \mathrm{~kg}$ & $644 \mathrm{~kg}$ \\
\hline Condición 7 & $75 \mathrm{~kg}$ & $482 \mathrm{~kg}$ \\
\hline
\end{tabular}

Tabla 4-8 Pesos resultantes del estabilizador horizontal para cada una de las maniobras simétricas. Fuente: elaboración propia.

El procedimiento que se ha seguido para el estabilizador horizontal se puede reproducir para el vertical. Simplemente se deben tomar las maniobras asimétricas y la geometría de la superficie de cola vertical, tanto en cuerda en la raíz, envergadura y parámetro de estrechamiento como en el perfil aerodinámico, que en este caso es un NACA 0009 (ver Figura 4-18). Los parámetros de ajuste de las regresiones se han tomado igual que los del estabilizador horizontal.

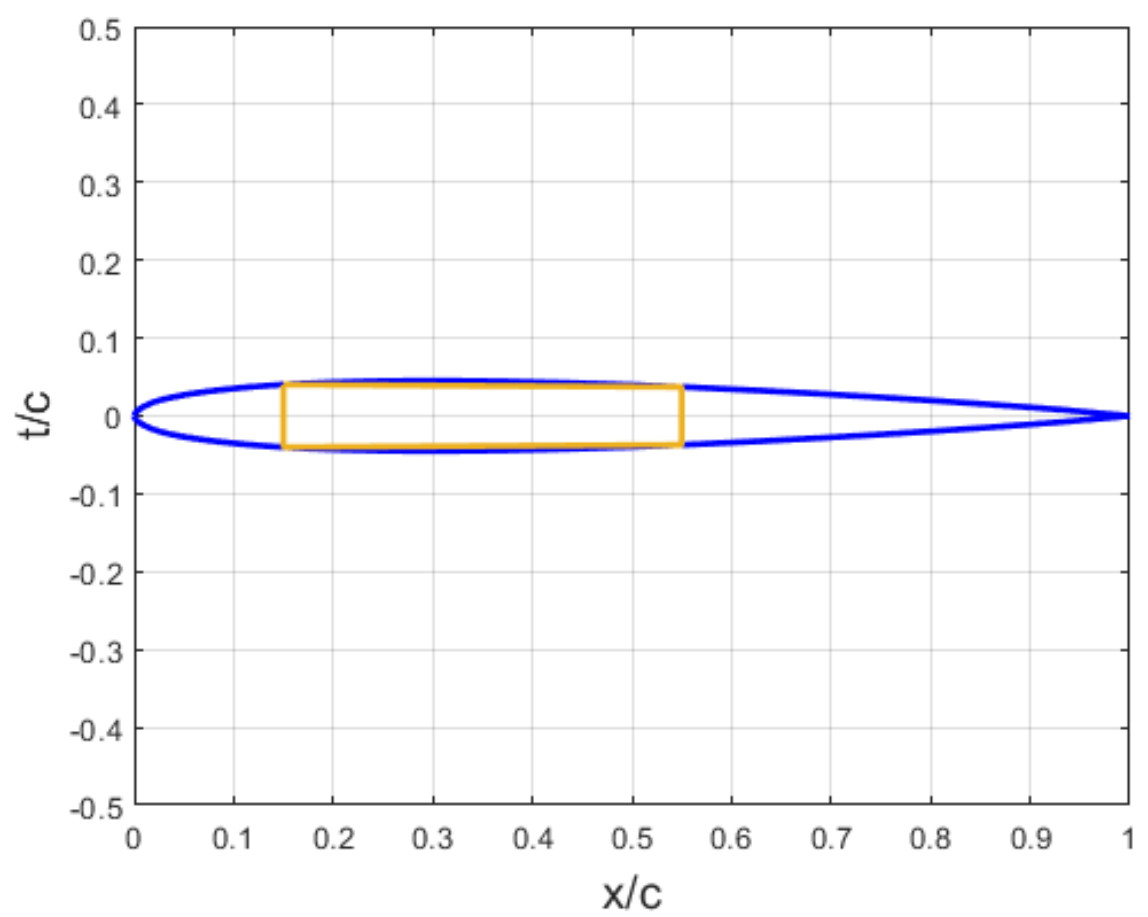

Figura 4-18 Perfil NACA 0009 del estabilizador vertical del CSR-01 adimensionalizado con la cuerda y remarcado el cajón de torsión interior. Fuente: elaboración propia.

Las cargas por unidad de longitud a compresión y del flujo cortante que inducen las maniobras asimétricas consideradas se muestran en la Figura 4-19. En este caso, el propio peso de la estructura introduce una carga de compresión distribuida a lo largo de la envergadura, debido a que el diedro del estabilizador vertical es de $90^{\circ}$. Los pesos resultantes del proceso iterativo tras alcanzar la convergencia se recogen en la Tabla 4-9. Se puede ver como las condiciones 4 y 5 son las que alcanzan los valores más coherentes en peso de cajón de torsión y, en consecuencia, en peso del estabilizador vertical. La base de datos CeRAS indica que el peso del estabilizador es de $522 \mathrm{~kg}$. A la vista de esto, se puede decir que la condición 5 es la que mejor se aproxima, ya que lo sobrestima en un 1,5\%. Con respecto a la condición 4 se puede decir que sobrestima demasiado el peso, concretamente en un $25 \%$ aproximadamente. Hay que recordar que la condición 4 es la que agrupa todas aquellas maniobras que suponen volar con un 
cierto ángulo de resbalamiento, que se ha decidido que se engloben todas en un resbalamiento de $10^{\circ}$. Esto quiere decir que la hipótesis de agrupar todas estas maniobras bajo el valor de $10^{\circ}$ ha sido demasiado conservativa, para el caso del estabilizador vertical del CSR-01.

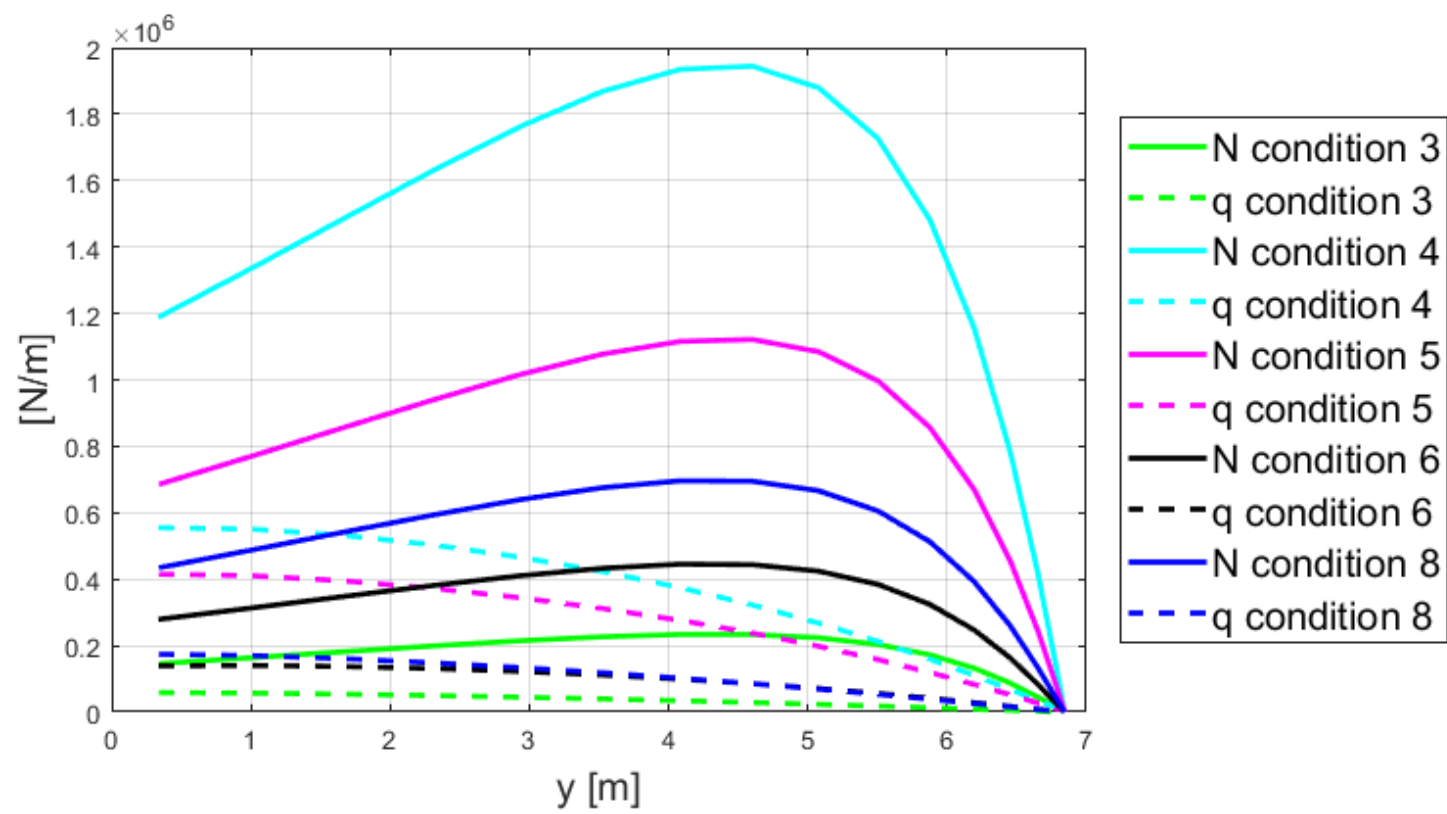

Figura 4-19 Carga por unidad de longitud y flujo cortante a lo largo de la envergadura del estabilizador vertical del CSR-01 para las maniobras asimétricas. Fuente: elaboración propia.

\section{Peso del cajón Peso del estabilizador}

\begin{tabular}{|lll|}
\hline Condición 3 & $53 \mathrm{~kg}$ & $450 \mathrm{~kg}$ \\
\hline Condición 4 & $262 \mathrm{~kg}$ & $656 \mathrm{~kg}$ \\
\hline Condición 5 & $136 \mathrm{~kg}$ & $530 \mathrm{~kg}$ \\
\hline Condición 6 & $79 \mathrm{~kg}$ & $473 \mathrm{~kg}$ \\
\hline Condición 7 & $94 \mathrm{~kg}$ & $488 \mathrm{~kg}$ \\
\hline
\end{tabular}

Tabla 4-9 Pesos resultantes del estabilizador vertical para cada una de las maniobras asimétricas. Fuente: elaboración propia.

De esta forma se puede concluir que el conjunto de maniobras seleccionadas, tanto simétricas como asimétricas, para dimensionar los estabilizadores de cola del CSR-01 es una buena representación de las cargas críticas, ya que los errores que se han obtenido en la estimación de pesos de las superficies de cola se encuentran en un margen adecuado para etapas de diseño conceptual. 


\subsection{DIMENSIONADO DE UNA CONFIGURACIÓN DE COLA EN V}

La configuración de cola que se va a seleccionar de entre todas las posibles para realizar un caso de aplicación de la metodología descrita será la cola en $\mathrm{V}$, ya que es la que supone un menor cambio en el aspecto exterior del avión, además de ser la más sencilla de entre las configuraciones no convencionales, lo que facilita las tareas de validación. El objetivo es dimensionar una cola en $V$ que desarrolle las mismas funciones que las de la cola convencional del avión de referencia. El capítulo se va a estructurar de tal forma que primeramente se va a justificar la selección de los parámetros de diseño que se van a considerar para caracterizar esta configuración. Posteriormente, se mostrará el espacio de diseño, limitado por las restricciones impuestas. Por último, se analizará la función objetivo y donde se encuentra el óptimo dentro del espacio de diseño restringido.

\subsubsection{Parámetros de diseño}

Los parámetros que caracterizan una cola en $\mathrm{V}$ son esencialmente los mismos que describen un estabilizador horizontal, con la única salvedad que el ángulo de diedro tiene un mayor rango de valores que puede tomar. Estos parámetros son los siguientes:

- Cuerda en la raíz.

- Parámetro de estrechamiento.

- Envergadura.

- Ángulo de diedro.

- Flecha de puntos $1 / 4$.

- Ley de torsión.

- Espesor relativo.

- Perfil aerodinámico.

Esta lista de parámetros se puede tratar de reducir tomando como constantes algunos de ellos. Esto se debe a que la función objetivo y las restricciones no son especialmente sensibles a los cambios de estos parámetros o a que se suelen tomar los mismos parámetros en etapas de diseño conceptual de aviones de transporte comercial. Por ejemplo, el perfil aerodinámico que se toma para las superficies de cola es simétrico, puesto que su función es equilibrar el avión, por lo que puede que tenga que sustentar hacia arriba en unos casos y hacia abajo en otros. En este caso, se ha tomado el mismo que el del estabilizador horizontal del avión de referencia, es decir, un NACA 0012. De la misma forma, el estabilizador horizontal no presenta torsión alguna, así que se ha considerado que la cola en $\mathrm{V}$ tampoco lo va a tener. Además, el espesor relativo se ha considerado también igual al del estabilizador horizontal. Por último, a la vista del efecto que tiene cambiar la flecha de puntos $1 / 4$ en las derivadas de estabilidad y en el peso, y sabiendo que suele tomar unos valores característicos situados alrededor de $25^{\circ} \circ 30^{\circ}$, se ha decidido mantener constante e igual al del estabilizador horizontal del CSR-01. En resumen, que el estudio de la configuración de cola en $\mathrm{V}$ se reduce a analizar la envergadura, diedro, parámetro de estrechamiento y cuerda en la raíz que se le debe dar al estabilizador horizontal del avión de referencia para cumplir con las restricciones y alcanzar el óptimo de la función objetivo. En la Figura 4-20 se muestran las definiciones de los parámetros sobre una cola en $\mathrm{V}$, salvo el parámetro de estrechamiento que se define como el cociente entre la cuerda en la punta y la cuerda en la raíz.

Además, una vez definida la geometría externa de la cola en $\mathrm{V}$, es necesario definir el tamaño y la posición de los controles longitudinal y lateral, es decir, el timón de profundidad y el de dirección. Como primera aproximación, los controles serán dos, 


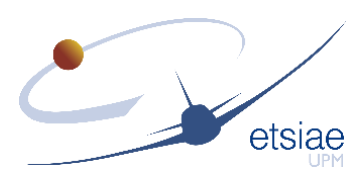

tendrán la misma envergadura y van a abarcar todo el borde de salida, como se muestra en la Figura 4-20, ocupando una fracción de cuerda del $25 \%$. Se van a contemplar dos casos: que el control interior (1 en la figura) sea el timón de profundidad, es decir, el control longitudinal y, en consecuencia, el exterior (2 en la figura) se el de dirección; o, justo al revés, que el interior sea el control lateral y el exterior el longitudinal. Analizando aeronaves que llevan esta configuración, principalmente aeronaves remotamente pilotadas como el Global Hawk, Predator B, RQ-7 Shadow, Milano o Atlante, se observa que el control exterior es el timón de profundidad, por lo que prevalecerá esta configuración, aunque se han querido contemplar las dos. Es cierto que la selección del control interior como el timón de dirección presenta la ventaja que el momento de balance que se genera como consecuencia de su deflexión es menor que si estuvieran situados en el control exterior, pudiendo ser este el motivo por el cual se hace esta selección.

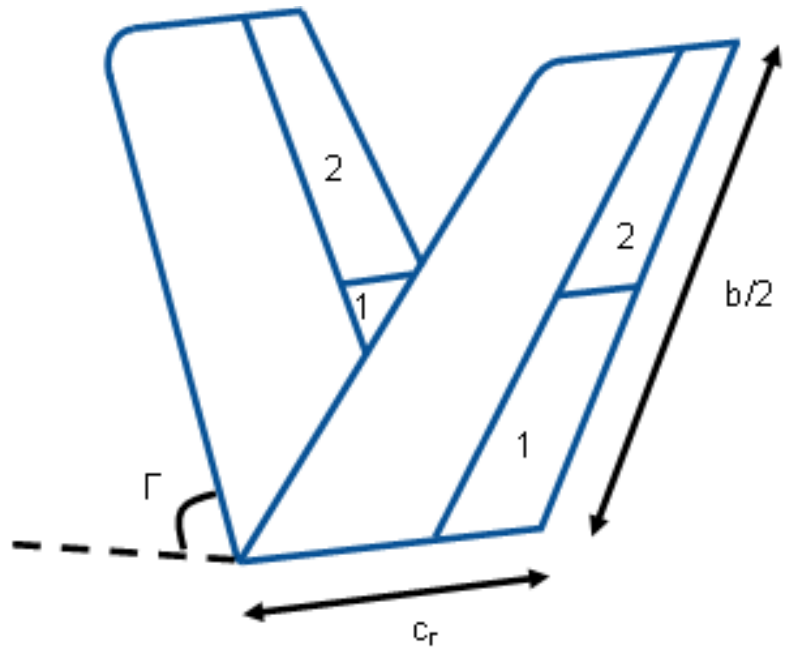

Figura 4-20 Definición de la envergadura, $b$, cuerda en la raíz, $C_{r}$ y ángulo de diedro, $\Gamma$, de una cola en $V$ y dónde se sitúan los controles. Fuente: elaboración propia.

El estudio de la configuración de cola en $V$ se llevará a cabo analizando el efecto de los cuatro parámetros geométricos propuestos (cuerda en la raíz, envergadura, parámetro de estrechamiento y diedro) y de las deflexiones necesarias para equilibrar la aeronave en las condiciones que se propongan. Así, es necesario limitar la búsqueda de valores de los parámetros y deflexiones para que el resultado tenga sentido físico, atendiendo a las recomendaciones expuestas en el capítulo 3.4 sobre que no se debe perder de vista el problema físico que se está resolviendo. La Tabla 4-10 recoge los límites de las variables. En el caso de los parámetros geométricos, los rangos se han establecido en base a estudios previos y análisis del estado del arte de configuraciones de cola en $\mathrm{V} y$ convencionales. Las deflexiones máximas y mínimas de los controles se han establecido acorde a las que se establecen para el Airbus A320 (Airbus 2005), asumiendo que el rango del control longitudinal es de $\left[-17^{\circ}, 30^{\circ}\right]$ y el del timón de dirección viene dado por la Figura 4-6. Se ha seleccionado este avión como referencia porque es un avión semejante en misión y configuración al avión CSR-01 que se encuentra en la base de datos CeRAS. 


\begin{tabular}{|ccc|}
\hline Parámetro & Unidades & Intervalo \\
\hline Envergadura & {$[\mathrm{m}]$} & {$[12-26]$} \\
\hline Cuerda en la raíz & {$[\mathrm{m}]$} & {$[3,3-4,5]$} \\
\hline Parámetro de estrechamiento & - & {$[0,28-0,36]$} \\
\hline Ángulo de diedro & $\left.{ }^{\circ}\right]$ & {$[0-50]$} \\
\hline
\end{tabular}

Tabla 4-10 Intervalo de búsqueda de los parámetros de diseño. Fuente: elaboración propia.

\subsubsection{Restricciones del espacio de diseño}

\subsubsection{Equilibrado longitudinal del avión}

El primer paso va a ser determinar el ángulo de ataque y la deflexión del timón de profundidad para equilibrar el peso del avión y el momento de cabeceo. Al igual que en el caso del avión de referencia, el sistema que hay que resolver es el [ 3-14 ]. Las condiciones de vuelo en las que se debe resolver son las de crucero, las de aterrizaje con viento cruzado y las de fallo del motor crítico en el segundo segmento, descritas en el apartado 0. Para cada una de las condiciones, se ha hecho un barrido de las variables del espacio de diseño dentro de los intervalos establecidos previamente. Comparando los resultados obtenidos, dentro de una misma condición de vuelo, apenas se perciben diferencias en los ángulos de ataque necesarios, ya que la contribución a la sustentación del avión de las superficies de cola no son grandes, inferiores al $10 \%$ del total (Torenbeek 1982).

En una primera aproximación al problema se va a considerar que en las tres condiciones de vuelo se tiene la misma posición del centro de gravedad, que será igual al $30 \%$ de la cuerda media aerodinámica, que el ángulo de equilibrado de la cola es nulo y el timón de profundidad corresponde al interior. Los valores medios obtenidos para cada una de las condiciones son $3,6^{\circ}$ para el crucero, $0,6^{\circ}$ para el aterrizaje y $1,9^{\circ}$ para el segundo segmento. En todos los casos, las variaciones en ángulo de ataque, comparando diferentes valores de los parámetros de diseño, se sitúan por debajo del $0,5 \%$.

Sin embargo, las diferencias en los ángulos del control para equilibrar la aeronave son más acuciadas. Analizando los resultados que se obtienen en esta primera aproximación, se concluye que es necesario deflectar el timón en un cierto sentido para equilibrar el avión. Si el diedro de la cola aumenta, sin cambiar el resto de parámetros, la sustentación de la cola es menor, ya que la fuerza proyectada verticalmente también es menor. De hecho, se ha visto en la ecuación [ 3-7 ] que esta fuerza decrece como el coseno al cuadrado del diedro. De ahí que la deflexión del control necesaria para alcanzar el valor de la sustentación que equilibra el peso debe reducirse a medida que aumenta el diedro de la cola en V. Esto se muestra gráficamente en la Figura 4-23, Figura 4-22 y Figura 4-23. Hay que indicar que este razonamiento es válido si el ángulo de equilibrado de la cola es nulo en los tres casos. Posteriormente se explicará lo que ocurre en la situación en la que el ángulo de equilibrado no sea nulo. El comportamiento que tiene la deflexión del control con los cambios con la cuerda en la raíz es un poco más difícil de explicar. Lo primero es recordar que para equilibrar el avión la cola necesita alcanzar una cierta sustentación, para cada condición de vuelo, independientemente de los valores de las variables de diseño. Esta sustentación de la cola va a recibir el nombre de sustentación objetivo de la cola. Este valor dependerá de la condición de vuelo, como es lógico, pero no de las variables de diseño de la cola en V. Centrándonos en una sola condición de vuelo, lo que cambia en función de las variables de diseño para alcanzar la sustentación objetivo de la cola es el ángulo de 


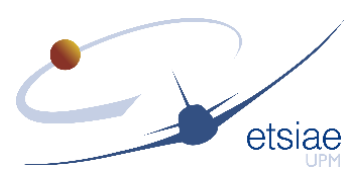

deflexión. En el caso de la cuerda en la raíz, sus variaciones afectan a la superficie de la cola. Esto supone que, para un mismo ángulo de ataque, valores mayores de la cuerda en la raíz llevan a menores valores del coeficiente de sustentación que necesita desarrollar la cola para alcanzar la sustentación objetivo. Además, la cuerda en la raíz afecta al alargamiento de la superficie por lo que se reduce la pendiente de la curva de sustentación al incrementar la cuerda. Sin embargo, aumentar la cuerda aumenta la derivada del momento de cabeceo con respecto al ángulo de deflexión del control. Estos dos últimos efectos son contrapuestos, pero se impone el segundo, por eso finalmente el aumentar la cuerda en la raíz resulta en que es necesario deflectar menos el timón de profundidad para equilibrar el avión.

Un razonamiento semejante se puede aplicar para explicar el efecto del parámetro de estrechamiento. Aumentarlo supone un incremento en el área y en la pendiente de la curva de sustentación debido a que el alargamiento aumenta también. Sin embargo, la derivada del momento de cabeceo con respecto al ángulo de deflexión del control apenas cambia. De ahí que, si el área y la pendiente son ligeramente mayores, la deflexión necesaria para alcanzar la sustentación objetivo de la cola sea algo menor. De todas formas, el efecto del parámetro de estrechamiento no es muy fuerte, como se puede ver en las curvas.
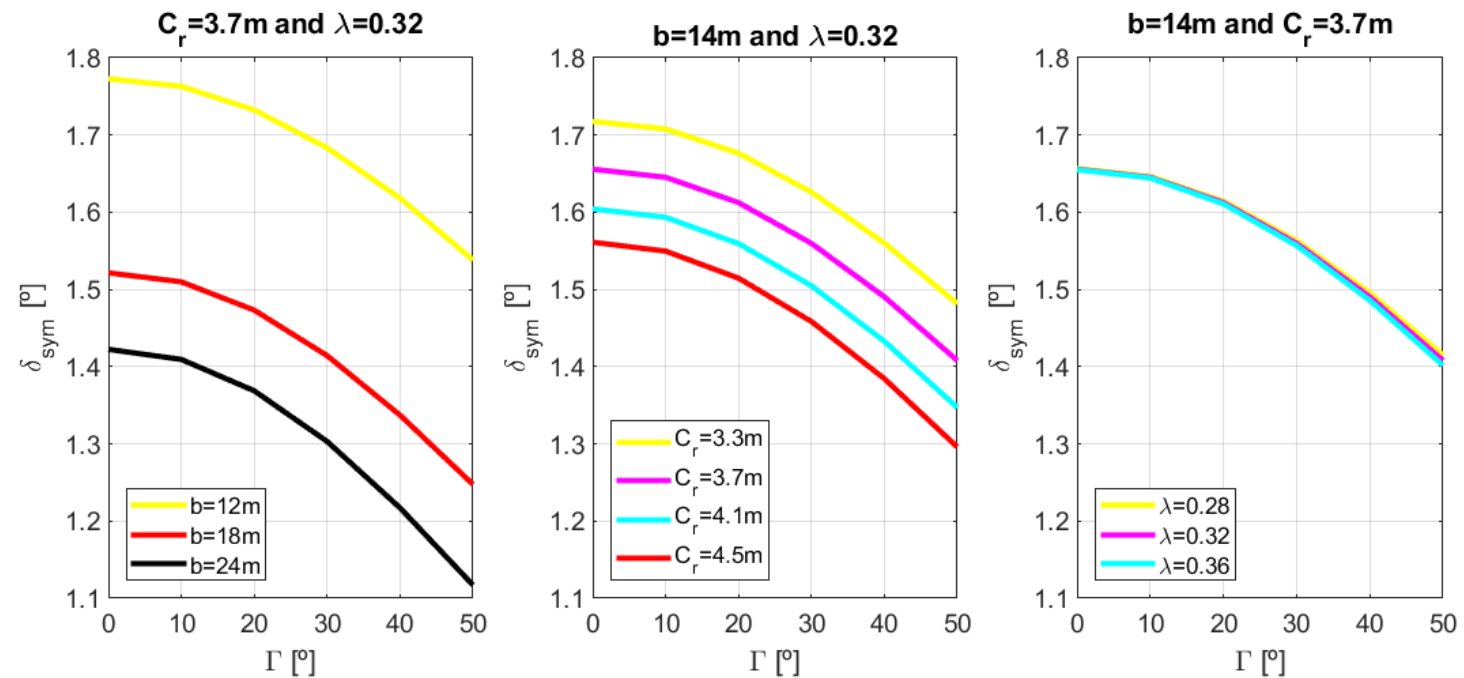

Figura 4-21 Análisis del efecto de la envergadura, cuerda en la raíz y el parámetro de estrechamiento al variar el ángulo de diedro de la cola en $V$ en la deflexión del timón de profundidad, situado en el control interior, para equilibrar en condiciones de aterrizaje. Fuente: elaboración propia. 

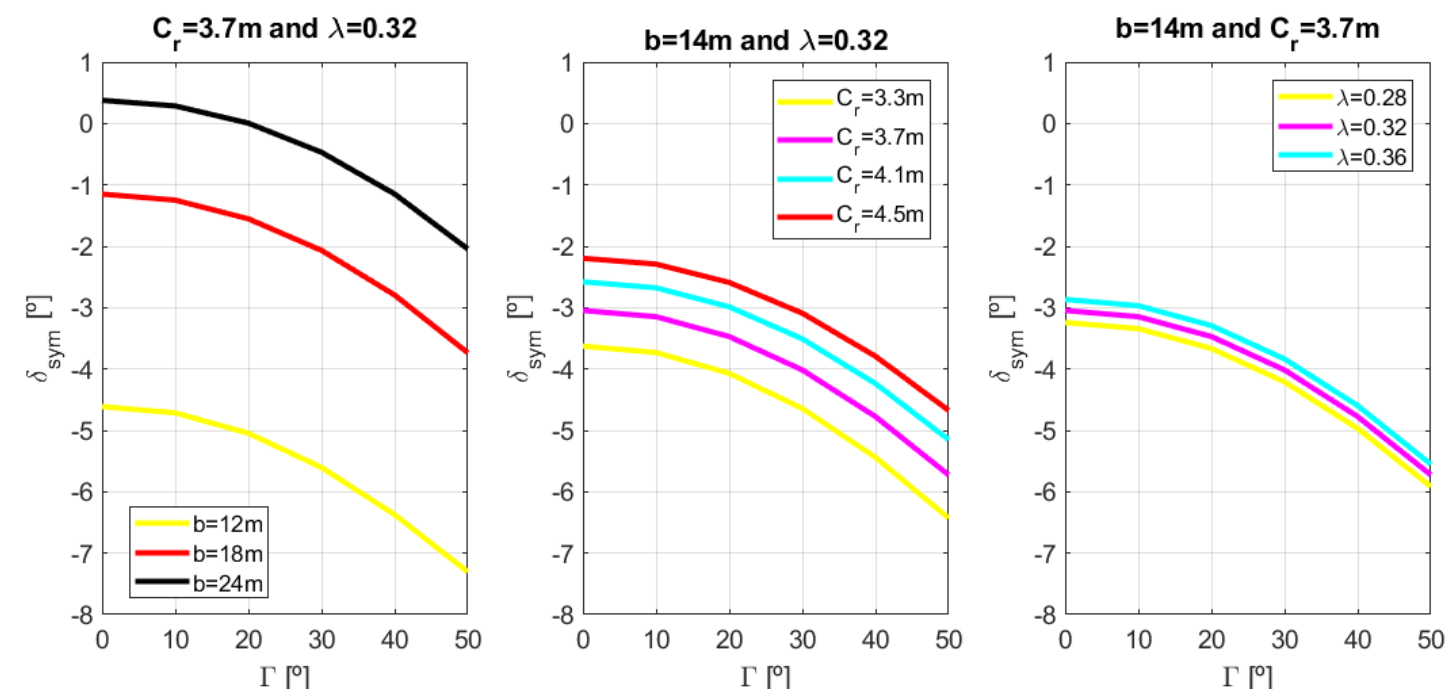

Figura 4-22 Análisis del efecto de la envergadura, cuerda en la raíz y el parámetro de estrechamiento al variar el ángulo de diedro de la cola en $V$ en la deflexión del timón de profundidad, situado en el control interior, para equilibrar en condiciones de segundo segmento.

Fuente: elaboración propia.
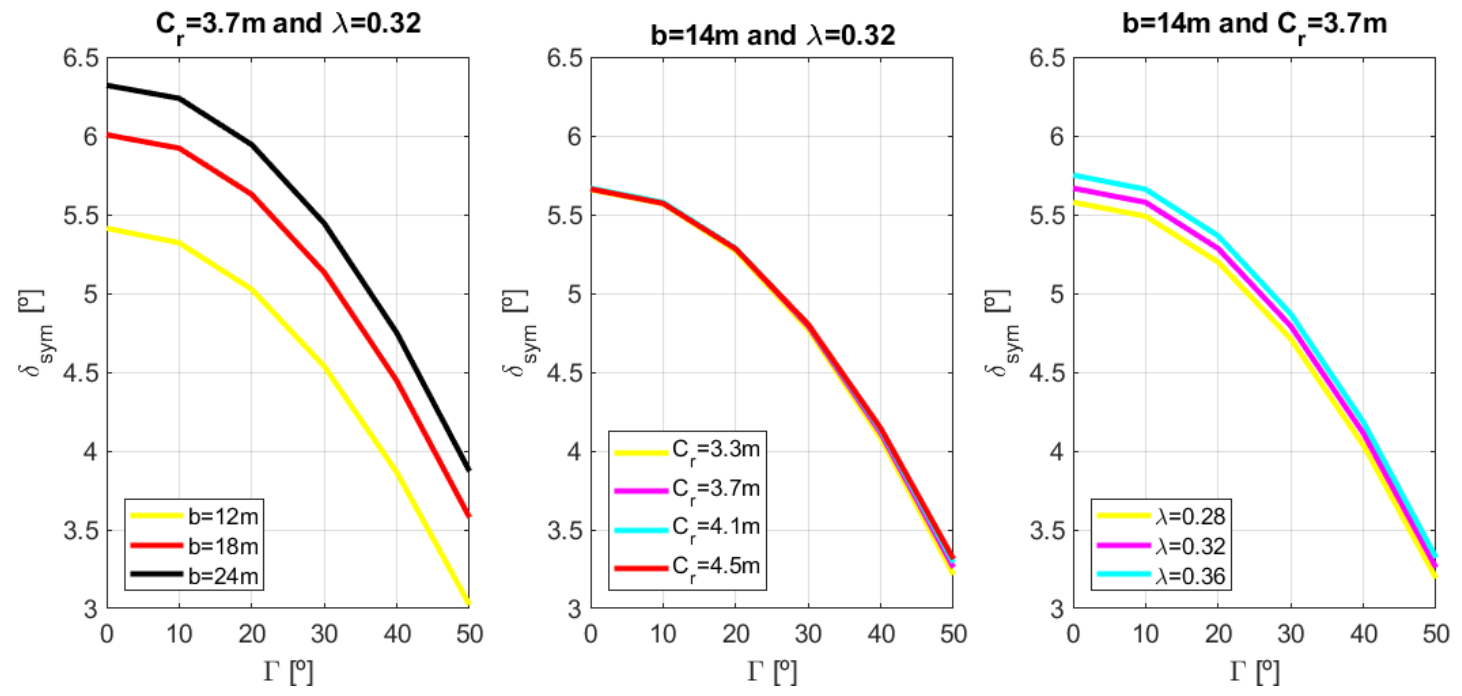

Figura 4-23 Análisis del efecto de la envergadura, cuerda en la raíz y el parámetro de estrechamiento al variar el ángulo de diedro de la cola en $V$ en la deflexión del timón de profundidad, situado en el control interior, para equilibrar en condiciones de crucero. Fuente: elaboración propia.

Sin embargo, el comportamiento con los cambios en la envergadura es diferente entre segundo segmento y crucero, por un lado, y aterrizaje, por otro. En las dos primeras condiciones, el equilibrio se produce para un coeficiente de sustentación bien distinto al de la otra condición debido al efecto del empuje. El empuje genera un momento que ayuda a equilibrar la aeronave. Esto significa que la sustentación objetivo en estas condiciones son diferentes con relación a la condición de aterrizaje; de hecho, cambia el signo entre una y otra. Por otro lado, aumentar la envergadura supone que la pendiente de la curva de sustentación también lo haga. En consecuencia, el coeficiente de sustentación de la cola es mayor en valor absoluto para un mismo ángulo de ataque. Por tanto, para reducir el valor del coeficiente de sustentación para alcanzar la sustentación objetivo, en el caso de la sustentación negativa es necesario reducir la deflexión del control; pero en el caso de la sustentación positiva es necesario aumentar dicha deflexión. De esta forma se explican los comportamientos experimentados en los 


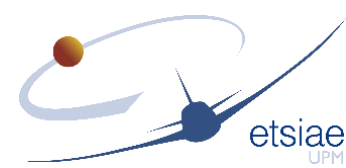

resultados, aumentando la deflexión del timón de profundidad en las condiciones de segundo segmento y crucero cuando aumenta la envergadura. $Y$ en el caso del aterrizaje, disminuyendo la deflexión del control con el aumento de envergadura. Esto se muestra de forma esquemática en la Figura 4-24.

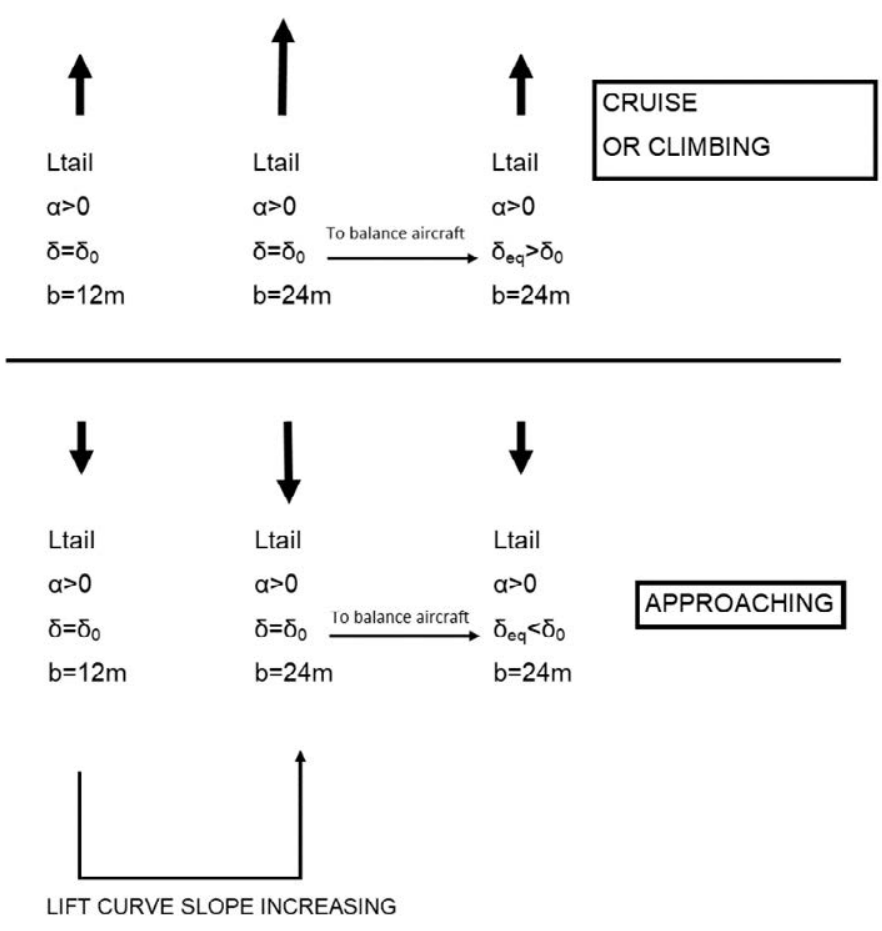

Figura 4-24 Esquema explicativo del efecto de variar la envergadura en la deflexión de equilibrado del control longitudinal en las tres condiciones de vuelo: crucero, segundo segmento y aterrizaje. Fuente: elaboración propia.

Una estimación más próxima a la realidad será si se considera que el control longitudinal es el exterior, el que se numera como 2 en la Figura 4-20 (García-Hernández, CuernoRejado y Pérez-Cortés 2017), en lugar del interior y, sobre todo, si se fija un ángulo de incidencia de la cola de tal forma que se equilibre el avión en un punto medio del crucero. El objetivo es resolver el equilibrado de fuerzas verticales y de momento de cabeceo del CSR-01, en lugar de calculando el ángulo de ataque y la deflexión del control necesarios, el ángulo de ataque y el ángulo de incidencia de la cola. Este ángulo de incidencia es el que se va a utilizar en el resto de condiciones de vuelo, ya que, como se ha comentado anteriormente, se va a considerar que la cola en $\mathrm{V}$ no tiene capacidad de incidencia variable, por lo que se determina para una cierta condición, que es precisamente la del punto medio del crucero (Torenbeek 1982).

El vuelo en crucero se realiza en las mismas condiciones que en el avión de referencia, pero ahora el centro de gravedad se ha estimado teniendo en cuenta el combustible que se ha consumido hasta el punto medio del crucero. Se ha supuesto que los tanques de combustible son una masa puntual. Además, el centro de gravedad del avión al peso vacío operativo se encuentra al $25,25 \%$, según la base de datos CeRAS, y la suma del peso vacío operativo y el peso máximo de combustible resultan en una posición del centro de gravedad del $25,8 \%$. De ahí, se puede calcular la posición del centro de gravedad de los tanques de combustible, que es al $27 \%$ de la cuerda media 
aerodinámica, aproximadamente. En cuanto a la carga de pago, se sabe dónde se encuentra el centro de gravedad del avión al peso máximo de despegue con la carga de pago de diseño, por tanto, se puede deducir la posición del centro de gravedad de la carga de pago de diseño, que resulta estar al $50 \%$ de la cuerda media aerodinámica. De esta forma, se puede estimar la posición del centro de gravedad del CSR-01 a medida que va consumiendo combustible y, en concreto, la que tendría en el punto medio del crucero. Esta posición corresponde con un valor del $30,6 \%$ de la cuerda media aerodinámica.

Una vez determinada la posición del centro de gravedad, se pasa a resolver el sistema [ 3-14 ], pero sustituyendo la deflexión del timón de profundidad por el ángulo de incidencia de la cola. Los resultados se muestran en la Figura 4-25. El ángulo de ataque resultante para cada una de las combinaciones de las variables de diseño apenas cambia de nuevo, situándose su valor medio alrededor de los $3,8^{\circ}$. Se observa como modificar las variables hacia una situación en la que el área de la cola aumenta, supone que el ángulo de incidencia se reduce en valor absoluto, es decir, la cola sustenta más en la dirección correspondiente y el ángulo de calado resultante se reduce. En el caso de variar el ángulo del diedro, la pendiente de la curva de sustentación de la cola se reduce, por tanto, el ángulo de incidencia necesario crece en valor absoluto. El signo del ángulo depende de la sustentación objetivo de la cola que se tiene que alcanzar, que en las situaciones que se muestran en la Figura 4-25, lleva a que el ángulo de incidencia tenga que ser negativo.
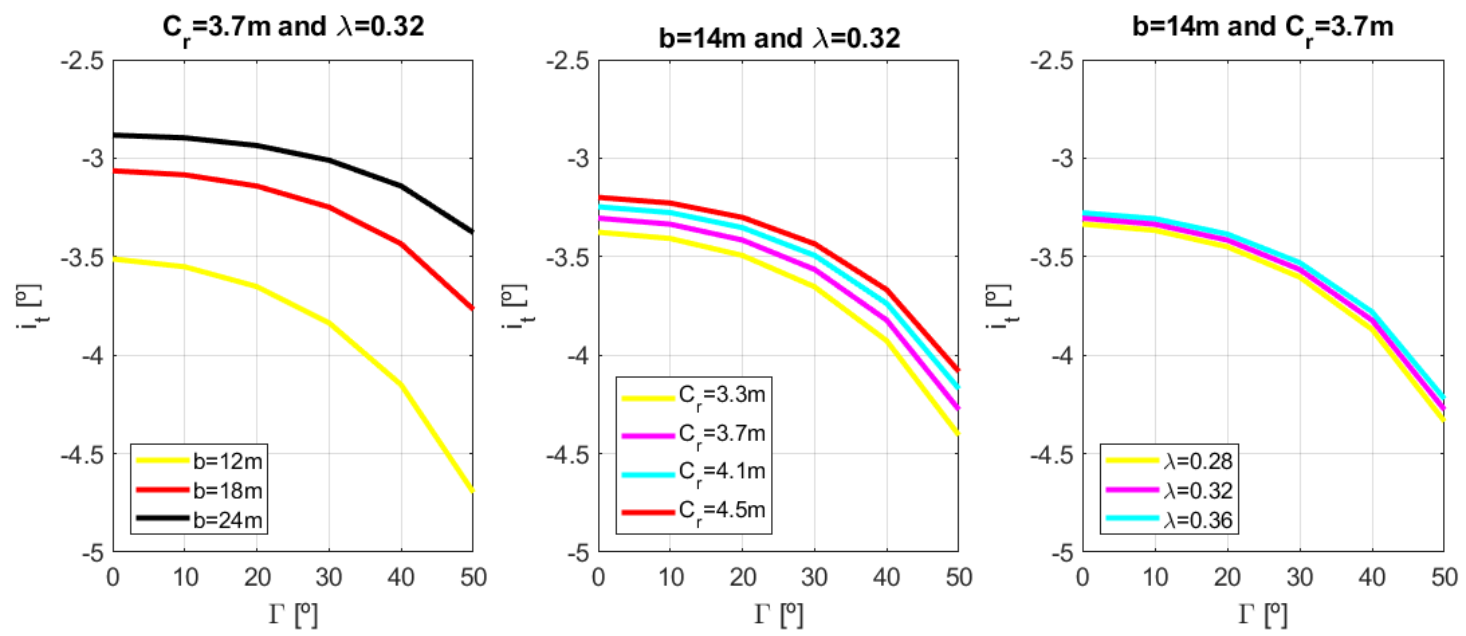

Figura 4-25 Ángulo de calado de la cola para equilibrar el CSR-01 en el punto medio de la condición de crucero. Fuente: elaboración propia.

A partir de aquí, se tomarán los ángulos de calado resultantes del equilibrio del punto medio del crucero como datos de entrada para resolver cualquier otra condición. Concretamente, se van a calcular los ángulos de deflexión del timón de profundidad necesarios para el equilibrio longitudinal del avión en las otras dos condiciones contempladas. Ahora sí, se tomarán los valores del centro de gravedad indicados en la Tabla 4-1, correspondientes al segundo segmento y aterrizaje. Analizando los resultados obtenidos, se reflejan dos comportamientos diferentes entre ambas condiciones, como se ven en la Figura 4-26 y en la Figura 4-27. Para entender lo que ocurre, primero hay que decir que el ángulo de ataque resultante en la condición de segundo segmento es aproximadamente $3,7^{\circ}$ para todos los valores de las variables de diseño, y para la de aterrizaje $0,87^{\circ}$. Entonces, el ángulo de incidencia determinado anteriormente, hace que el ángulo de ataque que ve la cola sea de diferente signo en cada una de las condiciones de vuelo. De ahí, que el comportamiento con el ángulo de 
diedro de la cola en $\mathrm{V}$ de la deflexión del timón de profundidad de equilibrio sea diferente. Aproximando la pendiente de la curva de sustentación de la cola por la que tendría si no tuviera diedro $\left(C_{L \alpha}\right)$ multiplicada por el cuadrado del coseno del diedro, la curva de sustentación de la cola quedaría, suponiendo aerodinámica lineal:

$$
c_{L \alpha} \alpha_{t} \cos ^{2} \Gamma+c_{L \delta} \delta_{\text {sym }}=c_{L T}
$$

Si se despeja la deflexión del timón de profundidad:

$$
\delta_{\text {sym }}=\frac{c_{L T}-c_{L \alpha} \alpha_{t} \cos ^{2} \Gamma}{c_{L \delta}}
$$

Representando la curva resultante de la ecuación [ 4-7 ] se obtiene el mismo comportamiento que los que se han determinado en la Figura 4-26 y en la Figura 4-27, dependiendo del ángulo de ataque que ve la cola.
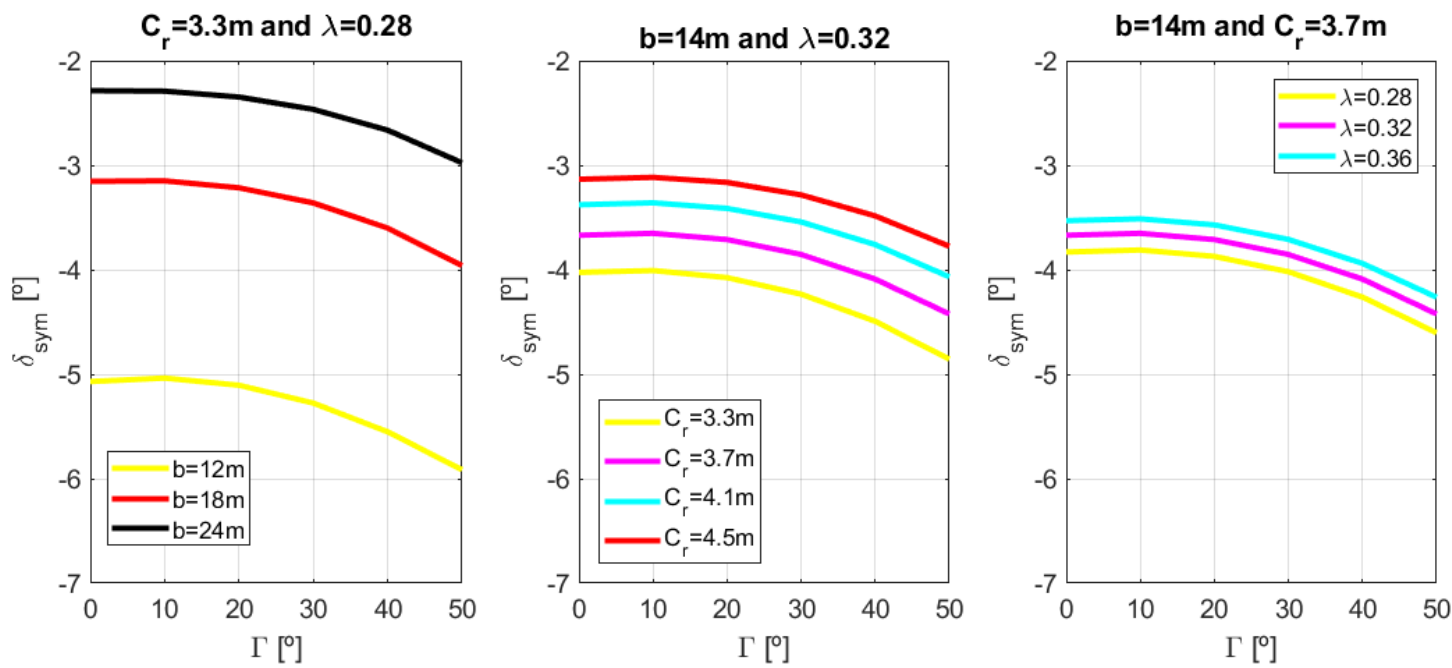

Figura 4-26 Ángulo de deflexión del timón de profundidad para equilibrar el avión en el segundo segmento, considerando el ángulo de calado de la cola determinado para la condición de crucero. Fuente: elaboración propia.
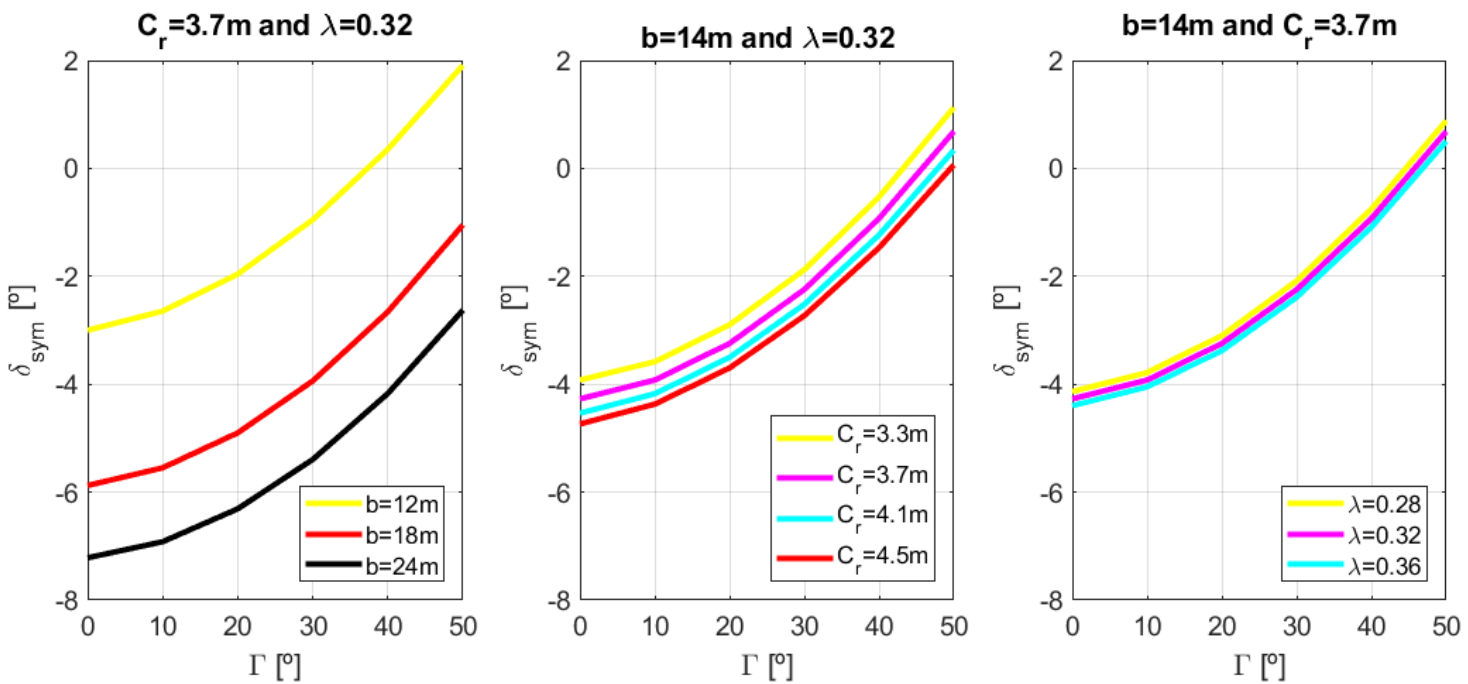

Figura 4-27 Ángulo de deflexión del timón de profundidad para equilibrar el avión en aterrizaje, considerando el ángulo de calado de la cola determinado para la condición de crucero. Fuente: elaboración propia. 


\subsubsection{Estabilidad estática en la condición de crucero}

En este apartado se va a analizar la variación de las derivadas de estabilidad estáticas en la condición de crucero del CSR-01 seleccionada. Hay que indicar que las derivadas se han determinado alrededor de la condición de equilibrio alcanzada con el ángulo de incidencia de la cola en la condición de crucero Los resultados se muestran en la Figura 4-28 y la Figura 4-29. En ambas figuras se indican con una línea discontinua roja los valores de las derivadas de estabilidad estática correspondientes al avión de referencia en la misma condición. Como se ha comentado anteriormente, la aeronave con la cola en $\mathrm{V}$ debe ser al menos tan estable estáticamente como el avión de referencia. Por tanto, en el caso de la estabilidad longitudinal, la zona donde es posible diseñar corresponde a la que se sitúa por debajo de la línea que marca la derivada de estabilidad estática longitudinal del CSR-01. Sin embargo, en el caso de la estabilidad lateral, la zona de diseño posible es la que se encuentra por encima de la recta que marca el avión de referencia. Como se espera, en las figuras se ve como aumentar el área de la cola aumenta la estabilidad estática, tanto longitudinal como lateral. Por otra parte, incrementar el diedro de la cola en $\mathrm{V}$ mejora la estabilidad estática lateral, pero empeora la longitudinal. Por ejemplo, en el caso de una envergadura de 24 metros, la condición de la derivada de estabilidad estática longitudinal impone que como máximo se puede tener un diedro de $45^{\circ}$ aproximadamente y la condición de la estabilidad lateral que debe estar por encima de $40^{\circ}$. Esto muestra que la derivada longitudinal es una restricción superior al ángulo de diedro y la derivada lateral lo es inferior.
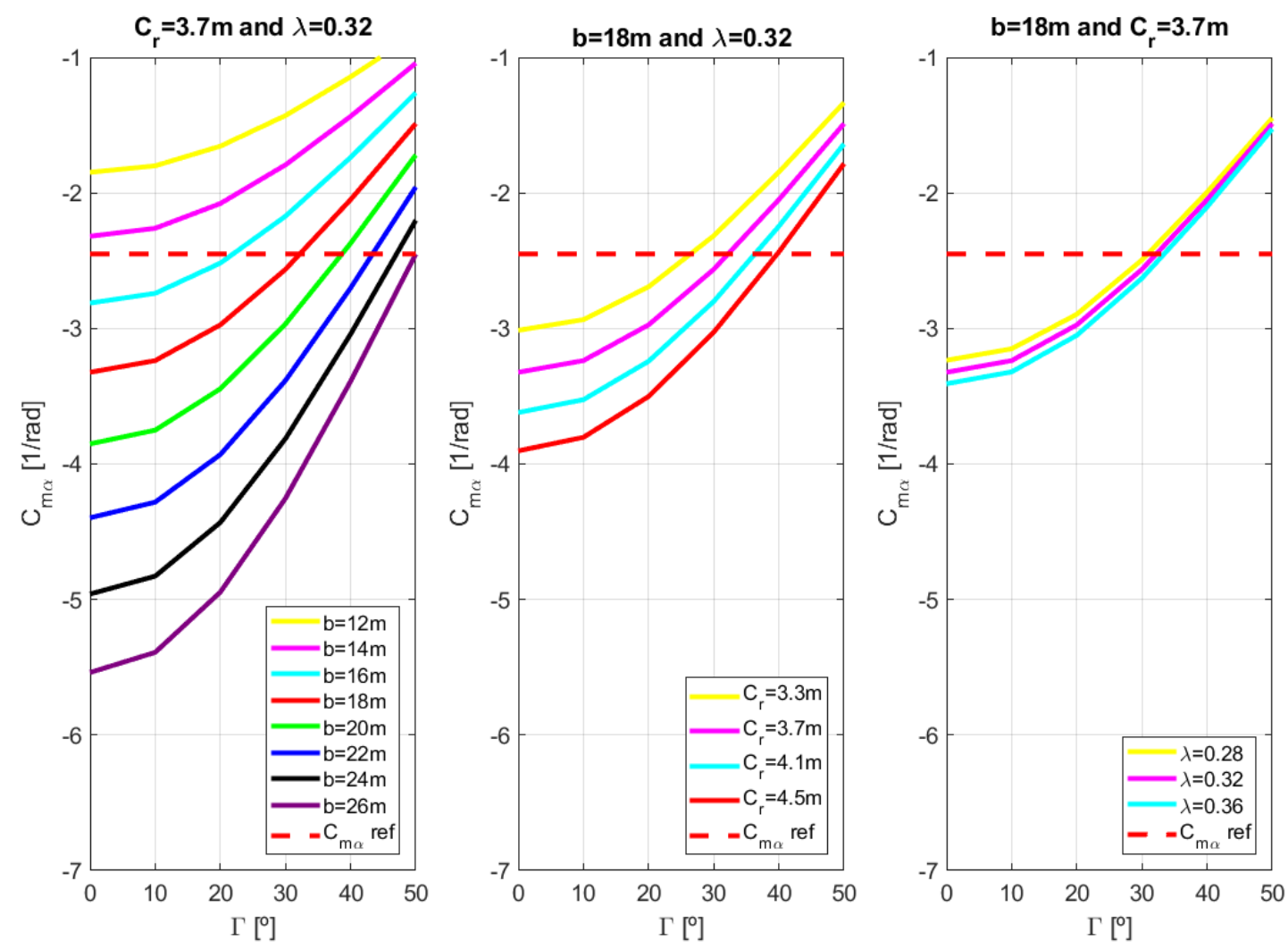

Figura 4-28 Análisis del efecto de la envergadura, la cuerda en la raíz, el parámetro de estrechamiento en función de la variación del diedro de la derivada estática longitudinal en la condición de crucero para una cola en V. Fuente: elaboración propia. 

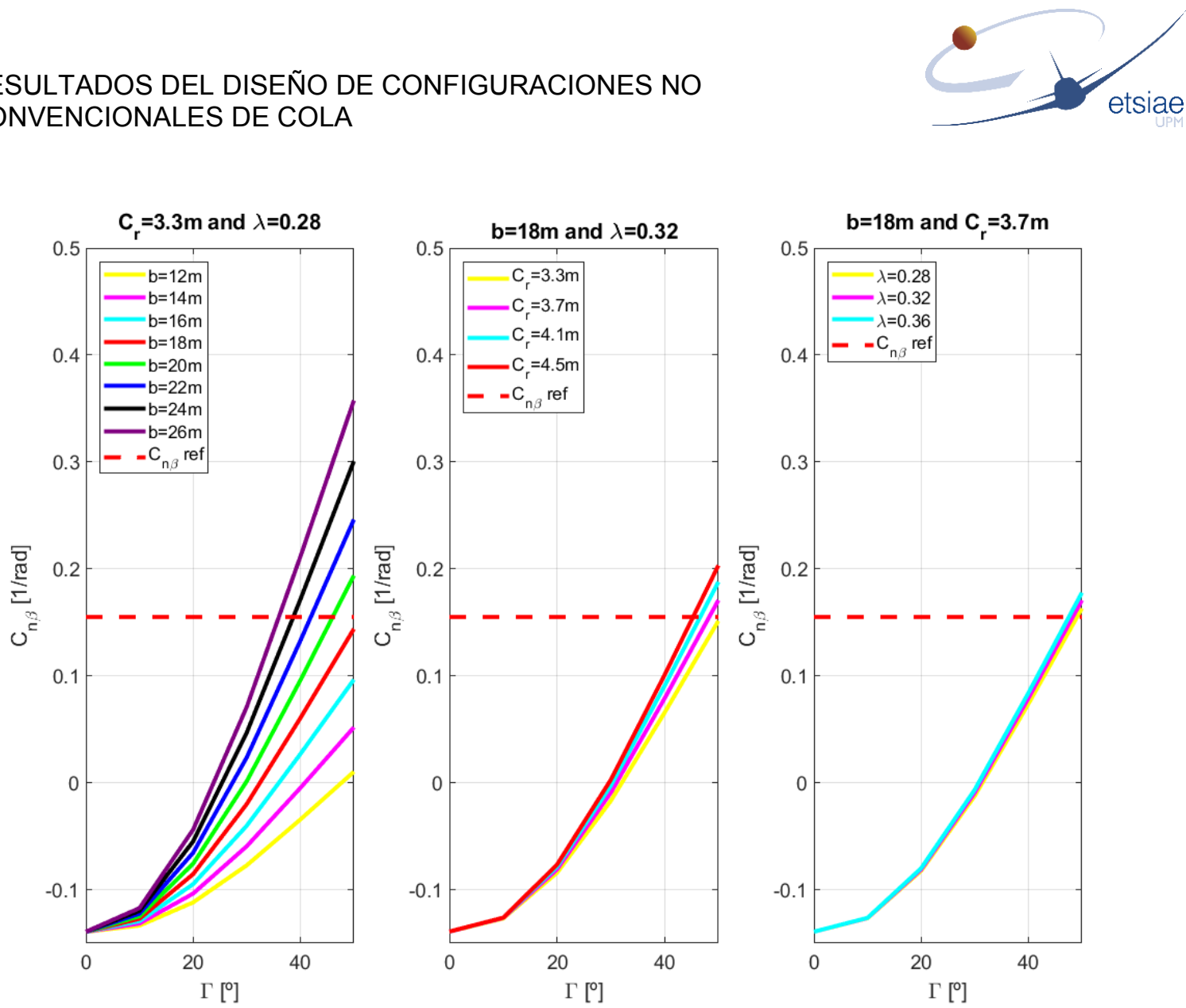

Figura 4-29 Análisis del efecto de la envergadura, la cuerda en la raíz, el parámetro de estrechamiento en función de la variación del diedro de la derivada estática lateral en la condición de crucero para una cola en V. Fuente: elaboración propia.

Para ambas derivadas de estabilidad, es posible determinar la red neuronal que ajusta los resultados de tal forma que permita interpolar las derivadas de estabilidad en puntos donde la malla no se ha evaluado aún. En el caso de la derivada de estabilidad estática longitudinal, el número de neuronas de la capa oculta de la red es de cinco, en cambio, para el caso de la derivada de estabilidad estática lateral, no se notaba mejoría en el ajuste al aumentar de tres, por tanto, se ha escogido tres neuronas en la capa oculta. La muestra inicial de puntos, en ambos conjuntos, se ha dividido en tres grupos, el de entrenamiento, validación y prueba en la proporción $70 \%, 15 \%$ y $15 \%$, respectivamente. El algoritmo que se ha utilizado para determinar los coeficientes de la red neuronal, tanto en el ajuste de la derivada lateral como en la longitudinal, es el de Levenberg-Marquardt; al igual que la función de activación, que es la sigmoidea en ambos casos. La condición inicial de los coeficientes para empezar a iterar con el algoritmo anterior son valores aleatorios que pertenecen al intervalo $[0 ; 1]$ en ambos casos. Los resultados que se obtienen tras realizar el ajuste de los resultados de la derivada estática longitudinal en crucero se muestran en Figura 4-30, y los que se obtienen para la derivada estática lateral en la Figura 4-31. Se puede observar como el coeficiente de correlación obtenido para las derivadas longitudinales son algo mayor que el correspondiente a las derivadas laterales, aunque en ambos casos se sitúan en todos los conjuntos por encima del $99,5 \%$. Además, se ve como los ajustes en los conjuntos de validación y de prueba siguen siendo buenos comparados con los de entrenamiento, lo cual es un signo de que no haya demasiado sobreajuste. Precisamente este es uno de los motivos por el cual no se ha incrementado el número de neuronas de la capa oculta del ajuste de la derivada lateral, ya que se vio que aparecía el fenómeno de overfitting si este número crecía demasiado. 

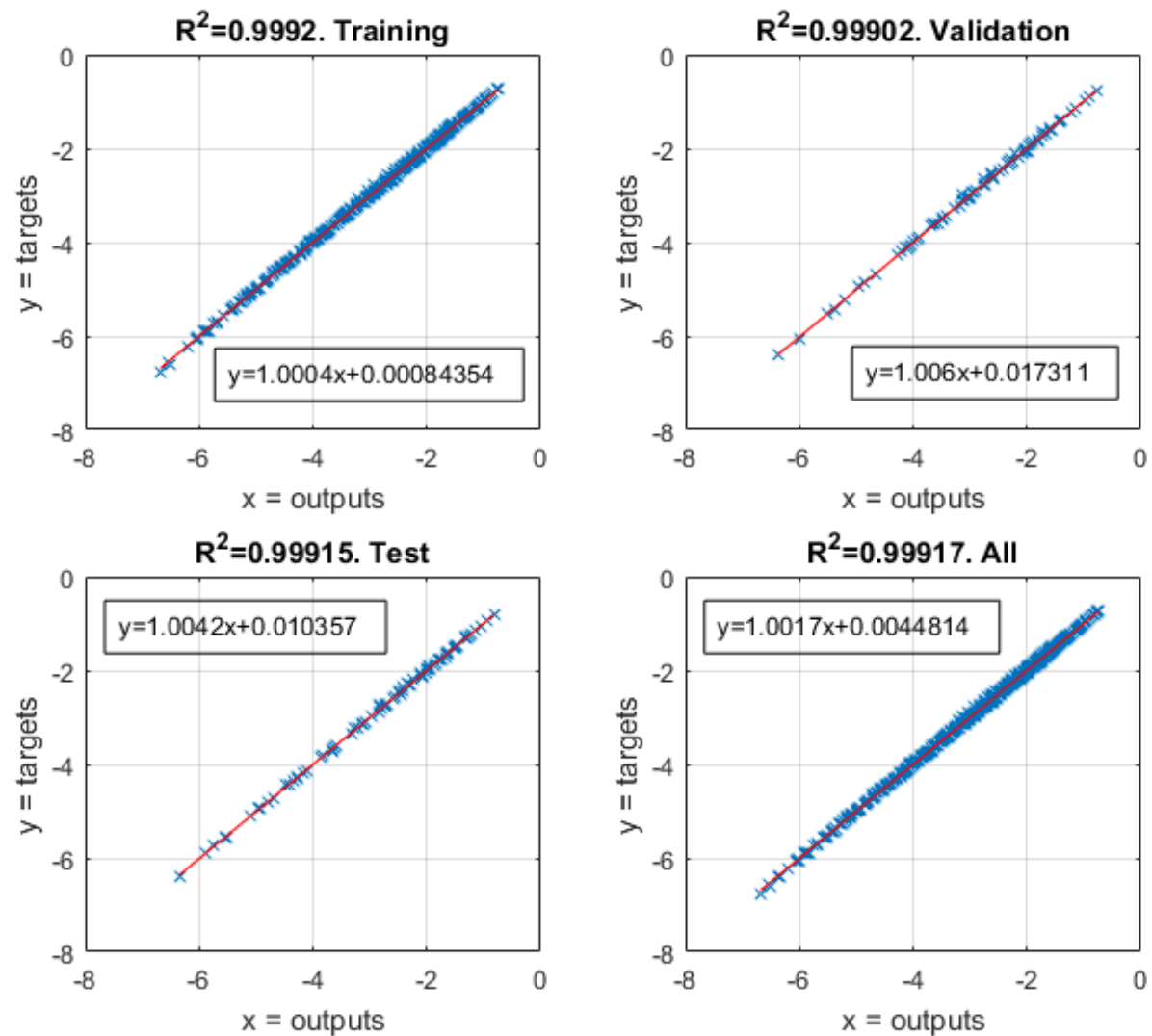

Figura 4-30 Resultados del ajuste de las derivadas de estabilidad estática longitudinal en crucero por medio de una red neuronal de una capa oculta con cinco neuronas. Fuente: elaboración propia.
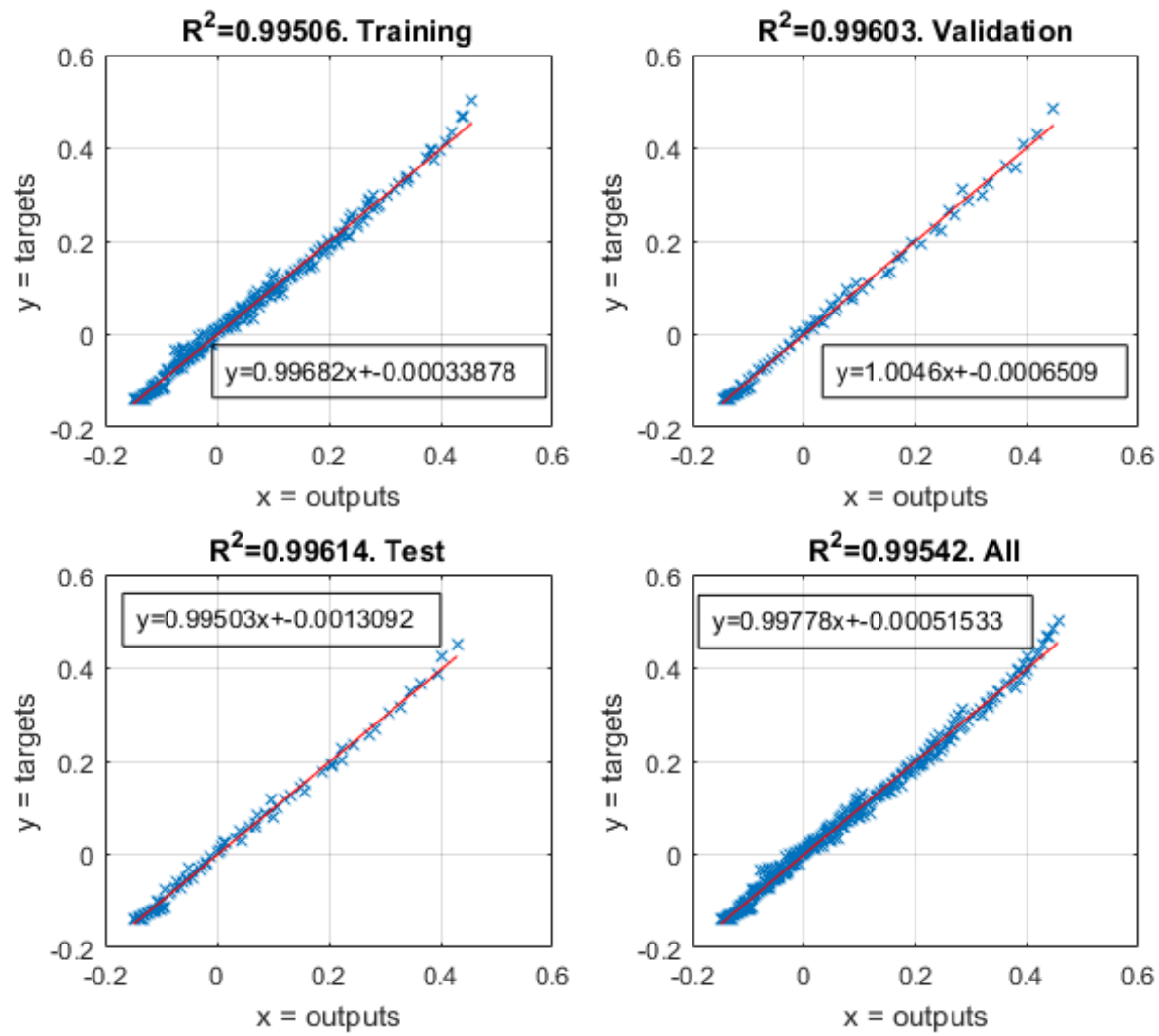

Figura 4-31 Resultados del ajuste de las derivadas de estabilidad estática lateral en crucero por medio de una red neuronal de una capa oculta con tres neuronas. Fuente: elaboración propia. 


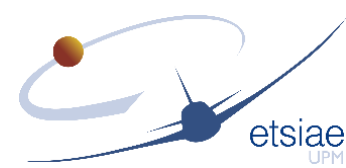

\subsubsection{Equilibrado del momento en guiñada del avión en la condición de fallo del motor crítico}

En la condición de fallo del motor crítico en segundo segmento el avión debe ser controlable gracias a la actuación de la cola. Esta condición requiere que la cola tenga un cierto tamaño para poder controlar el avión. El tamaño dependerá del ángulo de resbalamiento para el cual se quiere que se produzca el equilibrio de momentos. En este caso, se va a considerar que el ángulo de resbalamiento deba ser nulo y el equilibrio se efectúa por medio de la deflexión del timón de dirección. Al igual que en el caso anterior, la posición del timón de profundidad, interior o exterior de la cola en $\mathrm{V}$, afecta a los resultados de deflexión necesarias para anular el momento de guiñada generado por el fallo del motor crítico. Sin embargo, las diferencias no son muy llamativas, así que únicamente se van a mostrar los resultados obtenidos para el caso en el que el timón de profundidad corresponde al interior de la cola en $\mathrm{V}$ y para el ángulo de calado de la cola igual al determinado para la condición de crucero (Figura 4-32). El límite fijado por la deflexión máxima del control está marcado por la línea horizontal roja discontinua y se ha determinado de acuerdo con el correspondiente a la Figura 4-6 a la velocidad de vuelo de esta condición, tomando el valor de $18^{\circ}$. Esta restricción supone un límite inferior al ángulo de diedro de la cola, ya que, para una geometría fija, al reducir el diedro la deflexión necesaria excede el límite. Al igual que ocurría en el análisis de los resultados del equilibrio longitudinal del avión, si el área de la cola crece, esta restricción admite soluciones con un ángulo de diedro menor. Otro aspecto importante que se debe comprobar tras encontrar la solución de equilibrio en guiñada es el ángulo de balance al cual se produce el vuelo del avión. En ninguno de los casos se supera el límite establecido por la normativa de $5^{\circ}$ de ángulo de balance. Los resultados se encuentran entre $1,8^{\circ}$ y $2,2^{\circ}$. Estas pequeñas variaciones se deben a las variaciones que se encuentran en el coeficiente de fuerza lateral que equilibra la aeronave para cada una de las combinaciones de los parámetros de diseño que se han estudiado.
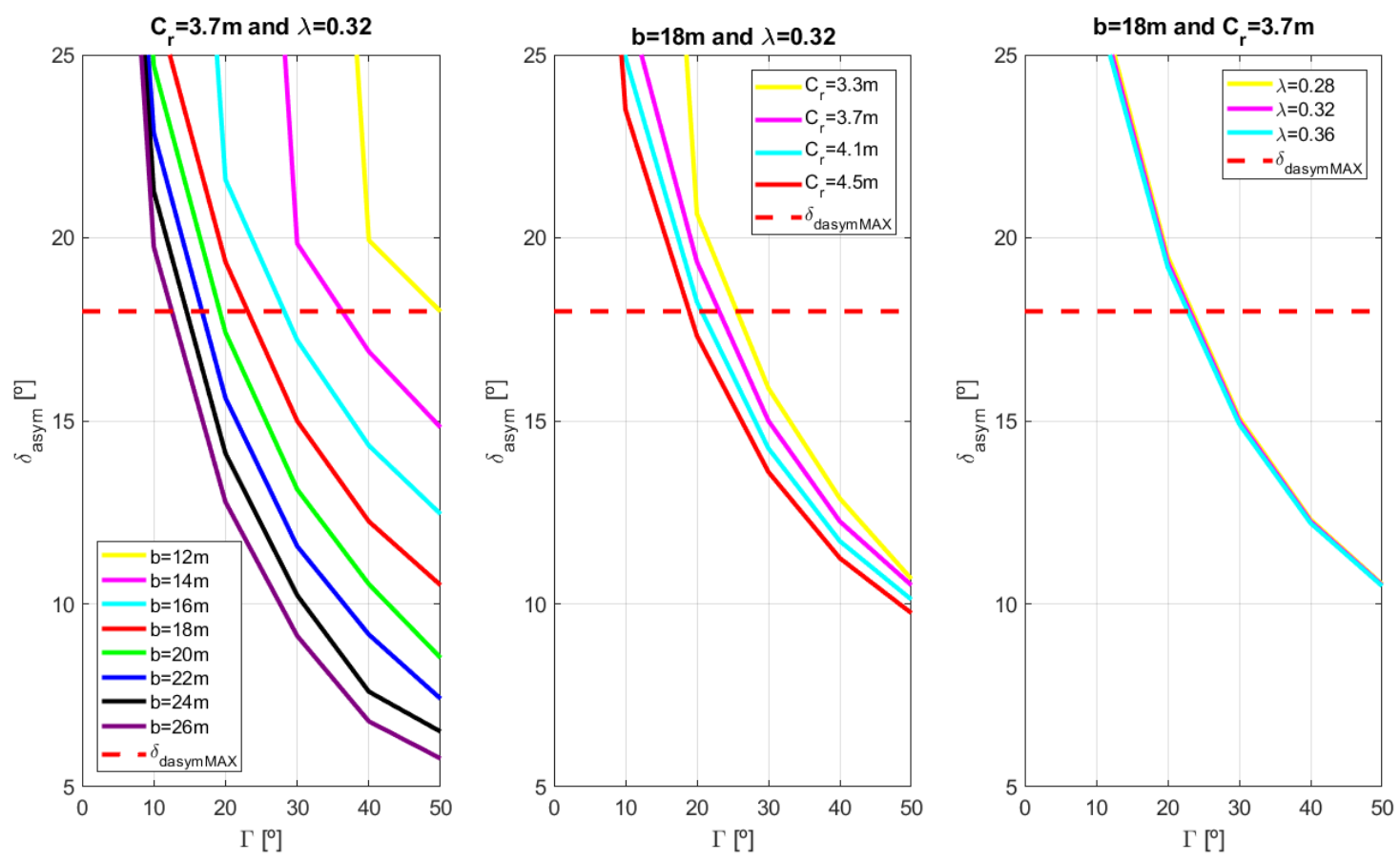

Figura 4-32 Análisis del efecto de la envergadura, cuerda en la raíz y parámetro de estrechamiento con la variación del diedro de la cola en $V$ en la condición del control de la aeronave tras el fallo del motor crítico en el segundo segmento. Fuente: elaboración propia. 
Los resultados obtenidos en este apartado se van a ajustar mediante una red neuronal para determinar una regresión que permita interpolar los ángulos de deflexión necesarios en combinaciones de los parámetros de diseño diferentes a las utilizadas para construir la malla inicial de puntos. La red neuronal de regresión construida en este caso presenta una sola capa oculta con seis neuronas en ella, con una función de activación del tipo sigmoidea. La muestra inicial de puntos se ha dividido en tres grupos, el de entrenamiento, validación y prueba en la proporción $70 \%, 15 \%$ y $15 \%$, respectivamente. El algoritmo que se ha utilizado para determinar los coeficientes de la red neuronal es el de Levenberg-Marquardt. La condición inicial de los coeficientes son valores aleatorios que pertenecen al intervalo [0;1]. Los resultados del proceso de ajuste de la red neuronal se muestran en la Figura 4-33. Como se puede observar, el coeficiente de correlación obtenido entre los resultados reales y los estimados es superior al $99,9 \%$ en los tres conjuntos en los que se ha dividido la muestra e incluso considerando todos los puntos en un mismo grupo. El hecho de que haya tan buenos resultados en los tres grupos, especialmente en el de prueba, representa que no se ha producido el fenómeno de overfitting. Los coeficientes que se han obtenido tras este ajuste son los que a partir de aquí van a utilizarse para representar los resultados de este apartado.
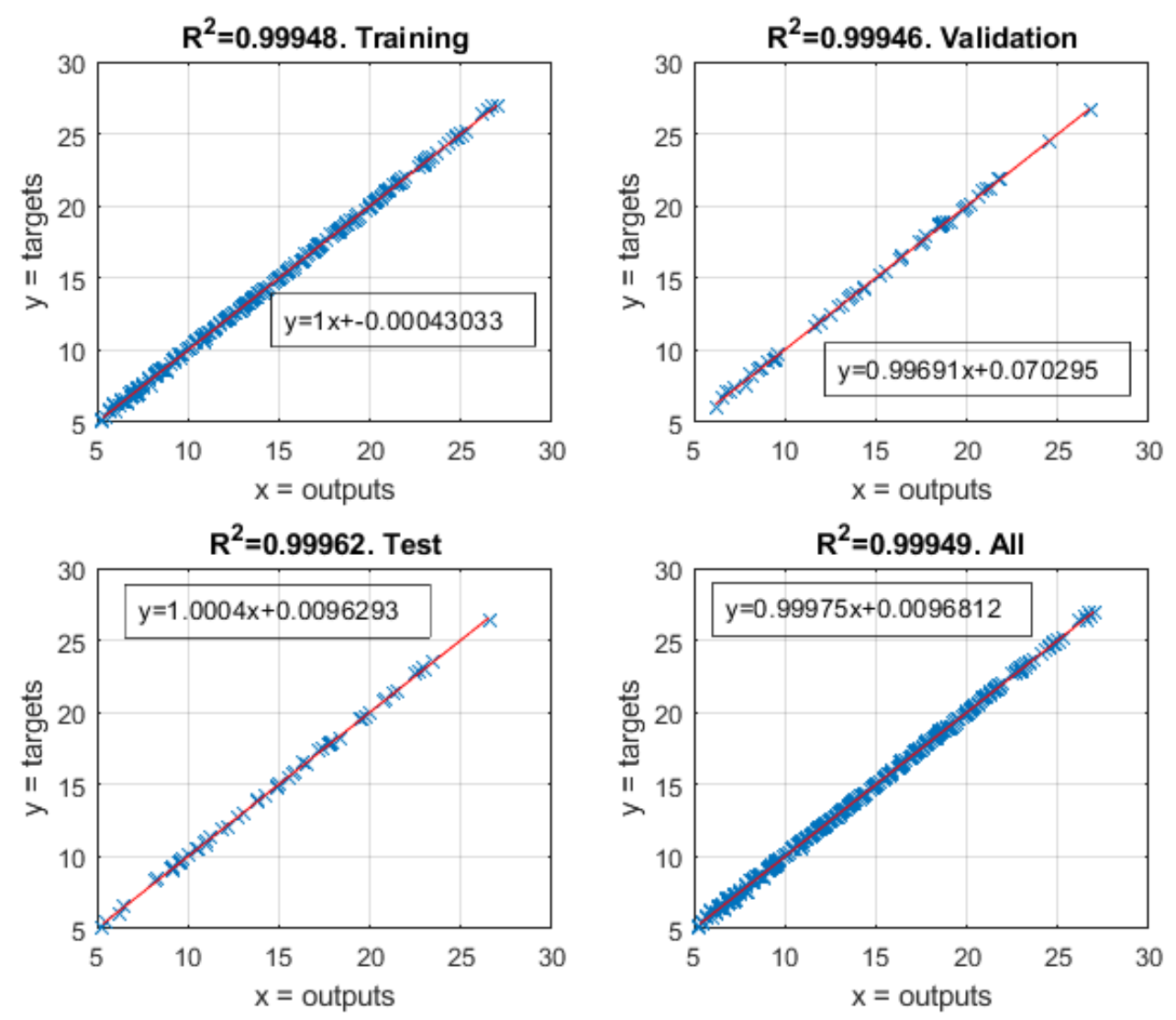

Figura 4-33 Resultados del ajuste de las deflexiones del timón de dirección para controlar el avión tras el fallo del motor crítico en el segundo segmento por medio de una red neuronal de una capa oculta con seis neuronas. Fuente: elaboración propia. 


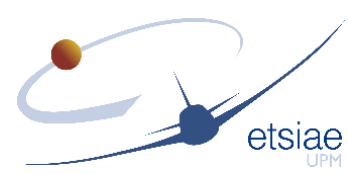

\subsubsection{Equilibrado del momento de guiñada del avión en la condición de aterrizaje con viento cruzado}

Para la condición de aterrizaje con viento cruzado, la deflexión del control necesaria para equilibrar la aeronave en guiñada se obtiene resolviendo la ecuación [ 3-15 ] para cada una de las combinaciones de los parámetros de diseño. Al igual que en el caso anterior, el avión se equilibra con el timón de dirección situado en la posición interior y considerando el ángulo de calado de la cola fijo e igual al obtenido en el equilibrado longitudinal de la condición de crucero. Los resultados se muestran en la Figura 4-34. La deflexión máxima del timón de dirección se ha determinado por medio de la Figura 4-6 para esta condición de vuelo, resultando en un valor máximo de $25^{\circ}$. Hay que recordar que este valor máximo coincide en valor absoluto con el valor mínimo de deflexión. Conviene tenerlo presente porque en esta condición de vuelo el equilibrio se produce para deflexiones tanto positivas como negativas. Como se observa en la figura, las deflexiones máximas positivas ejercen una limitación inferior al ángulo de diedro, en cambio, las deflexiones máximas negativas imponen un límite superior, a pesar de que en el caso mostrado ninguna combinación de parámetros alcanza dicho límite. Este comportamiento de los resultados con el ángulo de diedro tiene la siguiente explicación: para diedros bajos, la proyección vertical de la cola es lo suficientemente pequeña como para necesitar deflexiones positivas para anular el momento de guiñada, sin embargo, a medida que el diedro crece esta proyección también lo hace y el viento cruzado hace sustentar a la cola demasiado en el sentido contrario al necesario para equilibrar la aeronave, y, por tanto, es necesario deflectar el control en el sentido opuesto al anterior.
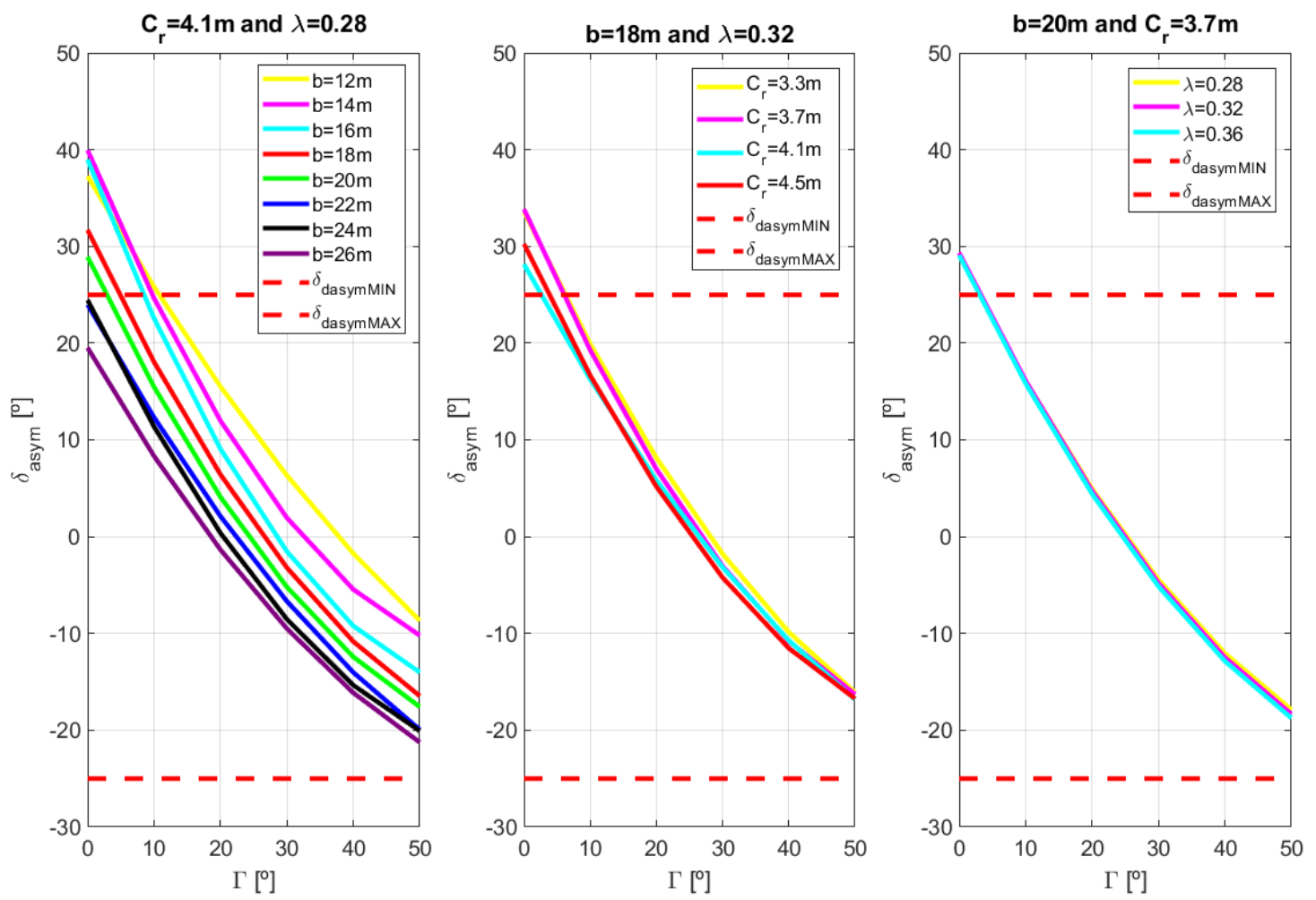

Figura 4-34 Análisis del efecto de la envergadura, cuerda en la raíz y parámetro de estrechamiento con la variación del diedro de la cola en $V$ en la condición del control de la aeronave en aterrizaje con viento cruzado. Fuente: elaboración propia.

Al igual que en el caso anterior, se van a presentar los resultados del ajuste de la red neuronal para las deflexiones necesarias para el control de la aeronave en el aterrizaje 
con viento cruzado. La red neuronal que se ha construido presenta cinco neuronas en la capa oculta y las demás propiedades son iguales a la red que se ha construido para el control del avión tras el fallo del motor crítico en el segundo segmento. En la Figura 4-35 se pueden ver los resultados del ajuste. Se observa como los coeficientes de correlación son superiores al $99 \%$ en todos los conjuntos.
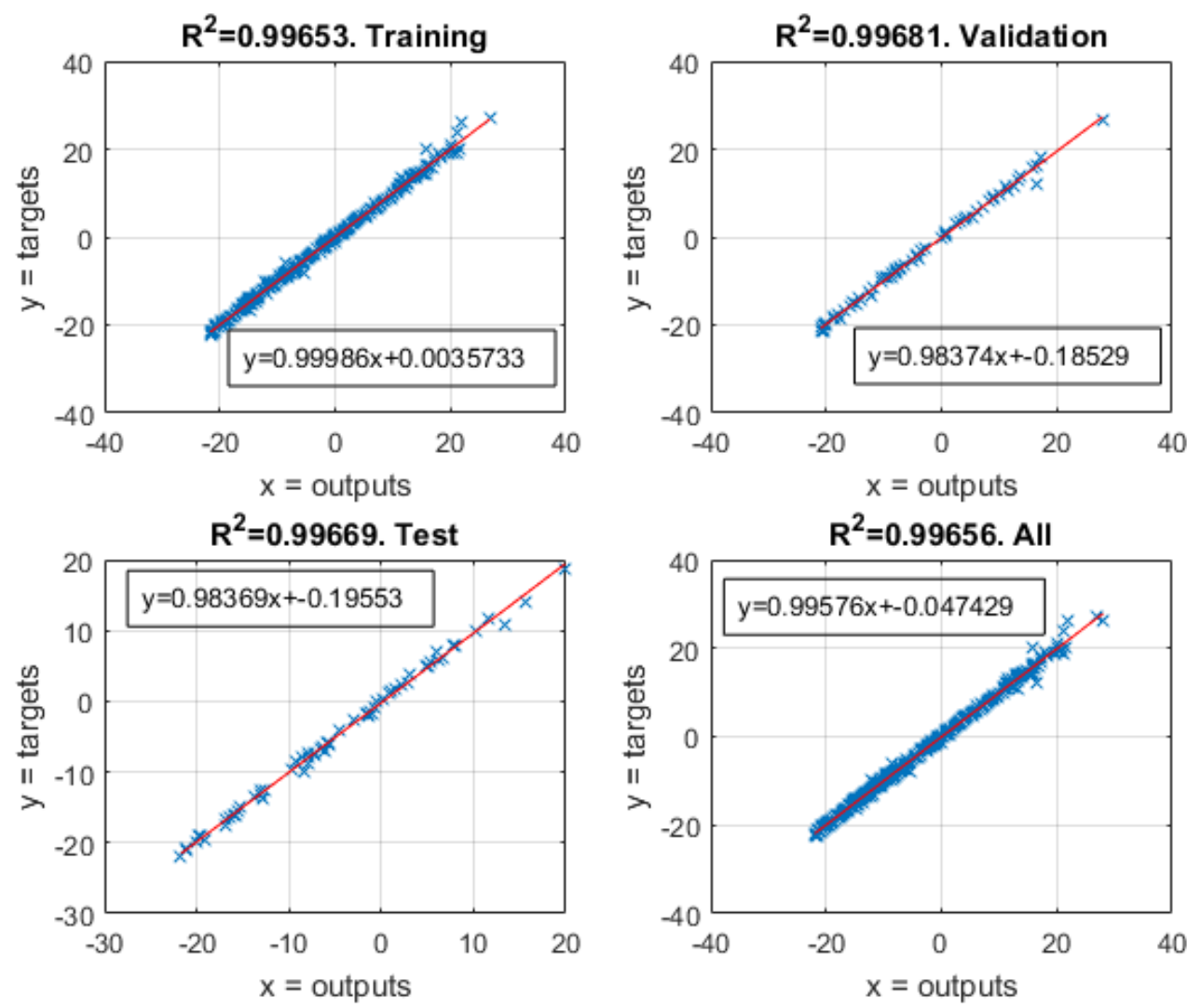

Figura 4-35 Resultados del ajuste de las deflexiones del timón de dirección para controlar el avión en aterrizaje con viento cruzado por medio de una red neuronal de una capa oculta con cinco neuronas. Fuente: elaboración propia.

\subsubsection{Espacio de diseño posible}

A la vista de los resultados obtenidos en cada una de las condiciones de vuelo estudiadas, es posible estudiar cuál de las restricciones está activa o es redundante. La primera conclusión es que el equilibrado longitudinal no introduce ninguna restricción, ya que no se alcanzan en ningún momento los máximos de las deflexiones de los controles longitudinales en ninguno de los casos estudiados: con ángulo de calado de la cola nulo y con el ángulo de calado de la cola que equilibra la condición de crucero.

Sin embargo, el caso de los equilibrios en guiñada y de las derivadas de estabilidad en crucero sí que introducen restricciones al problema de diseño. De hecho, se pueden determinar las relaciones que deben cumplir las variables de diseño precisamente en esos límites. Como las variables de diseño son cuatro, el problema tiene cuatro grados de libertad, así que, en las fronteras, las curvas tendrán tres grados de libertad. Las restricciones del problema vienen definidas por medio de una inecuación, es decir, dividen el espacio de diseño en dos subespacios: uno donde es posible el diseño y otro donde no. Por tanto, tomando las restricciones como igualdades, se puede relacionar una variable de diseño con las otras tres, de tal forma que se halle la curva frontera. Esta variable va a ser el diedro de la cola. 


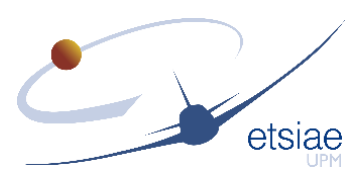

Primeramente, se van a considerar las restricciones impuestas por la derivada de estabilidad estática lateral en crucero, la derivada de estabilidad estática longitudinal en crucero y el ángulo de deflexión del timón de dirección tras el fallo del motor crítico en segundo segmento. En los tres casos, como se ha comentado anteriormente, se van a tomar las igualdades para determinar las curvas de la frontera, es decir, que se alcance exactamente la derivada de estabilidad estática lateral y longitudinal en crucero que el CSR-01 en las mismas condiciones, en el caso de las dos primeras restricciones; y que se alcance el ángulo de deflexión máximo del timón de dirección en el caso del control del avión tras el fallo del motor crítico en el segundo segmento. Los resultados se muestran en la Figura 4-36, donde se puede ver que la restricción de la derivada estática longitudinal en crucero es un límite superior del ángulo de diedro para el resto de parámetros constantes. Además, se observa como la restricción que nace de la condición de la derivada estática lateral es un límite inferior, al igual que la deflexión máxima para el control del avión tras el fallo del motor crítico. Analizando la actividad de las restricciones, las que vienen de las derivadas de estabilidad se puede decir que son activas y la que viene del fallo de motor crítico es redundante, ya que, si se prescinde de ella, el espacio de diseño no cambia y por tanto el óptimo tampoco.
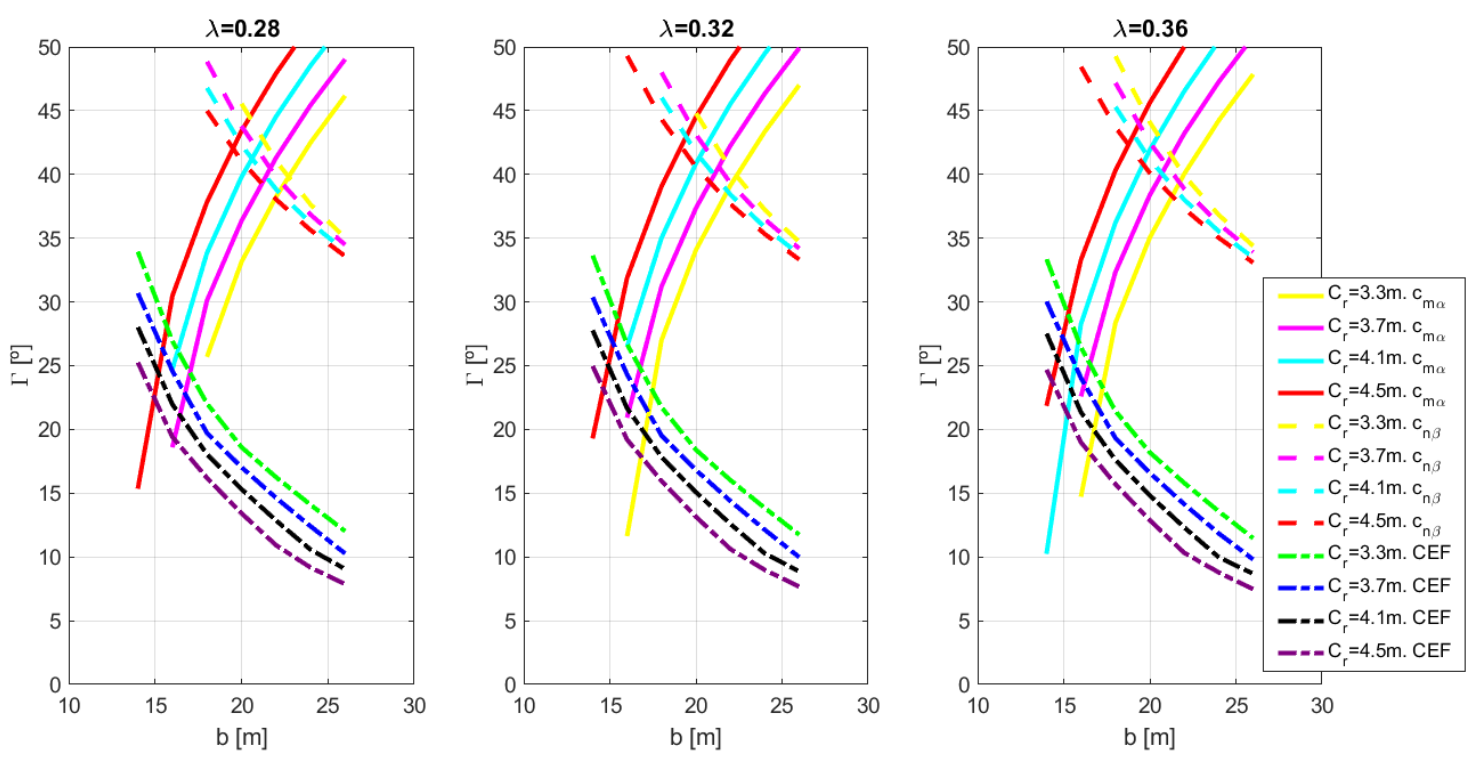

Figura 4-36 Espacio de diseño posible definido por las restricciones impuestas por las derivadas de estabilidad estáticas en crucero, tanto longitudinal $c_{m \alpha}$ y lateral $c_{n \beta}$, y por el control del avión tras el fallo del motor crítico en el segundo segmento (CEF). Fuente: elaboración propia.

Lo que se puede concluir de aquí es que el tamaño del timón de dirección es demasiado grande y no necesita trabajar cercano a su límite para cumplir la condición de control del avión tras el fallo del motor crítico. Ahora hay que analizar el caso del aterrizaje con viento cruzado para poder concluir fehacientemente si el control lateral está sobredimensionado.

Los resultados para el aterrizaje con viento cruzado imponen dos restricciones, tal y como se ha indicado en el apartado correspondiente, al alcanzar las deflexiones máximas positivas y negativas. Para los intervalos de parámetros que se han escogido, ninguna combinación de las variables de diseño hace sobrepasar la deflexión máxima negativa, pero sí la positiva. Los resultados se muestran en la Figura 4-37, junto con las restricciones impuestas por la estabilidad en crucero. A la vista de las curvas, se puede 
decir que la restricción impuesta por la deflexión positiva máxima en el aterrizaje con viento cruzado es también una restricción redundante. De hecho, está situada por debajo incluso de la restricción derivada del control del avión tras el fallo del motor crítico. Por tanto, la hipótesis de que el timón de dirección está sobredimensionado para cumplir con estas dos condiciones se confirma. En el apartado siguiente se presenta una posibilidad de redimensionado del timón de dirección.
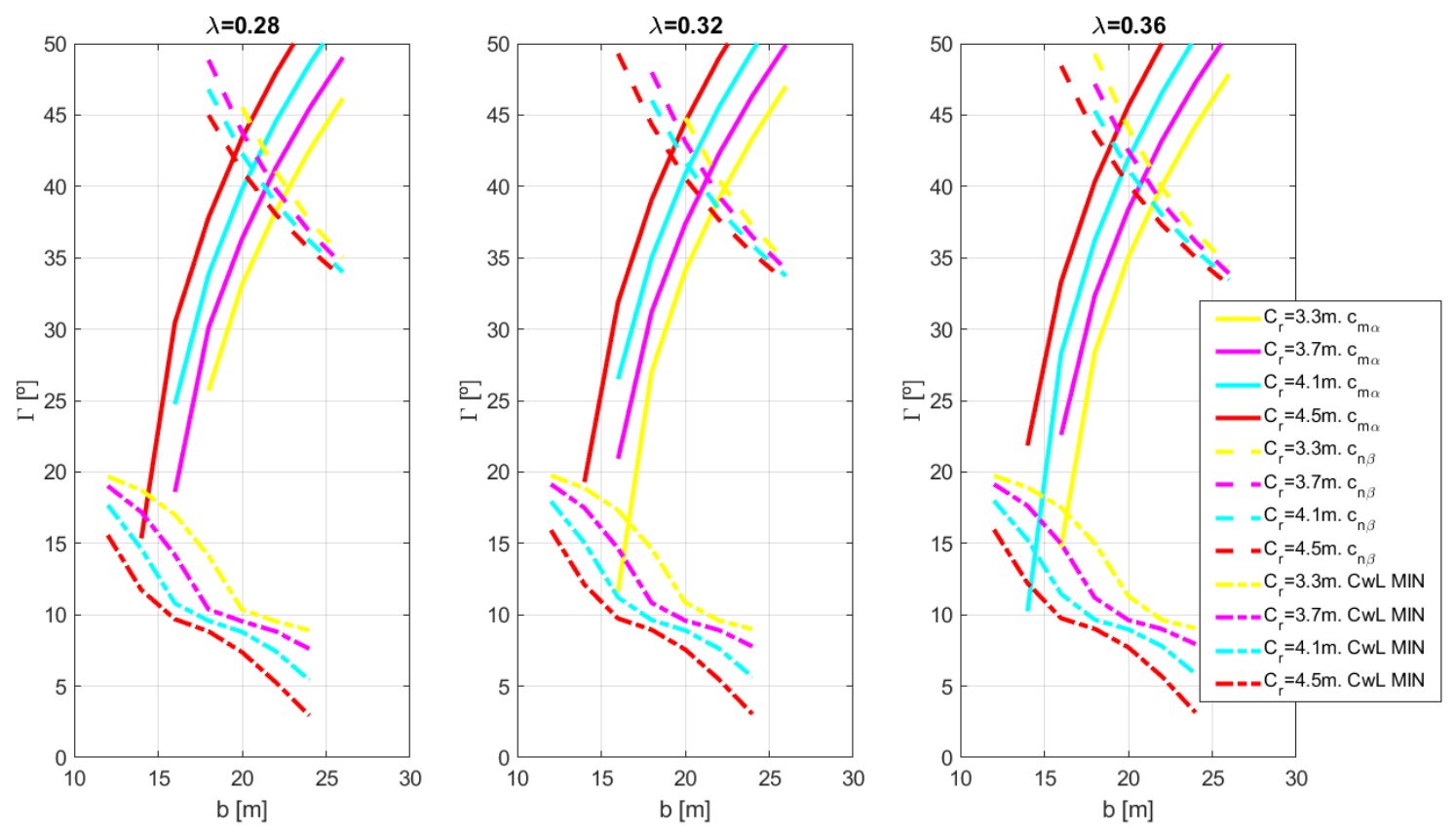

Figura 4-37 Espacio de diseño posible definido por las restricciones impuestas por las derivadas de estabilidad estáticas en crucero, tanto longitudinal $c_{m \alpha}$ y lateral $c_{n \beta}$, y por el control del avión en el aterrizaje con viento cruzado, definiendo un mínimo para el diedro (CwL MIN). Fuente: elaboración propia.

\subsubsection{Propuesta de dimensionado del timón de dirección}

A la vista del espacio de diseño, se puede proponer un rediseño del timón de dirección de tal forma que la restricción del control del avión tras el fallo del motor crítico pase a estar sobre la curva definida por la estabilidad estática lateral en crucero. Se ha escogido esta restricción en lugar de la del viento cruzado porque esta última es redundante respecto a la del fallo de motor crítico. La propuesta de rediseño del timón que se contempla es la de tratar de determinar el porcentaje de envergadura que debe ocupar el timón de dirección para que las restricciones del fallo de motor crítico y la de la estabilidad estática lateral en crucero estén superpuestas. El rediseño se hará aumentando la distancia entre el inicio del control y el plano de simetría del avión, tal y como se muestra en la Figura 4-38., manteniendo la fracción de cuerda que ocupa el control. El tamaño del control resultante se muestra en la Figura 4-39. Esta curva corresponde al tamaño del timón de dirección necesario para que al deflectar el control hasta el máximo, el avión anule el momento de guiñada para una cola con el diedro correspondiente a cada uno de los puntos de la curva definida por la restricción que impone la estabilidad estática lateral en crucero. Eso quiere decir que para cada uno de los puntos de las curvas de la Figura 4-39 se tiene un ángulo de diedro diferente. Por tanto, el cambio de un punto a otro de la curva lleva asociado varios efectos acoplados, como son el aumento de envergadura, y la reducción del diedro. Es por eso que las curvas no tienen un comportamiento homogéneo entre ellas, y se cruzan, ya que el que 


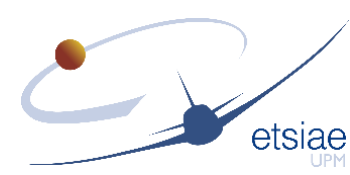

se representa es el tamaño del control correspondiente a la nueva combinación de los parámetros de diseño.

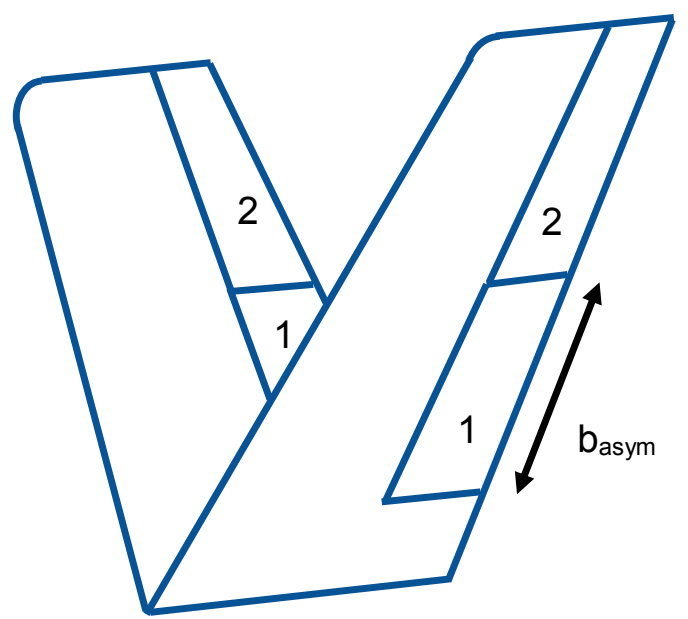

Figura 4-38 Definición de la envergadura del timón de dirección propuesto para ser rediseñado. Fuente: elaboración propia.
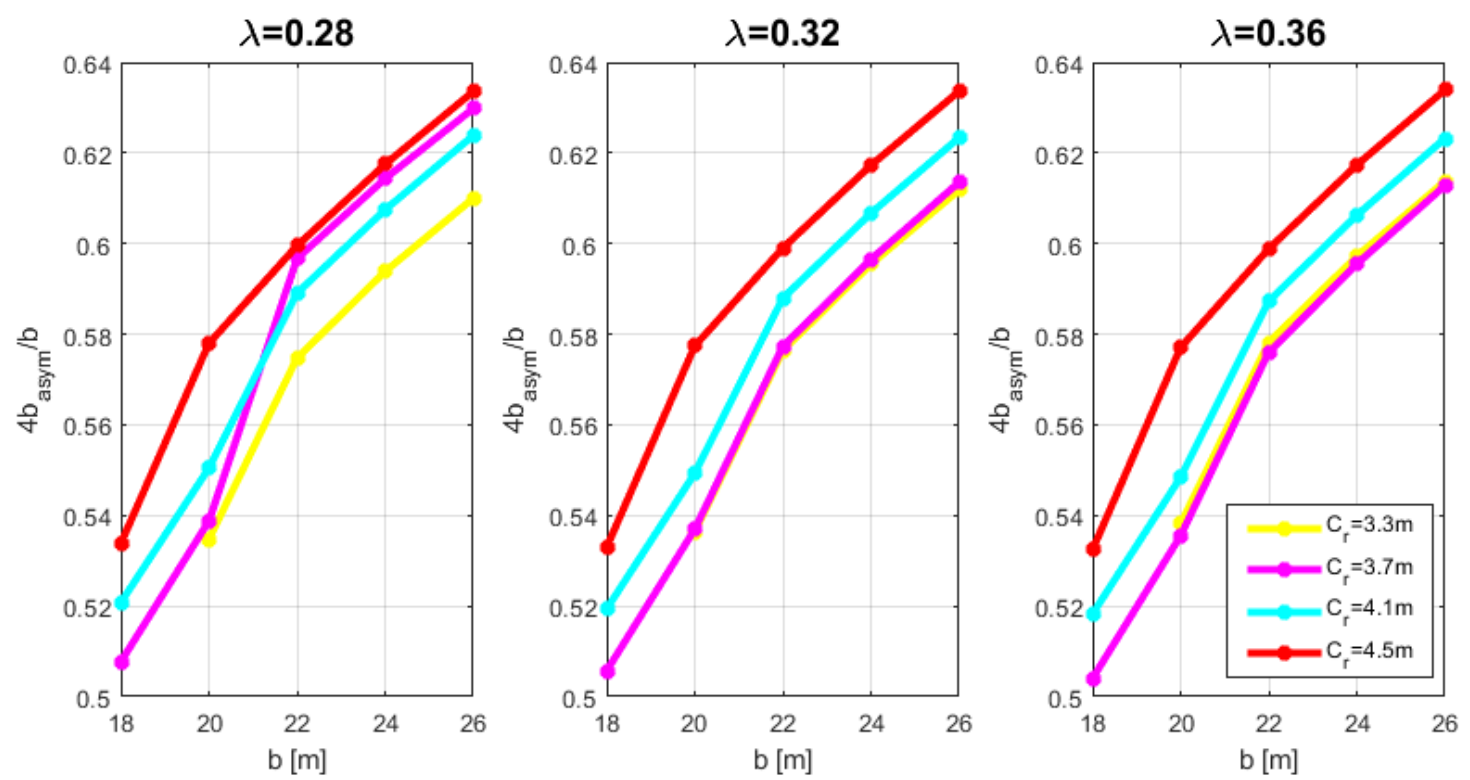

Figura 4-39 Tamaño mínimo del timón de dirección para que la restricción impuesta por el control del avión tras el fallo del motor crítico en el segundo segmento coincida con la restricción impuesta por la estabilidad estática lateral en crucero. Fuente: elaboración propia.

Sin embargo, aparece un inconveniente al redimensionar el timón de dirección de la forma que se ha propuesto, y es que la limitación por deflexión máxima negativa en el aterrizaje con viento cruzado pasa a estar activa, por lo que restringe el espacio de diseño acotando superiormente el valor del diedro, para una cierta combinación del resto de variables de diseño. De hecho, esta restricción reduce el espacio de diseño a cero, puesto que se sitúa por debajo de la limitación de la estabilidad estática lateral en crucero. Por tanto, las dimensiones del timón de dirección deben estar entre las que se indican en la Figura 4-39 y la correspondiente a la semienvergadura. Así que se ha decidido fijar el tamaño del timón de dirección igual al $75 \%$ de la semienvergadura, resultando en un espacio de diseño como el que se presenta en la Figura 4-40. En ella 
se ha sombreado en verde el espacio de diseño posible para el caso de cuerda en la raíz igual a 4,1 metros y parámetro de estrechamiento igual a 0,32.
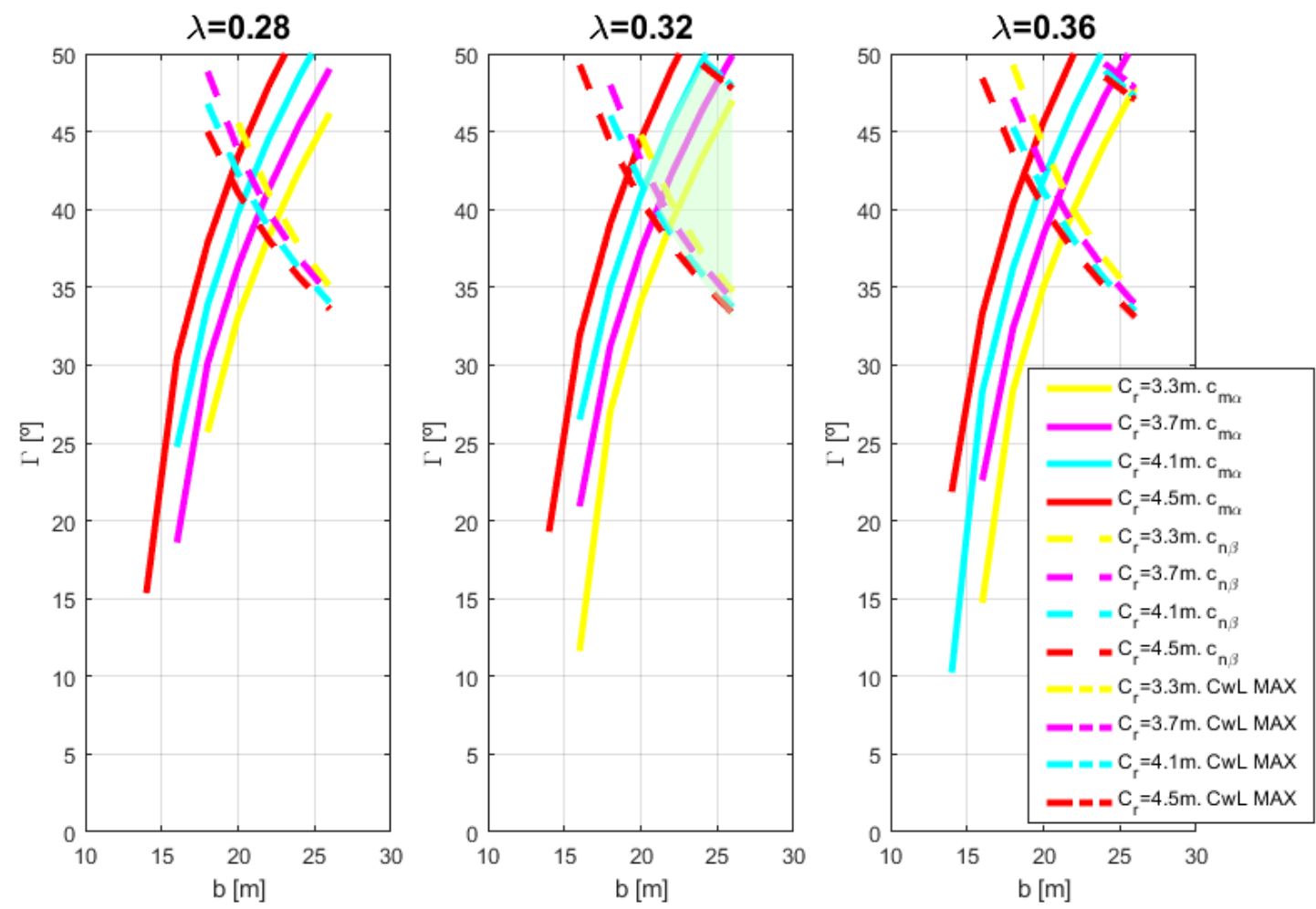

Figura 4-40 Espacio de diseño posible definido por las restricciones impuestas por las derivadas de estabilidad estáticas en crucero, tanto longitudinal $C_{m \alpha}$ y lateral $C_{n \beta}$, y por el control del avión en el aterrizaje con viento cruzado con el nuevo tamaño del timón de dirección, definiendo un máximo para el diedro (CwL MAX). Fuente: elaboración propia.

\subsubsection{Función objetivo: el peso}

\subsubsection{Método basado en regresiones estadísticas}

El peso de la cola en $V$ se va a determinar a partir de las ecuaciones presentadas para la configuración convencional en el apartado 3.3.1. El peso final será la suma del peso de un estabilizador horizontal determinado a partir de la proyección sobre el plano horizontal del avión de la cola en $\mathrm{V}$ y un estabilizador vertical generado a partir de la proyección vertical, siguiendo la hipótesis de que una cola en $\mathrm{V}$ se puede tratar como una configuración convencional equivalente con geometría igual a las proyecciones de la propia cola sobre los planos horizontal y vertical, respectivamente (Roskam 1999). Para determinar el peso de esta configuración de cola equivalente, es decir, de cada una de las proyecciones, se utilizarán las expresiones [ 3-28 ] y [ 3-29 ]. Los parámetros necesarios para poder evaluar las ecuaciones se pueden obtener a partir de los parámetros de la cola en V. Algunos de ellos se calculan de forma casi directa, como es el caso del área o la envergadura de la proyección. Se obtienen multiplicando por el coseno o el seno del ángulo de diedro de la cola en $\mathrm{V}$, para la proyección horizontal y vertical, respectivamente. A partir de ellos se pueden determinar otros, tales como la cuerda media aerodinámica o el alargamiento de las proyecciones. Por otro lado, la flecha de puntos $1 / 4$ de la proyección horizontal se obtiene por medio de la expresión siguiente, a partir de la flecha de la cola en V: 


$$
\tan \Lambda_{0,25 H}=\frac{\tan \Lambda_{0,25}}{\cos \Gamma}
$$

Para el caso de la flecha de la proyección vertical se debe sustituir el coseno por un seno. Desde el punto de vista del coeficiente de volumen, es necesario recalcular la posición de la cuerda media aerodinámica de la proyección para determinar el brazo de momentos, y, en consecuencia, el nuevo coeficiente de volumen.

Para comprobar si son aplicables los métodos rápidos descritos en el capítulo 3.4 de obtención del óptimo, se va a analizar la monotonía de la función objetivo con respecto cada una de las variables de diseño de la cola en $\mathrm{V}$. Se va a representar la dependencia del peso determinado como se ha indicado anteriormente con la cuerda en la raíz (Figura 4-41), el diedro (Figura 4-42), la envergadura (Figura 4-43) y el parámetro de estrechamiento (Figura 4-44). En ellas se puede ver como el peso es una función monótona respecto de todas las variables salvo el diedro. Analizando el signo del gradiente de la función, se puede comprobar que la función sí es monótona para cualquier valor del diedro superior a $20^{\circ}$. Puesto que las restricciones limitan el espacio de diseño en valores para el diedro superiores a los $20^{\circ}$, se puede decir que la función objetivo es monótona en el espacio de diseño con respecto al diedro también. A la vista del signo del gradiente, el mínimo se obtendría reduciendo la cuerda en la raíz, el parámetro de estrechamiento y la envergadura, pero aumentando el diedro. Puesto que el peso es una función monótona con respecto a todas las variables de diseño, el óptimo se alcanza sobre alguna de las restricciones.

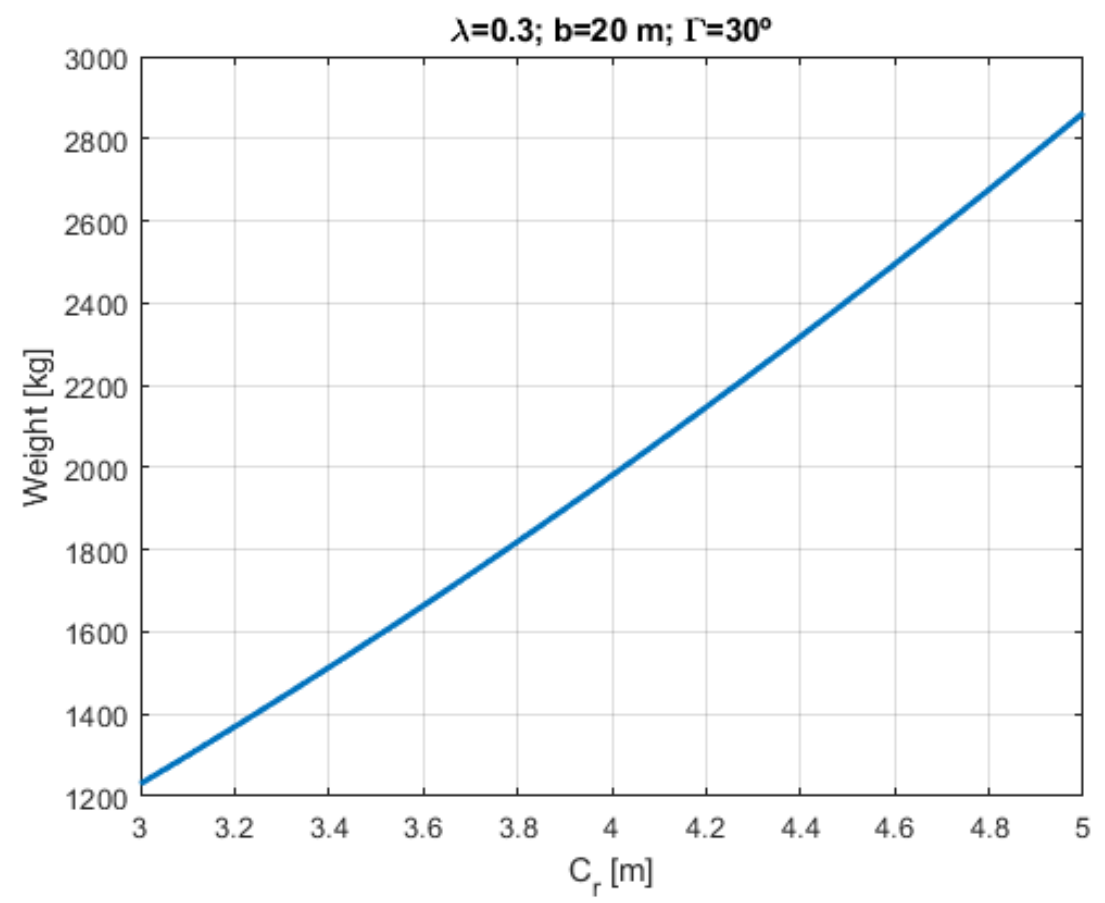

Figura 4-41 Dependencia del peso determinado combinando las fórmulas para colas convencionales en función de la cuerda en la raíz. Fuente: elaboración propia. 


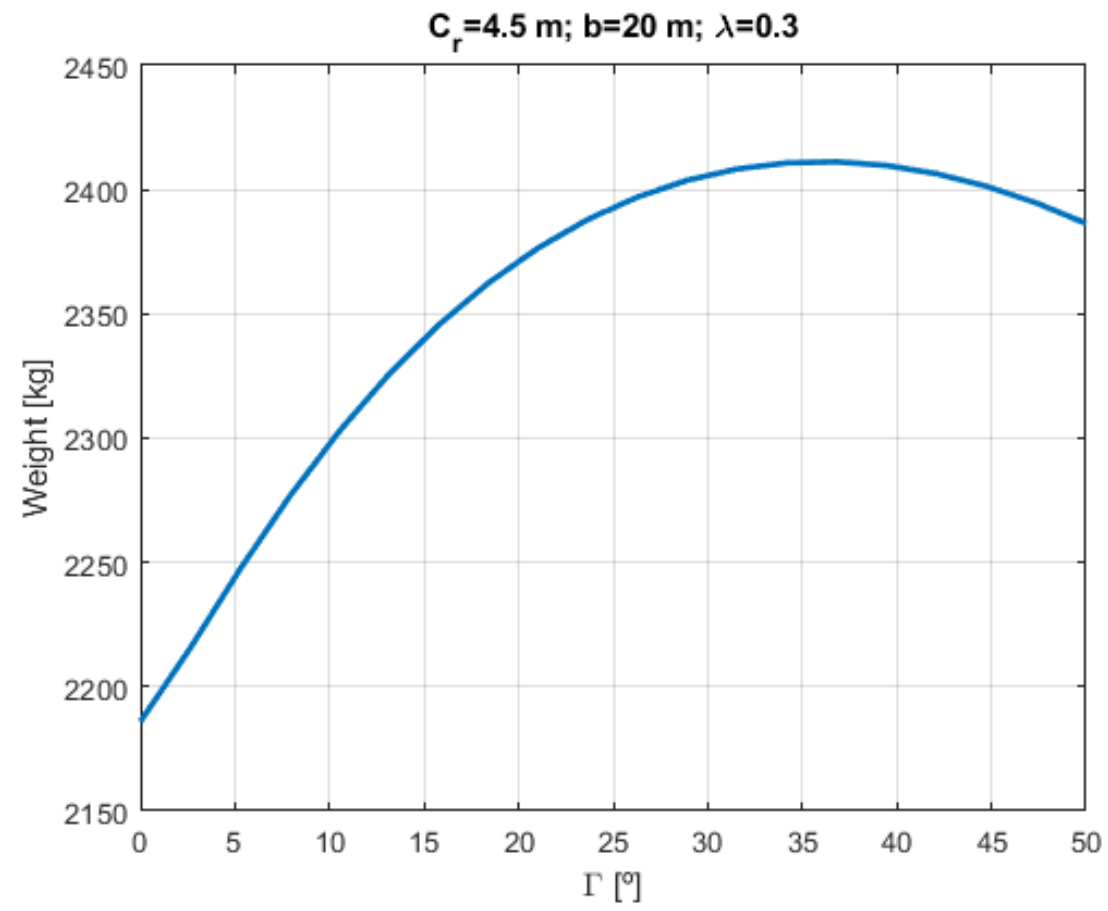

Figura 4-42 Dependencia del peso determinado combinando las fórmulas para colas convencionales en función del diedro. Fuente: elaboración propia.

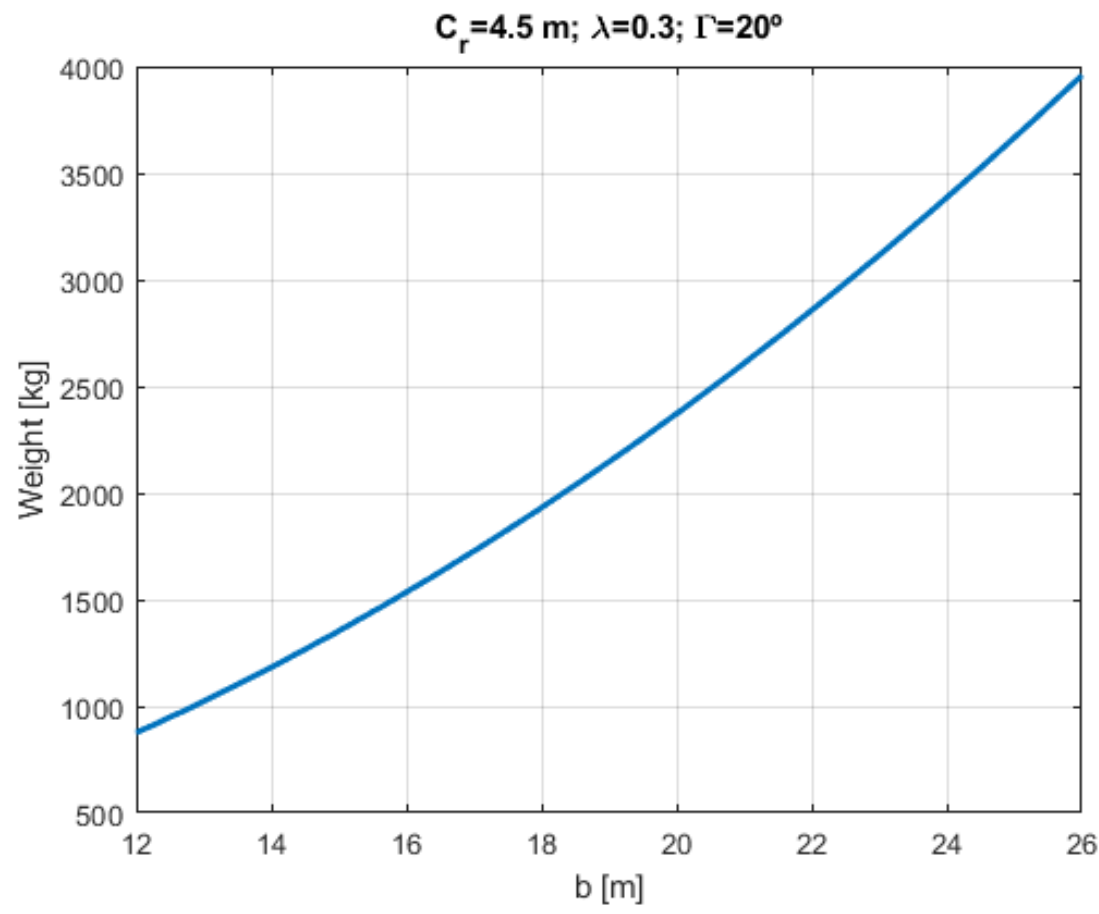

Figura 4-43 Dependencia del peso determinado combinando las fórmulas para colas convencionales en función de la envergadura. Fuente: elaboración propia. 


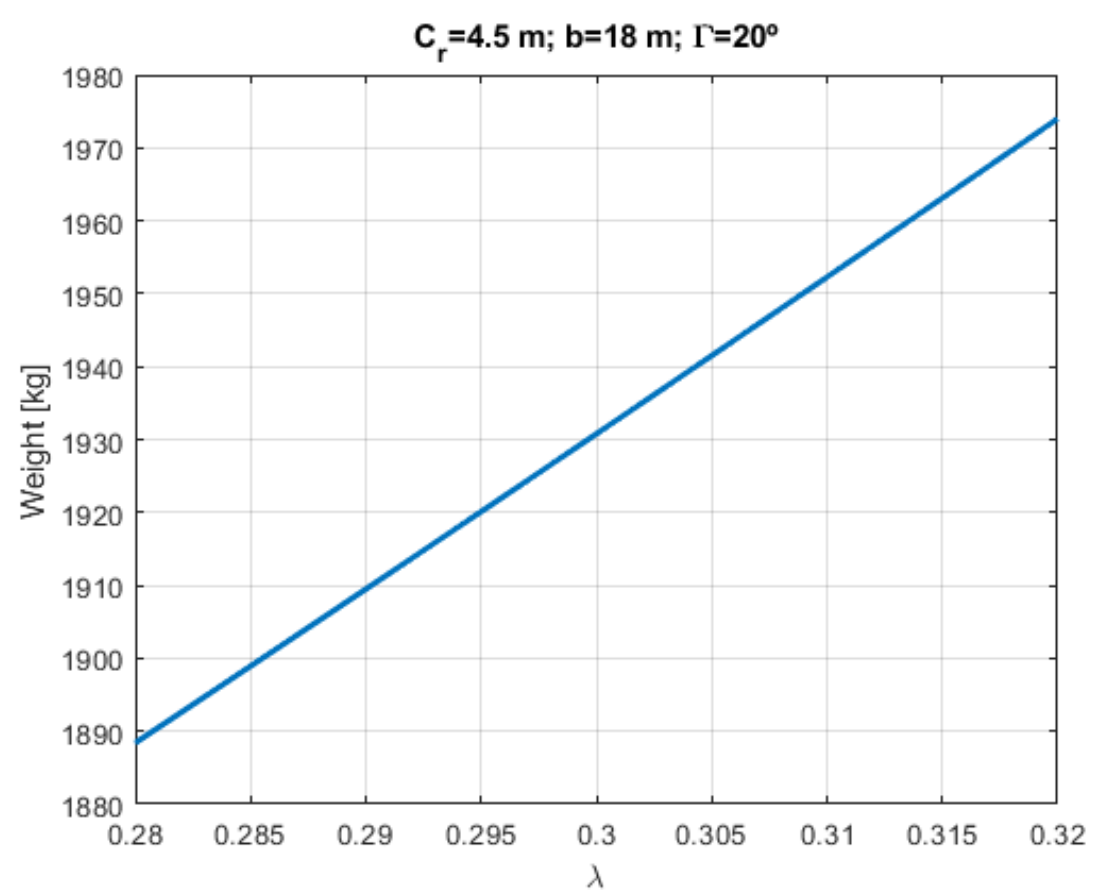

Figura 4-44 Dependencia del peso determinado combinando las fórmulas para colas convencionales en función del parámetro de estrechamiento. Fuente: elaboración propia.

Para determinar en qué punto de las restricciones se encuentra el óptimo, se debe analizar el gradiente sobre los subespacios definidos por estas restricciones. Se va a empezar por la impuesta por la estabilidad estática longitudinal en crucero. Para ello, se muestra cómo varía el peso al moverse por encima de esta restricción al variar la cuerda en la raíz (Figura 4-45), la envergadura (Figura 4-46) y el parámetro de estrechamiento (Figura 4-47), para el correspondiente valor del ángulo de diedro de la Figura 4-36.

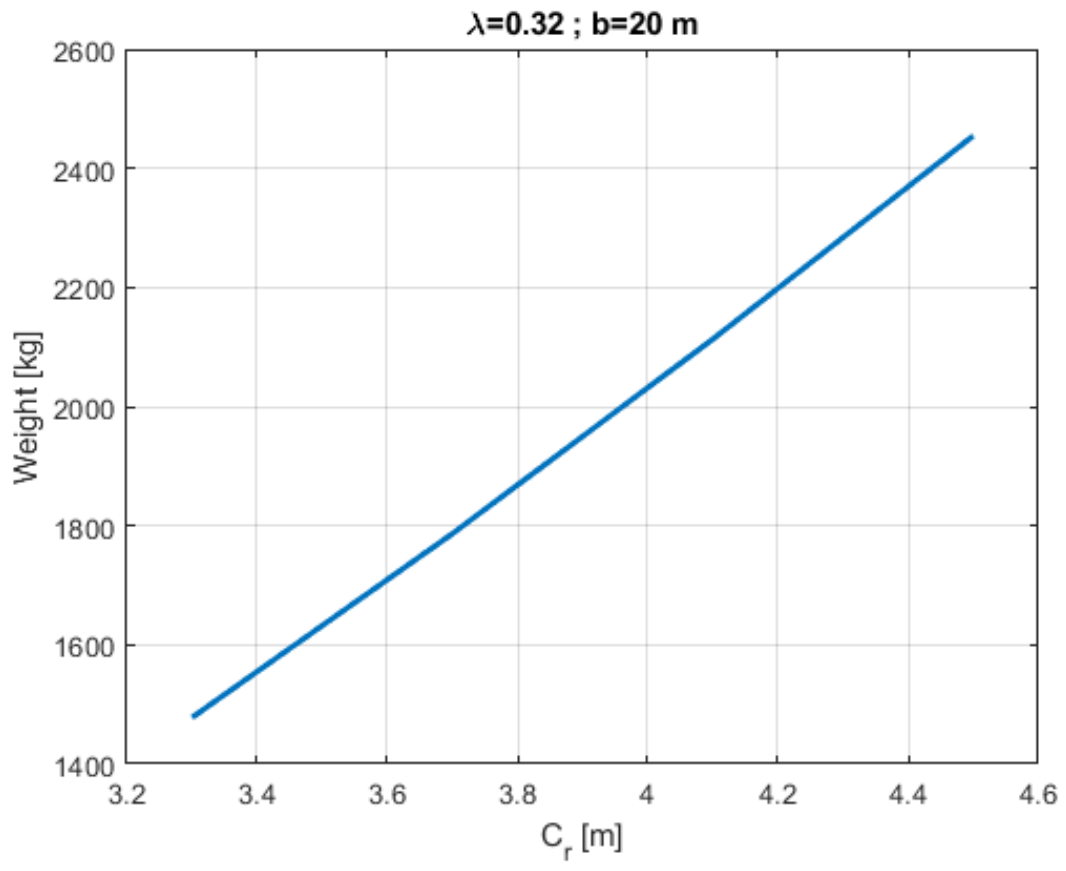

Figura 4-45 Dependencia del peso determinado combinando las fórmulas para colas convencionales en función de la cuerda en la raíz en puntos situados sobre la restricción impuesta por la derivada de estabilidad longitudinal en crucero. Fuente: elaboración propia. 


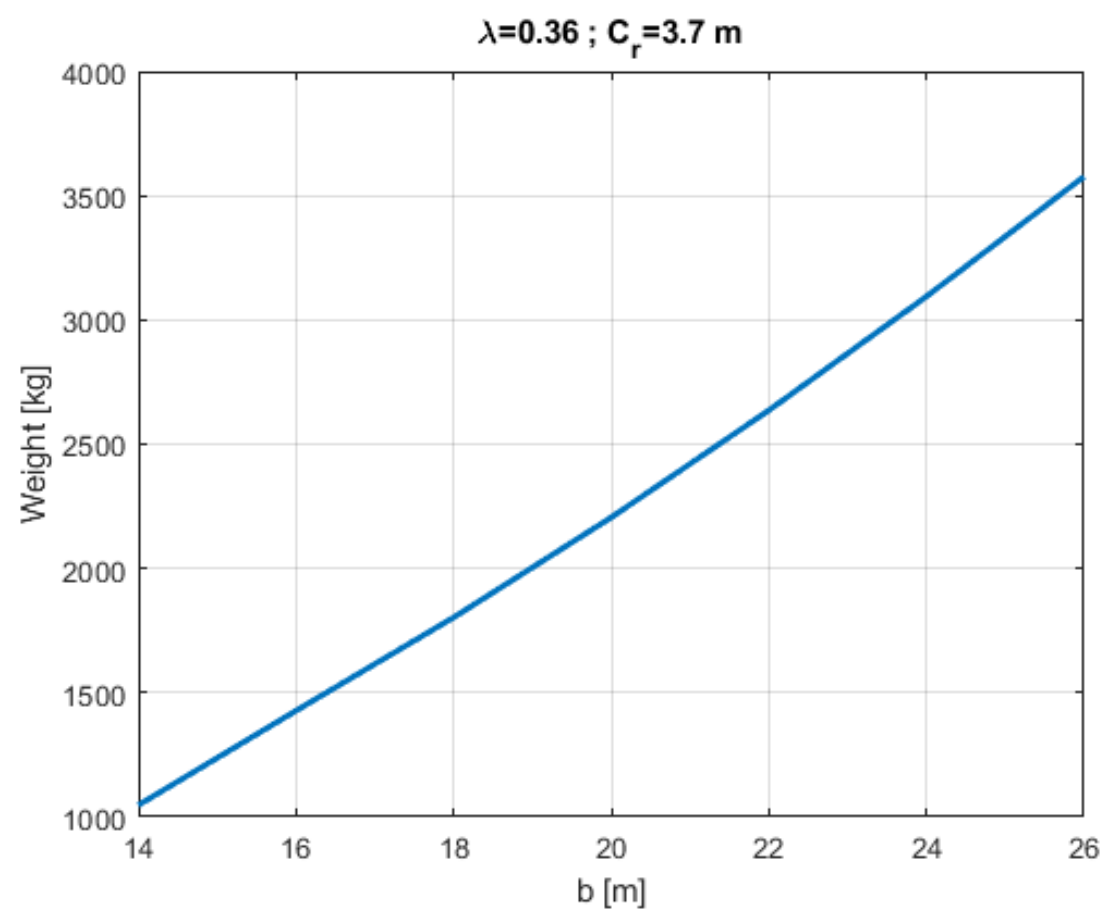

Figura 4-46 Dependencia del peso determinado combinando las fórmulas para colas convencionales en función de la envergadura en puntos situados sobre la restricción impuesta por la derivada de estabilidad longitudinal en crucero. Fuente: elaboración propia.

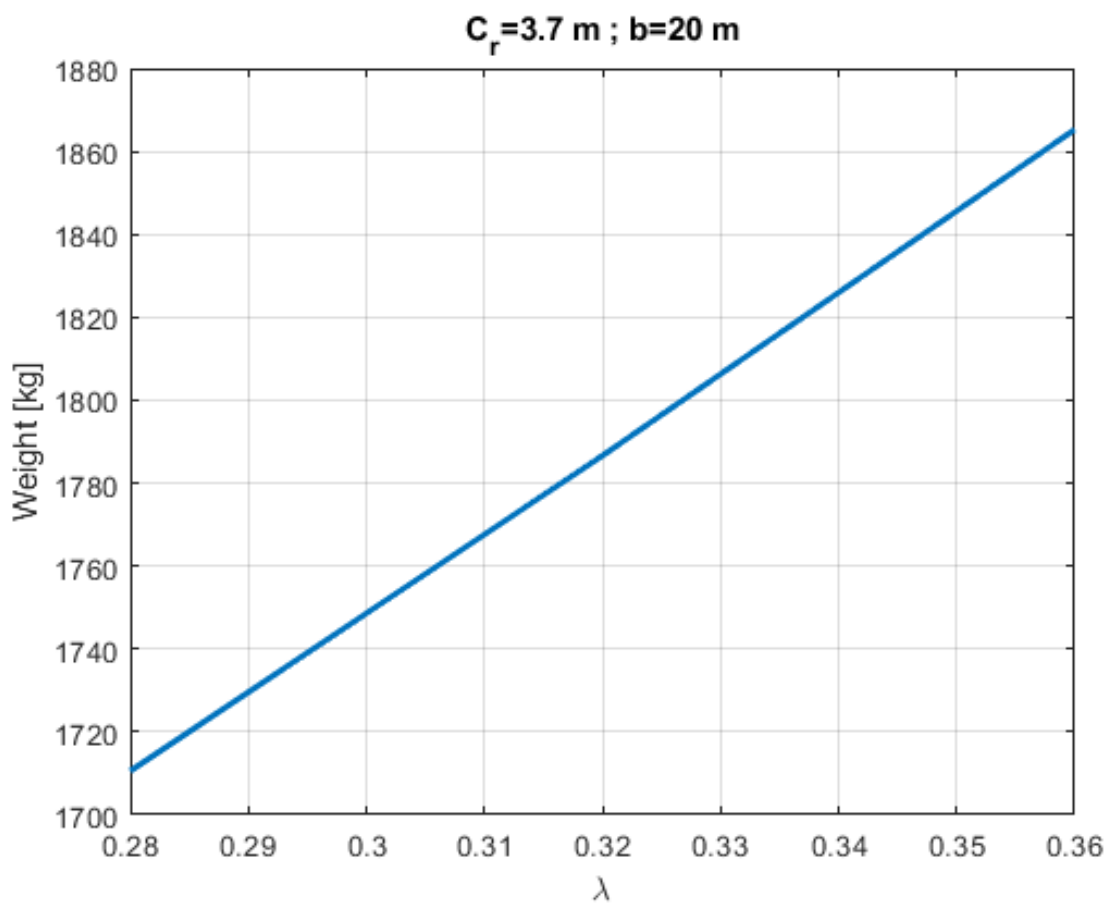

Figura 4-47 Dependencia del peso determinado combinando las fórmulas para colas convencionales en función del parámetro de estrechamiento en puntos situados sobre la restricción impuesta por la derivada de estabilidad longitudinal en crucero. Fuente: elaboración propia.

Como se puede ver en los ejemplos de las figuras y al analizar el signo del gradiente de la función de peso a lo largo de la limitación derivada de la estabilidad estática longitudinal en crucero, el peso se reduce a medida que cualquiera de las tres variables 


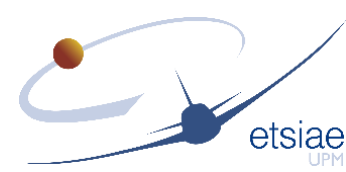

de diseño que se toman como grados de libertad (cuerda en la raíz, parámetro de estrechamiento y envergadura) reducen su valor. Esto quiere decir que el mínimo se obtendría al alcanzar los mínimos valores de estas variables que cumplan la restricción, alcanzando el correspondiente valor del ángulo de diedro.

Ahora bien, se debe considerar la restricción impuesta por la estabilidad estática lateral en crucero para concluir qué ocurre a lo largo de esta superficie. Se va a proceder de la misma forma, mostrándose en la Figura 4-48, la Figura 4-49 y la Figura 4-50 la variación del peso en función de la cuerda en la raíz, la envergadura y el parámetro de estrechamiento, respectivamente. Como se observa en todas ellas y se confirma analizando el signo del gradiente restringido al subespacio definido por la restricción, de nuevo ocurre que el peso se reduce a medida que se reducen los valores de las variables de los grados de libertad. Por tanto, se concluye que el mínimo se producirá para el menor valor de estas tres variables que cumplan las dos restricciones. A la vista de esta tendencia, la limitación superior que aparece en la Figura 4-40 debido a que se alcanza la deflexión máxima del timón de dirección para equilibrar el avión en el aterrizaje con viento cruzado es inactiva, ya que sí que restringe el espacio de diseño pero el mínimo no se alcanza sobre esa restricción, porque las otras dos restricciones permiten una combinación de las variables de diseño que suponen un valor menor de las mismas y, en consecuencia, un menor valor de la función objetivo.

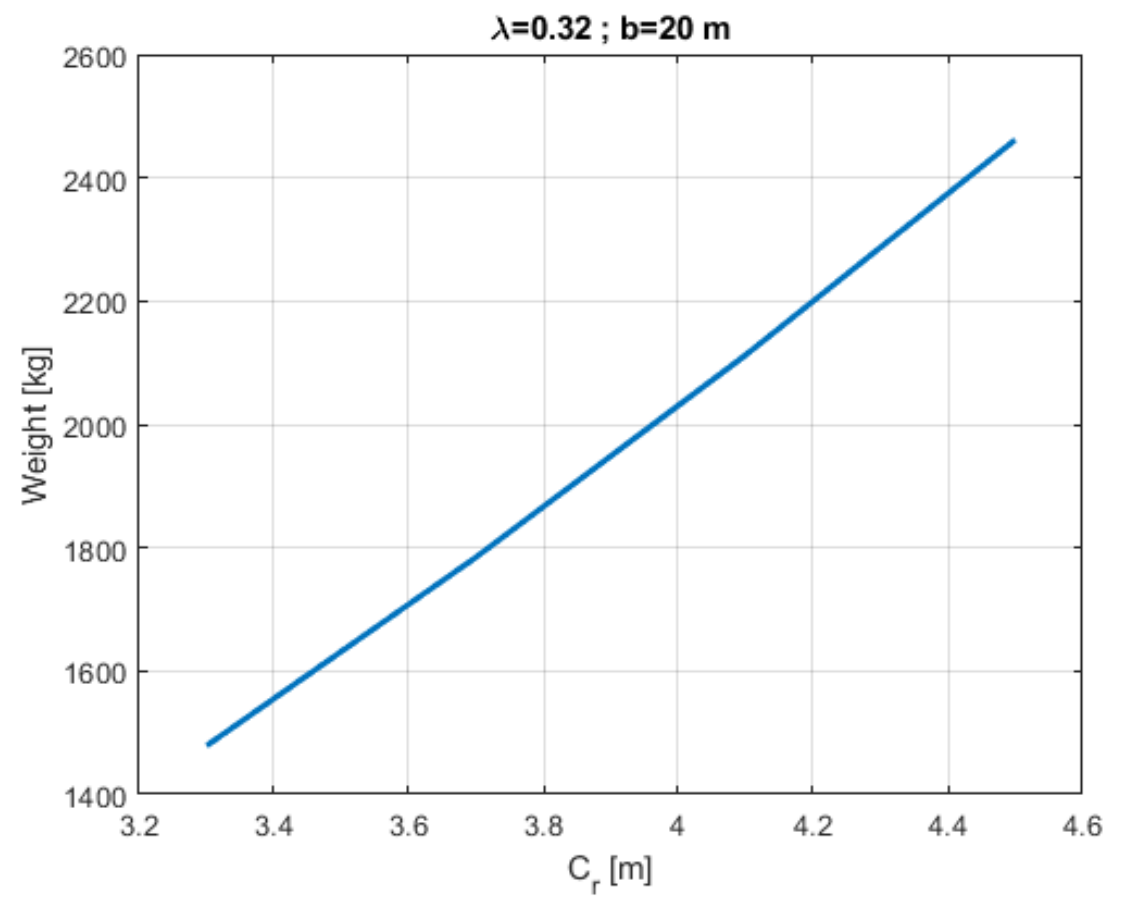

Figura 4-48 Dependencia del peso determinado combinando las fórmulas para colas convencionales en función de la cuerda en la raíz en puntos situados sobre la restricción impuesta por la derivada de estabilidad lateral en crucero. Fuente: elaboración propia. 


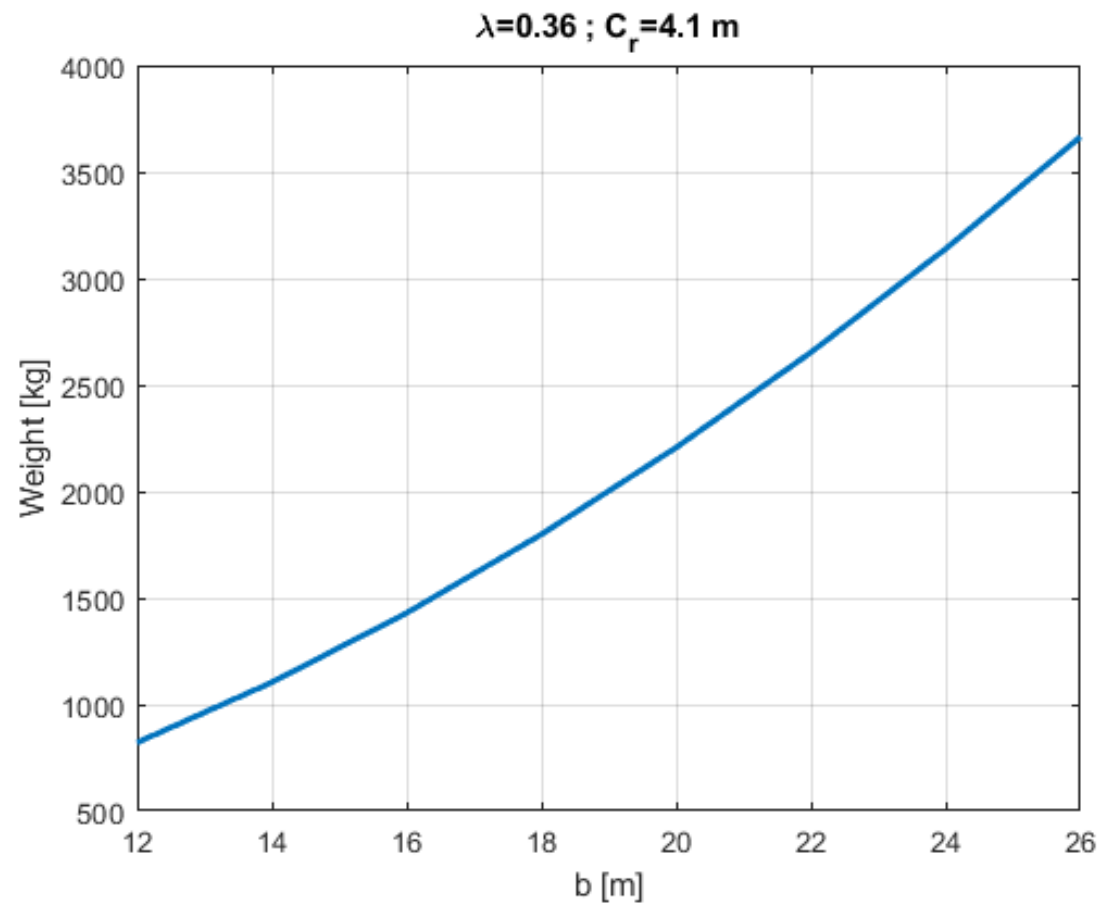

Figura 4-49 Dependencia del peso determinado combinando las fórmulas para colas convencionales en función de la envergadura en puntos situados sobre la restricción impuesta por la derivada de estabilidad lateral en crucero. Fuente: elaboración propia.

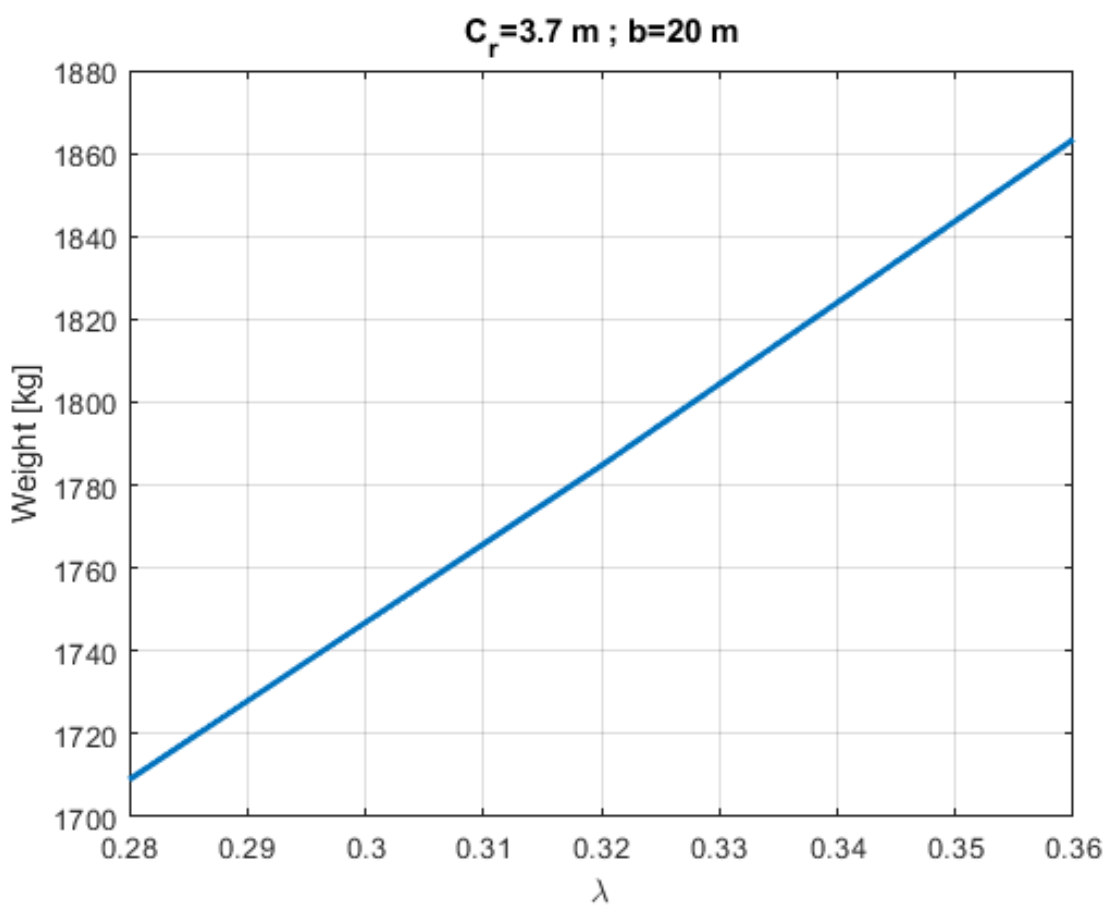

Figura 4-50 Dependencia del peso determinado combinando las fórmulas para colas convencionales en función del parámetro de estrechamiento en puntos situados sobre la restricción impuesta por la derivada de estabilidad lateral en crucero. Fuente: elaboración propia. 


\subsubsection{Método casi analítico para los estabilizadores}

La función de pesos determinada a partir del modelo casi analítico para dimensionar la estructura se aplica de la misma forma que se ha hecho para los estabilizadores del avión de referencia. En el caso de la cola en $\mathrm{V}$, debido a que el diedro es no nulo, aplican tanto las maniobras simétricas como las asimétricas. En principio, no se van a tener en cuenta más maniobras que las que se han escogido para validar el método con la configuración convencional del CSR-01. Las dimensiones del timón de dirección y profundidad son las que se han establecido en el apartado 4.3.2.6, es decir, el timón de profundidad ocupa la semienvergadura y el de dirección el $75 \%$ de la misma, situándose este último en el interior de la superficie.

En cuanto a las maniobras, se recuerda que las que se van a contemplar son las siguientes:

1. Equilibrado longitudinal del avión en un viraje estacionario a $V_{A}$ y a factor de carga límite, como maniobra simétrica estacionaria.

2. Deflexión súbita del timón de profundidad en una condición de equilibrio de vuelo de crucero a $\mathrm{V}_{\mathrm{A}}$, como maniobra simétrica con aceleración de cabeceo unchecked.

3. Equilibrado lateral del avión tras el fallo del motor crítico en segundo segmento con ángulo de resbalamiento nulo.

4. Vuelo a $\mathrm{V}_{\mathrm{A}}$ con ángulo de resbalamiento igual a $10^{\circ}$, sin deflexión del timón de dirección, que corresponde a la maniobra 2 de las expuestas en las maniobras asimétricas del apartado 3.2.8.

5. Vuelo a $V_{A}$ con ángulo de resbalamiento igual a $10^{\circ}$ y deflexión del timón de dirección máxima en el sentido que hiciera al avión moverse hacia ángulos de resbalamiento nulo, correspondiente a la maniobra 3 de las que se indican en el apartado 3.2.8.

6. Ráfaga lateral a $\mathrm{V}_{\mathrm{A}}$.

7. Ráfaga vertical positiva a $\vee_{A}$.

8. Vuelo a $V_{A}$ con ángulo de resbalamiento nulo, pero deflexión máxima del timón de dirección, como maniobra 1 dentro de las asimétricas de las que se recogen en el apartado 3.2.8.

Se debe destacar que con esta configuración de cola en $V$ está, acopladas las maniobras longitudinales y las laterales. Por eso, para resolver cualquier maniobra asimétrica necesita equilibrarse el avión longitudinalmente antes de aplicar el modelo simplificado de dicha maniobra, ya que este equilibrado introduce cargas en la misma superficie. El equilibrado a la velocidad $V_{A}$ de crucero se ha realizado a través del calado de la cola, siendo por medio del timón de profundidad en cualquier otra condición. La única maniobra que, a pesar de haberse calculado siguiendo el mismo procedimiento que para la configuración convencional de referencia, no es lo suficientemente exacta es la condición 1. Esta maniobra ya no es exclusivamente simétrica, puesto que, en un viraje estacionario, el timón de dirección se encarga de mantener girando al avión sin ángulo de resbalamiento, por lo que podría ser necesaria una deflexión del mismo que introduce nuevas cargas en la superficie de cola. Debido a esto, se ha considerado una nueva condición que corresponde a la deflexión máxima del timón de dirección y de profundidad, con el objetivo de ser conservativos y cubrir cualquier situación en la que sea necesario deflectar al máximo ambos timones. Esta condición se identificará con el número 9 : 
9. Deflexión máxima de los timones a $V_{\mathrm{A}}$ y factor de carga límite positivo de maniobra.

Así quedan cubiertas todas las situaciones que se puedan dar en las maniobras contempladas en el dimensionado de la configuración de cola convencional. Para la cola en $\mathrm{V}$, será necesario realizar un barrido a lo largo del intervalo de validez de las variables de diseño. Las distintas combinaciones de los parámetros de diseño resultan en geometrías distintas de la cola. Esto afecta a una de las condiciones consideradas, en concreto, a la de la ráfaga lateral. Esta condición introduce un ángulo de resbalamiento en la cola que depende del factor de atenuación de ráfagas que, para la ráfaga lateral, depende de la derivada de la fuerza lateral generada en la cola con respecto a ángulo de resbalamiento. En consecuencia, la fuerza lateral debida a la ráfaga lateral y el factor de carga inducido dependen de la combinación de las variables de diseño. Simplemente se quiere recalcar este hecho para no inducir a error y pensar que una misma ráfaga lateral introduce el mismo factor de carga en la cola, independientemente de la geometría de la misma.

Por lo demás, la modelización de las maniobras se ha llevado a cabo siguiendo las mismas hipótesis que se expusieron para el avión de referencia. En la Figura 4-51, Figura 4-52, Figura 4-53 y Figura 4-54 se muestran los resultados obtenidos, de tal forma que se pueden ver los efectos que tienen sobre las cargas la variación de los parámetros de diseño. Se observa cómo los cambios de las variables de diseño que llevan a aumentar la superficie del estabilizador también incrementan las cargas que se generan. En cuanto al efecto que tiene el diedro, por un lado se encuentran las maniobras asimétricas, que incrementan la carga a medida que el diedro crece. Por otro lado, se encuentran las maniobras simétricas a excepción de la condición 2 , que reducen las cargas según aumenta el diedro. La maniobra correspondiente a la condición 2 mantiene las cargas aproximadamente en los mismos valores debido a que se trata de la deflexión máxima del timón de profundidad. 

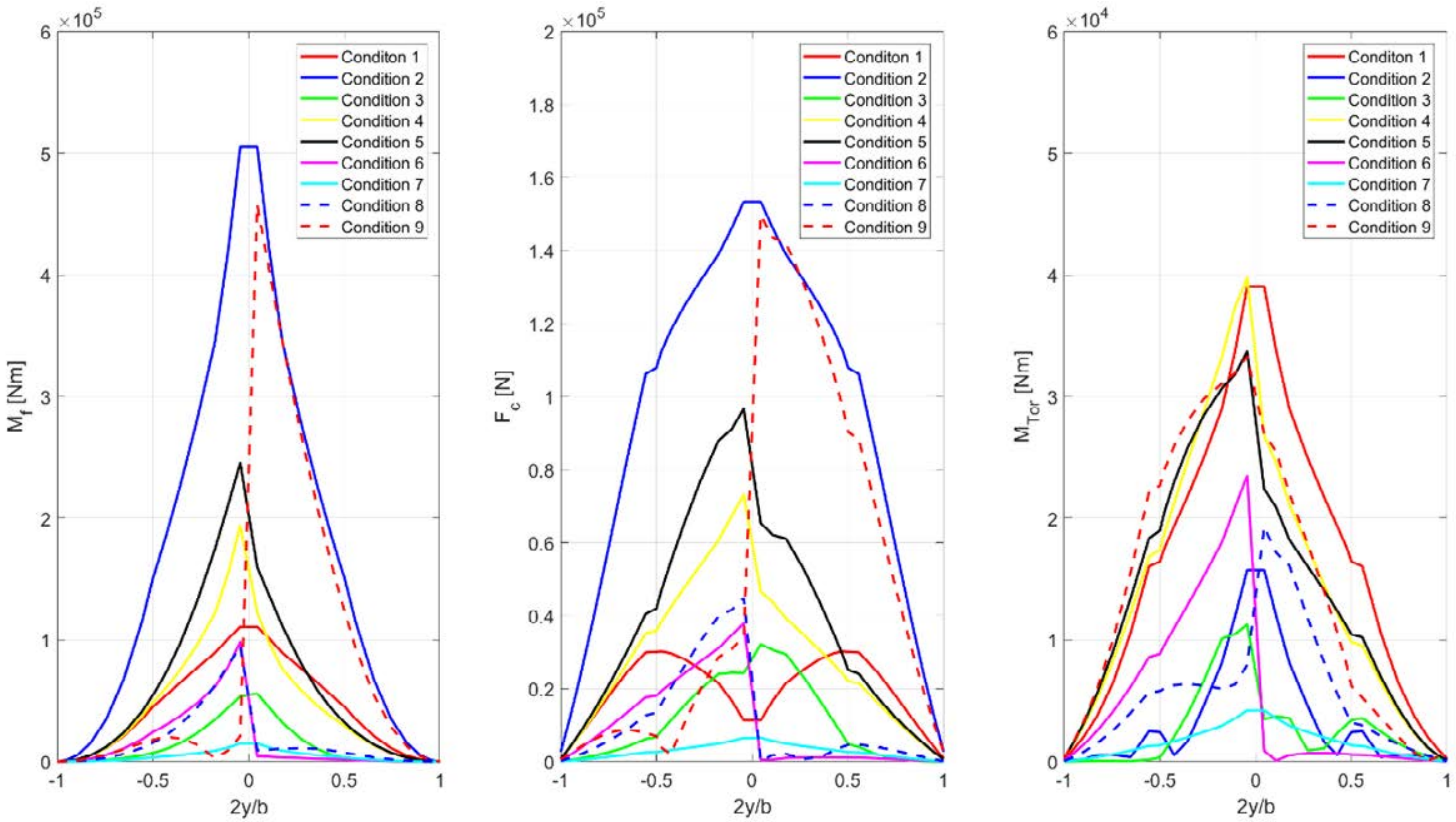

(a)
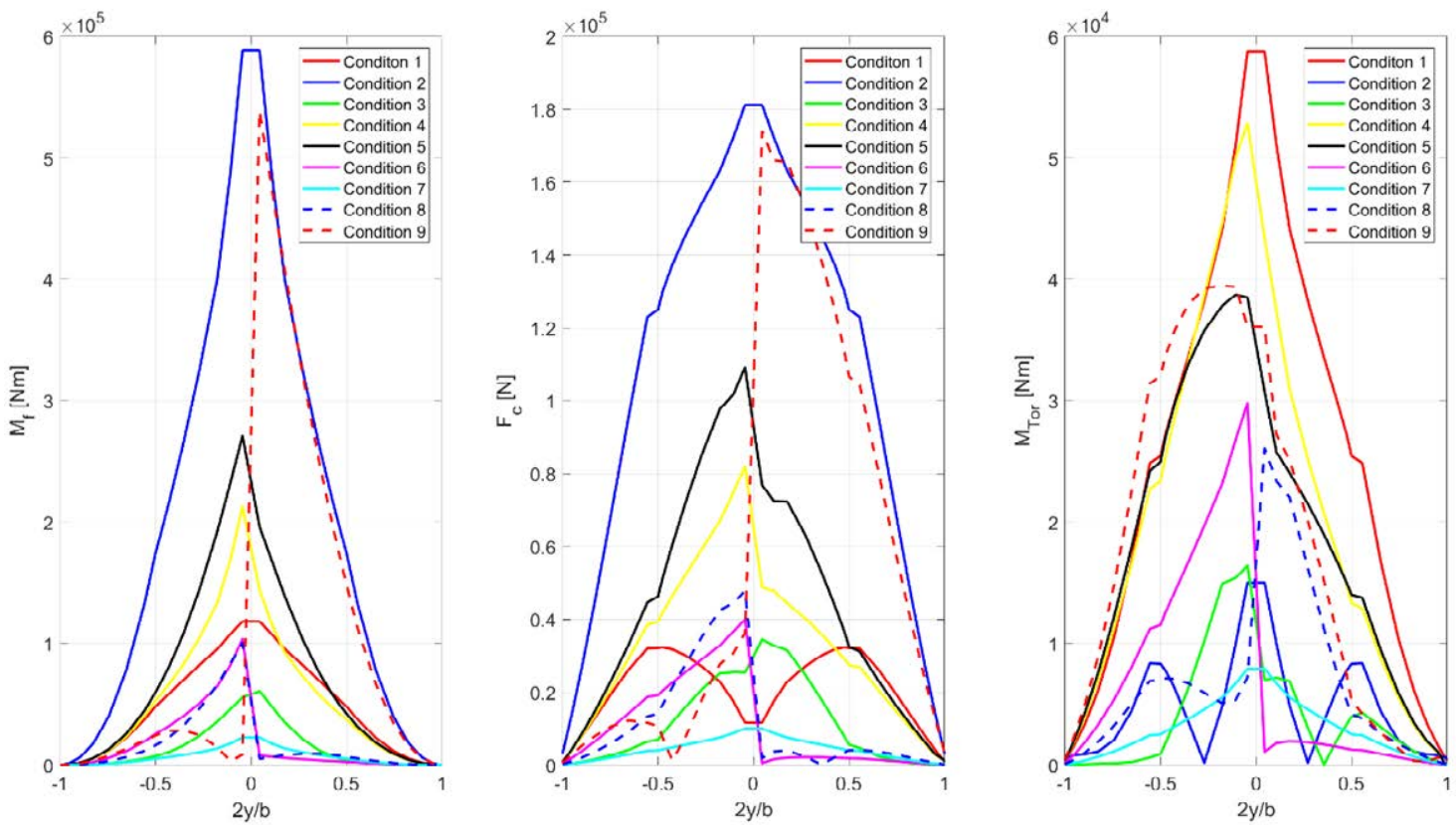

(b)

Figura 4-51 Cargas introducidas en la cola en las maniobras consideradas, analizando el efecto de variar la cuerda en la raíz, (a) para $\Gamma=30^{\circ}, C_{r}=3,7 \mathrm{~m}, b=12 \mathrm{~m}$ y $\lambda=0,32$ y (b) para $\Gamma=$ $30^{\circ}, C_{r}=4,5 m, b=12 m$ y $\lambda=0,32$. Fuente: elaboración propia. 

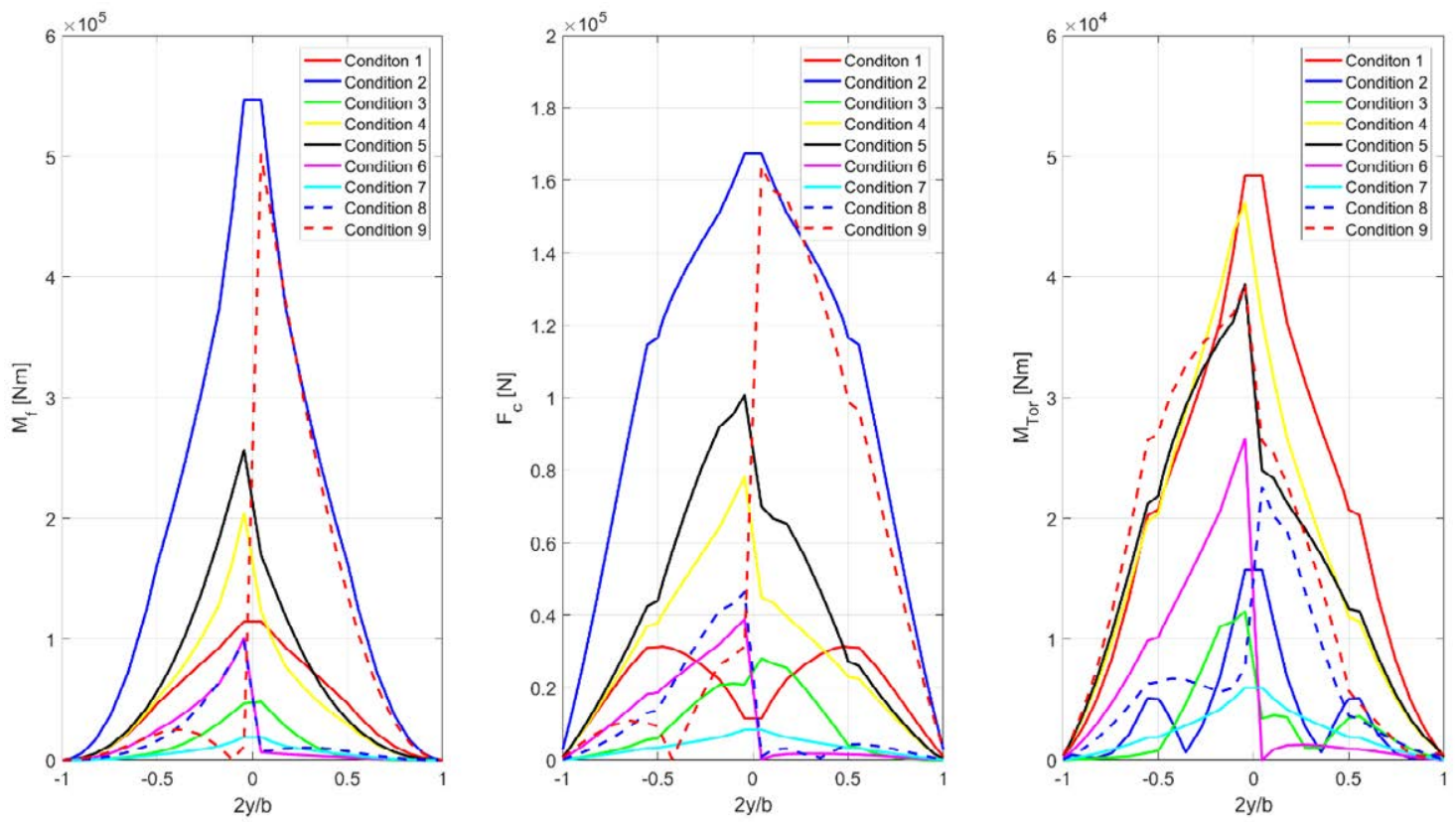

(a)
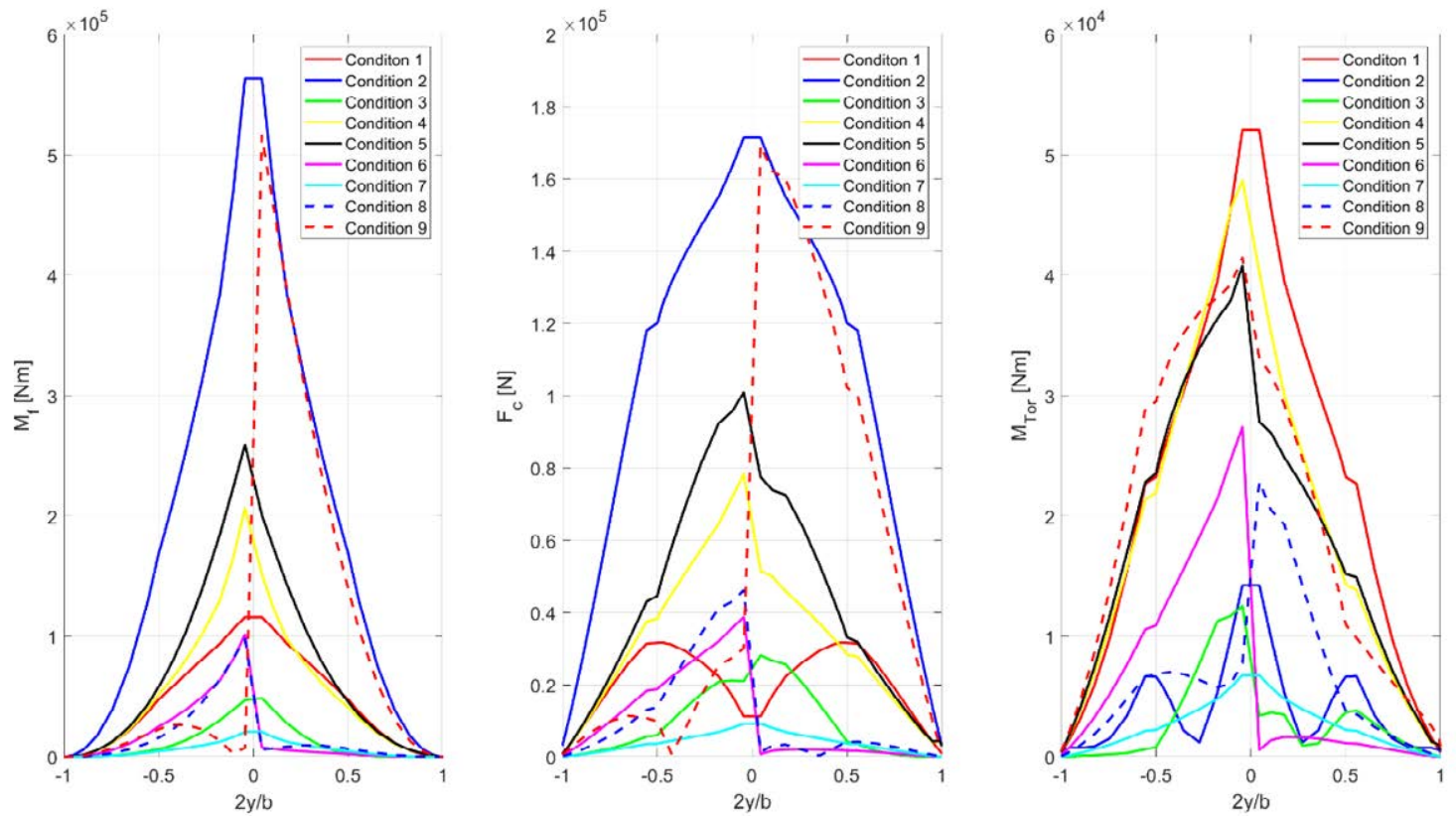

(b)

Figura 4-52 Cargas introducidas en la cola en las maniobras consideradas, analizando el efecto de variar el parámetro de estrechamiento, (a) para $\Gamma=30^{\circ}, C_{r}=4,5 \mathrm{~m}, b=12 \mathrm{~m}$ y $\lambda=0,32 \mathrm{y}$ (b) para $\Gamma=30^{\circ}, C_{r}=4,5 \mathrm{~m}, b=12 \mathrm{~m}$ y $\lambda=0,36$. Fuente: elaboración propia. 

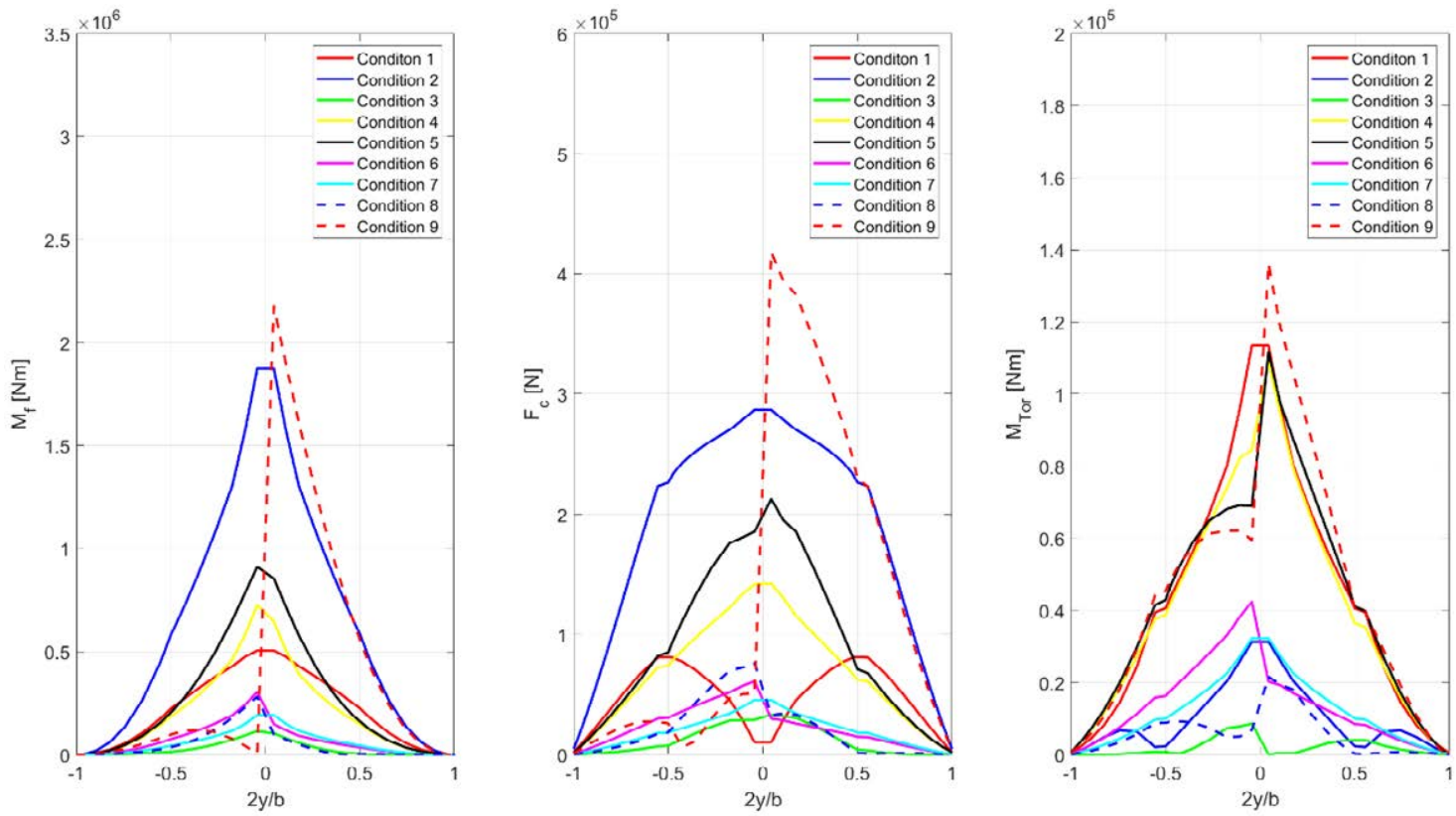

(a)
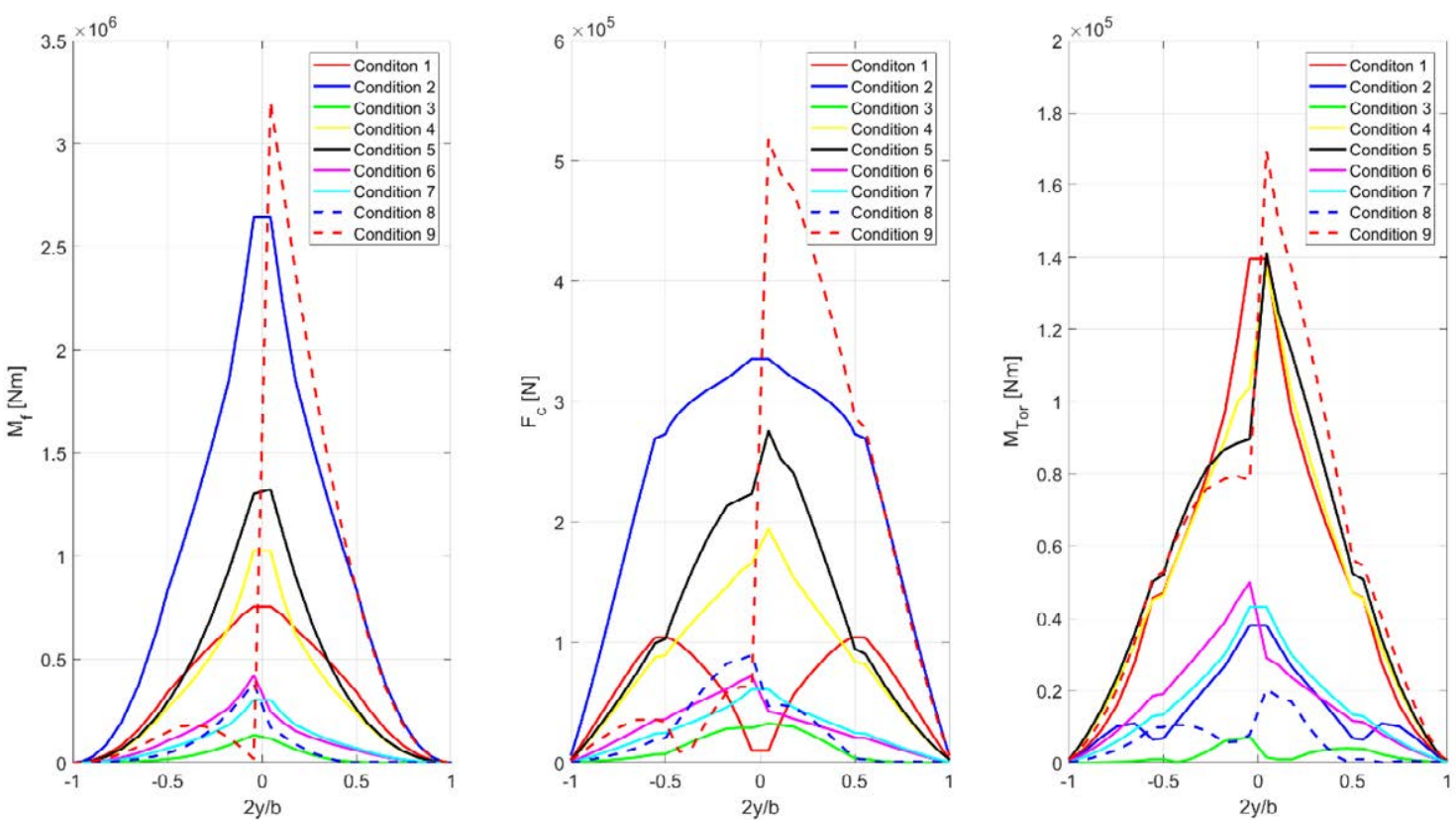

(b)

Figura 4-53 Cargas introducidas en la cola en las maniobras consideradas, analizando el efecto de variar la envergadura, (a) para $\Gamma=30^{\circ}, C_{r}=4,1 \mathrm{~m}, b=22 \mathrm{~m}$ y $\lambda=0,32$ y (b) para $\Gamma=30^{\circ}$, $C_{r}=4,1 m, b=26 m$ y $\lambda=0,32$. Fuente: elaboración propia. 

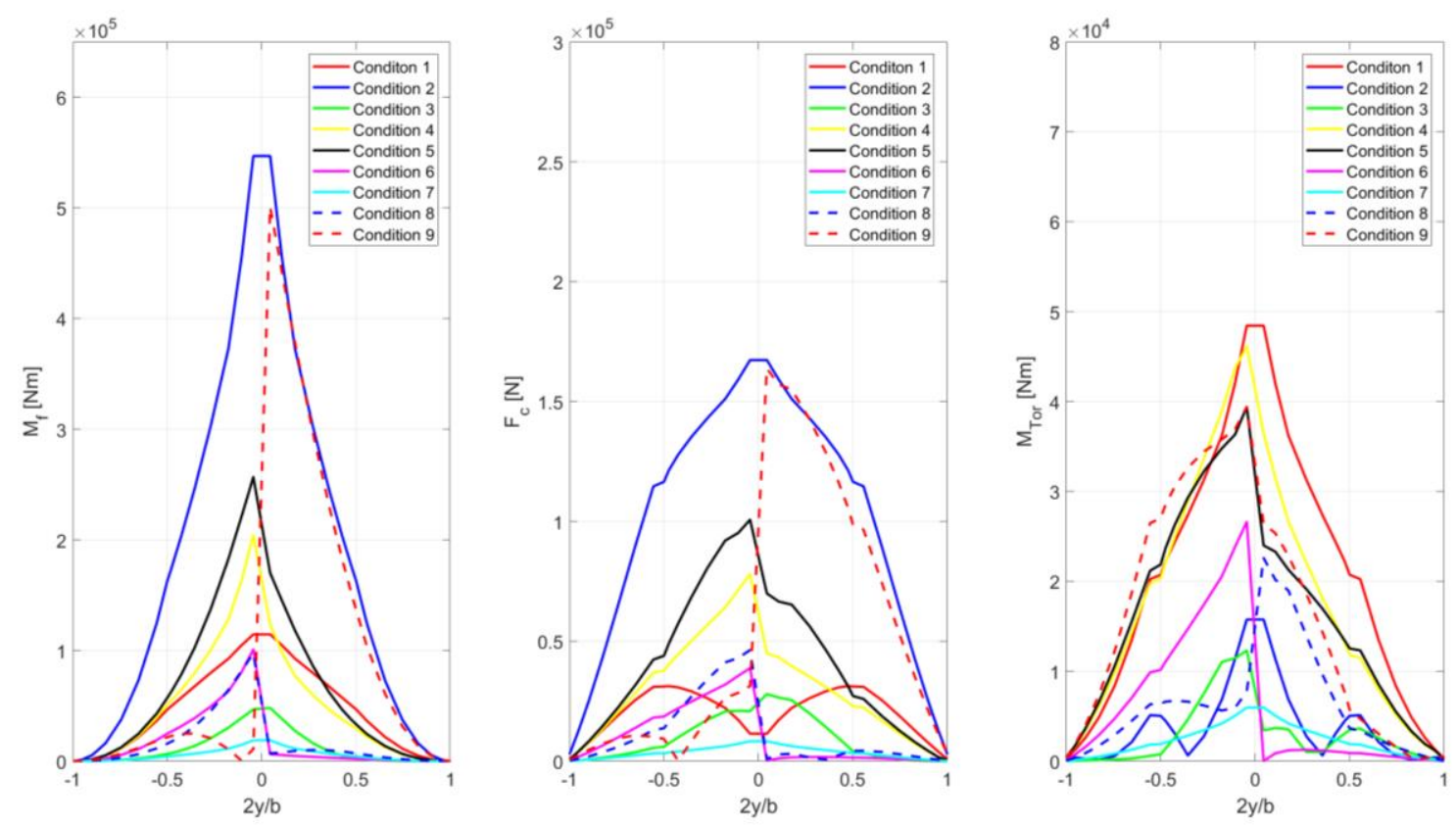

(a)
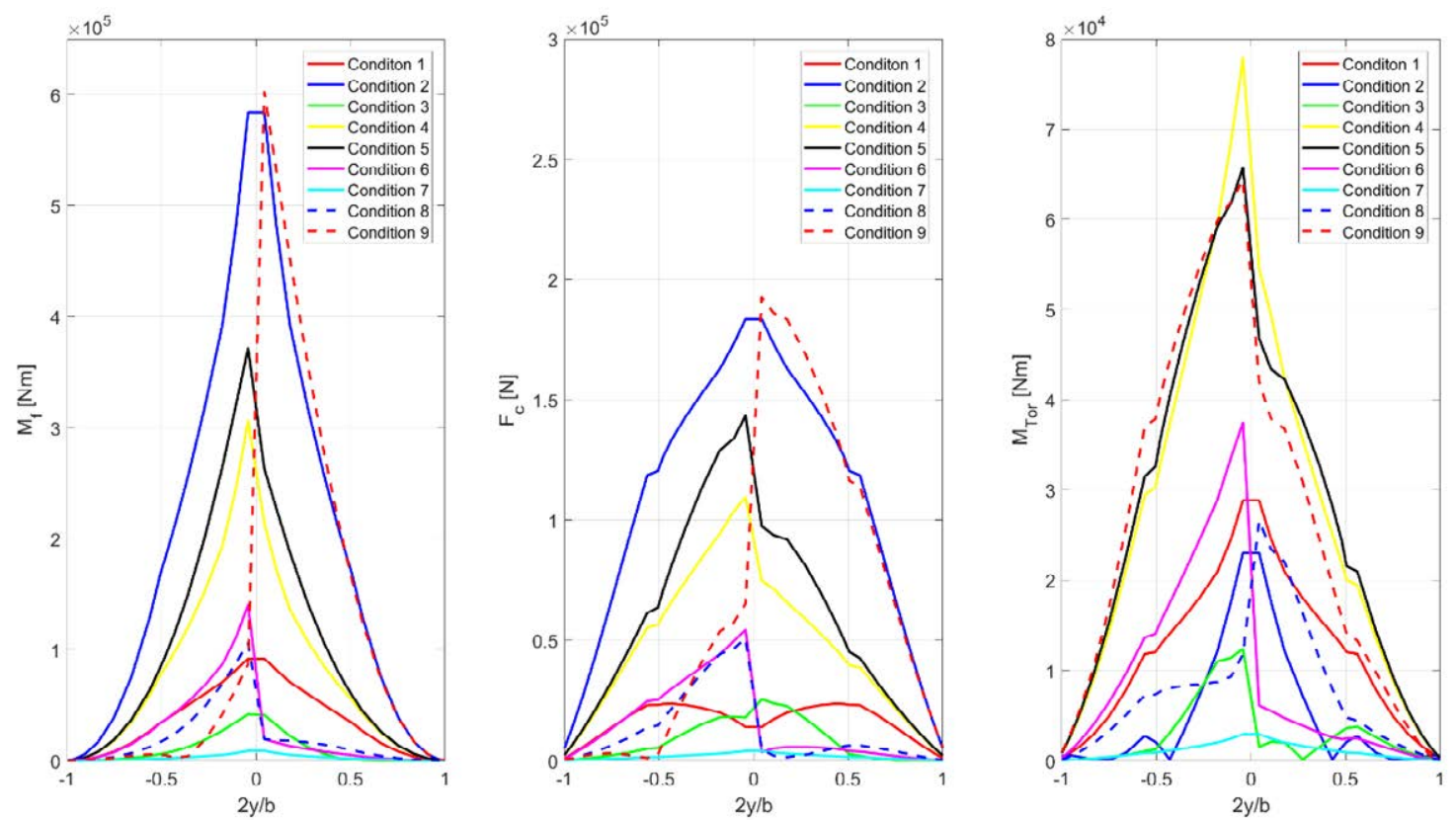

(b)

Figura 4-54 Cargas introducidas en la cola en las maniobras consideradas, analizando el efecto de variar el diedro, (a) para $\Gamma=30^{\circ}, C_{r}=4,1 \mathrm{~m}, b=12 \mathrm{~m}$ y $\lambda=0,32$ y (b) para $\Gamma=50^{\circ}, C_{r}=$ $4,1 m, b=12 m$ y $\lambda=0,32$. Fuente: elaboración propia.

Tras la presentación de los resultados de las cargas que se han generado en la cola en $V$ en las maniobras contempladas, se pasa al dimensionado de la estructura para determinar el peso del estabilizador de cola. La forma de proceder es exactamente igual que la que se siguió para la configuración de cola convencional del avión de referencia. A continuación, se muestran los resultados del peso de la estructura obtenidos. El peso final del estabilizador se corresponde con el máximo que se determine para cada una de las combinaciones de los parámetros de diseño de entre los casos de carga de estudio. Se presentan las dependencias que se han establecido entre el peso de cada 
uno de los casos de carga con los parámetros de diseño en la Figura 4-55, Figura 4-56, Figura 4-57 y Figura 4-58. Como se puede observar, el signo del gradiente de la función objetivo indica que el peso se reduce a medida que se reducen los parámetros de diseño. Por tanto, se concluye que el mínimo se encuentra en el punto que cumpla las restricciones que tenga el menor valor de las variables de diseño. También se muestra que la pendiente de la función con respecto al diedro es menor que para el resto de variables, esto implica que se deben minimizar las otras variables preferiblemente antes que el diedro.

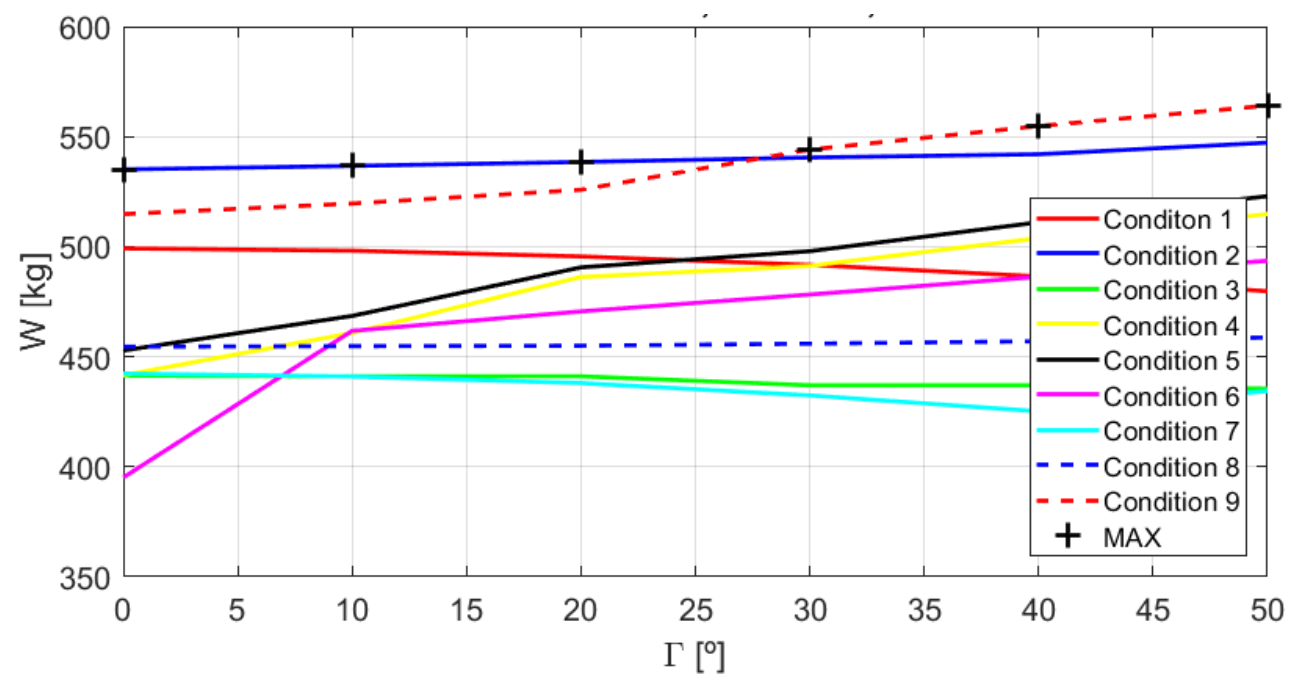

Figura 4-55 Peso del estabilizador en función del diedro para las maniobras seleccionadas. Fuente: elaboración propia.

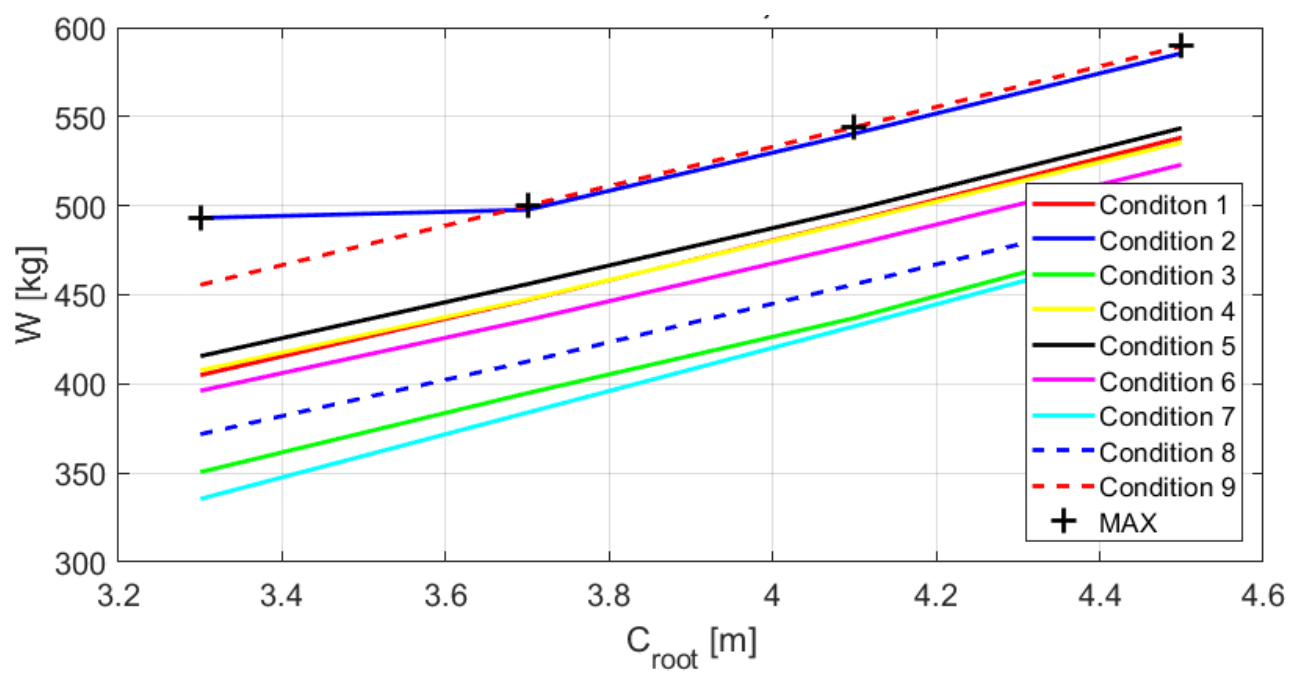

Figura 4-56 Peso del estabilizador en función de la cuerda en la raíz para las maniobras seleccionadas. Fuente: elaboración propia. 


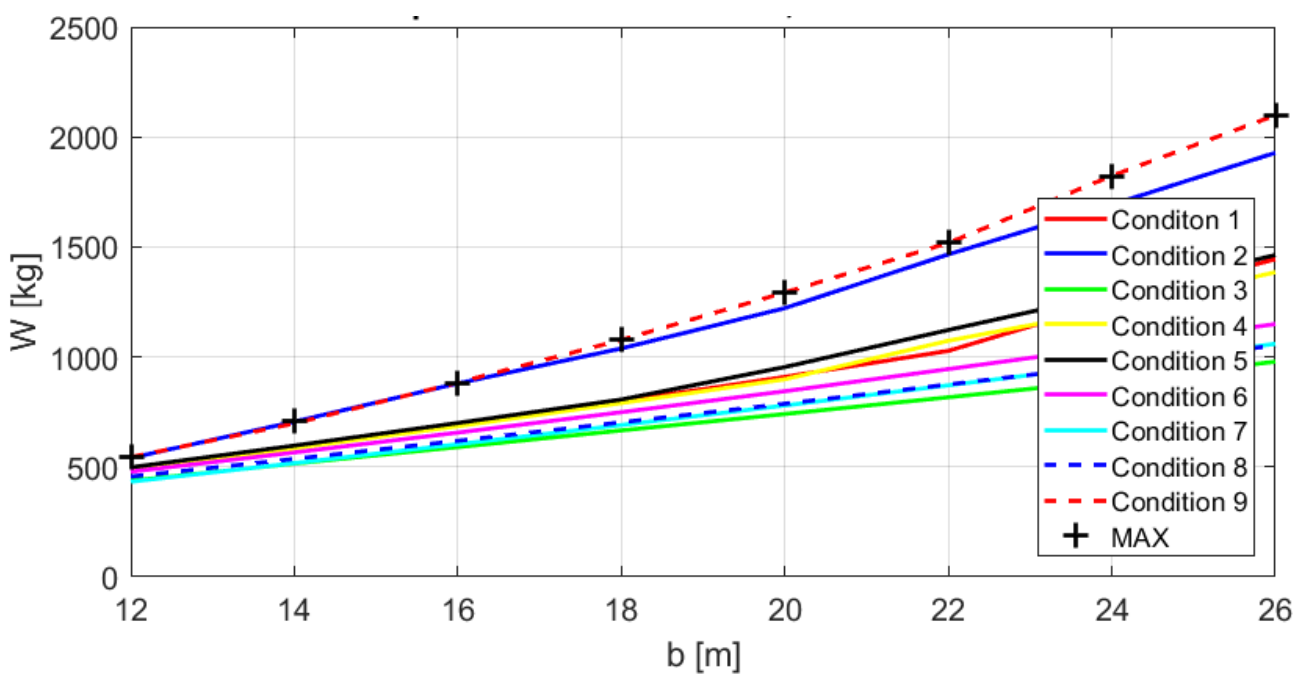

Figura 4-57 Peso del estabilizador en función de la envergadura para las maniobras seleccionadas. Fuente: elaboración propia.

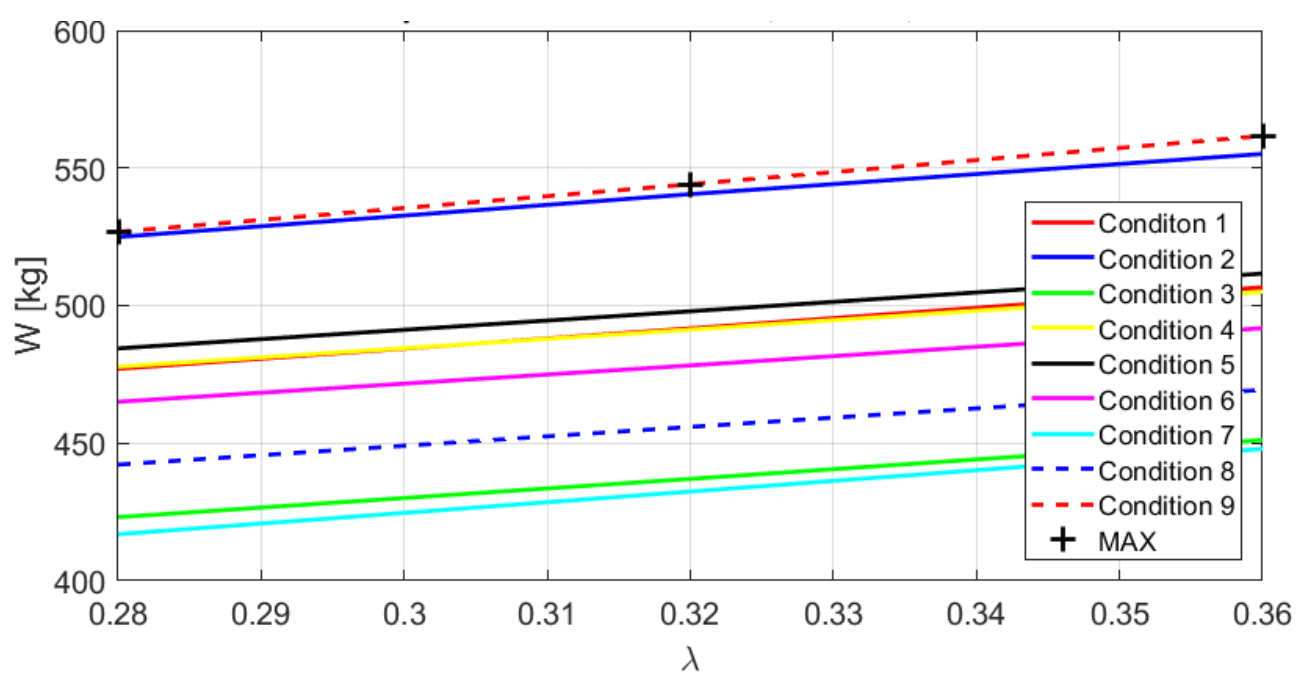

Figura 4-58 Peso del estabilizador en función del parámetro de estrechamiento para las maniobras seleccionadas. Fuente: elaboración propia.

\subsubsection{Resultados de diseño}

\subsubsection{Peso obtenido mediante el método basado en regresiones estadísticas}

La función de peso definida por el método que combina las regresiones estadísticas utilizadas para el estabilizador vertical y horizontal es monótona y alcanza su mínimo sobre una de las restricciones. Analizada la monotonía sobre las restricciones, se ha visto como las limitaciones activas son las impuestas por la estabilidad estática en crucero, tanto la longitudinal como la lateral. Además, los valores de las variables de diseño consideradas como grados de libertad, como son la cuerda en la raíz, parámetro de estrechamiento y envergadura deben tomar su menor valor posible que cumpla las restricciones para encontrar el mínimo de la función objetivo. A la vista del espacio de diseño definido en los apartados anteriores, el mínimo se encuentra en el lugar geométrico definido por la intersección entre las restricciones activas, porque es precisamente ahí donde se encuentran los valores mínimos de las variables de diseño. 


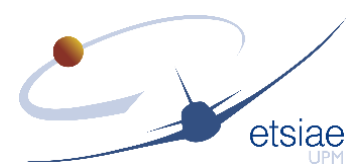

Esta intersección reduce a dos grados de libertad el problema, puesto que establece una nueva relación entre las variables de diseño. Esta relación se muestra de forma gráfica en la Figura 4-59, donde se ha tomado como nuevos grados de libertad la cuerda en la raíz y el parámetro de estrechamiento. Se presenta la dependencia de la envergadura y del diedro con los grados de libertad.
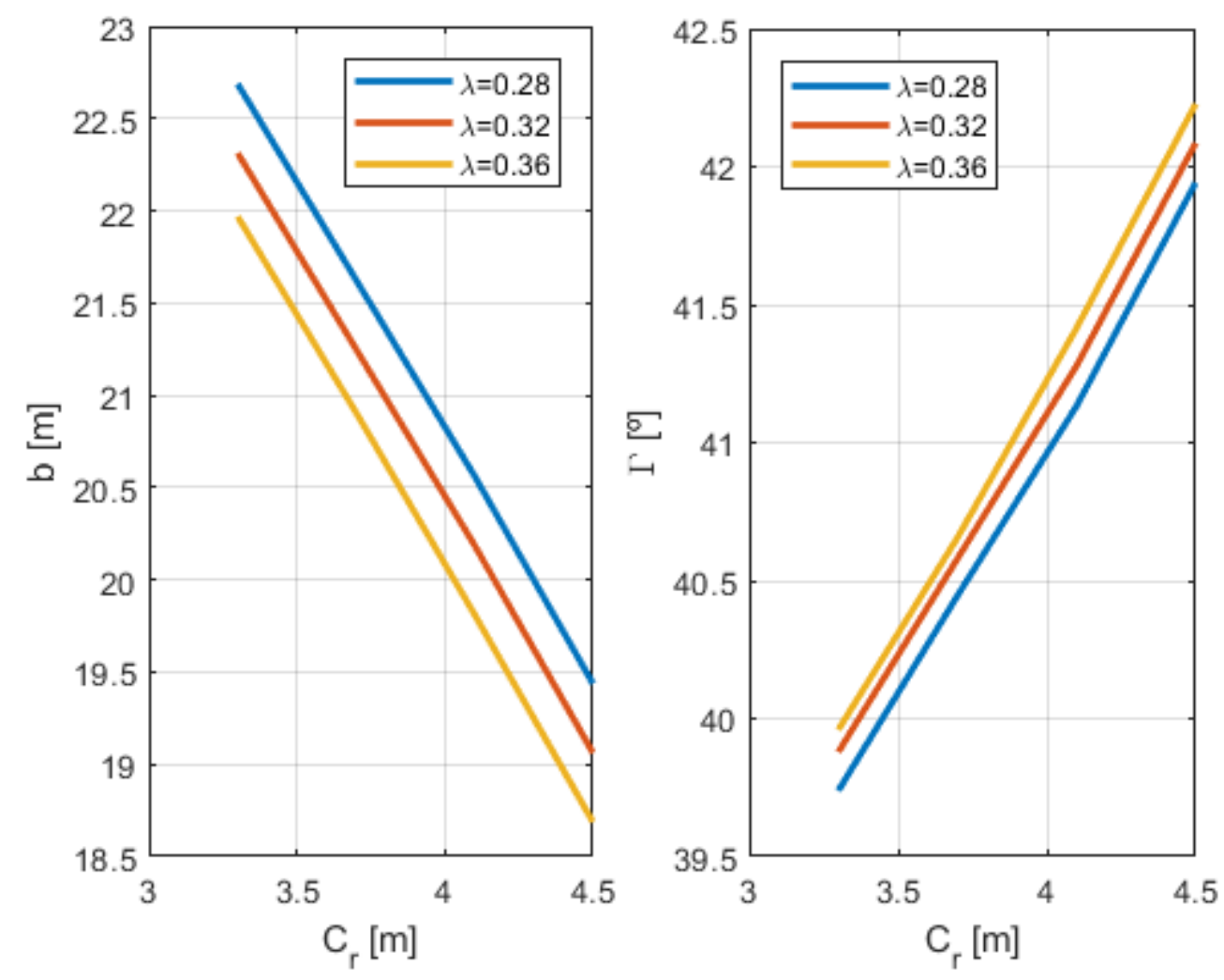

Figura 4-59 Relación que presentan las variables de diseño en los puntos de intersección entre la restricción impuesta por la derivada estática en crucero lateral y longitudinal. Fuente: elaboración propia.

Esto define una nueva restricción que debe analizarse con detenimiento, porque ahora no se puede cumplir el requisito de que se deben tomar el mínimo de las variables envergadura, cuerda en la raíz y parámetro de estrechamiento simultáneamente, ya que lo que se ve en la Figura 4-59 es que minimizar la cuerda en la raíz y el parámetro de estrechamiento, precisamente maximiza la envergadura. Por tanto, se debe analizar sobre esta restricción cómo se comporta la función objetivo. Este resultado se presenta en la Figura 4-60. Se puede ver que también en este caso el peso se reduce a medida que el parámetro de estrechamiento y la cuerda en la raíz toman valores menores. Es por esto que se puede concluir que el mínimo se alcanza para el mínimo valor de la cuerda en la raíz y el parámetro de estrechamiento, es decir, para 0,28 y 3,3 respectivamente. Esto corresponde a unos valores del diedro y de la envergadura de $39,7^{\circ}$ y $22,67 \mathrm{~m}$; y por tanto un peso mínimo de $1806 \mathrm{~kg}$. Este peso supone un incremento de un $50 \%$ aproximadamente respecto del peso de los estabilizadores de la cola convencional que presenta el CSR-01. 


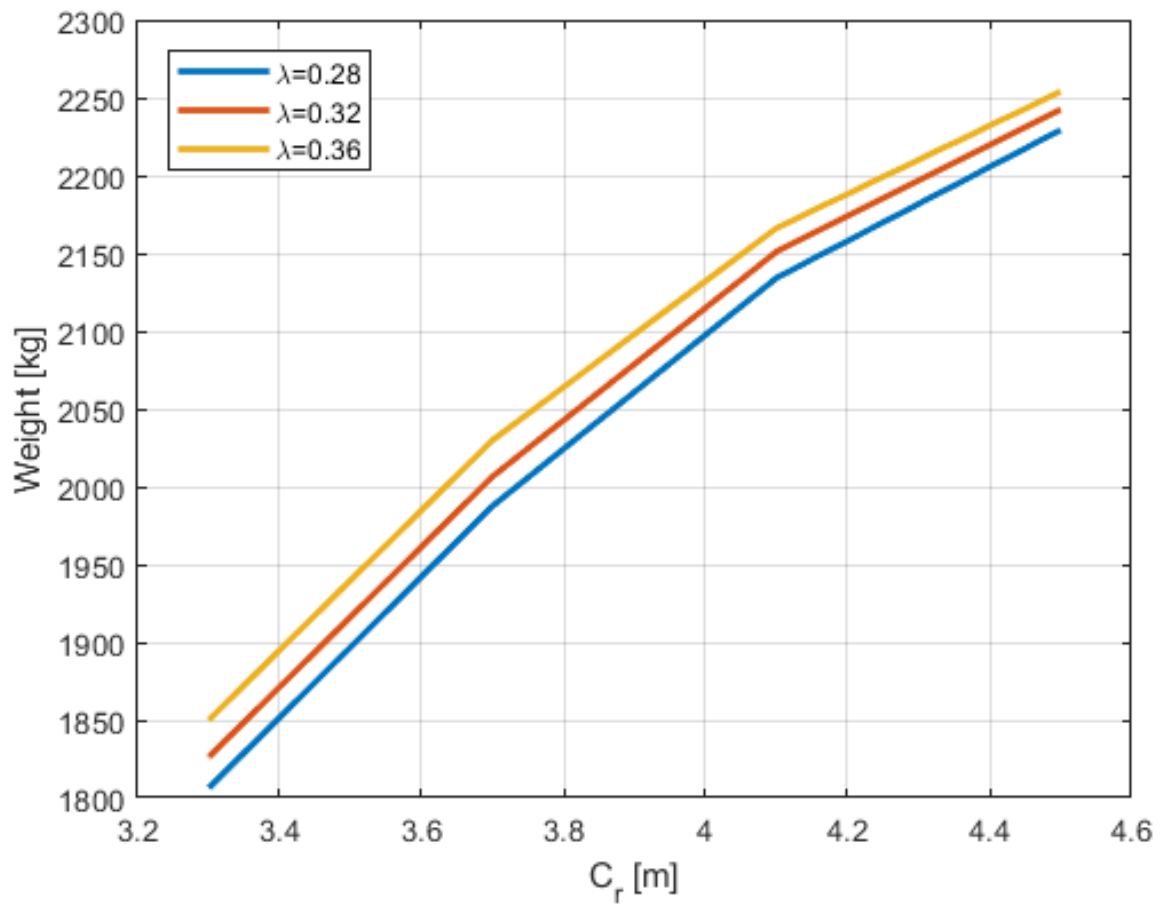

Figura 4-60 Peso estimado por el método basado en las regresiones para colas convencionales que presentan las variables de diseño en los puntos de intersección entre la restricción impuesta por la derivada estática en crucero lateral y longitudinal. Fuente: elaboración propia.

Este resultado no es representativo por sí solo, ya que lo que es realmente relevante es el efecto que tenga esta nueva configuración sobre el consumo de combustible. Esta nueva configuración presenta una reducción de superficie mojada de la cola, lo que a su vez supone una reducción de la resistencia aerodinámica. Por tanto, es necesario analizar si este mínimo de peso también supone un mínimo de resistencia de la cola. Para la estimación de la resistencia aerodinámica que supone la cola en $V$ se va a utilizar la misma expresión que se propone en (Torenbeek 1982) para los estabilizadores, indicada en la ecuación [ 4-1 ]. Para poder aplicarse a esta configuración, es necesario determinar el coeficiente de fricción de cada una de las combinaciones de los parámetros de diseño. Únicamente son necesarios la cuerda en la raíz y el parámetro de estrechamiento, ya que el coeficiente de fricción depende del número de Reynolds basado en la cuerda media geométrica, que se puede determinar de la siguiente forma para un ala de simple estrechamiento, como es el caso de la cola:

$$
C M G=\frac{C_{r}(1+\lambda)}{2}
$$

El espesor relativo se ha fijado en el $12 \%$ y la fecha de puntos $1 / 2$ es aproximadamente de $28^{\circ}$. El último término corresponde a la superficie del estabilizador, que se tomará como la que corresponde hasta prolongar las superficies hasta el plano de simetría del avión, de tal forma que el resultado de la ecuación incluya los fenómenos de interferencia con el fuselaje, aproximación que se recoge en la propia referencia. Por tanto, es necesario analizar la variación de la superficie de la cola en función de las variables de diseño, cuya dependencia es como se indica a continuación:

$$
S_{v e e}=\frac{C_{r}(1+\lambda)}{2} b
$$




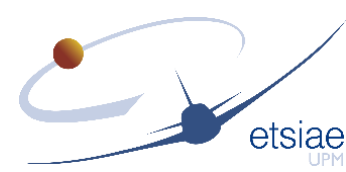

Como se puede observar en la anterior expresión, minimizar el área supone que las tres variables de diseño sean mínimas por separado. Sin embargo, es necesario tener en cuenta las restricciones del problema ya que plantean una dependencia entre las variables de diseño. La primera dependencia presentada en la Figura 4-37 o en la Figura 4-40 no afecta al valor de la superficie, ya que no depende del diedro de la cola. En cambio, la que se presenta en la Figura 4-59 sí que es de relevancia, puesto que esa dependencia se establece cuando se quiere buscar el mínimo de la función objetivo, pero también se alcanza la misma dependencia al querer buscar el mínimo de superficie. Es decir, que la Figura 4-59 también corresponde con la búsqueda de la cola de mínima superficie. Al igual que ocurría en el caso de la función de peso, el siguiente paso no es tan obvio ya que el área crece al aumentar la cuerda en la raíz, pero disminuye al reducirse la envergadura, que es precisamente lo que ocurre al moverse por las curvas presentadas en la figura. Es por ello por lo que se debe analizar cuál de los dos efectos es más acuciado. La Figura 4-61 muestra precisamente este resultado, observándose que es necesario moverse hacia puntos donde la cuerda en la raíz sea lo menor posible, porque la derivada con respecto a esta variable es mayor que con respecto a la envergadura.

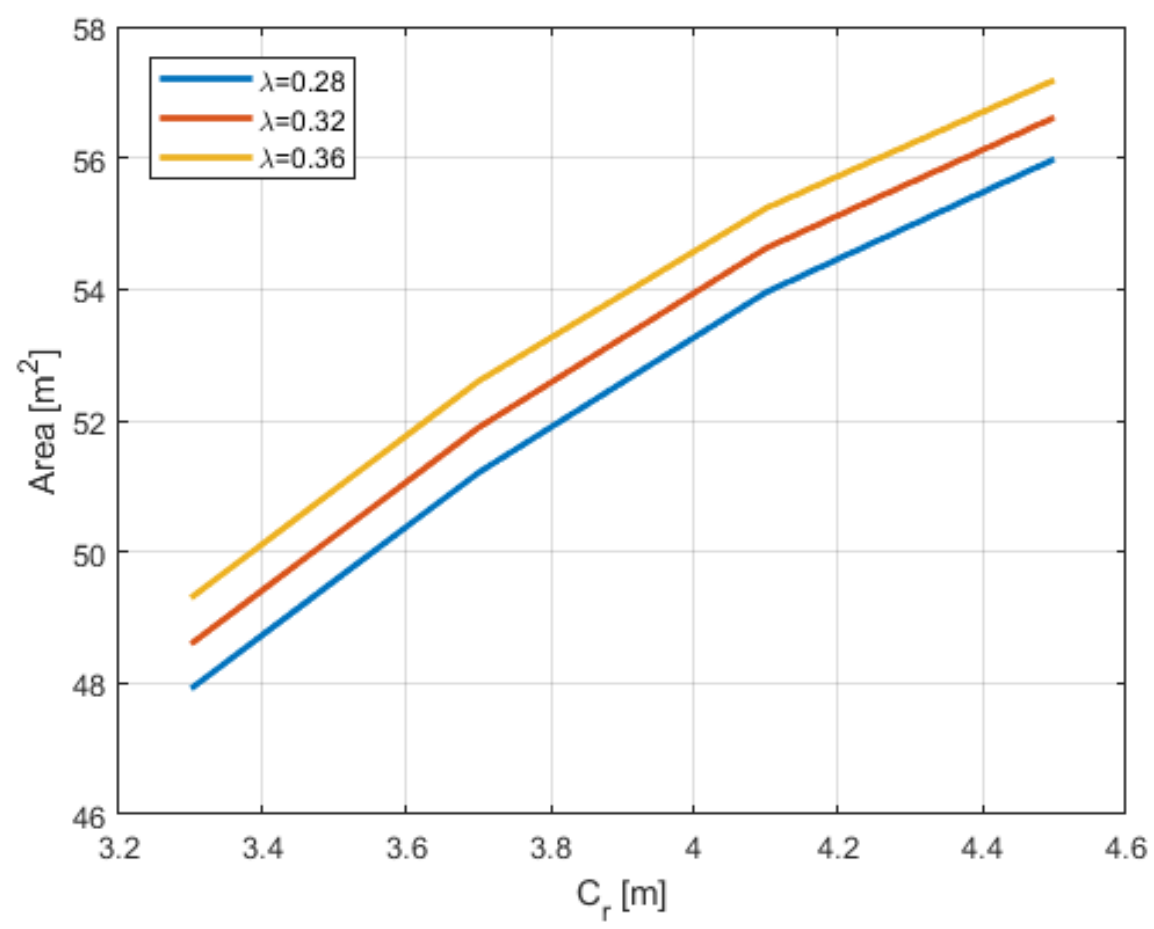

Figura 4-61 Superficie de la cola en V correspondiente a las variables de diseño en los puntos de intersección entre la restricción impuesta por la derivada estática en crucero lateral y longitudinal. Fuente: elaboración propia.

La reflexión que se debe hacer a continuación es si una cola de mínima superficie corresponde con la de mínima resistencia. A la vista de la ecuación que liga estas dos magnitudes, todos los términos que intervienen son constantes salvo el coeficiente de fricción, que depende del número de Reynolds y este, a su vez, de la cuerda media geométrica. Esta dependencia es muy suave, pero debe tenerse en cuenta. Los resultados que se obtienen para el coeficiente de resistencia sin sustentación de la cola se muestran en la Figura 4-62. Como se ha comentado que apenas varía el coeficiente de fricción, este término es prácticamente constante y el mínimo de área se corresponde con el mínimo de coeficiente de resistencia y de resistencia sin sustentación. Este 
mínimo supone una reducción del coeficiente de resistencia sin sustentación asociado al empenaje completo de un $12 \%$ aproximadamente.

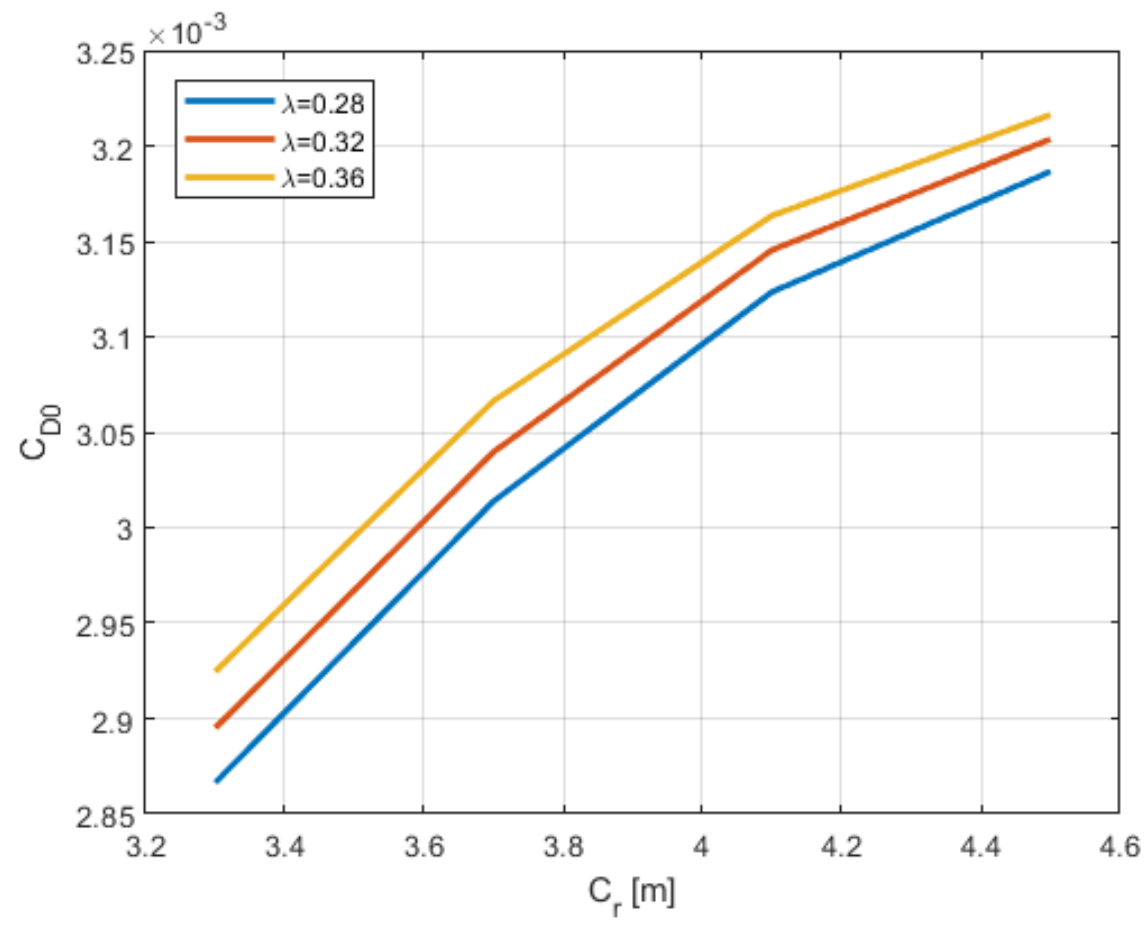

Figura 4-62 Coeficiente de resistencia sin sustentación de la cola en V correspondiente a las variables de diseño en los puntos de intersección entre la restricción impuesta por la derivada estática en crucero lateral y longitudinal. Fuente: elaboración propia.

Gracias a este resultado se puede concluir que la misma combinación de las variables de diseño que resultan en un mínimo de peso también corresponden a un mínimo de área y, en consecuencia, a un mínimo de resistencia sin sustentación de la cola.

Los resultados que se han obtenido tienen efectos opuestos en el consumo de combustible del avión. El incremento de peso supone un incremento de combustible necesario para la ruta de diseño, sin embargo, la reducción de la resistencia tiene consecuencias positivas en el consumo. Por tanto, un paso más que se puede dar para comprobar si esta configuración supone algún beneficio o no en el consumo de combustible, es analizar lo que ocurre en la misión de diseño en la configuración óptima encontrada.

En el apartado 4.2.1 se ha presentado una metodología para estimar el peso de combustible necesario para llevar a cabo la ruta de diseño. Ese método se puede aplicar al caso particular que se ha alcanzado tras la optimización. Procediendo de la misma forma que para el avión de referencia, se puede estimar el consumo de combustible del nuevo avión. Simplemente hay que considerar los cambios de peso y de coeficiente de resistencia que introducen la cola en $\mathrm{V}$. El peso vacío operativo se ve modificado porque la nueva cola se ve incrementada su peso en un $50 \%$, resultando en un nuevo peso vacío operativo de $42702 \mathrm{~kg}$, siendo el de referencia de $42100 \mathrm{~kg}$. El coeficiente de resistencia sin sustentación en crucero que se obtiene para la configuración óptima seleccionada es de 0,0184, siendo el de referencia de 0,0188.

Resolviendo de forma iterativa la ecuación [ 4-2 ] junto con la definición del parámetro de alcance $K$ en el punto medio del crucero, se obtiene un combustible consumido durante el viaje de $15011 \mathrm{~kg}$, para una $K$ de Breguet de $22673 \mathrm{~km}$. El combustible resultante supone un ahorro respecto al estimado por este mismo procedimiento para el 


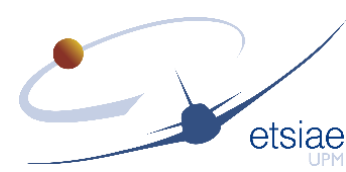

avión de referencia en la misión de diseño del 0,82\%. Para mostrar este resultado de forma gráfica, se ha realizado este procedimiento para todos y cada uno de los puntos representados en la Figura 4-62, y se van a ilustrar los incrementos del consumo de combustible que suponen cada una de esas combinaciones de las variables de diseño en la Figura 4-63.

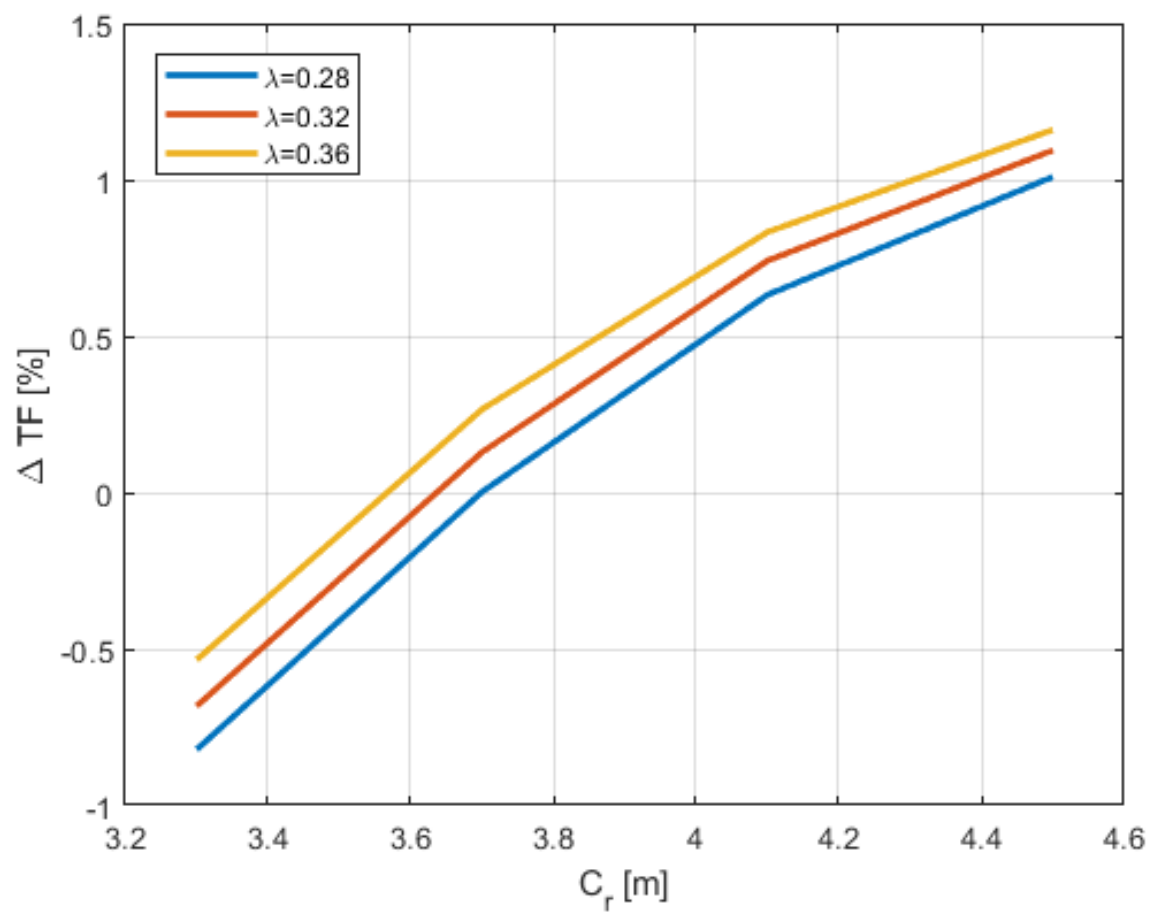

Figura 4-63 Incremento del combustible consumido en la ruta de diseño de la cola en $V$ correspondiente a las variables de diseño en los puntos de intersección entre la restricción impuesta por la derivada estática en crucero lateral y longitudinal calculando el peso por medio de las regresiones estadísticas. Fuente: elaboración propia.

\subsubsection{Peso obtenido mediante el método casi analítico para los estabilizadores}

El procedimiento para determinar el óptimo por medio del método casi analítico para la estimación del peso del estabilizador es el mismo que el que se ha seguido para el caso anterior. El gradiente indica que el mínimo se alcanza reduciendo los valores de las variables de diseño, por tanto, la relación que se muestra en la Figura 4-59 se mantiene para esta función objetivo. Sin embargo, en esta función de pesos el gradiente en la dirección de la envergadura es de mayor valor que en el de la cuerda y en el del parámetro de estrechamiento, lo cual lleva a minimizar la envergadura a pesar de que el parámetro de estrechamiento y la cuerda no lo hagan. Este resultado se representa en la Figura 4-64, donde se ve que el mínimo peso se alcanza para el máximo valor de cuerda en la raíz y de parámetro de estrechamiento. Este hecho lleva a que el punto donde se obtiene el mínimo peso no coincida con la mínima superficie ni la mínima resistencia aerodinámica. Por tanto, cobra aún más relevancia el análisis del combustible consumido en la misión de diseño, para comprobar para cuál de los dos mínimos se alcanza el óptimo desde el punto de vista del consumo de combustible. La dependencia del consumo de combustible con las variables de diseño que se toman como grados de libertad se refleja en la Figura 4-65, es decir, el parámetro de estrechamiento y la cuerda en la raíz. 


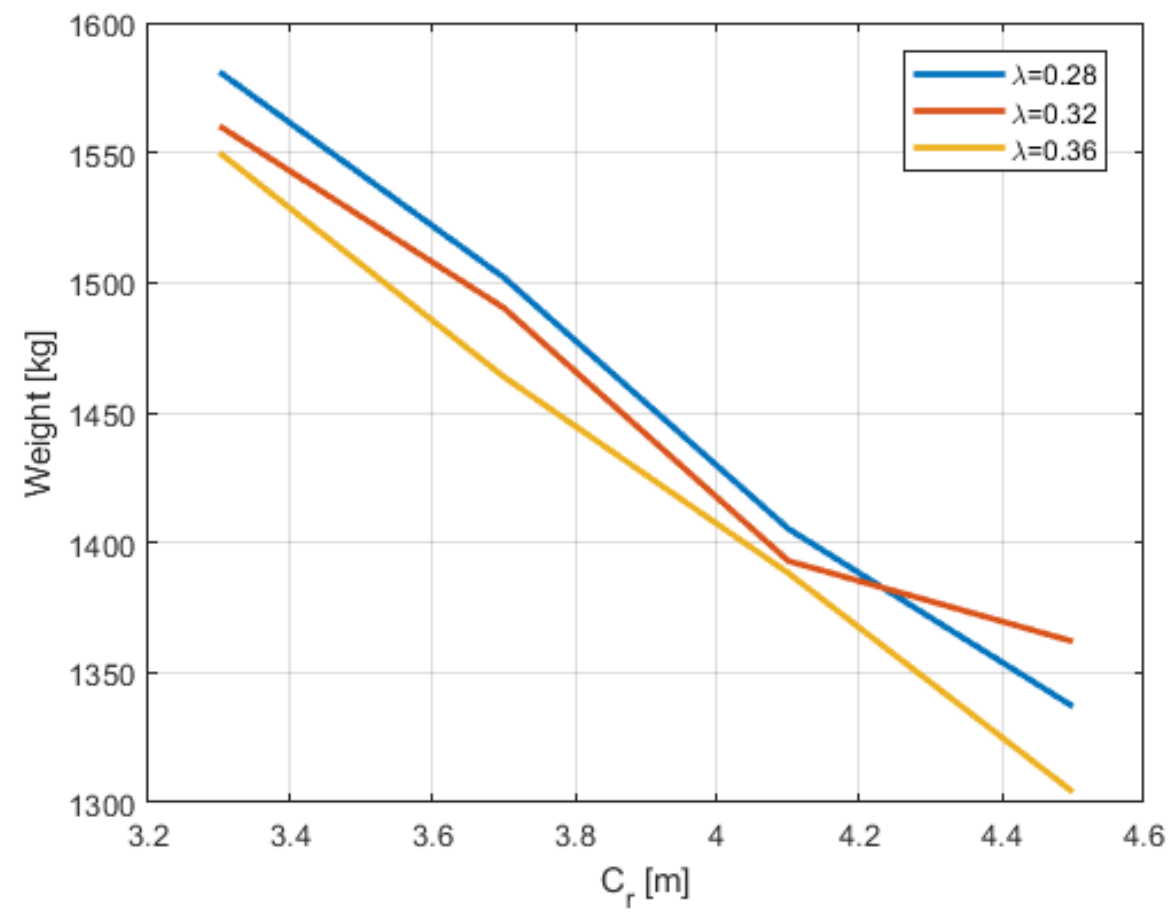

Figura 4-64 Peso estimado por el método basado los métodos casi analíticos que presentan las variables de diseño en los puntos de intersección entre la restricción impuesta por la derivada estática en crucero lateral y longitudinal. Fuente: elaboración propia.

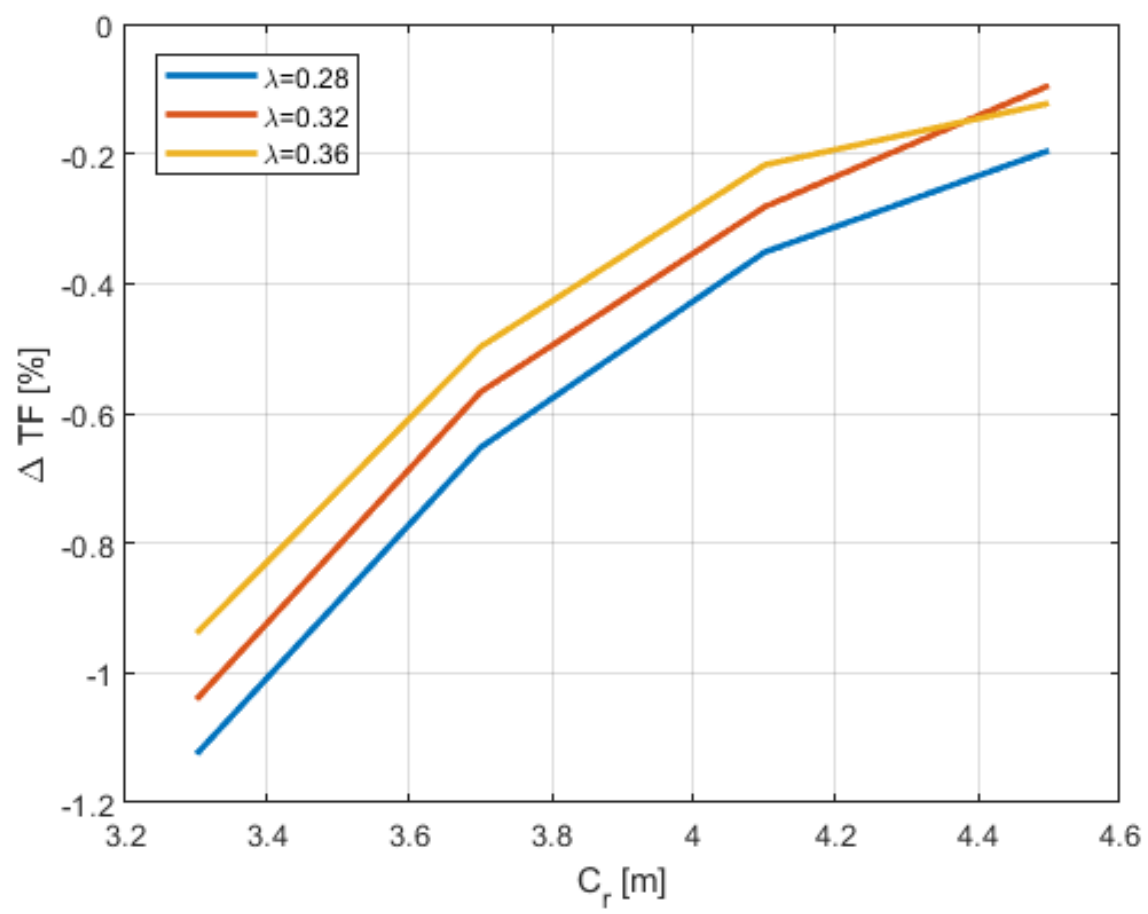

Figura 4-65 Incremento del combustible consumido en la ruta de diseño de la cola en $V$ correspondiente a las variables de diseño en los puntos de intersección entre la restricción impuesta por la derivada estática en crucero lateral y longitudinal calculando el peso por medio del método casi analítico. Fuente: elaboración propia.

Se observa como el óptimo coincide con el correspondiente al mínimo de resistencia aerodinámica de la cola. En conclusión, el óptimo es el mismo que el que se determinó con el otro método de peso. Sin embargo, el peso de la cola es algo menor por el procedimiento casi analítico que por el de regresiones estadísticas. La configuración de 
mínimo consumo de combustible incrementa el peso en un $30 \%$ con respecto a la cola de configuración convencional del avión de referencia. Por este motivo, el mínimo consumo de combustible es algo menor que el obtenido por el otro método, en este caso el incremento de combustible es del $-1,12 \%$ respecto al del avión de referencia. A pesar de esto, el parámetro de alcance resultante es prácticamente igual al que se ha determinado en el método de regresiones, es de $22653 \mathrm{~km}$. Por tanto, la reducción de combustible se debe al menor peso vacío operativo del avión, y no a un mayor parámetro de alcance. 


\subsection{APLICACIÓN A OTRAS CONFIGURACIONES NO CONVENCIONALES}

La metodología que se ha desarrollado en esta tesis es aplicable a cualquier configuración de cola en general. El esquema a seguir es el mismo que el que se ha presentado para la configuración de cola en V. El primero paso es identificar las variables de diseño que van a definir la geometría de la configuración. Posteriormente se debe analizar las restricciones que introducen en el dimensionado las condiciones de estabilidad estática en crucero, el control de la aeronave en el segundo segmento tras el fallo del motor crítico y el aterrizaje con viento cruzado. Una vez establecido el espacio de diseño se debe evaluar la función objetivo sobre las variables de diseño. En un caso general, el método de estimación de pesos por medio de regresiones estadísticas desarrolladas para la configuración de cola convencional, que se ha adaptado a la cola en $\mathrm{V}$ sin más que proyectando sobre los planos horizontal y vertical, debe tomarse con cuidado, ya que es muy posible que no se obtengan resultados tan adecuados como el caso de la cola en V. Por tanto, hay que determinar las cargas que se inducen en la estructura y dimensionar la misma siguiendo la metodología propuesta en el capítulo 3.3.2. De esta forma se tiene una estimación del peso de la estructura y se puede establecer la dependencia que tiene esta función con las variables de diseño, lo cual permite determinar la actividad de las restricciones.

Estudiando la monotonía sobre la función objetivo y, si procede, sobre las restricciones, se puede optimizar el peso de la configuración. Un dato importante es la comprobación de si en el caso de una configuración de cola no convencional distinta a la cola en $V$ el óptimo se produce simultáneamente para el mínimo de peso y de área, y, en consecuencia, para el mínimo consumo de combustible. Si ocurre que el mínimo del peso no coincide con el mínimo de área, se debería tomar como función objetivo el consumo de combustible de la misión de diseño, estimado como se ha hecho para la configuración de cola en $\mathrm{V}$ y como ha ocurrido en el caso del método de pesos casi analítico. Esto puede complicar ligeramente la operativa de cálculo, ya que el consumo de combustible de la misión de diseño está definido por medio de una función implícita que depende, a su vez, de la función de pesos. A pesar de esta complicación, la búsqueda del óptimo es conceptualmente semejante a la que se ha seguido para encontrar el mínimo de la función de pesos sometida a las restricciones activas.

Por tanto, a continuación, se presentan algunos ejemplos de configuraciones de entre las que se han podido ver en el estado del arte, sobre las cuales se muestran una serie de reflexiones, que principalmente afectan a la parametrización de la geometría. La primera que se va a tratar es la configuración de cola en U. Esta configuración se puede definir por medio de los parámetros que se indican en la Figura 4-66, además de por los parámetros de estrechamiento de cada uno de los dos tramos. En la figura se ha tomado el caso en el que el parámetro de estrechamiento del tramo 1 es distinto de uno pero en el tramo 2 el estrechamiento es nulo. La cola en $U$ que se muestra en la figura se construye sin más que extendiendo las puntas de la cola en $\mathrm{V}$ de forma vertical (diedro $90^{\circ}$ ), formando una especie de winglets. Si estas extensiones son lo suficientemente largas, se puede incluir en ellas los controles laterales, es decir, el timón de dirección. Habría que ver cuál debe ser la envergadura mínima de este segundo tramo para que el timón de dirección tenga la suficiente potencia de control como para controlar el avión en las condiciones de fallo de motor crítico y aterrizaje con viento cruzado. El tramo denominado como 1, es el que incluye el timón de profundidad, que en principio se ha supuesto que debe abarcar toda envergadura del tramo, a falta de dimensionarlo adecuadamente. 


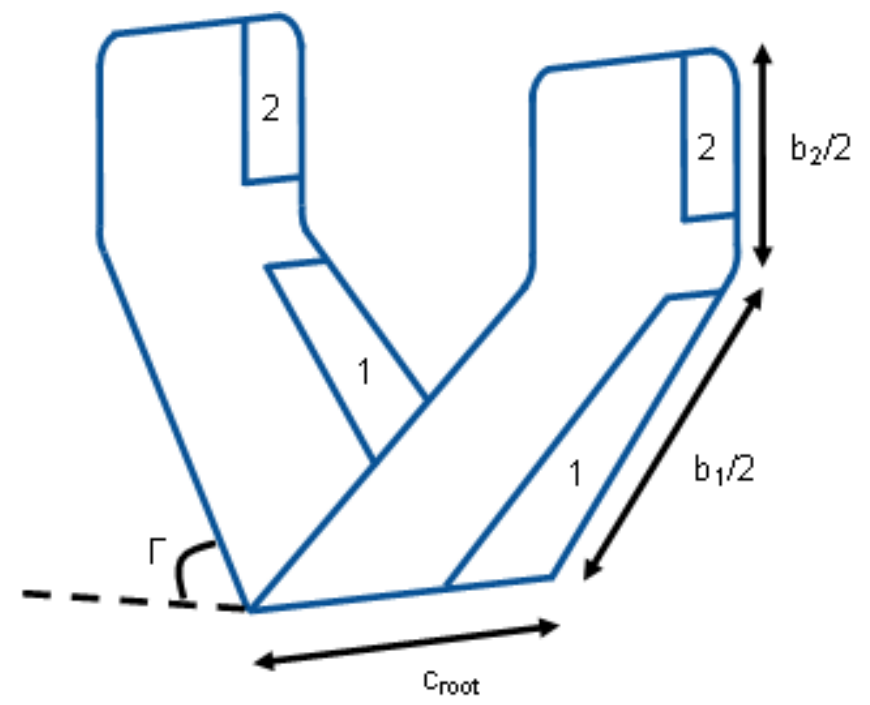

Figura 4-66 Definición de la envergadura, $b_{1}$ y $b_{2}$, cuerda en la raíz, $C_{\text {root }}$ y ángulo de diedro, $\Gamma$, de una cola en $U$ y dónde se sitúan los controles. Fuente: elaboración propia.

Otra de las posibilidades que se han presentado en el estado del arte son las configuraciones cerradas. La parametrización de estas superficies de cola es la misma que la de un estabilizador horizontal de una configuración convencional, pero añadiendo un parámetro adicional que mida la separación de las dos superficies paralelas, asumiendo que ambas superficies son iguales en geometría. Esta separación debería ser lo suficientemente amplia como para poder instalar unos timones de dirección en esas superficies de unión verticales, ya que no se encuentra otro sitio donde poder instalarse. El principal inconveniente que tendría esta configuración desde el punto de vista de la metodología de dimensionado es si el modelo aerodinámico que se ha utilizado en este estudio sería lo suficientemente preciso para este tipo de superficies de cola cerradas.

A pesar de las reflexiones que se han indicado y otras que puedan surgir dependiendo de la configuración de cola no convencional que se quiera dimensionar, el estudio presentado es perfectamente adaptable a cualquier otra geometría de cola. Además, siempre se podría sustituir cualquiera de los módulos por otro semejante que realice las mismas funciones y que sea más preciso o específico para una configuración de cola en concreto. 


\subsection{RESUMEN Y CONCLUSIONES}

En este capítulo se ha presentado una aplicación de la metodología de dimensionado de estabilizadores de cola, tanto para una configuración convencional de referencia como para una configuración no convencional como es la cola en V. Además, se han indicado una serie de directrices sobre cómo se aplicaría esta metodología a otras configuraciones no convencionales de cola.

Los métodos para determinar el peso de las superficies de cola que se han empleado presentan buenos resultados para la configuración convencional del avión de referencia, encontrándose diferencias con respecto a los pesos incluidos en la base de datos CeRAS inferiores al 6\%. Hay que decir que en ambos métodos aparecen una serie de factores que deben ajustarse empíricamente que se han tratado de adaptar al caso particular que se ha querido estudiar, de ahí que la precisión de los resultados sea tan buena. Sin embargo, la aplicación de ambos procedimientos de estimación de pesos para la cola en $\mathrm{V}$ ha reflejado una serie de diferencias. Ambas metodologías presentan un comportamiento cualitativo semejante con respecto a la variación de los parámetros de diseño, en concreto, que la reducción de la superficie de los estabilizadores supone una reducción del peso de la estructura. A pesar de ello, la dependencia con la envergadura es más acusada en el caso de la metodología casi analítica que la basada en regresiones. Esto lleva a que el óptimo del peso no se obtenga en el mismo punto.

Además, los pesos determinados por medio de la metodología de regresiones adaptada a la cola en $\mathrm{V}$ son mayores que los que se obtienen mediante el otro procedimiento. En principio, el método basado en las cargas aerodinámicas debería ser más fiel a la realidad que el de las regresiones, ya que el primero acomete el diseño de la estructura adaptada a las cargas que debe soportar. Sin embargo, no hay plenas garantías de que no haya otro caso de carga que no se haya podido modelizar siguiendo las directrices del módulo aerodinámico utilizado en esta tesis. Incluso, puesto que los casos de carga se han escogido entre los que contempla la normativa para el dimensionado de los estabilizadores de configuración convencional, podría existir otro caso de carga distinto a esos que sea más crítico para una configuración no convencional en concreto. Por todo esto, habría que realizar unos cálculos bastante más detallados para corroborar el peso de la estructura.

Analizando los resultados obtenidos por el método de Farrar, se quiere reflexionar sobre la decisión tomada de diseñar a factor de Farrar óptimo. Según la teoría desarrollada por Farrar, diseñar en este óptimo minimiza el peso de un panel para soportar unas cargas y con unas dimensiones generales del panel dadas. Este criterio de diseño resulta, generalmente en un panel con un número elevado de larguerillos, que puede llevar a un mayor coste de fabricación. Por eso, se puede valorar la posibilidad de tomar un factor de Farrar que sea menor que el óptimo para dimensionar una sección que sea más realista, a pesar de pesar más de acuerdo con la teoría de Farrar. Sin embargo, se quiere resaltar que el propio peso de la estructura afecta a las cargas que debe soportar el cajón de torsión. En esta tesis se ha seguido un procedimiento iterativo para considerar cómo afecta este peso resultante de la estructura a las propias cargas que debe soportar la superficie. Por tanto, un mayor peso de la estructura lleva a aliviar las cargas que debe soportar el cajón y podría resultar en un peso menor, a pesar de no tomar el factor de Farrar óptimo. Este hecho puede ser más relevante en las superficies de cola debido a que el peso del cajón de torsión, que es el que soporta las cargas, supone un mayor porcentaje del peso de la superficie total que en el caso del ala, si se lleva el combustible en los tanques integrales del cajón de torsión como es usual. Es por 
esto que puede verse más afectado en el proceso de convergencia las variaciones del peso del cajón con diferentes factores de Farrar. Simplemente se quiere resaltar esta reflexión, que deberá analizarse con más detenimiento en los futuros trabajos de esta línea de investigación.

A pesar de las diferencias que se encuentran entre los diferentes métodos, la optimización desde el punto de vista del consumo de combustible alcanza resultados muy parecidos. La configuración de cola en $\mathrm{V}$ ahorra alrededor de un $1 \%$ del combustible para la ruta de diseño, por lo menos a nivel de diseño conceptual. Esto está en línea con los resultados obtenidos por otros estudios que indican que el peso de la estructura es mayor pero que el ahorro en términos de combustible es prácticamente neutro (Frota et al. 2010). Por tanto, se puede concluir que el procedimiento de diseño presentado es una buena aproximación al problema de diseño de configuraciones de cola no convencionales en etapas iniciales de diseño conceptual de aviones de transporte comercial. 
RESUMEN Y CONCLUSIONES 


\section{CONCLUSIONES Y TRABAJOS}

FUTUROS 


\subsection{CARACTERIZACIÓN DE CONFIGURACIONES DE COLA CONVENCIONALES}

El estudio de caracterización de las configuraciones de cola convencionales que se ha presentado en esta tesis permite establecer tendencias en los diseños de este tipo de colas. Se pueden utilizar estas regresiones para realizar un dimensionado conceptual de los estabilizadores horizontal y vertical. Es cierto que las regresiones que exhiben un mejor comportamiento son las que se corresponden al estabilizador horizontal. Para el dimensionado del estabilizador vertical sería necesario profundizar en la regresión que se basa en el control de la aeronave tras el fallo del motor crítico en el segundo segmento, puesto que no se ha conseguido unos buenos resultados ni siquiera planteando las regresiones típicas basadas en parámetros adimensionales que han construido otros autores que representan este criterio.

Uno de los resultados más relevantes y diferenciadores que se han visto tras este estudio, es la alta probabilidad de que las estimaciones que se obtienen por medio de las regresiones sean representativas de cualquier avión que no se encuentre en la base de datos. Esto quiere decir que la muestra poblacional de aviones que se han seleccionado establece la tendencia de los diseños de las configuraciones de cola de toda la población de aviones completa con la misma configuración, lo que abala las conclusiones extraídas sobre los resultados.

\subsection{METODOLOGÍA DE DISEÑO DE CONFIGURACIONES DE COLA NO CONVENCIONALES}

Para poder llevar a cabo el dimensionado de las configuraciones no convencionales basándose en los requisitos de diseño inspirados en la normativa de certificación aplicable, son necesarias una serie de herramientas para estimar ciertas propiedades aerodinámicas, como pueden ser las derivadas de estabilidad, y para estimar el peso de las superficies estabilizadoras. Además, el peso depende de las cargas que debe soportar la estructura, por tanto, existe una interacción entre la aerodinámica de la cola y el peso resultante de la misma.

El método de estimación de las características aerodinámicas debe cumplir el principal requisito de rapidez de cálculo, indispensable para las etapas de diseño conceptual en las que se está enmarcado el trabajo. Típicamente, estos métodos son modelos físicomatemáticos que se corrigen mediante factores semi-empíricos para ajustar el modelo a la realidad. El principal inconveniente que tiene esta metodología es que son necesarios una gran variedad de ensayos y datos empíricos que permitan realizar ese ajuste. En el caso de las configuraciones no convencionales no es posible utilizar estos métodos precisamente por esa falta de información empírica que permita usarlos. Por tanto, hay que recurrir a otros métodos que se basen en estimar la aerodinámica a partir de la geometría de la superficie. La opción que se ha escogido en esta tesis es la de un software, Tornado, que estima las propiedades aerodinámicas por medio de métodos VLM. Este método cumple el requisito de rapidez necesario para estas etapas, pero tiene el principal inconveniente que no tiene la suficiente precisión en regímenes de vuelo transónico. Esta limitación supone una gran restricción para la resolución del problema de dimensionado, puesto que los aviones de transporte comercial deben diseñarse para el vuelo en régimen compresible. Sin embargo, las herramientas que proporcionan precisión en este régimen, como pueden ser los softwares basados en CFD, no tienen la suficiente flexibilidad y rapidez como para realizar muchos cálculos de diferentes geometrías en un tiempo reducido. 


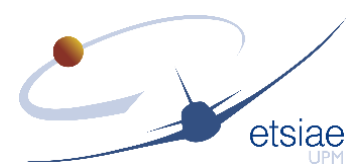

Tornado se utiliza con dos objetivos diferentes: determinar las derivadas de estabilidad en crucero y, por otro lado, estimar las fuerzas y momentos, tanto globales como la distribución de las mismas a lo largo de la envergadura, en diferentes condiciones de vuelo. Con respecto a las derivadas de estabilidad en crucero, la limitación en número de Mach impuesta por este tipo de programas lleva a seleccionar una condición de vuelo de crucero que no alcance el régimen transónico. A pesar de que el valor de la derivada de estabilidad estática en sí mismo no será el que tenga en la condición real de crucero, como el procedimiento presentado en esta tesis se basa en compararse con un avión de referencia, se pude asumir que las imprecisiones cometidas en el cálculo de estas derivadas en el avión de referencia también se comenten en el avión de configuración de cola no convencional. Es por esto que, al comparar los dos aviones, se están absorbiendo parte de los errores asociados a la metodología seleccionada. Diferente sería el caso si el valor de la derivada de estabilidad en crucero obtenida por esta metodología tuviese que ser el del crucero real en régimen compresible, ya que invalidaría por completo el método propuesto.

La normativa vigente impone requisitos no solo en el crucero sino también en otras condiciones que no se producen en régimen transónico. Es el caso del control del avión tras el fallo del motor crítico tras el despegue y en aterrizaje con viento cruzado. Estas condiciones introducen una serie de requisitos al dimensionado de la cola no convencional. Estas condiciones afectan al tanto al tamaño de la cola como al diseño del timón de dirección, ya que debe ser posible el control de la aeronave sin sobrepasar la deflexión máxima del timón. El análisis de estos requisitos lleva a cuestionarse la validez del software Tornado en la estimación de las fuerzas generadas en una superficie cuando el control alcanza deflexiones elevadas, donde los efectos no lineales empiezan a ser considerables. Por este motivo, la propuesta de modificar los resultados del Tornado adecuándose a los resultados que se obtienen por medio de un método semi-empírico clásico para altas deflexiones del control se considera muy adecuada y necesaria. De esta forma, los resultados obtenidos para estas condiciones de vuelo se aproximan más a la realidad y suplen las imprecisiones de los métodos VLM.

Donde se pueden ver claramente las limitaciones de la metodología seleccionada es en la estimación de las cargas generadas sobre la cola. La imposibilidad de analizar las cargas que se producen en régimen transónico o en situaciones en las que la superficie de cola esté próxima a la pérdida, lleva a que las cargas determinadas como críticas tienen muchas probabilidades de no ser las críticas si se hubieran podido analizar estas otras condiciones. De hecho, los resultados de pesos obtenidos por medio de la metodología denominada como casi analítica, tanto para material metálico como para material compuesto, son más optimistas que los que se obtienen por medio de las regresiones estadísticas, tanto en el caso del avión de referencia como en el caso de estudio de configuración no convencional. Este es el principal aspecto por mejorar de la metodología implementada, tratar de establecer un método rápido de estimación de las cargas críticas generadas sobre la cola que pueda abarcar tanto el régimen transónico como subsónico. Puede que este requisito no sea fácil de cumplir, puesto que estos métodos suelen necesitar de tiempos de cálculo elevados. Es cierto que existen algunas técnicas que tratan de reducir estos tiempos sin perjudicar la precisión de los métodos de CFD, como por ejemplo un método de orden reducido basado en descomposición en valores singulares. Estas metodologías no evitan el uso de programas de CFD, pero consiguen que la aplicación al diseño conceptual no suponga un tiempo de cálculo tan elevado. A pesar de esto, el procedimiento propuesto en esta tesis puede servir para reducir el espacio de diseño a unas pocas configuraciones objetivo, de tal forma que sean las seleccionadas las que únicamente se pasen a un programa de CFD más 
preciso que los de VLM utilizados aquí. Es decir, que esta metodología casi analítica para la estimación de pesos debe tomarse como una primera aproximación al problema y debe utilizarse para extraer conclusiones cualitativas, no cuantitativas. Es por eso que tiene vital importancia que se comparen los resultados con los obtenidos para el avión de referencia.

Entrando al análisis específico del procedimiento del dimensionado estructural para la posterior estimación del peso, se ha propuesto una metodología adaptada para las colas que se basa en las que se han desarrollado clásicamente para dimensionar la estructura del ala principal. La mayoría de los elementos estructurales del ala están fabricados en materiales metálicos, principalmente en aleaciones de aluminio. El procedimiento inicial propone una serie de correcciones para considerar que ciertos elementos se fabrican en material compuesto. Precisamente esta es una de las limitaciones que tiene la aplicación de esta metodología a la estructura de la cola, ya que casi todos los elementos estructurales son de material compuesto. Es por ello que se ha esbozado por donde debe evolucionar esta metodología para que esté desarrollada plenamente para este tipo de materiales.

El procedimiento de estimación de pesos presentado no es totalmente analítico, es decir, hay ciertos elementos que se determina su peso mediante regresiones estadísticas, como es el caso de las costillas o los remaches, por ejemplo. Por tanto, estas regresiones deberían de construirse de forma específica para los elementos equivalentes de la cola, para estar seguros de que la regresión es aplicable o, al menos, adaptable. La parte que sí se dimensiona analíticamente corresponde a los paneles del revestimiento y de los largueros que forman el cajón de torsión. Por eso, la adaptación de esta metodología a un panel que se encuentra en la cola es directa, siempre que esté sometido al mismo estado de tensiones. Este estado de tensiones es diferente entre los paneles del revestimiento y los del larguero. El revestimiento está sometido a una combinación de compresión y cortadura y el larguero se encuentra en un estado de tensión diagonal. El planteamiento del dimensionado de un panel de material compuesto sometido a una combinación de compresión y cortadura, a nivel de diseño conceptual, es el que se ha acometido en esta tesis. Habría que desarrollar un procedimiento semejante para el dimensionado de un panel que esté en un estado de tensión diagonal que cumpla con las necesidades del diseño conceptual. La falta de este paso es el que ha impedido implementar la metodología completa de estimación de pesos de la cola plenamente basada en materiales compuestos, sin coeficientes estadísticos de ajuste de los resultados.

Tanto el dimensionado del panel del revestimiento llevado a cabo por el procedimiento para material metálico como el presentado para material compuesto, resultan en un número de rigidizadores bastante elevado, superior al que se puede encontrar en las estructuras reales. Este resultado es consecuencia de las hipótesis de partida, que se impone que el pandeo global y local de la estructura ocurran al mismo tiempo. Si se permite que el tramo de panel situado entre los rigidizadores pandee, se puede alcanzar unas estructuras que contengan un menor número de rigidizadores, aunque no necesariamente un menor peso que con la condición anterior. Este criterio puede ser una buena incorporación para una futura evolución de la metodología presentada.

A pesar de todas las limitaciones y restricciones que imponen las metodologías de estimación de características aerodinámicas y de peso de la estructura que se han seleccionado, suponen de gran ayuda para el diseñador. Este procedimiento permite realizar una primera aproximación al problema de dimensionado de colas no convencionales, con el objetivo de acometer estudios de viabilidad de las 


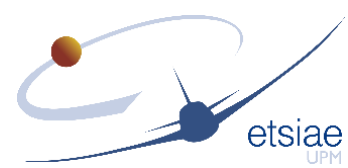

configuraciones, permitiendo descartar y/o seleccionar configuraciones que sean analizadas en mayor profundidad y por medio de herramientas más precisas en etapas más avanzadas de un proyecto de diseño de un avión de transporte comercial.

\subsection{RESULTADOS PARA LA COLA EN V}

El procedimiento de optimización propuesto para la cola en $\mathrm{V}$ ha resultado en que el diseño presenta dos grados de libertad, como son la cuerda en la raíz y el parámetro de estrechamiento. Tanto la envergadura como el ángulo de diedro dependen de los grados de libertad debido a las restricciones activas que restringen el diseño. Estas restricciones son dos y corresponden a imponer un mínimo en las derivadas de estabilidad estática en crucero, tanto la longitudinal como la lateral. Esta imposición nace del resultado de estimar estas derivadas para el avión de referencia. Es de suponer que el avión de referencia cumple con todos los requisitos impuestos por la normativa de certificación vigente. Por tanto, el hecho de que precisamente sean los requisitos en derivadas de estabilidad los que reflejan las restricciones activas es un resultado esperable, ya que esos mismos valores cumplen los requisitos de certificación con un cierto margen, como se ha podido comprobar para el avión de referencia. Al imponer los requisitos basados en ciertos artículos de la normativa se ha visto como no introducen restricciones que reduzcan aún más el espacio de diseño, simplemente sirven para comprobar que cumplen estos requisitos. Es cierto que sí que se pueden utilizar para dimensionar el timón de profundidad, aprovechando el margen que tienen estas restricciones para dejar de ser redundantes.

En cuanto al espacio de diseño resultante, los parámetros de diseño que no son libres, es decir, el diedro y la envergadura toman valores semejantes a los que se pueden encontrar en otras configuraciones de cola en $\mathrm{V}$ en aeronaves pilotadas por control remoto de gran tamaño. En este tipo de aeronaves, los ángulos de diedro se encuentran entre los $25^{\circ}$, como es el caso del Predator $B, y$ los $45^{\circ}$, como puede ser el caso del Global Hawk. Precisamente el espacio de diseño posible establece unos ángulos de diedro que se encuentran en ese intervalo. Además, se puede analizar el cociente entre la envergadura de la cola y las dimensiones del fuselaje, como puede ser la altura del propio fuselaje. Esta magnitud se ha escogido porque afecta a la estabilidad estática lateral de la aeronave, que es la que también se ve afectada al aumentar el diedro de la cola y la envergadura de la misma. Este cociente se sitúa en valores que se encuentran en el intervalo de [5-7,5], siendo el cociente mayor para las aeronaves que presentan un menor diedro. El espacio de diseño posible, que se limita por las restricciones activas, presenta los cocientes entre la envergadura de la cola en $\mathrm{V}$ y la altura del fuselaje en este intervalo aproximadamente. De hecho, la cola del Global Hawk toma el valor de este cociente alrededor de 5,25. El óptimo que se obtiene minimizando la función de peso se corresponde con un diedro que se encuentra muy próximo al de esta aeronave $y$, por tanto, el cociente de la envergadura y el ancho del fuselaje es de 5,47, situándose también muy cercano al de dicha aeronave. Esta comparativa con el estado del arte de configuraciones en $\mathrm{V}$ permite concluir que los resultados aquí obtenidos no son descabellados, a pesar de que son necesarias metodologías más detalladas para poder asegurar los beneficios de esta configuración con mayor exactitud.

Un resultado relevante que se quiere hacer hincapié es que la minimización de la función objetivo, el peso, determinada por medio de las regresiones pensadas para configuraciones convencionales adaptadas a la configuración de cola en $\mathrm{V}$ coincide con minimizar el área de la cola y, por tanto, la resistencia de la misma. Esto supone que minimizar el peso es equivalente a minimizar el consumo de combustible, que es 
realmente el objetivo que se busca con el diseño óptimo de aviones. Si la función objetivo sometida a las restricciones no llevase a este resultado, habría que realizar la optimización sobre el consumo de combustible, en lugar de sobre el peso únicamente. La función de consumo de combustible se construiría de la misma forma que se ha hecho en este estudio para determinar los beneficios en el consumo de la misión de diseño. Esto complicaría ligeramente el problema, ya que el consumo de combustible viene dado por una función implícita, que se debe resolver de forma iterativa. Aunque siempre pueden presentarse formas de simplificar el problema, analizando por separado el impacto sobre la resistencia y el peso y luego integrándolo sobre el consumo de combustible, como se ha hecho para la metodología de pesos casi analítica presentada en la tesis.

Analizando en mayor profundidad la configuración óptima resultante, esta lleva asociada un incremento de peso con respecto de la cola convencional de la que se partió y una reducción de la resistencia con respecto a esta misma. Estos resultados están en la línea de otros estudios de viabilidad centrados en esta configuración realizados previamente por otros autores, como es el caso del proyecto FP5 NEFA, que se describió someramente en la introducción de la tesis. En él se predice que la cola en $\mathrm{V}$ conlleva una reducción del área del empenaje con respecto a la configuración convencional de un $20 \%$ aproximadamente. En la metodología aplicada a la cola en $\mathrm{V}$ que se ha presentado en esta tesis, la reducción de superficie obtenida es del $21 \%$, que es congruente con los resultados que se estimaron en el proyecto NEFA. Los resultados de este proyecto europeo muestran que la configuración de cola en $\mathrm{V}$ supone un incremento de peso del fuselaje posterior, tal y como se ha podido comprobar aquí. Por último, las conclusiones del proyecto NEFA indican que esta configuración resulta en un pequeño beneficio en términos de consumo de combustible, aunque se podría considerar casi neutro. De nuevo, este resultado coincide con el que se ha alcanzado en esta tesis, que el ahorro de combustible se encuentra por debajo del $1 \%$. Esta comparativa de resultados sirve de validación de la metodología presentada para las configuraciones no convencionales, especialmente para la cola en $\mathrm{V}$.

Los métodos presentados en este estudio están condicionados por los requisitos necesarios para las etapas de diseño conceptual iniciales de un proyecto de diseño de avión, por tanto, la precisión de los mismos es menor debido a que pesa más la necesidad de que se utilicen procedimientos rápidos. Con esto lo que se quiere resaltar es que no son muy relevantes los resultados obtenidos en términos de beneficio de consumo de combustible. La reducción del consumo de combustible se encuentra por debajo del $1 \%$, que está muy por debajo de la precisión que se contempla en las metodologías presentadas, que puede estar entre un 5 o un 10 por ciento. En consecuencia, sería necesario dar un paso más en las etapas de diseño, entrando ya en diseño preliminar y detallado, para poder corroborar si este ahorro es real o no. Lo que sí parece más claro es que esta configuración supone un incremento de peso del empenaje y una reducción de la superficie mojada de la cola y de la resistencia sin sustentación, aunque los porcentajes determinados aquí no sean exactamente los que finalmente se obtengan tras un diseño detallado.

Por todo lo que se ha comentado aquí, los resultados del dimensionado de la cola en $\mathrm{V}$ no permiten concluir fehacientemente si su instalación en un avión de transporte comercial supondría un ahorro de combustible y, consecuentemente, una menor emisión de gases contaminantes, con respecto a la configuración convencional. Lo que sí se puede decir es que, a nivel de diseño conceptual, se podrían considerar como equivalentes. 


\subsection{FUTUROS TRABAJOS}

El desarrollo de esta tesis doctoral ha sentado las bases para alcanzar un objetivo más ambicioso como es el de establecer una metodología de diseño conceptual para configuraciones no convencionales, no centradas exclusivamente en los estabilizadores de cola. Para ello, se debe perfeccionar el procedimiento que se ha presentado intentando suplir las carencias que se presentan en alguno de los aspectos de la investigación y que se han resaltado a lo largo del estudio.

Uno de los principales puntos de mejora que puede dar lugar a que la estimación de pesos aumente su precisión es el método de cálculo de las cargas aerodinámicas. Los casos de carga que deberían dimensionar los estabilizadores están identificados y se han expuesto en la tesis, el problema es que muchos de esos casos obligan a que la herramienta de cálculo sea aplicable en condiciones de vuelo a régimen transónico. Habría que realizar un estudio del estado del arte de técnicas de estimación de cargas aerodinámicas sobre superficies sustentadoras, de tal forma que se seleccionaría aquella que cumpla con los requisitos necesarios para las primeras etapas de diseño conceptual y preliminar, donde el principal de ellos es la rapidez de cálculo, aunque sea en perjuicio de la precisión, pero que ofrezcan mejores resultados en las estimaciones respecto a las obtenidas con los métodos VLM que se han utilizado en la tesis. Además, la posible ampliación de los casos de carga permitiría identificar con mayor seguridad los casos críticos y, por lo tanto, serviría para validar con mayor fiabilidad el procedimiento de diseño estructural presentado.

Otro de los aspectos que derivan en un futuro trabajo que mejoraría el procedimiento de diseño presentado en esta tesis es el dimensionado de los largueros directamente en material compuesto, al igual que el caso de los paneles rigidizados sometidos a una combinación de cortadura y compresión. El alma del larguero se encuentra en un estado de tensión diagonal, por tanto, habría que analizar los procedimientos de diseño de paneles que se encuentran en este estado de tensiones que estén publicados por la comunidad científica. Las conclusiones de esta exploración deberían marcar la dirección de cómo avanzar en esta línea, bien porque haya un procedimiento directamente adaptable o bien porque haya que desarrollar uno que sea válido para los propósitos que se buscan, basándose en las hipótesis sobre el diseño de paneles sometidos a un estado de tensión diagonal que sí se pueden encontrar para paneles metálicos. La aplicación de esta metodología permitiría comparar el procedimiento de dimensionado del cajón de torsión basado en Farrar con el que se establezca íntegramente en material compuesto. De esta forma se podría concluir cuánto de buena es la aproximación que se ha tomado en este estudio.

Por último, sería necesario ampliar los casos de estudio de tal forma que se abarquen otras configuraciones de cola. Esta ampliación se debe acometer con dos propósitos principales. El primero de ellos sería extraer conclusiones de diseño sobre esas nuevas configuraciones seleccionadas, por si alguna de ellas resultara en un beneficio en términos de consumo de combustible, principalmente, y se tuviera que contemplar su posibilidad real de ser instalada en un avión de transporte comercial. Por otra parte, el otro objetivo con el que se debe ampliar el conjunto de configuraciones es que la aplicación del procedimiento de diseño puede destapar alguna debilidad en el mismo que no se haya identificado hasta ahora.

Con todas estas líneas de trabajo que se plantean y que han sido identificadas por el autor, la metodología presentada mejoraría en calidad y precisión, siendo un complemento perfecto para los diseñadores que quieran contemplar la viabilidad de 
emprender un proyecto de avión de configuración de cola no convencional. Además, tal y como se ha expuesto anteriormente, esta metodología es la base de una herramienta aún más general que permita estudiar la aplicación de las configuraciones no convencionales a un avión de transporte comercial. El principal valor añadido que tiene el enfoque que se ha presentado es que se toma como referencia un avión comercial existente, de tal forma que la nueva configuración es fácilmente comparable con la referencia, pudiendo analizar potenciales beneficios que pudieran presentar las configuraciones no convencionales a nivel de diseño conceptual. 
ABBAS, A., DIAS, J. y CABELLO, J.A., 2004. REMFI Rear-Fuselage and Empennage Flow Investigation. Final Publishable Activity Report. S.I.: EC 6th Framework Programme.

ACARE (THE ADVISORY COUNCIL FOR AERONAUTICS RESEARCH IN EUROPE), 2010. Aeronautics and Air Transport Beyond Vision 2020 (Towards 2050). . Brussels: European Commission.

AFSAR, R. y SALAM, A., 2013. CEASIOM: An Open Source Multi Module Conceptual Aircraft Design Tool. International Journal of Engineering Rsearch \&Technology (IJERT), vol. 2, no. 7, pp. 1807-1813.

AIRBUS, 2005. Flight Crew Operational Manual A320. 2005. S.I.: Airbus Training.

ANDERSON, J.D., 1999. Aircraft Performance and Design. Boston: WCB McGraw-Hill. ISBN 0-07-001971-1.

ANDERSON, J.D., 2002. The airplane: a history of its technology. Reston, Virigina: AIAA. ISBN 978-1-56347-525-2.

ARGYRIS, J.H., 1947. The Initial Instability Stress of Flat Panels with Z-Section Stringers. Structures Data Sheet 02.01.25. 1947. S.I.: Royal Aeronautical Society.

BAKER, A.A. y SCOTT, M.L., 2016. Composite Materials for Aircraft Structures. Third. Reston, VA: AIAA Education Series. ISBN 978-1-62410-326-1.

BAZARAA, M.W. y SETTY, C.M., 1979. Nonlinear Programming - Theory and Algorithms. New York, NY: Harper \& Row. ISBN 978-0471486008.

BEALE, M. y DEMUTH, H., 1994. The Neural Net Toolbox User's Guide. Natick, MA: MathWorks.

BIL, C., 1988. Development and application of a computer-based system for conceptual aircraft design. 1988. Delft: Delft University Press. ISBN 90-6275-484-8.

BISHOP, A.W. y PAGE, A.N., 1973. An Approach to Design Integration. AGARD CP147, vol. 1, no. Paper No. 5 October.

BOEING, 2017. Current Market Outlook - 2017-2036. 2017. Seattle, WA: Boeing Commercial Airplanes.

BOYD, S. y VANDENBERGHE, L., 2009. Convex Optimization. New York: Cambridge University Press. ISBN 978-0-521-83378-3.

BRAUN, R.D., MOORE, A.A. y KROO, I.M., 1997. Collaborative Approach to Launch Vehicle Design. Journal of Spacecraft and Rockets, vol. 34, no. 4, pp. 478-486. ISSN 0022-4650. DOI 10.2514/2.3237.

BROYDEN, C.G., 1970. The convergence of a class of double rank minimization algorithms: parts I and II. Journal of the Institute of Mathematics and its Application, vol. 6 , pp. $76-90,222-231$.

BUSQUIN, P., ARGÜELLES, P., BISCHOFF, M., DROSTE, B. a. C., EVANS, S.R.H., KRÖLL, W., LAGARDĖRE, J.-L., LINA, A., LUMSDEN, J., RANQUE, D., RASMUSSEN, S., REUTLINGER, P., ROBINS, S.R., TERHO, H. y WITTLÖW, A., 2001. European aeronautics: a vision for 2020 - a synopsis. Air \& Space Europe, vol. 3, no. s 3-4, pp. 16-18. ISSN 12900958. DOI 10.1016/S1290-0958(01)900425 .

CAI, G., FANG, J., ZHENG, Y., TONG, X., CHEN, J. y WANG, J., 2010. Optimization of System Parameters for Liquid Rocket Engines with Gas-Generator Cycles. Journal 
of Propulsion and Power, vol. 26, no. 1, pp. 113-119. ISSN 0748-4658. DOI 10.2514/1.40649.

CARRIER, G. y GEBHARDT, L., 2005. A joint DLR-ONERA contribution to CFD based investigations of unconventional empennages for future civil transport aircraft. . S.I.:

CASTILLO-ACERO, M.Á., CUERNO-REJADO, C. y GÓMEZ-TIERNO, M.Á., 2014. Aerodynamic Modelling for a Morphing Rudder. RAeS Aerodynamics Conference. Advanced Aero Concepts, Design and Operations. Bristol, UK: 22-24 July,

CAVAGNA, L., RICCI, S. y TRAVAGLINI, L., 2011. NeoCASS: An integrated tool for structural sizing, aeroelastic analysis and MDO at conceptual design level. Progress in Aerospace Sciences, vol. 47, no. 8, pp. 621-635. DOI 10.1016/J.PAEROSCI.2011.08.006.

CAZALS, O., 2009a. AIRCRAFT TAIL. Patent. US D594,811 S. United States. US D594,811 S.

CAZALS, O., 2009b. AIRCRAFT TAIL. Patent. US D595,211 S. United States. US D595,211 S.

CAZALS, O., 2009c. AIRCRAFT TAIL. Patent. US D595,206 S. United States. US D595,206 S.

CAZALS, O., 2009d. AIRCRAFT TAIL. Patent. US D594,809 S. United States. US D594,809 S.

CAZALS, O. y DRUOT, T., 2012. AIRCRAFT WITH REAR ANNULAR TAIL. Patent. US 2012/0325958 A1. United States. US 2012/0325958 A1.

CAZALS, O. y SAGNE, J.G. de la, 2010. AIRCRAFT TAIL. Patent. US D621,334 S. United States. US D621,334 S.

CEASIOM - Conceptual Aircraft Design Tool. [en línea], [sin fecha]. [Consulta: 2 septiembre 2018]. Disponible en: https://www.ceasiom.com/wp/.

CERUTI, A., VOLOSHIN, V. y MARZOCCA, P., 2014. Heuristic Algorithms Applied to Multidisciplinary Design Optimization of Unconventional Airship Configuration. Journal of Aircraft, vol. 51, no. 6, pp. 1758-1772. ISSN 0021-8669. DOI 10.2514/1.C032439.

CESNIK, C.E.S., SU, W. y FELLOW, A., 2005. Nonlinear Aeroelastic Modeling and Analysis of Fully Flexible Aircraft. AIAA 2005-2169. Austin, Texas: s.n., pp. 1-27.

CHAN, K.-Y., 2006. Monotonicity, activity and sequential linearizations in probabilistic design optimization. S.I.: University of Michigan.

CILIBERTI, D., NICOLOSI, F. y DELLA VECCHIA, P., 2014. Aerodynamic Interference Issues in Aircraft Directional Control. Journal of Aerospace Engineering, vol. 28, no. 1. DOI 10.1061/(ASCE)AS.1943-5525.0000379.

CINAR, G., EMENETH, M. y MAVRIS, D.N., 2016. A Methodology for Sizing and Analysis of Electric Propulsion Subsystems for Unmanned Aerial Vehicles. 54th AIAA Aerospace Sciences Meeting. San Diego, California: 4-8 January. American Institute of Aeronautics and Astronautics, ISBN 978-1-62410-393-3. DOI 10.2514/6.2016-0216.

CINAR, G., MAVRIS, D.N., EMENETH, M., SCHNEEGANS, A., RIEDIGER, C., FEFERMANN, Y. y ISIKVEREN, A., 2017. Sizing, Integration and Performance Evaluation of Hybrid Electric Propulsion Subsystem Architectures. 55th AIAA Aerospace Sciences Meeting. Grapevine, Texas: 9-13 January. American Institute 
of Aeronautics and Astronautics, ISBN 978-1-62410-447-3. DOI 10.2514/6.20171183.

Code of Federal Regulations: Title 14 - Chapter I - Subchapter C - Part 25 [en línea], 2017. 2017. Federal Aviation Administration. United States of America: s.n. Disponible en: https://www.ecfr.gov/cgi-bin/textidx?SID=9860dcdaeb43952f61ea90922b90edc1\&mc=true\&tpl=/ecfrbrowse/Title1 4/14cfr25_main_02.tpl.

CONE, C.D., 1962. The Theory of Induced Lift and Minimum Induced Drag of Nonplanar Lifting Systems. . S.I.: NASA. Technical Report R-139.

COUFFIGNAL, J.-P. y VINCHES, F., 2012. AIRCRAFT HORIZONTAL STABILIZER. Patent. US 8,196,863 B2. United States. US 8,196,863 B2.

CUERNO-REJADO, C. y SANCHEZ-CARMONA, A., 2016. Preliminary Sizing Correlations for the Rear-End of Transport Aircraft. Aircraft Engineering and Aerospace Technology, vol. 88, no. 1, pp. 24-32. DOI 10.1108/AEAT-04-20140051.

DAR CORPORATION, [sin fecha]. Advanced Aircraft Analysis: AAA. [en línea]. [Consulta: 2 septiembre 2018]. Disponible en: http://www.darcorp.com/Software/AAA/.

DARECKI, M., EDELSTENNE, C., ENDERS, T., FERNANDEZ, E., HARTMAN, P., HERTEMAN, J.-P., KERKLOH, M., KING, I., KY, P., MATHIEU, M., ORSI, G., SCHOTMAN, G., SMITH, C. y WÖRNER, J.-D., 2011. Flightpath 2050 Europe's Vision for Aviation. Report of the High Level Group on Aviation Research Policy [en línea]. 2011. S.I.: s.n. ISBN 9789279197246. Disponible en: http://ec.europa.eu/transport/modes/air/doc/flightpath2050.pdf.

DAVIDON, W.C., 1959. Variable Metric Method for Minimization. US Atomic Energy Commission Research and Development Report no. ANL-5990. Argonne, IL: Argonne National Laboratory. Report no. ANL-5990.

DEGENHARDT, R., KLING, A., ROHWER, K., ORIFICI, A.C. y THOMSON, R.S., 2008. Design and analysis of stiffened composite panels including post-buckling and collapse. Computers \& Structures, vol. 86, no. 9, pp. 919-929. ISSN 00457949. DOI 10.1016/j.compstruc.2007.04.022.

DIEDRICH, A.J. y WILLCOX, K., 2005. The Multidisciplinary Design and Optimization of an Unconventional, Extremely Quiet Transport Aircraft. S.I.: Thesis in Massachusetts Institute of Technology.

DLR - Air Tansportation Systems - CPACS. [en línea], [sin fecha]. [Consulta: 2 septiembre 2018]. Disponible en: https://www.dlr.de/lk/en/desktopdefault.aspx/tabid-4469/7258_read-39713/.

DRAPER, N.R., 1981. Applied Regression Analysis. New York, NY: Wiley.

DUPUIS, R., JOUHAUD, J.-C. y SAGAUT, P., 2018. Surrogate Modeling of Aerodynamic Simulations for Multiple Operating Conditions Using Machine Learning. AIAA Journal, vol. 56, no. 9, pp. 3622-3635. ISSN 0001-1452. DOI 10.2514/1.J056405.

EASA, 2018. Certification Specifications and Acceptance Means of Compilance for Large Aeroplanes. CS-25, no. March, pp. 750. ISSN 0921-0296. DOI 10.1002/9780470664797.

EUROCONTROL, 2018. EUROCONTROL Seven-Year Forecast Network Manager nominated by the European Commission [en línea]. 2018. S.I.: s.n. Disponible en: 
https://www.eurocontrol.int/sites/default/files/content/documents/officialdocuments/forecasts/seven-year-flights-service-units-forecast-2018-2024Feb2018.pdf.

EUROPEAN COMMISSION, D.-G. for C.A., 2017. Proposal for a REGULATION OF THE EUROPEAN PARLIAMENT AND OF THE COUNCIL amending Directive 2003/87/EC to continue current limitations of scope for aviation activities and to prepare to implement a global market-based measure from 2021. 2017. COM/2017/054 final - 2017/017 (COD): s.n.

EUROPEAN COMMISSION, S.-G., 2016. COMMUNICATION FROM THE COMMISSION TO THE EUROPEAN PARLIAMENT AND THE COUNCIL The Road from Paris: assessing the implications of the Paris Agreement and accompanying the proposal for a Council decision on the signing, on behalf of the European Union, of th. 2016. COM/2016/0110 final: s.n.

EUROPEAN PARLIAMENT, C. of the E.U., 2009. Directive 2008/101/EC of the European Parliament and of the Council of 19 November 2008 amending Directive $2003 / 87 / E C$ so as to include aviation activities in the scheme for greenhouse gas emission allowance trading within the Community. Official Journal of the European Union, vol. 52, no. L8, pp. 3-21. DOI ISSN 1725-2555.

FARRAR, D.J., 1949. The design of compression structures for minimum weight. Journal of the Royal Aeronautical Society, vol. November, pp. 1041-1052.

FLETCHER, R., 1970. A new approach to variable metrics algorithms. Computer Journal, vol. 13, pp. 317-322.

FLETCHER, R. y POWEL, M.J.D., 1963. A rapidly convergent descent method for minimization. Computer Journal, vol. 6, pp. 163-168.

FROTA, J., NICHOLLS, K., WHURR, J., MÜLLER, M., GALL, P.-E., LOERKE, J., MACGREGOR, K., SCHMOLLGRUBER, P., RUSSELL, J., HEPPERLE, M., RODRIGUEZ, S., TAUPIN, K., GODARD, J.-L., DRON, S., PLÖTNER, K. y GALLANT, G., 2010. Final Activity Report. New Aircraft Concept Research (NACRE). . S.I.: SIXTH FRAMEWORK PROGRAMME PRIORITY 4 Aeronautics and Space FP6-2003-AERO-1.

GALL, P.E., 2010a. AIRCRAFT TAIL. Patent. US D622,206 S. United States. US D622,206 S.

GALL, P.E., 2010b. AIRCRAFT TAIL. Patent. US D622,205 S. United States. US D622,205 S.

GANESH, R. V, 2018. Development and application of WASPE for conceptual design of HEDiP aircraft. , no. July, pp. 24-26.

GARCIA-BENITEZ, J., CUERNO-REJADO, C. y GOMEZ-BLANCO, R., 2016. Conceptual design of a nonplanar wing airliner. Aircraft Engineering and Aerospace Technology, vol. 88, no. 4, pp. 561-571. ISSN 0002-2667. DOI 10.1108/AEAT-112014-0204.

GARCÍA-HERNÁNDEZ, L., CUERNO-REJADO, C. y PÉREZ-CORTÉS, M., 2017. Dynamics and Failure Models for a V-Tail Remotely Piloted Aircraft System. Journal of Guidance, Control, and Dynamics, vol. 41, no. 2, pp. 506-514. ISSN 0731-5090. DOI 10.2514/1.G003069.

GENGISKANHG, 2004. Archivo:RedNeuronalArtificial.png - Wikipedia. [en línea]. [Consulta: 24 septiembre 2018]. Disponible en: https://es.m.wikipedia.org/wiki/Archivo:RedNeuronalArtificial.png. 


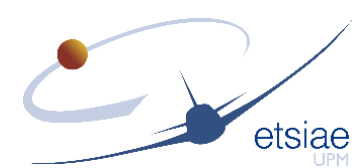

GILBERT, R.D., 1995. AIRCRAFT TAIL SURFACE SPOILERS. Patent. 5,445,346. United States. 5,445,346.

GILL, P.E. y MURRAY, W., 1972. Quasi-Newton methods for unconstrained optimization. Journal of the Institute of Mathematics ans its Applications, vol. 9, pp. 91-108.

GILL, P.E. y MURRAY, W., 1978. Algorithms for the solution of the nonlinear leastsquares problem. SIAM Journal on Numerical Analysis, vol. 15, no. 5, pp. 977-992.

GIPSON, L., 2015. Outside the Box, Sort of. NASA/Lockheed Martin [en línea]. [Consulta: 13 septiembre 2018]. Disponible en: https://www.nasa.gov/content/outside-the-box-sort-of.

GOLDFARB, D., 1970. A family of variable metric methods derived by variational means. Mathematics of Computation, vol. 24, pp. 23-26.

GOODFELLOW, I., BENGIO, Y. y COURVILLE, A., 2016. Deep Learning. Cambridge, MA: MIT Press. ISBN 978-0262035613.

HAGAN, M.T., DEMUTH, H.B. y BEALE, M.H., 1996. Neural Network Design. Boston, MA: PWS Publishing Company.

HANSEN, P., JAUMARD, B. y LU, S.H., 1989. Some further results on monotonicity in globally optimal design. ASME Journal of Mechanisms, Transmissions and Automation in Design, vol. 111, no. 3, pp. 345-352.

HARE, W., NUTINI, J. y TESFAMARIAM, S., 2013. A survey of non-gradient optimization methods in structural engineering. Advances in Engineering Software, vol. 59, pp. 19-28. ISSN 0965-9978. DOI 10.1016/J.ADVENGSOFT.2013.03.001.

HEPPERLE, M., 2008. MDO of Forward Swept Wings. KATnet II Workshop. Braunschweig, Germany: 28-29 January,

HOLT, A., 1982. On Making Things the Best-Aeronautical Uses of Optimization. Journal of Aircraft, vol. 19, no. 1, pp. 5-28. ISSN 0021-8669. DOI 10.2514/3.57350.

IBRAHIM, K., 2004. Selecting Principal Parameters of Baseline Design Configuration for Twin Turboprop Transport Aircraft. 22nd Applied Aerodynamics Conference and Exhibit. Providence, Rhode Island: 16-19 August,

IBRAHIM, K. y MOHNOT, A., 2006. Selecting Principal Parameters of Baseline Design Configuration for Light General Aviation Aircraft. 24th Applied Aerodynamics Conference. San Francisco, California: 5-8 June, pp. 1-11.

ICAO, 2016. Historic agreement reached to mitigate international aviation emissions. ICAO News Release [en línea]. Montreal, 2016. Disponible en: http://www.icao.int/Newsroom/Pages/Historic-agreement-reached-to-mitigateinternational-aviation-emissions.aspx.

ILR y RWTH-AECHEN-UNIVERSITY, 2014. Central Reference Aircraft data System CeRAS. [en línea]. [Consulta: 3 agosto 2017]. Disponible en: https://ceras.ilr.rwthaachen.de/.

JAYARAM, S., MYKLEBUST, A. y GELHAUSEN, P., 1992. ACSYNT - A standardsbased system for parametric, computer aided conceptual design of aircraft. AIAA, Aerospace Design Conference. Irvine, CA: 3-6 October, pp. 14.

JENKINSON, L., SIMPKIN, P. y RHODES, D., 1999. Civil Jet Aircraft Design. Elsiever L. Reston, VA: Elsiever Ltd. ISBN 034074152X. 
JONES, P.K., WEIR, J., BONENFANT, D., BOYD, E.A., BURGIN, K., CARTER, E.C. y DOVEY, J.R.J., 1993. Contribution of fin to sideforce, yawing moment and rolling moment derivatives due to sideslip, $(\mathrm{Yv}) \mathrm{F},(\mathrm{Nv}) \mathrm{F},(\mathrm{Lv}) \mathrm{F}$, in the presence of body, wing and tailplane. . S.I.: Royal Aeronautical Society. ESDU 82010.

JULES, K. y LIN, P.P., 2002. Artificial neural networks applications: From aircraft design optimization to orbiting spacecraft on-board environment monitoring. NASA/TM2002-211811. Hanover, MD: Glenn Research Center.

KALAVALAPALLY, R., PENMETSA, R. y GRANDHI, R., 2006. Multidisciplinary optimization of a lightweight torpedo structure subjected to an underwater explosion. Finite Elements in Analysis and Design, vol. 43, no. 2, pp. 103-111. ISSN 0168-874X. DOI 10.1016/J.FINEL.2006.07.005.

KALLINDERIS, Y., VOUVAKOS, X. y MENOUNOU, P., 2009. Linear correlations of principal parameters for the preliminary design of twin civil jet aircraft. Aircraft Engineering and Aerospace Technology, vol. 81, no. 6, pp. 508-515. ISSN 00022667. DOI 10.1108/00022660910997801.

KASSAPOGLOU, C., 1997. Simultaneous cost and weight minimization of compositestiffened panel under compression and shear. Composites Part A: Applied Science and Manufacturing, vol. 28, no. 5, pp. 419-435. DOI 10.1016/S1359835X(96)00141-8.

KASSAPOGLOU, C., 2013. Design and analysis of composite structures: with applications to aerospace structures. Chichester, UK: Wiley. Aerospace Series. ISBN 978-1118401606.

KAUFMANN, M., 2008. Cost Optimization of Aircraft Structures. S.I.: Thesis. KTH School of Engineering Sciences.

KOKKOLARAS, M., LOUCA, L., DELAGRAMMATIKAS, G., MICHELENA, N., FILIPI, Z., PAPALAMBROS, P., STEIN, J. y ASSANIS, D., 2004. Simulation-based optimal design of heavy trucks by model-based decomposition: an extensive analytical target cascading case study. International Journal of Heavy Vehicle Systems, vol. 11, no. 3/4, pp. 403. ISSN 1744-232X. DOI 10.1504/IJHVS.2004.005456.

KROO, I., 2005. Nonplanar Wing Concepts for Increased Aircraft Efficiency. VKI lecture series of Innovative Configurations and Advanced Concepts for Future Civil Aircraft. Sint-Genesius-Rode, Belgium: 6-10 June, pp. 1-29.

KUHN, P., 1956. Stresses in aircraft and shell structures. New York: Mac Graw-Hill.

KUHN, P., PETERSON, J.P. y LEVIN, L.R., 1952. A Summary of Diagonal Tension. Part I: Methods of Analysis. 1952. S.I.: NACA Technical Note 2661.

KUMAR, R. y GHOSH, A.K., 2014. Estimation of aerodynamic derivatives using neural network based method. Third International Conference on Advances in Control and Optimizatin of Dynamical Systems. Kanapur, India: March 13-15, pp. 897-904. DOI 10.3182/20140313-3-IN-3024.00057.

LAI, W.H., KEK, S.L. y TAY, K.G., 2017. Solving Nonlinear Least Squares Problem Using Gauss-Newton Method. IJISET - International Journal of Innovative Science, Engineering \& Technology, vol. 4, no. 1, pp. 258-262.

LAW, B., 2006. WING UNIT, IN PARTICULAR SPAR BOX, FOR FORMING AERODYNAMICALLY ACTIVE SURFACES OF AN AIRCRAFT, IN PARTICULAR AIRFOILS, HORIZONTAL TAIL UNITS OR RUDDER UNITS OF A PLANE. Patent. US 2006/0226291 A1. United States. US 2006/0226291 A1. 


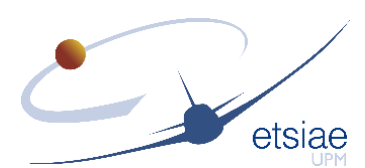

LAWSON, C.L. y HANSON, R.J., 1995. Solving Least Squares Problems. Philadelphia, PA: SIAM.

LEE, D.S., PITARI, G., GREWE, V., GIERENS, K., PENNER, J.E., PETZOLD, A., PRATHER, M.J., SCHUMANN, U., BAIS, A. y BERNTSEN, T., 2010. Transport impacts on atmosphere and climate: Aviation. Atmospheric Environment, vol. 44, no. 37, pp. 4678-4734. ISSN 13522310. DOI 10.1016/j.atmosenv.2009.06.005.

LEVENBERG, K., 1944. A method for the solution of certain nonlinear problems in least squares. Quarterly Journal of Applied Mathematics, vol. 2, pp. 164-168.

LIEM, R.P., MADER, C.A. y MARTINS, J.R.R.A., 2015. Surrogate models and mixtures of experts in aerodynamic performance prediction for aircraft mission analysis. Aerospace Science and Technology, vol. 43. ISSN 12709638. DOI 10.1016/j.procs.2010.04.019.

LIU, W., BUTLER, R. y KIM, H.A., 2008. Optimization of composite stiffened panels subject to compression and lateral pressure using a bi-level approach. Structural and Multidisciplinary Optimization, vol. 36, no. 3, pp. 235-245. ISSN 1615147X. DOI 10.1007/s00158-007-0156-9.

LOMAX, T.L., 1996. Structural Loads Analysis for Commercial Transport Aircraft: Theory and Practice. Reston, Virigina: AIAA Education Series.

LOUGHLAN, J., 1994. The buckling performance of composite stiffened panel structures subjected to combined in-plane compression and shear loading. Composite Structures, vol. 29, no. 2, pp. 197-212. ISSN 02638223. DOI 10.1016/02638223(94)90100-7.

LUCAS, S. De, VELAZQUEZ, A. y VEGA, J.M., 2011. An Optimization Method for an Aircraft Rear-end Conceptual Design Based on Surrogate Models. Proceedings of the World Congress of Engineering. London, United Kingdom: 6-8 July, pp. 26102615. ISBN 9789881925152.

LUCIANO, D., ANTONIO, D. y GIOVANNI, M., 2014. An Invariant Formulation for the Minimum Induced Drag Conditions of Non-planar Wing Systems. 52nd Aerospace Sciences Meeting. National Harbor, Maryland: 13-17 January. AIAA SciTech,

LYU, Z. y MARTINS, J.R.R. a., 2014. Aerodynamic Design Optimization Studies of a Blended-Wing-Body Aircraft. Journal of Aircraft, vol. 51, no. 5, pp. 1604-1617. ISSN 0021-8669. DOI 10.2514/1.C032491.

MACINTOSH, A. y WALLACE, L., 2009. International aviation emissions to 2025: Can emissions be stabilised without restricting demand? Energy Policy, vol. 37, no. 1, pp. 264-273. ISSN 03014215. DOI 10.1016/j.enpol.2008.08.029.

MARLETT, K., NG, Y., TOMBLIN, J. y HOOPER, E., 2011. Hexcel 8552 AS4 Unidirectional Prepreg at $190 \mathrm{gsm} \& 35 \%$ RC Qualification Material Property Data Report. NCAMP Test Report Number: CAM-RP-2010-002 Rev A. 2011. Fairmount, Wichita, KS: s.n.

MARQUARDT, D.W., 1963. An algorithm for least-squares estimation of nonlinear parameters. Journal of the Society for Industrial and Applied Mathematics, vol. 11, no. 2, pp. 431-441.

MARTÍNEZ-ESTUDILLO, A.C., 2005. Modelos de Regresión Basados en Redes Neuronales de Unidades Producto Diseñadas y Entrenadas Mediante Algoritmos de Optimización Híbrida . S.I.: Thesis. Universidad de Granada.

MARTÍNEZ-VAL, R., CUERNO-REJADO, C., PÉREZ, E. y MUÑOZ, T., 1994. Design 
constraints in the payload-range diagram of ultrahigh capacity transport airplanes. Journal of Aircraft, vol. 31, no. 6, pp. 1268-1272. DOI 10.2514/3.46646.

MARTINEZ-VAL, R., PALACIN, J.F. y PEREZ, E., 2008. The evolution of jet airliners explained through the range equation. Proceedings of the Institution of Mechanical Engineers, Part G: Journal of Aerospace Engineering, vol. 222, no. 6, pp. 915-919. ISSN 0954-4100. DOI 10.1243/09544100JAERO338.

MARTINEZ-VAL, R. y PEREZ, E., 2009. Aeronautics and astronautics: recent progress and future trends. Proceedings of the Institution of Mechanical Engineers, Part C: Journal of Mechanical Engineering Science, vol. 223, no. 12, pp. 2767-2820. ISSN 0954-4062. DOI 10.1243/09544062JMES1546.

MARTíNEZ-VAL, R., PÉREZ, E., MUNOZ, T. y CUERNO, C., 1994. Design constraints in the payload-range diagram of ultrahigh capacity transport airplanes. Journal of Aircraft, vol. 31, no. 6, pp. 1268-1281.

MARTINS, J.R.R.A., KENWAY, G.K.W. y BROOKS, T.R., 2016. Multidisciplinary Design Optimization of Aircraft Configurations Part 1: A modular coupled adjoint approach. . Sint-Genesius-Rode, Belgium: Lecture series, Von Karman Institute for Fluid Dynamics, DOI 10.13140/RG.2.2.10513.22886.

MARTINS, J.R.R.A. y LAMBE, A.B., 2013. Multidisciplinary Design Optimization: A Survey of Architectures. AIAA Journal, vol. 51, no. 9. ISSN 0001-1452. DOI 10.2514/1.J051895.

MASON, R.L., GUNST, R.F. y HESS, J.L., 2003. Statistical Design and Analysis of Experiments. Second. Hoboken, New Jersey: Wiley-Interscience. ISBN 0-47137216-1.

MATLAB, 1997. Matlab: The Neural Net Toolbox [en línea]. Natick, MA.: s.n. Disponible en: http://www.mathworks.com/products/matlab.

MCALLISTER, C.D. y SIMPSON, T.W., 2003. Multidisciplinary Robust Design Optimization of an Internal Combustion Engine. Journal of Mechanical Design, vol. 125, no. 1, pp. 124. ISSN 10500472. DOI 10.1115/1.1543978.

MCCULLERS, L.A., 1984. Aicraft Configuration Optimization including Optimized Flight Profiles. NASA Symposium on Recent Experiences in Multidisciplinary Analysis and Optimization. Hampton, Virginia: 24-26 April, pp. 395-412.

MCLEAN, D., 2005. Wingtip Devices : What They Do and How They Do It. Performance and Flight Operations ENgineering Conference. S.I.: s.n., pp. 1-20.

MEISTER, J. (Buxtehude), PFENNIG, J. (Marfeld) y HELD, W. (Skye), 1999. RUDDER ASSEMBLY WITH A CONTROLLED BOUNDARY LAYER CONTROL FOR AN AIRCRAFT. Patent. 5,899,419. United States. 5,899,419.

MELIN, T., 2000. A vortex lattice MATLAB implementation for linear aerodynamic wing applications. S.I.: Master Thesis in Royal Institute of Technology (KTH).

MIETTINEN, K., 1999. Nonlinear multiobjective optimization. New York, NY: Kluwer Academic Publishers. ISBN 0792382781.

MIRAT, J.-J., 2006. NEFA -New Empennage For Aircraft, An FP5 project to enhance the knowledge on V-tail configuration. 2006. Vienna: Airbus.

MØLLER, M.F., 1993. A scaled conjugate gradient algorithm for fast supervised learning. Neural Networks, vol. 6, no. 4, pp. 525-533. ISSN 08936080. DOI 10.1016/S08936080(05)80056-5. 
MOORE, K.T., NAYLOR, B. a y GRAY, J.S., 2008. The Development of an Open Source Framework for Multidisciplinary Analysis \& Optimization. 12th AIAA/ISSMO Multidisciplinary Analysis and Optimization Conference. Victoria, British Columbia Canada: 10-12 September, pp. 1-13. ISBN 9781563479472. DOI 10.2514/6.20086069.

MUSA, N.A., MANSOR, S., ALI, A., HASRIZAM, M., MAN, C., ZAIDI, W. y OMAR, W., 2015. Effect of Tail Dihedral Angle on Lateral Directional Stability due to Sideslip Angles. 53rd AIAA Aerospace Sciences Meeting. Kissimmee, Florida, United Sates of America: s.n., ISBN 9781624103438. DOI 10.2514/6.2015-1498.

MUSA, N.A., MANSOR, S., ALI, A., WAN OMAR, W.Z., ABDUL LATIF, A. y PERUMAL, K., 2014. Effects of Aircraft Tail Configurations on Sensitivity to Yaw Disturbances. Applied Mechanics and Materials, vol. 629, no. February, pp. 197-201. ISSN 16627482. DOI 10.4028/www.scientific.net/AMM.629.197.

NASA, 2011. Lockheed box wing aircraft concept. [en línea]. [Consulta: 19 septiembre 2018]. Disponible en: https://commons.wikimedia.org/wiki/File:Lockheed_box_wing_aircraft_concept_20 11_2.jpg.

NICOLAI, L.M., 1975. Fundamentals of Aircraft Design. Dayton, Ohio: METS Inc.

NICOLAI, L.M. y CARICHNER, G.E., 2010. Fundamentals of aircraft and airship design. Volume I: Aircraft Design. AIAA Educa. Blacksburg, Virginia: American Institute of Aeronautics and Astronautics, Inc.

NICOLOSI, F. y PADUANO, G., 2011. Development of a Software for Aircraft Preliminary Design and Analysis. CEAS 3rd Air and Space Conference - XXI AIDAA Congress. Venice, Italy: 17-21 October, pp. 702-714.

NIELSEN, M.A., 2015. Neural Networks and Deep Learning [en línea]. S.I.: Determination Press. [Consulta: 3 septiembre 2018]. Disponible en: http://neuralnetworksanddeeplearning.com/.

NIU, M.C., 1988. Airframe Structural Design. Second. Hong Kong: Conmilit Press Ltd. ISBN 962-7128-04-X.

NIU, M.C.Y., 1992. Composite Airframe Structures. Third. Hong Kong: Conmilit Press Ltd. ISBN 9627128066.

NORGAARD, M., JORGENSEN, C.C. y ROSS, J.C., 1997. Neural network prediction of new aircraft design coefficients. NASA Technical Memorandum 112197. Moffett Field, California: Ames Research Center.

Pacelab SysArc - TXT. [en línea], [sin fecha]. [Consulta: 2 septiembre 2018]. Disponible en: https://www.txtgroup.com/markets/solutions/pacelab-sysarc/.

PAPAGEORGIOU, A., TARKIAN, M., AMADORI, K. y ÖLVANDER, J., 2018. Multidisciplinary Optimization of Unmanned Aircraft Considering Radar Signature, Sensors, and Trajectory Constraints. Journal of Aircraft, vol. 55, no. 4, pp. 1-12. ISSN 0021-8669. DOI 10.2514/1.C034314.

PAPALAMBROS, P., 1979. Monotonicity analysis in engineering design optimization. S.I.: Stanford University.

PAPALAMBROS, P.Y. y WILDE, D.J., 2017. Principles of optimal design : modeling and computation. Third. Cambridge: Cambridge University Press. ISBN 9781107132672. 
PARK, H.U., LEE, J.W., CHUNG, J. y BEHDINAN, K., 2015. Uncertainty-based MDO for aircraft conceptual design. Aircraft Engineering and Aerospace Technology, vol. 87, no. 4, pp. 345-356. ISSN 00022667. DOI 10.1108/AEAT-07-2013-0128.

PERI, D. y CAMPANA, E.F., 2003. Multidisciplinary Design Optimization of a Naval Surface Combatant. Journal of Ship Research, vol. 47, no. 1, pp. 1-12.

PERKINS, C.D. y HAGE, R.E., 1949. Airplane Performance Stability and Control. S.I.: John Wiley \& Sons, Ltd. ISBN 047168046 X.

PHILLIPS, W.F., HANSEN, a. B. y NELSON, W.M., 2006. Effects of Tail Dihedral on Static Stability. Journal of Aircraft, vol. 43, no. 6, pp. 1829-1837. ISSN 0021-8669. DOI 10.2514/1.20683.

POLHAMUS, E.C., 1971. Charts for predicting the subsonic vortex-lift characteristics of arrow, delta, and diamond wings. . S.I.: NASA TN D-6243.

PRATT, K.G., 1953. A Revised Formula for Caluclation of Gust Loads. . S.I.: NACA TN 2964.

PRATT, K.G. y WALKER, W.G., 1953. Revised Gust Load Formula and a Re-Evaluation of V-G Data Taken on Civil Transport Airplanes from 1933 to 1950. . S.I.: NACA Rept. 1206.

PURSER, P.E. y CAMPBELL, J.P., 1945. Experimental verification of a simplified veetail theory and analysis of available data on complete models with vee-tails. . S.I.: NACA Report No.823.

RAMÍREZ BLANCO, G., BARROSO VLOEDGRAVEN, D., LOZANO GARCÍA, J.L. y LUQUE BUZO, M., 2012. LIFTING STRUCTURE FOR AIRCRAFT. Patent. US 8,091,829 B2. United States. US 8,091,829 B2.

RAYMER, D.P., 1989. Aircraft Design: A Conceptual Approach. First. Washington, D.C.: American Institute of Aeronautics and Astronautics. ISBN 1563472813.

RAYMER, D.P., 2002. Enhancing Aircraft Conceptual Design Using Multidisciplinary Optimization. S.I.: Doctoral Thesis. Royal Institute of Technology.

RAYMER, D.P., 2012. Aircraft Design: A Conceptual Approach. Sylmar, Calofornia: AIAA Education Series. ISBN 1600869114.

RAYMER, D.P., 2016. RDSwin : Seamlessly-Integrated Aircraft Conceptual Design for Students \& Professionals. 54th AIAA Aerospace Sciences Meeting [en línea]. San Diego, California: 4-8 January, pp. 1-17. DOI doi:10.2514/6.2016-1277. Disponible en: http://dx.doi.org/10.2514/6.2016-1277.

RICHARDSON, T.S., MCFARLANE, C., ISIKVEREN, A., BADCOCK, K. y DA RONCH, A., 2011. Analysis of Conventional and Asymmetric Aircraft Configurations Using CEASIOM. Progress in Aerospace Sciences, vol. 47, no. 8, pp. 647-659. ISSN 0376-0421. DOI 10.1016/j.paerosci.2011.08.008.

Ring Tail debuts. General Aviation News [en línea], 2011. Disponible en: https://generalaviationnews.com/2011/05/03/ring-tail-debuts/.

RISSE, K., 2014. Design of CeRAS CSR-01 Technical Report. . S.I.: ILR RWTH Aachen University.

RISSE, K., ANTON, E., LAMMERING, T., SCHÄFER, K. y HÖRNSCHEMEYER, R., 2012. An Integrated Environment for Preliminary Aircraft Design and Optimization. 8th AIAA Multidisciplanry Design Optimization Specialist COnference, AIAA. Honolulu, HI: April, ISBN 978-1-60086-937-2. 
ROSKAM, J., 1986. Rapid Sizing method for airplanes. Journal of Aircraft, vol. 23, no. 7 , pp. 554-560. DOI 10.2514/3.45343.

ROSKAM, J., 1987. Airplane Design, Part VI: Preliminary Calculation of Aerodynamic, Thrust and Power Characteristics. Ottawa, Kansas: Roskam Aviation and Engineering Corporation.

ROSKAM, J., 1989. Airplane Design, Part II: Preliminary Configuration Design and Integration of the Propulsion System. First. Ottawa, Kansas: Roskam Aviation and Engineering Corporation. ISBN 1122620632.

ROSKAM, J., 1999. Airplane Deisgn. Part V: Component Weight Estimation. Ottawa, Kansas: DAR Corporation. ISBN 1884885500.

SADRAEY, M.H., 2013. Aircraft Design. A Systems Engineering Approach. First edit. Chichester, West Sussex, UK: John Wiley \& Sons, Ltd. ISBN 978-1-119-95340-1.

SANCHEZ-CARMONA, A., 2014. ESTUDIO DE DEPENDENCIA DE PARÁMETROS PARA EL DISEÑO CONCEPTUAL DEL FUSELAJE POSTERIOR Y SUPERFICIES ESTABILIZADORAS DE AVIONES DE TRANSPORTE COMERCIAL. S.I.: Trabajo Fin de Máster. Universidad Politécnica de Madrid.

SANCHEZ-CARMONA, A. y CUERNO-REJADO, C., 2018a. Composite stiffened panel sizing for conceptual tail design. Aircraft Engineering and Aerospace Technology,

SANCHEZ-CARMONA, A. y CUERNO-REJADO, C., 2018b. Vee-tail conceptual design criteria for commercial transport aeroplanes. Chinese Journal of Aeronautics [en línea], ISSN 10009361. DOI 10.1016/j.cja.2018.06.012. Disponible en: https://doi.org/10.1016/j.cja.2018.06.012.

SASENA, M.J., PAPALAMBROS, P.Y. y GOOVAERTS, P., 2001. The use of surrogate modeling algorithms to exploit disparities in function computation time within simulation-based optimization. 4th Congress on Structural and Multidisciplinary Optmimization. Dalian, China: June 4-8,

SCHULZ, E. (Airbus), 2018. Global Networks, Global Citizens. Global Market Forecast 2018-2037. 2018. S.I.: Airbus.

SECCO, N.R. y MATTOS, B.S., 2015. Artificial Neural Networks Applied to Airplane Design. 53rd AIAA Aerospace Sciences Meeting. Kissimmee, Florida: 5-9 January, ISBN 978-1-62410-343-8. DOI 10.2514/6.2015-1013.

SEECKT, K. y SCHOLZ, D., 2010. Application of the Aircraft Preliminary Sizing Tool Presto To Kerosene and Liquid Hydrogen Fueled Regional Freighter Aircraft. DGLR: Deutscher Luft- und Raumfahrtkongress. Hamburg: 31 August - 02 September, pp. 137-148.

SEELE, R., GRAFF, E., GHARIB, M., TAUBERT, L., LIN, J. y WYGNANSKI, I., 2012. Improving Rudder Effectiveness with Sweeping Jet Actuators. 6th AIAA Flow Control Conference. S.I.: s.n., pp. 1-12.

SEUBERT, R., 1997. The Preliminary Aircraft Design and Optimization Program for Supersonic Commercial Transport Aircraft PrADO-Sup. En: H. KÖRNER y R. HILBIG (eds.), New Results in Numerical and Experimental Fluid Mechanics. Notes on Numerical Fluid Mechanics (NNFM) [en línea]. S.I.: Vieweg+Teubner Verlag, Wiesbaden, pp. 311-318. Disponible en: http://link.springer.com/10.1007/978-3322-86573-1 40.

SHANNO, D.F., 1970a. Conditioning of quasi-Newton methods for function minimization. Mathematics of Computation, vol. 24, pp. 647-656. 
SHANNO, D.F., 1970b. Conditioning of quasi-Newton mtethods for function minimization. Mathematics of Computation, vol. 24, pp. 647-656.

SHARMA, R., 2015. Basic Aerodynamic Study of Joined Wing. S.I.: Amity School of Engineering and Technology. Amity University Haryana.

SOBIESZCZANSKI-SOBIESKI, J., 1995. Multidisciplinary Design Optimization: An Emerging New Engineering Discipline. Advances in Structural Optimization. the Netherlands: Kluwer Academic Publishers, pp. 483-496.

SOBIESZCZANSKI-SOBIESKI, J. y HAFTKA, R.T., 1997. Multidisciplinary aerospace design optimization: survey of recent developments. Structural Optimization, vol. 14, no. 1, pp. 1-23.

SONG, L., YANG, H., ZHANG, Y., ZHANG, H. y HUANG, J., 2014. Dihedral influence on lateral-directional dynamic stability on large aspect ratio tailless flying wing aircraft. Chinese Journal of Aeronautics, vol. 27, no. 5, pp. 1149-1155. ISSN 10009361. DOI 10.1016/j.cja.2014.08.003.

THOMAS, C., 2009. Blended Wing Body Aircraft. The Boeing Company \& NASA [en línea]. [Consulta: 24 septiembre 2018]. Disponible en: https://www.nasa.gov/centers/dryden/news/X-

Press/50th_anniversary/on_the_horizon/bwb.html.

TORENBEEK, E., 1982. Synthesis Of Subsonic Airplane Design. Dordrecht / Boston / London: Delft University Press. Kluwer Academic Publishers.

TORENBEEK, E., 2007. Blended wing body and all-wing airliners. European Workshop on Aircraft Design Education (EWADE). Samara, Russia: May-June,

TORENBEEK, E., 2013. Advanced Aircraft Design. Chichester, West Sussex, UK: John Wiley \& Sons, Ltd. ISBN 9781119969303.

TRAPANI, M. y GUO, S., 2008. Development of a Rudderless Aeroelastic Fin Technology. 26th INTERNATIONAL CONGRESS OF THE AERONAUTICAL SCIENCES (ICAS). S.I.: s.n., pp. 1-9.

TSAI, S.W. y WU, E.M., 1971. A General Theory of Strength for Anisotropic Materials. Journal of Composite Materials, vol. 5, no. 1, pp. 58-80. ISSN 0021-9983. DOI 10.1177/002199837100500106.

VISWANATH, P., 1996. Flow management techniques for base and after body drag reduction. Progress in Aerospace Sciences, vol. 32, no. 2-3, pp. 79-129.

VOUVAKOS, X., KALLINDERIS, Y. y MENOUNOU, P., 2010. Preliminary design correlations for twin civil turboprops and comparison with jet aircraft. Aircraft Engineering and Aerospace Technology, vol. 82, no. 2, pp. 126-133. ISSN 00022667. DOI 10.1108/00022661011053427.

WILDE, D.J., 1975. Monotonicity and dominance in optimal hydraulic cylinder design. Transactions of the ASME Journal og Engineering for Industry, vol. 94, no. 4, pp. 1390-1394.

WILDE, D.J., 1978. Globally Optimal Design. New York, NY: Wiley-Interscience.

WRIGHT, W. y WRIGHT, O., 2001. The Papers of Wilbur and Orville Wright. New York, NY: McGraw-Hill Co.

XFOIL 6.99. Subsonic Airfoil Development System. 2013 [en línea], 2013. [Consulta: 14 diciembre 2017]. Disponible en: http://web.mit.edu/drela/Public/web/xfoil/. 
YATES, A.H., 1952. Notes on the Mean Aerodynamic Chord and the Mean Aerodynamic Centre of a Wing. Journal of Royal Aeronautical Society, vol. June, pp. 461-475.

ZHANG, G., YANG, S., XU, Y. y SONG, Q., 2008. Numerical Simulation of the Aerodynamic Characteristics of Vee-tail Based on Cluster System. ITESS. S.I.: s.n., pp. 823-827.

ZHANG, G.Q., YU, S.C.M., CHIEN, A. y XU, Y., 2013. Investigation of the Tail Dihedral Effects on the Aerodynamic Characteristics for the Low Speed Aircraft. Advances in Mechanical Engineering, vol. 2013, pp. 1-12. ISSN 1687-8132. DOI $10.1155 / 2013 / 308582$.

ZUNIGA SAGREDO, J., 2008. RIB STRUCTURE FOR TORSION BOXES OF A WING OR HORIZONTAL STABILISER OF AN AIRCRAFT. US 2008/0283675 A1. United States. US 2008/0283675 A1. 
REFERENCIAS 